\title{
Hydrologic Data for Long Valley Caldera, Mono County, California, 1987-93
}

\author{
By James F. Howle and Christopher D. Farrar
}

U.S. Geological Survey

Open-File Report 96-382 


\title{
U.S. DEPARTMENT OF THE INTERIOR BRUCE BABBITT, Secretary
}

\author{
U.S. GEOLOGICAL SURVEY \\ Gordon P. Eaton, Director
}

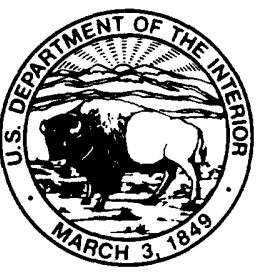

Any use of trade, product, or firm names in this publication is for descriptive purposes only and does not imply endorsement by the U.S. Government.

For sale by the U.S. Geological Survey

Branch of Information Services

Box 25286

Denver Federal Center

Denver, CO 80225-0286

For additional information write to:

District Chief

U.S. Geological Survey

Federal Building, Room W-2233

2800 Cottage Way

Sacramento, CA 95825 


\section{CONTENTS}

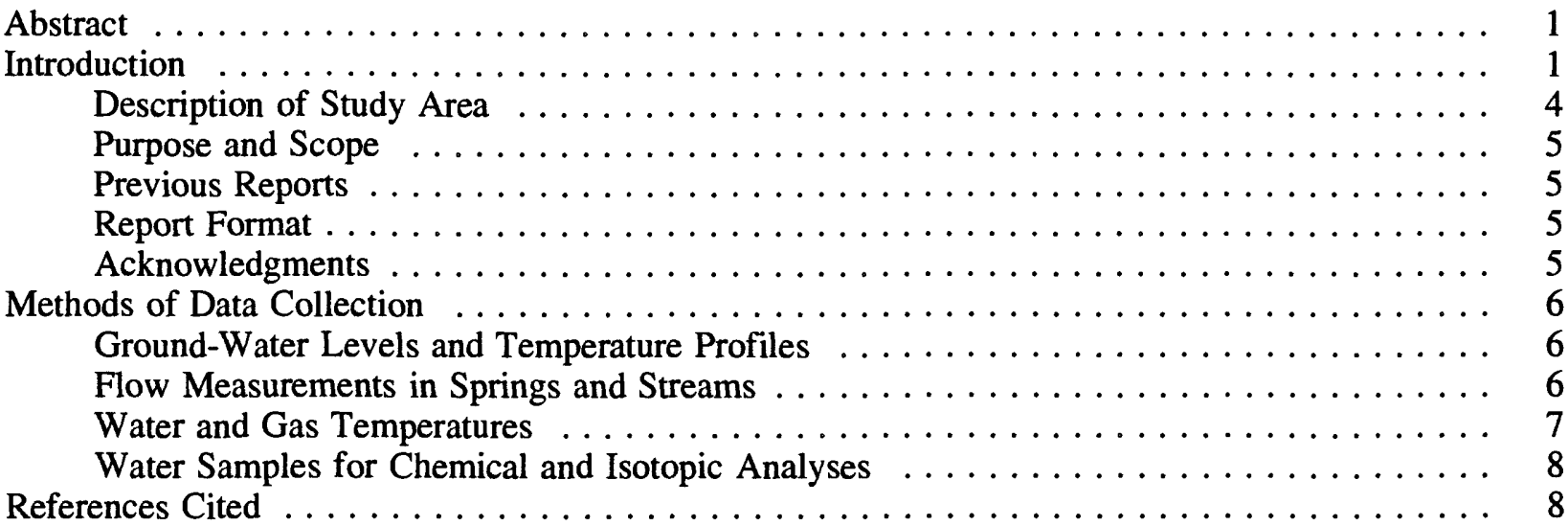

FIGURES

1. Map showing location of Long Valley study area, Mono County, California ........ 2

2. Map showing key geographic features, Long Valley Caldera, Mono County, California . . 3

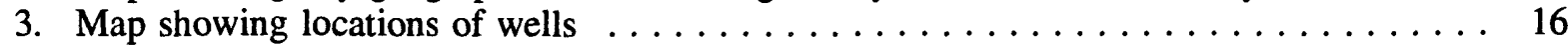

4-20. Graphs showing:

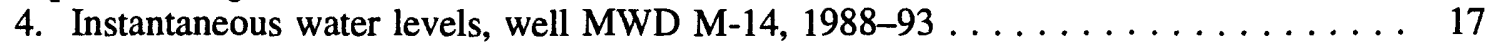

5. Daily mean water levels, Lookout Mountain well (LKT), 1987-93 . . . . . . . . . 17

6. Daily mean and instantaneous water levels, Lookout Mountain well (LKT), 1987,

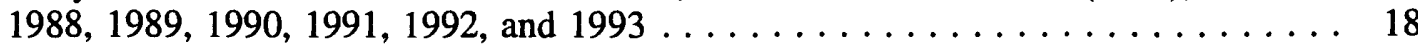

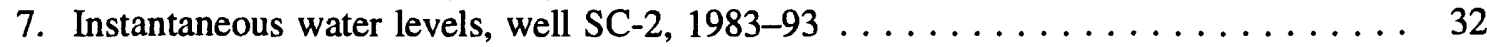

8. Daily mean water levels, well $\mathrm{CH}-1,1987-92 \ldots \ldots \ldots \ldots \ldots \ldots \ldots \ldots$

9. Daily mean and instantaneous water levels, well CH-1, 1987, 1988, 1989, 1990,

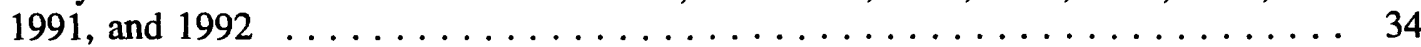

10. Temperature $\log$ of well RDO-8, June $19,1987 \ldots \ldots \ldots \ldots \ldots \ldots \ldots \ldots \ldots$

11. Daily mean water levels, well RDO-8, 1988-93 . . . . . . . . . . . . 46

12. Daily mean and instantaneous water levels, well RDO-8, 1989, 1990, 1991, 1992,

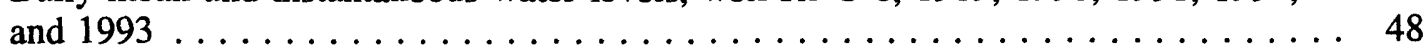

13. Temperature log of well CM-2, July $22,1989 \ldots \ldots \ldots \ldots \ldots \ldots$

14. Daily mean water levels, well CM-2, 1987-92 . . . . . . . . . . . . . . . 59

15. Daily mean water levels, well CM-2, 1987, 1988, 1989, 1990, and $1992 \ldots \ldots$

16. Instantaneous water levels, 1988-91, and mean daily water levels, 1992-93, well CW-3 . . . . . . . . . . . . . . . . . . . . . . . . 70

17. Daily mean water levels, well CW-3, 1992 and $1993 \ldots \ldots \ldots \ldots \ldots \ldots$

18. Temperature log of well $\mathrm{CH}-10 \mathrm{~B}$, December $19,1988 \ldots \ldots \ldots \ldots \ldots$

19. Daily mean water levels, well $\mathrm{CH}-10 \mathrm{~B}, 1987-93 \ldots \ldots \ldots \ldots$. . . . . . . . . 77

20. Daily mean and instantaneous water levels, well CH-10B, 1987, 1988, 1989,

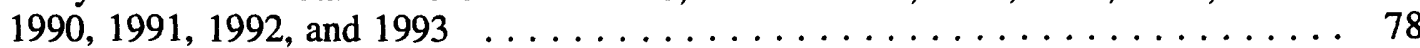

21. Map showing locations of springs $\ldots \ldots \ldots \ldots \ldots \ldots \ldots \ldots \ldots \ldots \ldots \ldots$

22. Graph showing daily mean discharge, Laurel Spring (LS), $1987 \ldots \ldots \ldots \ldots \ldots$

23. Map showing locations of data-collection sites at Hot Creek Fish Hatchery . . . . . . 119

24-38. Graphs showing:

24. Daily mean discharge, Fish Hatchery Spring AB (FHAB), weir site, 1990-93 . . . 120

25. Daily mean discharge, Fish Hatchery Spring AB (FHAB), weir site, 1991, 1992,

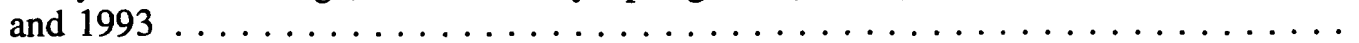


26. Daily mean water temperature, Fish Hatchery Spring AB (FHAB), upper site, 1988-92

27. Daily mean water temperature, Fish Hatchery Spring AB (FHAB), upper site, 1988, 1989, 1990, 1991, and 1992 and weir site, October 1992 through

December 1993 . . . . . . . . . . . . . . . . . . . . . . . . . . . 128

28. Daily mean water temperature, Fish Hatchery Spring CD (FHCD-T), 1988-93 _ . . 142

29. Daily mean water temperature, Fish Hatchery Spring CD (FHCD-T) October 1988 through December 1989, 1990, 1991, 1992, and $1993 \ldots \ldots \ldots \ldots \ldots \ldots$

30. Daily mean discharge, Fish Hatchery Spring 23 (FH23-Q), 1987-93 . . . . . . . 154

31. Daily mean discharge, Fish Hatchery Spring 23 (FH23-Q), 1987, 1988, 1989, 1990, 1991, 1992, and $1993 \ldots \ldots \ldots \ldots \ldots \ldots \ldots \ldots \ldots \ldots \ldots \ldots$

32. Daily mean water temperature, Fish Hatchery Spring 23 (FH23-T), 1988-93 . . . . 169

33. Daily mean water temperature, Fish Hatchery Spring 23 (FH23-T), 1988, 1989,

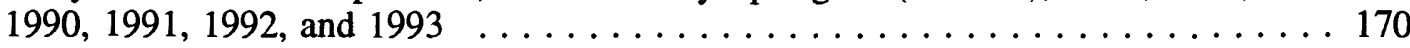

34. Daily mean discharge, Colton Spring (CS), $1987-91 \ldots \ldots \ldots \ldots \ldots \ldots$. . . . . 182

35. Daily mean and instantaneous measured gage heights, Hot Bubbling Pool (HBP), 1989-93

36. Daily mean and instantaneous measured gage heights, Hot Bubbling Pool (HBP),

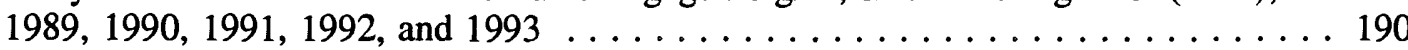

37. Daily mean water temperature, Hot Bubbling Pool (HBP), 1989, 1990, and $1991 \ldots 200$

38. Daily mean discharge, Little Hot Creek Spring (LHC-SP), 1987-93 . . . . . . . . 206

39. Map showing locations of streamflow sites . . . . . . . . . . . . . . . . 209

40-45. Graphs showing:

40. Daily mean discharge, Hot Creek Flume (HCF), $1987-93 \ldots \ldots \ldots \ldots \ldots$. . . . 213

41. Daily mean discharge, Hot Creek Flume (HCF), 1987, 1988, 1989, 1990, 1991,

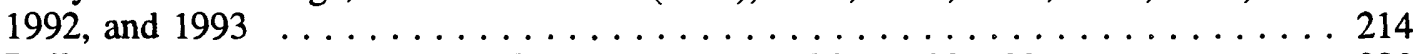

42. Daily mean water temperature, Hot Creek Flume (HCF), 1987-88 . . . . . . . 228

43. Daily mean specific conductance, Hot Creek Flume (HCF), 1987-88 . . . . . . . 232

44. Daily mean discharge, Little Hot Creek downstream from Little Hot Creek Spring

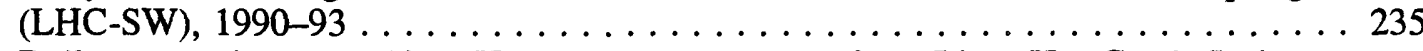

45. Daily mean discharge, Little Hot Creek downstream from Little Hot Creek Spring

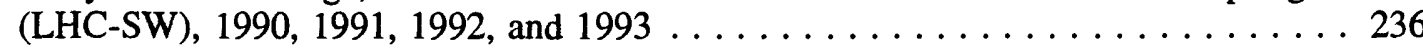

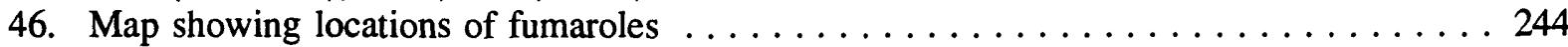

47. Graph showing daily mean vent gas temperature, Mammoth Mountain Fumarole (MMF), 1991-93 . . . . . . . . . . . . . . . . . . . . . . . . . 245

48. Graph showing daily mean vent gas temperature, Mammoth Mountain Fumarole

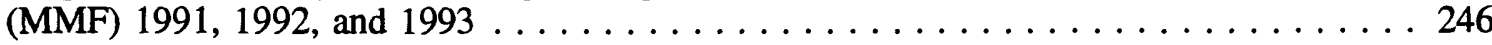

49. Graph showing daily mean and instantaneous measured vent gas temperatures,

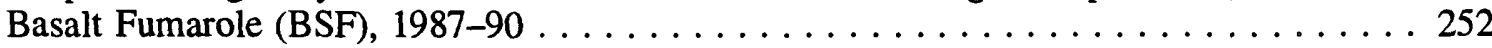

50. Map showing locations of precipitation gages $\ldots \ldots \ldots \ldots \ldots \ldots \ldots \ldots \ldots \ldots$

51. Graph showing annual precipitation at Little Hot Creek (LHC-P), Lookout Mountain

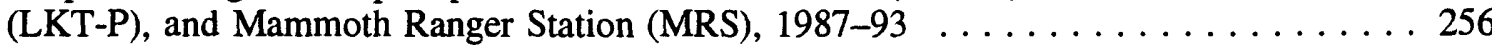

52. Map showing locations of water-quality sampling sites $\ldots \ldots \ldots \ldots \ldots \ldots \ldots$

\section{TABLES}

1. Descriptions of data-collection sites, Long Valley Caldera, Mono County, California . . . 13 2-8. Daily mean water levels, Lookout Mountain well (LKT):

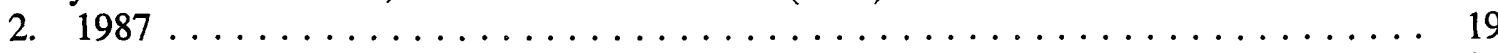

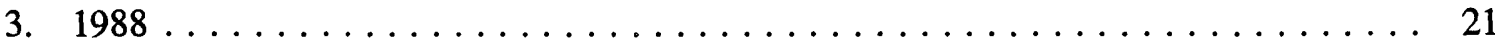

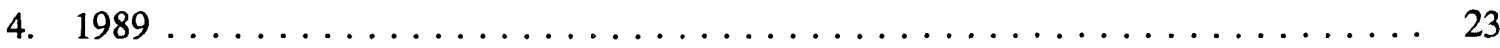

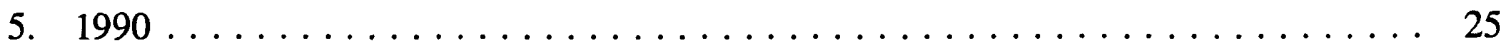

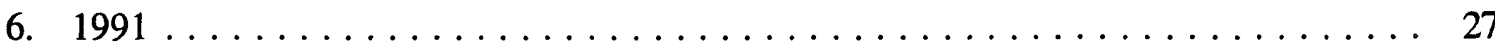

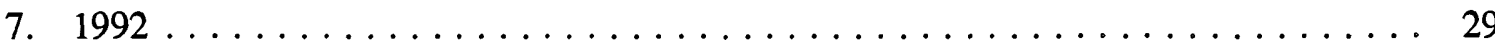

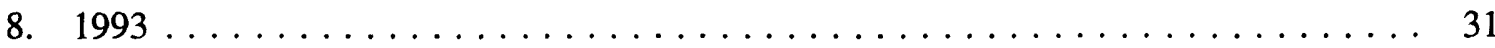


9-14. Daily mean water levels, well $\mathrm{CH}-1$ :

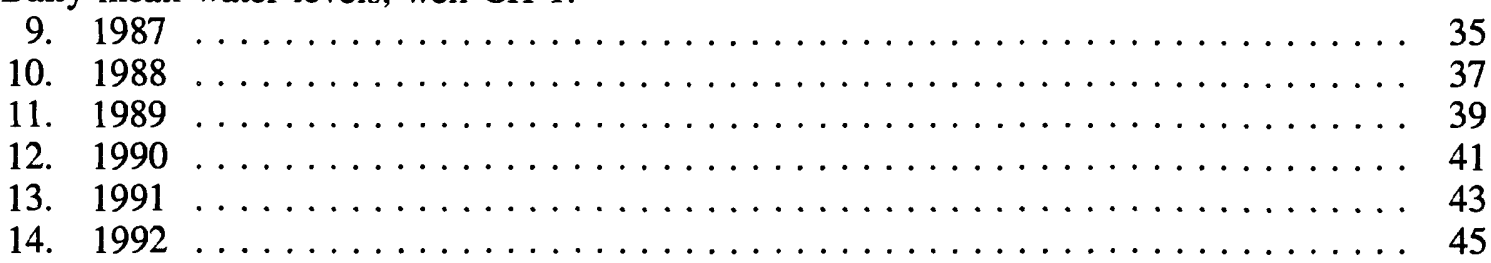

15-20. Daily mean water levels, well RDO-8:

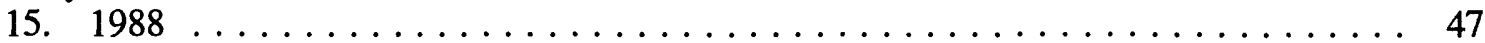

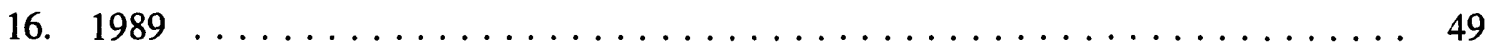

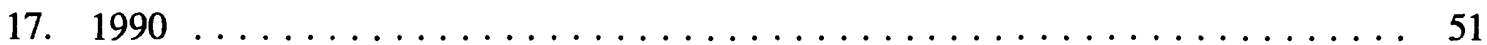

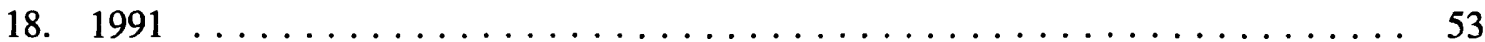

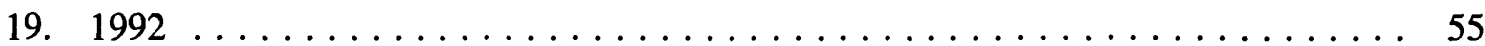

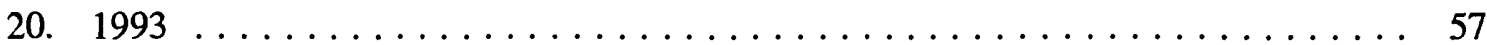

21-25. Daily mean water levels, well CM-2:

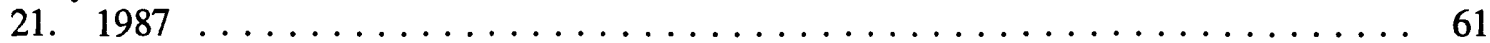

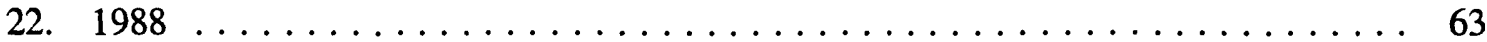

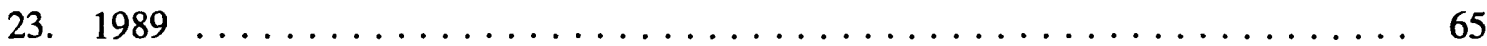

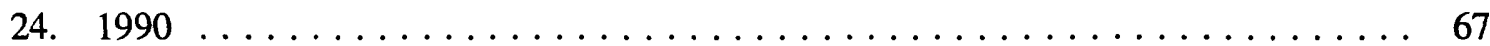

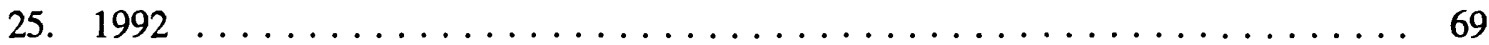

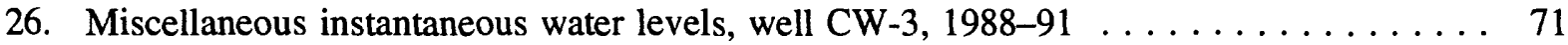

27. Daily mean water levels, well CW-3, $1992 \ldots \ldots \ldots \ldots \ldots \ldots \ldots \ldots$

28. Daily mean water levels, well CW-3, $1993 \ldots \ldots \ldots \ldots \ldots \ldots \ldots \ldots \ldots$

29-35. Daily mean water levels, well CH-10B:

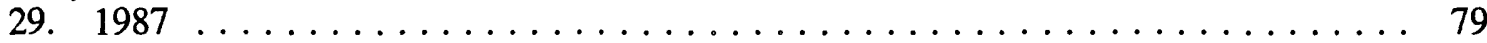

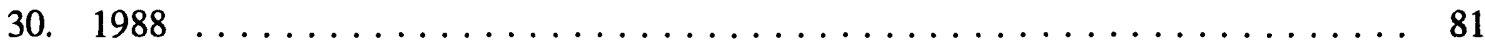

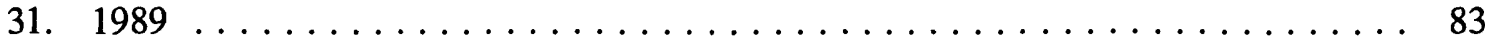

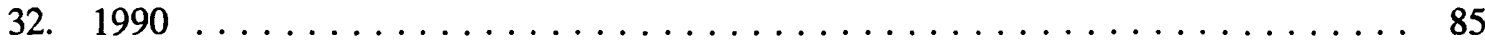

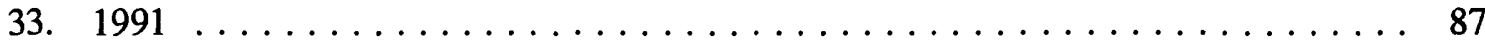

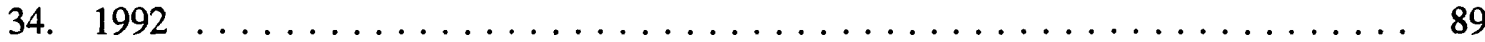

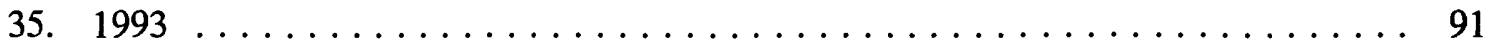

36. Ground-water levels in selected wells, $1987-93 \ldots \ldots \ldots \ldots \ldots \ldots \ldots \ldots$

37. Daily mean discharge, Laurel Spring (LS), $1987 \ldots \ldots \ldots \ldots \ldots \ldots \ldots$. . . . . . . . 117

38. Miscellaneous instantaneous discharge measurements, Laurel Spring (LS), 1991-92 _ . . 118

39-42. Daily mean discharge, Fish Hatchery Spring AB (FHAB), weir site:

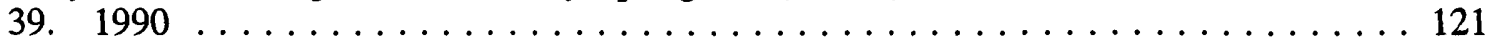

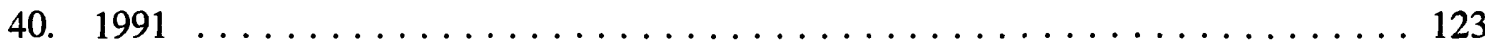

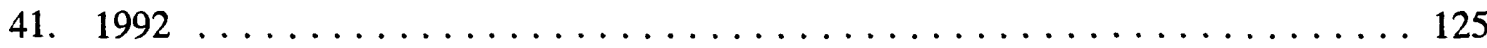

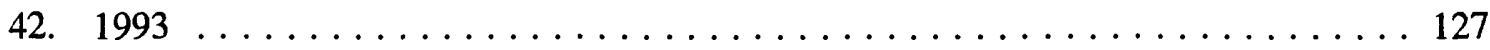

43-49. Daily mean water temperature, Fish Hatchery Spring AB (FHAB):

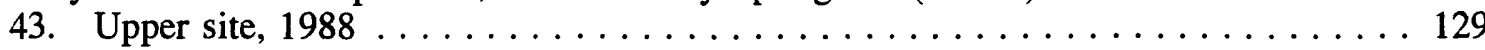

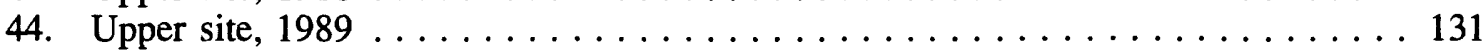

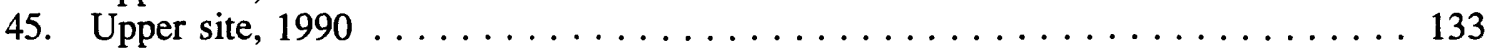

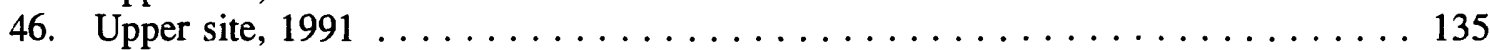

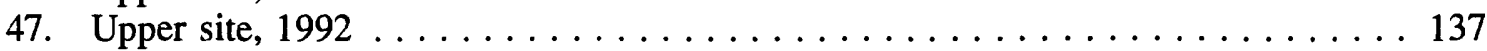

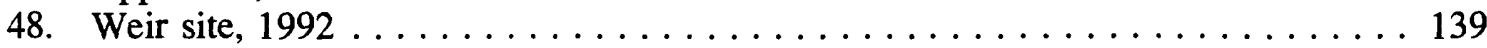

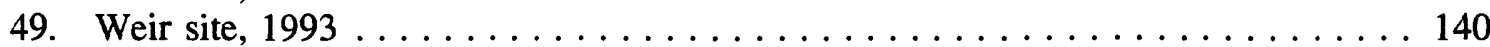

50. Instantaneous discharge measurements, Fish Hatchery Spring CD (FHCD-Q), 1988-93 . . 141

51-56. Daily mean water temperature, Fish Hatchery Spring CD (FHCD-T):

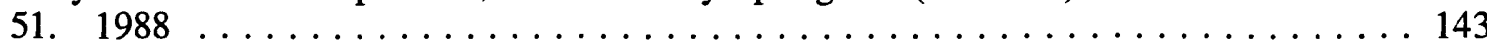

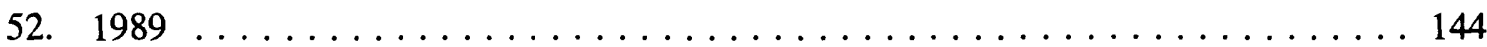

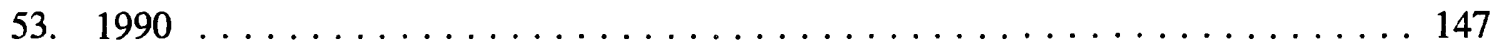

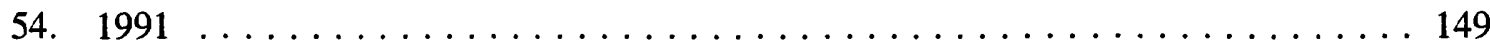

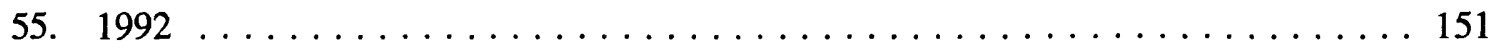

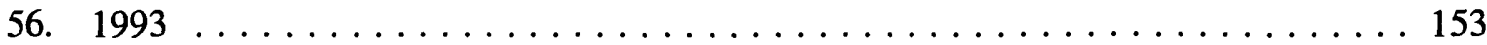


57-63. Daily mean discharge, Fish Hatchery Spring 23 (FH23-Q):

57. 1987

58. 1988

59. 1989

60. 1990

61. 1991

62. 1992

63. 1993

64-69. Daily mean water temperature, Fish Hatchery Spring 23 (FH23-T):

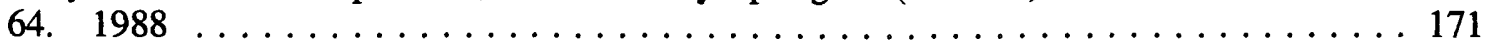

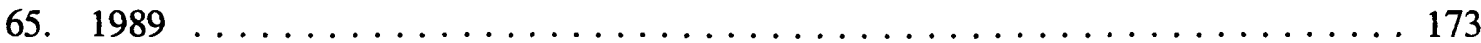

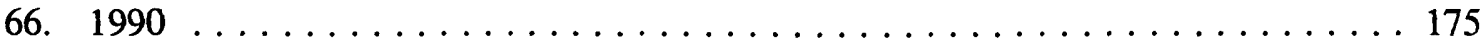

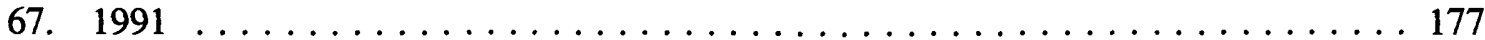

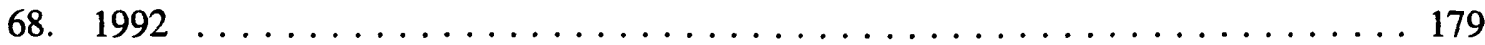

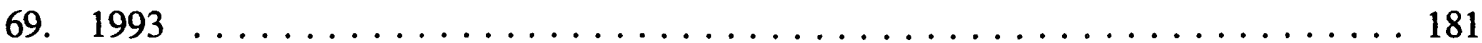

70-74. Daily mean discharge, Colton Spring (CS):

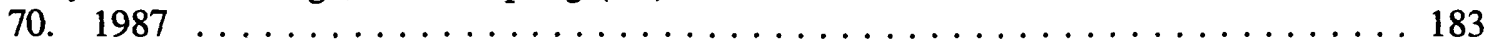

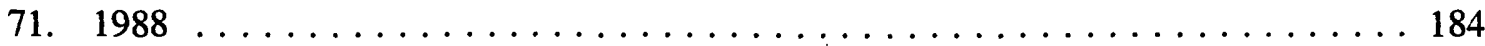

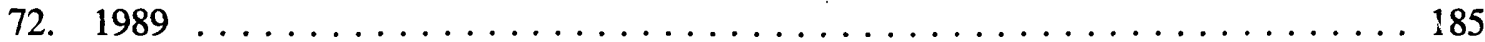

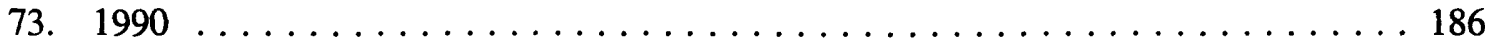

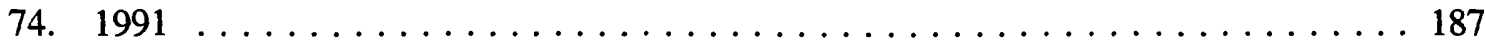

75-79. Daily mean gage height, Hot Bubbling Pool (HBP):

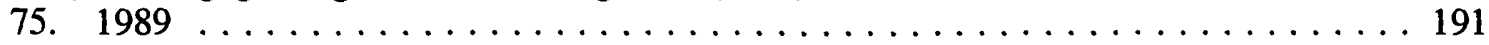

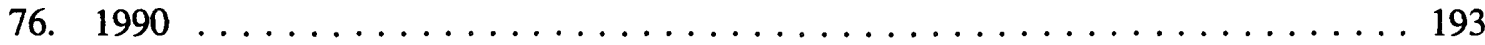

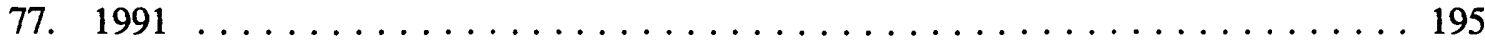

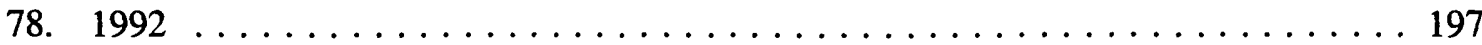

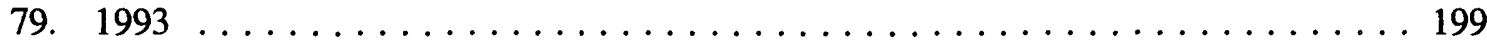

80. Daily mean water temperature, Hot Bubbling Pool (HBP), $1989 \ldots \ldots \ldots \ldots \ldots \ldots$

81. Daily mean water temperature, Hot Bubbling Pool (HBP), $1990 \ldots \ldots \ldots \ldots \ldots$. . . . . 203

82. Daily mean water temperature, Hot Bubbling Pool (HBP), $1991 \ldots \ldots \ldots \ldots . \ldots \ldots 5$

83. Monthly mean discharge, Little Hot Creek Spring (LHC-SP), 1987-93 . . . . . . . . . 207

84. Miscellaneous discharge and water temperature, Big Alkali Lake Spring

(BAL), 1988-93 . . . . . . . . . . . . . . . . . . . . . . . . . . . . 208

85. Instantaneous discharge measurements, Mammoth Creek at Highway 395

(MC395), 1988-93 . . . . . . . . . . . . . . . . . . . . . 210

86. Instantaneous discharge measurements and gage heights, Mammoth

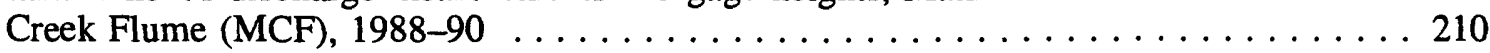

87. Instantaneous discharge measurements, Mammoth Creek upstream of Hot Creek Fish Hatchery (MCAF), 1988-93 . . . . . . . . . . . . . . . . . . . . 210

88. Instantaneous discharge measurements, Hot Creek above Gorge Geyser

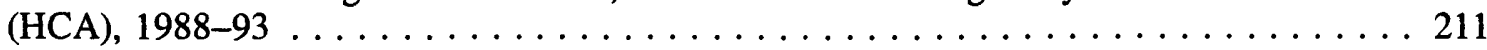

89-95. Daily mean discharge, Hot Creek Flume (HCF):

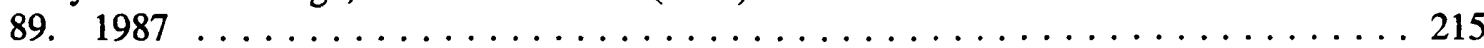

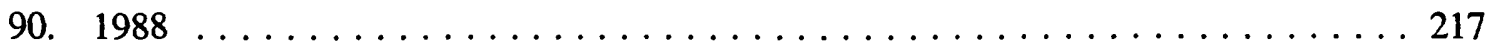

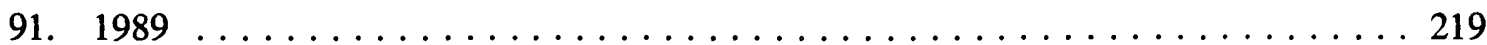

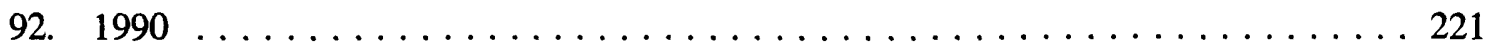

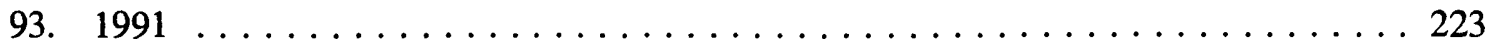

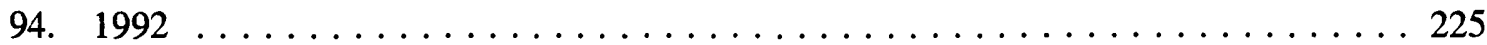

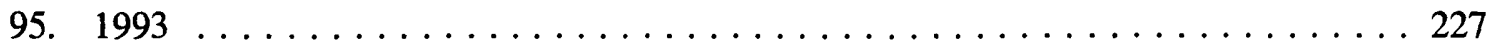

96. Daily mean water temperature, Hot Creek Flume (HCF), $1987 \ldots \ldots \ldots \ldots$. . . . . . 229

97. Daily mean water temperature, Hot Creek Flume (HCF), $1988 \ldots \ldots \ldots \ldots \ldots \ldots \ldots$

98. Daily mean specific conductance, Hot Creek Flume (HCF), $1987 \ldots \ldots \ldots \ldots \ldots$

99. Daily mean specific conductance, Hot Creek Flume (HCF), $1988 \ldots \ldots \ldots \ldots \ldots$ 
100-103. Daily mean discharge, Little Hot Creek downstream of Little Hot Creek Spring (LHC-SW):

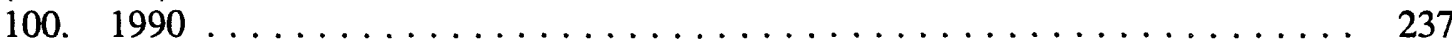

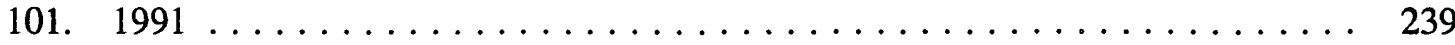

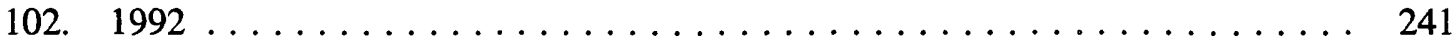

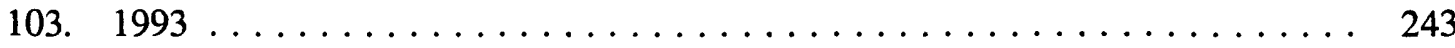

104. Daily mean vent gas temperature, Mammoth Mountain Fumarole (MMF), 1991 . . . 247

105. Daily mean vent gas temperature, Mammoth Mountain Fumarole (MMF), 1992 . . . 249

106. Daily mean vent gas temperature, Mammoth Mountain Fumarole (MMF), 1993 . . . 251

107. Daily mean vent gas temperature, Basalt Fumarole (BSF), $1987 \ldots \ldots$. . . . . . . 253

108. Miscellaneous instantaneous vent gas temperatures, Basalt Fumarole (BSF), 1987-90 254

109. Daily precipitation Little Hot Creek gage (LHC-P), $1987 \ldots \ldots \ldots \ldots \ldots \ldots \ldots$

110. Daily precipitation Little Hot Creek gage (LHC-P), $1988 \ldots \ldots \ldots \ldots \ldots \ldots \ldots$

111. Daily precipitation, Lookout Mountain gage (LKT-P), $1993 \ldots \ldots \ldots$. . . . . . 259

112. Monthly precipitation, Mammoth Ranger Station gage (MRS), 1987-93 . . . . . . . 260

113. Chemical and isotopic analyses of water samples collected at selected surface-water

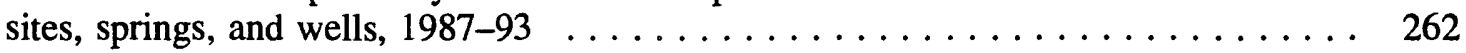

114. Partial chemical analyses of water samples collected at selected surface-water sites

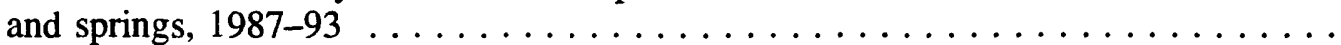

CONVERSION FACTORS, ABBREVIATIONS, AND VERTICAL DATUM Conversion Factors

\begin{tabular}{rcl}
\hline Multiply & By & To obtain \\
\hline foot $(\mathrm{ft})$ & 0.3048 & meter \\
foot squared $\left(\mathrm{ft}^{2}\right)$ & 0.09290 & square meter \\
cubic foot per second $\left(\mathrm{ft}^{3} / \mathrm{s}\right)$ & 0.02832 & cubic meter per second \\
inch (in.) & 25.4 & millimeter \\
mile (mi) & 1.609 & kilometer
\end{tabular}

Temperature is given in degrees Celsius $\left({ }^{\circ} \mathrm{C}\right)$, which can be converted to degrees Fahrenheit $\left({ }^{\circ} \mathrm{F}\right)$ by the following equation:

$$
{ }^{\circ} \mathrm{F}=1.8\left({ }^{\circ} \mathrm{C}\right)+32
$$

\section{Abbreviations}

$\mu \mathrm{m}=$ micrometer

$\mathrm{mL}=$ milliliter

\section{Vertical Datum}

Sea Level: In this report, "sea level" refers to the National Geodetic Vertical Datum of 1929 (NGVD of 1929)-a geodetic datum derived from a general adjustment of the first-order level nets of both the United States and Canada, formerly called Sea Level Datum of 1929. 


\title{
Hydrologic Data for Long Valley Caldera, Mono County, California, 1987-93
}

\author{
By James F. Howle and Christopher D. Farrar
}

\begin{abstract}
Hydrologic data were collected during 1987-93 as part of the U.S. Geological Survey's long-term Volcanic Hazards Monitoring Program of the Long Valley Caldera, Mono County, California. The data are presented in graphs or tables. Data collected for the Long Valley Hydrologic Advisory Committee monitoring program also are presented. Hydrologic data collected include continuous record of ground-water levels in 6 wells, instantaneous measurement of ground-water levels in 55 wells, continuous record of discharge at 2 streams and 5 springs, instantaneous discharge measurements at 4 streams and 2 springs, ground-water temperature profiles of 3 thermal wells, continuous record of water temperature in 1 stream and 5 springs, vent gas temperatures at 2 fumaroles, and chemical and isotopic analyses of water samples collected at 13 ground-water and 6 surface-water sites. Precipitation amounts at three sites also are included. A diskette of data tables is provided.
\end{abstract}

\section{INTRODUCTION}

Long Valley Caldera (fig. 1) is a site of geologically recent volcanism (Bailey, 1989). The high rates of seismicity and crustal deformation recorded in Long Valley Caldera since 1980 probably are related to magmatic intrusions (Rundle and Hill, 1988). The volcanic history and recent crustal unrest have been the motivation for the U.S. Geological Survey and other agencies to monitor a number of geologic and geophysical parameters to assess the potential of volcanic eruption in the near term (Hill and others, 1991).

The volcanic activity has provided heat for a hydrothermal system that underlies the western, southern, and eastern parts of Long Valley Caldera. Ground-water temperatures greater than $200{ }^{\circ} \mathrm{C}$ have been measured in the western part of the caldera. Flow in the hydrothermal system is from west to east along the hydraulic gradient (Sorey and others, 1991). Thermal springs located in the southern and eastern parts of the caldera are natural discharge points for the hydrothermal system.

The thermal springs provide unique environments for wildlife and plants and are used for recreational bathing by thousands of tourists each year. The largest direct use of geothermal resources in the study area is at the Hot Creek Fish Hatchery (fig. 2), operated by the State of California since 1932. The hatchery uses between 15 and $25 \mathrm{ft}^{3} / \mathrm{s}$ of water from low-temperature $\left(15-20^{\circ} \mathrm{C}\right)$ thermal springs for raising trout. Use of geothermal resources for electric power generation began in 1985. By 1992, power production totaled about 40 megawatts from three plants located at Casa Diablo (fig. 2). 


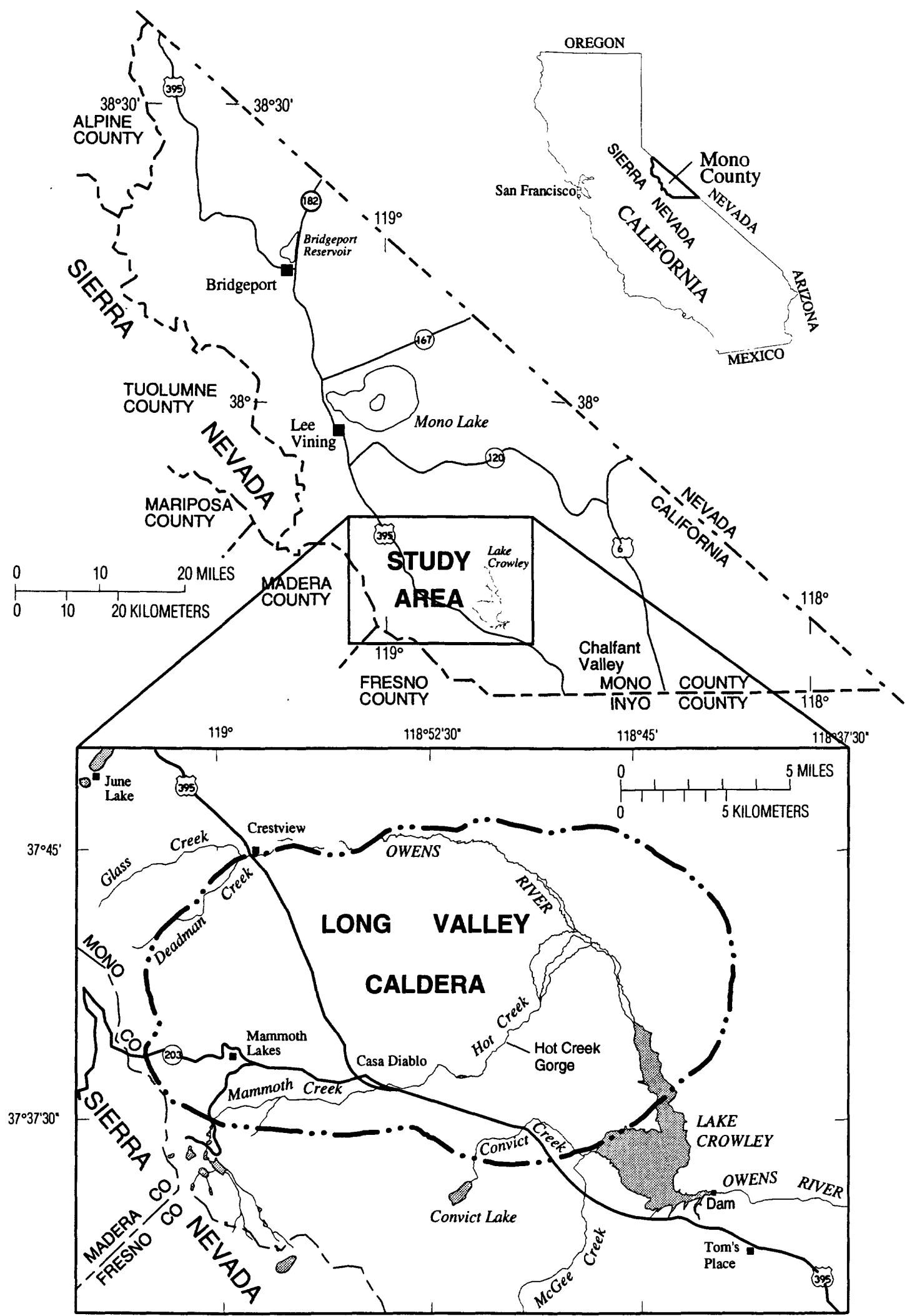

Figure 1. Location of Long Valley study area, Mono County, California. 


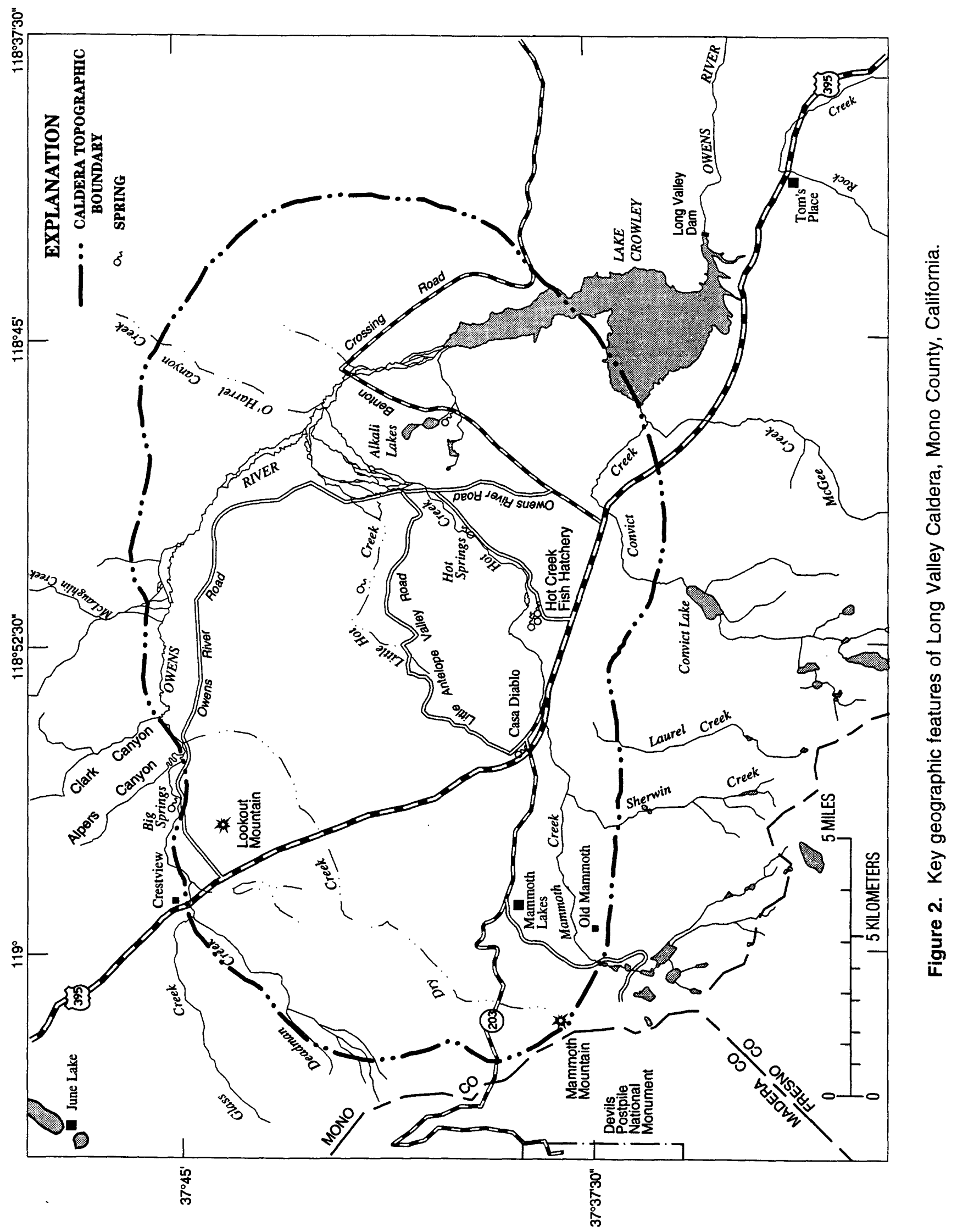


A long-term hydrologic monitoring program was started in 1982 as part of the U.S. Geological Survey's Volcanic Hazards Monitoring Program. The purpose of the hydrologic monitoring is to document changes in the hydrologic system that are related to volcanic processes and seismicity. Hydrologic data collected for this purpose include ground-water levels, discharge rates of streams and springs, ground-water temperature profiles, water temperatures of streams and springs, vent gas temperatures of fumaroles, chemical and isotopic analyses of ground water and surface water, and precipitation amounts. The number of data-collection sites and frequency of data collection have varied over the years in response to changes in hydrologic conditions, the level of geologic unrest, fulfilling the needs for baseline records, and funding levels.

A second hydrologic monitoring program began in 1988 in cooperation with Mono County, California. The monitoring program was implemented to provide data to the Long Valley Hydrologic Advisory Committee (LVHAC). The LVHAC was formed in 1986 to provide Mono County with advice concerning hydrologic environmental issues related to resource developments (Farrar and Lyster, 1990). The monitoring program parallels the volcanic hazard monitoring, but is specifically directed at detecting hydrologic changes that are caused by geothermal resource utilization and ground-water pumping for potable water supply for the town of Mammoth Lakes.

The LVHAC has identified three key hydrologic features of concern for the county: Mammoth Creek, the thermal springs at Hot Creek Fish Hatchery (fig.2), and the thermal springs in Hot Creek Gorge. Hydrologic monitoring carried out by the U.S. Geological Survey for the LVHAC and Mono County is primarily at sites along Mammoth Creek, Hot Creek, and at Hot Creek Fish Hatchery (fig. 2).

\section{Description of Study Area}

Long Valley Caldera is in Mono County in eastern California along the steep eastern front of the Sierra Nevada about $20 \mathrm{mi}$ south-southeast of Mono Lake (fig. 1). The caldera is an oval-shaped topographic depression measuring approximately $18 \mathrm{mi}$ from east to west and about $12 \mathrm{mi}$ north to south. The topographic depression formed as the result of a massive volcanic eruption about 760 thousand years ago (Sarna-Wojcicki and Pringle, 1992) and subsequent erosion. All monitoring sites for which data are presented in this report lie within the boundary of the Long Valley Caldera.

The caldera floor has a gentle topographic relief in the eastern part where altitudes range from about 6,800 to $7,200 \mathrm{ft}$ above sea level. The central and western parts are more mountainous with altitudes that range up to about $9,000 \mathrm{ft}$. Prominent mountains, reaching altitudes of over $13,000 \mathrm{ft}$, enclose the caldera on the south, west, and north. The eastern boundary is of lower relief.

The caldera forms a well-defined surface-water drainage basin with a single outlet by way of Owens River in the southeastern part of the area (fig. 2). A number of perennial and intermittent streams drain from the high mountains that border the southern and western sides of the caldera. Fewer streams drain the northern mountains because precipitation amounts are less. These peripheral streams drain toward one of two main east-flowing streams on the caldera floor, Mammoth Creek on the south and Owens River on the north (fig. 2). Lake Crowley is an artificial reservoir formed by the Long Valley Dam across the Owens River. The reservoir receives water from Owens River, Mammoth Creek, and a few streams that drain smaller areas. In addition, the reservoir receives water imported from the Mono Basin located north of Long Valley Caldera. The imported water is transported $11 \mathrm{mi}$ through a tunnel and discharged into the Owens River about $15 \mathrm{mi}$ upstream of Lake Crowley. The reservoir is part of the water-supply system for the City of Los Angeles. 


\section{Purpose and Scope}

This report provides a compilation of hydrologic data collected in Long Valley Caldera by the U.S. Geological Survey during 1987-93. Data collected for both the volcanic hazards monitoring program and the LVHAC are presented in this report. The data include ground-water levels; discharge measurements; water temperatures in wells, streams, and springs; vent gas temperatures; precipitation amounts; and chemical and isotopic analyses of water samples collected at selected sites. Hydrologic records for most of the monitoring sites span a few years and in some cases span the entire 7-year period. In many cases, these multi-year records indicate annual cycles and long-term trends.

Although no interpretations of the data are provided in this report, the data will be useful to researchers who may want to relate hydrologic changes to geodetic and geophysical measurements collected during the study of volcanic and seismic processes active during recent years. The report also provides a summary of hydrologic data relevant to some of the environmental concerns of local, State, and Federal agencies, as well as individuals.

\section{Previous Reports}

Hydrologic data from the volcanic hazards monitoring program for years prior to 1987 have been published in reports by Farrar and others $(1985 ; 1987 ; 1989)$. Other significant data compilations include: California Department of Water Resources (1967; 1973), Lewis (1974), Willey and others (1974), Mariner and Willey (1976), and Setmire (1984). In addition, the U.S. Forest Service has published annual reports containing hydrologic data for 1980 through 1985.

\section{Report Format}

The data tables and figures in this report are grouped by site type (wells, springs, streams, fumaroles, and precipitation gages) and are further subdivided into nonthermal or thermal within the groups for wells and springs. Individual maps show the locations of sites by site type.

The presentation of data differs according to site type and the level of variability of the data over the collection period. In general, each graph is followed by the table of data used to construct the graph. For sites with several years of record and where the variability of data is great, graphs for individual years are provided in addition to the multi-year graphs.

Each site has been assigned a short alpha or alphanumeric identifier that is used in figures and tables. Table 1 lists all sites, grouped by site type, and provides the U.S. Geological Survey station identifier, station number, latitude, longitude, altitude, and information about the data presented in this report.

A high density, 1.4 Megabyte, 3-1/2-in. diskette containing ASCII files of the data tables is included at the back of the report. The files on the diskette are labeled to match the table numbers in the report.

\section{Acknowledgments}

The cooperation of many well owners and landowners in allowing access to collect data is greatly appreciated. Thom Heller, U.S. Forest Service, provided the precipitation data for the Mammoth Ranger Station gage. Gary Sisson was especially helpful in providing information and access to some of the Mammoth Community Water District wells. Donald Barnett, Intermountain Water Consultants, provided water-level data for well CW-3 for 1988-91. Chris Boone and Dennis Redfern, California Department of Fish and Game, cooperated in many ways that assisted data collection at Hot Creek Fish Hatchery. 


\section{METHODS OF DATA COLLECTION}

\section{Ground-Water Levels and Temperature Profiles}

Ground-water levels were measured three times per year in a network of approximately 55 wells (tables 1 and 36, at back of report). The actual number of wells varied from year to year because of access conditions and the addition of new wells. The measurements generally were made in April, July, and November. These three months were chosen to provide values reasonably close to actual maximum and minimum water levels and to avoid difficult access conditions in the winter months. Beginning in 1988, water levels were measured monthly in about 10 wells selected from the larger network.

The water levels were measured using chalked steel tapes or electric sounding devices. Electric sounding devices were used only when conditions in the well bore prevented accurate steel tape measurements. Conditions that required the use of electric sounders were high water temperatures $\left(>30^{\circ} \mathrm{C}\right)$ at the water surface, which would cause a high evaporation rate as the tape was withdrawn from the well, and wet casings above the water level, which would give false indications of the true water level. The general procedures for making accurate water-level measurements discussed in a report by Garber and Koopman (1968) were followed.

The water levels were measured with reference to a convenient measuring point on the well head and then were adjusted to land-surface datum using a constant difference between the measuring point and the land surface at the well. Land-surface altitudes were determined for each well site by spirit leveling from a point of known altitude or approximated from topographic maps (table 1). Altitudes that have a precision of less than $1 \mathrm{ft}$ are from leveling; those that have a precision of $1 \mathrm{ft}$ or more are from topographic maps.

Daily mean values of water levels were computed from data recorded at hourly or shorter intervals. Water levels were recorded by a variety of means (table 1). A system consisting of a float, tape, and counter weight suspended over a pulley was used for shallow water levels. The data were recorded on graphic charts, digital punch tapes, or electronically using a shaft-encoder and data logger. For deeper water levels (more than $20 \mathrm{ft}$ ), pressure transducers were used to sense the depth of water above an arbitrary datum. In wells that had water temperatures at the water surface of less than $50{ }^{\circ} \mathrm{C}$, submersible transducers were used. The transducers were suspended by weighted cables that held the sensing port at a constant depth. In wells that had water temperatures greater than $50{ }^{\circ} \mathrm{C}$, a bubbler system was used with the transducer at the surface. The system consisted of a nitrogen tank, pressure regulator, and tubing. One end of the tubing was set at a fixed depth and nitrogen was allowed to bubble out of the tube below the water surface. The back-pressure on this tube was sensed by a transducer and was related to the height of the water column above the tube orifice. The pressure data were recorded by a data logger and were converted to water levels in feet below land surface.

Temperature profiles in wells were made using portable logging equipment consisting of a thermistor attached to a cable and a digital meter to display the resistance. Resistance was converted to the equivalent temperature using a polynomial equation derived from calibration measurements performed in a laboratory. The profiles consist of a number of readings made at discrete points, variably spaced depending on the temperature gradient and depth of the well. Temperatures are accurate to $0.1{ }^{\circ} \mathrm{C}$ or better.

\section{Flow Measurements in Springs and Streams}

Measurements of flow from springs and at cross sections in streams were made using current velocity meters, weirs, and flumes. Procedures used are described in reports by Buchanan and Somers (1969) and Kilpatrick and Schneider (1983). 
Daily mean values of flow were computed from stage records and a stage-discharge relation equation (Rantz and others, 1982; Kennedy, 1983, 1984). Stage was measured using a float, tape, and counter weight suspended over a pulley and was recorded on digital punch tape, graphic chart, or electronically using a shaft-encoder and data logger.

\section{Water and Gas Temperatures}

Daily mean values of water temperature in springs and streams and vent gas temperatures for fumaroles were computed from values recorded at hourly or more frequent intervals. The temperatures were sensed by using either thermistors or platinum resistance devices, and the data were recorded using data loggers. The accuracy of the temperature values is about $0.2{ }^{\circ} \mathrm{C}$ for the sites at Hot Creek Fish Hatchery and Hot Creek Flume. The accuracy of the values for Basalt Fumarole, Hot Bubbling Pool, and Mammoth Mountain Fumarole is about $0.5^{\circ} \mathrm{C}$. Water temperatures were measured using the guidelines given in a report by Stevens and others (1975).

At Fish Hatchery Spring AB (fig. 23), several spring vents discharge subaqueously to a pool about $400 \mathrm{ft}$ long and 10 to $25 \mathrm{ft}$ wide. From June 1988 to October 1992, water temperatures were measured with a thermistor placed at the west end of the pool $0.1 \mathrm{ft}$ above the pool floor and above a subaqueous spring vent. The flow from the vent declined during January 1992 and, within a few weeks, there was no measurable evidence of discharge. A radical departure of the general water temperature trend (shown in fig. 26) occurred during the period January to March 1992 because flow from the spring vents at the west end of the pool had ceased, and the stagnant water was cooled by winter air temperatures. The temperature site was moved to the east end of the pool near the weir at the outflow point. At this site, the temperature recorded is a flow-weighted average temperature from all the individual vents. This flow-weighted temperature is affected by variations in ambient air and soil temperatures, as well as variations in water temperatures at the spring vents.

Fish Hatchery Spring $C D$ is similar to Fish Hatchery Spring $A B$, in that several spring vents discharge subaqueously to a collection pool about $700 \mathrm{ft}$ in length. Temperature measurements at this site were made at the west end of the pool. A thermistor was placed in the subaqueous discharge stream of an individual vent that issues from between basalt boulders lining the pool side.

At the Fish Hatchery Spring 23 site, several springs, both subaqueous and subaerial, dis charge to a collection pool. A thermistor was placed in a subaerial vent along the southeast side of the pool.

The Hot Bubbling Pool site is a thermal pool that receives discharge from several subaqueous spring vents. The pool is roughly elliptical in shape and has varied in surface area from about 5,000 $\mathrm{ft}^{2}$ in 1987 to about $800 \mathrm{ft}^{2}$ in 1993 . Water temperatures were measured at a depth of about $3 \mathrm{ft}$ below the water surface along the north side of the pool.

Temperatures of the gas discharged from Basalt Fumarole and Mammoth Mountain Fumarole were measured by placing sensors in 1/2-in.-diameter pipes inserted into the side of the vent at a depth of approximately $1 \mathrm{ft}$ below land surface. The discharge point for the main flow of gas at both fumaroles shifted a few inches from time to time because of minor slumping of the soil or disruption of the vent by curious visitors. At times, these disruptions of the vents left the temperature sensor a few inches from the main discharge stream. When the sensor was not positioned in the main discharge stream, recorded temperatures deviated from temperatures measured directly in the vent by as much as 2 or $3^{\circ} \mathrm{C}$. 


\section{Water Samples for Chemical and Isotopic Analyses}

Water samples from subaerial springs were collected by dipping a polyethylene container into the discharge stream as near the vent as possible. Samples from subaqueous springs and streams were collected using a depth-integrated sampler and equal-width stationing. Samples of water in both vapor and liquid phase were collected from the discharge pipe of geothermal wells while the wells were being pumped. The vapor-fluid sample was condensed to a liquid in a coil of stainless steel tubing immersed in an ice-water bath and was cooled from about $170^{\circ} \mathrm{C}$ to $30^{\circ} \mathrm{C}$.

Aliquots of the samples were used to determine alkalinity, $\mathrm{pH}$, and specific conductance in the field. Aliquots for laboratory analyses were prepared in the field by filtering through a $0.45-\mu \mathrm{m}$ acetate filter. Filtered, unacidified water was placed in polyethylene bottles for anion analyses, and filtered water acidified with nitric acid was placed in polyethylene bottles for cation analyses. Water for mercury analysis was placed in flint glass bottles to which $10 \mathrm{~mL}$ of nitric acid and potassium dichromate was added. Samples for nitrogen and phosphorous analyses were placed in opaque, brown polyethylene bottles, treated with mercuric chloride, and chilled to $4{ }^{\circ} \mathrm{C}$. This procedure was done to inhibit biologic activity. Laboratory methods used for analyses are described in a report by Fishman and Friedman (1989).

\section{REFERENCES CITED}

Bailey, R.A., 1989, Geologic map of Long Valley caldera, Mono-Inyo Craters Volcanic Chain, and vicinity, eastern California: U.S. Geological Survey Map Series I-1933, 1 sheet plus 11 p. of text, scale $1: 62,500$.

Buchanan, T.J., and Somers, W.P., 1969, Discharge measurements at gaging stations: U.S. Geological Survey Techniques of Water-Resources Investigations, book 3, chap. A8, 65 p.

California Department of Water Resources, 1967, Investigation of geothermal waters in the Long Valley area, Mono County: California Department of Water Resources, $141 \mathrm{p}$.

1973, Mammoth Basin water resources environmental study (final report): California Department of Water Resources, $70 \mathrm{p}$.

Farrar, C.D., and Lyster, D.L., 1990, Monitoring the hydrologic system for potential effects of geothermal and ground-water development in the Long Valley caldera, Mono County, California, U.S.A.: Geothermal Resources Council Transactions, v. 14, pt. 1, p. 669-674.

Farrar, C.D., Sorey, M.L., Rojstaczer, S.A., Janik, C.J., Mariner, R.H., Winnett, T.L., and Clark, M.D., 1985, Hydrologic and geochemical monitoring in Long Valley Caldera, Mono County, California: U.S. Geological Survey Water-Resources Investigations Report 85-4183, 137 p.

Farrar, C.D., Sorey, M.L., Rojstaczer, S.A., Janik, C.J., Winnett, T.L., and Clark, M.D., 1987, Hydrologic and geochemical monitoring in Long Valley caldera, Mono County, California, 1985: U.S. Geological Survey Water-Resources Investigations Report 87-4090, 71 p.

Farrar, C.D., Sorey, M.L., Rojstaczer, S.A., Steinemann, A.C., and Clark, M.D., 1989, Hydrologic and geochemical monitoring in Long Valley caldera, Mono County, California, 1986: U.S. Geological Survey Water-Resources Investigations Report 89-4033, 69 p.

Fishman, M.J., and Friedman, L.C., 1989, Methods for determination of inorganic substances in water and fluvial sediments (3d ed): U.S. Geological Survey Techniques of Water-Resources Investigations, book 5 , chap. A1, 545 p.

Garber, M.S., and Koopman, F.C., 1968, Methods of measuring water levels in deep wells: U.S. Geological Survey Techniques of Water-Resources Investigations, book 8, chap. A1, 23 p.

Hill, D.P., Johnston, M.J.S., Langbein, J.O., McNutt, S.R., Miller, C.D., Mortensen, C.E., Pitt, A.M., and Rojstaczer, S., 1991, Response plans for volcanic hazards in the Long Valley caldera and Mono Craters area, California: U.S. Geological Survey Open-File Report 91-270, 64 p.

Kennedy, E.J., 1983, Computation of continuous records of streamflow: U.S. Geological Survey Techniques of Water-Resources Investigations, book 3, chap. A13, 53 p.

1984, Discharge ratings at gaging stations: U.S. Geological Survey Techniques of Water-Resources Investigations, book 3 , chap. A10, 59 p. 
Kilpatrick, F.A., and Schneider, V.R., 1983, Use of flumes in measuring discharge: U.S. Geological Survey Techniques of Water-Resources Investigations, book 3, chap. A14, 46 p.

Lewis, R.E., 1974, Data on wells, springs, and thermal springs in Long Valley, Mono County, California: U.S. Geological Survey Open-File Report, 52 p.

Mariner, R.H., and Willey, L.M., 1976, Geochemistry of thermal waters in Long Valley, Mono County, California: Journal of Geophysical Research, v. 81, no. 5, p. 792-800.

Rantz, S.E., and others, 1982, Measurement and computation of streamflow: Volume 2. Computation of discharge: U.S. Geological Survey Water-Supply Paper 2175, 631 p.

Rundle, J.B., and Hill, D.P., 1988, The geophysics of a restless caldera-Long Valley, California: Palo Alto, Calif., Annual Reviews Inc., Annual Review of Earth and Planetary Sciences, v. 16, p. 251-271.

Sarna-Wojcicki, A.M., and Pringle, M.S., Jr., 1992, Laser-fusion ${ }^{40} \mathrm{Ar} /{ }^{39} \mathrm{Ar}$ ages of the Tuff of Taylor Canyon and Bishop Tuff, E. California-W. Nevada: Eos, American Geophysical Union, Transactions, v. 73, no. 43, p. 633.

Setmire, J.G., 1984, Water-quality appraisal of Mammoth Creek and Hot Creek, Mono County, California: U.S. Geological Survey Water-Resources Investigations Report 84-4060, 50 p.

Stevens, H.H., Jr., Ficke, J.F., and Smoot, G.F., 1975, Water temperature-influential factors, field measurement, and data presentation: U.S. Geological Survey Techniques of Water-Resources Investigations, book 1, chap. D1, $65 \mathrm{p}$.

Sorey, M.L., Suemnicht, G.A., Sturchio, N.C., and Nordquist, G.A., 1991, New evidence on the hydrochermal system in Long Valley caldera, California, from wells, fluid sampling, electrical geophysics, and age determinations of hot-spring deposits: Journal of Volcanology and Geothermal Research, v. 48, p. 229-263.

U.S. Forest Service, 1980-1985, Water resources data for Mammoth Ranger District, Inyo National Forest, California: Published annually.

Willey, L.M., O'Neil, J.R., and Rapp, J.B., 1974, Chemistry of thermal waters in Long Valley, Mono County, California: U.S. Geological Survey Open-File Report, 19 p. 

TABLES 1-114

AND

FIGURES 3-52 
Table 1. Descriptions of data-collection sites, Long Valley Caldera, Mono County, California

[Station identifier: Short alphanumeric code used as reference in figures and tables.

USGS station number: Unique numeric code used in U.S. Geological Survey data bases.

Data types: The types of data shown in this report are denoted by codes (CD-daily specific conductance, F-miscellaneous discharge measurements, FD-daily discharge, L-miscellaneous ground-water levels, LD—daily ground-water levels computed from values recorded at least hourly, PD-daily precipitation, PM-monthly precipitation (daily values available from U.S. Forest Service), Q-chemical or isotopic analyses, SD-daily gage height, Tmiscellaneous temperatures, TD-daily water temperatures, TP-temperature profiles in a well).

Equipment types: The types of equipment used at recording sites to collect data are given by codes (C-conductivity sensor and data logger, DR - digital recorder, GR-graphic chart recorder, PT-pressure transducer with data logger, SE-shaft encoder with data logger, T-thermister with data logger, TB-tipping bucket gage with data logger, W-weir or flume, WB-weighing bucket gage and chart recorder).

Data on pages: pages in this report showing data for the site]

\begin{tabular}{|c|c|c|c|c|c|c|c|}
\hline $\begin{array}{l}\text { Station } \\
\text { identifier }\end{array}$ & $\begin{array}{l}\text { USGS } \\
\text { station } \\
\text { no. }\end{array}$ & Latitude & Longitude & $\begin{array}{c}\text { Altitude } \\
\text { of land } \\
\text { surface } \\
\text { (feet above } \\
\text { sea level) }\end{array}$ & $\begin{array}{l}\text { Data } \\
\text { type }\end{array}$ & $\begin{array}{l}\text { Equipment } \\
\text { type }\end{array}$ & $\begin{array}{c}\text { Data } \\
\text { on page(s) }\end{array}$ \\
\hline \multicolumn{8}{|c|}{$\begin{array}{c}\text { Well sites } \\
\text { (Location map on page 16) }\end{array}$} \\
\hline $\begin{array}{l}2 \mathrm{~A} \\
27 \mathrm{R} 1 \\
28 \mathrm{P} 1 \\
30 \mathrm{~N} 2 \\
31 \mathrm{P} 1\end{array}$ & $\begin{array}{l}374315118441201 \\
374420118515501 \\
374420118532501 \\
374420118493301 \\
374332118491001\end{array}$ & $\begin{array}{l}37^{\circ} 43^{\prime} 15^{\prime \prime} \\
37^{\circ} 44^{\prime} 20^{\prime \prime} \\
37^{\circ} 44^{\prime} 20^{\prime \prime} \\
37^{\circ} 44^{\prime} 20^{\prime \prime} \\
37^{\circ} 43^{\prime} 32^{\prime \prime}\end{array}$ & $\begin{array}{l}118^{\circ} 44^{\prime} 12^{\prime \prime} \\
118^{\circ} 51^{\prime} 55^{\prime \prime} \\
118^{\circ} 53^{\prime} 25^{\prime \prime} \\
118^{\circ} 49^{\prime} 33^{\prime \prime} \\
118^{\circ} 49^{\prime} 10^{\prime \prime}\end{array}$ & $\begin{array}{l}6,922 \\
7,039.18 \\
7,109.02 \\
6,913.21 \\
6,914.01\end{array}$ & $\begin{array}{l}\text { L } \\
\text { L } \\
\text { L } \\
\text { L } \\
\text { L }\end{array}$ & & $\begin{array}{l}92 \\
92 \\
92 \\
93 \\
93\end{array}$ \\
\hline $\begin{array}{l}31 \mathrm{P} 2 \\
35 \mathrm{~N} 1 \\
36 \mathrm{P} 1 \\
\mathrm{AP} \\
\mathrm{CD}-2\end{array}$ & $\begin{array}{l}374332118491002 \\
374327118445901 \\
374335118434101 \\
373737118501701 \\
373847118545802\end{array}$ & $\begin{array}{l}37^{\circ} 43^{\prime} 32^{\prime \prime} \\
37^{\circ} 43^{\prime} 27^{\prime \prime} \\
37^{\circ} 43^{\prime} 35^{\prime \prime} \\
37^{\circ} 37^{\prime} 37^{\prime \prime} \\
37^{\circ} 38^{\prime} 47^{\prime \prime}\end{array}$ & $\begin{array}{l}118^{\circ} 49^{\prime} 10^{\prime \prime} \\
118^{\circ} 44^{\prime} 59^{\prime \prime} \\
118^{\circ} 43^{\prime} 41^{\prime \prime} \\
118^{\circ} 50^{\prime} 17^{\prime \prime} \\
118^{\circ} 54^{\prime} 58^{\prime \prime}\end{array}$ & $\begin{array}{l}6,915.7 \\
6,914 \\
6,995 \\
7,120 \\
7,316\end{array}$ & $\begin{array}{l}\text { L } \\
\text { L } \\
\text { L } \\
\text { L } \\
\text { L }\end{array}$ & & $\begin{array}{l}93 \\
94 \\
94 \\
94 \\
95\end{array}$ \\
\hline $\begin{array}{l}\mathrm{CH}-1 \\
\mathrm{CH}-10 \\
\mathrm{CH}-10 \mathrm{~A} \\
\mathrm{CH}-10 \mathrm{~B} \\
\mathrm{CH}-3\end{array}$ & $\begin{array}{l}374045118491001 \\
373932118491501 \\
373930118491601 \\
373930118491602 \\
373929118455405\end{array}$ & $\begin{array}{l}37^{\circ} 40^{\prime} 45^{\prime \prime} \\
37^{\circ} 39^{\prime} 32^{\prime \prime} \\
37^{\circ} 39^{\prime} 30^{\prime \prime} \\
37^{\circ} 39^{\prime} 30^{\prime \prime} \\
37^{\circ} 39^{\prime} 29^{\prime \prime}\end{array}$ & $\begin{array}{l}118^{\circ} 49^{\prime} 10^{\prime \prime} \\
118^{\circ} 49^{\prime} 15^{\prime \prime} \\
118^{\circ} 49^{\prime} 16^{\prime \prime} \\
118^{\circ} 49^{\prime} 16^{\prime \prime} \\
118^{\circ} 45^{\prime} 54^{\prime \prime}\end{array}$ & $\begin{array}{l}6,969.93 \\
7,074.88 \\
7,078.80 \\
7,078.99 \\
6,870\end{array}$ & $\begin{array}{l}\text { L,LD } \\
\text { L } \\
\text { L } \\
\text { L,LD,TP } \\
\text { L }\end{array}$ & PT & $\begin{array}{l}33,95 \\
96 \\
96 \\
76,97 \\
97\end{array}$ \\
\hline $\begin{array}{l}\mathrm{CH}-5 \\
\mathrm{CH}-6 \\
\text { CH-7 } \\
\text { CHURCH } \\
\text { CM-2 }\end{array}$ & $\begin{array}{l}373759118474101 \\
374040118522501 \\
374000118483102 \\
373707118491701 \\
373906118522301\end{array}$ & $\begin{array}{l}37^{\circ} 37^{\prime} 59^{\prime \prime} \\
37^{\circ} 40^{\prime} 40^{\prime \prime} \\
37^{\circ} 40^{\prime} 00^{\prime \prime} \\
37^{\circ} 37^{\prime} 07^{\prime \prime} \\
37^{\circ} 39^{\prime} 06^{\prime \prime}\end{array}$ & $\begin{array}{l}118^{\circ} 47^{\prime} 41^{\prime \prime} \\
118^{\circ} 52^{\prime} 25^{\prime \prime} \\
118^{\circ} 48^{\prime} 31^{\prime \prime} \\
118^{\circ} 49^{\prime} 17^{\prime \prime} \\
118^{\circ} 52^{\prime} 23^{\prime \prime}\end{array}$ & $\begin{array}{l}6,918 \\
7,248.23 \\
6,956.89 \\
7,035 \\
7,077\end{array}$ & $\begin{array}{l}\text { L } \\
\text { L } \\
\text { L } \\
\text { L } \\
\text { L,LD,TP }\end{array}$ & DR & $\begin{array}{l}98 \\
98 \\
98 \\
99 \\
58,99\end{array}$ \\
\hline $\begin{array}{l}\text { CR } \\
\text { CT } \\
\text { CW-1 } \\
\text { CW-3 } \\
\text { DC }\end{array}$ & $\begin{array}{l}373816118532001 \\
374511118585801 \\
373850118513601 \\
373849118513001 \\
374410118583401\end{array}$ & $\begin{array}{l}37^{\circ} 38^{\prime} 16^{\prime \prime} \\
37^{\circ} 45^{\prime} 11^{\prime \prime} \\
37^{\circ} 38^{\prime} 50^{\prime \prime} \\
37^{\circ} 38^{\prime} 49^{\prime \prime} \\
37^{\circ} 44^{\prime} 10^{\prime \prime}\end{array}$ & $\begin{array}{l}118^{\circ} 53^{\prime} 20^{\prime \prime} \\
118^{\circ} 58^{\prime} 58^{\prime \prime} \\
118^{\circ} 51^{\prime} 36^{\prime \prime} \\
118^{\circ} 51^{\prime} 30^{\prime \prime} \\
118^{\circ} 58^{\prime} 34^{\prime \prime}\end{array}$ & $\begin{array}{l}7,161.76 \\
7,520 \\
7,084.99 \\
7,062.20 \\
7,515.27\end{array}$ & $\begin{array}{l}\mathrm{L} \\
\mathrm{L} \\
\mathrm{L} \\
\mathrm{L}, \mathrm{LD} \\
\mathrm{L}\end{array}$ & GR,SE & $\begin{array}{l}99 \\
100 \\
100 \\
70,101 \\
101\end{array}$ \\
\hline $\begin{array}{l}\text { ESO } \\
\text { LAV-1 } \\
\text { LKT }\end{array}$ & $\begin{array}{l}373818118514502 \\
374051118523301 \\
374300118554401\end{array}$ & $\begin{array}{l}37^{\circ} 38^{\prime} 18^{\prime \prime} \\
37^{\circ} 40^{\prime} 51^{\prime \prime} \\
37^{\circ} 43^{\prime} 00^{\prime \prime}\end{array}$ & $\begin{array}{l}118^{\circ} 51^{\prime} 45^{\prime \prime} \\
118^{\circ} 52^{\prime} 33^{\prime \prime} \\
118^{\circ} 55^{\prime} 44^{\prime \prime}\end{array}$ & $\begin{array}{l}7,090 \\
7,200 \\
7,350\end{array}$ & $\begin{array}{l}\mathrm{L} \\
\mathrm{L} \\
\mathrm{L}, \mathrm{LD}, \mathrm{PD}\end{array}$ & $\mathrm{PT}, \mathrm{SE}$ & $\begin{array}{l}102 \\
102 \\
17,103, \\
256,259\end{array}$ \\
\hline $\begin{array}{l}\text { LV-15 } \\
\text { LV-18 }\end{array}$ & $\begin{array}{l}373913118551401 \\
373918118481901\end{array}$ & $\begin{array}{l}37^{\circ} 39^{\prime} 13^{\prime \prime} \\
37^{\circ} 39^{\prime} 18^{\prime \prime}\end{array}$ & $\begin{array}{l}118^{\circ} 55^{\prime} 14^{\prime \prime} \\
118^{\circ} 48^{\prime} 19^{\prime \prime}\end{array}$ & $\begin{array}{l}7,340 \\
6,966.00\end{array}$ & $\begin{array}{l}\mathrm{L} \\
\mathrm{L}\end{array}$ & & $\begin{array}{l}103 \\
104\end{array}$ \\
\hline
\end{tabular}


Table 1. Descriptions of data-collection sites, Long Valley Caldera, Mono County, California-Continued

\begin{tabular}{|c|c|c|c|c|c|c|c|}
\hline $\begin{array}{c}\text { Station } \\
\text { identifier }\end{array}$ & $\begin{array}{c}\text { USGS } \\
\text { station } \\
\text { no. }\end{array}$ & Latitude & Longitude & $\begin{array}{l}\text { Altitude } \\
\text { of land } \\
\text { surface } \\
\text { (feet above } \\
\text { sea level) } \\
\end{array}$ & $\begin{array}{l}\text { Data } \\
\text { type }\end{array}$ & $\begin{array}{l}\text { Equipment } \\
\text { type }\end{array}$ & $\begin{array}{c}\text { Data } \\
\text { on page(s) }\end{array}$ \\
\hline \multicolumn{8}{|c|}{ Well sites-Continued } \\
\hline $\begin{array}{l}\text { LV-19 } \\
\text { LV-2 } \\
\text { LV-25 } \\
\text { LV-3 } \\
\text { LV-30 }\end{array}$ & $\begin{array}{l}373754118501701 \\
373729118532802 \\
374233118482701 \\
373759118474101 \\
374109118484101\end{array}$ & $\begin{array}{l}37^{\circ} 37^{\prime} 54^{\prime \prime} \\
37^{\circ} 37^{\prime} 29^{\prime \prime} \\
37^{\circ} 42^{\prime} 33^{\prime \prime} \\
37^{\circ} 37^{\prime} 59^{\prime \prime} \\
37^{\circ} 41^{\prime} 09^{\prime \prime}\end{array}$ & $\begin{array}{l}118^{\circ} 50^{\prime} 17^{\prime \prime} \\
118^{\circ} 53^{\prime} 28^{\prime \prime} \\
118^{\circ} 48^{\prime} 27^{\prime \prime} \\
118^{\circ} 47^{\prime} 41^{\prime \prime} \\
118^{\circ} 48^{\prime} 41^{\prime \prime}\end{array}$ & $\begin{array}{l}7,090.40 \\
7,167.39 \\
6,873.81 \\
6,918 \\
6,895.62\end{array}$ & $\begin{array}{l}\text { L } \\
\text { L } \\
\text { L } \\
\text { L } \\
\text { L }\end{array}$ & & $\begin{array}{l}104 \\
105 \\
105 \\
105 \\
106\end{array}$ \\
\hline $\begin{array}{l}\text { LV-44 } \\
\text { LV-45 } \\
\text { MBP-1 } \\
\text { MBP-3 } \\
\text { MW-1 }\end{array}$ & $\begin{array}{l}373917118551402 \\
373914118472201 \\
373849118545501 \\
373840118545001 \\
373816118523901\end{array}$ & $\begin{array}{l}37^{\circ} 39^{\prime} 17^{\prime \prime} \\
37^{\circ} 39^{\prime} 14^{\prime \prime} \\
37^{\circ} 38^{\prime} 49^{\prime \prime} \\
37^{\circ} 38^{\prime} 40^{\prime \prime} \\
37^{\circ} 38^{\prime} 16^{\prime \prime}\end{array}$ & $\begin{array}{l}118^{\circ} 55^{\prime} 14^{\prime \prime} \\
118^{\circ} 47^{\prime} 22^{\prime \prime} \\
118^{\circ} 54^{\prime} 55^{\prime \prime} \\
118^{\circ} 54^{\prime} 50^{\prime \prime} \\
118^{\circ} 52^{\prime} 39^{\prime \prime}\end{array}$ & $\begin{array}{l}7,340 \\
6,985.38 \\
7,343 \\
7,310 \\
7,046.43\end{array}$ & $\begin{array}{l}\text { L } \\
\text { L } \\
Q \\
Q \\
\text { L }\end{array}$ & & $\begin{array}{l}106 \\
106 \\
275 \\
275 \\
107\end{array}$ \\
\hline $\begin{array}{l}\text { MW-2 } \\
\text { MW-4 } \\
\text { MW-5 } \\
\text { MWD 5 } \\
\text { MWD M-10 }\end{array}$ & $\begin{array}{l}373841118512902 \\
373907118503801 \\
373841118512901 \\
373757118591801 \\
373719118585301\end{array}$ & $\begin{array}{l}37^{\circ} 38^{\prime} 41^{\prime \prime} \\
37^{\circ} 39^{\prime} 07^{\prime \prime} \\
37^{\circ} 38^{\prime} 41^{\prime \prime} \\
37^{\circ} 37^{\prime} 57^{\prime \prime} \\
37^{\circ} 37^{\prime} 19^{\prime \prime}\end{array}$ & $\begin{array}{l}118^{\circ} 51^{\prime} 29^{\prime \prime} \\
118^{\circ} 50^{\prime} 38^{\prime \prime} \\
118^{\circ} 51 ' 29^{\prime \prime} \\
118^{\circ} 59^{\prime} 18^{\prime \prime} \\
118^{\circ} 58^{\prime} 53^{\prime \prime}\end{array}$ & $\begin{array}{l}7,057.08 \\
7,081.26 \\
7,054.12 \\
7,985.28 \\
7,939.63\end{array}$ & $\begin{array}{l}\text { L } \\
L \\
L \\
L \\
L\end{array}$ & & $\begin{array}{l}107 \\
107 \\
108 \\
108 \\
108\end{array}$ \\
\hline $\begin{array}{l}\text { MWD M-11 } \\
\text { MWD M-12 } \\
\text { MWD M-14 } \\
\text { PD } \\
\text { PLV-2 }\end{array}$ & $\begin{array}{l}373705118585601 \\
373714118585501 \\
373739118582101 \\
373732118490001 \\
374249118584501\end{array}$ & $\begin{array}{l}37^{\circ} 37^{\prime} 05^{\prime \prime} \\
37^{\circ} 37^{\prime} 14^{\prime \prime} \\
37^{\circ} 37^{\prime} 39^{\prime \prime} \\
37^{\circ} 37^{\prime} 32^{\prime \prime} \\
37^{\circ} 42^{\prime} 49^{\prime \prime}\end{array}$ & $\begin{array}{l}118^{\circ} 58^{\prime} 56^{\prime \prime} \\
118^{\circ} 58^{\prime} 55^{\prime \prime} \\
118^{\circ} 58^{\prime} 21^{\prime \prime} \\
118^{\circ} 49^{\prime} 00^{\prime \prime} \\
118^{\circ} 58^{\prime} 45^{\prime \prime}\end{array}$ & $\begin{array}{l}7,968.56 \\
7,952.92 \\
7,877.79 \\
7,014 \\
7,734\end{array}$ & $\begin{array}{l}\text { L } \\
L \\
L \\
L \\
L\end{array}$ & & $\begin{array}{l}109 \\
109 \\
17,110 \\
110 \\
110\end{array}$ \\
\hline $\begin{array}{l}\text { RDO-8 } \\
\text { SC-1 } \\
\text { SC-2 } \\
\text { SNARL } \\
\text { SQ }\end{array}$ & $\begin{array}{l}373924118571201 \\
373745118554001 \\
373745118554002 \\
373654118495401 \\
373742118515703\end{array}$ & $\begin{array}{l}37^{\circ} 39^{\prime} 24^{\prime \prime} \\
37^{\circ} 37^{\prime} 45^{\prime \prime} \\
37^{\circ} 37^{\prime} 45^{\prime \prime} \\
37^{\circ} 36^{\prime} 54^{\prime \prime} \\
37^{\circ} 37^{\prime} 42^{\prime \prime}\end{array}$ & $\begin{array}{l}118^{\circ} 57^{\prime} 14^{\prime \prime} \\
118^{\circ} 55^{\prime} 40^{\prime \prime} \\
118^{\circ} 55^{\prime} 40^{\prime \prime} \\
118^{\circ} 49^{\prime} 54^{\prime \prime} \\
118^{\circ} 51^{\prime} 57^{\prime \prime}\end{array}$ & $\begin{array}{l}7,788.4 \\
7,469.84 \\
7,470.65 \\
7,095 \\
7,102.22\end{array}$ & $\begin{array}{l}\text { L,LD,TP } \\
\text { L } \\
\text { L } \\
\text { L } \\
\text { L }\end{array}$ & PT & $\begin{array}{l}46,111 \\
112 \\
32,112 \\
113 \\
113\end{array}$ \\
\hline $\begin{array}{l}\text { SS-1 } \\
\text { SS-2 }\end{array}$ & $\begin{array}{l}373818118533201 \\
373818118533202 \\
\end{array}$ & $\begin{array}{l}37^{\circ} 38^{\prime} 18^{\prime \prime} \\
37^{\circ} 38^{\prime} 18^{\prime \prime} \\
\end{array}$ & $\begin{array}{l}118^{\circ} 53^{\prime} 32^{\prime \prime} \\
118^{\circ} 53^{\prime} 32^{\prime \prime}\end{array}$ & $\begin{array}{l}7,180.61 \\
7,179.13 \\
\end{array}$ & $\begin{array}{l}\mathrm{L} \\
\mathrm{L}\end{array}$ & & $\begin{array}{l}114 \\
114 \\
\end{array}$ \\
\hline \multicolumn{8}{|c|}{$\begin{array}{c}\text { Spring sites } \\
\text { (Location map on pages } 115 \text { and 261) }\end{array}$} \\
\hline $\begin{array}{l}\text { BAL } \\
\text { BS } \\
\text { CS } \\
\text { FHAB-UPPER } \\
\text { FHAB-WEIR }\end{array}$ & $\begin{array}{l}374010118465301 \\
374501118561501 \\
373821118531901 \\
373819118514901 \\
373822118514401\end{array}$ & $\begin{array}{l}37^{\circ} 40^{\prime} 10^{\prime \prime} \\
37^{\circ} 45^{\prime} 01^{\prime \prime} \\
37^{\circ} 38^{\prime} 21^{\prime \prime} \\
37^{\circ} 38^{\prime} 19^{\prime \prime} \\
37^{\circ} 38^{\prime} 22^{\prime \prime}\end{array}$ & $\begin{array}{l}118^{\circ} 46^{\prime} 53^{\prime \prime} \\
118^{\circ} 56^{\prime} 15^{\prime \prime} \\
118^{\circ} 53^{\prime} 19^{\prime \prime} \\
118^{\circ} 51^{\prime} 49^{\prime \prime} \\
118^{\circ} 51^{\prime} 44^{\prime \prime}\end{array}$ & $\begin{array}{l}6,880 \\
7,230 \\
7,200 \\
7,051 \\
7,050\end{array}$ & $\begin{array}{l}\text { F,T,Q } \\
Q \\
\text { FD,Q } \\
\text { TD } \\
\text { FD,TD,Q }\end{array}$ & $\begin{array}{l}\text { W } \\
\text { GR,W } \\
\text { T } \\
\text { DR,SE,T }\end{array}$ & $\begin{array}{l}208,277 \\
277 \\
182,268 \\
128 \\
120,138 \\
268,285\end{array}$ \\
\hline FHCD-Q & 373818118513301 & $37^{\circ} 38^{\prime} 18^{\prime \prime}$ & $118^{\circ} 51^{\prime} 33^{\prime \prime}$ & 7,050 & $\mathrm{~F}, \mathrm{Q}$ & & $\begin{array}{l}141,268 \\
284\end{array}$ \\
\hline FHCD-T & 373817118514101 & $37^{\circ} 38^{\prime} 17^{\prime \prime}$ & $118^{\circ} 51^{\prime} 41^{\prime \prime}$ & 7,053 & TD & $\mathrm{T}$ & 142 \\
\hline & 373829118505801 & $37^{\circ} 38^{\prime} 29^{\prime \prime}$ & $118^{\circ} 50^{\prime} 56^{\prime \prime}$ & 7,050 & FD & $\begin{array}{l}\text { DR,GR, } \\
\text { SE,W }\end{array}$ & 154 \\
\hline FH23-T & 373829118505801 & $37^{\circ} 38^{\prime} 29^{\prime \prime}$ & $118^{\circ} 50^{\prime} 58^{\prime \prime}$ & 7,065 & $\mathrm{TD}, \mathrm{Q}$ & $\mathrm{T}$ & $\begin{array}{l}169,271 \\
285\end{array}$ \\
\hline HBP & 373850118513501 & $37^{\circ} 38^{\prime} 50^{\prime \prime}$ & $118^{\circ} 51^{\prime} 35^{\prime \prime}$ & 7,070 & $\mathrm{SD}, \mathrm{TD}, \mathrm{Q}$ & PT,T & $\begin{array}{l}189,200 \\
274\end{array}$ \\
\hline $\mathrm{HC}-3$ & 373942118494201 & $37^{\circ} 39^{\prime} 42^{\prime \prime}$ & $118^{\circ} 49^{\prime} 42^{\prime \prime}$ & 6,960 & Q & & 274,286 \\
\hline $\mathrm{HC}-4$ & 373941118494301 & $37^{\circ} 39^{\prime} 41^{\prime \prime}$ & $118^{\circ} 49^{\prime} 37^{\prime \prime}$ & 6,960 & Q & & 286 \\
\hline HC-5L & 373940118494001 & $37^{\circ} 39^{\prime} 40^{\prime \prime}$ & $118^{\circ} 49^{\prime} 41^{\prime \prime}$ & 6,970 & $\mathrm{Q}$ & & 274,286 \\
\hline $\begin{array}{l}\text { LHC-SP } \\
\text { LS }\end{array}$ & $\begin{array}{l}374125118502501 \\
373701118533701\end{array}$ & $\begin{array}{l}37^{\circ} 41^{\prime} 25^{\prime \prime} \\
37^{\circ} 37^{\prime} 01^{\prime \prime}\end{array}$ & $\begin{array}{l}118^{\circ} 50^{\prime} 25^{\prime \prime} \\
118^{\circ} 53^{\prime} 37^{\prime \prime}\end{array}$ & $\begin{array}{l}7,035 \\
7,200\end{array}$ & $\begin{array}{l}\text { FD,Q } \\
\text { F.FD }\end{array}$ & $\begin{array}{l}\text { GR,W } \\
\text { GR,W }\end{array}$ & $\begin{array}{l}206 \\
116\end{array}$ \\
\hline
\end{tabular}


Table 1. Descriptions of data-collection sites, Long Valley Caldera, Mono County, California-Continued

\begin{tabular}{|c|c|c|c|c|c|c|c|}
\hline $\begin{array}{c}\text { Station } \\
\text { identifier }\end{array}$ & $\begin{array}{c}\text { USGS } \\
\text { station } \\
\text { no. }\end{array}$ & Latitude & Longitude & $\begin{array}{l}\text { Altitude } \\
\text { of land } \\
\text { surface } \\
\text { (feet above } \\
\text { sea level) }\end{array}$ & $\begin{array}{l}\text { Data } \\
\text { type }\end{array}$ & $\begin{array}{l}\text { Equipment } \\
\text { type }\end{array}$ & $\begin{array}{c}\text { Data } \\
\text { on page(s) }\end{array}$ \\
\hline \multicolumn{8}{|c|}{$\begin{array}{c}\text { Stream sites } \\
\text { (Location map on page 209) }\end{array}$} \\
\hline$\overline{\mathrm{HCA}}$ & 10265147 & $37^{\circ} 39^{\prime} 27^{\prime \prime}$ & $118^{\circ} 49^{\prime} 54^{\prime \prime}$ & 7,035 & $F, Q$ & & $\begin{array}{l}211,265 \\
280\end{array}$ \\
\hline $\mathrm{HCF}$ & 10265150 & $37^{\circ} 40^{\prime} 08^{\prime \prime}$ & $118^{\circ} 49^{\prime} 00^{\prime \prime}$ & 6,950 & $\begin{array}{l}\text { FD,TD, } \\
\text { CD,Q }\end{array}$ & $\begin{array}{l}\text { DR,SE, } \\
\text { T,C,W }\end{array}$ & $\begin{array}{l}213,228 \\
232,265 \\
282\end{array}$ \\
\hline LHC-SW & 10265160 & $37^{\circ} 41^{\prime} 25^{\prime \prime}$ & $118^{\circ} 50^{\prime} 31^{\prime \prime}$ & 6,990 & $\mathrm{FD}, \mathrm{Q}$ & $\mathrm{DR}, \mathrm{W}$ & 235 \\
\hline MC395 & 10265130 & $37^{\circ} 38^{\prime} 16^{\prime \prime}$ & $118^{\circ} 54^{\prime} 10^{\prime \prime}$ & 7,200 & $\mathrm{~F}, \mathrm{Q}$ & & 210,262 \\
\hline MCAF & 10265143 & $37^{\circ} 38^{\prime} 37^{\prime \prime}$ & $118^{\circ} 51^{\prime} 11^{\prime \prime}$ & 7,052 & $F, Q$ & & 210,262 \\
\hline MCF & 10265140 & $37^{\circ} 38^{\prime} 26^{\prime \prime}$ & $118^{\circ} 53^{\prime} 58^{\prime \prime}$ & 7,193 & $\mathrm{~F}, \mathrm{Q}$ & & 210,262 \\
\hline \multicolumn{8}{|c|}{$\begin{array}{c}\text { Fumarole sites } \\
\text { (Location map on page 244) }\end{array}$} \\
\hline BSF & 373843118554601 & $37^{\circ} 38^{\prime} 43^{\prime \prime}$ & $118^{\circ} 55^{\prime} 46^{\prime \prime}$ & 7,515 & $\mathrm{~T}, \mathrm{TD}$ & $T$ & 252,254 \\
\hline MMF & 373819119014201 & $37^{\circ} 38^{\prime} 19^{\prime \prime}$ & $119^{\circ} 01^{\prime} 42^{\prime \prime}$ & 9,900 & $\mathrm{TD}$ & $\mathrm{T}$ & 245 \\
\hline \multicolumn{8}{|c|}{$\begin{array}{c}\text { Precipitation sites } \\
\text { (Location map on page } 255 \text { ) }\end{array}$} \\
\hline LHC-P & 374125118503101 & $37^{\circ} 41^{\prime} 25^{\prime \prime}$ & $118^{\circ} 50^{\prime} 31^{\prime \prime}$ & 6,990 & PD & WB & 256 \\
\hline LKT-P & 374300118554401 & $37^{\circ} 43^{\prime} 00^{\prime \prime}$ & $118^{\circ} 55^{\prime} 44^{\prime \prime}$ & 7,350 & PD & TB & 256,259 \\
\hline MRS & none & $37^{\circ} 38^{\prime} 52^{\prime \prime}$ & $118^{\circ} 57^{\prime} 35^{\prime \prime}$ & 7,800 & PM & WB & 256,260 \\
\hline
\end{tabular}




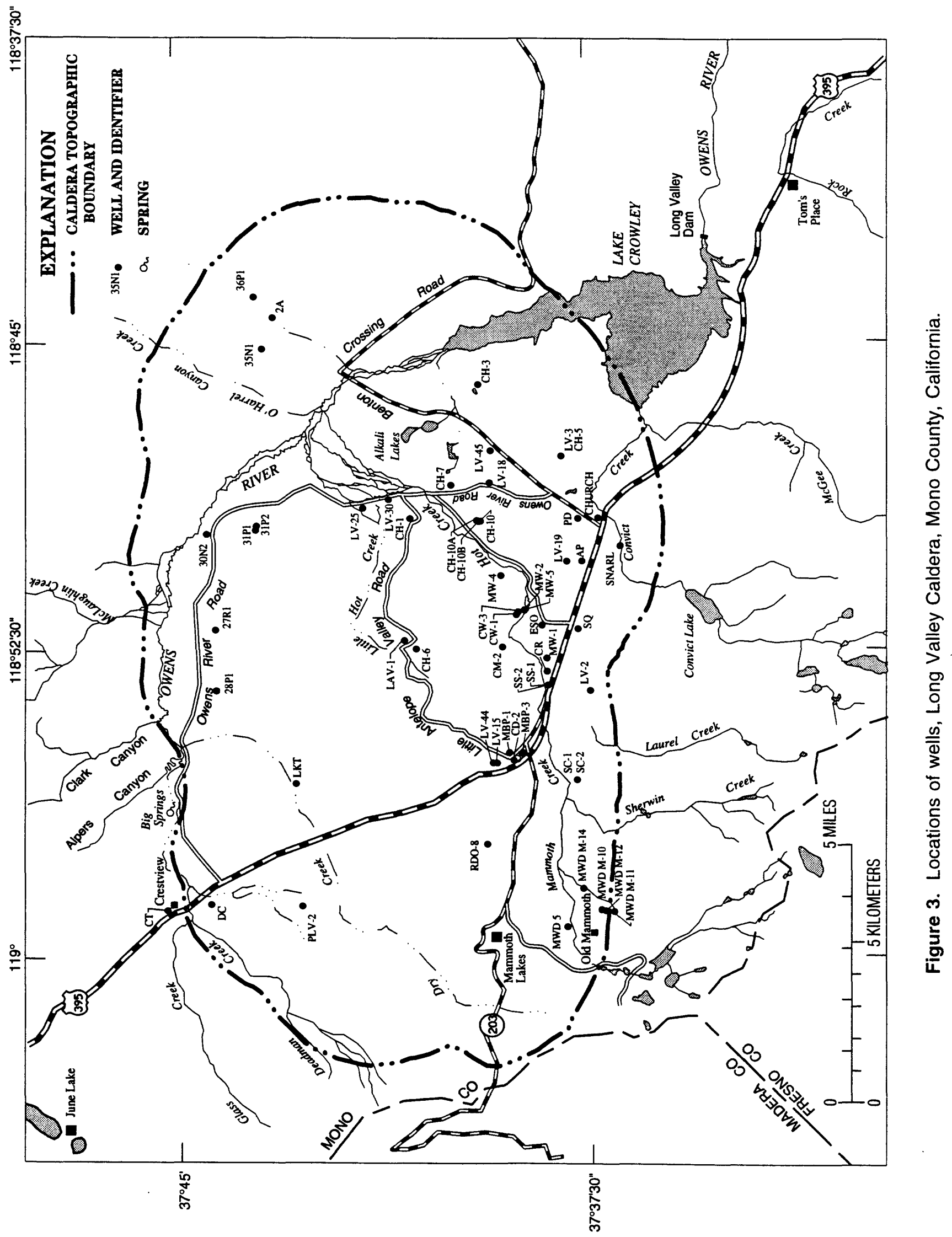




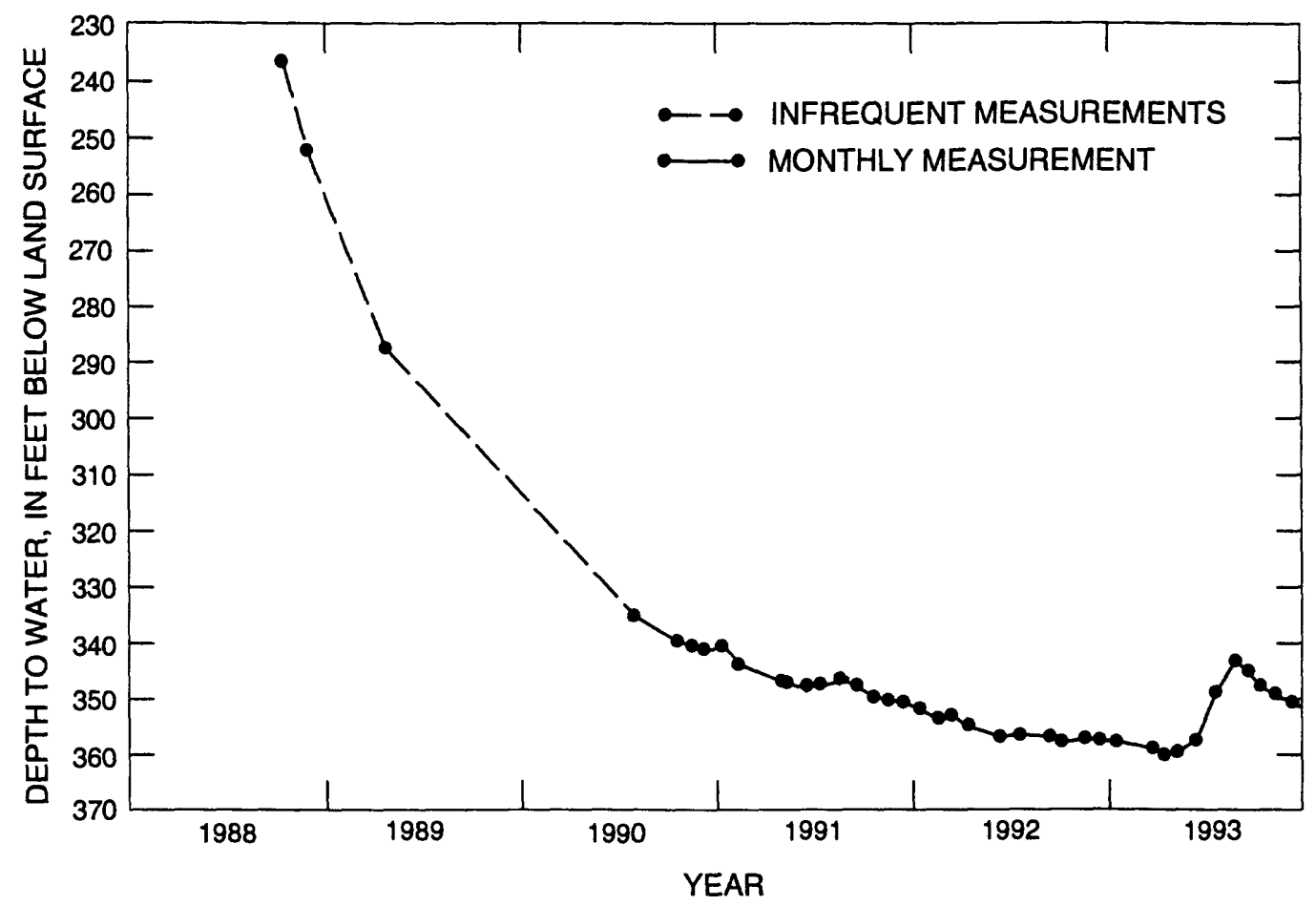

Figure 4. Instantaneous water levels, well MWD M-14, Long Valley Caldera, Mono County, California, 1988-93.

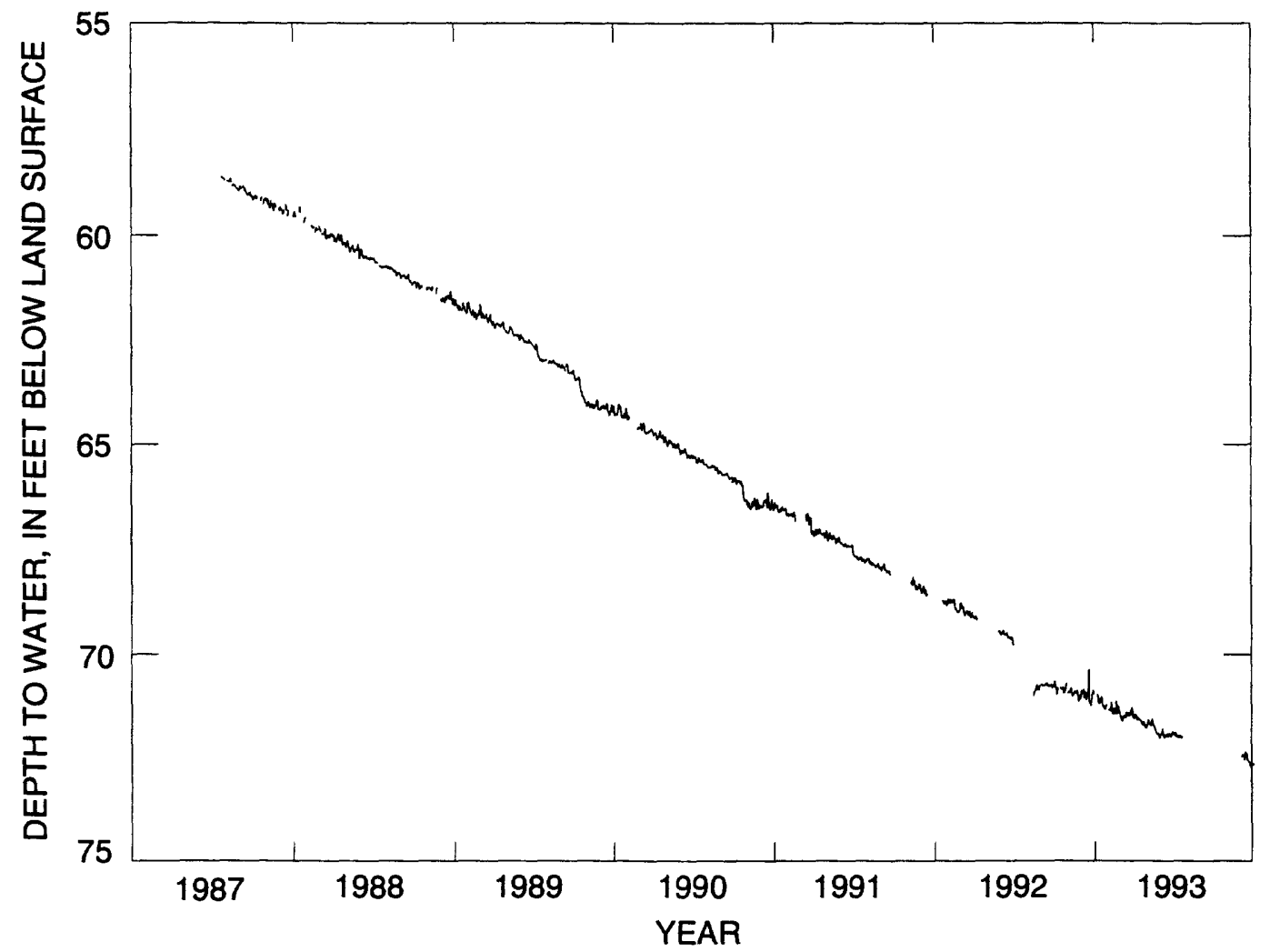

Figure 5. Daily mean water levels, Lookout Mountain well (LKT), Long Valley Caldera, Mono County, California, 1987-93. 


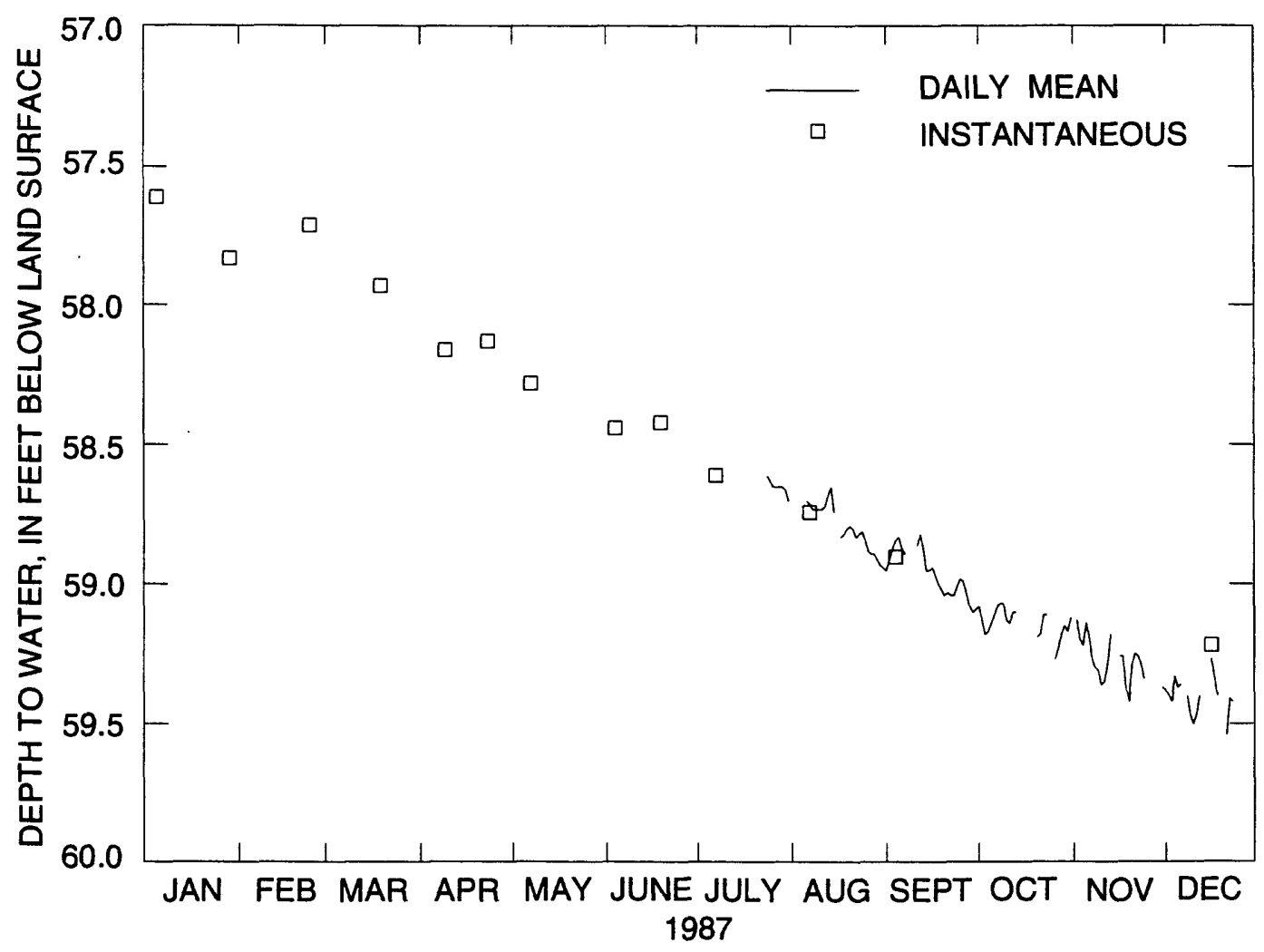

Figure 6. (A) Daily mean and instantaneous water levels, Lookout Mountain well (LKT), Long Valley Caldera, Mono County, California, 1987. 
Table 2. Daily mean water levels, Lookout Mountain well (LKT), Long Valley Caldera, Mono County, California, 1987

[Values are in feet below land surface. Max, maximum; Min, minimum; -, no data]

\begin{tabular}{|c|c|c|c|c|c|c|c|c|c|c|c|c|}
\hline Day & Jan & Feb & Mar & Apr & May & June & July & Aug & Sept & Oct & Nov & Dec \\
\hline 1 & - & - & - & - & - & - & - & - & 58.95 & 59.08 & - & 59.38 \\
\hline 2 & - & - & - & - & - & - & - & - & 58.91 & 59.13 & 59.13 & 59.40 \\
\hline 3 & - & - & - & - & - & - & - & - & 58.87 & 59.18 & 59.20 & 59.42 \\
\hline 4 & - & - & - & - & - & - & - & - & 58.84 & 59.17 & 59.22 & 59.33 \\
\hline 5 & - & - & - & - & - & - & - & - & 58.83 & 59.14 & 59.14 & 59.37 \\
\hline 6 & - & - & - & - & - & - & - & 58.70 & 58.87 & 59.11 & 59.19 & 59.36 \\
\hline 7 & - & - & - & - & - & - & - & 58.71 & 58.89 & 59.08 & 59.27 & - \\
\hline 8 & - & - & - & - & - & - & - & 58.73 & - & 59.07 & 59.30 & 59.40 \\
\hline 9 & - & - & - & - & - & - & - & 58.73 & - & 59.07 & 59.31 & 59.47 \\
\hline 10 & - & - & - & - & - & - & - & 58.73 & - & 59.13 & 59.36 & 59.50 \\
\hline 11 & - & - & - & - & - & - & - & 58.73 & 58.86 & 59.14 & 59.35 & 59.47 \\
\hline 12 & - & - & - & - & - & - & - & 58.72 & 58.82 & 59.10 & 59.29 & 59.40 \\
\hline 13 & - & - & - & - & - & - & - & 58.68 & 58.87 & 59.10 & 59.18 & - \\
\hline 14 & - & - & - & - & - & - & - & 58.65 & 58.95 & - & - & - \\
\hline 15 & - & - & - & - & - & - & - & 58.74 & 58.95 & 59.13 & - & - \\
\hline 16 & - & - & - & - & - & - & - & - & 58.94 & - & 59.26 & 59.27 \\
\hline 17 & - & - & - & - & - & - & - & 58.83 & 58.97 & 59.21 & 59.26 & 59.33 \\
\hline 18 & - & - & - & - & - & - & - & 58.82 & 59.00 & - & 59.37 & 59.40 \\
\hline 19 & - & - & - & - & - & - & - & 58.80 & 59.02 & - & 59.42 & - \\
\hline 20 & - & - & - & - & - & - & - & 58.79 & 59.04 & 59.19 & 59.29 & - \\
\hline 21 & - & - & - & - & - & - & - & 58.80 & 59.03 & 59.18 & .59 .25 & 59.54 \\
\hline 22 & - & - & - & - & - & - & - & 58.83 & 59.04 & 59.11 & 59.26 & 59.41 \\
\hline 23 & - & - & - & - & - & - & - & 58.82 & 59.04 & 59.11 & 59.29 & 59.42 \\
\hline 24 & - & - & - & - & - & - & 58.61 & 58.81 & 59.01 & - & 59.34 & - \\
\hline 25 & - & - & - & - & - & - & 58.63 & 58.84 & 58.98 & - & - & - \\
\hline 26 & - & - & - & - & - & - & 58.65 & 58.88 & 58.99 & 59.27 & - & - \\
\hline 27 & - & - & - & - & - & - & 58.65 & 58.89 & 59.03 & 59.23 & - & - \\
\hline 28 & - & - & - & - & - & - & 58.65 & 58.89 & 59.08 & 59.18 & - & 59.49 \\
\hline 29 & - & - & - & - & - & - & 58.65 & 58.91 & 59.10 & 59.15 & - & - \\
\hline 30 & - & - & - & - & - & - & 58.66 & 58.93 & 59.09 & 59.17 & 59.37 & - \\
\hline 31 & - & - & - & - & - & - & 58.70 & 58.94 & - & 59.12 & - & - \\
\hline Mean & - & - & - & - & - & - & 58.65 & 58.80 & 58.96 & 59.14 & 59.27 & 59.41 \\
\hline $\operatorname{Max}$ & - & - & - & - & - & - & 58.70 & 58.94 & 59.10 & 59.27 & 59.42 & 59.54 \\
\hline Min & - & - & - & - & - & - & 58.61 & 58.65 & 58.82 & 59.07 & 59.13 & 59.27 \\
\hline
\end{tabular}




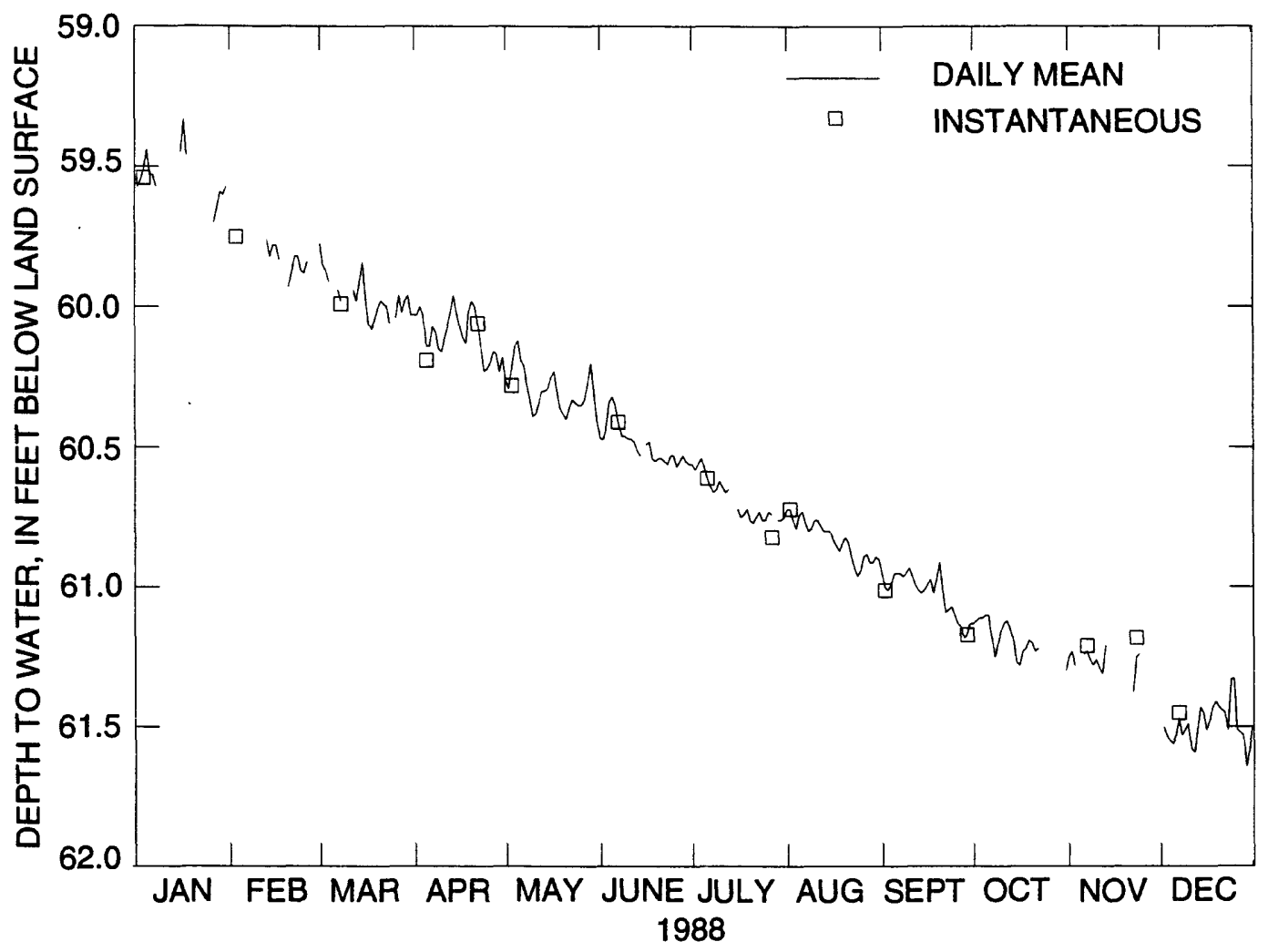

Figure 6. (B) Daily mean and instantaneous water levels, Lookout Mountain well (LKT), Long Valley Caldera, Mono County, California, 1988. 
Table 3. Daily mean water levels, Lookout Mountain well (LKT), Long Valley Caldera, Mono County, California, 1988

[Values are in feet below land surface. Max, maximum; Min, minimum; - , no data]

\begin{tabular}{|c|c|c|c|c|c|c|c|c|c|c|c|c|}
\hline Day & Jan & Feb & Mar & Apr & May & June & July & Aug & Sept & Oct & Nov & Dec \\
\hline 1 & - & - & 59.77 & 60.03 & 60.26 & 60.47 & 60.56 & 60.72 & 60.95 & 61.13 & 61.25 & - \\
\hline 2 & 59.57 & - & 59.85 & 60.03 & 60.29 & 60.47 & 60.58 & 60.72 & 61.00 & 61.12 & 61.23 & 61.50 \\
\hline 3 & 59.55 & - & 59.87 & 60.00 & 60.22 & 60.43 & 60.56 & 60.76 & 61.01 & 61.11 & 61.28 & 61.53 \\
\hline 4 & 59.51 & - & 59.91 & 60.03 & 60.14 & 60.34 & 60.54 & 60.79 & 60.99 & 61.11 & - & 61.55 \\
\hline 5 & 59.44 & - & - & 60.14 & 60.12 & 60.32 & 60.57 & 60.74 & 60.95 & 61.10 & - & 61.56 \\
\hline 6 & 59.53 & - & - & 60.14 & 60.19 & 60.35 & 60.61 & 60.73 & 60.95 & 61.10 & 61.24 & 61.53 \\
\hline 7 & 59.53 & - & 59.94 & 60.07 & 60.21 & 60.41 & 60.64 & 60.77 & 60.95 & 61.18 & 61.23 & 61.47 \\
\hline 8 & 59.57 & - & 59.98 & 60.09 & 60.28 & 60.46 & 60.66 & 60.80 & 60.96 & 61.25 & 61.26 & 61.53 \\
\hline 9 & - & - & - & 60.15 & 60.33 & 60.46 & 60.65 & 60.79 & 60.95 & 61.20 & 61.28 & 61.51 \\
\hline 10 & - & 59.86 & - & 60.16 & 60.39 & 60.47 & 60.62 & 60.76 & 60.93 & 61.16 & 61.26 & 61.49 \\
\hline 11 & - & - & - & 60.11 & 60.38 & 60.47 & 60.64 & 60.76 & 60.96 & 61.13 & 61.29 & 61.58 \\
\hline 12 & - & - & 59.94 & 60.07 & 60.34 & 60.48 & 60.66 & 60.78 & 60.99 & 61.12 & 61.31 & 61.59 \\
\hline 13 & - & 59.76 & 59.98 & 60.02 & 60.30 & 60.51 & 60.65 & 60.80 & 61.01 & 61.15 & 61.21 & 61.52 \\
\hline 14 & 59.67 & 59.82 & 59.91 & 59.96 & 60.30 & 60.53 & - & 60.80 & 61.02 & 61.19 & - & 61.43 \\
\hline 15 & - & 59.78 & 59.84 & 60.02 & 60.29 & - & - & 60.80 & 61.01 & 61.27 & - & 61.45 \\
\hline 16 & 59.45 & 59.78 & 59.96 & 60.07 & 60.25 & 60.49 & 60.72 & 60.83 & 60.99 & 61.28 & - & 61.51 \\
\hline 17 & 59.33 & 59.83 & 60.06 & 60.11 & 60.23 & 60.48 & 60.75 & 60.85 & 60 & .23 & 61.26 & 61.48 \\
\hline 18 & 59.46 & - & 60.08 & 60.13 & 60.30 & 60.54 & 60.74 & 60.87 & 61.02 & 61.22 & - & 61.43 \\
\hline 19 & - & - & 60.05 & 60.02 & 60.36 & 60.55 & 60.72 & 60.84 & 60.97 & 61.19 & - & 61.41 \\
\hline 20 & - & 59.93 & 60.01 & 59.98 & 60.38 & 60.54 & 60.76 & 60.82 & 60.91 & 61.20 & - & 61.43 \\
\hline 21 & - & 59.88 & 59.98 & 60.00 & 60.40 & 60. & 60.77 & 60.84 & 61 & 1.23 & - & 61.44 \\
\hline 22 & - & .82 & 59.99 & 60.06 & 60.36 & 60.55 & 60.75 & 60.89 & 61.09 & 61.22 & 61.38 & 61.45 \\
\hline 23 & - & 59.82 & 60.00 & 60.15 & 60.33 & 60.56 & 60.73 & 60.93 & 61.08 & - & 61.25 & 61.51 \\
\hline 24 & - & 59.87 & 60.06 & 60.23 & 60.34 & 60.53 & 60.76 & 60.96 & 61.07 & - & 61.24 & 61.33 \\
\hline 25 & - & 59.88 & - & 60.22 & 60.35 & 60.53 & 60.76 & 60.94 & 61.10 & - & - & 61.33 \\
\hline 26 & - & 59.84 & 60.04 & 60.20 & 60.35 & 60. & 60.73 & 60.89 & 61 & - & - & 61.51 \\
\hline 27 & 59.70 & - & 59.96 & 60.16 & 60.33 & 60.55 & 60.74 & 60.88 & 61.14 & - & 61.49 & 61.52 \\
\hline 28 & 59.65 & - & 60.02 & 60.17 & 60.27 & 60.53 & - & 60.91 & 61.18 & 61.21 & - & 61.53 \\
\hline 29 & 59.59 & - & 59.98 & 60.23 & 60.20 & 60.55 & 60.76 & 60.91 & 61.17 & - & - & 61.64 \\
\hline 30 & 59.60 & - & 59.96 & 60.18 & 60.30 & 60.56 & 60.76 & 60.89 & 61.13 & - & - & 61.58 \\
\hline 31 & 59.57 & - & 60.03 & - & 60.40 & - & 60.75 & 60.90 & - & 61.30 & - & 61.50 \\
\hline Mean & 59.54 & 59.84 & 59.97 & 60.10 & 60.30 & 60.49 & 60.68 & 6 & 61.02 & & 61.28 & 61.49 \\
\hline $\operatorname{Max}$ & 59.70 & 59.93 & 60.08 & 60.23 & 60.40 & 60.57 & 60.77 & 60.96 & 61.18 & 61.30 & 61.49 & 61.64 \\
\hline Min & 59.33 & 59.76 & 59.77 & 59.96 & 60.12 & 60.32 & 60.54 & 60.72 & 60.91 & 61.10 & 61.21 & 61.33 \\
\hline
\end{tabular}




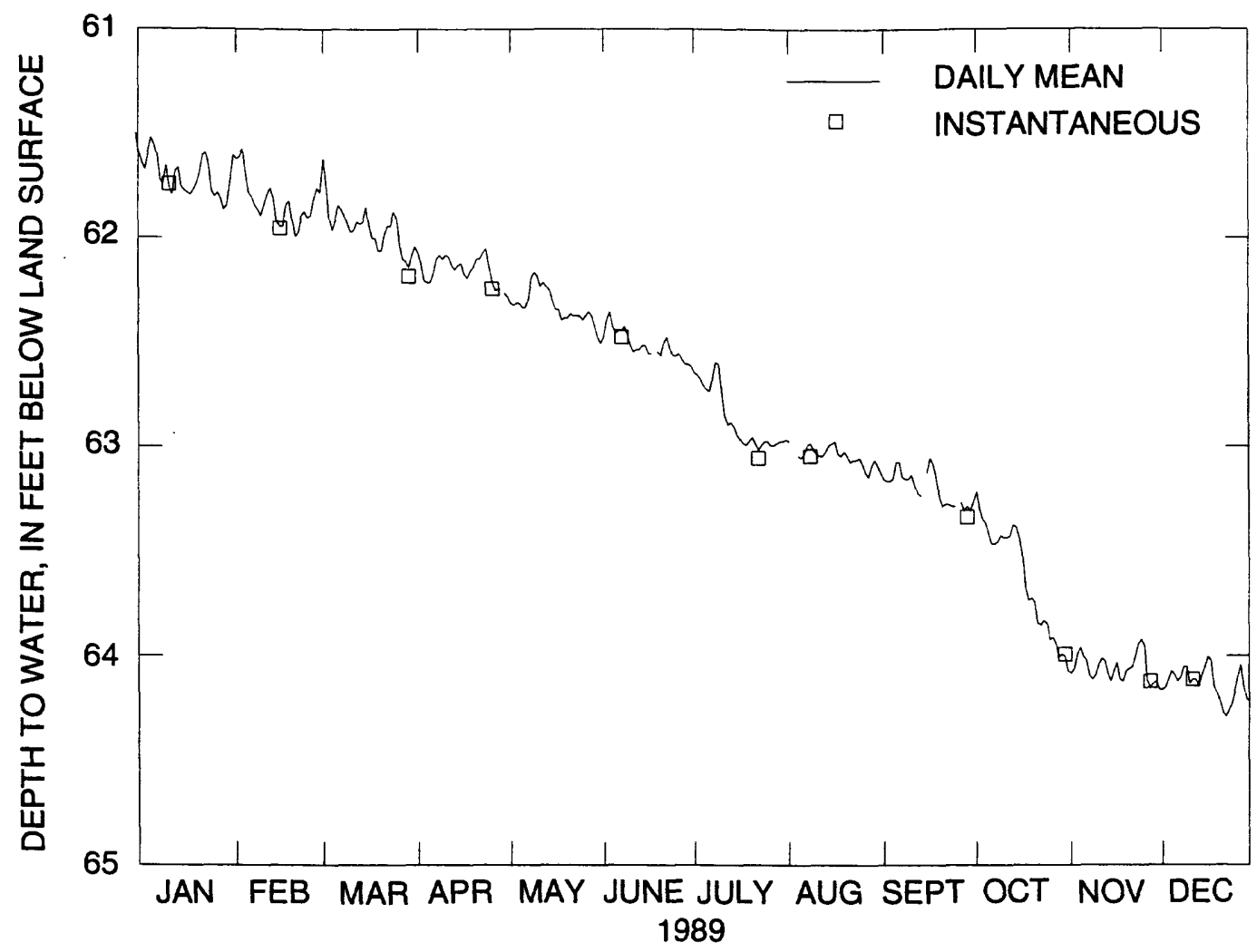

Figure 6. (C) Daily mean and instantaneous water levels, Lookout Mountain well (LKT), Long Valley Caldera, Mono County, California, 1989. 
Table 4. Daily mean water levels, Lookout Mountain well (LKT), Long Valley Caldera, Mono County, California, 1989

[Values are in feet below land surface. Max, maximum; Min, minimum; -, no data]

\begin{tabular}{|c|c|c|c|c|c|c|c|c|c|c|c|c|}
\hline Day & Jan & Feb & Mar & Apr & May & June & July & Aug & Sept & Oct & Nov & Dec \\
\hline 1 & 61.59 & 61.60 & 61.78 & 62.04 & 62.28 & 62.50 & 62.61 & 62.96 & 63.12 & 63.26 & 64.07 & 64.16 \\
\hline 2 & 61.64 & 61.62 & 61.62 & 62.07 & 62.31 & 62.47 & 62.64 & 62.97 & 63.15 & 63.21 & 64.08 & 64.16 \\
\hline 3 & 61.67 & 61.61 & 61.74 & 62.12 & 62.32 & 62.39 & 62.65 & - & 63.16 & 63.29 & 64.06 & 64.15 \\
\hline 4 & 61.59 & 61.57 & 61.90 & 62.20 & 62.31 & 62.35 & 62.67 & - & 63.16 & 63.34 & 63.99 & 64.11 \\
\hline 5 & 61.52 & 61.69 & 61.96 & 62.21 & 62.31 & 62.42 & 62.70 & 63.04 & 63.15 & 63.36 & 63.96 & 64.07 \\
\hline 6 & 61.55 & 61.78 & 61.91 & 62.21 & 62.33 & 62.45 & 62.72 & 63.05 & 63.07 & 63.41 & 64.00 & 64.09 \\
\hline 7 & 61.60 & 61.80 & 61.84 & 62.17 & 62.33 & 62.44 & 62.73 & 63.03 & 63.07 & 63.46 & 64.02 & 64.12 \\
\hline 8 & 61.72 & 61.84 & 61.86 & 62.10 & 62.29 & 62.44 & 62.67 & 62.99 & 63.14 & 63.46 & 64.09 & 64.10 \\
\hline 9 & 61.74 & 61.86 & 61.89 & 62.08 & 62.19 & 62.42 & 62.59 & 62.98 & 63.15 & 63.45 & 64.11 & 64.05 \\
\hline 10 & 61.65 & 61.89 & 61.93 & 62.10 & 62.16 & 62.46 & 62.60 & 63.01 & 63.15 & 63.42 & 64.09 & 64.05 \\
\hline 11 & 61.75 & 61.84 & 61.97 & 62.08 & 62.18 & 62.51 & 62.72 & 63.03 & 63.13 & 63.43 & 64.04 & 64.13 \\
\hline 12 & 61.79 & 61.79 & 61.96 & 62.09 & 62.23 & 62.54 & 62.84 & 63.04 & 63.18 & 63.43 & 64.01 & 64.11 \\
\hline 13 & 61.68 & 61.76 & 61.92 & 62.13 & 62.21 & 62.53 & 62.89 & 63.04 & 63.22 & 63.42 & 64.02 & 64.11 \\
\hline 14 & 61.66 & 61.81 & 61.93 & 62.15 & 62.23 & 62.53 & 62.88 & 63.02 & 63.23 & 63.37 & 64.08 & 64.14 \\
\hline 15 & 61.75 & 61.92 & 61.92 & 62.13 & 62.25 & 62.51 & 62.90 & 62.99 & - & 63.38 & 64.12 & 64.09 \\
\hline 16 & 61.77 & 61.94 & 61.85 & 62.12 & 62.30 & 62.51 & 62.94 & 62.98 & 63.12 & 63.43 & 64.07 & 64.05 \\
\hline 17 & 61.78 & 61.94 & 61.94 & 62.17 & 62.34 & 62.55 & 62.96 & 62.97 & 63.05 & 63.51 & 64.03 & 64.00 \\
\hline 18 & 61.79 & 61.84 & 62.00 & 62.19 & 62.34 & 62.55 & 62.98 & 63.03 & 63.08 & 63.67 & 64.11 & 64.02 \\
\hline 19 & 61.77 & 61.82 & 62.00 & 62.16 & 62.39 & - & 62.99 & 63.04 & 63.14 & 63.73 & 64.12 & 64.14 \\
\hline 20 & 61.74 & 61.91 & 62.06 & 62.14 & 62.38 & 62.54 & 62.97 & 63.02 & 63.24 & 63.72 & 64.07 & 64.18 \\
\hline 21 & 61.69 & 61.99 & 62.06 & 62.10 & 62.38 & 62.56 & 62.95 & 63.04 & 63.28 & 63.74 & 64.06 & 64.22 \\
\hline 22 & 61.60 & 61.97 & 61.98 & 62.10 & 62.36 & 62.50 & 62.98 & 63.07 & 63.27 & 63.84 & 64.05 & 64.27 \\
\hline 23 & 61.59 & 61.89 & 61.94 & 62.07 & 62.37 & 62.47 & 63.01 & 63.06 & 63.27 & 63.85 & 64.00 & 64.29 \\
\hline 24 & 61.65 & 61.87 & 61.94 & 62.05 & 62.37 & 62.52 & 62.99 & 63.06 & 63.28 & 63.83 & 63.94 & 64.26 \\
\hline 25 & 61.77 & 61.90 & 61.87 & 62.13 & 62.37 & 62.56 & 62.97 & 63.05 & 63.28 & 63.84 & 63.92 & 64.23 \\
\hline 26 & 61.80 & 61.89 & 61.90 & 62.20 & 62.39 & 62.56 & 62.97 & 63.08 & - & 63.92 & 63.95 & 64.17 \\
\hline 27 & 61.78 & 61.81 & 62.03 & 62.25 & 62.37 & 62.55 & 62.99 & 63.12 & 63.26 & 63.91 & 64.12 & 64.10 \\
\hline 28 & 61.81 & 61.76 & 62.10 & 62.24 & 62.35 & 62.58 & 62.99 & 63.14 & 63.30 & 63.94 & 64.15 & 64.04 \\
\hline 29 & 61.86 & - & 62.11 & - & 62.37 & 62.60 & 62.98 & 63.09 & 63.28 & 64.00 & 64.13 & 64.14 \\
\hline 30 & 61.84 & - & 62.14 & 62.26 & 62.42 & 62.60 & 62.97 & 63.06 & 63.30 & 63.99 & 64.12 & 64.21 \\
\hline 31 & 61.73 & - & 62.08 & - & 62.47 & - & 62.97 & 63.09 & - & 64.00 & - & 64.21 \\
\hline Mean & 61.71 & 61.82 & 61.94 & 62.14 & 62.32 & 62.50 & 62.85 & 63.04 & 63.19 & 63.60 & 64.05 & 64.13 \\
\hline $\operatorname{Max}$ & 61.86 & 61.99 & 62.14 & 62.26 & 62.47 & 62.60 & 63.01 & 63.14 & 63.30 & 64.00 & 64.15 & 64.29 \\
\hline Min & 61.52 & 61.57 & 61.62 & 62.04 & 62.16 & 62.35 & 62.59 & 62.96 & 63.05 & 63.21 & 63.92 & 64.00 \\
\hline
\end{tabular}




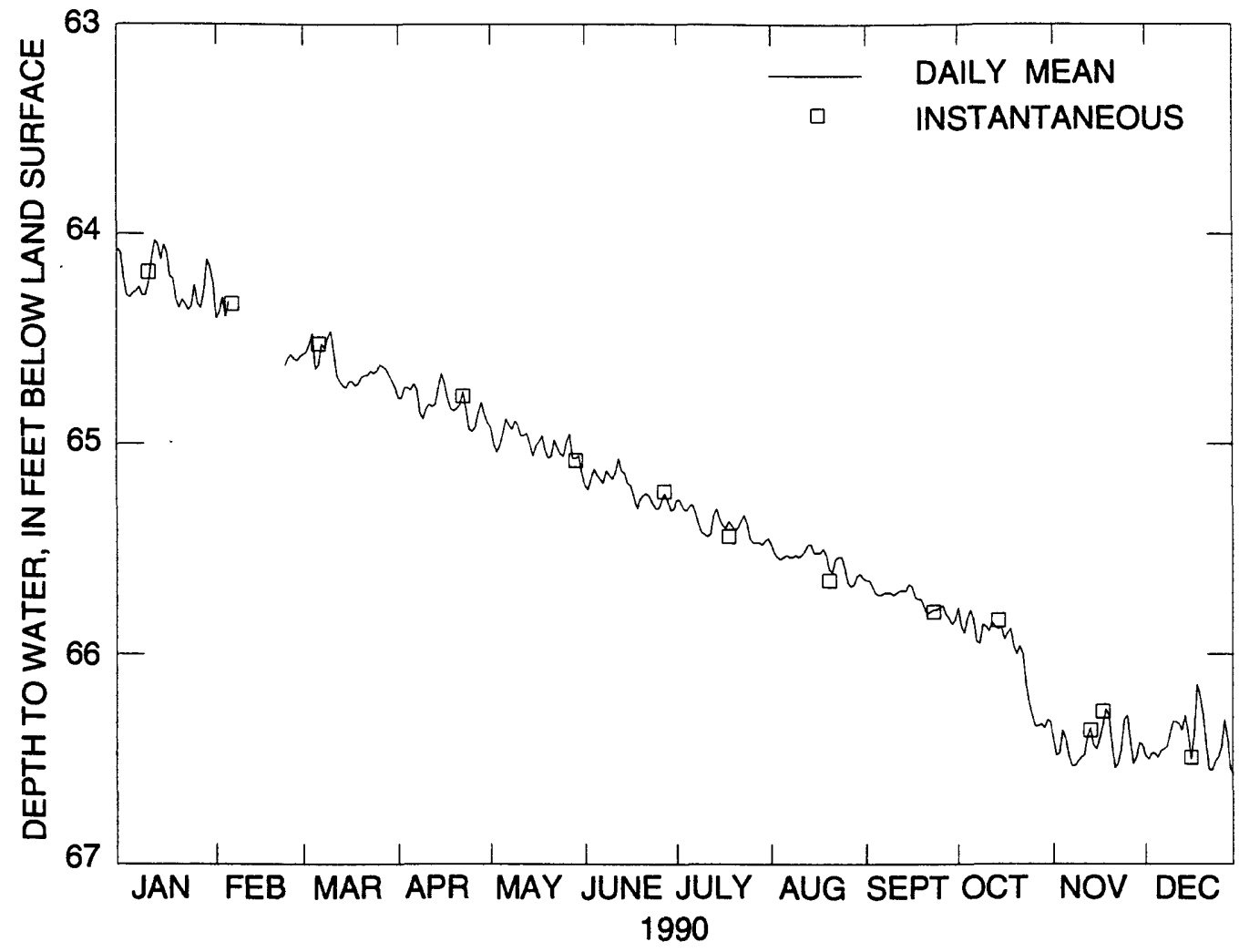

Figure 6. (D) Daily mean and instantaneous water levels, Lookout Mountain well (LKT), Long Valley Caldera, Mono County, California, 1990. 
Table 5. Daily mean water levels, Lookout Mountain well (LKT), Long Valley Caldera, Mono County, California, 1990

[Values are in feet below land surface. Max, maximum; Min. minimum; - , no data]

\begin{tabular}{|c|c|c|c|c|c|c|c|c|c|c|c|c|}
\hline Day & Jan & Feb & Mar & Apr & May & June & July & Aug & Sept & Oct & Nov & Dec \\
\hline 1 & 64.07 & 64.23 & 64.58 & 64.73 & 64.90 & 65.14 & 65.31 & 65.45 & 65.64 & 65.84 & 66.32 & 66.43 \\
\hline 2 & 64.09 & 64.40 & 64.57 & 64.78 & 64.92 & 65.20 & 65.27 & 65.48 & 65.65 & 65.78 & 66.40 & 66.48 \\
\hline 3 & 64.21 & 64.37 & 64.56 & 64.78 & 65.00 & 65.22 & 65.27 & 65.52 & 65.65 & 65.87 & 66.48 & 66.50 \\
\hline 4 & 64.29 & 64.30 & 64.52 & 64.73 & 65.04 & 65.17 & 65.31 & 65.54 & 65.68 & 65.90 & 66.47 & 66.47 \\
\hline 5 & 64.30 & 64.39 & 64.47 & 64.73 & 65.01 & 65.12 & 65.32 & 65.55 & 65.71 & 65.83 & 66.36 & 66.47 \\
\hline 6 & 64.28 & 64.32 & 64.64 & 64.74 & 64.95 & 65.15 & 65.30 & 65.54 & 65.72 & 65.79 & 66.40 & 66.49 \\
\hline 7 & 64.27 & - & 64.62 & 64.71 & 64.88 & 65.17 & 65.29 & 65.53 & 65.72 & 65.84 & 66.48 & 66.46 \\
\hline 8 & 64.25 & - & 64.52 & 64.74 & 64.91 & 65.19 & 65.33 & 65.54 & 65.71 & 65.94 & 66.53 & 66.45 \\
\hline 9 & 64.29 & - & 64.54 & 64.85 & 64.93 & 65.13 & 65.38 & 65.54 & 65.71 & 65.95 & 66.53 & 66.44 \\
\hline 10 & 64.29 & - & 64.49 & 64.88 & 64.89 & 65.15 & 65.42 & 65.53 & 65.71 & 65.86 & 66.51 & 66.38 \\
\hline 11 & 64.23 & - & 64.46 & 64.83 & 64.91 & 65.17 & 65.43 & 65.54 & 65.72 & 65.87 & 66.49 & 66.32 \\
\hline 12 & 64.12 & - & 64.56 & 64.81 & 64.96 & 65.14 & 65.44 & 65.53 & 65.71 & 65.89 & 66.48 & 66.32 \\
\hline 13 & 64.03 & - & 64.67 & 64.82 & 64.96 & 65.07 & 65.43 & 65.51 & 65.70 & 65.85 & 66.39 & 66.33 \\
\hline 14 & 64.05 & - & 64.70 & 64.81 & 64.95 & 65.13 & 65.34 & 65.48 & 65.70 & 65.87 & 66.35 & 66.36 \\
\hline 15 & 64.12 & - & 64.72 & 64.73 & 65.00 & 65.14 & 65.31 & 65.48 & 65.70 & 65.88 & 66.43 & 66.29 \\
\hline 16 & 64.05 & - & 64.73 & 64.66 & 65.06 & 9 & 65.36 & 5.52 & 65.67 & 65.87 & 66.45 & 66.37 \\
\hline 17 & 64.09 & - & 64.70 & 64.71 & 65.01 & 20 & 65.39 & 65.52 & 65.68 & 65.93 & 66.40 & 66.50 \\
\hline 18 & 64.20 & - & 64.70 & 64.78 & 64.99 & 65.25 & 65.40 & 52 & 73 & 65.90 & 66.34 & 66.38 \\
\hline 19 & 64.21 & - & 64.72 & 64.83 & 64.96 & 65.31 & 65.37 & 65.50 & 65.74 & 65.88 & 66.26 & 66.14 \\
\hline 20 & 64.31 & - & 64.71 & 64.84 & 65.03 & 65.27 & 65.39 & 65.53 & 65.74 & 65.96 & 66.29 & 66.20 \\
\hline 21 & 64.35 & - & 64.68 & 64.83 & 65.07 & 65.25 & 65.41 & 65.60 & 65.78 & 66.00 & 66.43 & 66.29 \\
\hline 22 & 64.31 & - & 7 & 64 & 65.06 & 65.24 & 65.40 & 61 & 81 & .96 & 66.54 & 66.43 \\
\hline 23 & 64.33 & - & 64.67 & 64.75 & 64.98 & 65.25 & 65.37 & 65.55 & 65.80 & 66.00 & 66.52 & 66.55 \\
\hline 24 & 64.36 & 64.63 & 64.65 & 64.83 & 65.02 & 65.28 & 65.34 & 65.54 & 65.79 & 66.14 & 66.45 & 66.55 \\
\hline 25 & 64.34 & 64.59 & 64.66 & 64.93 & 65.05 & 65.31 & 65.38 & 65.54 & 65.79 & 66.23 & 66.31 & 66.51 \\
\hline 26 & 64.24 & 64.57 & 64.65 & 64.94 & 65.06 & 65. & 65.45 & 65.59 & 65.78 & 66.29 & 66.29 & 66.49 \\
\hline 27 & 64.33 & 64.59 & 64.62 & 64.92 & 64.99 & 65.28 & 65.47 & 65.66 & 65.77 & 66.34 & 66.41 & 66.44 \\
\hline 28 & 64.35 & 64.60 & 64.63 & 64.85 & 64.95 & 65.24 & 65.47 & 65.68 & 65.81 & 66.34 & 66.52 & 66.31 \\
\hline 29 & 64.27 & - & 64.64 & 64.80 & 65.07 & 65.27 & 65.47 & 65.67 & 65.83 & 66.33 & 66.49 & 66.40 \\
\hline 30 & 64.12 & - & 64.67 & 64.86 & 65.07 & 65.32 & 65.48 & 65.63 & 65.86 & 66.35 & 66.42 & 66.54 \\
\hline 31 & 64.16 & - & 64.70 & - & 65.06 & - & 65.46 & 65.62 & - & 66.31 & - & 66.57 \\
\hline Mean & 64.22 & 64.45 & 64.63 & 64.80 & 64.99 & 65.21 & 65.38 & 65.55 & 65.73 & 65.99 & 66.42 & 66.41 \\
\hline Max & 64.36 & 64.63 & 64.73 & 64.94 & 65.07 & 65.32 & 65.48 & 65.68 & 65.86 & 66.35 & 66.54 & 66.57 \\
\hline Min & 64.03 & 64.23 & 64.46 & 64.66 & 64.88 & 65.07 & 65.27 & 65.45 & 65.64 & 65.78 & 66.26 & 66.14 \\
\hline
\end{tabular}




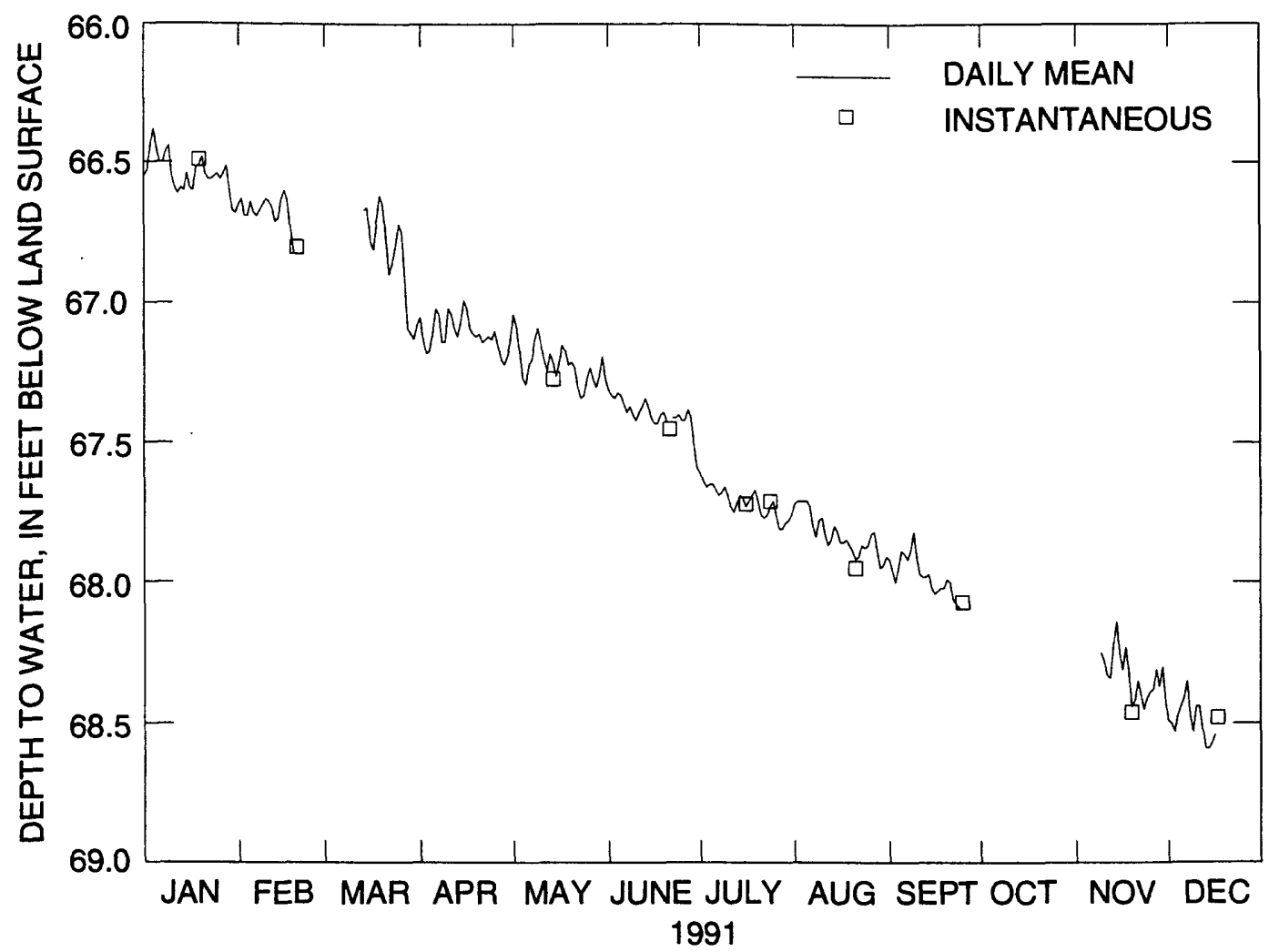

Figure 6. (E) Daily mean and instantaneous water levels, Lookout Mountain well (LKT), Long Valley Caldera, Mono County, California, 1991. 
Table 6. Daily mean water levels, Lookout Mountain well (LKT), Long Valley Caldera, Mono County, California, 1991

[Values are in feet below land surface. Max, maximum; Min, minimum; -, no data]

\begin{tabular}{|c|c|c|c|c|c|c|c|c|c|c|c|c|}
\hline Day & Jan & Feb & Mar & Apr & May & June & July & Aug & Sept & Oct & Nov & Dec \\
\hline 1 & 66.55 & 66.65 & - & 67.05 & 67.04 & 67.31 & 67.61 & 67.72 & 67.92 & - & - & 68.49 \\
\hline 2 & 66.53 & 66.63 & - & 67.13 & 67.08 & 67.33 & 67.64 & 67.71 & 67.96 & - & - & 68.50 \\
\hline 3 & 66.44 & 66.69 & - & 67.18 & 67.17 & 67.34 & 67.66 & 67.71 & 68.00 & - & - & 68.53 \\
\hline 4 & 66.38 & 66.69 & - & 67.17 & 67.27 & 67.32 & 67.65 & 67.71 & 67.95 & - & - & 68.47 \\
\hline 5 & 66.45 & 66.64 & - & 67.11 & 67.29 & 67.33 & 67.65 & 67.71 & 67.89 & - & - & 68.44 \\
\hline 6 & 66.50 & 66.68 & - & 67.02 & 67.22 & 67.36 & 67.67 & 67.73 & 67.90 & - & - & 68.41 \\
\hline 7 & 66.50 & 66.69 & - & 67.04 & 67.20 & 67.39 & 67.69 & 67.80 & 67.92 & - & - & 68.35 \\
\hline 8 & 66.46 & 66.67 & - & 67.14 & 67.13 & 67.37 & 67.68 & 67.84 & 67.89 & - & - & 68.47 \\
\hline 9 & 66.44 & 66.65 & - & 67.14 & 67.09 & 67.40 & 67.66 & 67.78 & 67.82 & - & 68.25 & 68.53 \\
\hline 10 & 66.55 & 66.63 & - & 67.02 & 67.14 & 67.42 & 67.69 & 67.77 & 67.91 & - & 68.28 & 68.44 \\
\hline 11 & 66.59 & 66.64 & - & 67.04 & 67.20 & 67.39 & 67.73 & 67.83 & 67.97 & - & 68.33 & 68.44 \\
\hline 12 & 66.61 & 66.66 & - & 67.09 & 67.24 & 67.37 & 67.75 & 67.87 & 67.98 & - & 68.34 & 68.52 \\
\hline 13 & 66.59 & 66.71 & - & 67.12 & 67.18 & 67.34 & 67.72 & 67.85 & 67.98 & - & 68.22 & 68.59 \\
\hline 14 & 66.60 & 66.70 & 66.67 & 67.07 & 67.21 & 67.37 & 67.69 & 67.80 & 67.97 & - & 68.14 & 68.59 \\
\hline 15 & 66.54 & 66.63 & 66.66 & 66.99 & 67.26 & 67.41 & 67.70 & 67.82 & 68.02 & - & 68.25 & 68.57 \\
\hline 16 & 66.59 & 66.60 & 66.78 & 67.02 & 67.21 & 67.43 & 67.73 & 67.86 & 68.04 & - & 68.31 & 68.54 \\
\hline 17 & 66.60 & 66.64 & 66.81 & 67.09 & 67.15 & 67.43 & 67.71 & 67.86 & 68.03 & - & 68.23 & - \\
\hline 18 & 66.52 & 66.74 & 66.71 & 67.11 & 67.17 & 67.40 & 67.69 & 67.85 & 68.02 & - & 68.32 & - \\
\hline 19 & 66.51 & 66.82 & 66.62 & 67.12 & 67.22 & 67.39 & 67.67 & 67.87 & 68.02 & - & 68.44 & - \\
\hline 20 & 66.48 & - & 66.65 & 67.11 & 67.21 & 67.42 & 67.71 & 67.89 & 67.99 & - & 68.42 & - \\
\hline 21 & 66.54 & - & 66.77 & 67.14 & 67.23 & - & 67.76 & 67.92 & 68.00 & - & 68.35 & - \\
\hline 22 & 66.56 & - & 66.90 & 67.13 & 67.30 & 67.41 & 67.77 & 67.91 & 68.06 & - & 68.40 & - \\
\hline 23 & 66.56 & - & 66.86 & 67.12 & 67.34 & 67.41 & 67.76 & 67.87 & 68.08 & - & 68.45 & - \\
\hline 24 & 66.55 & - & 66.80 & 67.13 & 67.33 & 67.40 & 67.73 & 67.88 & 68.09 & - & 68.41 & - \\
\hline 25 & 66.54 & - & 66.72 & 67.10 & 67.27 & 67.42 & 67.71 & 67.87 & - & - & 68.39 & - \\
\hline 26 & 66.56 & - & 66.75 & 67.15 & 67.23 & 67.42 & 67.76 & 67.83 & - & - & 68.38 & - \\
\hline 27 & 66.54 & - & 66.91 & 67.20 & 67.27 & 67.38 & 67.81 & 67.82 & - & - & 68.31 & - \\
\hline 28 & 66.51 & - & 67.09 & 67.22 & 67.30 & 67.41 & 67.81 & 67.89 & - & - & 68.37 & - \\
\hline 29 & 66.60 & - & 67.11 & 67.19 & 67.26 & 67.51 & 67.79 & 67.95 & - & - & 68.30 & - \\
\hline 30 & 66.67 & - & 67.13 & 67.14 & 67.19 & 67.59 & 67.78 & 67.94 & - & - & 68.42 & - \\
\hline 31 & 66.68 & - & 67.08 & - & 67.27 & - & 67.76 & 67.91 & - & - & - & - \\
\hline Mean & 66.54 & 66.67 & 66.83 & 67.11 & 67.22 & 67.40 & 67.71 & 67.83 & 67.98 & - & 68.33 & 68.49 \\
\hline $\operatorname{Max}$ & 66.68 & 66.82 & 67.13 & 67.22 & 67.34 & 67.59 & 67.81 & 67.95 & 68.09 & - & 68.45 & 68.59 \\
\hline Min & 66.38 & 66.60 & 66.62 & 66.99 & 67.04 & 67.31 & 67.61 & 67.71 & 67.82 & - & 68.14 & 68.35 \\
\hline
\end{tabular}




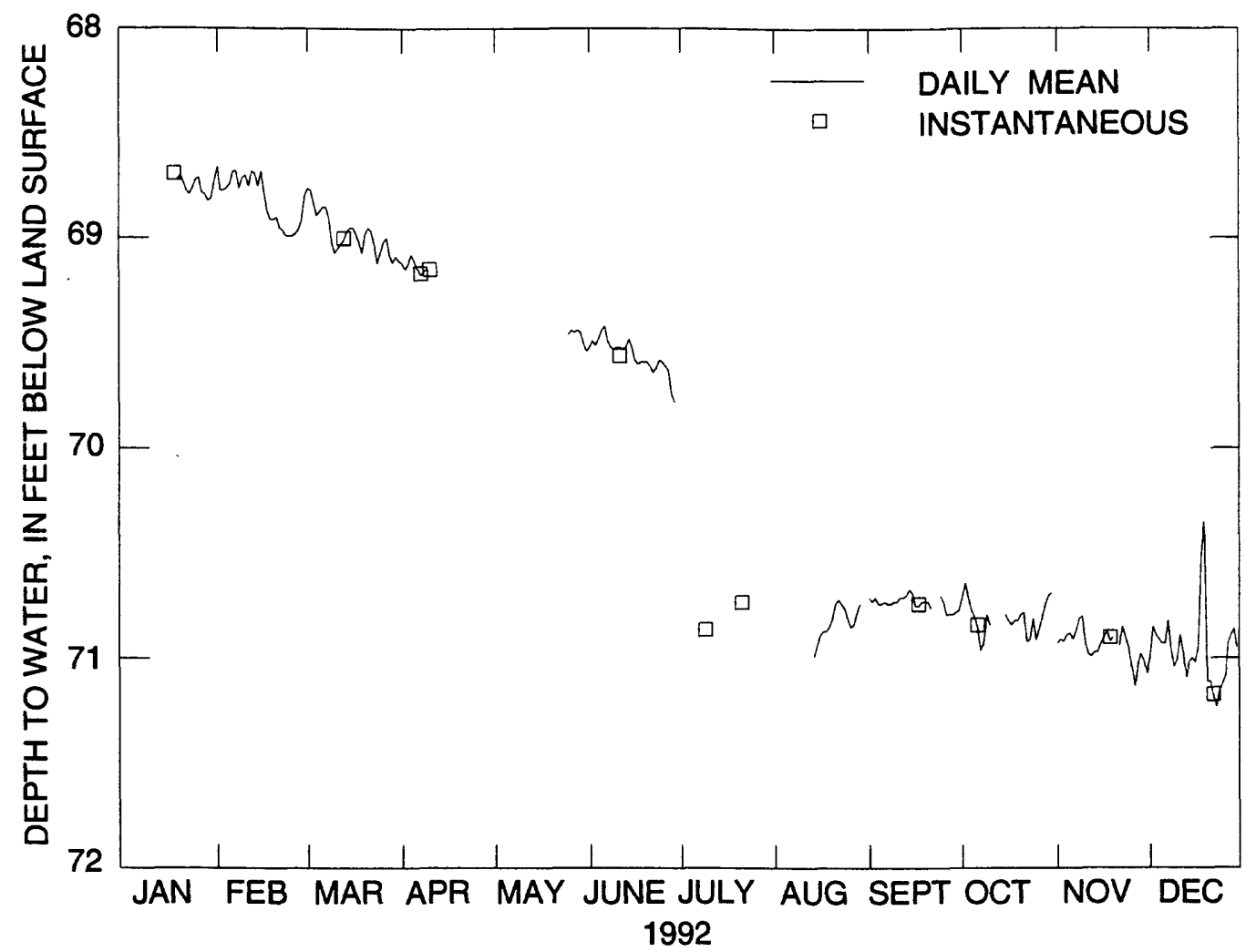

Figure 6. (F) Daily mean and instantaneous water levels, Lookout Mountain well (LKT), Long Valley Caldera, Mono County, California, 1992. 
Table 7. Daily mean water levels, Lookout Mountain well (LKT), Long Valley Caldera, Mono County, California, 1992

[Values are in feet below land surface. Max, maximum; Min, minimum; -, no data]

\begin{tabular}{|c|c|c|c|c|c|c|c|c|c|c|c|c|}
\hline Day & Jan & Feb & Mar & Apr & May & June & July & Aug & Sept & Oct & Nov & Dec \\
\hline 1 & - & 68.66 & 68.76 & 69.12 & - & 69.52 & - & - & 70.71 & 70.71 & 70.93 & 71.00 \\
\hline 2 & - & 68.77 & 68.77 & 69.15 & - & 69.49 & - & - & 70.73 & 70.64 & 70.91 & 70.85 \\
\hline 3 & - & 68.77 & 68.83 & 69.13 & - & 69.51 & - & - & 70.71 & 70.71 & 70.92 & 70.89 \\
\hline 4 & - & 68.76 & 68.89 & 69.08 & - & 69.48 & - & - & 70.74 & 70.77 & 70.89 & 70.91 \\
\hline 5 & - & 68.74 & 68.87 & 69.11 & - & 69.44 & - & - & 70.74 & 70.80 & 70.88 & 70.93 \\
\hline 6 & - & 68.68 & 68.85 & 69.15 & - & 69.42 & - & - & 70.73 & 70.85 & 70.91 & 70.93 \\
\hline 7 & - & 68.68 & 68.85 & 69.18 & - & 69.49 & - & - & 70.74 & 70.96 & 70.87 & 70.82 \\
\hline 8 & - & 68.76 & 68.90 & 69.16 & - & 69.52 & - & - & 70.74 & 70.93 & 70.81 & 70.96 \\
\hline 9 & - & 68.71 & 69.02 & 69.15 & - & 69.53 & - & - & 70.73 & 70.79 & 70.80 & 71.04 \\
\hline 10 & - & 68.70 & 69.07 & - & - & 69.52 & - & - & 70.73 & 70.84 & 70.93 & 71.01 \\
\hline 11 & - & 68.75 & 69.05 & - & - & 69.52 & - & - & 70.71 & - & 70.98 & 70.89 \\
\hline 12 & - & 68.68 & 69.03 & - & - & 69.53 & - & - & 70.71 & - & 70.99 & 70.97 \\
\hline 13 & - & 68.69 & 69.01 & - & - & 69.52 & - & - & 70.70 & 70.74 & 70.97 & 71.09 \\
\hline 14 & - & 68.75 & 68.97 & - & - & 69.48 & - & 70.99 & 70.67 & - & 70.97 & 71.02 \\
\hline 15 & - & 68.68 & 68.95 & - & - & 69.52 & - & 70.94 & 70.69 & 70.79 & 70.93 & 71.00 \\
\hline 16 & - & 68.78 & 68.95 & - & - & 69.58 & - & 70.89 & 70.75 & 70.82 & 70.90 & 71.02 \\
\hline 17 & - & 68.87 & 68.98 & - & - & 69.60 & - & 70.87 & 70.75 & 70.84 & 70.87 & 70.95 \\
\hline 18 & - & 68.91 & 69.02 & - & - & 69.59 & - & 70.87 & 70.73 & 70.82 & 70.92 & 70.50 \\
\hline 19 & 68.72 & 68.91 & 69.07 & - & - & 69.59 & - & 70.85 & 70.73 & 70.82 & 70.90 & 70.35 \\
\hline 20 & 68.70 & 68.90 & 68.98 & - & - & 69.59 & - & 70.81 & 70.73 & 70.79 & - & 71.11 \\
\hline 21 & 68.73 & 68.95 & 68.95 & - & - & 69.61 & - & 70.74 & 70.76 & 70.78 & 70.94 & 71.11 \\
\hline 22 & 68.77 & 68.96 & 68.97 & - & - & 69.64 & - & 70.72 & - & 70.92 & 70.85 & 71.18 \\
\hline 23 & 68.79 & 68.99 & 69.03 & - & - & 69.62 & - & 70.74 & - & 70.91 & 70.90 & 71.23 \\
\hline 24 & 68.76 & 68.99 & 69.12 & - & - & 69.58 & - & 70.76 & 70.70 & 70.81 & 70.95 & 71.15 \\
\hline 25 & 68.72 & 68.99 & 69.07 & - & 69.46 & 69.59 & - & 70.81 & 70.73 & 70.91 & 71.04 & 71.12 \\
\hline 26 & 68.71 & 68.98 & 69.02 & - & 69.44 & 69.61 & - & 70.85 & 70.79 & 70.86 & 71.13 & 71.08 \\
\hline 27 & 68.78 & 68.96 & 69.00 & - & 69.45 & 69.63 & - & 70.84 & 70.79 & 70.80 & 71.04 & 70.93 \\
\hline 28 & 68.79 & 68.92 & 69.08 & - & 69.44 & - & - & 70.78 & 70.79 & 70.74 & 70.98 & 70.89 \\
\hline 29 & 68.82 & 68.80 & 69.12 & - & 69.45 & - & - & 70.74 & 70.78 & 70.70 & 71.01 & 70.86 \\
\hline 30 & 68.81 & - & 69.09 & - & 69.50 & - & - & - & 70.77 & 70.69 & 71.07 & 70.95 \\
\hline 31 & 68.73 & - & 69.11 & - & 69.54 & - & - & - & - & - & - & - \\
\hline Mean & 68.76 & 68.82 & 68.98 & 69.14 & 69.47 & 69.64 & - & 70.82 & 70.73 & 70.81 & 70.94 & 70.96 \\
\hline $\operatorname{Max}$ & 68.82 & 68.99 & 69.12 & 69.18 & 69.54 & 69.55 & - & 70.99 & 70.79 & 70.96 & 71.13 & 71.23 \\
\hline Min & 68.70 & 68.66 & 68.76 & 69.08 & 69.44 & 69.42 & - & 70.72 & 70.67 & 70.64 & 70.80 & 70.35 \\
\hline
\end{tabular}




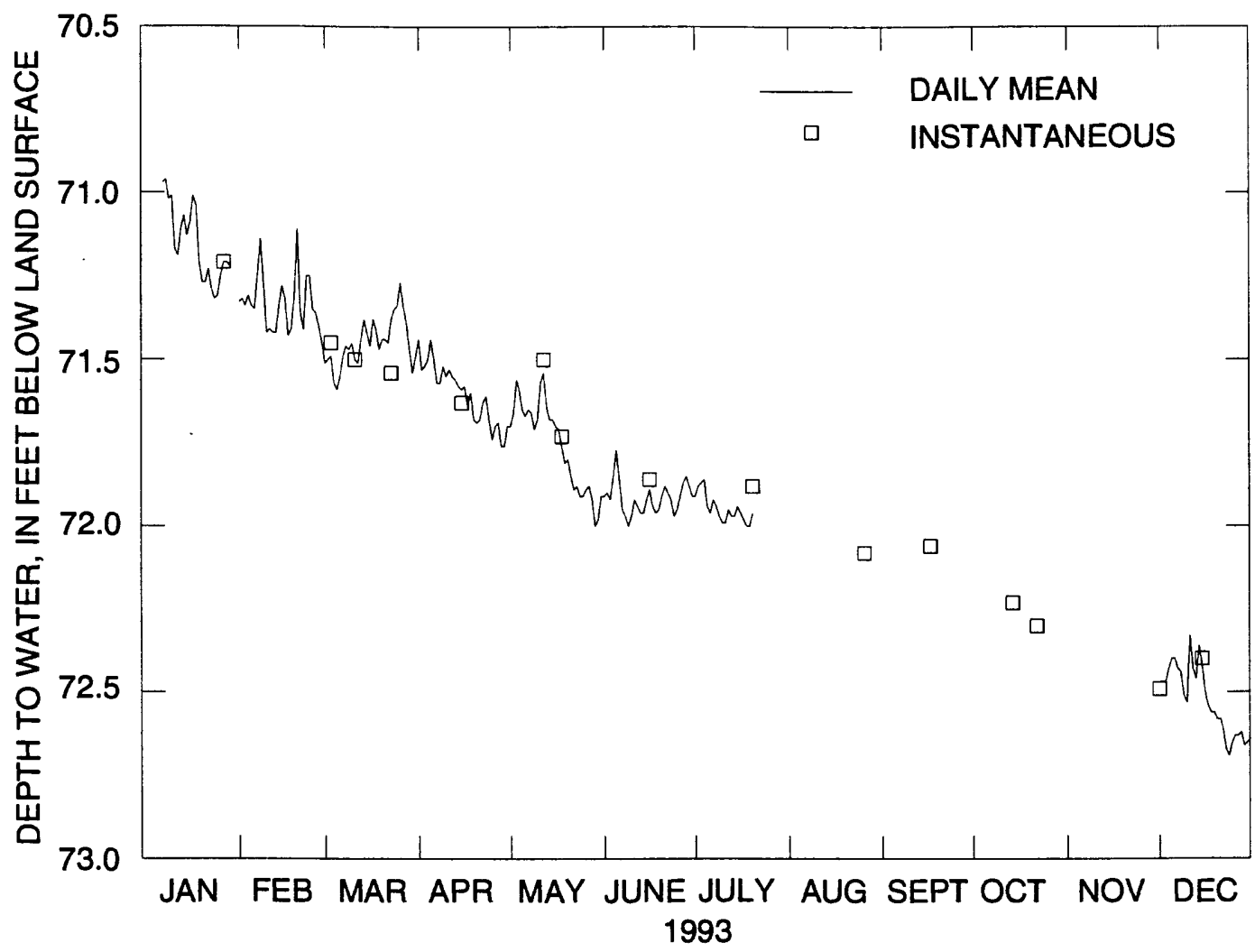

Figure 6. (G) Daily mean and instantaneous water levels, Lookout Mountain well (LKT), Long Valley Caldera, Mono County, California, 1993. 
Table 8. Daily mean water levels, Lookout Mountain well (LKT), Long Valley Caldera, Mono County, California, 1993

[Values are in feet below land surface. Max, maximum; Min, minimum; -, no data]

\begin{tabular}{|c|c|c|c|c|c|c|c|c|c|c|c|c|}
\hline Day & Jan & Feb & Mar & Apr & May & June & July & Aug & Sept & Oct & Nov & Dec \\
\hline 1 & & - & 71.33 & 71.51 & 71.44 & 71.70 & 71.91 & 71.91 & - & - & - & - \\
\hline 2 & - & 71.32 & 71.50 & 71.53 & 71.66 & 71.90 & 71.88 & - & - & - & - & - \\
\hline 3 & - & 71.34 & 71.49 & 71.52 & 71.56 & 71.92 & 71.87 & - & - & - & - & 72.47 \\
\hline 4 & - & 71.31 & 71.57 & 71.50 & 71.59 & 71.85 & 71.86 & - & - & - & - & 72.43 \\
\hline 5 & - & 71.34 & 71.59 & 71.44 & 71.65 & 71.77 & 71.94 & - & - & - & - & 72.40 \\
\hline 6 & - & 71.35 & 71.55 & 71.50 & 71.67 & 71.86 & 71.96 & - & - & - & - & 72.40 \\
\hline 7 & 70.97 & 71.25 & 71.49 & 71.57 & 71.65 & 71.95 & 71.92 & - & - & - & - & 72.43 \\
\hline 8 & 70.96 & 71.14 & 71.46 & 71.57 & 71.66 & 71.97 & 71.94 & - & - & - & - & 72.44 \\
\hline 9 & 71.02 & 71.27 & 71.47 & 71.52 & 71.71 & 72.00 & 71.97 & - & - & - & - & 72.51 \\
\hline 10 & 71.01 & 71.42 & 71.45 & 71.55 & 71.68 & 71.97 & 71.99 & - & - & - & - & 72.53 \\
\hline 11 & 71.17 & 71.41 & 71.50 & 71.53 & 71.57 & 71.92 & 71.99 & - & - & - & - & 72.33 \\
\hline 12 & 71.19 & 71.42 & 71.51 & 71.55 & 71.54 & 71.94 & 71.95 & - & - & - & - & 72.43 \\
\hline 13 & 71.11 & 71.42 & 71.44 & 71.56 & 71.64 & 71.96 & 71.97 & - & - & - & - & 72.46 \\
\hline 14 & 71.07 & 71.34 & 71.38 & 71.58 & 71.68 & 71.96 & 71.97 & - & - & - & - & 72.36 \\
\hline 15 & 71.13 & 71.28 & 71.42 & 71.59 & 71.68 & 71.92 & 71.94 & - & - & - & - & 72.42 \\
\hline 16 & 71.09 & 71.32 & 71.46 & 71.58 & 71.70 & 71.89 & 71.96 & - & - & - & - & 72.50 \\
\hline 17 & 71.01 & 71.43 & 71.38 & 71.64 & 71.71 & 71.94 & 71.98 & - & - & - & - & 72.54 \\
\hline 18 & 71.04 & 71.41 & 71.41 & 71.60 & 71.76 & 71.96 & 72.00 & - & - & - & - & 72.56 \\
\hline 19 & 71.21 & 71.31 & 71.47 & 71.68 & 71.81 & 71.95 & 72.00 & - & - & - & - & 72.56 \\
\hline 20 & 71.27 & 71.11 & 71.44 & 71.69 & 71.80 & 71.91 & 71.96 & - & - & - & - & 72.58 \\
\hline 21 & 71.27 & 71.36 & 71.44 & 71.68 & 71.85 & 71.88 & - & - & - & - & - & 72.58 \\
\hline 22 & 71.23 & 71.41 & 71.45 & 71.63 & 71.89 & 71.90 & - & - & - & - & - & 72.61 \\
\hline 23 & 71.29 & 71.25 & 71.38 & 71.61 & 71.88 & 71.92 & - & - & - & - & - & 72.67 \\
\hline 24 & 71.32 & 71.25 & 71.35 & 71.68 & 71.91 & 71.97 & - & - & - & - & - & 72.69 \\
\hline 25 & 71.31 & 71.35 & 71.34 & 71.74 & 71.91 & 71.95 & - & - & - & - & - & 72.65 \\
\hline 26 & 71.25 & 71.36 & 71.27 & 71.70 & 71.89 & 71.91 & - & - & - & - & - & 72.63 \\
\hline 27 & 71.21 & 71.40 & 71.34 & 71.69 & 71.88 & 71.87 & - & - & - & - & - & 72.63 \\
\hline 28 & 71.21 & 71.45 & 71.39 & 71.76 & 71.92 & 71.85 & - & - & - & - & - & 72.62 \\
\hline 29 & 71.22 & - & 71.47 & 71.76 & 72.00 & 71.88 & - & - & - & - & - & 72.66 \\
\hline 30 & - & - & 71.54 & 71.70 & 71.98 & 71.91 & - & - & - & - & - & 72.65 \\
\hline 31 & - & - & 71.49 & - & 71.91 & - & - & - & - & - & - & 72.64 \\
\hline Mean & 71.15 & 71.33 & 71.45 & 71.60 & 71.76 & 71.92 & 71.95 & - & - & - & - & 72.53 \\
\hline Max & 71.32 & 71.45 & 71.59 & 71.76 & 72.00 & 72.00 & 72.00 & - & - & - & - & 72.69 \\
\hline Min & 70.96 & 71.11 & 71.27 & 71.44 & 71.54 & 71.77 & 71.86 & - & - & - & - & 72.33 \\
\hline
\end{tabular}




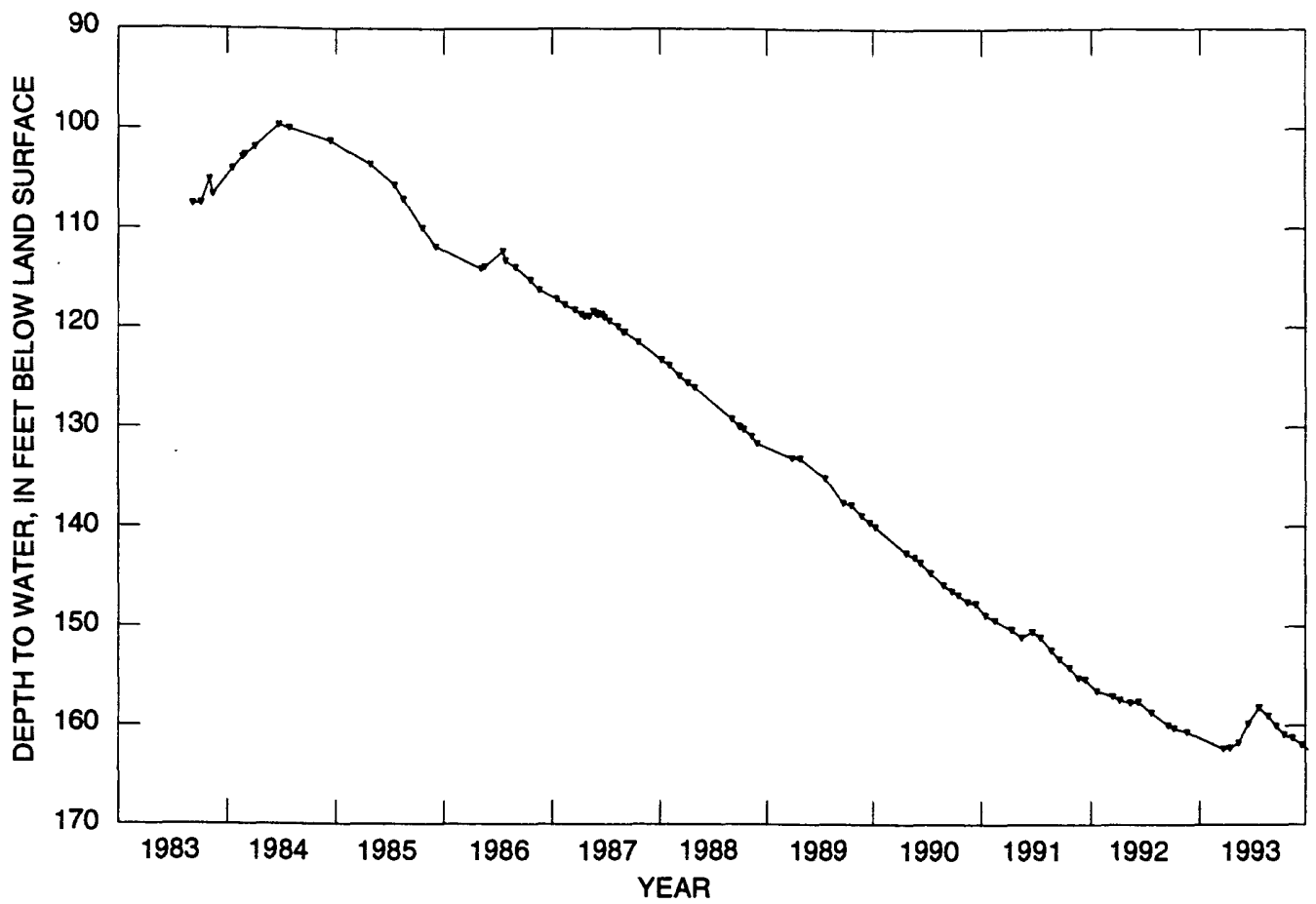

Figure 7. Instantaneous water levels, well SC-2, Long Valley Caldera, Mono County, California, 1983-93 


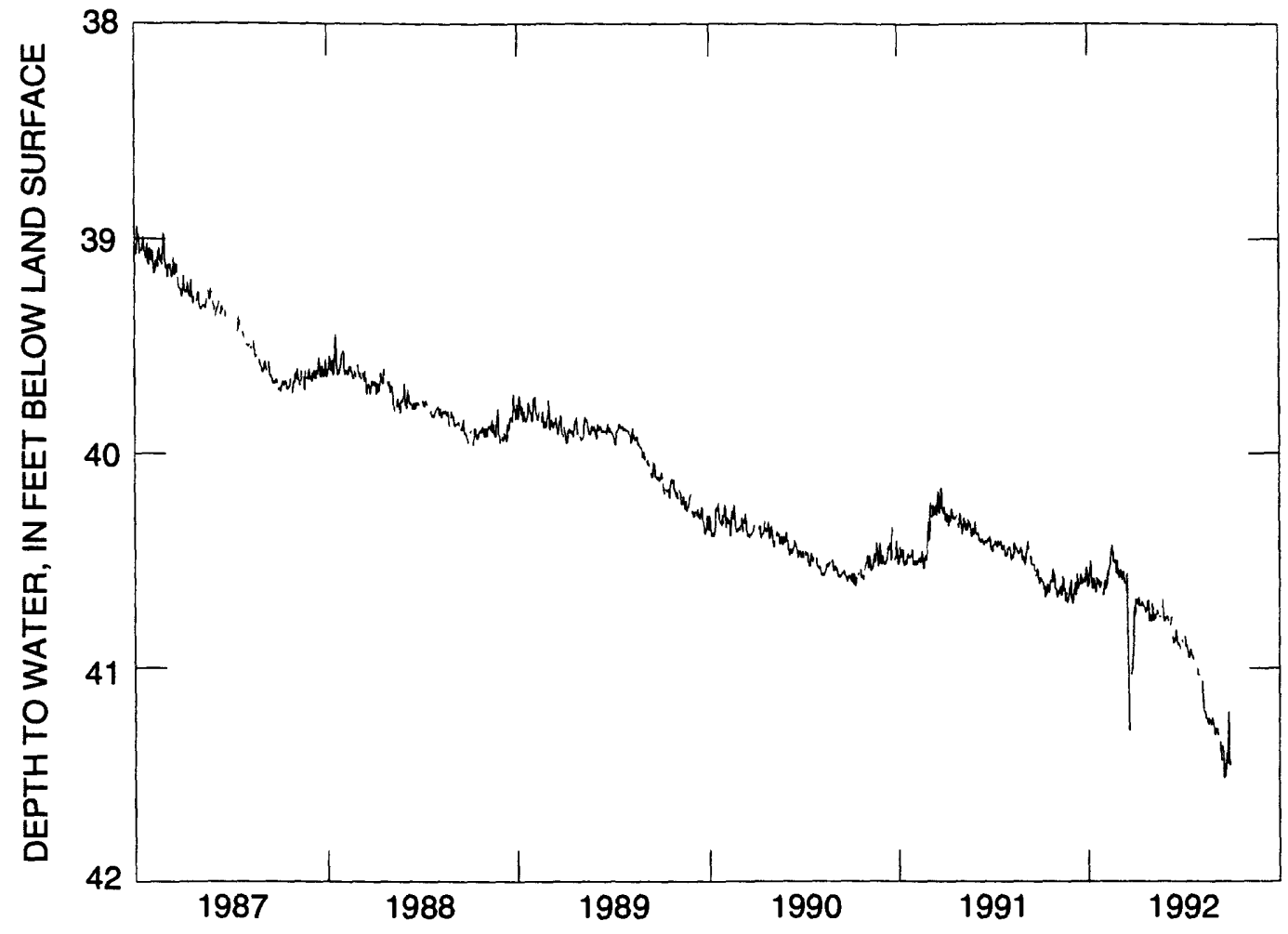

Figure 8. Daily mean water levels, well CH-1, Long Valley Caldera, Mono County, California, 1987-92. 


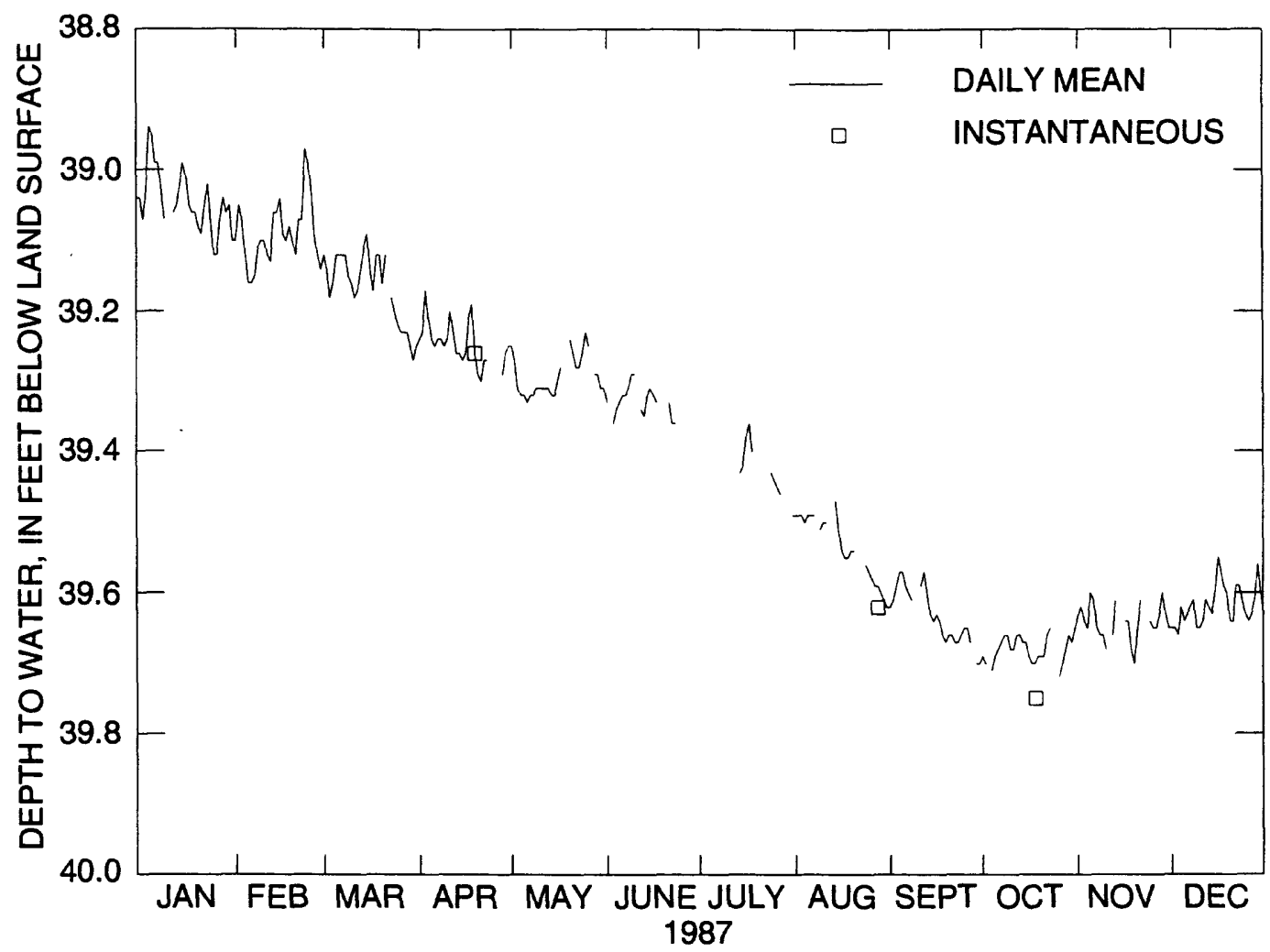

Figure 9. (A) Daily mean and instantaneous water levels, well CH-1, Long Valley Caldera, Mono County, California, 1987. 
Table 9. Daily mean water levels, well $\mathrm{CH}-1$, Long Valley Caldera, Mono County, California, 1987

[Values are in feet below land surface. Max, maximum; Min, minimum; - , no data]

\begin{tabular}{|c|c|c|c|c|c|c|c|c|c|c|c|c|}
\hline Day & Jan & Feb & Mar & Apr & May & June & July & Aug & Sept & Oct & Nov & Dec \\
\hline 1 & 39.04 & 39.10 & 39.12 & 39.24 & 39.25 & 39.33 & - & 39.49 & 39.62 & 39.69 & 39.63 & 39.65 \\
\hline 2 & 39.07 & 39.05 & 39.14 & 39.23 & 39.27 & - & 39.36 & 39.49 & 39.61 & 39.70 & 39.62 & 39.65 \\
\hline 3 & 39.03 & 39.07 & 39.18 & 39.17 & 39.31 & 39.36 & - & 39.49 & 39.59 & - & 39.64 & 39.66 \\
\hline 4 & 38.94 & 39.12 & 39.16 & 39.21 & 39.32 & 39.34 & 39.35 & 39.50 & 39.57 & 39.71 & 39.65 & 39.62 \\
\hline 5 & 38.95 & 39.16 & 39.12 & 39.24 & 39.32 & 39.33 & - & 39.49 & 39.57 & 39.69 & 39.60 & 39.64 \\
\hline 6 & 38.99 & 39.16 & 39.12 & 39.25 & 39.33 & 39.32 & - & 39.49 & 39.59 & 39.68 & 39.61 & 39.63 \\
\hline 7 & 38.99 & 39.15 & 39.12 & 39.24 & 39.32 & 39.32 & - & - & 39.61 & 39.66 & 39.66 & 39.61 \\
\hline 8 & 39.03 & 39.11 & 39.12 & 39.24 & 39.32 & 39.31 & - & - & 39.61 & 39.66 & 39.66 & 39.61 \\
\hline 9 & 39.07 & 39.10 & 39.15 & 39.25 & 39.31 & 39.29 & 39.37 & 39.51 & - & 39.66 & 39.66 & 39.65 \\
\hline 10 & - & 39.10 & 39.16 & 39.24 & 39.31 & 39.29 & - & 39.50 & - & 39.68 & 39.68 & 39.65 \\
\hline 11 & - & 39.12 & 39.18 & 39.2 & 39.31 & - & - & 39.50 & 39.59 & 39.68 & - & 39.64 \\
\hline 12 & 39.06 & 39.13 & 39.17 & 39.23 & 39.31 & 39.34 & - & - & 39.57 & 39.66 & 39.66 & 39.61 \\
\hline 13 & 39.05 & 39.06 & 39.14 & 39.26 & 39.31 & 39.35 & - & - & 39.60 & 39.66 & 39.61 & 39.62 \\
\hline 14 & 39.02 & 39.06 & 39.11 & 39.26 & 39.32 & 39.32 & 39.43 & 39.47 & 39.63 & 39.67 & - & 39.63 \\
\hline 15 & 38.99 & 39.04 & 39.09 & 39.27 & 39.32 & 39.31 & 39.42 & 39.51 & 39.64 & 39.67 & - & 39.60 \\
\hline 16 & 39.01 & 39.09 & 39.14 & 39.2 & 39.3 & 39.32 & 39.3 & 39.54 & 39. & 39.69 & 39.64 & 39.55 \\
\hline 17 & 39.05 & 39.10 & 39.17 & 39.21 & 39.28 & 39.33 & 39.36 & 39 & 39. & 39.70 & 39.64 & 39.57 \\
\hline 18 & 39.06 & 39.08 & 39.12 & 39.19 & - & - & 39.40 & 39.55 & 39.66 & 39.70 & 39.68 & 39.59 \\
\hline 19 & 39.06 & 39.10 & 39.12 & 39.26 & - & 39.33 & - & 39.54 & 39.67 & 39.69 & 39.70 & 39.60 \\
\hline 20 & 39.08 & 39.12 & 39.16 & 39.29 & 39.24 & - & - & 39.54 & 39.66 & 39.69 & 39.65 & 39.64 \\
\hline 21 & 39.09 & 39.07 & 39.12 & 39. & 39. & 39. & - & - & 39 & 39.69 & 39.61 & 39.64 \\
\hline 22 & 39.05 & 39.07 & - & 39.27 & 39.28 & 39.36 & 39.43 & 39.56 & 39 & 39.66 & - & 39.59 \\
\hline 23 & 39.02 & 38.97 & 39.18 & 39.27 & 39 & 39.36 & - & 一 & 39 & 39.65 & - & 39.59 \\
\hline 24 & 39.07 & 38.99 & 39.20 & - & 39.26 & - & 39.43 & 39.56 & 39.66 & - & 39.64 & 39.61 \\
\hline 25 & 39.12 & 39.03 & 39.22 & - & 39.23 & - & 39.44 & 39.57 & 39.65 & - & 39.65 & 39.63 \\
\hline 26 & 39.12 & 3 & 35 & 39.30 & 39.25 & 39.40 & 39 & & & 39.72 & 65 & 39.64 \\
\hline 27 & 39.07 & 39.12 & 39.23 & - & - & - & 39.46 & 39 & 39.67 & 39.70 & 39.63 & 39.63 \\
\hline 28 & 39.04 & 39.14 & 39.23 & 39.29 & 39.29 & - & - & 39.59 & - & 39.68 & 39.60 & 39.61 \\
\hline 29 & 39.06 & - & 39.25 & 39.26 & 39.29 & - & - & 39.60 & 39.70 & 39.66 & 39.63 & 39.56 \\
\hline 30 & 39.05 & - & 39.27 & 39.25 & 39.31 & - & - & 39.61 & 39.70 & 39.67 & 39.65 & 39.60 \\
\hline 31 & 39.10 & - & 39.25 & - & 39.31 & - & 39.49 & 39.62 & - & 39.65 & - & 39.63 \\
\hline Mean & 39.04 & 3 & & & & & & & & & 39.64 & 39.62 \\
\hline $\operatorname{Max}$ & 39.12 & 39.16 & 39.27 & 39.30 & 39. & 39.40 & 39. & 39.62 & 39.70 & 39.72 & 39.70 & 39.66 \\
\hline Min & 38.94 & 38.97 & 39.09 & 39.17 & 39.23 & 39.29 & 39.35 & 39.47 & 39.57 & 39.65 & 39.60 & 39.55 \\
\hline
\end{tabular}




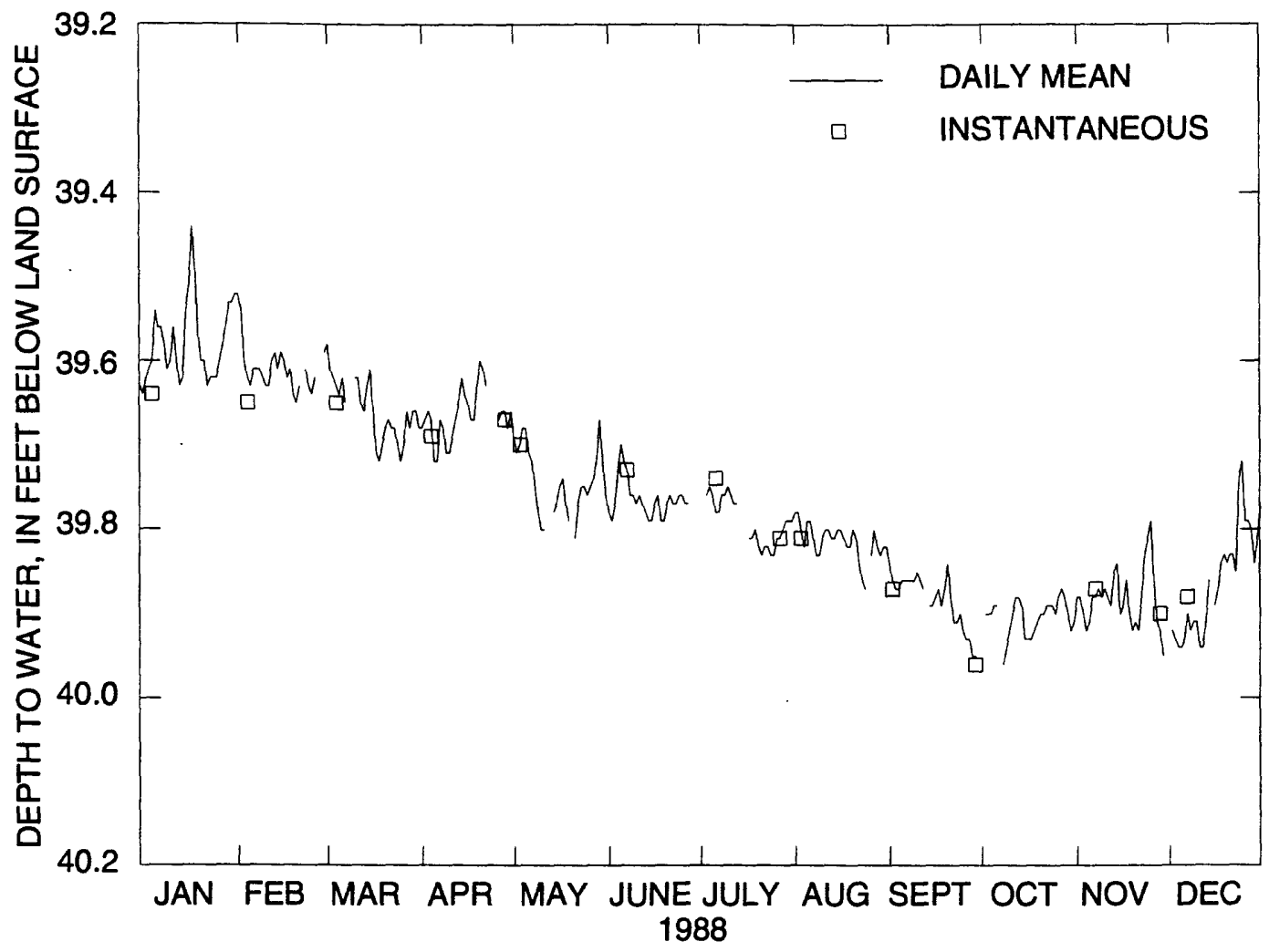

Figure 9. (B) Daily mean and instantaneous water levels, well $\mathrm{CH}-1$, Long Valley Caldera, Mono County, California, 1988. 
Table 10. Daily mean water levels, well CH-1, Long Valley Caldera, Mono County, California, 1988

[Values are in feet below land surface. Max. maximum; Min. minimum; - - no data]

\begin{tabular}{|c|c|c|c|c|c|c|c|c|c|c|c|c|}
\hline Day & Jan & Feb & Mar & Apr & May & June & July & Aug & Sept & Oct & Nov & Dec \\
\hline 1 & 39.64 & 39.52 & 39.58 & 39.68 & 39.69 & 39.78 & 39.77 & 39.78 & 39.84 & - & 39.88 & - \\
\hline 2 & 39.62 & 39.54 & 39.61 & 39.67 & 39.71 & 39.79 & - & 39.78 & 39.86 & 39.90 & & 39.92 \\
\hline 3 & 39.61 & 39.60 & 39.62 & 39.66 & 39.70 & 39.77 & 39.76 & 39.80 & & 39.90 & 39.90 & 39.93 \\
\hline 4 & 39.60 & 39.62 & 39.63 & 39.67 & 39.68 & 39.73 & 39.75 & 39.82 & 39.87 & 39.90 & 39.92 & 39.94 \\
\hline 5 & 39.54 & 39.63 & 39.64 & 39.72 & 39.68 & 39.70 & 39.76 & 39.79 & 39.86 & 39.89 & 39.91 & 39.94 \\
\hline 6 & 39.56 & 39.61 & 39.62 & 39.72 & 39.71 & 39.72 & 39.78 & 39.79 & 39.86 & 39.89 & 39.88 & 39.93 \\
\hline 7 & 39.56 & 39.61 & 39.65 & 39.67 & 39.72 & 39.73 & 39.78 & 9.81 & 39.86 & - & 39.88 & 39.90 \\
\hline 8 & 39.58 & 39.61 & - & 39.68 & 39.75 & 39.76 & 39.76 & 39.83 & 39.86 & 39.96 & 39.87 & 39.92 \\
\hline 9 & 39.61 & 39.62 & - & 39.71 & 39 & 39.76 & 39.76 & 39.83 & 39.86 & 39.94 & 39.88 & 39.91 \\
\hline 10 & 39.60 & 39.63 & 39.62 & 39.71 & 39.80 & 39.77 & 39.75 & 39.81 & 39.85 & 39.92 & 39.87 & 39.91 \\
\hline 11 & 39.56 & 39.63 & 39.62 & 39.69 & 39.80 & 39.76 & 39.76 & 0 & 39.86 & 39.90 & 39.88 & 39.94 \\
\hline 12 & 39.60 & 39.60 & 39.65 & 39. & - & 7 & 39.77 & & 39.87 & 8 & 39 & 39.94 \\
\hline 13 & 39.63 & 39.59 & 39.66 & 39.65 & - & 39.78 & 39.77 & 1 & - & 39 & 39.85 & 39.91 \\
\hline 14 & 39.62 & 39.61 & 39.63 & 39.62 & 39.78 & 39.79 & - & 39.81 & 39.89 & 39.89 & 39.84 & 39.86 \\
\hline 15 & 39.54 & 39.59 & 39.61 & 39.64 & 39.77 & 39.79 & - & 39.80 & 39.89 & 39.93 & 39.90 & 一 \\
\hline 16 & 39.51 & 39.6 & 39.66 & 39. & 3 & 39.77 & - & & 39 & & 39 & 9.89 \\
\hline 17 & 39.44 & 39.62 & 39.71 & 39.67 & 39.74 & 39.76 & 39.81 & 1 & 39.87 & 39.93 & 39.86 & 39.87 \\
\hline 18 & 39.49 & 39.61 & 39.72 & 39.67 & 39.77 & 39.79 & 39.81 & 39 & 39.89 & 39.92 & 39.90 & 39.84 \\
\hline 19 & 39.57 & 39.64 & 39.70 & 39.63 & 39.79 & 39.79 & 39.80 & 39.82 & 39.87 & 39.91 & 39.92 & 39.83 \\
\hline 20 & 39.60 & 39.65 & 39.68 & 39.60 & - & 39.77 & 39.82 & 39.80 & 39.84 & 39.90 & 39.91 & 39.84 \\
\hline 21 & 39.60 & 39.63 & 39.67 & 39.6 & 39. & 6 & 39.83 & & 39 & & 92 & 9.83 \\
\hline 22 & 39.63 & - & 39.68 & 39.63 & 39.77 & 39.77 & 39.82 & 39 & 39.91 & 39.89 & 39.88 & 39.83 \\
\hline 23 & 39.62 & 39.61 & 39.68 & - & 39.75 & 39.77 & 39.82 & 39.86 & 39.91 & 39.89 & 39.83 & 39.85 \\
\hline 24 & 39.62 & 39.63 & 39.70 & - & 39.75 & 39.76 & 39.83 & 39.87 & 39.90 & 39.89 & 39.81 & 39.74 \\
\hline 25 & 39.62 & 39.64 & 39.72 & - & 39.76 & 39.76 & 39.83 & - & 39.92 & 39.90 & 39.79 & 39.72 \\
\hline 26 & 39.60 & 39.62 & 39.70 & 39.67 & 39 & 39.77 & 39.81 & & 39.93 & 39 & 39.86 & 39.79 \\
\hline 27 & 39.58 & - & 39.66 & 39.66 & 39.74 & 39.77 & 39.81 & 39.80 & 39.93 & 39.87 & 39.91 & 39.79 \\
\hline 28 & 39.56 & - & 39.68 & 39.66 & 39.72 & - & 39.80 & 39.82 & 39.95 & 39.88 & 39.92 & 39.80 \\
\hline 29 & 39.53 & 39.59 & 39.66 & 39.68 & 39.67 & - & 39.79 & 39.83 & 39.95 & 39.90 & 39.95 & 39.84 \\
\hline 30 & 39.53 & - & 39.66 & 39.66 & 39.72 & - & 39.79 & 39.82 & - & 39.92 & - & 39.81 \\
\hline 31 & 39.52 & - & 39.68 & - & 39.76 & - & 39.79 & 39.82 & - & 39.91 & - & 39.77 \\
\hline Mean & 39.58 & 39.6 & 39.66 & 39. & 39 & 39.76 & 39.79 & & 39.88 & & 39.88 & 39.86 \\
\hline $\operatorname{Max}$ & 39.64 & 39.6 & 39.72 & 39. & 39. & 39.79 & 39.83 & 39 & 39.95 & 39.96 & 39.95 & 39.94 \\
\hline Min & 39.44 & 39.52 & 39.58 & 39.60 & 39.67 & 39.70 & 39.75 & 39.78 & 39.84 & 39.87 & 39.79 & 39.72 \\
\hline
\end{tabular}




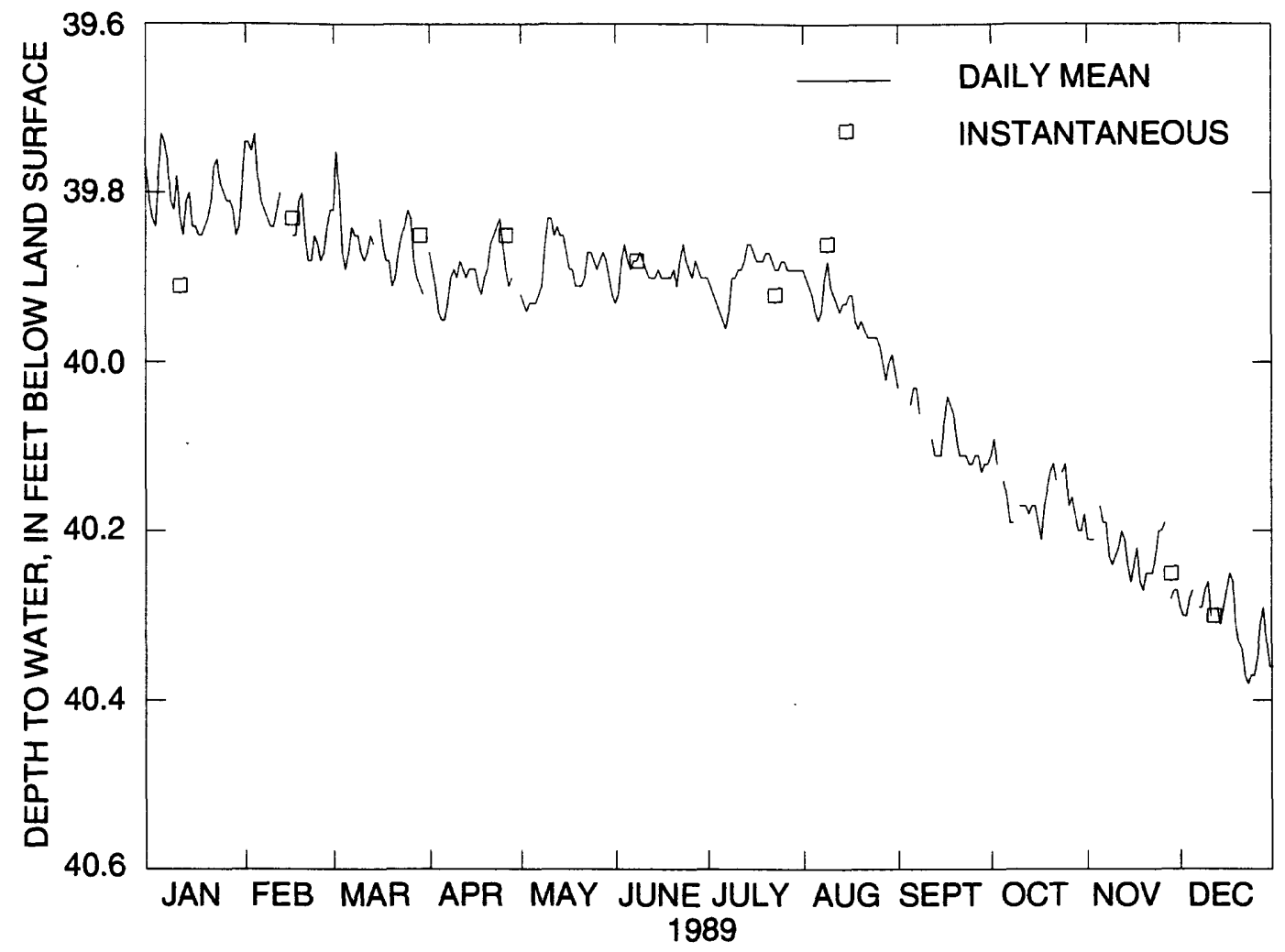

Figure 9. (C) Daily mean and instantaneous water levels, well CH-1, Long Valley Caldera, Mono County, California, 1989. 
Table 11. Daily mean water levels, well $\mathrm{CH}-1$, Long Valley Caldera, Mono County, California, 1989

[Values are in feet below land surface. Max, maximum; Min, minimum; -, no data]

\begin{tabular}{|c|c|c|c|c|c|c|c|c|c|c|c|c|}
\hline Day & Jan & Feb & Mar & Apr & May & June & July & Aug & Sept & Oct & Nov & Dec \\
\hline 1 & 39.81 & 39.74 & 39.82 & 39.87 & 39.92 & 39.93 & 39.90 & 39.89 & 40.03 & 40.11 & 40.21 & 40.29 \\
\hline 2 & 39.83 & 39.74 & & & & & & & - & 40.09 & 40.21 & 40.30 \\
\hline 3 & 39.84 & 39.75 & 0 & 39.91 & 94 & 39.88 & 39.92 & 39.91 & 40.05 & 40.12 & 40.21 & 40.30 \\
\hline 4 & 39.78 & 39.73 & 39.87 & 39.94 & 39.93 & 39.86 & 39.93 & 39.92 & - & - & - & 40.28 \\
\hline 5 & 39.73 & 39.78 & 39.89 & 39.95 & 39.93 & 39.88 & 39.94 & 39.94 & 40.05 & 40.14 & 40.17 & 40.27 \\
\hline 6 & 39.74 & 39.81 & 39.87 & 39.95 & 39.93 & 39.89 & 39.95 & 39.95 & 40.03 & 40.16 & 40.19 & - \\
\hline 7 & 39.76 & 39. & 39.84 & 39.93 & 2 & 39.88 & 39.96 & 39.94 & 40.03 & 40.19 & 40.19 & 40.29 \\
\hline 8 & 39.81 & 39.83 & 39.85 & 39.90 & 1 & 39.88 & 39.94 & & 40.06 & 40.19 & 40.23 & 40.29 \\
\hline 9 & 39.82 & 39.84 & 39.85 & 39.89 & 39.86 & 39.87 & 39.90 & 39.88 & - & - & 40.24 & 40.27 \\
\hline 10 & 39.78 & 39.84 & 39.87 & 39.90 & 39.83 & 39.88 & 39.90 & 39.91 & - & 40.17 & 40.23 & 40.26 \\
\hline 11 & 39.83 & 39.8 & 39.88 & 39.8 & 39.83 & 39.89 & 39.89 & 39.92 & - & 40.17 & 40.22 & 40.30 \\
\hline 12 & 39.85 & 39.8 & 39. & 39. & 3 & 39.90 & 39.89 & 39 & 40.09 & 40.17 & 40.20 & - \\
\hline 13 & 39.81 & - & 39.85 & 39.90 & 39.84 & 39.90 & 39.88 & 39.94 & 40.11 & 40.18 & 21 & 40.29 \\
\hline 14 & 39.80 & - & 39.86 & 39. & 39. & 39.90 & 39.86 & 39.93 & 40.11 & 40.17 & & 40.31 \\
\hline 15 & 39.84 & - & - & 39.89 & 39.85 & 39.89 & 39.86 & 39.93 & 40.11 & 40.17 & 40.26 & 40.29 \\
\hline 16 & 39.84 & 39.8 & 39.8 & 39. & & 9.90 & 39.87 & & 4 & 40.19 & & 40.27 \\
\hline 17 & 39.85 & 39.85 & 39.86 & 39.91 & 39.89 & 39.90 & 39.88 & & 40 & 40.21 & & 40.25 \\
\hline 18 & 39.85 & 39.81 & 39.88 & 39.92 & 39 & 39.90 & 39.88 & 39 & 40.05 & 40.17 & 40.26 & 40.26 \\
\hline 19 & 39.84 & 39. & 39.88 & 39 & 1 & 39.90 & 39.88 & 39 & 66 & 15 & 27 & 40.31 \\
\hline 20 & 39.83 & 39.85 & 39.91 & 39.89 & 39.91 & 39.89 & 39.87 & 39.95 & 40.09 & 40.13 & 40.25 & 40.33 \\
\hline 21 & & & & & & 91 & 37 & & 40.11 & 40.12 & 40.25 & 40.34 \\
\hline 22 & 39.77 & 39. & 39.87 & 39 & 39.90 & 39.88 & 39.88 & 39 & 40.11 & 40.14 & 40.25 & 40.37 \\
\hline 23 & 39.76 & 39. & 39.85 & 39 & 39 & 39.86 & 39.89 & 7 & 40.11 & - & 40 & 40.38 \\
\hline 24 & 39.79 & 39. & 39.84 & 39.83 & 39.87 & 39.88 & 39.89 & 39 & 40.12 & 40.13 & 40.20 & 40.37 \\
\hline 25 & 39.80 & 39.88 & 39.82 & 39.86 & 39.88 & 39.89 & 39.88 & 39.97 & 40.12 & 40.12 & 40.20 & 40.37 \\
\hline 26 & 39.81 & & & 3 & 80 & 90 & 39.88 & & 1 & 7 & 40.19 & 40.35 \\
\hline 27 & 39.81 & 39. & 39.8 & 39. & 39.88 & 39.88 & 39.89 & 40 & 40 & 40.16 & - & 40.31 \\
\hline 28 & 39.82 & 39.82 & 39.90 & 39.90 & 39.87 & 39.89 & 39.89 & 40 & 40.13 & 40.18 & 40.28 & 40.29 \\
\hline 29 & 39.85 & - & 39.91 & - & 39.88 & 39.90 & 39.89 & 40.00 & 40.12 & 40.20 & 40.27 & $<0.33$ \\
\hline 30 & 39.84 & - & 39.92 & - & 39.90 & 39.90 & 39.89 & 39.99 & 40.12 & 40.20 & 40.27 & 40.36 \\
\hline 31 & 39.79 & - & - & - & 39.92 & - & 39.89 & 40.01 & - & 40.18 & - & 40.36 \\
\hline & 39. & & & 39. & & & & & 40 & 40.16 & 40.23 & 40.31 \\
\hline Max & 39.85 & 39.8 & 39. & 39. & 39.94 & 39.93 & 39.96 & 40.02 & 40.13 & 40.21 & 40.28 & 40.38 \\
\hline Min & 39.73 & 39.73 & 39.75 & 39.83 & 39.83 & 39.86 & 39.86 & 39.88 & 40.03 & 40.09 & 40.17 & 40.25 \\
\hline
\end{tabular}




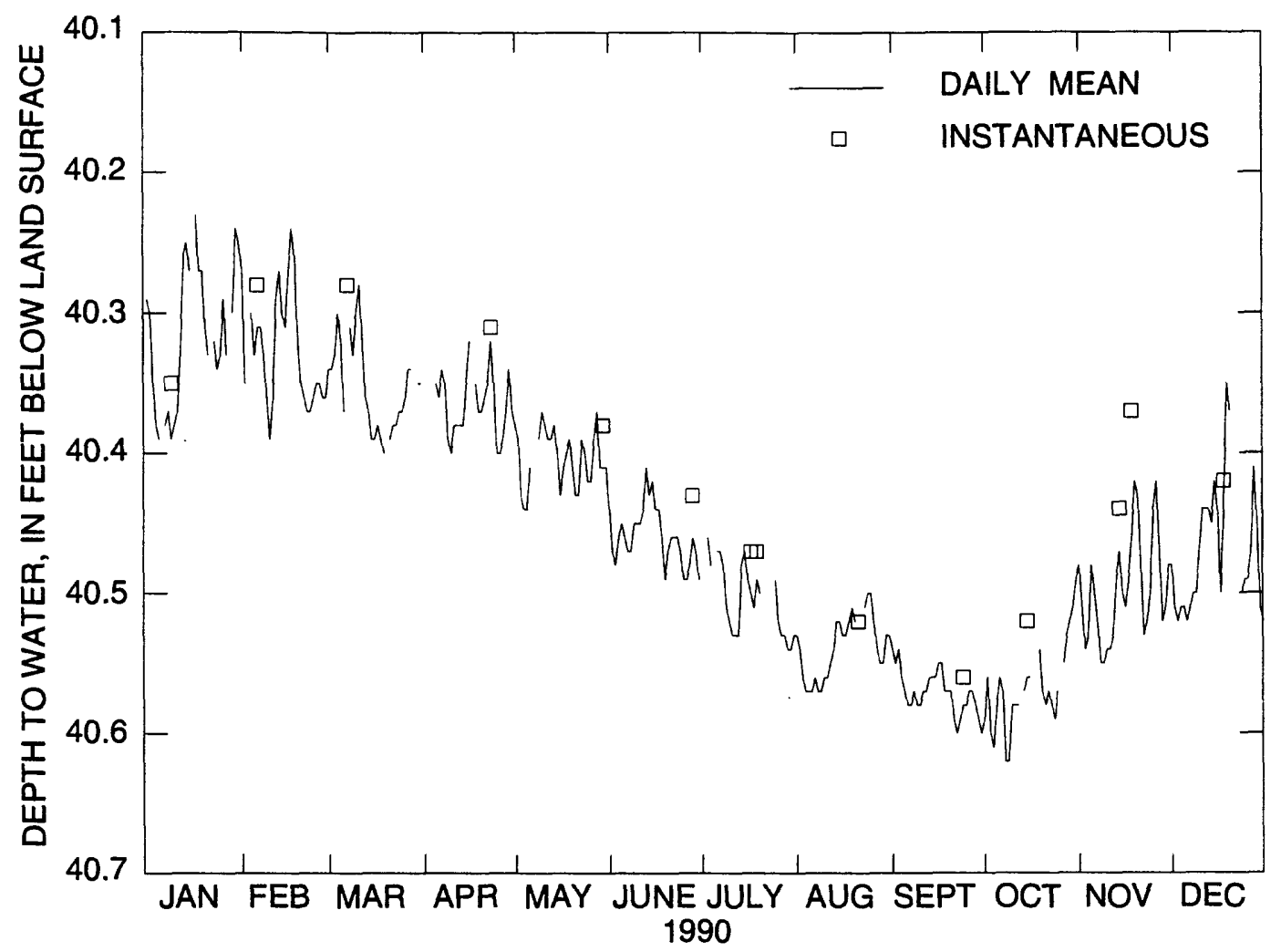

Figure 9. (D) Daily mean and instantaneous water levels, well CH-1, Long Valley Caldera, Mono County, California, 1990. 
Table 12. Daily mean water levels, well CH-1, Long Valley Caldera, Mono County, California, 1990

[Values are in feet below land surface. Max, maximum: Min, minimum; -, no data]

\begin{tabular}{|c|c|c|c|c|c|c|c|c|c|c|c|c|}
\hline Day & Jan & Feb & Mar & Apr & May & June & July & Aug & Sept & Oct & Nov & Dec \\
\hline 1 & 40.29 & 40.27 & 40.34 & - & 40.38 & 40.44 & - & 40.53 & 40.54 & 40.59 & 40.48 & 40.48 \\
\hline 2 & 40.30 & 40.35 & 40.34 & 40.39 & 40.39 & 40.47 & - & 40.54 & 40.55 & 40.56 & 40.51 & 40.51 \\
\hline 3 & 40.35 & - & 40.33 & - & 40.43 & 40.48 & 40.46 & 40.56 & 40.54 & 40.60 & 40.54 & 40.52 \\
\hline 4 & 40.38 & 40.30 & 40.30 & - & 40.44 & 40.46 & 40.48 & 40.57 & 40.56 & 40.61 & 40.53 & 40.51 \\
\hline 5 & 40.39 & 40.33 & 40.32 & 40.35 & 40.44 & 40.45 & - & 40.57 & 40.57 & 40.58 & 40.48 & 40.51 \\
\hline 6 & - & 40.31 & 40.37 & 40.36 & 40.41 & 40.46 & 40.47 & 40.57 & 40.58 & 40.56 & 40.50 & 40.52 \\
\hline 7 & 40.38 & 40.31 & - & 40.34 & - & 40.47 & 40.47 & 40.56 & 40.58 & 40.57 & 40.52 & 40.51 \\
\hline 8 & 40.37 & 40.33 & 40.31 & 40.35 & - & 40.47 & 40.48 & 40.57 & 40.57 & 40.62 & 40.55 & 40.50 \\
\hline 9 & 40.39 & 40.36 & 40.33 & 40.39 & 40.39 & 40.45 & 40.51 & 40.57 & 40.58 & 40.62 & 40.55 & 40.50 \\
\hline 10 & 40.38 & 40.39 & 40.30 & 40.40 & 40.37 & 40.45 & 40.52 & 40.56 & 40.58 & 40.58 & 40.54 & 40.47 \\
\hline 11 & 40.37 & 40.36 & 40.28 & 40.38 & 40.38 & 40.45 & 40.53 & 40.56 & 40.57 & 40.58 & 40.54 & 40.44 \\
\hline 12 & 40.33 & 40.29 & 40.32 & 40.38 & 40.39 & 40.44 & 40.53 & 40.55 & 40.57 & 40.58 & 40.53 & 40.44 \\
\hline 13 & 40.26 & 40.27 & 40.36 & 40.38 & 40.39 & 40.41 & 40.53 & 40.54 & 40.56 & - & 40.49 & 40.44 \\
\hline 14 & 40.25 & 40.30 & 40.37 & 40.38 & 40.38 & 40.43 & 40.48 & 40.52 & 40.56 & 40.57 & 40.47 & 40.45 \\
\hline 15 & 40.27 & 40.31 & 40.39 & 40.35 & 40.40 & 40.42 & 40.47 & 40.52 & 40.56 & 40.56 & 40.50 & 40.42 \\
\hline 16 & - & 40.27 & 40.39 & 40.32 & 40.43 & 40.44 & 40.49 & 40.53 & 40.55 & 40.56 & 40.51 & 40.44 \\
\hline 17 & 40.23 & 40.24 & 40.38 & - & 40.41 & 40.44 & 40.50 & 40.53 & 40.55 & - & 40.49 & 40.50 \\
\hline 18 & 40.27 & 40.26 & 40.39 & 40.35 & 40.40 & 40.46 & 40.51 & 40.52 & 40.57 & - & 40.46 & 40.44 \\
\hline 19 & 40.27 & 40.32 & 40.40 & 40.37 & 40.39 & 40.49 & 40.49 & 40.51 & 40.57 & 40.54 & 40.42 & 40.35 \\
\hline 20 & 40.31 & 40.35 & - & 40.37 & 40.41 & 40.47 & 40.50 & 40.52 & 40.57 & 40.57 & 40.43 & 40.37 \\
\hline 21 & 40.33 & 40.36 & 40.39 & 40.36 & 40.43 & 40.46 & - & - & 40.59 & 40.58 & 40.48 & - \\
\hline 22 & - & 40.37 & 40.38 & 40.35 & 40.43 & 40.46 & 40.50 & - & 40.60 & 40.57 & 40.53 & - \\
\hline 23 & 40.32 & 40.37 & 40.38 & 40.32 & 40.39 & 40.46 & - & 40.51 & 40.59 & 40.58 & 40.52 & 40.50 \\
\hline 24 & 40.34 & 40.36 & 40.37 & 40.35 & 40.40 & 40.47 & - & 40.50 & 40.58 & 40.59 & 40.50 & 40.50 \\
\hline 25 & 40.33 & 40.35 & 40.37 & 40.40 & 40.42 & 40.49 & 40.49 & 40.50 & 40.58 & 40.57 & 40.44 & 40.49 \\
\hline 26 & 40.29 & 40.35 & 40.36 & 40.40 & 40.42 & 40.49 & 40.52 & 40.52 & 40.57 & - & 40.42 & 40.49 \\
\hline 27 & 40.33 & 40.36 & 40.34 & 40.39 & 40.39 & 40.48 & 40.53 & 40.54 & 40.57 & 40.55 & 40.47 & 40.47 \\
\hline 28 & - & 40.36 & 40.34 & 40.37 & 40.37 & 40.46 & 40.53 & 40.55 & 40.58 & 40.53 & 40.52 & 40.41 \\
\hline 29 & 40.30 & - & - & 40.34 & 40.41 & 40.47 & 40.54 & 40.55 & 40.59 & 40.52 & 40.51 & 40.45 \\
\hline 30 & 40.24 & - & 40.35 & 40.37 & 40.41 & 40.49 & 40.54 & 40.53 & 40.60 & 40.51 & 40.48 & 40.51 \\
\hline 31 & 40.25 & - & 40.35 & - & 40.41 & - & 40.53 & 40.53 & - & 40.49 & 一 & 40.52 \\
\hline Mean & 40.32 & 40.33 & 40.35 & 40.37 & 40.40 & 40.46 & 40.50 & 40.54 & 40.57 & 40.57 & 40.50 & 40.47 \\
\hline $\operatorname{Max}$ & 40.39 & 40.39 & 40.40 & 40.40 & 40.44 & 40.49 & 40.54 & 40.57 & 40.60 & 40.62 & 40.55 & 40.52 \\
\hline Min & 40.23 & 40.24 & 40.28 & 40.32 & 40.37 & 40.41 & 40.46 & 40.50 & 40.54 & 40.49 & 40.42 & 40.35 \\
\hline
\end{tabular}




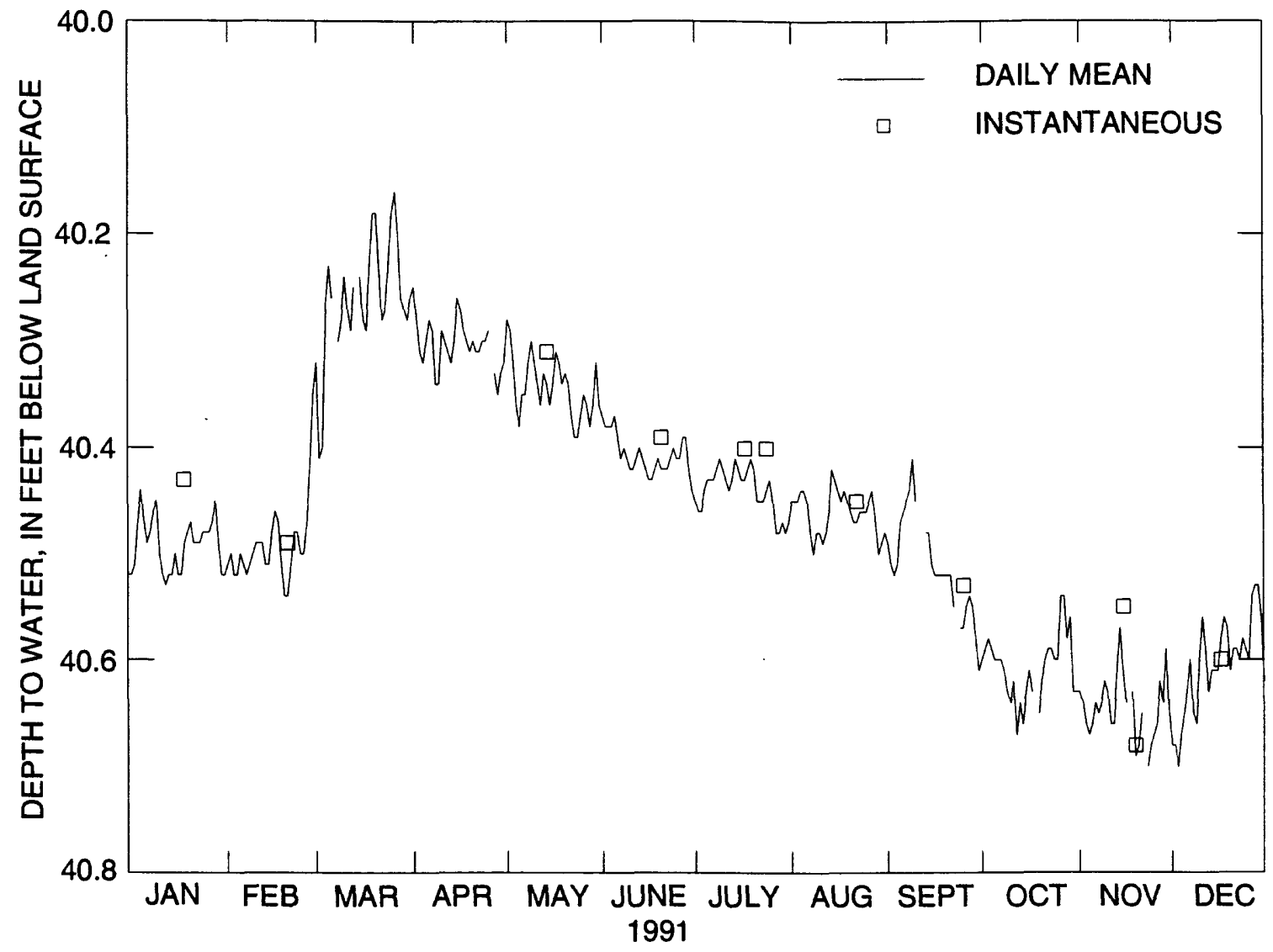

Figure 9. (E) Daily mean and instantaneous water levels, well CH-1, Long Valley Caldera, Mono County, California, 1991. 
Table 13. Daily mean water levels, well CH-1, Long Valley Caldera, Mono County, California, 1991

[Values are in feet below land surface. Max, maximum; Min, minimum; -, no data]

\begin{tabular}{|c|c|c|c|c|c|c|c|c|c|c|c|c|}
\hline Day & Jan & Feb & Mar & Apr & May & June & July & Aug & Sept & Oct & Nov & Dec \\
\hline 1 & 40.52 & 40.51 & 40.32 & 40.25 & 40.28 & 40.37 & 40.45 & 40.45 & 40.49 & 40.60 & 40.63 & 40.68 \\
\hline 2 & 40.51 & 40.50 & 40.41 & 40 & 40.29 & 40.38 & 40.46 & 40.45 & 40.51 & 40.59 & 40.64 & 40.68 \\
\hline 3 & 40.47 & 40.52 & 40.40 & 40.31 & 40.32 & 40.38 & 40.46 & 40.45 & 40.52 & 40.58 & 40.66 & 40.70 \\
\hline 4 & 40.44 & 40.52 & 40.27 & 40.32 & 40.36 & 40.38 & 40.44 & 40.44 & 40.51 & 40.59 & 40.67 & 40.67 \\
\hline 5 & 40.47 & 40.50 & 40.23 & 40.30 & 40.38 & 40.37 & 40.43 & 40.44 & 40.47 & 40.60 & 40.66 & 40.65 \\
\hline 6 & 40.49 & 40.51 & 40.26 & 40.28 & 40.35 & 40.39 & 40.43 & 40.45 & 40.46 & 40.60 & 40.64 & 40.63 \\
\hline 7 & 40.48 & 40.52 & - & 40.29 & 40.35 & 40.41 & 40.43 & 40.48 & 40.45 & 40.60 & 40.65 & 40.60 \\
\hline 8 & 40.46 & 40.51 & 40.30 & 40.34 & 40.32 & 40.40 & 40.42 & 40.50 & 40.44 & 40.61 & 40.64 & 40.65 \\
\hline 9 & 40.45 & 40.50 & 40.28 & 40 & 40.30 & 40.41 & 40.41 & 40.48 & 40.41 & 40.63 & 40.62 & 40.66 \\
\hline 10 & 40.50 & 40.49 & 40.24 & 40.29 & 40.32 & 40.42 & 40.42 & 40.48 & 40.45 & 40.64 & 40.63 & 40.60 \\
\hline 11 & 40.52 & 40.49 & 40.27 & 40.30 & 40.34 & 40.42 & 40.43 & 40.49 & - & 40.62 & 40.66 & 40.56 \\
\hline 12 & 40.53 & 40.49 & 40.29 & 40. & 40.36 & 40.41 & 40.44 & 40.48 & - & 40.67 & 40.66 & 40.59 \\
\hline 13 & 40.52 & 40.51 & 40.25 & 40.32 & 40.33 & 40.40 & 40.43 & 40.46 & 40.48 & 40.64 & 40.61 & 40.63 \\
\hline 14 & 40.52 & 40.51 & - & 40.30 & 40.34 & 40.41 & 40.41 & 40.42 & 40.48 & 40.66 & 40.57 & 40.61 \\
\hline 15 & 40.50 & 40.48 & 40.24 & 40.26 & 40.36 & 40.42 & 40.42 & 40.43 & 40.51 & 40.63 & 40.61 & 40.61 \\
\hline 16 & 40.52 & 40.46 & 40.28 & 40.27 & 40.34 & 40.43 & 40.43 & 40.44 & 40 & 40.61 & 40.64 & 40.61 \\
\hline 17 & 40.52 & 40.47 & 40.29 & 40.29 & 40.31 & 40.43 & 40.43 & 40.45 & 40.52 & 40.63 & - & 40.58 \\
\hline 18 & 40.49 & 40.51 & 40.23 & 40.30 & 40.32 & 40.42 & 40.42 & 40.44 & 40.52 & - & 40.63 & 40.56 \\
\hline 19 & 40.48 & 40.54 & 40.18 & 40.31 & 40.34 & 40.41 & 40.41 & 40.45 & 40.52 & 40.65 & 40.69 & 40.57 \\
\hline 20 & 40.47 & 40.54 & 40.18 & 40.30 & 40.33 & 40.42 & 40.42 & 40.46 & 40.52 & 40.62 & 40.68 & 40.61 \\
\hline 21 & 40.49 & 40.51 & 40.23 & 40.31 & 40.34 & & 40. & & & 60 & 40.65 & 0.59 \\
\hline 22 & 40.49 & 40.48 & 40.28 & 40.31 & 40.37 & 40.42 & 40.45 & 40.47 & 40.55 & 40.59 & - & 40.59 \\
\hline 23 & 40.49 & 40.48 & 40.27 & 40.30 & 40.39 & 40.41 & 40.45 & 40.46 & - & 40.59 & 40.70 & 40.60 \\
\hline 24 & 40.48 & 40.50 & 40.23 & 40.30 & 40.39 & 40.40 & 40.44 & 40.46 & 40.57 & 40.60 & 40.68 & 40.58 \\
\hline 25 & 40.48 & 40.50 & 40.18 & 40.29 & 40.37 & 40.41 & 40.43 & 40.46 & 40.57 & 40.60 & 40.67 & 40.59 \\
\hline 26 & 40.48 & 40.47 & 40.16 & - & 40.35 & 40.41 & 40.45 & 40.45 & & 40.54 & 40.66 & 40.60 \\
\hline 27 & 40.47 & 40.42 & 40.20 & 40.33 & 40.36 & 40.39 & 40.48 & 40.44 & 40.54 & 40.54 & 40.62 & 40.54 \\
\hline 28 & 40.45 & 40.35 & 40.26 & 40.35 & 40.38 & 40.39 & 40.48 & 40.47 & 40.55 & 40.58 & 40.64 & 40.53 \\
\hline 29 & 40.49 & - & 40.27 & 40.33 & 40.36 & 40.42 & 40.47 & 40.50 & 40.58 & 40.56 & 40.59 & 40.53 \\
\hline 30 & 40.52 & - & 40.28 & 40.32 & 40.32 & 40.44 & 40.48 & 40.49 & 40.61 & 40.63 & 40.65 & 40.56 \\
\hline 31 & 40.52 & - & 40.26 & - & 40.36 & - & 40.47 & 40.48 & - & 40.63 & - & 40.62 \\
\hline Mean & 40.49 & 40.49 & 40.26 & 40.30 & & 40.41 & 40 & 40 & 40 & 40.61 & 40.64 & 40.61 \\
\hline $\operatorname{Max}$ & 40.53 & 40.54 & 40.41 & 40.35 & 40.39 & 40.44 & 40.48 & 40.50 & 40.61 & 40.67 & 40.70 & 40.70 \\
\hline Min & 40.44 & 40.35 & 40.16 & 40.25 & 40.28 & 40.37 & 40.41 & 40.42 & 40.41 & 40.54 & 40.57 & 40.53 \\
\hline
\end{tabular}




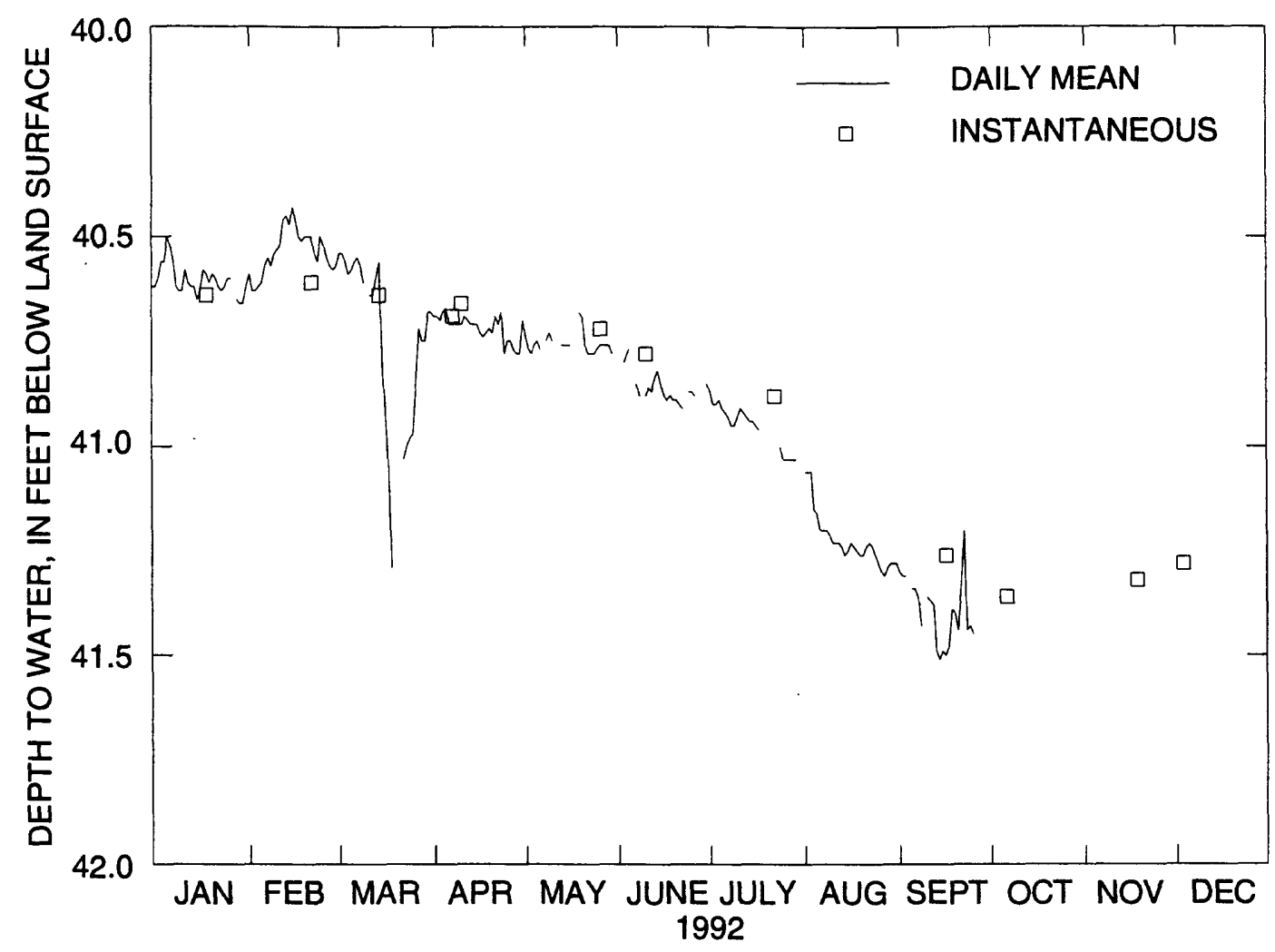

Figure 9. (F) Daily mean and instantaneous water levels, well $\mathrm{CH}-1$, Long Valley Caldera, Mono County, California, 1992. 
Table 14 . Daily mean water levels, well $\mathrm{CH}-1$, Long Valley Caldera, Mono County, California, 1992

[Values are in feet below land surface. Max, maximum: Min, minimum; - , no data]

\begin{tabular}{|c|c|c|c|c|c|c|c|c|c|c|c|c|}
\hline Day & Jan & Feb & Mar & Apr & May & June & July & Aug & Sept & Oct & Nov & Dec \\
\hline 1 & 40.62 & 40.59 & 40.54 & 40.69 & 40.74 & 40.79 & 40.87 & 41.06 & 41.30 & - & - & - \\
\hline 2 & 40.60 & 40.63 & 40.54 & 40.69 & 40.77 & - & 40.90 & 41.06 & 41.31 & - & - & - \\
\hline 3 & 40.56 & 40.63 & 40.56 & 40.70 & 40.78 & 40.80 & 40.90 & 41.06 & 41.31 & - & - & - \\
\hline 4 & 40.56 & 40.62 & 40.59 & 40.68 & 40.76 & 40.78 & 40.89 & 41.15 & - & - & - & - \\
\hline 5 & 40.50 & 40.61 & 40.58 & 40.67 & 40.75 & 40.76 & 40.91 & 41.16 & 41.34 & - & - & - \\
\hline 6 & 40.52 & 40.57 & 40.56 & 40.71 & 40.77 & - & 40.92 & 41.20 & 41.34 & - & - & - \\
\hline 7 & 40.56 & 40.55 & 40.55 & 40.71 & - & 40.85 & 40.93 & 41.20 & 41.36 & - & - & - \\
\hline 8 & 40.62 & 40.57 & 40.57 & 40.71 & 40.75 & 40.88 & 40.95 & 41.20 & 41.43 & - & - & - \\
\hline 9 & 40.63 & 40.54 & 40.61 & 40.71 & 40.73 & - & 40.95 & 41.21 & - & - & - & - \\
\hline 10 & 40.63 & 40.53 & - & 40.71 & 40.75 & 40.88 & 40.93 & 41.23 & 41.36 & - & - & - \\
\hline 11 & 40.58 & 40.52 & 40.64 & 40.69 & - & 40.86 & 40.91 & 41.23 & 41.37 & - & - & - \\
\hline 12 & 40.61 & 40.46 & 40.64 & 40.70 & - & 40.87 & 40.92 & 41.23 & 41.38 & - & - & - \\
\hline 13 & 40.62 & 40.45 & 40.60 & 40.71 & 40.76 & 40.84 & 40.93 & 41.24 & 41.49 & - & - & - \\
\hline 14 & 40.62 & 40.47 & 40.56 & 40.71 & 40.76 & 40.82 & 40.94 & 41.26 & 41.51 & - & - & - \\
\hline 15 & 40.65 & 40.43 & 40.80 & 40.71 & 40.76 & 40.85 & 40.94 & 41.25 & 41.49 & - & - & - \\
\hline 16 & 40.63 & 40.46 & 40.92 & 40.73 & 40.76 & 40.88 & 40.95 & 41.23 & 41.50 & - & - & - \\
\hline 17 & 40.58 & 40.50 & 41.05 & 40.74 & - & 40.89 & 40.96 & 41.24 & 41.48 & - & - & - \\
\hline 18 & 40.59 & 40.51 & 41.29 & 40.73 & - & 40.88 & - & 41.25 & 41.39 & - & - & - \\
\hline 19 & 40.61 & 40.50 & - & 40.72 & 40.68 & 40.89 & - & 41.26 & 41.40 & - & - & - \\
\hline 20 & 40.59 & 40.50 & 41.17 & 40.73 & 40.69 & 40.89 & - & 41.26 & 41.44 & - & - & - \\
\hline 21 & 40.60 & 40.50 & - & 40.69 & 40.76 & 40.90 & - & 41.24 & 41.34 & - & - & - \\
\hline 22 & 40.62 & 40.54 & 41.03 & 40.71 & 40.78 & 40.91 & - & 41.23 & 41.20 & - & - & - \\
\hline 23 & 40.63 & 40.56 & 41.00 & 40.68 & 40.78 & - & - & 41.24 & 41.44 & - & - & - \\
\hline 24 & 40.62 & 40.50 & 40.98 & 40.78 & 40.78 & 40.87 & 41.00 & 41.26 & 41.43 & - & - & - \\
\hline 25 & 40.60 & 40.52 & 40.97 & 40.75 & 40.77 & 40.87 & 41.03 & 41.28 & 41.45 & - & - & - \\
\hline 26 & 40.60 & 40.55 & 40.85 & 40.75 & 40.76 & 40.88 & 41.03 & 41.30 & - & - & - & - \\
\hline 27 & & - & 40.57 & 40.72 & 40.77 & 40.76 & - & 41.03 & 41.31 & - & - & - \\
\hline 28 & 40.65 & 40.58 & 40.75 & 40.78 & 40.76 & - & 41.03 & 41.29 & - & - & - & - \\
\hline 29 & 40.66 & 40.57 & 40.75 & 40.78 & 40.76 & - & 41.03 & 41.28 & - & - & - & - \\
\hline 30 & 40.66 & - & 40.68 & 40.70 & 40.78 & 40.85 & - & 41.28 & - & - & - & - \\
\hline 31 & 40.62 & - & 40.68 & - & - & - & - & 41.28 & - & - & - & - \\
\hline Mean & 40.60 & 40.54 & 40.76 & 40.72 & 40.76 & 40.86 & 40.95 & 41.22 & 41.39 & - & - & - \\
\hline Max & 40.66 & 40.63 & 41.29 & 40.78 & 40.78 & 40.91 & 41.03 & 41.31 & 41.51 & - & - & - \\
\hline Min & 40.50 & 40.43 & 40.54 & 40.67 & 40.68 & 40.76 & 40.87 & 41.06 & 41.20 & - & - & - \\
\hline
\end{tabular}




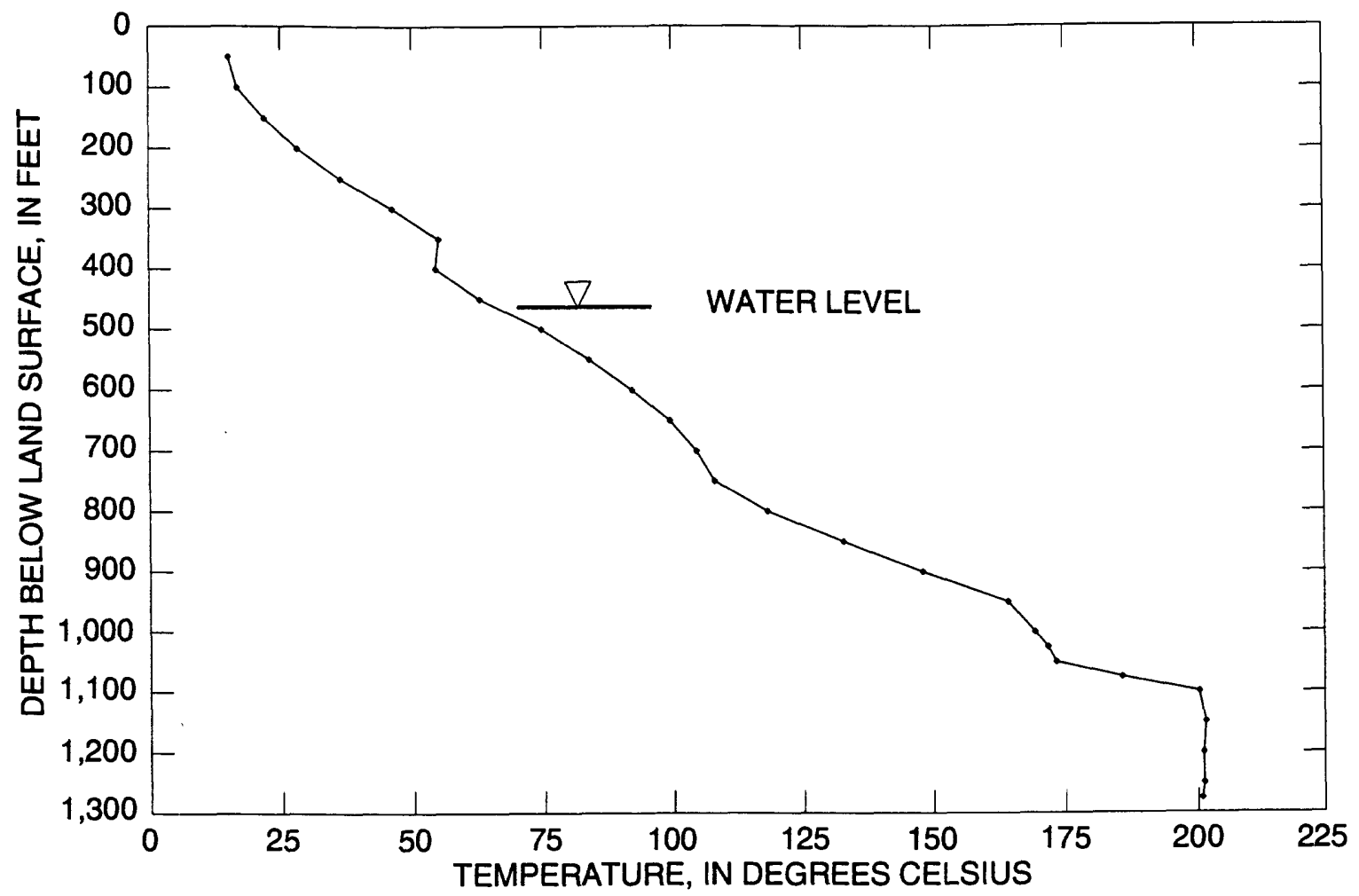

Figure 10. Temperature log of well RDO-8, Long Valley Caldera, Mono County, California, June 19, 1987.

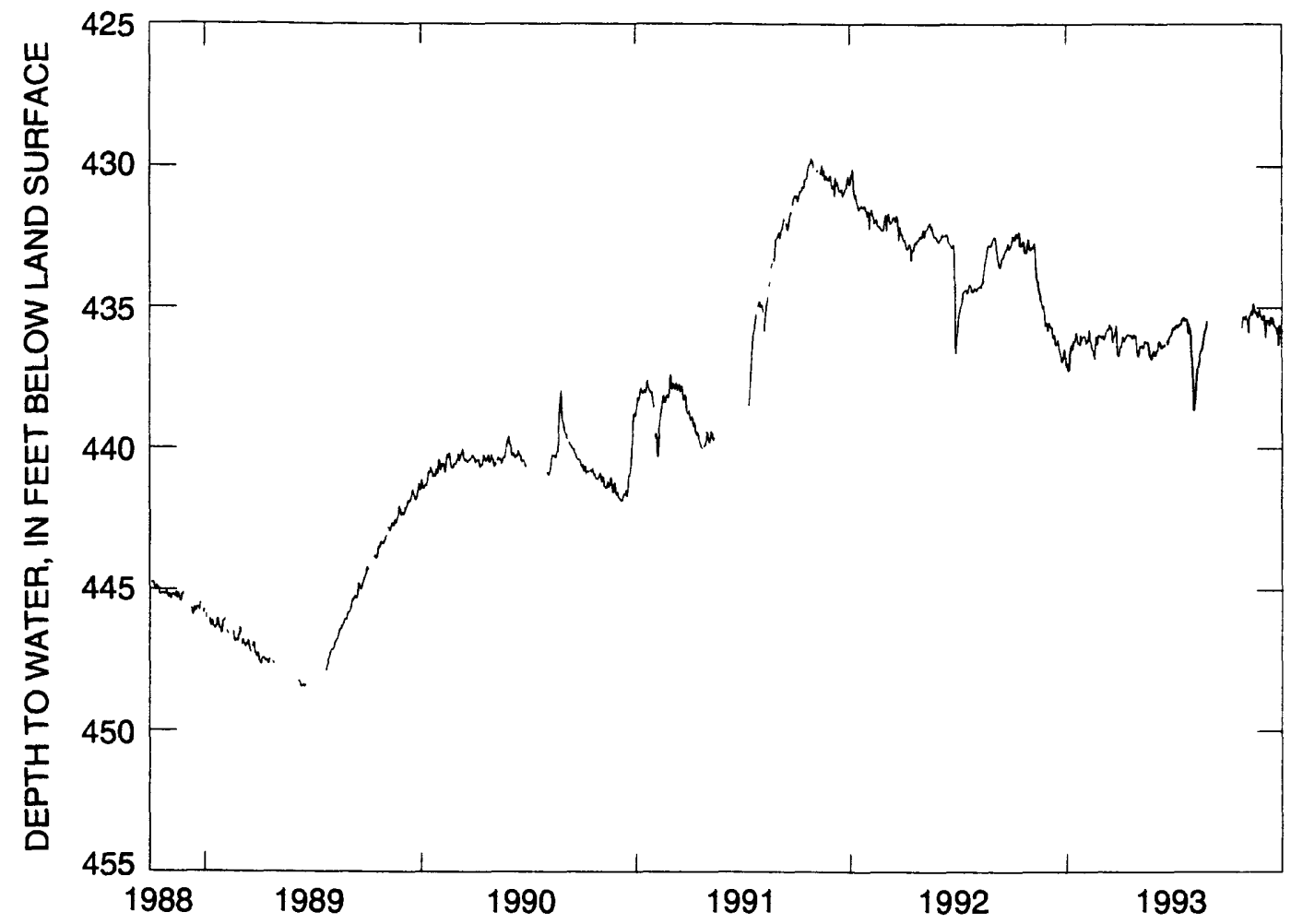

Figure 11. Daily mean water levels, well RDO-8, Long Valley Caldera, Mono County California, 1988-93. 
Table 15. Daily water levels, well RDO-8, Long Valley Caldera, Mono County, California, 1988

[Values are in feet below land surface. Max, maximum; Min, minimum; - , no data]

\begin{tabular}{|c|c|c|c|c|c|c|c|c|c|c|c|c|}
\hline Day & Jan & Feb & Mar & Apr & May & June & July & Aug & Sept & Oct & Nov & Dec \\
\hline 1 & - & - & - & - & - & - & - & - & - & - & 445.17 & - \\
\hline 2 & - & - & - & - & - & - & - & - & - & 444.76 & 445.16 & - \\
\hline 3 & - & - & - & - & - & - & - & - & - & 444.76 & 445.25 & - \\
\hline 4 & - & - & - & - & - & - & - & - & - & 444.76 & 445.35 & - \\
\hline 5 & - & - & - & - & - & - & - & - & - & 444.72 & 445.30 & - \\
\hline 6 & - & - & - & - & - & - & - & - & - & 444.73 & 445.19 & - \\
\hline 7 & - & - & - & - & - & - & - & - & - & 444.86 & 445.15 & - \\
\hline 8 & - & - & - & - & - & - & - & - & - & 445.01 & 445.19 & 445.68 \\
\hline 9 & - & - & - & - & - & - & - & - & - & 444.95 & 445.22 & 445.69 \\
\hline 10 & - & - & - & - & - & - & - & - & - & 444.89 & 445.18 & 445.70 \\
\hline 11 & - & - & - & - & - & - & - & - & - & 444.85 & 445.24 & 445.88 \\
\hline 12 & - & - & - & - & - & - & - & - & - & 444.87 & 445.27 & 445.91 \\
\hline 13 & - & - & - & - & - & - & - & - & - & 444.89 & 445.10 & 445.81 \\
\hline 14 & - & - & - & - & - & - & - & - & - & 444.97 & 445.11 & 445.63 \\
\hline 15 & - & - & - & - & - & - & - & - & - & 445.14 & 445.32 & 445.64 \\
\hline 16 & - & - & - & - & - & - & - & - & - & 445.19 & 445.24 & 445.77 \\
\hline 17 & - & - & - & - & - & - & - & - & 444.43 & 445.17 & 445.12 & 445.75 \\
\hline 18 & - & - & - & - & - & - & - & - & 444.51 & 445.17 & 445.30 & 445.66 \\
\hline 19 & - & - & - & - & - & - & - & - & 444.42 & 445.12 & 445.37 & 445.61 \\
\hline 20 & - & - & - & - & - & - & - & - & 444.32 & 445.12 & 445.38 & 445.62 \\
\hline 21 & - & - & - & - & - & - & - & - & 444.44 & 445.17 & 445.43 & 445.65 \\
\hline 22 & - & - & - & - & - & - & - & - & - & 445.15 & 445.36 & 445.64 \\
\hline 23 & - & - & - & - & - & - & - & - & - & 445.15 & 445.17 & 445.74 \\
\hline 24 & - & - & - & - & - & - & - & - & - & 445.15 & 445.17 & 445.46 \\
\hline 25 & - & - & - & - & - & - & - & - & 444.62 & 445.17 & 445.11 & - \\
\hline 26 & - & - & - & - & - & - & - & - & 444.69 & 445.12 & - & - \\
\hline 27 & - & - & - & - & - & - & - & - & 444.73 & 445.04 & - & - \\
\hline 28 & - & - & - & - & - & - & - & - & 444.81 & 445.10 & - & - \\
\hline 29 & - & - & - & - & - & - & - & - & 444.82 & 445.19 & - & - \\
\hline 30 & - & - & - & - & - & - & - & - & 444.76 & 445.28 & - & 445.83 \\
\hline 31 & - & - & - & - & - & - & - & - & - & 445.27 & - & 445.70 \\
\hline Mean & - & - & - & - & - & - & - & - & 444.60 & 445.02 & 445.23 & 445.70 \\
\hline Max & - & - & - & - & - & - & - & - & 444.82 & 445.28 & 445.43 & 445.91 \\
\hline Min & - & - & - & - & - & - & - & - & 444.32 & 444.72 & 445.10 & 445.46 \\
\hline
\end{tabular}




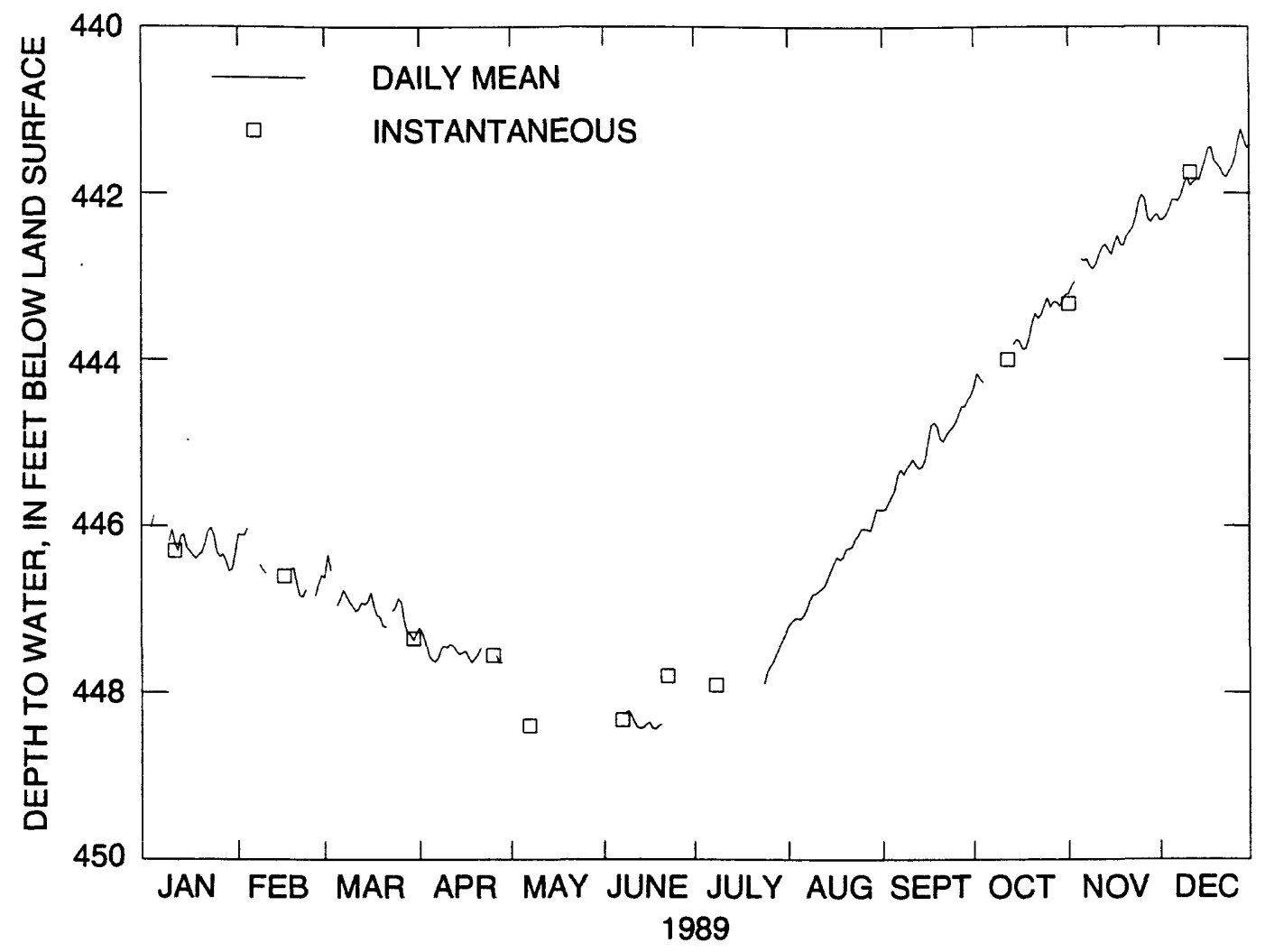

Figure 12. (A) Daily mean and instantaneous water levels, well RDO-8, Long Valley Caldera, Mono County, California, 1989. 
Table 16. Daily mean water levels, well RDO-8, Long Valley Caldera, Mono County, California, 1989

[Values are in feet below land surface. Max, maximum; Min, minimum; -, no data]

\begin{tabular}{|c|c|c|c|c|c|c|c|c|c|c|c|c|}
\hline Day & Jan & Feb & Mar & Apr & May & June & July & Aug & Sept & Oct & Nov & Dec \\
\hline 1 & - & 446.10 & 446.62 & 447.22 & 447.77 & - & - & 447.20 & 445.80 & 444.33 & 443.20 & 442.32 \\
\hline 2 & - & 446.11 & 446.34 & 447.29 & - & - & - & 447.15 & 445.79 & 444.16 & 43.11 & .31 \\
\hline 3 & 446.02 & 446.11 & 446.53 & 447.40 & - & - & - & 447.11 & 445.71 & 444.22 & 443.05 & 42.27 \\
\hline 4 & 445.87 & 446.03 & - & 447.56 & - & - & - & 447.10 & 445.64 & 444.27 & - & 42.19 \\
\hline 5 & - & - & 446.96 & 447.61 & - & - & - & 447.11 & 445.56 & - & 442.78 & 442.08 \\
\hline 6 & - & - & 446.88 & 447.63 & - & - & - & 447.07 & 445.38 & - & 442.80 & 442.08 \\
\hline 7 & - & - & 446.77 & 447.58 & - & - & - & 447.00 & 445.31 & - & & 442.09 \\
\hline 8 & - & 446.46 & 446.84 & 447.47 & - & 448.25 & - & 446.89 & 37 & - & 87 & 442.02 \\
\hline 9 & 446.19 & 446.52 & 446.91 & 447.44 & - & 448.22 & - & 82 & 44 & - & 90 & .89 \\
\hline 10 & 446.04 & 446.57 & 446.96 & 447.46 & - & 448.28 & - & 446.81 & 445.25 & - & 442.84 & 441.81 \\
\hline 11 & 446.21 & - & 447 & 447 & - & 6 & - & & 445.19 & - & 73 & 441.91 \\
\hline 12 & 446.30 & 446.40 & 447.00 & 447 & - & 2 & - & & & - & 64 & 441.87 \\
\hline 13 & 446.13 & - & 446.92 & 447. & - & 448 & - & & 30 & - & 61 & 83 \\
\hline 14 & 446.10 & - & 446.94 & 447.54 & - & 44 & - & 52 & 28 & 443.81 & 67 & 441.84 \\
\hline 15 & 446.26 & - & 446.91 & 447.52 & - & 448.38 & - & 446.53 & 445.20 & 443.75 & 442.73 & 441.72 \\
\hline 16 & 446.30 & 446.68 & 446 & 44 & - & & - & & 8 & & & \\
\hline 17 & 4 & - & 446 & 447 & - & & - & & 8 & & 50 & 46 \\
\hline 18 & 446.39 & 446.52 & 447.07 & 447.64 & - & 4 & - & 0 & 44 & 44 & 61 & 441.45 \\
\hline 19 & 446.35 & 446.50 & 447.09 & 447.59 & - & 44 & - & 7 & 44 & 443.74 & 62 & 441.61 \\
\hline 20 & 446.32 & 446.68 & 447.20 & 447.54 & - & 448.38 & - & 446.27 & 444.95 & 443.56 & 442.51 & 441.65 \\
\hline 21 & 6.22 & 446 & 447.21 & 447.46 & - & - & 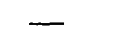 & & & & & \\
\hline 22 & 446.07 & 446.85 & - & - & - & - & - & 446.24 & 444.90 & 443 & .41 & 41.78 \\
\hline 23 & 446.02 & 446.76 & 447.02 & - & - & - & - & 446.15 & 444.85 & 443 & .29 & 41.81 \\
\hline 24 & 446.11 & - & 446.98 & - & - & - & 447.89 & 446.10 & 444.81 & 443.34 & 442.10 & 441.74 \\
\hline 25 & 446.31 & - & 446.87 & - & - & - & 447.75 & 446.03 & 444.75 & 443.25 & 442.02 & 441.68 \\
\hline 26 & & 4 & & & 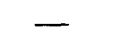 & - & & & & & & \\
\hline 27 & 446.34 & 446.70 & 447.15 & 447.64 & - & - & 62 & 446.04 & 444.56 & & .30 & 441.38 \\
\hline 28 & 446.42 & 446.59 & 447.28 & 447.64 & - & - & 47.53 & & 444.56 & & .34 & 441.23 \\
\hline 29 & 446.54 & - & 447.30 & - & - & - & 447.45 & & & & 442.28 & 441.35 \\
\hline 30 & 446.52 & - & 447.37 & - & - & - & 447.38 & 445.79 & 444.43 & 44 & 442.25 & 441.45 \\
\hline 31 & 446.33 & - & 447.29 & - & - & - & 447.30 & 445.80 & - & 443.21 & - & 441.42 \\
\hline Mean & & & & & & & & & & & & \\
\hline Max & 446.54 & 446. & 447 & 447.64 & 447.77 & & & & & & 143.20 & 442.32 \\
\hline Min & 445.87 & 446.03 & 446.34 & 447.22 & 447.77 & 448.22 & 447.30 & 445.79 & 444.43 & 443.21 & 442.02 & 441.23 \\
\hline
\end{tabular}




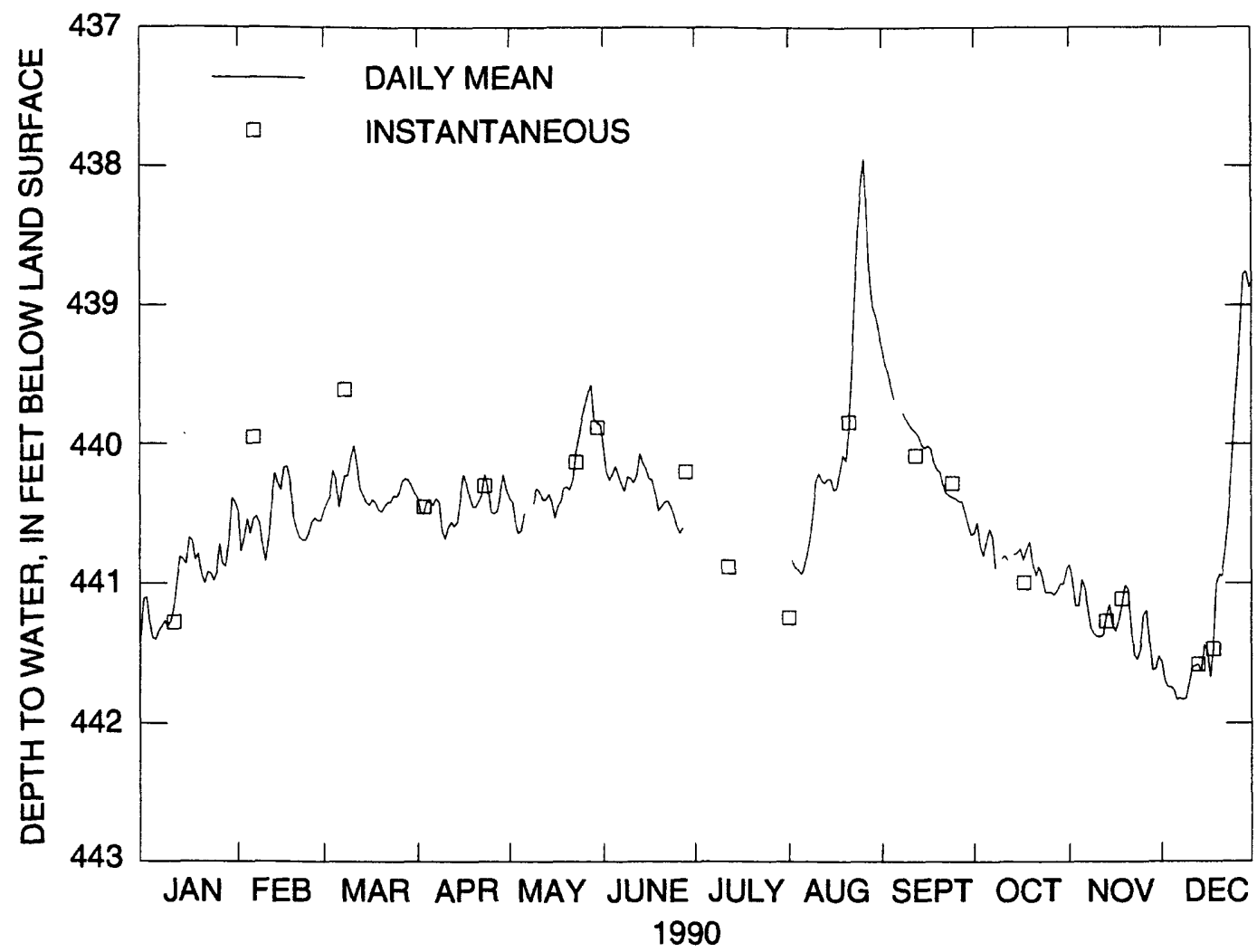

Figure 12. (B) Daily mean and instantaneous water levels, well RDO-8, Long Valley Caldera, Mono County, California, 1990. 
Table 17. Daily mean water levels, well RDO-8, Long Valley Caldera, Mono County, California, 1990

[Values are in feet below land surface. Max, maximum; Min, minimum; - , no data]

\begin{tabular}{|c|c|c|c|c|c|c|c|c|c|c|c|c|}
\hline Day & Jan & Feb & Mar & Apr & May & June & July & Aug & Sept & Oct & Nov & Dec \\
\hline 1 & 441.11 & 440.48 & 440.47 & 440.39 & 440.39 & 440.02 & - & - & 439.31 & 440.64 & 440.86 & 441.56 \\
\hline 2 & 441.10 & 440.77 & 440.42 & 440.50 & 440.42 & 440.20 & - & 440.83 & 439.42 & 440.56 & 440.99 & 41.69 \\
\hline 3 & 441.28 & 440.68 & 440.38 & 440.49 & 440.56 & 440.26 & - & 440.88 & 439.48 & 440.73 & 441.16 & 41.74 \\
\hline 4 & 441.39 & 440.54 & 440.19 & 440.41 & 440.64 & 440.22 & - & 440.90 & 439.58 & 440.80 & 441.16 & 441.74 \\
\hline 5 & 441.40 & 440.64 & 440.25 & 440.42 & 440.62 & 440.16 & - & 440.93 & 439.67 & 440.70 & 440.97 & 441.76 \\
\hline 6 & 441.34 & 440.54 & 440.45 & 440.44 & 440.50 & 440.23 & - & 440.89 & - & 440.61 & 441.03 & 41.83 \\
\hline 7 & 441.31 & 440.52 & 440.34 & 440.39 & - & 440.29 & - & 440.80 & - & 440.67 & 441.19 & 41.82 \\
\hline 8 & 441.27 & 440.57 & 440.23 & 440.42 & - & 440.34 & - & 440.68 & 439.77 & 440.89 & 441.32 & 41.83 \\
\hline 9 & 441.31 & 440.72 & 440.23 & 440.62 & 440.43 & 440.24 & - & 440.49 & 439.82 & - & 441.36 & 41.82 \\
\hline 10 & 441.27 & 440.84 & 440.10 & 440.68 & 440.32 & 440.25 & - & 440.27 & 439.86 & 440.82 & 441.38 & 441.72 \\
\hline 11 & 441.16 & 440.68 & 440.01 & 440.60 & 440.34 & 44 & - & .21 & 439.89 & 440.80 & 41.38 & +1.60 \\
\hline 12 & 440.96 & 440.42 & 440.14 & 440.56 & 440.40 & 44 & - & 440.26 & 439.91 & 440.83 & 41.37 & 1.59 \\
\hline 13 & 440.81 & 440.21 & 440.31 & 440.59 & 440.40 & 440 & - & 440.28 & 439.94 & - & 41.23 & 441.58 \\
\hline 14 & 440.83 & 440 & 440.37 & 440.56 & 440.36 & 44 & - & .25 & 440.00 & 440.79 & 41.15 & 1.63 \\
\hline 15 & 440.86 & 440.33 & 440.42 & 440.39 & 440.42 & 440.18 & - & 440.25 & 440.03 & 440.78 & 441.30 & 41.44 \\
\hline 16 & 440.67 & 440.17 & 440.44 & 440.22 & 440.53 & 440 & - & .33 & 440.01 & 440.75 & 1.34 & 441.48 \\
\hline 17 & 440.70 & 440.16 & 440.40 & 440.28 & 440.45 & 440 & - & .32 & 440.03 & 440.83 & 1.27 & 41.67 \\
\hline 18 & 440.83 & 440 & 440.42 & 440.38 & 440.41 & 44 & - & .20 & 440.13 & 440.76 & 41.16 & .46 \\
\hline 19 & 440.79 & 440.52 & 440.47 & 440.45 & 440.32 & 440 & - & 440.08 & 440.18 & 440.70 & 441.01 & 441.00 \\
\hline 20 & 440.94 & 440.61 & 440.49 & 440.45 & 440.31 & 440.45 & - & 440.12 & 440.20 & 440.85 & 441.04 & 440.94 \\
\hline 21 & .00 & 4 & 0.45 & 440.41 & 440.33 & & - & & 440.29 & 440.94 & .29 & \\
\hline 22 & 440.92 & 440.69 & 440.42 & 440.36 & 440.26 & 44 & - & .43 & 440.35 & 440.88 & 11.51 & 40.78 \\
\hline 23 & 440.93 & 440.69 & 440.42 & 440.22 & 440.06 & 440 & - & 438.87 & 440.37 & 440.95 & 41.54 & 440.56 \\
\hline 24 & 440.98 & 440.64 & 440.37 & 440.31 & 439.94 & 44 & - & 438.45 & 440.38 & 441.06 & 441.47 & 440.23 \\
\hline 25 & 440.92 & 440.56 & 440.38 & 440.49 & 439.81 & 440.59 & - & 438.12 & 440.39 & 441.06 & 441.23 & 439.83 \\
\hline 26 & .72 & 4 & 5 & & 439.72 & & - & 94 & 440.41 & .06 & .19 & .52 \\
\hline 27 & 440.86 & 440 & 440.27 & 440.48 & 439.63 & 440.60 & - & .40 & 440.41 & 441.08 & 1.41 & 439.20 \\
\hline 28 & 440.88 & 440.55 & 440.25 & 440.36 & 439.57 & - & - & 438.83 & 440.49 & 441.05 & 441.62 & 438.78 \\
\hline 29 & 440.70 & - & 440.26 & 440.22 & 439.82 & - & - & 439.01 & 440.57 & 441.00 & 441.61 & 438.76 \\
\hline 30 & 440.39 & - & 440.30 & 440.32 & 439.85 & - & - & 439.07 & 440.65 & 441.00 & 441.52 & 438.87 \\
\hline 31 & 440.42 & - & 440.35 & - & 439.86 & - & - & 439.17 & 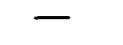 & 440.90 & - & 438.82 \\
\hline Mean & 440.97 & 440.53 & 440.33 & 440.43 & 440.23 & & - & & 440.05 & 440.84 & 441.27 & 440.91 \\
\hline $\operatorname{Max}$ & 441.40 & 440.84 & 440.49 & 440.68 & 440.64 & 440.64 & - & 440.93 & 440.65 & 441.08 & 441.62 & 441.83 \\
\hline Min & 440.39 & 440.16 & 440.01 & 440.22 & 439.57 & 440.02 & - & 437.94 & 439.31 & 440.56 & 440.86 & 438.76 \\
\hline
\end{tabular}




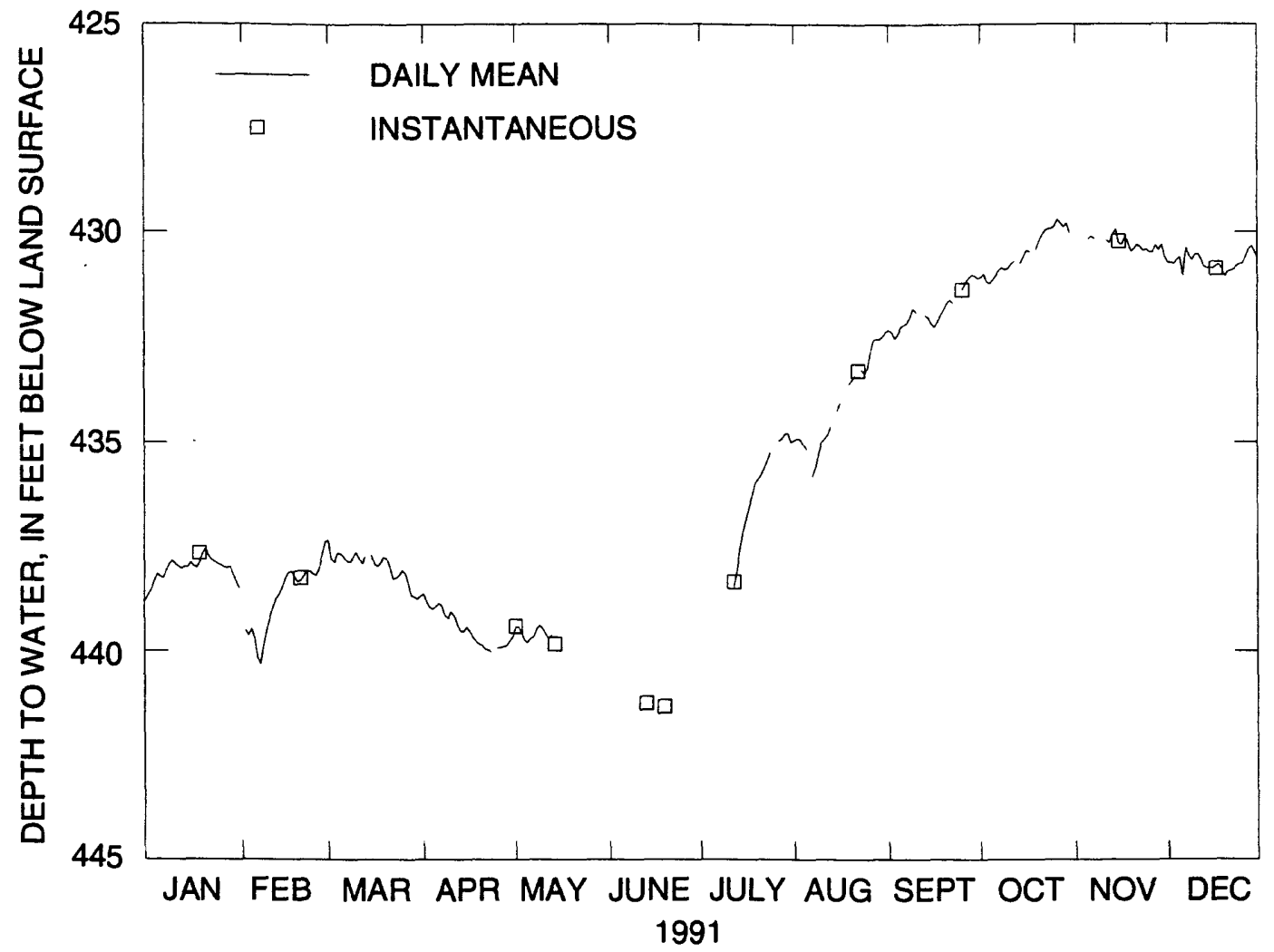

Figure 12. (C) Daily mean and instantaneous water levels, well RDO-8, Long Valley Caldera, Mono County, California, 1991. 
Table 18. Daily mean water levels, well RDO-8, Long Valley Caldera, Mono County, California, 1991

[Values are in feet below land surface. Max, maximum; Min, minimum; - , no data]

\begin{tabular}{|c|c|c|c|c|c|c|c|c|c|c|c|c|}
\hline Day & Jan & Feb & Mar & Apr & May & June & July & Aug & Sept & Oct & Nov & Dec \\
\hline 1 & 438.68 & - & 437.37 & 438.65 & 439.42 & - & - & 434.95 & 432.32 & 431.07 & 430.11 & 430.73 \\
\hline 2 & 438.56 & 439.51 & 437.82 & 438.83 & 439.43 & - & - & 90 & 432.38 & 431.00 & - & 30.73 \\
\hline 3 & 438.35 & 439.63 & 437.90 & 438.96 & 439.56 & - & - & 434.92 & 432.53 & 431.18 & 430.21 & 430.76 \\
\hline 4 & 438.18 & 439.48 & 437.68 & 439.01 & 439.73 & - & - & 435.04 & 432.42 & 431.22 & - & 430.66 \\
\hline 5 & 438.25 & 439.70 & 437.69 & 438.95 & 439.80 & - & - & 435.15 & 432.25 & 431.13 & 430.17 & 430.60 \\
\hline 6 & 438.28 & 440.16 & 437.75 & 438.87 & 439.70 & - & - & - & 432.20 & 431.03 & 430.10 & 431.03 \\
\hline 7 & 438.09 & 440.31 & 437.87 & 438.93 & 439 & - & - & 435.82 & 432.16 & 430.91 & 430.15 & 430.37 \\
\hline 8 & 437.94 & 439.85 & 437.89 & 439.15 & 439.48 & - & - & 435.61 & 432.02 & 430.84 & - & 430.56 \\
\hline 9 & 437.86 & 439.48 & 437.80 & 439.23 & 439.39 & - & - & 435.25 & 431.81 & 430.89 & 430.03 & 430.66 \\
\hline 10 & 437.95 & 439.22 & 437.66 & 439.07 & 439.47 & - & - & 434.99 & 431.90 & 430.86 & - & 430.53 \\
\hline 11 & .00 & 4 & 30 & 439.16 & 439 & - & - & 434.91 & - & 430.75 & 430.20 & 0.52 \\
\hline 12 & 438.05 & 43 & 437.93 & 439.38 & 439 & - & 438.47 & 434.81 & - & 430.69 & 430.26 & 0.64 \\
\hline 13 & 438.02 & 438.67 & 437.77 & 439.53 & 439.6 & - & 438.09 & 434.61 & 431.98 & - & 430.05 & 430.82 \\
\hline 14 & 438.02 & 438.50 & - & 439.53 & - & - & 437.58 & - & 432.03 & 430.74 & 429.94 & 430.85 \\
\hline 15 & 437.91 & 438.29 & 437.71 & 439.43 & - & - & 437.17 & 434.23 & 432.16 & 430.57 & 430.27 & 430.85 \\
\hline 16 & .99 & 4 & 437.94 & 439.53 & $\ldots$ & - & 436 & 434.06 & 432 & 430.43 & 430.30 & 430.87 \\
\hline 17 & 438.03 & 438.12 & 437.99 & 439.67 & - & - & 436.57 & - & 432.13 & 430.46 & 430.11 & 430.81 \\
\hline 18 & 437.90 & 438.24 & 437.90 & 439.76 & - & - & 436.32 & - & 431.97 & - & 430.25 & 430.77 \\
\hline 19 & 437.70 & 37 & 437.78 & 439.83 & - & - & 436.01 & 433.60 & 43 & 430.41 & 430.46 & 430.87 \\
\hline 20 & 437.57 & 438.34 & 437.81 & 439.86 & - & - & 435.90 & 433.50 & 431.68 & 430.22 & 430.40 & 431.05 \\
\hline 21 & 7.74 & & & 439 & - & - & 43 & 433.41 & 431 & 43 & 430.30 & 430.95 \\
\hline 22 & 437.84 & 438.10 & 438.29 & 439.97 & - & - & 435.63 & - & 431.68 & 429.96 & 430.36 & 430.92 \\
\hline 23 & 4 & 4 & 438.27 & 440.02 & - & - & 43 & 433.25 & - & 429.93 & 430.44 & 430.90 \\
\hline 24 & 437.94 & 438.18 & 438.21 & - & - & - & 435.24 & 433.34 & - & 429.91 & 430.41 & 430.80 \\
\hline 25 & 437.97 & 438.21 & 438.09 & 439.93 & - & - & - & 433.23 & 431.36 & 429.87 & 430.47 & 430.76 \\
\hline 26 & & & & & - & - & - & & & & 43 & 430.74 \\
\hline 27 & 438.03 & 43 & 40 & 439.89 & - & - & 434.9 & 432.56 & 431.10 & 429.78 & 430.32 & 430.57 \\
\hline 28 & 438.00 & 437.40 & 438.69 & 439.87 & 一 & - & 434.90 & 432.54 & 43 & 429.88 & 430.43 & 430.40 \\
\hline 29 & 438.20 & - & 438.73 & 439.76 & - & - & 434.79 & 432.54 & 431.03 & 429.79 & 430.30 & 430.33 \\
\hline 30 & 438.36 & - & 438.77 & 439.66 & - & - & 434.77 & 432.47 & 431.09 & 430.02 & 430.59 & 430.48 \\
\hline 31 & 438.52 & - & 438.70 & - & - & - & 434.99 & 432.36 & - & - & - & 430.62 \\
\hline Mean & & & & & 43 & - & & & & & 430.27 & 430.71 \\
\hline $\operatorname{Max}$ & 438.68 & 440.31 & 438.77 & 440.02 & 439. & - & 438 & 435.82 & 432 & 431.22 & 430.59 & 431.05 \\
\hline Min & 437.57 & 437.40 & 437.37 & 438.65 & 439.39 & - & 434.77 & 432.36 & 431.02 & 429.70 & 429.94 & 430.33 \\
\hline
\end{tabular}




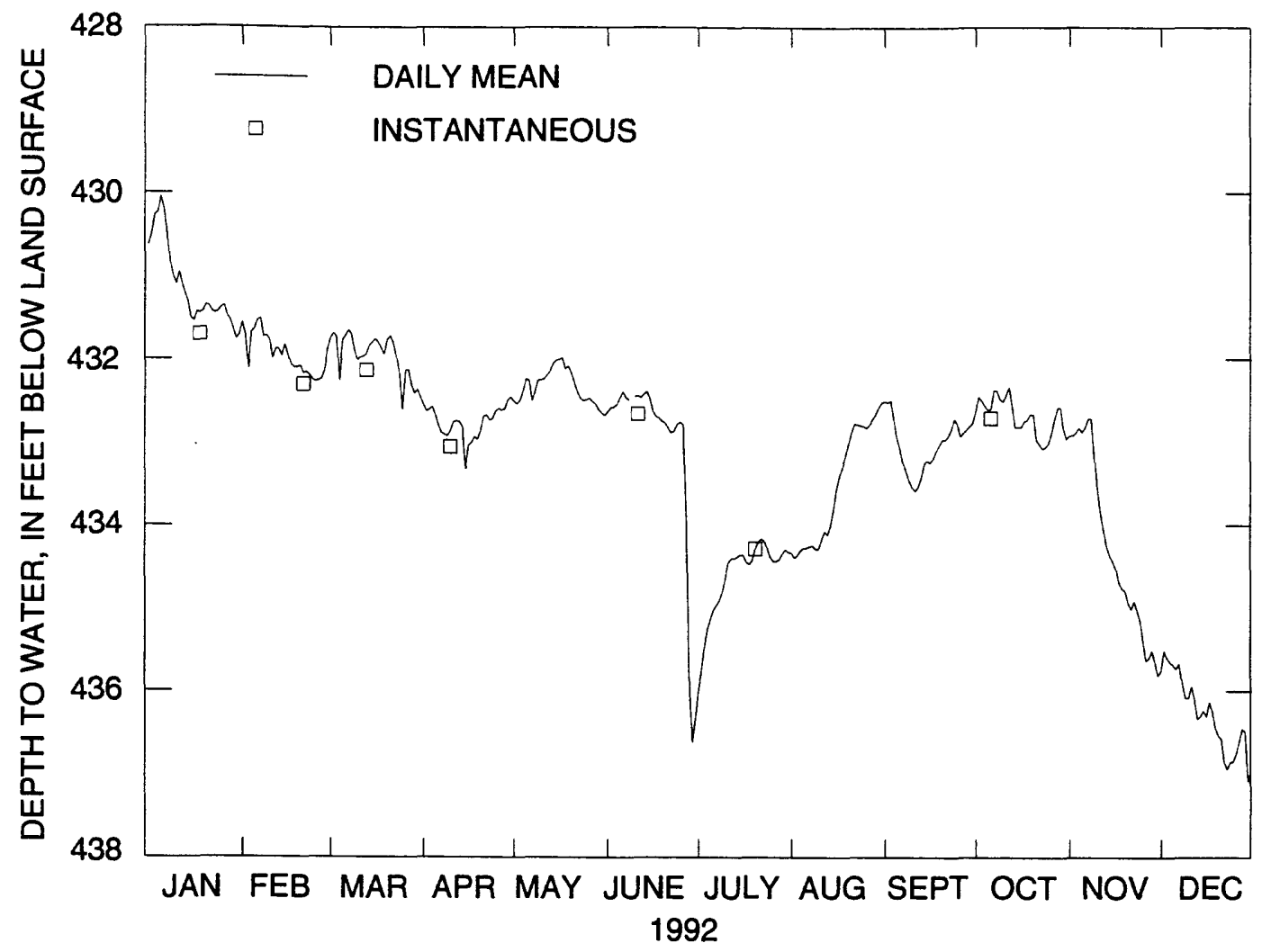

Figure 12. (D) Daily mean and instantaneous water levels, well RDO-8, Long Valley Caldera, Mono County, California, 1992. 
Table 19. Daily mean water levels, well RDO-8, Long Valley Caldera, Mono County, California, 1992

[Values are in feet below land surface. Max, maximum; Min, minimum; - , no data]

\begin{tabular}{|c|c|c|c|c|c|c|c|c|c|c|c|c|}
\hline Day & Jan & Feb & Mar & Apr & May & June & July & Aug & Sept & Oct & Nov & Dec \\
\hline 1 & 430.63 & 431.56 & 431.75 & - & 43 & 43 & 8 & 4 & 0 & 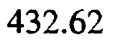 & 11 & 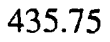 \\
\hline 2 & 430.50 & 431.71 & 431.69 & 432.62 & 432.53 & 432 & 435 & 434.39 & & & & 35.51 \\
\hline 3 & 430.27 & 432.11 & - & 432.60 & 432.49 & 432.57 & 435.47 & 434.36 & 9 & & & 435.60 \\
\hline 4 & 430.24 & 431.68 & 432.25 & 432.57 & 432.37 & 432.54 & 435.25 & 434.30 & 432.71 & 432.55 & & 435.65 \\
\hline 5 & 430.05 & 431.64 & 431.78 & 432.67 & 432.23 & 432.46 & 435.13 & 434.27 & 432.93 & 432.60 & 432.87 & 435.68 \\
\hline 6 & 430.20 & 4 & 4 & 9 & - & 432.38 & 2 & 7 & 6 & 32.59 & 32.82 & 435.73 \\
\hline 7 & 430.48 & 431.51 & 431 & 38 & 432.49 & 432.45 & 96 & 434.25 & 24 & 36 & & 435.67 \\
\hline 8 & 430.84 & 431.73 & 431.70 & 432.90 & 432.40 & 432.48 & 434.90 & 434.24 & 433.35 & 432.37 & .71 & 435.92 \\
\hline 9 & 431.01 & 431.72 & 431.89 & 432.92 & 432.25 & - & 434.79 & 434.28 & 433.46 & 432.47 & 433.16 & 436.08 \\
\hline 10 & 431.10 & 431.77 & 432.01 & 432.86 & 432.24 & 432.44 & 434.62 & 434.28 & 433.54 & 432.50 & 433.57 & 436.08 \\
\hline 11 & 0.96 & 4 & 8 & & 432.23 & 432.43 & 45 & 17 & & 2 & & 35.94 \\
\hline 12 & 431.10 & 431.88 & 431.97 & 432.74 & - & 432.45 & 434.40 & 434.07 & 53 & 32 & 34.06 & 436.09 \\
\hline 13 & 431.22 & - & 431.94 & 432.75 & 432.14 & 432.41 & 434.40 & 34.11 & 41 & 55 & 34.25 & 436.33 \\
\hline 14 & 431.32 & 431.96 & 431.84 & 432.81 & 432.05 & 432.38 & 434.38 & 433.99 & & 31 & & 436.30 \\
\hline 15 & 431.51 & 431.83 & 431.80 & 433.32 & 432.01 & 432.47 & 434.35 & 433.79 & 433.21 & 432.81 & 434.43 & 436.23 \\
\hline 20 & 431.54 & & & & 32.00 & 2 & & & & & & 436.30 \\
\hline 17 & 431 & 432 & 431. & 00 & 一 & 432 & 44 & & & & & 436.13 \\
\hline 18 & 5 & 4. & 7 & & 一 & 0 & 47 & 29 & & & & 36.24 \\
\hline 19 & 1.43 & 10 & 43 & 96 & 432.08 & 432.74 & 42 & 16 & & & 79 & 436.44 \\
\hline 20 & 431.35 & 432.08 & 431.77 & 432.86 & 432.16 & 432.76 & 4.28 & 33.01 & 32.96 & 432.66 & 34.93 & 436.53 \\
\hline 21 & 37 & & 2 & & 27 & & & & & & & 6.57 \\
\hline 22 & 3 & & 1 & & 38 & & & & & & & 436.85 \\
\hline 23 & 1.45 & 4 & 7 & .73 & 432.46 & 432.86 & 19 & .78 & 84 & 07 & 03 & 36.93 \\
\hline 24 & 431.43 & 43 & 43 & 71 & 432.49 & 43 & & & & 05 & 15 & 436.85 \\
\hline 25 & 431.38 & 432.26 & 432.60 & 432.62 & 432.48 & 432.75 & 434.38 & 432.80 & 432.77 & 433.00 & 435.40 & 436.84 \\
\hline 26 & 431.3 & & & & & & & & & & & 6.74 \\
\hline 27 & - & 4 & $4 ?$ & & 432.50 & 434 & & 78 & & 70 & & 36.61 \\
\hline 28 & 431.53 & 432.1 & 432. & 432.59 & 432.53 & 435.96 & 11 & 432.71 & & 57 & & 436.45 \\
\hline 29 & 431.64 & 431.8 & 432.40 & 432.48 & 432.60 & 436.61 & & 32.65 & & & & 36.48 \\
\hline 30 & .76 & - & & 432.45 & & 436.32 & & & 432.76 & & 435.81 & 437.07 \\
\hline 31 & 431.71 & - & 432.45 & - & 432.67 & - & 434.32 & 432.51 & - & 432.95 & - & 36.97 \\
\hline $\mathrm{Me}_{\mathrm{c}}$ & 4 & & & & & & & & & & & 436.28 \\
\hline $\mathrm{Ma}$ & 431.76 & & 432 & & 432.67 & 436.61 & 435.98 & & & & & 437.07 \\
\hline Min & 430.05 & 431.51 & 431.65 & 432.45 & 431.98 & 432.38 & 434.16 & 432.51 & 432.49 & 432.32 & 432.71 & 435.51 \\
\hline
\end{tabular}




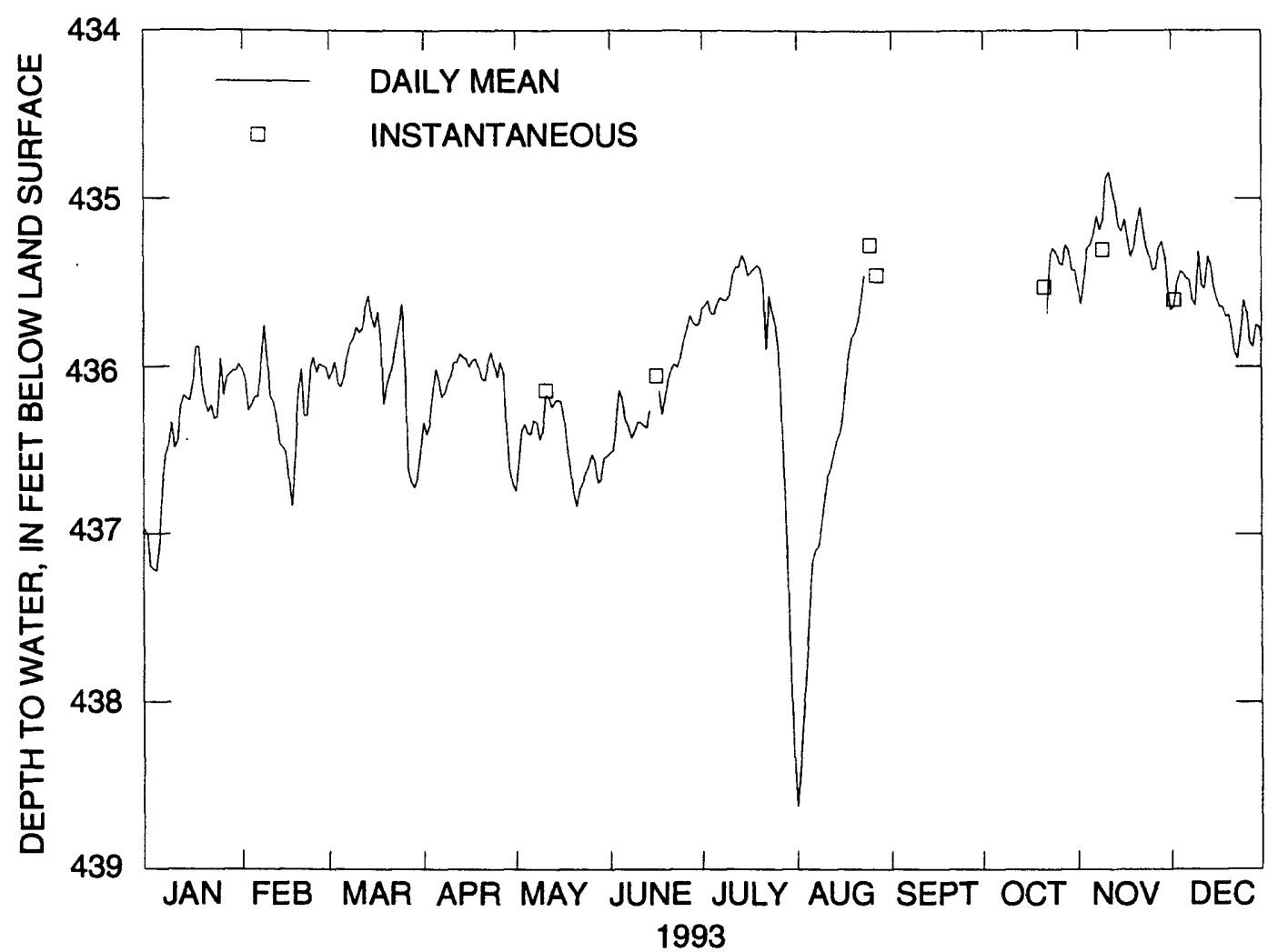

Figure 12. (E) Daily mean and instantaneous water levels, well RDO-8, Long Valley Caldera, Mono County, California, 1993. 
Table 20. Daily mean water levels, well RDO-8, Long Valley Caldera, Mono County, California, 1993

[Values are in feet below land surface. Max, maximum; Min, minimum; -, no data]

\begin{tabular}{|c|c|c|c|c|c|c|c|c|c|c|c|c|}
\hline Day & Jan & Feb & Mar & Apr & May & June & July & Aug & Sept & Oct & Nov & Dec \\
\hline 1 & 437.00 & 436.02 & 436.07 & 436.33 & 436.74 & 436.51 & 435.65 & 438.62 & 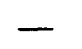 & - & 435.52 & 435.66 \\
\hline 2 & 437.19 & 436.08 & 436.03 & 436.40 & 436.61 & 436.50 & 435.63 & 438.39 & - & - & 435.62 & 435.64 \\
\hline 3 & 437.21 & 436.26 & 435.97 & 436.35 & 436.38 & 436.33 & 435.60 & 438.07 & - & - & 435.47 & 435.49 \\
\hline 4 & 437.22 & 436.23 & 436.10 & 436.16 & 436.34 & 436.14 & 435.68 & 437.81 & - & - & 435.29 & 435.43 \\
\hline 5 & 437.05 & 436.18 & 436.12 & 436.01 & 436.39 & 436.19 & 435.68 & 437.44 & - & - & 435.27 & 435.44 \\
\hline 6 & 436.71 & 436.18 & 436.05 & 436.08 & 436.40 & 436.31 & 435.62 & 437.16 & - & - & 435.21 & 435.47 \\
\hline 7 & 436.53 & 435.99 & 435.94 & 436.18 & 436.32 & 436.36 & 435.58 & $43^{\prime}$ & - & - & 435.10 & 435.48 \\
\hline 8 & 436.47 & 435.75 & 435.86 & 436.16 & 436.33 & 436.42 & 435.60 & 437.07 & - & - & 435.18 & 435.60 \\
\hline 9 & 436.33 & 435.94 & 435.83 & 436.09 & 436.43 & 436.38 & 435.60 & 436.95 & - & - & 435.12 & 435.63 \\
\hline 10 & 436.48 & 436.18 & 435.76 & 436.05 & 436.38 & 436.33 & 435.57 & 436.79 & - & - & 434.88 & 435.31 \\
\hline 11 & 436.44 & 436.21 & 435.79 & 435.97 & 436 & 436 & .45 & 43 & - & - & 34.84 & 35.51 \\
\hline 12 & 436.24 & 436.30 & 435.77 & 435.97 & 436.18 & 436.35 & 435.40 & 436.61 & - & - & 34.94 & 435.53 \\
\hline 13 & 436.17 & 436.46 & 435.64 & 435.92 & 436.24 & 436.36 & 435.40 & 436.52 & - & - & 435.03 & 435.34 \\
\hline 14 & 436.19 & 436.48 & 435.57 & 435.94 & 436.21 & 436.26 & 435.33 & 436.44 & - & - & 435.16 & 435.39 \\
\hline 15 & 436.20 & 436.51 & 435.70 & 435.95 & 436.20 & - & 435.37 & 436.39 & - & - & 435.19 & 435.52 \\
\hline 16 & 436.09 & 436.67 & 435.76 & 436.00 & 436.21 & - & .45 & 436 & - & - & 35.12 & 435.59 \\
\hline 17 & 435.88 & 436.83 & 435.67 & 435.96 & 436.31 & 436.14 & 435.43 & 436.08 & - & - & 35.23 & 35.64 \\
\hline 18 & 435.88 & 436.56 & 435.83 & 435.95 & 436.48 & 436.28 & 435.41 & 435.92 & - & - & 435.34 & 435.64 \\
\hline 19 & 436.11 & 436.15 & 436.22 & 436.00 & 436.60 & 436.18 & 435.39 & 82 & - & - & 435.29 & 435.70 \\
\hline 20 & 436.21 & 436.01 & 436.11 & 436.07 & 436.75 & 436.07 & 435.41 & 435.79 & - & - & 435.15 & 435.69 \\
\hline 21 & 436.27 & 29 & 436.04 & 436.08 & 436.83 & 43 & .50 & 435.72 & - & - & 435.05 & 435.78 \\
\hline 22 & 436.23 & 29 & 435.99 & 435.98 & 436.73 & 43 & 435.89 & 435.61 & - & 135 & 435.18 & 435.91 \\
\hline 23 & 436.31 & 01 & 435.86 & 435.91 & 436.69 & 436.00 & 435.57 & 435.45 & - & 435.34 & 435.28 & 435.95 \\
\hline 24 & 436.30 & 435.94 & 435.75 & 435.98 & 436.63 & 435.94 & 435.67 & - & - & 435.29 & 435.34 & 435.79 \\
\hline 25 & 435.95 & 436.03 & 435.62 & 436.06 & 436.59 & 435.84 & 435.75 & - & - & 435.32 & 435.42 & 435.60 \\
\hline 26 & 17 & 43 & 10 & 43 & 436 & & 90 & - & - & 435 & 435.41 & 435.67 \\
\hline 27 & 436.06 & 435.99 & 436.60 & 436.04 & 436.56 & 435.69 & 436.29 & - & - & 435.39 & 435.29 & 435.85 \\
\hline 28 & 436.04 & 436.00 & 436.69 & 436.37 & 436.69 & 435.73 & 436.73 & - & - & 435.27 & 435.25 & 435.88 \\
\hline 29 & 436.02 & - & 436.72 & 436.60 & 436.67 & 435.75 & 437.26 & - & - & 435.30 & 435.33 & 435.75 \\
\hline 30 & 436.02 & - & 436.66 & 436.70 & 436.54 & 435.74 & 437.87 & - & - & 435.42 & 435.54 & 435.76 \\
\hline 31 & 435.98 & - & 436.51 & - & 436.53 & - & 438.33 & - & - & 435.42 & - & 435.84 \\
\hline Mean & 436 & 4 & & & & & & & - & & 435.23 & 435.63 \\
\hline $\operatorname{Max}$ & 437.22 & 436.83 & 436.72 & 436.70 & 436.83 & 436.51 & 438.33 & 438.62 & - & 435.68 & 435.62 & 435.95 \\
\hline Min & 435.88 & 435.75 & 435.57 & 435.91 & 436.17 & 435.69 & 435.33 & 435.45 & - & 435.27 & 434.84 & 435.31 \\
\hline
\end{tabular}




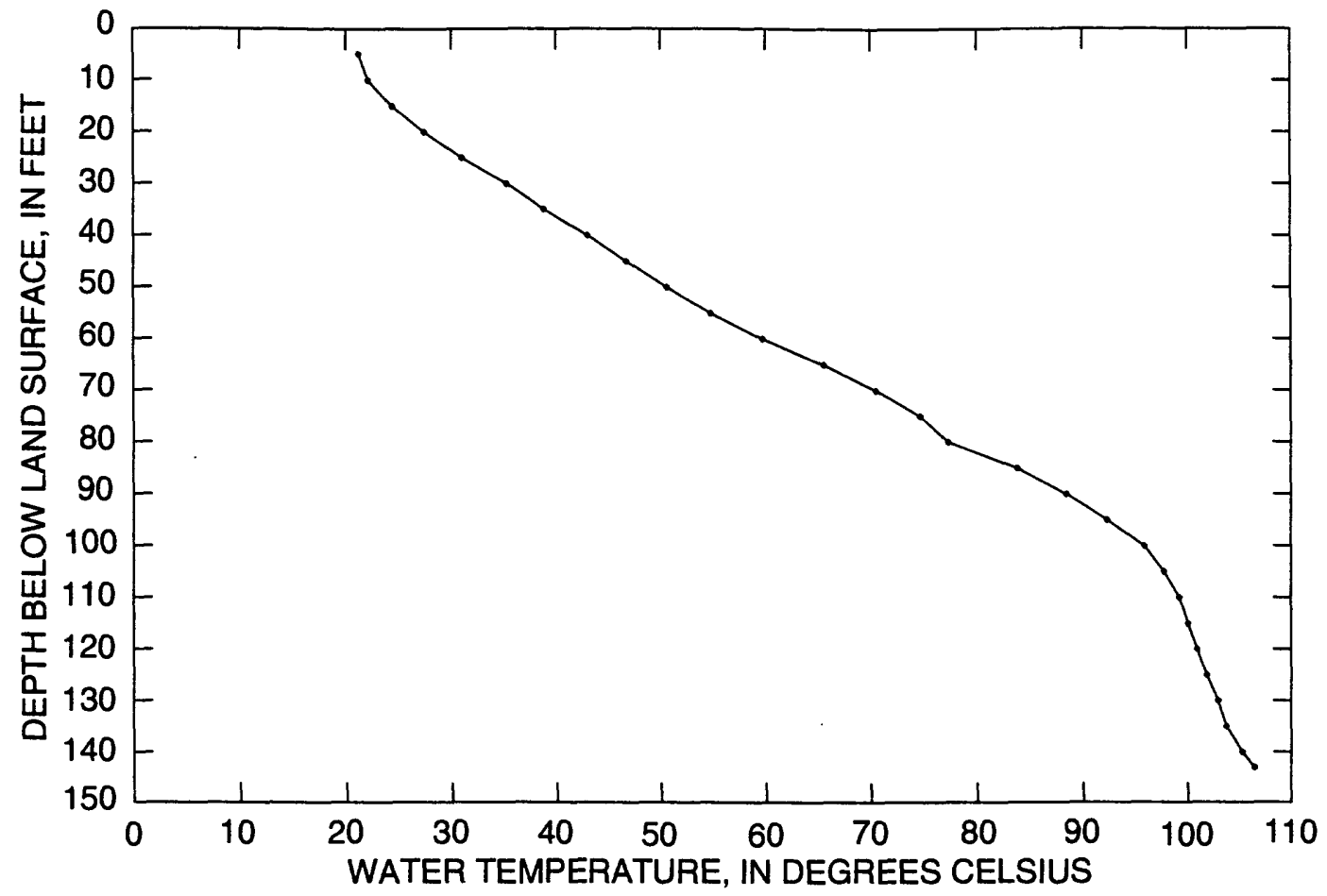

Figure 13. Temperature log of well CM-2, Long Valley Caldera, Mono County, California, July 22, 1989. 


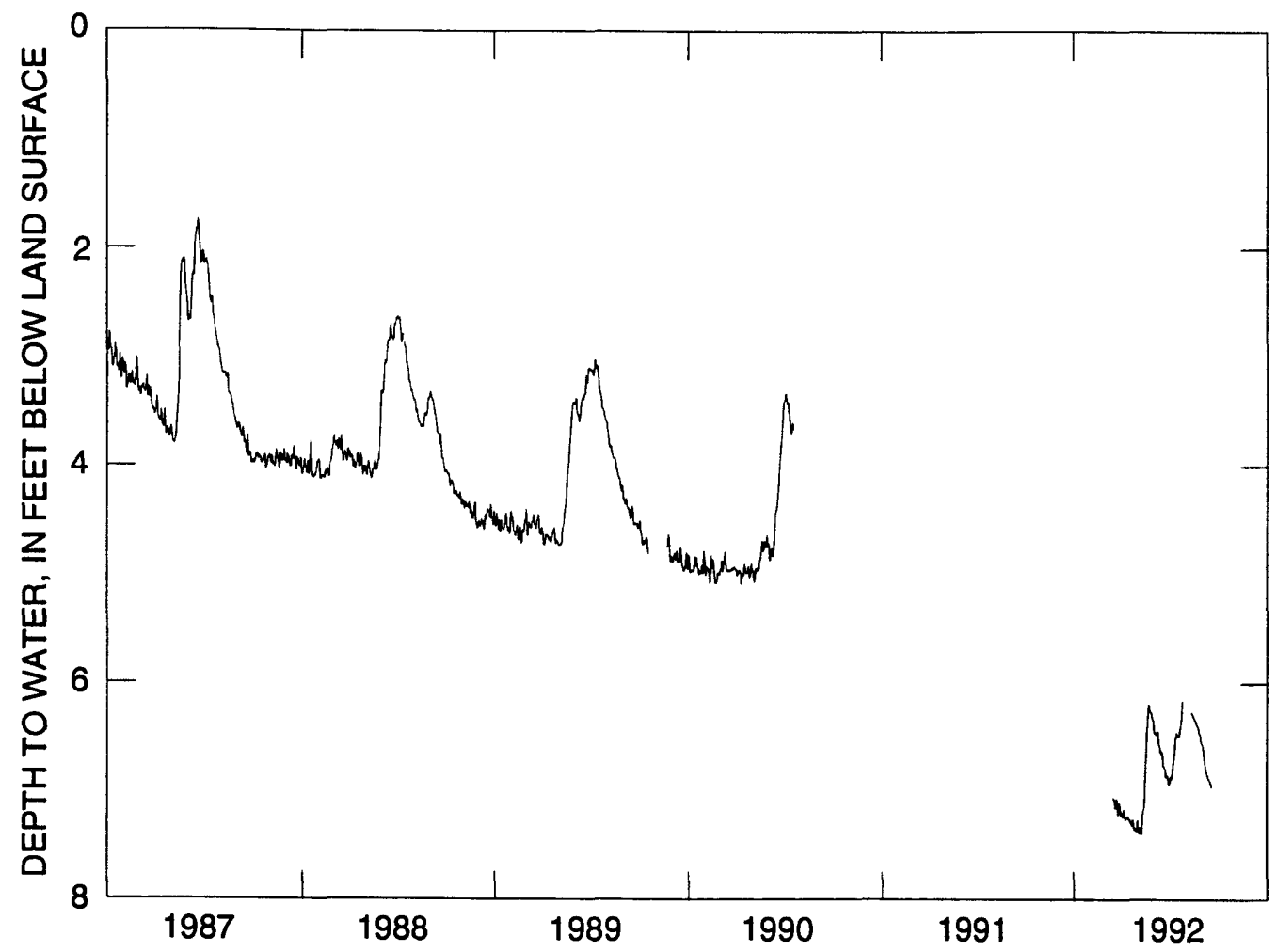

Figure 14. Daily mean water levels, well CM-2, Long Valley Caldera, Mono County California, 1987-92. 


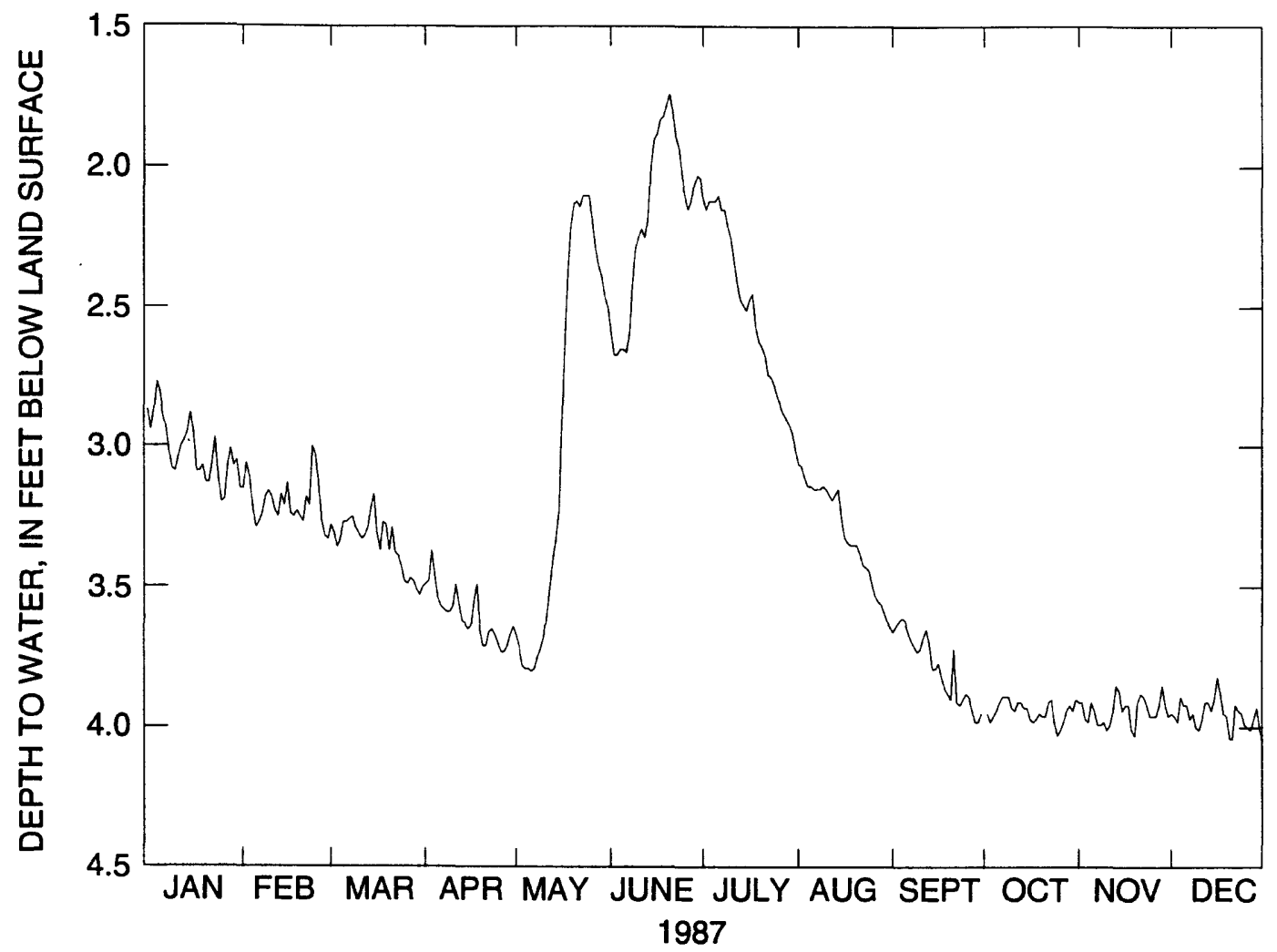

Figure 15. (A) Daily mean water levels, well CM-2, Long Valley Caldera, Mono County, California, 1987. 
Table 21. Daily mean water leveis, well CM-2, Long Valiey Caldera, Mono County, California, 1987 [Values are in feet below land surface. Max, maximum; Min, minimum; -, no data]

\begin{tabular}{|c|c|c|c|c|c|c|c|c|c|c|c|c|}
\hline Day & Jan & Feb & Mar & Apr & May & June & July & Aug & Sept & Oct & Nov & Dec \\
\hline 1 & 2.87 & 3.15 & 3.28 & 3.49 & 3.67 & 2.59 & 2.11 & 3.06 & 3.66 & - & 3.91 & 3.95 \\
\hline 2 & 2.94 & 3.06 & 3.31 & 3.48 & 3.71 & 2.67 & 2.15 & 3.07 & 3.64 & 3.95 & 3.91 & 3.96 \\
\hline 3 & 2.87 & 3.11 & 3.36 & 3.37 & 3.78 & 2.67 & 2.12 & 3.11 & 3.62 & 3.98 & 3.97 & 3.98 \\
\hline 4 & 2.77 & 3.22 & 3.33 & 3.46 & 3.79 & 2.65 & 2.12 & 3.14 & 3.61 & 3.96 & 3.98 & 3.89 \\
\hline 5 & 2.81 & 3.29 & 3.27 & 3.54 & 3.79 & 2.65 & 2.12 & 3.14 & 3.62 & 3.94 & 3.91 & 3.92 \\
\hline 6 & 2.90 & 3.27 & 3.27 & 3.57 & 3.80 & 2.66 & 2.10 & 3.15 & 3.66 & 3.91 & 3.94 & 3.92 \\
\hline 7 & 2.93 & 3.24 & 3.26 & 3.58 & 3.79 & 2.59 & 2.15 & 3.15 & 3.69 & 3.89 & 3.99 & 3.97 \\
\hline 8 & 3.02 & 3.18 & 3.25 & 3.59 & 3.75 & 2.41 & 2.15 & 3.15 & 3.71 & 3.89 & 3.99 & 3.95 \\
\hline 9 & 3.08 & 3.16 & 3.29 & 3.59 & 3.72 & 2.29 & 2.21 & 3.14 & 3.73 & 3.89 & 3.98 & 4.00 \\
\hline 10 & 3.09 & 3.18 & 3.31 & 3.57 & 3.68 & 2.25 & 2.25 & 3.15 & 3.72 & 3.93 & 4.01 & 4.01 \\
\hline 11 & 3.04 & 3.23 & 3.33 & 3.49 & 3.60 & 2.22 & 2.33 & 3.17 & 3.68 & 3.94 & 3.99 & 3.97 \\
\hline 12 & 3.00 & 3.25 & 3.32 & 3.56 & 3.50 & 2.25 & 2.41 & 3.19 & 3.65 & 3.91 & 3.94 & 3.91 \\
\hline 13 & 2.98 & 3.17 & 3.29 & 3.62 & 3.40 & 2.19 & 2.47 & 3.17 & 3.70 & 3.91 & 3.85 & 3.91 \\
\hline 14 & 2.95 & 3.21 & 3.22 & 3.63 & 3.33 & 1.99 & 2.49 & 3.15 & 3.79 & 3.93 & 3.87 & 3.94 \\
\hline 15 & 2.88 & 3.13 & 3.17 & 3.65 & 3.23 & 1.90 & 2.51 & 3.25 & 3.79 & 3.93 & 3.94 & 3.90 \\
\hline 16 & 2.95 & 3.24 & 3.31 & 3.64 & 2.91 & 1.88 & 2.47 & 3.32 & 3.77 & 3.97 & 3.92 & 3.82 \\
\hline 17 & 3.09 & 3.25 & 3.37 & 3.55 & 2.60 & 1.83 & 2.45 & 3.34 & 3.82 & 3.98 & 3.92 & 3.88 \\
\hline 18 & 3.09 & 3.23 & 3.27 & 3.49 & 2.37 & 1.82 & 2.57 & 3.35 & 3.86 & 3.97 & 4.01 & 3.95 \\
\hline 19 & 3.07 & 3.25 & 3.28 & 3.65 & 2.21 & 1.78 & 2.62 & 3.35 & 3.88 & 3.95 & 4.03 & 3.96 \\
\hline 20 & 3.13 & 3.27 & 3.37 & 3.71 & 2.13 & 1.74 & 2.64 & 3.35 & 3.90 & 3.96 & 3.91 & 4.04 \\
\hline 21 & 3.13 & 3.18 & 3.29 & 3.71 & 2.12 & 1.80 & 2.67 & 3.38 & 3.72 & 3.96 & 3.88 & 4.04 \\
\hline 22 & 3.06 & 3.21 & 3.38 & 3.66 & 2.14 & 1.89 & 2.74 & 3.42 & 3.91 & 3.91 & 3.89 & 3.92 \\
\hline 23 & 2.97 & 3.00 & 3.39 & 3.65 & 2.10 & 1.93 & 2.75 & 3.43 & 3.92 & 3.90 & 3.92 & 3.94 \\
\hline 24 & 3.11 & 3.03 & 3.43 & 3.67 & 2.10 & 2.03 & 2.78 & 3.44 & 3.90 & 3.98 & 3.96 & 3.95 \\
\hline 25 & 3.20 & 3.14 & 3.48 & 3.70 & 2.10 & 2.10 & 2.82 & 3.49 & 3.88 & 4.03 & 3.96 & 3.99 \\
\hline 26 & 3.19 & 3.27 & 3.49 & 3.73 & 2.19 & 2.15 & 2.85 & 3.53 & 3.89 & 4.01 & 3.96 & 4.00 \\
\hline 27 & 3.07 & 3.32 & 3.47 & 3.73 & 2.29 & 2.12 & 2.88 & 3.55 & 3.94 & 3.98 & 3.92 & 4.01 \\
\hline 28 & 3.01 & 3.33 & 3.48 & 3.71 & 2.35 & 2.06 & 2.90 & 3.56 & 3.98 & 3.94 & 3.85 & 3.97 \\
\hline 29 & 3.07 & - & 3.51 & 3.67 & 2.39 & 2.03 & 2.92 & 3.59 & 3.98 & 3.92 & 3.92 & 3.93 \\
\hline 30 & 3.05 & - & 3.53 & 3.64 & 2.46 & 2.04 & 2.95 & 3.62 & 3.95 & 3.94 & 3.96 & 4.01 \\
\hline 31 & 3.15 & - & 3.50 & - & 2.50 & - & 3.01 & 3.64 & - & 3.90 & - & 4.08 \\
\hline Mean & 3.02 & 3.20 & 3.35 & 3.60 & 2.95 & 2.17 & 2.51 & 3.31 & 3.79 & 3.94 & 3.94 & 3.96 \\
\hline $\operatorname{Max}$ & 3.20 & 3.33 & 3.53 & 3.73 & 3.80 & 2.67 & 3.01 & 3.64 & 3.98 & 4.03 & 4.03 & 4.08 \\
\hline Min & 2.77 & 3.00 & 3.17 & 3.37 & 2.10 & 1.74 & 2.10 & 3.06 & 3.61 & 3.89 & 3.85 & 3.82 \\
\hline
\end{tabular}




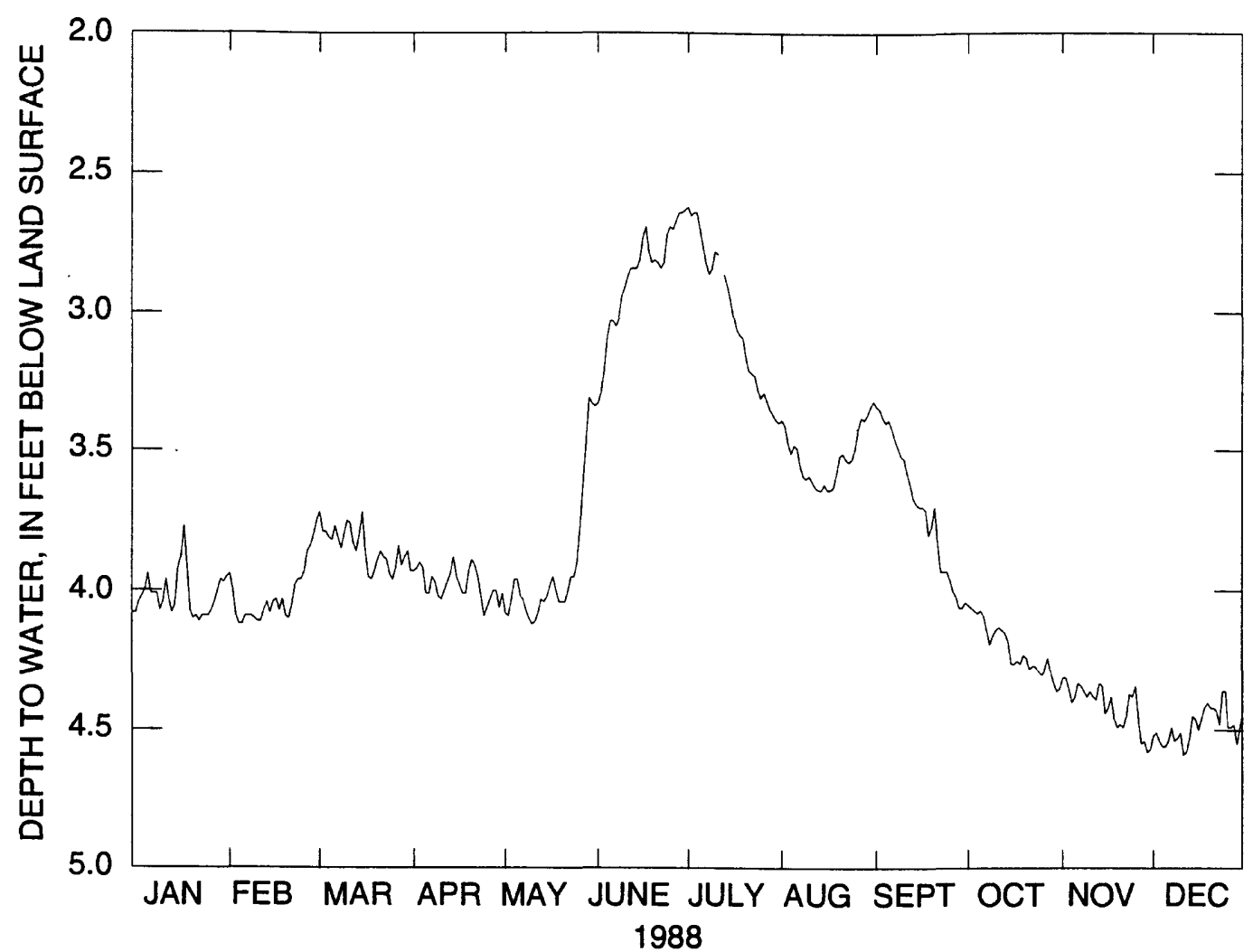

Figure 15. (B) Daily mean water levels, well CM-2, Long Valley Caldera, Mono County, California, 1988. 
Table 22. Daily mean water levels, well CM-2, Long Valley Caldera, Mono County, California, 1988

[Values are in feet below land surface. Max, maximum; Min, minimum; -, no data]

\begin{tabular}{|c|c|c|c|c|c|c|c|c|c|c|c|c|}
\hline Day & Jan & Feb & Mar & Apr & May & June & July & Aug & Sept & Oct & Nov & Dec \\
\hline 1 & 4.08 & 3.94 & 3.72 & 3.93 & 4.08 & 3.33 & 2.62 & 3.39 & 3.34 & 4.05 & 4.31 & 4.52 \\
\hline 2 & 4.04 & 4.00 & 3.79 & 3.92 & 4.09 & 3.29 & 2.65 & 3.41 & 3.35 & 4.06 & 4.31 & 4.51 \\
\hline 3 & 4.02 & 4.09 & 3.79 & 3.90 & 4.03 & 3.21 & 2.64 & 3.47 & 3.38 & 4.07 & 4.35 & 4.54 \\
\hline 4 & 4.00 & 4.12 & 3.81 & 3.92 & 3.96 & 3.09 & 2.64 & 3.51 & 3.40 & 4.08 & 4.40 & 4.56 \\
\hline 5 & 3.94 & 4.12 & 3.82 & 4.01 & 3.96 & 3.03 & 2.70 & 3.48 & 3.39 & 4.07 & 4.38 & 4.56 \\
\hline 6 & 4.01 & 4.09 & 3.77 & 4.01 & 4.02 & 3.03 & 2.76 & 3.49 & 3.42 & 4.09 & 4.33 & 4.54 \\
\hline 7 & 4.01 & 4.09 & 3.81 & 3.95 & 4.03 & 3.05 & 2.82 & 3.55 & 3.46 & 4.14 & 4.34 & 4.49 \\
\hline 8 & 4.01 & 4.09 & 3.85 & 3.97 & 4.07 & 3.02 & 2.86 & 3.59 & 3.49 & 4.19 & 4.36 & 4.54 \\
\hline 9 & 4.07 & 4.10 & 3.80 & 4.02 & 4.10 & 2.94 & 2.84 & 3.60 & 3.52 & 4.16 & 4.38 & 4.53 \\
\hline 10 & 4.04 & 4.11 & 3.75 & 4.03 & 4.12 & 2.91 & 2.78 & 3.59 & 3.53 & 4.14 & 4.36 & 4.51 \\
\hline 11 & 3.96 & 4.11 & 3.76 & 4.00 & 4.11 & 2.87 & 2.79 & 3.61 & 3.58 & 4.13 & 4.38 & 4.59 \\
\hline 12 & 4.03 & 4.07 & 3.83 & 3.97 & 4.08 & 2.84 & - & 3.63 & 3.62 & 4.14 & 4.39 & 4.58 \\
\hline 13 & 4.08 & 4.04 & 3.86 & 3.94 & 4.03 & 2.84 & 2.86 & 3.64 & 3.67 & 4.15 & 4.33 & 4.53 \\
\hline 14 & 4.05 & 4.08 & 3.80 & 3.88 & 4.04 & 2.84 & 2.90 & 3.64 & 3.69 & 4.18 & 4.34 & 4.45 \\
\hline 15 & 3.92 & 4.04 & 3.72 & 3.95 & 4.02 & 2.81 & 2.95 & 3.62 & 3.70 & 4.26 & 4.44 & 4.46 \\
\hline 16 & 3.88 & 4.03 & 3.86 & 3.98 & 3.98 & 2.73 & 3.01 & 3.64 & 3.70 & 4.26 & 4.42 & 4.50 \\
\hline 17 & 3.77 & 4.07 & 3.95 & 4.01 & 3.95 & 2.69 & 3.06 & 3.64 & 3.71 & 4.25 & 4.38 & 4.46 \\
\hline 18 & 3.91 & 4.03 & 3.96 & 4.01 & 4.00 & 2.78 & 3.08 & 3.63 & 3.80 & 4.26 & 4.46 & 4.42 \\
\hline 19 & 4.07 & 4.09 & 3.93 & 3.93 & 4.04 & 2.82 & 3.09 & 3.58 & 3.77 & 4.23 & 4.49 & 4.40 \\
\hline 20 & 4.10 & 4.10 & 3.89 & 3.89 & 4.04 & 2.81 & 3.16 & 3.52 & 3.70 & 4.24 & 4.48 & 4.42 \\
\hline 21 & 4.09 & 4.05 & 3.86 & 3.91 & 4.04 & 2.82 & 3.21 & 3.51 & 3.83 & 4.28 & 4.49 & 4.42 \\
\hline 22 & 4.11 & 3.98 & 3.88 & 3.95 & 4.00 & 2.84 & 3.22 & 3.53 & 3.93 & 4.27 & 4.45 & 4.43 \\
\hline 23 & 4.09 & 3.96 & 3.89 & 4.02 & 3.95 & 2.82 & 3.23 & 3.54 & 3.93 & 4.27 & 4.37 & 4.48 \\
\hline 24 & 4.09 & 3.96 & 3.94 & 4.09 & 3.95 & 2.72 & 3.28 & 3.53 & 3.93 & 4.29 & 4.38 & 4.36 \\
\hline 25 & 4.09 & 3.93 & 3.96 & 4.06 & 3.90 & 2.69 & 3.31 & 3.49 & 3.96 & 4.30 & 4.34 & 4.36 \\
\hline 26 & 4.07 & 3.86 & 3.92 & 4.03 & 3.77 & 2.70 & 3.29 & 3.42 & 4.00 & 4.28 & 4.46 & 4.49 \\
\hline 27 & 4.04 & 3.84 & 3.84 & 4.00 & 3.62 & 2.67 & 3.32 & 3.38 & 4.02 & 4.24 & 4.55 & 4.49 \\
\hline 28 & 4.00 & 3.80 & 3.91 & 4.00 & 3.47 & 2.64 & 3.35 & 3.39 & 4.06 & 4.29 & 4.54 & 4.48 \\
\hline 29 & 3.96 & 3.75 & 3.88 & 4.06 & 3.31 & 2.64 & 3.37 & 3.37 & 4.06 & 4.33 & 4.58 & 4.55 \\
\hline 30 & 3.97 & - & 3.86 & 4.01 & 3.33 & 2.63 & 3.39 & 3.34 & 4.04 & 4.36 & 4.57 & 4.49 \\
\hline 31 & 3.95 & - & 3.93 & - & 3.34 & - & 3.40 & 3.32 & - & 4.35 & - & 4.42 \\
\hline Mean & 4.01 & 4.02 & 3.85 & 3.98 & 3.92 & 2.87 & 3.02 & 3.52 & 3.70 & 4.21 & 4.41 & 4.49 \\
\hline Max & 4.11 & 4.12 & 3.96 & 4.09 & 4.12 & 3.33 & 3.40 & 3.64 & 4.06 & 4.36 & 4.58 & 4.59 \\
\hline Min & 3.77 & 3.75 & 3.72 & 3.88 & 3.31 & 2.63 & 2.62 & 3.32 & 3.34 & 4.05 & 4.31 & 4.36 \\
\hline
\end{tabular}




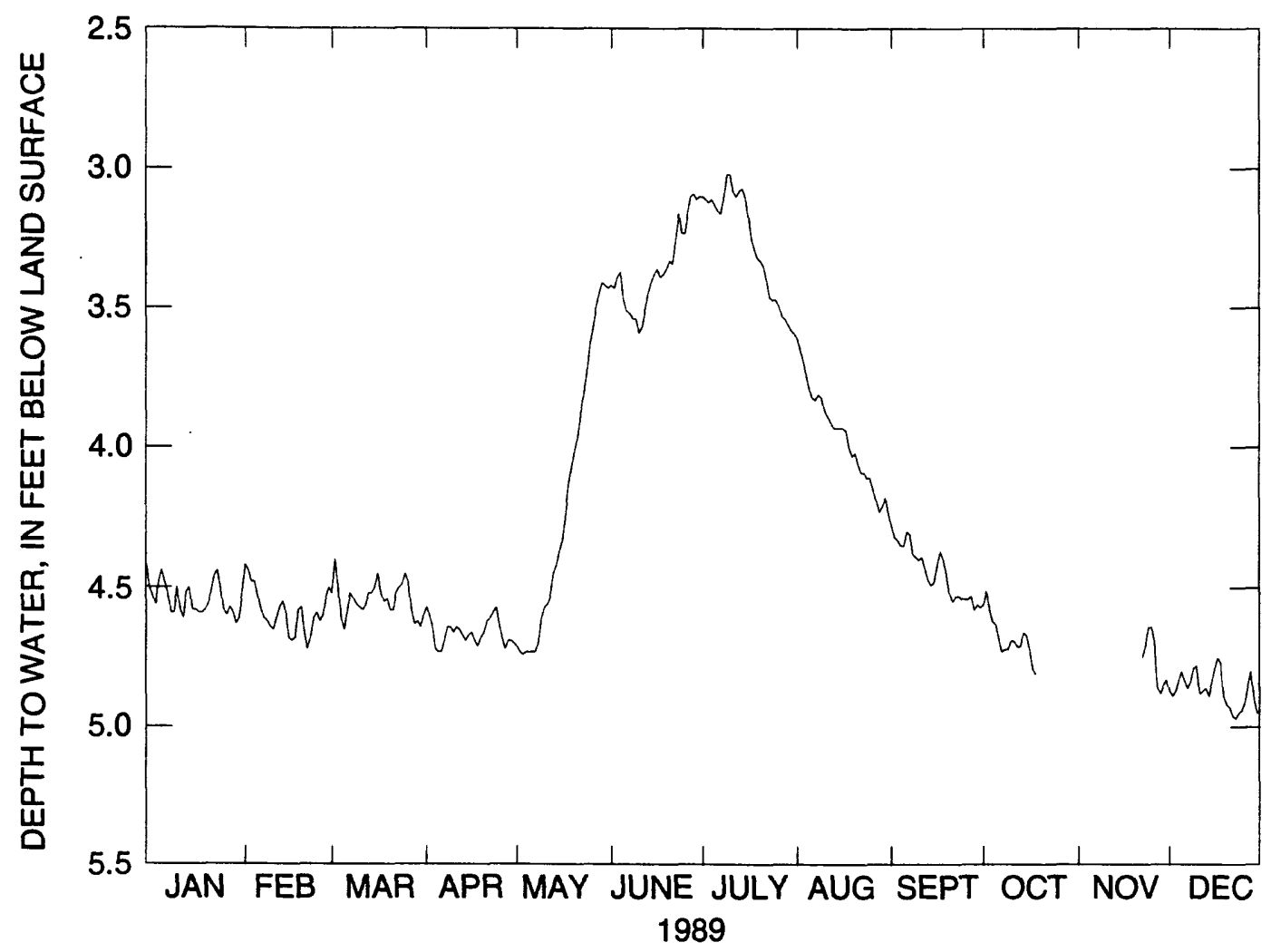

Figure 15. (C) Daily mean water levels, well CM-2, Long Valley Caldera, Mono County, California, 1989. 
Table 23. Daily mean water levels, well CM-2, Long Valley Caldera, Mono County, California, 1989

[Values are in feet below land surface. Max, maximum; Min, minimum; -, no data]

\begin{tabular}{|c|c|c|c|c|c|c|c|c|c|c|c|c|}
\hline Day & Jan & $\mathrm{Feb}$ & Mar & Apr & May & June & July & Aug & Sept & Oct & Nov & Dec \\
\hline 1 & 4.50 & 4.42 & 4.52 & 4.57 & 4.71 & 3.42 & 3.10 & 3.61 & 4.28 & 4.56 & - & 4.87 \\
\hline 2 & 4.53 & 4.44 & 4.40 & 4.60 & 4.73 & 3.43 & 3.11 & 3.65 & 4.32 & 4.51 & - & 4.89 \\
\hline 3 & 4.56 & 4.48 & 4.49 & 4.64 & 4.74 & 3.39 & 3.12 & 3.69 & 4.33 & 4.58 & - & 4.87 \\
\hline 4 & 4.48 & 4.48 & 4.61 & 4.72 & 4.73 & 3.37 & 3.11 & 3.74 & 4.35 & 4.62 & - & 4.84 \\
\hline 5 & 4.44 & 4.53 & 4.65 & 4.73 & 4.73 & 3.47 & 3.13 & 3.79 & 4.35 & 4.63 & - & 4.80 \\
\hline 6 & 4.48 & 4.58 & 4.58 & 4.73 & 4.73 & 3.51 & 3.15 & 3.82 & 4.30 & 4.68 & - & 4.83 \\
\hline 7 & 4.52 & 4.61 & 4.52 & 4.69 & 4.73 & 3.52 & 3.16 & 3.83 & 4.31 & 4.73 & - & 4.86 \\
\hline 8 & 4.59 & 4.62 & 4.54 & 4.64 & 4.70 & 3.54 & 3.10 & 3.81 & 4.38 & 4.72 & - & 4.84 \\
\hline 9 & 4.59 & 4.64 & 4.56 & 4.64 & 4.61 & 3.54 & 3.02 & 3.82 & 4.39 & 4.72 & - & 4.79 \\
\hline 10 & 4.50 & 4.65 & 4.57 & 4.66 & 4.57 & 3.59 & 3.02 & 3.87 & 4.40 & 4.69 & - & 4.78 \\
\hline 11 & 4.58 & 4.61 & 4.58 & 4.64 & 4.56 & 3.57 & 3.08 & 3.89 & 4.39 & 4.69 & - & 4.88 \\
\hline 12 & 4.61 & 4.57 & 4.56 & 4.65 & 4.53 & 3.51 & 3.10 & 3.91 & 4.43 & 4.71 & - & 4.87 \\
\hline 13 & 4.52 & 4.55 & 4.52 & 4.67 & 4.45 & 3.45 & 3.08 & 3.93 & 4.47 & 4.71 & - & 4.86 \\
\hline 14 & 4.50 & 4.59 & 4.52 & 4.69 & 4.42 & 3.41 & 3.07 & 3.93 & 4.49 & 4.66 & - & 4.89 \\
\hline 15 & 4.58 & 4.68 & 4.50 & 4.67 & 4.37 & 3.38 & 3.10 & 3.93 & 4.48 & 4.67 & - & 4.84 \\
\hline 16 & 4.58 & 4.69 & 4.45 & 4.66 & 4.33 & 3.36 & 3.17 & 3.93 & 4.42 & 4.72 & - & 4.79 \\
\hline 17 & 4.59 & 4.68 & 4.52 & 4.69 & 4.24 & 3.39 & 3.25 & 3.94 & 4.37 & 4.79 & - & 4.75 \\
\hline 18 & 4.59 & 4.58 & 4.55 & 4.71 & 4.13 & 3.38 & 3.29 & 4.00 & 4.40 & 4.81 & - & 4.77 \\
\hline 19 & 4.58 & 4.57 & 4.54 & 4.68 & 4.07 & 3.36 & 3.32 & 4.03 & 4.45 & - & - & 4.89 \\
\hline 20 & 4.56 & 4.66 & 4.58 & 4.66 & 4.01 & 3.33 & 3.33 & 4.02 & 4.52 & - & - & 4.92 \\
\hline 21 & 4.52 & 4.72 & 4.58 & 4.62 & 3.96 & 3.34 & 3.35 & 4.06 & 4.55 & - & - & 4.93 \\
\hline 22 & 4.46 & 4.68 & 4.52 & 4.61 & 3.87 & 3.25 & 3.40 & 4.09 & 4.53 & - & 4.75 & 4.96 \\
\hline 23 & 4.44 & 4.61 & 4.50 & 4.59 & 3.81 & 3.16 & 3.46 & 4.09 & 4.53 & - & 4.71 & 4.97 \\
\hline 24 & 4.50 & 4.59 & 4.49 & 4.57 & 3.73 & 3.23 & 3.47 & 4.11 & 4.54 & - & 4.64 & 4.95 \\
\hline 25 & 4.58 & 4.62 & 4.45 & 4.63 & 3.63 & 3.23 & 3.47 & 4.11 & 4.54 & - & 4.64 & 4.94 \\
\hline 26 & 4.60 & 4.60 & 4.48 & 4.68 & 3.57 & 3.16 & 3.49 & 4.15 & 4.54 & - & 4.69 & 4.91 \\
\hline 27 & 4.57 & 4.54 & 4.58 & 4.72 & 3.50 & 3.10 & 3.53 & 4.19 & 4.53 & - & 4.86 & 4.85 \\
\hline 28 & 4.59 & 4.50 & 4.63 & 4.69 & 3.45 & 3.09 & 3.54 & 4.23 & 4.58 & - & 4.88 & 4.80 \\
\hline 29 & 4.63 & - & 4.62 & 4.69 & 3.41 & 3.11 & 3.56 & 4.21 & 4.56 & - & 4.85 & 4.90 \\
\hline 30 & 4.61 & - & 4.64 & 4.70 & 3.42 & 3.10 & 3.58 & 4.18 & 4.57 & - & 4.83 & 4.95 \\
\hline 31 & 4.52 & - & 4.60 & - & 3.43 & - & 3.59 & 4.24 & - & - & - & 4.94 \\
\hline Mean & 4.55 & 4.59 & 4.54 & 4.66 & 4.19 & 3.36 & 3.27 & 3.96 & 4.44 & 4.68 & 4.76 & 4.87 \\
\hline $\operatorname{Max}$ & 4.63 & 4.72 & 4.65 & 4.73 & 4.74 & 3.59 & 3.59 & 4.24 & 4.58 & 4.81 & 4.88 & 4.97 \\
\hline Min & 4.44 & 4.42 & 4.40 & 4.57 & 3.41 & 3.09 & 3.02 & 3.61 & 4.28 & 4.51 & 4.64 & 4.75 \\
\hline
\end{tabular}




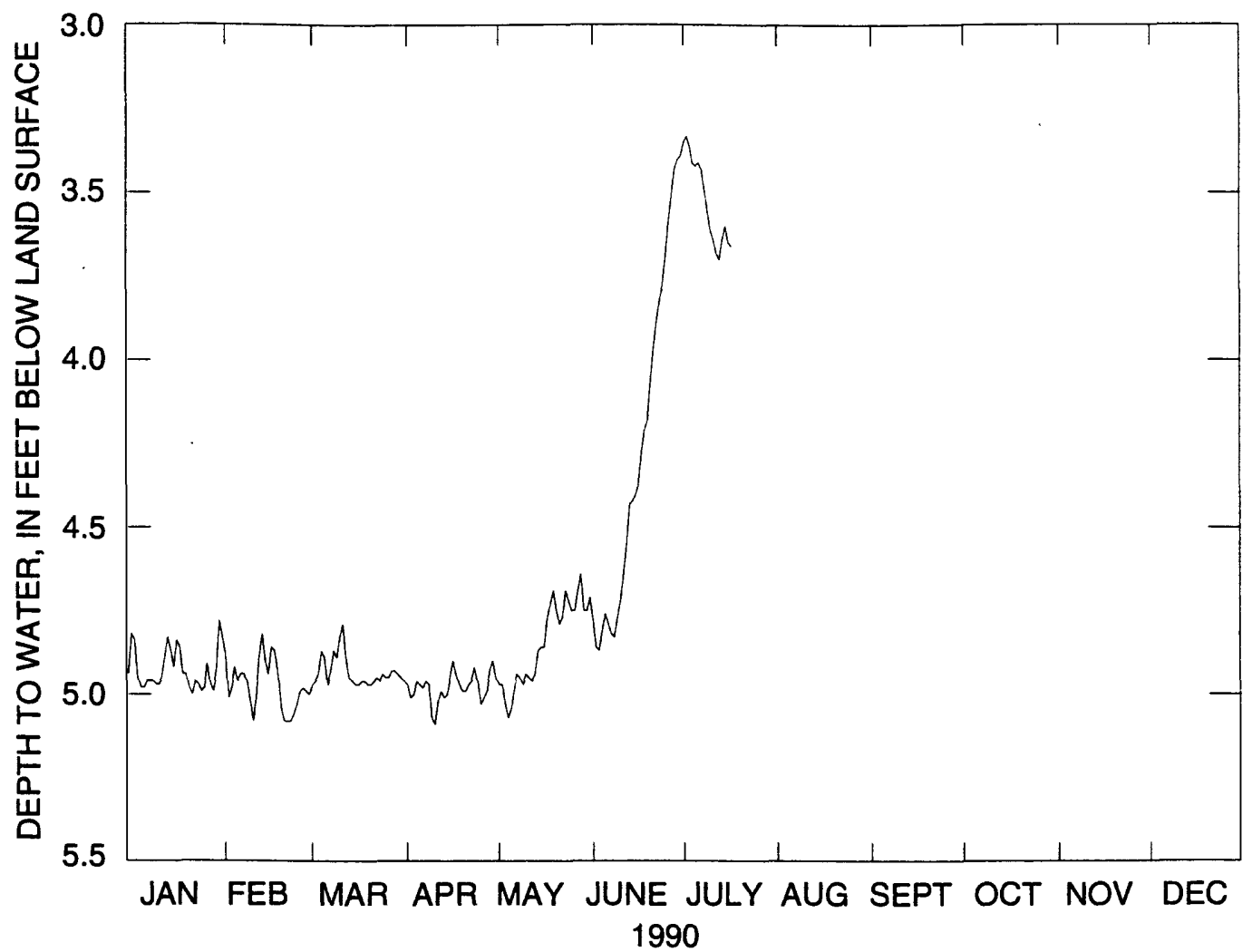

Figure 15. (D) Daily mean water levels, well CM-2, Long Valley Caldera, Mono County, California, 1990. 
Table 24. Daily mean water levels, well CM-2, Long Valley Caldera, Mono County, California, 1990

[Values are in feet below land surface. Max, maximum; Min, minimum; -, no data]

\begin{tabular}{|c|c|c|c|c|c|c|c|c|c|c|c|c|}
\hline Day & Jan & Feb & Mar & Apr & May & June & July & Aug & Sept & Oct & Nov & Dec \\
\hline 1 & 4.82 & 4.88 & 4.97 & 4.97 & 4.97 & 4.78 & 3.35 & - & - & - & - & - \\
\hline 2 & 4.84 & 5.01 & 4.96 & 5.01 & 4.97 & 4.86 & 3.33 & - & - & - & - & - \\
\hline 3 & 4.95 & 4.98 & 4.94 & 5.00 & 5.03 & 4.87 & 3.36 & - & - & - & - & - \\
\hline 4 & 4.98 & 4.92 & 4.87 & 4.96 & 5.07 & 4.81 & 3.41 & - & - & - & - & - \\
\hline 5 & 4.98 & 4.96 & 4.89 & 4.97 & 5.04 & 4.76 & 3.42 & - & - & - & - & - \\
\hline 6 & 4.96 & 4.94 & 4.97 & 4.98 & 4.98 & 4.79 & 3.41 & - & - & - & - & - \\
\hline 7 & 4.96 & 4.94 & 4.93 & 4.96 & 4.94 & 4.82 & 3.43 & - & - & - & - & - \\
\hline 8 & 4.96 & 4.96 & 4.87 & 4.97 & 4.95 & 4.83 & 3.49 & - & - & - & - & - \\
\hline 9 & 4.97 & 5.02 & 4.89 & 5.07 & 4.97 & 4.77 & 3.55 & - & - & - & - & - \\
\hline 10 & 4.97 & 5.08 & 4.83 & 5.09 & 4.94 & 4.72 & 3.61 & - & - & - & - & - \\
\hline 11 & 4.95 & 5.00 & 4.79 & 5.02 & 4.95 & 4.64 & 3.64 & - & - & - & - & - \\
\hline 12 & 4.89 & 4.89 & 4.89 & 4.99 & 4.96 & 4.55 & 3.68 & - & - & - & - & - \\
\hline 13 & 4.83 & 4.82 & 4.95 & 5.01 & 4.94 & 4.43 & 3.70 & - & - & - & - & - \\
\hline 14 & 4.87 & 4.90 & 4.96 & 5.00 & 4.87 & 4.42 & 3.64 & - & - & - & - & - \\
\hline 15 & 4.92 & 4.94 & 4.97 & 4.95 & 4.86 & 4.40 & 3.60 & - & - & - & - & - \\
\hline 16 & 4.84 & 4.86 & 4.97 & 4.90 & 4.86 & 4.37 & 3.65 & - & - & - & - & - \\
\hline 17 & 4.86 & 4.87 & 4.96 & 4.94 & 4.77 & 4.28 & 3.66 & - & - & - & - & - \\
\hline 18 & 4.94 & 4.93 & 4.96 & 4.97 & 4.73 & 4.21 & - & - & - & - & - & - \\
\hline 19 & 4.94 & 5.04 & 4.97 & 4.99 & 4.69 & 4.18 & - & - & - & - & - & - \\
\hline 20 & 4.98 & 5.08 & 4.97 & 4.99 & 4.75 & 4.06 & - & - & - & - & - & - \\
\hline 21 & 5.00 & 5.08 & 4.96 & 4.97 & 4.79 & 3.96 & - & - & - & - & - & - \\
\hline 22 & 4.96 & 5.08 & 4.95 & 4.96 & 4.77 & 3.88 & - & - & - & - & - & - \\
\hline 23 & 4.97 & 5.06 & 4.96 & 4.92 & 4.69 & 3.82 & - & - & - & - & - & - \\
\hline 24 & 4.99 & 5.03 & 4.94 & 4.96 & 4.72 & 3.78 & - & - & - & - & - & - \\
\hline 25 & 4.98 & 4.99 & 4.95 & 5.03 & 4.75 & 3.70 & - & - & - & - & - & - \\
\hline 26 & 4.91 & 4.98 & 4.95 & 5.01 & 4.75 & 3.59 & - & - & - & - & - & - \\
\hline 27 & 4.97 & 4.99 & 4.93 & 4.99 & 4.69 & 3.51 & - & - & - & - & - & - \\
\hline 28 & 4.99 & 5.00 & 4.93 & 4.93 & 4.64 & 3.43 & 一 & - & - & - & - & - \\
\hline 29 & 4.93 & - & 4.94 & 4.90 & 4.75 & 3.40 & - & - & - & - & - & - \\
\hline 30 & 4.78 & - & 4.95 & 4.95 & 4.75 & 3.39 & - & - & - & - & - & - \\
\hline 31 & 4.83 & - & 4.96 & - & 4.71 & - & - & - & - & - & - & - \\
\hline Mean & 4.93 & 4.97 & 4.93 & 4.98 & 4.85 & 4.27 & 3.53 & - & - & - & - & - \\
\hline $\operatorname{Max}$ & 5.00 & 5.08 & 4.97 & 5.09 & 5.07 & 4.87 & 3.70 & - & - & - & - & - \\
\hline Min & 4.78 & 4.82 & 4.79 & 4.90 & 4.64 & 3.39 & 3.33 & 一 & - & - & - & - \\
\hline
\end{tabular}




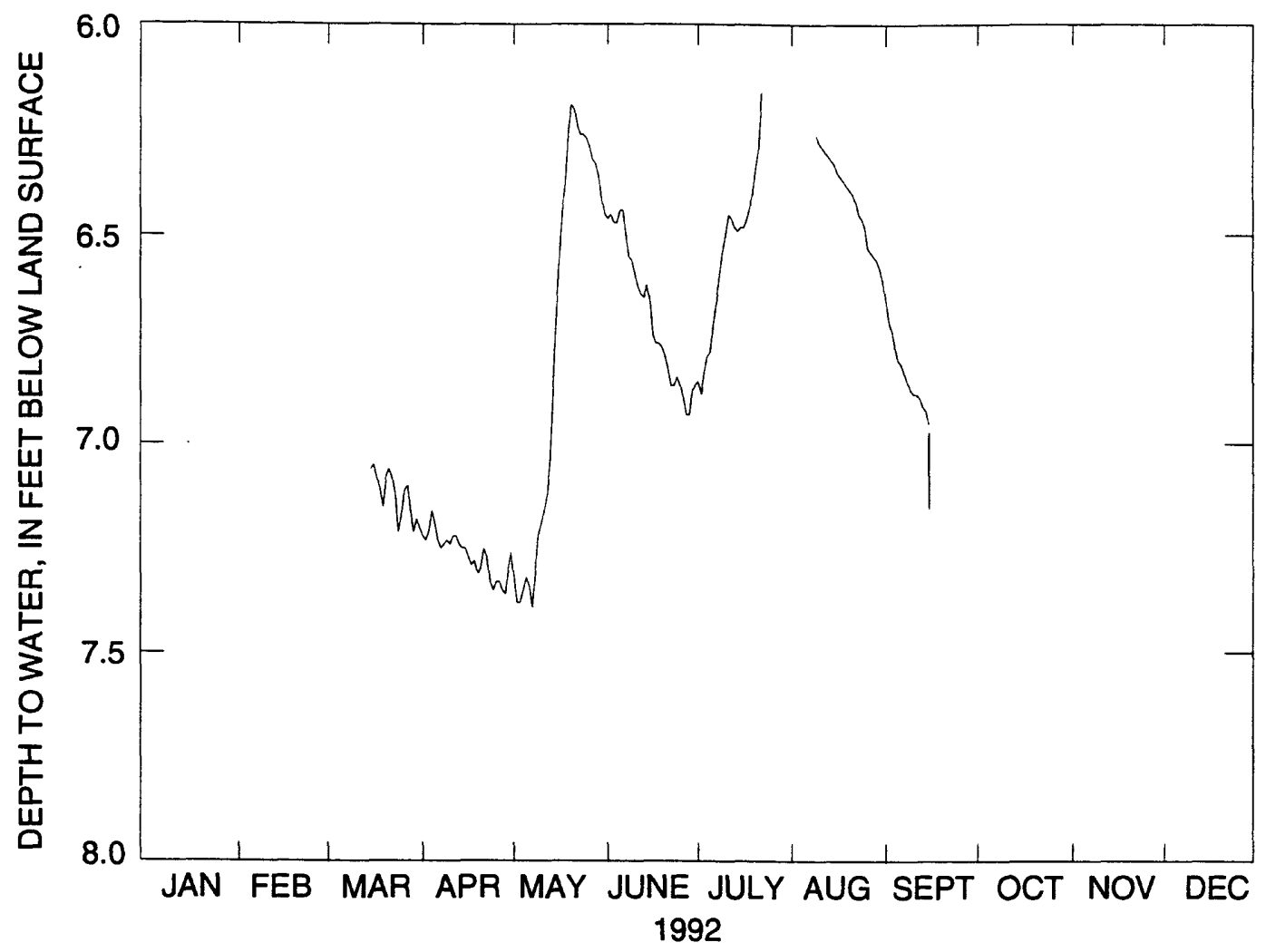

Figure 15. (E) Daily mean water levels, well CM-2, Long Valley Caldera, Mono County, California, 1992. 
Table 25. Daily mean water levels, well CM-2, Long Valley Caldera, Mono County, California, 1992

[Values are in feet below land surface. Max, maximum; Min, minimum; -, no data]

\begin{tabular}{|c|c|c|c|c|c|c|c|c|c|c|c|c|}
\hline Day & Jan & Feb & Mar & Apr & May & June & July & Aug & Sept & Oct & Nov & Dec \\
\hline 1 & - & - & - & 7.22 & 7.32 & 6.46 & 6.85 & - & 6.66 & - & - & - \\
\hline 2 & - & 一 & - & 7.23 & 7.38 & 6.45 & 6.88 & - & 6.71 & - & - & - \\
\hline 3 & - & - & - & 7.21 & 7.38 & 6.47 & 6.83 & - & 6.73 & - & - & 一 \\
\hline 4 & - & - & - & 7.16 & 7.35 & 6.47 & 6.79 & - & 6.77 & - & - & - \\
\hline 5 & - & - & - & 7.19 & 7.32 & 6.44 & 6.78 & - & 6.80 & - & - & - \\
\hline 6 & - & - & - & 7.23 & 7.34 & 6.44 & 6.71 & - & 6.81 & - & - & - \\
\hline 7 & - & - & - & 7.25 & 7.39 & 6.50 & 6.66 & - & 6.83 & - & - & - \\
\hline 8 & - & - & - & 7.24 & 7.31 & 6.55 & 6.60 & - & 6.85 & - & - & - \\
\hline 9 & - & - & - & 7.23 & 7.22 & 6.56 & 6.54 & 6.26 & 6.87 & - & - & - \\
\hline 10 & - & - & - & 7.24 & 7.19 & 6.59 & 6.50 & 6.28 & 6.88 & - & - & - \\
\hline 11 & - & - & - & 7.22 & 7.16 & 6.62 & 6.45 & 6.29 & 6.88 & - & - & - \\
\hline 12 & - & - & - & 7.22 & 7.12 & 6.64 & 6.46 & 6.30 & 6.89 & - & - & - \\
\hline 13 & - & - & - & 7.24 & 7.04 & 6.65 & 6.48 & 6.31 & 6.91 & - & - & - \\
\hline 14 & - & - & - & 7.25 & 6.86 & 6.62 & 6.49 & 6.32 & 6.92 & - & - & - \\
\hline 15 & - & - & 7.06 & 7.25 & 6.69 & 6.66 & 6.48 & 6.33 & 6.95 & - & - & - \\
\hline 16 & - & - & 7.05 & 7.27 & 6.56 & 6.74 & 6.48 & 6.35 & - & - & - & - \\
\hline 17 & - & - & 7.08 & 7.29 & 6.44 & 6.76 & 6.46 & 6.36 & - & - & - & - \\
\hline 18 & - & - & 7.11 & 7.28 & 6.38 & 6.76 & 6.43 & 6.37 & - & - & - & - \\
\hline 19 & - & - & 7.15 & 7.31 & 6.25 & 6.77 & 6.40 & 6.38 & - & - & - & - \\
\hline 20 & - & - & 7.08 & 7.30 & 6.19 & 6.79 & 6.34 & 6.39 & - & - & - & - \\
\hline 21 & - & - & 7.06 & 7.25 & 6.20 & 6.82 & 6.29 & 6.40 & - & - & - & - \\
\hline 22 & - & - & 7.08 & 7.27 & 6.24 & 6.86 & 6.16 & 6.42 & - & - & - & - \\
\hline 23 & - & - & 7.12 & 7.33 & 6.26 & 6.86 & - & 6.45 & - & - & - & - \\
\hline 24 & - & - & 7.21 & 7.35 & 6.26 & 6.84 & - & 6.46 & - & - & - & - \\
\hline 25 & - & - & 7.17 & 7.33 & 6.27 & 6.86 & - & 6.48 & - & - & - & - \\
\hline 26 & - & - & 7.11 & 7.33 & 6.29 & 6.89 & - & 6.53 & - & - & - & - \\
\hline 27 & - & - & 7.10 & 7.35 & 6.32 & 6.93 & - & 6.54 & - & - & - & - \\
\hline 28 & - & - & 7.16 & 7.36 & 6.33 & 6.93 & - & 6.55 & - & - & - & - \\
\hline 29 & - & - & 7.21 & 7.30 & 6.36 & 6.87 & - & 6.56 & - & - & - & - \\
\hline 30 & - & - & 7.18 & 7.26 & 6.41 & 6.86 & - & 6.58 & - & - & - & - \\
\hline 31 & - & - & 7.20 & - & 6.45 & - & - & 6.61 & - & - & - & - \\
\hline Mean & - & - & 7.13 & 7.27 & 6.75 & 6.69 & 6.55 & 6.41 & 6.83 & - & - & - \\
\hline Max & - & - & 7.21 & 7.36 & 7.39 & 6.93 & 6.88 & 6.61 & 6.95 & - & - & - \\
\hline Min & - & - & 7.05 & 7.16 & 6.19 & 6.44 & 6.16 & 6.26 & 6.66 & - & - & - \\
\hline
\end{tabular}




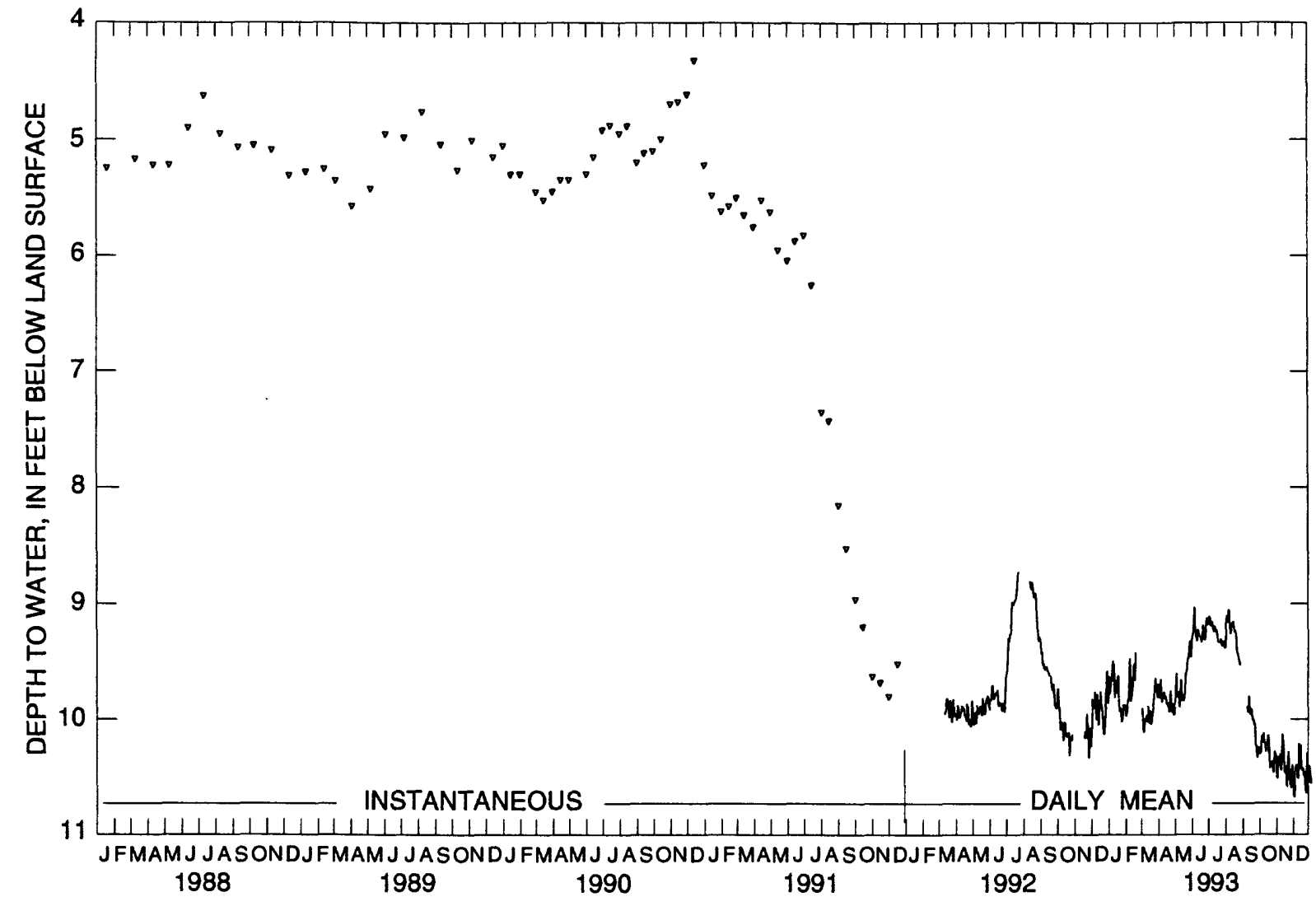

Figure 16. Instantaneous water levels, 1988-91, and daily mean water levels, 1992-93, well CW-3, Long Valley Caldera, Mono County, California. [Data for 1988-91 provided by Donald Barnett, Intermountain Water Consultants (written commun., 1993).] 
Table 26. Miscellaneous instantaneous water levels, well CW-3, Long Valley Caldera, Mono County, California, 1988-91

[Water levels are feet below land surface. Data provided by Donald Barnett, Intermountain Water Consultants (written commun., 1993)]

\begin{tabular}{cccccc}
\hline Date & Water level & Date & Water level & Date & Water level \\
\hline $01-19-88$ & 5.25 & $01-01-90$ & 5.05 & $01-15-91$ & 5.48 \\
$03-10-88$ & 5.17 & $01-15-90$ & 5.30 & $02-01-91$ & 5.62 \\
$04-11-88$ & 5.22 & $02-01-90$ & 5.30 & $02-15-91$ & 5.57 \\
$05-10-88$ & 5.22 & $03-01-90$ & 5.45 & $03-01-91$ & 5.50 \\
$06-13-88$ & 4.90 & $03-15-90$ & 5.52 & $03-15-91$ & 5.65 \\
$07-11-88$ & 4.63 & $04-01-90$ & 5.45 & $04-01-91$ & 5.75 \\
$08-10-88$ & 4.95 & $04-15-90$ & 5.35 & $04-15-91$ & 5.52 \\
$09-12-88$ & 5.07 & $05-01-90$ & 5.35 & $05-01-91$ & 5.62 \\
$10-10-88$ & 5.05 & $06-01-90$ & 5.30 & $05-15-91$ & 5.95 \\
$11-11-88$ & 5.09 & $06-15-90$ & 5.15 & $06-01-91$ & 5.04 \\
$12-12-88$ & 5.31 & $07-01-90$ & 4.92 & $06-15-91$ & 5.82 \\
$01-11-89$ & 5.28 & $07-15-90$ & 4.88 & $07-01-91$ & 6.25 \\
$02-13-89$ & 5.25 & $08-01-90$ & 4.95 & $07-15-91$ & 7.35 \\
$03-05-89$ & 5.35 & $08-15-90$ & 4.89 & $08-01-91$ & $08-42$ \\
$04-04-89$ & 5.57 & $09-01-90$ & 5.20 & $08-15-91$ & 8.15 \\
$05-07-89$ & 5.42 & $09-15-90$ & 5.12 & $09-01-91$ & 8.96 \\
$06-04-89$ & 4.95 & $10-01-90$ & 5.10 & $09-15-91$ & 9.20 \\
$07-08-89$ & 4.98 & $10-15-90$ & 5.00 & $10-01-91$ & 9.63 \\
$08-09-89$ & 4.76 & $11-01-90$ & 4.70 & $10-15-91$ & 9.68 \\
$09-12-89$ & 5.04 & $11-15-90$ & 4.68 & $11-01-91$ & 9.80 \\
$10-12-89$ & 5.26 & $12-01-90$ & 4.62 & $11-15-91$ & 9.52 \\
$11-07-89$ & 5.01 & $12-15-90$ & 4.33 & $12-01-91$ & $12-17-91$ \\
$12-15-89$ & 5.15 & $01-01-91$ & 5.22 & & \\
\hline
\end{tabular}




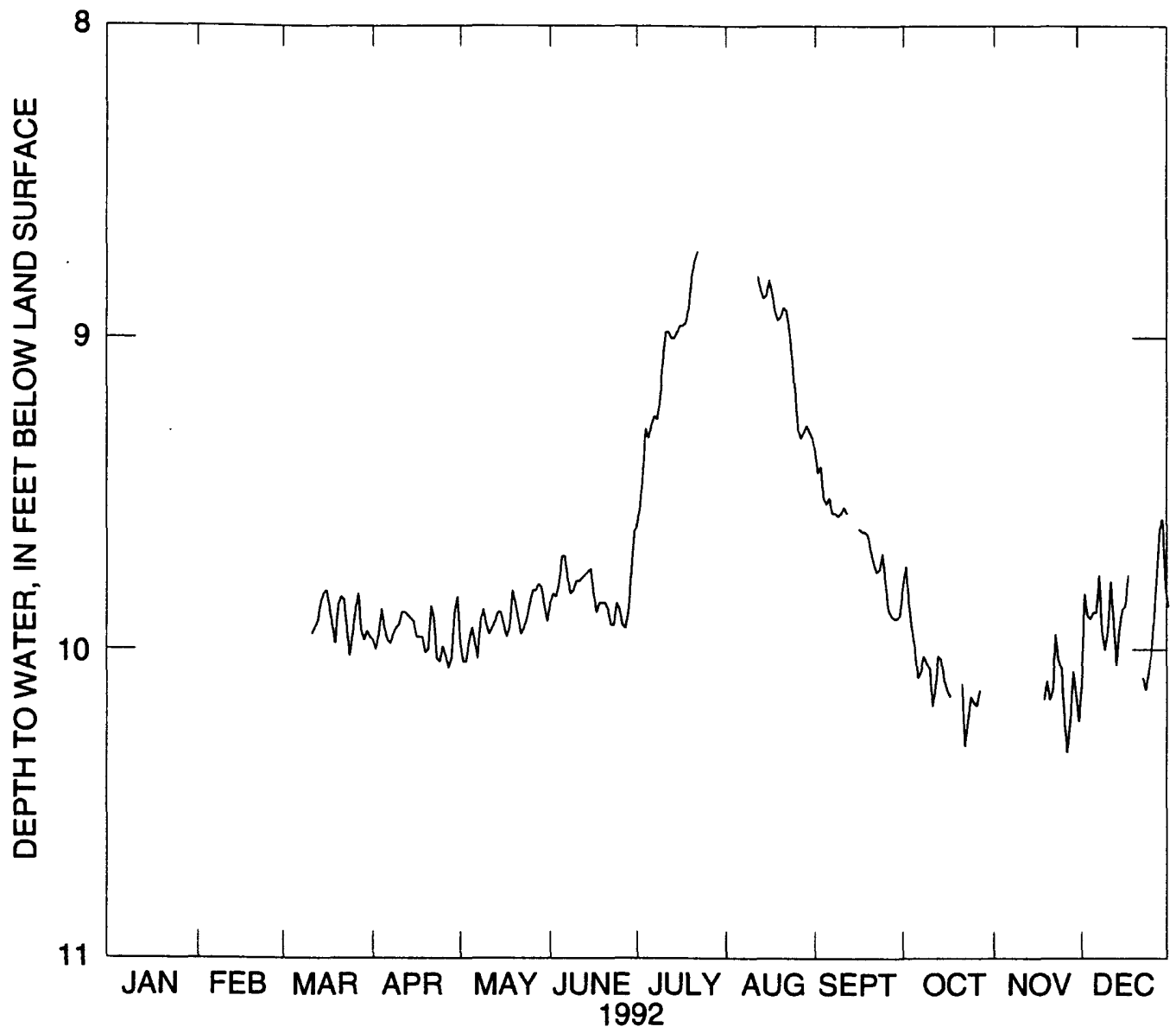

Figure 17. (A) Daily mean water levels, well CW-3, Long Valley Caldera, Mono County, California, 1992. 
Table 27. Daily mean water levels, well CW-3, Long Valley Caldera, Mono County, California, 1992

[Values are in feet below land surface. Max, maximum; Min, minimum; -, no data]

\begin{tabular}{|c|c|c|c|c|c|c|c|c|c|c|c|c|}
\hline Day & Jan & $\mathrm{Feb}$ & Mar & Apr & May & June & July & Aug & Sept & Oct & Nov & Dec \\
\hline 1 & - & - & - & 9.97 & 9.98 & 9.85 & 9.60 & 8.78 & 9.36 & 9.79 & - & 10.10 \\
\hline 2 & - & - & - & 10.00 & 10.04 & 9.82 & 9.55 & - & 9.43 & 9.73 & - & 9.82 \\
\hline 3 & - & - & - & 9.95 & 10.04 & 9.83 & 9.45 & - & 9.41 & 9.85 & - & 9.89 \\
\hline 4 & - & - & - & 9.87 & 9.97 & 9.79 & 9.29 & - & 9.51 & 9.93 & - & 9.90 \\
\hline 5 & - & - & - & 9.93 & 9.93 & 9.70 & 9.32 & - & 9.53 & 9.99 & - & 9.88 \\
\hline 6 & - & - & - & 9.97 & 9.98 & 9.70 & 9.28 & - & 9.51 & 10.09 & - & 9.88 \\
\hline 7 & - & - & - & 9.98 & 10.03 & 9.77 & 9.25 & - & 9.56 & 10.07 & - & 9.76 \\
\hline 8 & - & - & - & 9.95 & 9.91 & 9.82 & 9.26 & - & 9.56 & 10.02 & - & 9.94 \\
\hline 9 & - & - & - & 9.93 & 9.87 & 9.81 & 9.20 & - & 9.57 & 10.04 & - & 10.00 \\
\hline 10 & - & - & - & 9.92 & 9.92 & 9.78 & 9.08 & - & 9.56 & 10.06 & - & 9.94 \\
\hline 11 & - & - & 9.95 & 9.88 & 9.95 & 9.78 & 8.98 & - & 9.54 & 10.18 & - & 9.78 \\
\hline 12 & - & - & 9.93 & 9.88 & 9.93 & 9.77 & 8.98 & 8.80 & 9.56 & 10.12 & - & 9.91 \\
\hline 13 & - & - & 9.91 & 9.89 & 9.91 & - & 9.00 & 8.84 & - & 10.02 & - & 10.05 \\
\hline 14 & - & - & 9.85 & 9.90 & 9.88 & - & 9.00 & 8.87 & 一 & 10.03 & - & 9.93 \\
\hline 15 & - & - & 9.82 & 9.91 & 9.88 & 9.74 & 8.98 & 8.86 & - & 10.10 & - & 9.87 \\
\hline 16 & - & - & 9.81 & 9.96 & 9.92 & 9.82 & 8.96 & 8.81 & 9.61 & 10.13 & - & 9.86 \\
\hline 17 & - & - & 9.86 & 9.96 & 9.96 & 9.88 & 8.96 & 8.85 & 9.62 & 10.15 & - & 9.76 \\
\hline 18 & - & - & 9.92 & 9.96 & 9.93 & 9.85 & 8.95 & 8.91 & 9.62 & - & 10.16 & - \\
\hline 19 & - & - & 9.98 & 10.01 & 9.81 & 9.85 & 8.90 & 8.94 & 9.63 & - & 10.10 & - \\
\hline 20 & - & - & 9.86 & 10.00 & 9.85 & 9.85 & 8.80 & 8.93 & 9.68 & - & 10.16 & - \\
\hline 21 & - & - & 9.83 & 9.86 & 9.90 & 9.87 & 8.75 & 8.90 & 9.72 & 10.11 & 10.13 & - \\
\hline 22 & - & - & 9.84 & 9.90 & 9.95 & 9.92 & 8.72 & 8.91 & 9.75 & 10.31 & 9.95 & 10.09 \\
\hline 23 & - & - & 9.93 & 10.03 & 9.93 & 9.92 & - & 8.97 & 9.74 & - & 10.03 & 10.13 \\
\hline 24 & - & - & 10.02 & 10.04 & 9.90 & 9.85 & 一 & 9.06 & 9.69 & 10.15 & 10.06 & 10.07 \\
\hline 25 & - & - & 9.95 & 9.99 & 9.85 & 9.87 & - & 9.17 & 9.78 & 10.17 & 10.22 & 10.02 \\
\hline 26 & - & - & 9.87 & 10.02 & 9.81 & 9.92 & - & 9.29 & 9.87 & 10.18 & 10.33 & 9.91 \\
\hline 27 & - & - & 9.82 & 10.06 & 9.81 & 9.93 & 一 & 9.32 & 9.89 & 10.13 & 10.23 & 9.76 \\
\hline 28 & - & - & 9.94 & 10.03 & 9.79 & 9.88 & - & 9.30 & 9.90 & - & 10.07 & 9.61 \\
\hline 29 & - & - & 9.97 & 9.88 & 9.80 & 9.75 & 一 & 9.28 & 9.90 & - & 10.14 & 9.58 \\
\hline 30 & - & - & 9.94 & 9.83 & 9.86 & 9.62 & - & 9.30 & 9.89 & - & 10.23 & 9.79 \\
\hline 31 & - & - & 9.96 & - & 9.91 & - & - & 9.32 & 一 & - & - & 9.86 \\
\hline Mean & - & - & 9.90 & 9.95 & 9.91 & 9.82 & 9.10 & 9.02 & 9.64 & 10.06 & 10.14 & 9.89 \\
\hline $\operatorname{Max}$ & - & - & 10.02 & 10.06 & 10.04 & 9.93 & 9.60 & 9.32 & 9.90 & 10.31 & 10.33 & 10.13 \\
\hline Min & - & - & 9.81 & 9.83 & 9.79 & 9.62 & 8.72 & 8.78 & 9.36 & 9.73 & 9.95 & 9.58 \\
\hline
\end{tabular}




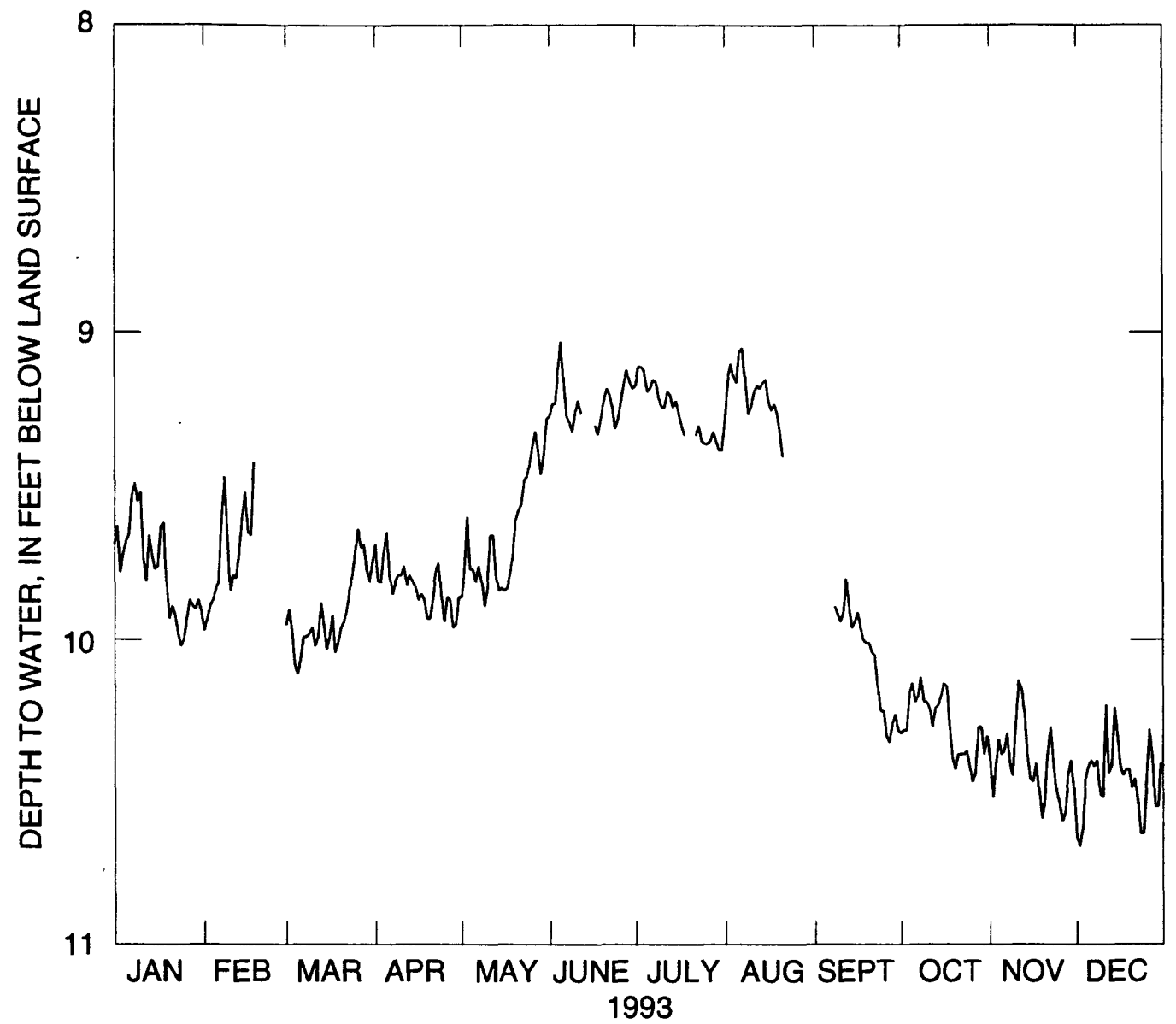

Figure 17. (B) Daily mean water levels, well CW-3, Long Valley Caldera, Mono County, California, 1993. 
Table 28. Daily mean water levels, well CW-3, Long Valley Caldera, Mono County, California, 1993

[Values are in feet below land surface. Max, maximum; Min, minimum; -, no data]

\begin{tabular}{|c|c|c|c|c|c|c|c|c|c|c|c|c|}
\hline Day & Jan & Feb & Mar & Apr & May & June & July & Aug & Sept & Oct & Nov & Dec \\
\hline 1 & 9.69 & 9.97 & 9.95 & 9.69 & 9.86 & 9.27 & 9.17 & 9.28 & - & 10.30 & 10.38 & 10.64 \\
\hline 2 & 9.63 & 9.93 & 9.90 & 9.81 & 9.78 & 9.23 & 9.11 & 9.15 & - & 10.29 & 10.51 & 10.67 \\
\hline 3 & 9.78 & 9.89 & 9.97 & 9.81 & 9.60 & 9.23 & 9.11 & 9.10 & - & 10.29 & 10.41 & 10.61 \\
\hline 4 & 9.72 & 9.87 & 10.08 & 9.71 & 9.77 & 9.12 & 9.12 & 9.14 & - & 10.17 & 10.32 & 10.45 \\
\hline 5 & 9.68 & 9.83 & 10.11 & 9.65 & 9.77 & 9.03 & 9.19 & 9.16 & - & 10.14 & 10.37 & 10.41 \\
\hline 6 & 9.66 & 9.81 & 10.06 & 9.79 & 9.81 & 9.16 & 9.18 & 9.06 & - & 10.20 & 10.36 & 10.39 \\
\hline 7 & 9.53 & 9.60 & 9.99 & 9.85 & 9.76 & 9.27 & 9.15 & 9.05 & - & 10.18 & 10.30 & 10.41 \\
\hline 8 & 9.49 & 9.47 & 9.99 & 9.81 & 9.81 & 9.29 & 9.16 & 9.15 & 9.89 & 10.12 & 10.40 & 10.39 \\
\hline 9 & 9.55 & 9.67 & 9.98 & 9.79 & 9.89 & 9.32 & 9.21 & 9.26 & 9.92 & 10.20 & 10.44 & 10.50 \\
\hline 10 & 9.52 & 9.84 & 9.96 & 9.79 & 9.83 & 9.26 & 9.24 & 9.24 & 9.94 & 10.20 & 10.26 & 10.51 \\
\hline 11 & 9.73 & 9.79 & 10.02 & 9.76 & 9.66 & 9.22 & 9.24 & 9.19 & 9.91 & 10.22 & 10.13 & 10.21 \\
\hline 12 & 9.81 & 9.80 & 9.99 & 9.82 & 9.66 & 9.26 & 9.19 & 9.17 & 9.80 & 10.28 & 10.16 & 10.43 \\
\hline 13 & 9.66 & 9.72 & 9.88 & 9.79 & 9.79 & - & 9.20 & 9.18 & 9.89 & 10.22 & 10.24 & 10.41 \\
\hline 14 & 9.72 & 9.60 & 9.95 & 9.81 & 9.84 & - & 9.24 & 9.16 & 9.96 & 10.21 & 10.37 & 10.22 \\
\hline 15 & 9.77 & 9.52 & 10.03 & 9.83 & 9.83 & - & 9.22 & 9.15 & 9.94 & 10.18 & 10.45 & 10.31 \\
\hline 16 & 9.76 & 9.65 & 9.99 & 9.87 & 9.84 & - & 9.26 & 9.22 & 9.91 & 10.14 & 10.46 & 10.41 \\
\hline 17 & 9.63 & 9.66 & 9.92 & 9.85 & 9.83 & 9.30 & 9.30 & 9.25 & 9.96 & 10.15 & 10.40 & 10.44 \\
\hline 18 & 9.62 & 9.42 & 10.04 & 9.87 & 9.79 & 9.33 & 9.33 & 9.23 & 10.00 & 10.28 & 10.49 & 10.42 \\
\hline 19 & 9.82 & - & 10.01 & 9.93 & 9.73 & 9.28 & - & 9.26 & 10.01 & 10.38 & 10.58 & 10.42 \\
\hline 20 & 9.93 & - & 9.96 & 9.93 & 9.61 & 9.22 & - & 9.32 & 10.01 & 10.42 & 10.51 & 10.48 \\
\hline 21 & 9.89 & - & 9.94 & 9.87 & 9.58 & 9.18 & - & 9.40 & 10.04 & 10.37 & 10.36 & 10.45 \\
\hline 22 & 9.92 & - & 9.90 & 9.78 & 9.56 & 9.20 & 9.33 & - & 10.05 & 10.37 & 10.28 & 10.53 \\
\hline 23 & 9.98 & - & 9.83 & 9.75 & 9.48 & 9.24 & 9.30 & - & 10.16 & 10.37 & 10.40 & 10.63 \\
\hline 24 & 10.02 & - & 9.79 & 9.86 & 9.47 & 9.31 & 9.35 & - & 10.23 & 10.36 & 10.49 & 10.63 \\
\hline 25 & 10.00 & - & 9.71 & 9.94 & 9.43 & 9.28 & 9.36 & - & 10.23 & 10.41 & 10.53 & 10.46 \\
\hline 26 & 9.93 & - & 9.64 & 9.86 & 9.37 & 9.22 & 9.36 & - & 10.31 & 10.46 & 10.59 & 10.29 \\
\hline 27 & 9.87 & - & 9.70 & 9.87 & 9.32 & 9.16 & 9.35 & 9.53 & 10.33 & 10.43 & 10.56 & 10.37 \\
\hline 28 & 9.89 & - & 9.69 & 9.96 & 9.38 & 9.12 & 9.32 & - & 10.27 & 10.28 & 10.44 & 10.54 \\
\hline 29 & 9.90 & - & 9.77 & 9.95 & 9.46 & 9.16 & 9.35 & - & 10.24 & 10.28 & 10.39 & 10.54 \\
\hline 30 & 9.87 & - & 9.81 & 9.86 & 9.40 & 9.18 & 9.38 & - & 10.29 & 10.37 & 10.48 & 10.40 \\
\hline 31 & 9.91 & - & 9.75 & - & 9.28 & - & 9.38 & - & - & 10.31 & - & 10.41 \\
\hline Mean & 9.77 & 9.72 & 9.91 & 9.83 & 9.65 & 9.22 & 9.25 & 9.21 & 10.06 & 10.28 & 10.40 & 10.45 \\
\hline Max & 10.02 & 9.97 & 10.11 & 9.96 & 9.89 & 9.33 & 9.38 & 9.53 & 10.33 & 10.46 & 10.59 & 10.67 \\
\hline Min & 9.49 & 9.42 & 9.64 & 9.65 & 9.28 & 9.03 & 9.11 & 9.05 & 9.80 & 10.12 & 10.13 & 10.21 \\
\hline
\end{tabular}




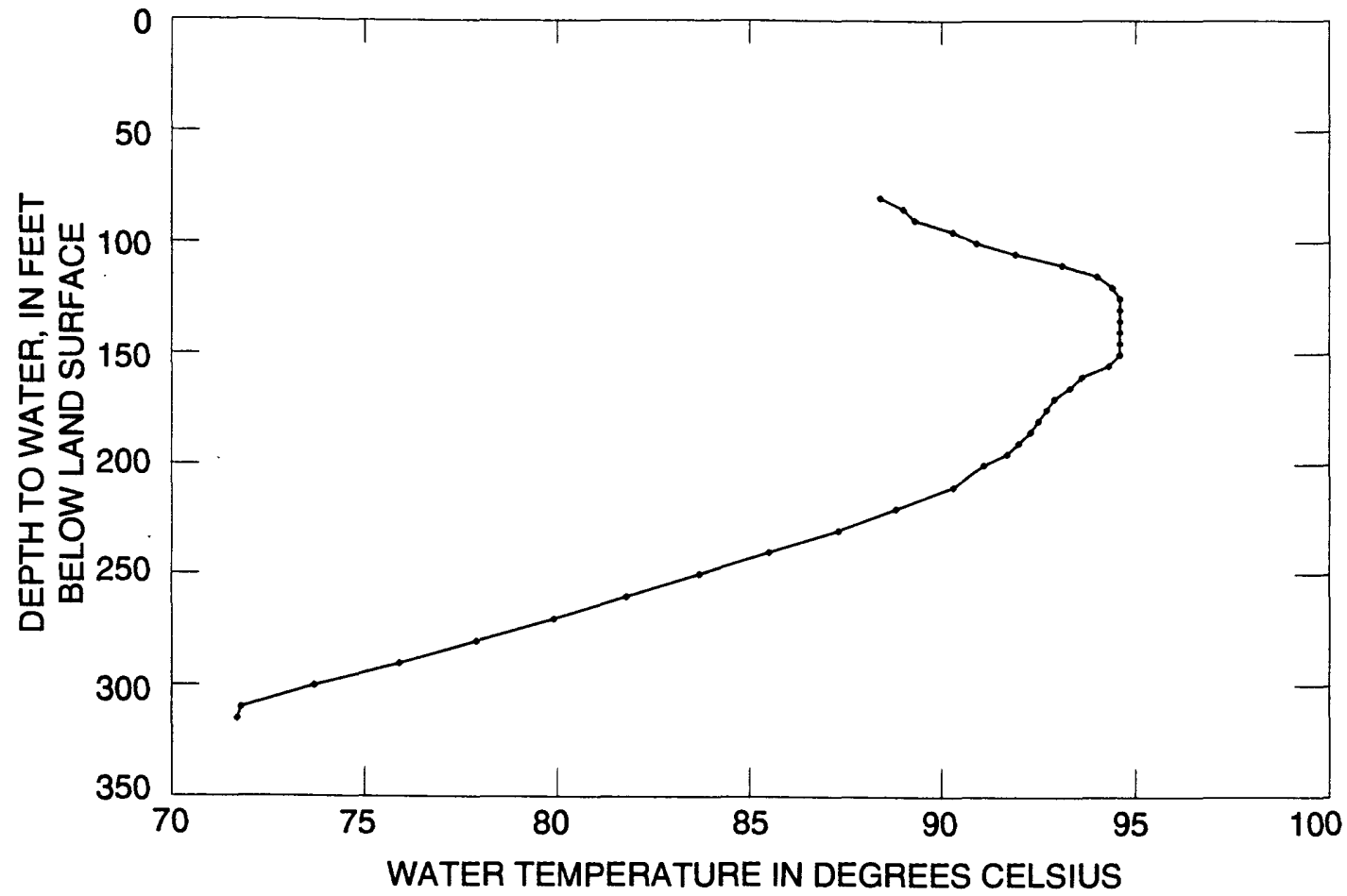

Figure 18. Temperature log of well $\mathrm{CH}-10 \mathrm{~B}$, Long Valley Caldera, Mono County, California, December 19, 1988. 


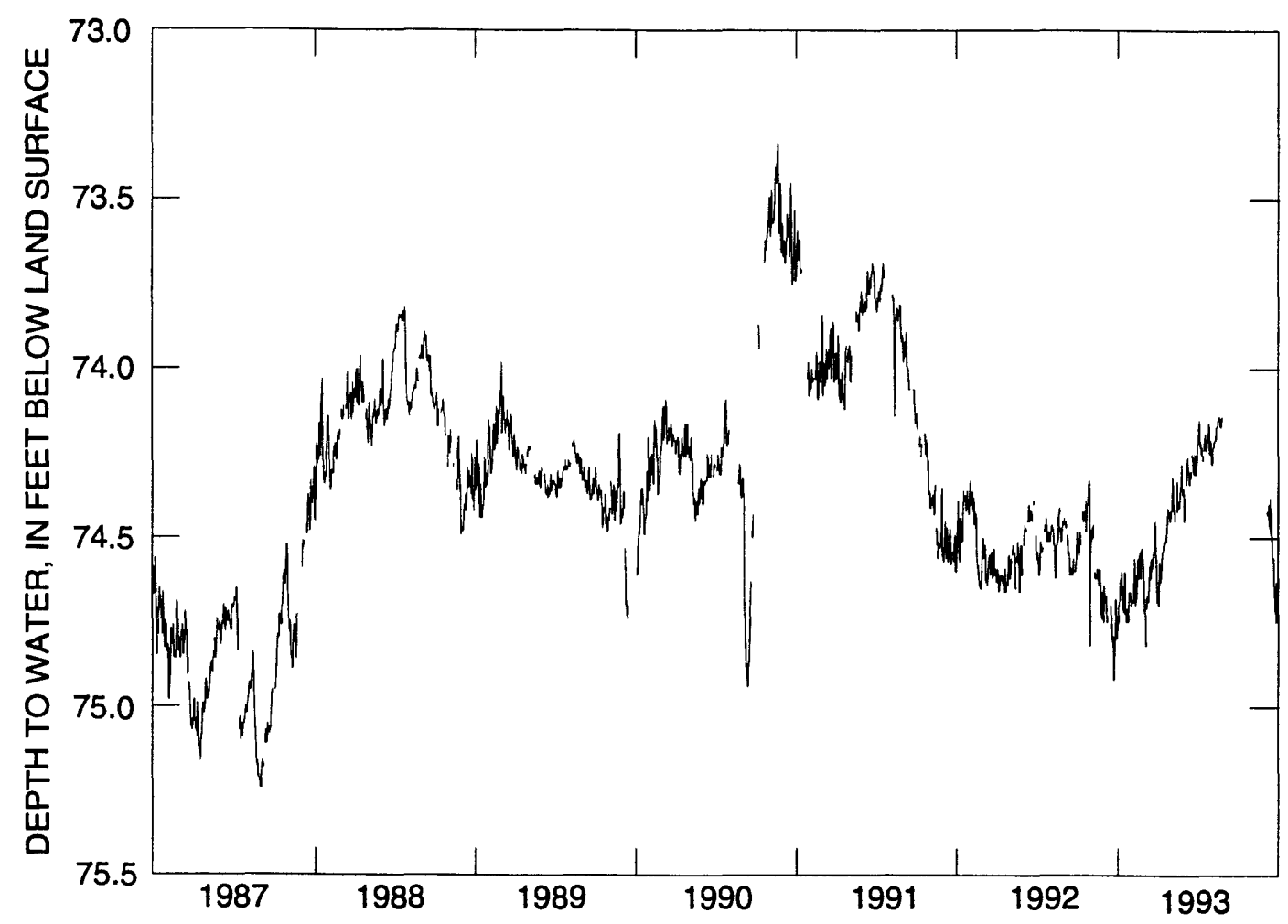

Figure 19. Daily mean water levels, well $\mathrm{CH}-10 \mathrm{~B}$, Long Valley Caldera, Mono County, California, 1987-93. 


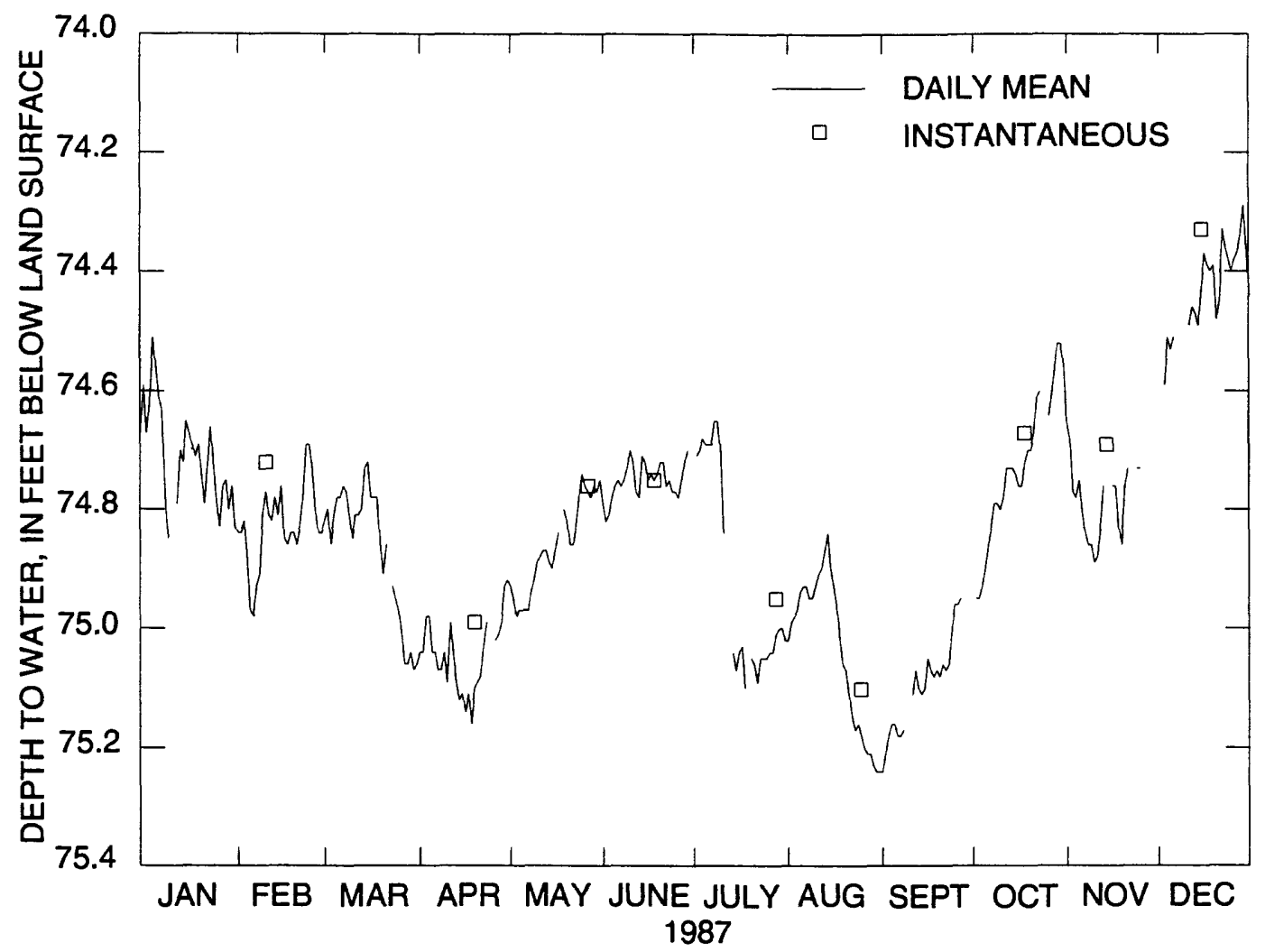

Figure 20. (A) Daily mean and instantaneous water levels, well CH-10B, Long Valley Caldera, Mono County, California, 1987. 
Table 29. Daily mean water levels, well CH-10B, Long Valley Caldera, Mono County, California, 1987

[Values are in feet below land surface. Max, maximum; Min, minimum; -, no data]

\begin{tabular}{|c|c|c|c|c|c|c|c|c|c|c|c|c|}
\hline Day & Jan & Feb & Mar & Apr & May & June & July & Aug & Sept & Oct & Nov & Dec \\
\hline 1 & 74.59 & 74.84 & 74.82 & 75.04 & 74.93 & 74.79 & - & 75.02 & 75.24 & - & 74.65 & - \\
\hline 2 & 74.67 & 74.84 & 74.80 & 75.04 & 74.95 & 74.82 & 74.71 & 74.99 & 75.21 & 74.95 & 74.68 & - \\
\hline 3 & 74.62 & 74.82 & 74.86 & 74.98 & 74.98 & 74.81 & 74.70 & 74.98 & 75.18 & 74.95 & 74.77 & 74.59 \\
\hline 4 & 74.51 & 74.89 & 74.81 & 74.98 & 74.97 & 74.78 & 74.68 & 74.97 & 75.16 & 74.93 & 74.78 & 74.51 \\
\hline 5 & 74.56 & 74.97 & 74.78 & 75.04 & 74.97 & 74.76 & 74.69 & 74.94 & 75.16 & 74.90 & 74.75 & 74.53 \\
\hline 6 & 74.61 & 74.98 & 74.78 & 75.04 & 74.97 & 74.75 & 74.69 & 74.93 & 75.18 & 74.86 & 74.80 & 74.51 \\
\hline 7 & 74.63 & 74.93 & 74.76 & 75.07 & 74.97 & 74.76 & 74.69 & 74.93 & 75.18 & 74.83 & 74.84 & - \\
\hline 8 & 74.77 & 74.91 & 74.77 & 75.07 & 74.94 & 74.75 & 74.65 & 74.95 & 75.17 & 74.79 & 74.86 & - \\
\hline 9 & 74.85 & 74.81 & 74.81 & 75.04 & 74.92 & 74.73 & 74.65 & 74.95 & - & 74.79 & 74.86 & - \\
\hline 10 & - & 74.77 & 74.85 & 75.09 & 74.89 & 74.70 & 74.70 & 74.93 & - & 74.80 & 74.89 & - \\
\hline 11 & - & 74.81 & 74.81 & 74.99 & 74.88 & 74.72 & 74.84 & 74.91 & 75.11 & 74.78 & 74.88 & 74.49 \\
\hline 12 & 74.79 & 74.82 & 74.81 & 75.04 & 74.87 & 74.77 & - & 74.90 & 75.07 & 74.73 & 74.83 & 74.46 \\
\hline 13 & 74.70 & 74.78 & 74.80 & 75.09 & 74.87 & 74.78 & - & 74.87 & 75.10 & 74.73 & 74.76 & 74.47 \\
\hline 14 & 74.72 & 74.81 & 74.73 & 75.12 & 74.89 & 74.71 & 75.04 & 74.84 & 75.11 & 74.73 & - & 74.49 \\
\hline 15 & 74.65 & 74.76 & 74.72 & 75.11 & 74.90 & 74.72 & 75.07 & 74.90 & 75.10 & 74.74 & - & 74.43 \\
\hline 16 & 74.67 & 74.85 & 74.78 & 75.14 & 74.87 & 74.75 & 75.04 & 74.93 & 75.05 & 74.76 & 74.76 & 74.37 \\
\hline 17 & 74.69 & 74.86 & 74.78 & 75.11 & 74.84 & 74.74 & 75.03 & 74.96 & 75.07 & 74.76 & 74.76 & 74.39 \\
\hline 18 & 74.71 & 74.84 & 74.78 & 75.16 & - & 74.75 & 75.10 & 75.02 & 75.08 & 74.72 & 74.83 & 74.40 \\
\hline 19 & 74.69 & 74.84 & 74.86 & 75.10 & 74.80 & 74.74 & - & 75.06 & 75.07 & 74.70 & 74.86 & 74.39 \\
\hline 20 & 74.74 & 74.86 & 74.91 & 75.09 & 74.82 & 74.72 & 75.05 & 75.07 & 75.08 & 74.70 & 74.76 & 74.48 \\
\hline 21 & 74.79 & 74.83 & 74.86 & 75.08 & 74.86 & 74.72 & 75.06 & 75.11 & 75.06 & 74.67 & 74.73 & 74.45 \\
\hline 22 & 74.72 & 74.78 & - & 75.03 & 74.86 & 74.76 & 75.09 & 75.15 & 75.07 & 74.61 & - & 74.33 \\
\hline 23 & 74.66 & 74.69 & 74.93 & 74.99 & 74.83 & 74.75 & 75.05 & 75.17 & 75.06 & 74.60 & - & 74.36 \\
\hline 24 & 74.73 & 74.69 & 74.95 & - & 74.78 & 74.77 & 75.05 & 75.16 & 75.00 & - & 74.73 & 74.38 \\
\hline 25 & 74.79 & 74.73 & 74.97 & - & 74.74 & 74.77 & 75.05 & 75.18 & 74.96 & - & 74.73 & 74.40 \\
\hline 26 & 74.83 & 74.80 & 75. & 75.02 & 74.76 & 74.78 & 75.04 & 75.20 & 74.96 & 74.64 & - & 74.38 \\
\hline 27 & 74.76 & 74.84 & 75.06 & 75.01 & 74.77 & 74.75 & 75.04 & 75.21 & 74.95 & 74.60 & - & 74.37 \\
\hline 28 & 74.75 & 74.84 & 75.06 & 74.99 & 74.78 & 74.72 & 75.01 & 75.21 & - & 74.55 & - & 74.34 \\
\hline 29 & 74.80 & - & 75.04 & 74.93 & 74.76 & 74.70 & 75.00 & 75.23 & - & 74.52 & - & 74.29 \\
\hline 30 & 74.76 & - & 75.07 & 74.92 & 74.77 & - & 75.00 & 75.24 & - & 74.52 & - & 74.36 \\
\hline 31 & 74.83 & - & 75.06 & - & 74.75 & - & 75.02 & 75.24 & - & 74.56 & - & 74.44 \\
\hline Mean & 74.71 & 74.83 & 74.87 & 75.05 & 74.86 & 74.75 & 74.92 & 75.04 & 75.10 & 74.73 & 74.79 & 74.42 \\
\hline $\operatorname{Max}$ & 74.85 & 74.98 & 75.07 & 75.16 & 74.98 & 74.82 & 75.10 & 75.24 & 75.24 & 74.95 & 74.89 & 74.59 \\
\hline Min & 74.51 & 74.69 & 74.72 & 74.92 & 74.74 & 74.70 & 74.65 & 74.84 & 74.95 & 74.52 & 74.65 & 74.29 \\
\hline
\end{tabular}




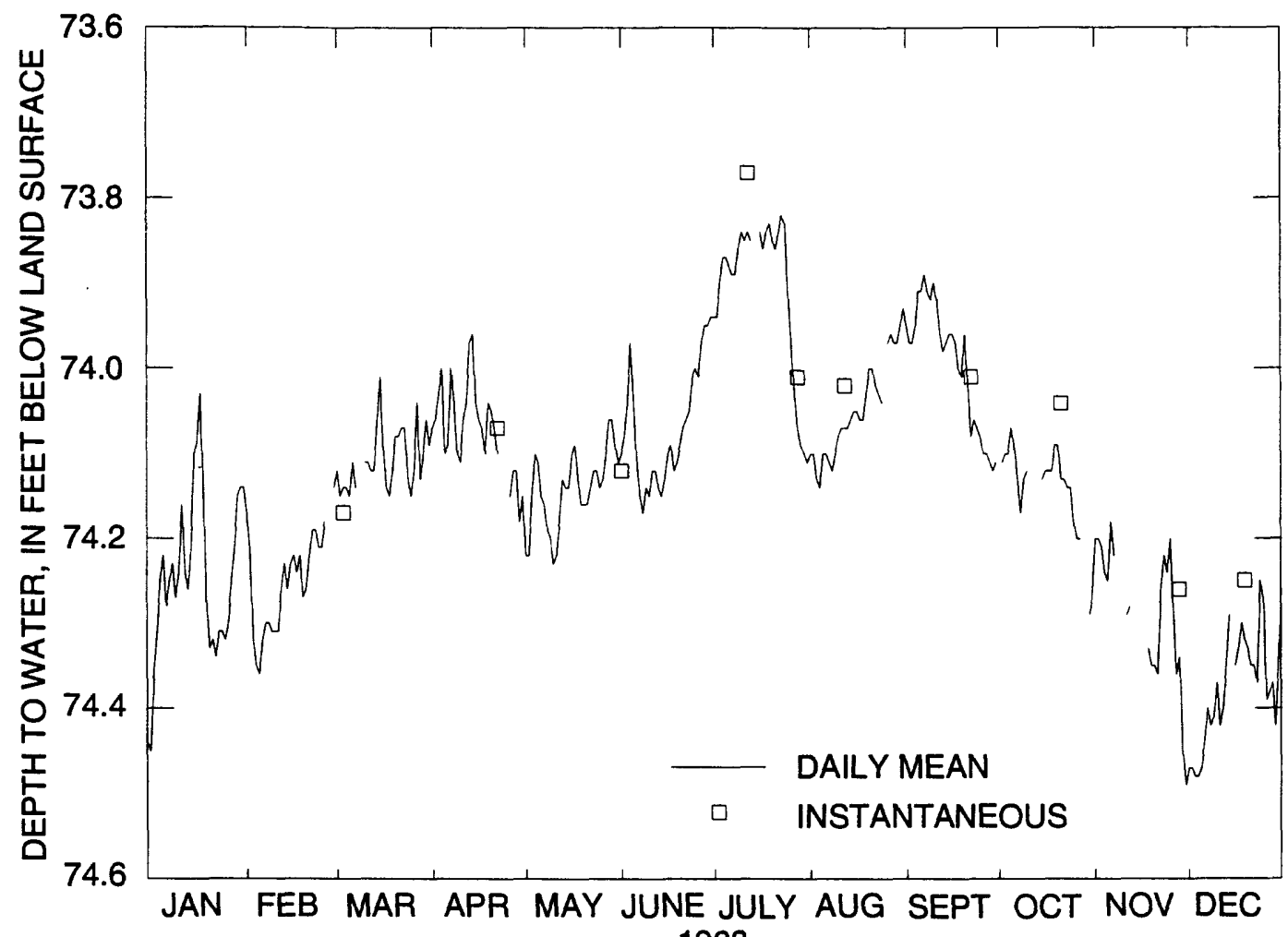
1988

Figure 20. (B) Daily mean and instantaneous water levels, well CH-10B, Long Valley Caldera, Mono County, California, 1988. 
Table 30. Daily mean water levels, well $\mathrm{CH}-10 \mathrm{~B}$, Long Valley Caldera, Mono County, California, 1988

[Values are in feet below land surface. Max, maximum; Min, minimum; -, no data]

\begin{tabular}{|c|c|c|c|c|c|c|c|c|c|c|c|c|}
\hline Day & Jan & Feb & Mar & Apr & May & June & July & Aug & Sept & Oct & Nov & Dec \\
\hline 1 & 74.45 & 74.17 & 74.12 & 74.07 & 74.22 & 74.10 & 73.94 & 74.10 & 73.95 & - & 74.20 & 74.47 \\
\hline 2 & 74.36 & 74.22 & 74.15 & 74.06 & 74.22 & 74.08 & 73.94 & 74.10 & 73.97 & 74.11 & 74.20 & 74.47 \\
\hline 3 & 74.31 & 74.32 & 74.14 & 74.03 & 74.15 & 74.04 & 73.90 & 74.13 & 73.97 & 74.10 & 74.21 & 74.48 \\
\hline 4 & 74.25 & 74.35 & 74.14 & 74.00 & 74.10 & 73.97 & 73.87 & 74.14 & 73.95 & 74.10 & 74.24 & 74.48 \\
\hline 5 & 74.22 & 74.36 & 74.15 & 74.10 & 74.11 & 74.04 & 73.87 & 74.10 & 73.91 & 74.07 & 74.25 & 74.47 \\
\hline 6 & 74.28 & 74.32 & 74.11 & 74.09 & 74.15 & 74.11 & 73.88 & 74.10 & 73.91 & 74.09 & 74.18 & 74.44 \\
\hline 7 & 74.25 & 74.30 & 74.14 & 74.00 & 74.16 & 74.15 & 73.89 & 74.11 & 73.89 & 74.13 & 74.22 & 74.40 \\
\hline 8 & 74.23 & 74.30 & - & 74.04 & 74.19 & 74.17 & 73.89 & 74.12 & 73.91 & 74.17 & - & 74.42 \\
\hline 9 & 74.27 & 74.31 & - & 74.10 & 74.20 & 74.14 & 73.86 & 74.10 & 73.92 & 74.13 & - & 74.41 \\
\hline 10 & 74.24 & 74.31 & 74.11 & 74.11 & 74.23 & 74.15 & 73.84 & 74.08 & 73.90 & 74.12 & - & 74.37 \\
\hline 11 & 74.16 & 74.31 & 74.11 & 74.06 & 74.22 & 74.12 & 73.85 & 74.07 & 73.92 & - & 74.29 & 74.42 \\
\hline 12 & 74.24 & 74.26 & 74.12 & 74.04 & 74.18 & 74.12 & 73.84 & 74.07 & 73.96 & - & 74.28 & 74.40 \\
\hline 13 & 74.26 & 74.23 & 74.12 & 73.97 & 74.13 & 74.14 & 73.85 & 74.07 & 73.98 & - & - & 74.35 \\
\hline 14 & 74.21 & 74.26 & 74.07 & 73.96 & 74.14 & 74.15 & - & 74.06 & 73.97 & - & - & 74.29 \\
\hline 15 & 74.10 & 74.23 & 74.01 & 74.04 & 74.14 & 74.13 & - & 74.05 & 73.96 & 74.13 & 74.35 & - \\
\hline 16 & 74.09 & 74.22 & 74.09 & 74.06 & 74.10 & 74.10 & 73.84 & 74.05 & 73.96 & 74.12 & - & 74.35 \\
\hline 17 & 74.03 & 74.24 & 74.14 & 74.07 & 74.09 & 74.09 & 73.86 & 74.06 & 73.97 & 74.12 & - & 74.33 \\
\hline 18 & 74.14 & 74.22 & 74.15 & 74.10 & 74.13 & 74.12 & 73.84 & 74.06 & 74.00 & 74.12 & 74.33 & 74.30 \\
\hline 19 & 74.28 & 74.27 & 74.12 & 74.04 & 74.16 & 74.11 & 73.83 & 74.03 & 74.01 & 74.09 & 74.35 & 74.32 \\
\hline 20 & 74.33 & 74.26 & 74.08 & 74.05 & 74.16 & 74.09 & 73.85 & 74.00 & 73.96 & 74.09 & 74.35 & 74.33 \\
\hline 21 & 74.32 & 74.22 & 74.08 & 74.07 & 74.16 & 74.07 & 73.86 & 74.00 & 74.02 & 74.13 & 74.36 & 74.35 \\
\hline 22 & 74.34 & 74.19 & 74.07 & 74.10 & 74.14 & 74.06 & 73.84 & 74.02 & 74.08 & 74.13 & 74.26 & 74.35 \\
\hline 23 & 74.31 & 74.19 & 74.07 & - & 74.12 & 74.05 & 73.82 & 74.03 & 74.06 & 74.14 & 74.22 & 74.37 \\
\hline 24 & 74.31 & 74.21 & 74.13 & - & 74.12 & 74.01 & 73.83 & 74.04 & 74.07 & 74.14 & 74.24 & 74.25 \\
\hline 25 & 74.32 & 74.21 & 74.15 & - & 74.14 & 74.00 & 73.91 & - & 74.08 & 74.18 & 74.20 & 74.27 \\
\hline 26 & 74.30 & 74.18 & 74.12 & 74.15 & 74.13 & 74.01 & 73.97 & 73.97 & 74.10 & 74.20 & 74.29 & 74.39 \\
\hline 27 & 74.25 & - & 74.04 & 74.12 & 74.10 & 73.97 & 74.03 & 73.96 & 74.10 & 74.20 & 74.36 & 74.38 \\
\hline 28 & 74.21 & - & 74.13 & 74.12 & 74.06 & 73.95 & 74.07 & 73.97 & 74.11 & - & 74.34 & 74.37 \\
\hline 29 & 74.15 & 74.14 & 74.10 & 74.18 & 74.06 & 73.95 & 74.09 & 73.97 & 74.12 & - & 74.45 & 74.42 \\
\hline 30 & 74.14 & - & 74.06 & 74.15 & 74.09 & 73.94 & 74.10 & 73.95 & 74.11 & 74.29 & 74.49 & 74.34 \\
\hline 31 & 74.14 & - & 74.09 & - & 74.11 & - & 74.11 & 73.93 & - & 74.27 & - & 74.26 \\
\hline Mean & 74.24 & 74.25 & 74.11 & 74.07 & 74.14 & 74.07 & 73.91 & 74.05 & 73.99 & 74.14 & 74.29 & 74.37 \\
\hline $\operatorname{Max}$ & 74.45 & 74.36 & 74.15 & 74.18 & 74.23 & 74.17 & 74.11 & 74.14 & 74.12 & 74.29 & 74.49 & 74.48 \\
\hline Min & 74.03 & 74.14 & 74.01 & 73.96 & 74.06 & 73.94 & 73.82 & 73.93 & 73.89 & 74.07 & 74.18 & 74.25 \\
\hline
\end{tabular}




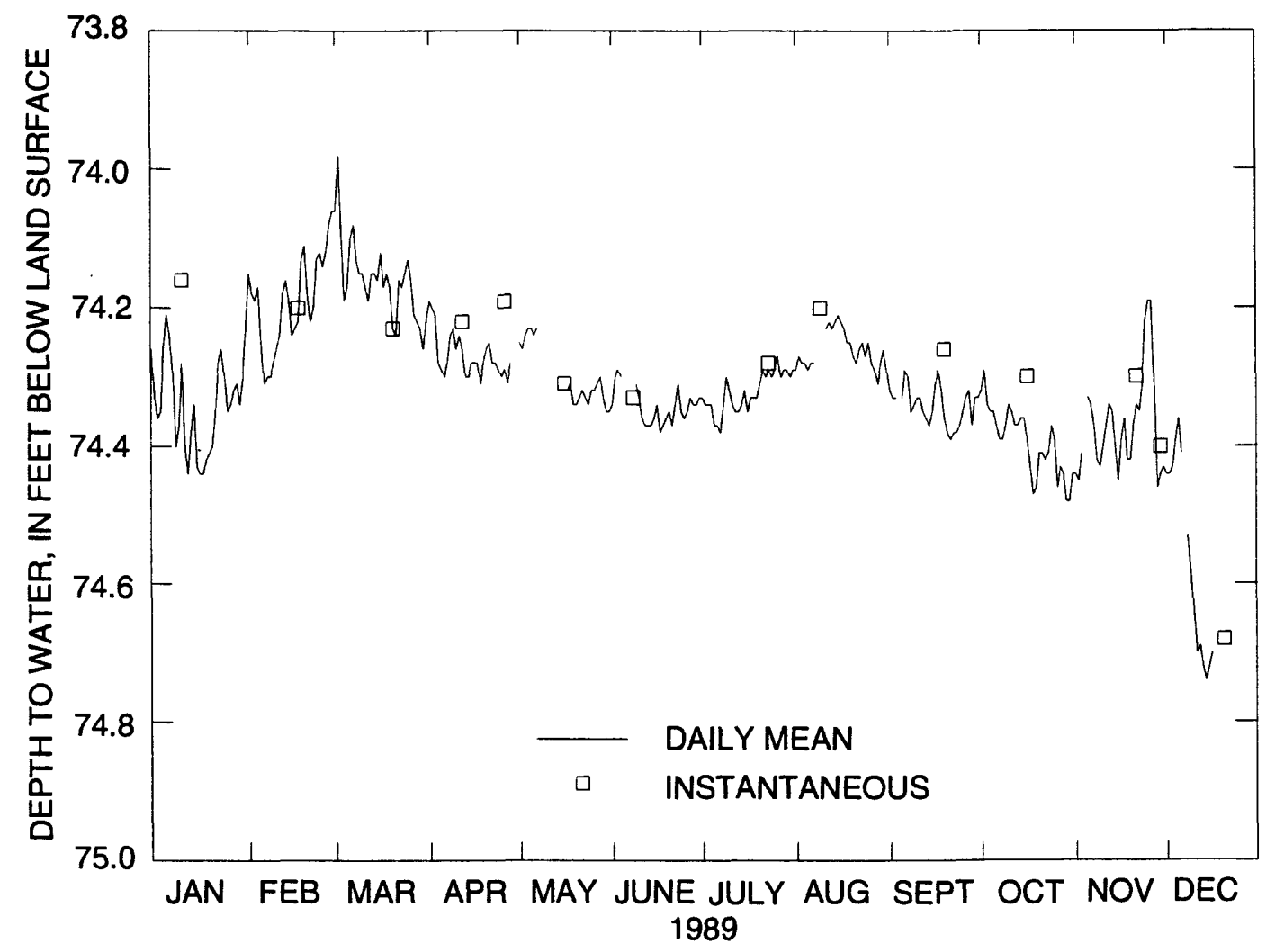

Figure 20. (C) Daily mean and instantaneous water levels, well CH-10B, Long Valley Caldera, Mono County, California, 1989. 
Table 31. Daily mean water levels, well $\mathrm{CH}-10 \mathrm{~B}$, Long Valley Caldera, Mono County, California, 1989

[Values are in feet below land surface. Max, maximum; Min, minimum; -, no data]

\begin{tabular}{|c|c|c|c|c|c|c|c|c|c|c|c|c|}
\hline Day & Jan & Feb & Mar & Apr & May & June & July & Aug & Sept & Oct & Nov & Dec \\
\hline 1 & 74.33 & 74.15 & 74.06 & 74.19 & 74.25 & 74.34 & 74.33 & 74.29 & 74.32 & 74.32 & 74.44 & 74.44 \\
\hline 2 & 74.36 & 74.18 & 73.98 & 74.20 & 74.26 & 74.30 & 74.34 & 74.27 & 74.33 & 74.29 & 74.45 & 74.44 \\
\hline 3 & 74.35 & 74.19 & 74.09 & 74.21 & 74.24 & 74.29 & 74.34 & 74.28 & 74.33 & 74.34 & 74.41 & 74.43 \\
\hline 4 & 74.26 & 74.17 & 74.19 & 74.28 & 74.23 & 74.30 & 74.34 & 74.28 & - & 74.35 & - & 74.39 \\
\hline 5 & 74.21 & 74.25 & 74.17 & 74.29 & 74.23 & - & 74.37 & 74.29 & 74.33 & 74.35 & 74.33 & 74.36 \\
\hline 6 & 74.24 & 74.31 & 74.10 & 74.30 & 74.24 & - & 74.37 & 74.28 & 74.29 & 74.37 & 74.34 & 74.41 \\
\hline 7 & 74.29 & 74.30 & 74.08 & 74.28 & 74.23 & - & 74.38 & 74.28 & 74.30 & 74.39 & 74.37 & - \\
\hline 8 & 74.40 & 74.30 & 74.13 & 74.24 & - & - & 74.34 & - & 74.35 & 74.39 & 74.42 & 74.53 \\
\hline 9 & 74.37 & 74.28 & 74.15 & 74.23 & - & 74.31 & 74.30 & - & 74.34 & 74.37 & 74.43 & 74.58 \\
\hline 10 & 74.28 & 74.26 & 74.15 & 74.26 & - & 74.33 & 74.32 & - & 74.33 & 74.34 & 74.40 & 74.64 \\
\hline 11 & 74.40 & 74.24 & 74.17 & 74.24 & - & 74.36 & 74.34 & 74.23 & 74.33 & 74.35 & 74.37 & 74.70 \\
\hline 12 & 74.44 & 74.18 & 74.19 & 74.26 & - & 74.37 & 74.35 & 74.22 & 74.35 & 74.37 & 74.34 & 74.69 \\
\hline 13 & 74.38 & 74.16 & 74.15 & 74.30 & - & 74.37 & 74.35 & 74.23 & 74.36 & 74.37 & 74.35 & 74.72 \\
\hline 14 & 74.34 & 74.19 & 74.15 & 74.30 & - & 74.37 & 74.34 & 74.22 & 74.37 & 74.36 & 74.40 & 74.74 \\
\hline 15 & 74.43 & 74.24 & 74.16 & 74.28 & - & 74.36 & 74.32 & 74.21 & 74.35 & 74.36 & 74.45 & 74.72 \\
\hline 16 & 74.44 & 74.23 & 74.12 & 74.28 & - & 74.34 & 74.35 & 74.22 & 74.31 & 74.39 & 74.39 & 74.70 \\
\hline 17 & 74.44 & 74.22 & 74.17 & 74.28 & 74.32 & 74.38 & 74.33 & 74.23 & 74.29 & 74.43 & 74.36 & - \\
\hline 18 & 74.42 & 74.13 & 74.15 & 74.31 & 74.31 & 74.37 & 74.33 & 74.25 & 74.32 & 74.47 & 74.42 & - \\
\hline 19 & 74.41 & 74.11 & 74.17 & 74.28 & 74.34 & 74.36 & 74.33 & 74.25 & 74.36 & 74.46 & 74.42 & - \\
\hline 20 & 74.40 & 74.18 & 74.23 & 74.26 & 74.34 & 74.35 & 74.31 & 74.27 & 74.38 & 74.41 & 74.37 & - \\
\hline 21 & 74.35 & 74.22 & 74.24 & 74.25 & 74.33 & 74.37 & 74.29 & 74.28 & 39 & 74 & & - \\
\hline 22 & 74.28 & 74.20 & 74.16 & 74.28 & 74.32 & 74.34 & 74.30 & 74.26 & 74.38 & 74.42 & 74.35 & - \\
\hline 23 & 74.26 & 74.13 & 74.17 & 74.28 & 74.33 & 74.31 & 74.29 & 74.25 & 74.38 & 74.41 & 74.31 & - \\
\hline 24 & 74.30 & 74.12 & 74.15 & 74.29 & 74.34 & 74.35 & 74.30 & 74.27 & 74.37 & 74.37 & 74.22 & - \\
\hline 25 & 74.35 & 74.14 & 74.13 & 74.30 & 74.32 & 74.36 & 74.29 & 74.25 & 74.35 & 74.39 & 74.19 & - \\
\hline 26 & 74.34 & 74.12 & 74.16 & 74.29 & 74.32 & 74.35 & 74.27 & 74.28 & 74.33 & 74.46 & 74.19 & - \\
\hline 27 & 74.32 & 74.08 & 74.21 & 74.31 & 74.31 & 74.33 & 74.30 & 74.29 & 74.32 & 74.43 & 74.31 & - \\
\hline 28 & 74.31 & 74.06 & 74.22 & 74.28 & 74.30 & 74.34 & 74.29 & 74.31 & 74.37 & 74.44 & 74.46 & - \\
\hline 29 & 74.34 & - & 74.23 & - & 74.33 & 74.34 & 74.29 & 74.28 & 74.33 & 74.48 & 74.44 & - \\
\hline 30 & 74.30 & - & 74.26 & - & 74.35 & 74.33 & 74.30 & 74.26 & 74.33 & 74.48 & 74.43 & - \\
\hline 31 & 74.23 & - & 74.22 & - & 74.35 & - & 74.29 & 74.29 & - & 74.44 & - & - \\
\hline Mean & 74.34 & 74.19 & 74.16 & 74.27 & 74.30 & 74.34 & 74.32 & 74.26 & 74.34 & 74.39 & 74.37 & 74.57 \\
\hline $\operatorname{Max}$ & 74.44 & 74.31 & 74.26 & 74.31 & 74.35 & 74.38 & 74.38 & 74.31 & 74.39 & 74.48 & 74.46 & 74.74 \\
\hline Min & 74.21 & 74.06 & 73.98 & 74.19 & 74.23 & 74.29 & 74.27 & 74.21 & 74.29 & 74.29 & 74.19 & 74.36 \\
\hline
\end{tabular}




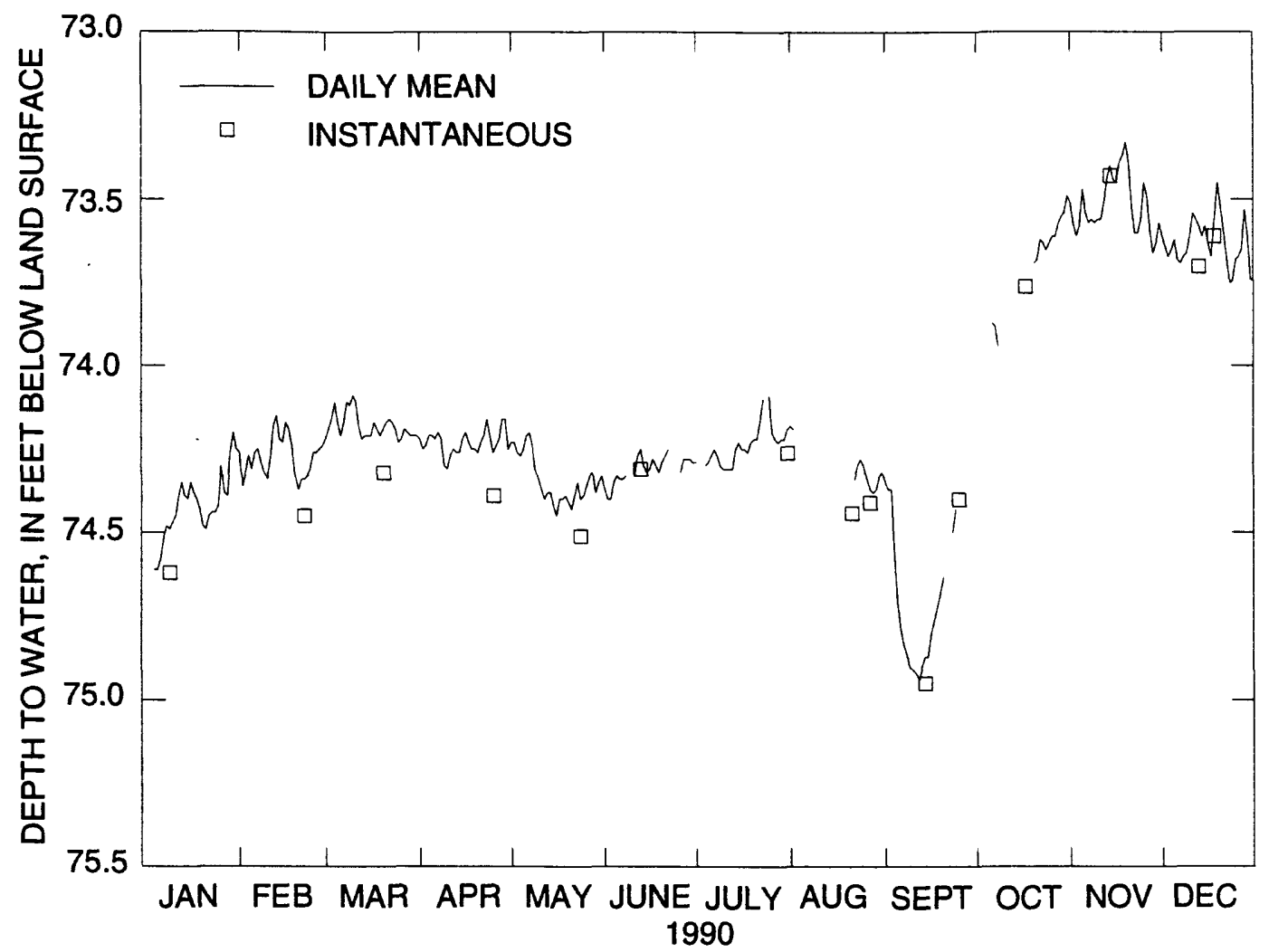

Figure 20. (D) Daily mean and instantaneous water levels, well CH-10B, Long Valley Caldera, Mono County, California, 1990. 
Table 32. Daily mean water levels, well CH-10B, Long Valley Caldera, Mono County, California, 1990

[Values are in feet below land surface. Max, maximum; Min, minimum; -, no data]

\begin{tabular}{|c|c|c|c|c|c|c|c|c|c|c|c|c|}
\hline Day & Jan & Feb & Mar & Apr & May & June & July & Aug & Sept & Oct & Nov & Dec \\
\hline 1 & - & 74.26 & 74.22 & 74.22 & 74.23 & 74.37 & 74.29 & 74.18 & 74.35 & - & 73.51 & 73.61 \\
\hline 2 & - & 74.36 & 74.19 & 74.25 & 74.23 & 74.40 & - & 74.19 & 74.37 & - & 73.57 & 73.64 \\
\hline 3 & - & 74.32 & 74.16 & 74.24 & 74.26 & 74.40 & - & - & 74.37 & - & 73.61 & 73.67 \\
\hline 4 & 74.61 & 74.27 & 74.11 & 74.21 & 74.27 & 74.35 & 74.30 & - & 74.56 & - & 73.58 & 73.65 \\
\hline 5 & 74.61 & 74.31 & 74.17 & 74.21 & 74.25 & 74.33 & 74.29 & - & 74.70 & - & 73.47 & 73.62 \\
\hline 6 & 74.58 & 74.26 & 74.21 & 74.22 & 74.21 & 74.34 & 74.27 & - & 74.78 & 73.87 & 73.54 & 73.68 \\
\hline 7 & 74.52 & 74.25 & 74.17 & 74.20 & 74.20 & 74.34 & 74.25 & - & 74.83 & 73.88 & 73.57 & 73.69 \\
\hline 8 & 74.48 & 74.29 & 74.11 & 74.22 & 74.24 & 74.33 & 74.27 & - & 74.86 & 73.94 & 73.56 & 73.67 \\
\hline 9 & 74.49 & 74.32 & 74.12 & 74.30 & 74.31 & - & 74.30 & - & 74.90 & - & 73.57 & 73.66 \\
\hline 10 & 74.47 & 74.34 & 74.09 & 74.31 & 74.34 & - & 74.31 & - & 74.91 & - & 73.56 & 73.61 \\
\hline 11 & 74.45 & 74.28 & 74.11 & 74.27 & 74.37 & 74.33 & 74.31 & - & 74.92 & - & 73.56 & 73.54 \\
\hline 12 & 74.39 & 74.18 & 74.18 & 74.25 & 74.40 & 74.27 & 74.31 & - & 74.94 & - & 73.51 & 73.56 \\
\hline 13 & 74.35 & 74.15 & 74.22 & 74.26 & 74.38 & 74.25 & 74.31 & - & 74.90 & - & 73.44 & 73.58 \\
\hline 14 & 74.39 & 74.22 & 74.21 & 74.26 & 74.38 & 74.30 & 74.25 & - & 74.87 & - & 73.40 & 73.61 \\
\hline 15 & 74.40 & 74.23 & 74.21 & 74.22 & 74.42 & 74.32 & 74.23 & - & 74.87 & - & 73.44 & 73.58 \\
\hline 16 & 74.35 & 74.17 & 74.21 & 74.20 & 74.45 & 74.31 & 74.25 & - & 74.80 & - & 73.45 & 73.63 \\
\hline 17 & 74.38 & 74.19 & 74.17 & 74.23 & 74.40 & 74.28 & 74.25 & - & 74.76 & - & 73.39 & 73.67 \\
\hline 18 & 74.40 & 74.24 & 74.19 & 74.25 & 74.40 & 74.30 & 74.26 & - & 74.72 & - & 73.37 & 73.56 \\
\hline 19 & 74.43 & 74.33 & 74.21 & 74.25 & 74.39 & 74.32 & 74.23 & - & 74.68 & - & 73.33 & 73.45 \\
\hline 20 & 74.48 & 74.37 & 74.19 & 74.26 & 74.41 & 74.29 & 74.22 & - & 74.63 & 73.69 & 73.39 & 73.52 \\
\hline 21 & 74.49 & 74.34 & 74.17 & 74.23 & 74.43 & 74.27 & 74.22 & - & - & 73.68 & 73.52 & 73.59 \\
\hline 22 & 74.45 & 74.34 & 74.16 & 74.21 & 74.39 & 74.25 & 74.17 & 74.34 & - & 73.62 & 73.60 & 73.67 \\
\hline 23 & 74.44 & 74.33 & 74.17 & 74.16 & 74.35 & - & 74.10 & 74.30 & 74.50 & 73.63 & 73.60 & 73.75 \\
\hline 24 & 74.44 & 74.31 & 74.19 & 74.21 & 74.40 & - & - & 74.28 & 74.43 & 73.65 & 73.56 & 73.74 \\
\hline 25 & 74.42 & 74.26 & 74.23 & 74.26 & 74.39 & - & 74.09 & 74.30 & - & 73.63 & 73.45 & 73.68 \\
\hline 26 & 74.30 & 74.26 & 74.22 & 74.24 & 74.36 & 74.32 & 74.20 & 74.34 & - & 73.61 & 73.49 & 73.67 \\
\hline 27 & 74.38 & 74.25 & 74.19 & 74.22 & 74.33 & 74.28 & 74.22 & 74.37 & - & 73.61 & 73.59 & 73.65 \\
\hline 28 & 74.39 & 74.24 & 74.20 & 74.16 & 74.32 & 74.28 & 74.23 & 74.38 & - & 73.57 & 73.66 & 73.53 \\
\hline 29 & 74.26 & - & 74.21 & 74.16 & 74.38 & 74.28 & 74.22 & 74.37 & - & 73.55 & 73.63 & 73.61 \\
\hline 30 & 74.20 & - & 74.21 & 74.25 & 74.35 & 74.29 & 74.22 & 74.33 & - & 73.54 & 73.57 & 73.74 \\
\hline 31 & 74.25 & - & 74.21 & - & 74.33 & - & 74.19 & 74.32 & - & 73.49 & - & 73.74 \\
\hline Mean & 74.42 & 74.27 & 74.18 & 74.23 & 74.34 & 74.31 & 74.24 & 74.31 & 74.71 & 73.66 & 73.52 & 73.63 \\
\hline Max & 74.61 & 74.37 & 74.23 & 74.31 & 74.45 & 74.40 & 74.31 & 74.38 & 74.94 & 73.94 & 73.66 & 73.75 \\
\hline Min & 74.20 & 74.15 & 74.09 & 74.16 & 74.20 & 74.25 & 74.09 & 74.18 & 74.35 & 73.49 & 73.33 & 73.45 \\
\hline
\end{tabular}




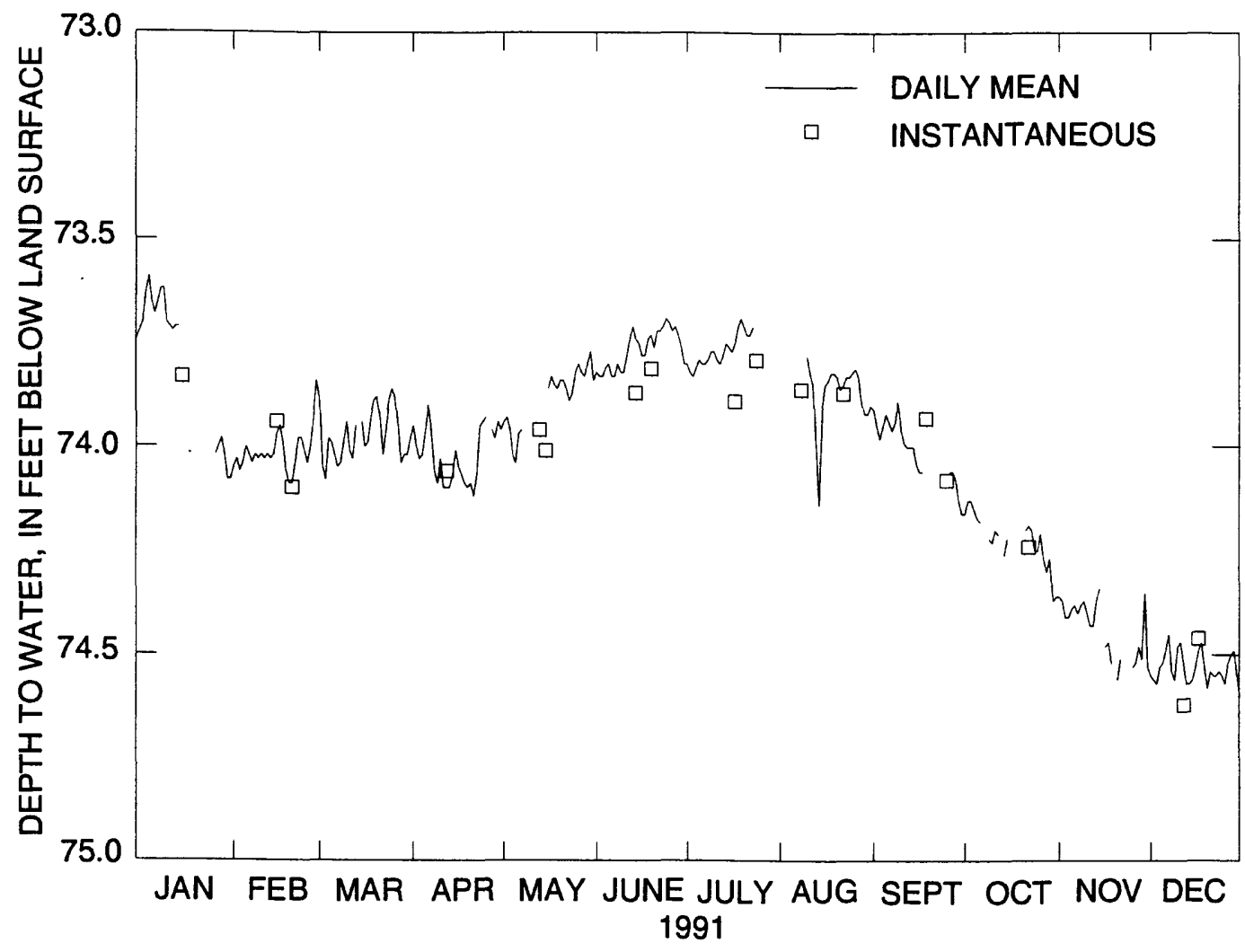

Figure 20. (E) Daily mean and instantaneous water levels, well CH-10B, Long Valley Caldera, Mono County, California, 1991. 
Table 33. Daily mean water levels, well $\mathrm{CH}-10 \mathrm{~B}$, Long Valley Caldera, Mono County, California, 1991

[Values are in feet below land surface. Max, maximum; Min, minimum; -, no data]

\begin{tabular}{|c|c|c|c|c|c|c|c|c|c|c|c|c|}
\hline Day & Jan & Feb & Mar & Apr & May & June & July & Aug & Sept & Oct & Nov & Dec \\
\hline 1 & 73.72 & 74.05 & 73.88 & 73.95 & 73.94 & 73.82 & 73.80 & - & 73.91 & 74.16 & 74.36 & 74.55 \\
\hline 2 & 73.70 & 74.03 & 74.05 & 74.00 & 73.93 & 73.83 & 73.82 & - & 73.95 & 74.13 & 74.37 & 74.56 \\
\hline 3 & 73.63 & 74.06 & 74.08 & 74.03 & 73.96 & 73.83 & 73.83 & - & 73.98 & 74.13 & 74.41 & 74.57 \\
\hline 4 & 73.59 & 74.04 & 73.98 & 74.02 & 74.02 & 73.81 & 73.81 & - & 73.95 & 74.15 & 74.41 & 74.53 \\
\hline 5 & 73.65 & 74.00 & 73.99 & 73.96 & 74.04 & 73.80 & 73.79 & - & 73.92 & 74.17 & 74.39 & 74.52 \\
\hline 6 & 73.68 & 74.02 & 74.02 & 73.90 & 73.97 & 73.83 & 73.80 & - & 73.94 & 74.18 & 74.38 & 74.49 \\
\hline 7 & 73.65 & 74.04 & 74.05 & 73.97 & 73.96 & 73.83 & 73.80 & - & 73.96 & - & 74.40 & 74.45 \\
\hline 8 & 73.62 & 74.02 & 74.04 & 74.06 & - & 73.80 & 73.79 & - & 73.94 & - & 74.38 & 74.54 \\
\hline 9 & 73.62 & 74.03 & 73.99 & 74.09 & - & 73.82 & 73.77 & - & 73.89 & 74.22 & 74.37 & 74.56 \\
\hline 10 & 73.70 & 74.02 & 73.94 & 74.03 & - & 73.82 & 73.77 & 73.78 & 73.96 & 74.23 & 74.40 & 74.48 \\
\hline 11 & 73.71 & 74.03 & 74.01 & 74.10 & - & 73.78 & 73.79 & 73.82 & 73.99 & 74.20 & 74.43 & 74.47 \\
\hline 12 & 73.72 & 74.02 & 74.03 & 74.10 & - & 73.74 & 73.80 & 73.85 & 74.00 & 74.21 & 74.43 & 74.52 \\
\hline 13 & 73.71 & 74.03 & 73.95 & 74.10 & - & 73.71 & 73.78 & 74.00 & 74.00 & - & 74.37 & 74.57 \\
\hline 14 & 73.71 & 74.02 & - & 74.07 & - & 73.74 & 73.75 & 74.14 & 74.00 & 74.26 & 74.34 & 74.57 \\
\hline 15 & - & 73.97 & 73.94 & 74.01 & - & 73.75 & 73.76 & 73.91 & 74.04 & 74.22 & - & 74.56 \\
\hline 16 & - & 73.95 & 74.00 & 74.05 & 73.86 & 73.78 & 73.77 & 73.85 & 74.06 & - & 74.48 & 74.53 \\
\hline 17 & - & 73.99 & 73.99 & 74.07 & 73.83 & 73.78 & 73.75 & 73.84 & 74.06 & - & 74.47 & 74.49 \\
\hline 18 & - & 74.06 & 73.93 & 74.09 & 73.85 & 73.74 & 73.71 & 73.82 & - & - & 74.52 & 74.47 \\
\hline 19 & - & 74.09 & 73.89 & 74.10 & 73.86 & 73.73 & 73.69 & 73.82 & - & - & - & 74.53 \\
\hline 20 & - & 74.09 & 73.88 & 74.09 & 73.84 & 73.76 & 73.71 & 73.83 & - & - & 74.56 & 74.58 \\
\hline 21 & - & 74.04 & 73.93 & 74.12 & 73.84 & 73.72 & 73.73 & 73.86 & - & 74.20 & 74.51 & 74.54 \\
\hline 22 & - & 73.98 & 74.02 & 74.07 & 73.86 & 73.72 & 73.73 & 73.85 & - & 74.19 & - & 74.55 \\
\hline 23 & - & 73.98 & 73.96 & 73.95 & 73.89 & 73.71 & 73.71 & 73.83 & - & 74.20 & 74.58 & 74.55 \\
\hline 24 & - & 74.01 & 73.89 & 73.94 & 73.87 & 73.69 & - & 73.83 & - & 74.25 & - & 74.54 \\
\hline 25 & - & 74.04 & 73.86 & 73.93 & 73.82 & 73.70 & - & 73.82 & - & 74.25 & 74.53 & 74.55 \\
\hline 26 & 74.02 & 74.00 & 73.88 & - & 73.80 & 73.72 & - & 73.81 & 74.06 & 74.21 & 74.52 & 74.57 \\
\hline 27 & 74.00 & 73.93 & 73.95 & 73.96 & 73.82 & 73.71 & - & 73.83 & 74.06 & 74.27 & 74.48 & 74.52 \\
\hline 28 & 73.98 & 73.84 & 74.04 & 73.98 & 73.83 & 73.73 & - & 73.89 & 74.08 & 74.30 & 74.51 & 74.50 \\
\hline 29 & 74.02 & - & 74.02 & 73.94 & 73.80 & 73.76 & - & 73.92 & 74.13 & 74.27 & 74.35 & 74.49 \\
\hline 30 & 74.08 & - & 74.02 & 73.96 & 73.77 & 73.80 & - & 73.92 & 74.16 & 74.37 & 74.53 & 74.55 \\
\hline 31 & 74.08 & - & 73.98 & - & 73.84 & - & - & 73.90 & - & 74.36 & - & 74.60 \\
\hline Mean & 73.78 & 74.01 & 73.97 & 74.02 & 73.88 & 73.77 & 73.77 & 73.87 & 74.00 & 74.22 & 74.44 & 74.53 \\
\hline Max & 74.08 & 74.09 & 74.08 & 74.12 & 74.04 & 73.83 & 73.83 & 74.14 & 74.16 & 74.37 & 74.58 & 74.60 \\
\hline Min & 73.59 & 73.84 & 73.86 & 73.90 & 73.77 & 73.69 & 73.69 & 73.78 & 73.89 & 74.13 & 74.34 & 74.45 \\
\hline
\end{tabular}




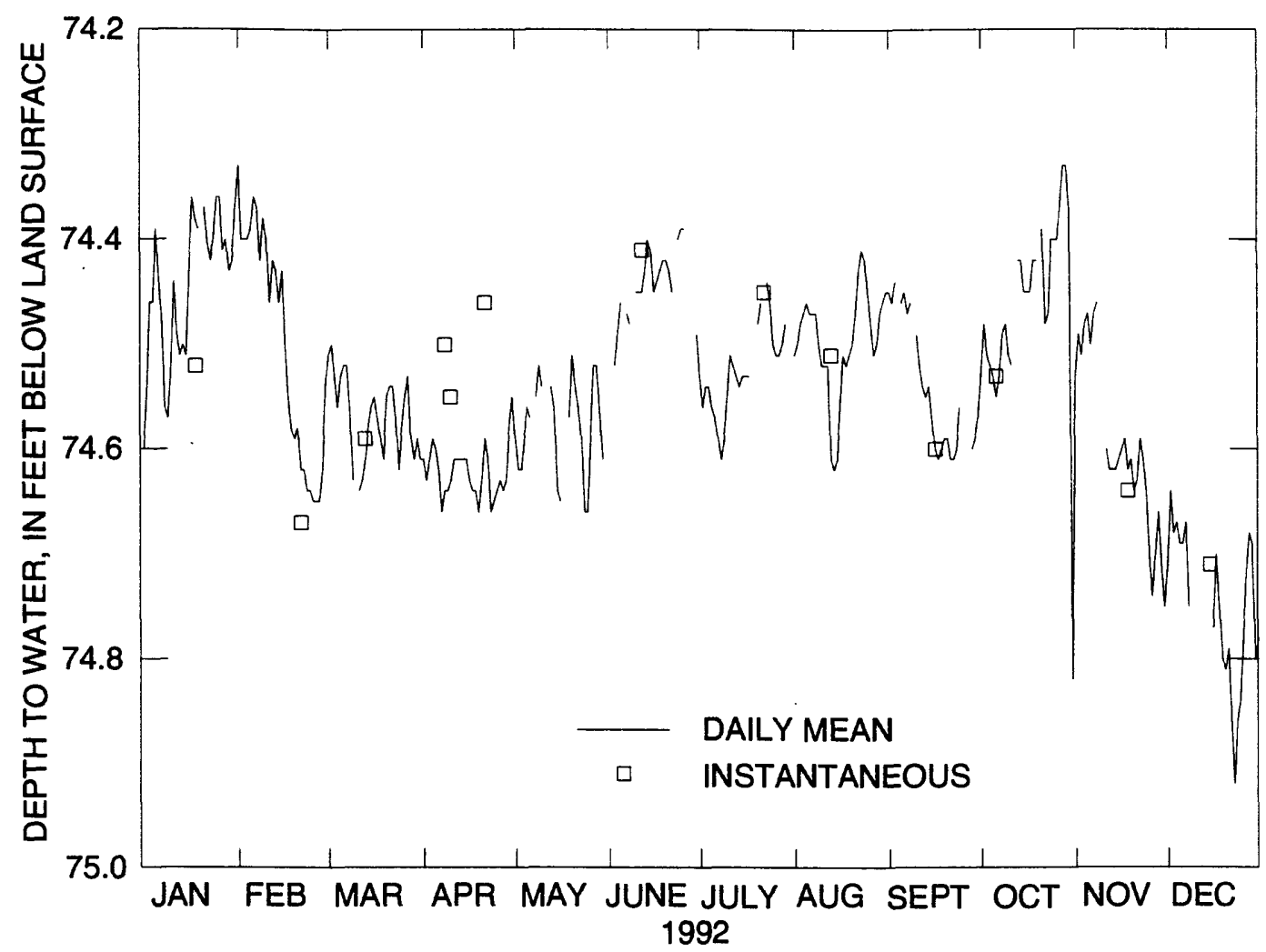

Figure 20. (F) Daily mean and instantaneous water levels, well CH-10B, Long Valley Caldera, Mono County, California, 1992. 
Table 34. Daily mean water levels, well CH-10B, Long Valley Caldera, Mono County, California, 1992

[Values are in feet below land surface. Max, maximum: Min. minimum; -, no data]

\begin{tabular}{|c|c|c|c|c|c|c|c|c|c|c|c|c|}
\hline Day & Jan & Feb & Mar & Apr & May & June & July & Aug & Sept & Oct & Nov & Dec \\
\hline 1 & 74.60 & 74.33 & 74.51 & 74.61 & 74.59 & 74.55 & 74.53 & 74.51 & 74.45 & 74.53 & 74.53 & 74.71 \\
\hline 2 & 74.55 & 74.40 & 74.50 & 74.63 & 74.62 & - & 74.56 & 74.50 & 74.46 & 74.48 & 74.49 & 74.64 \\
\hline 3 & 74.46 & 74.40 & 74.53 & 74.61 & 74.62 & 74.52 & 74.54 & 74.48 & 74.44 & 74.51 & 74.51 & 74.68 \\
\hline 4 & 74.46 & 74.40 & 74.56 & 74.59 & 74.59 & 74.49 & 74.54 & 74.47 & - & 74.52 & 74.48 & 74.67 \\
\hline 5 & 74.39 & 74.39 & 74.53 & 74.60 & 74.56 & 74.46 & 74.56 & 74.46 & 74.46 & 74.53 & 74.47 & 74.69 \\
\hline 6 & 74.44 & 74.36 & 74.52 & 74.62 & 74.57 & - & 74.57 & 74.47 & 74.45 & 74.55 & 74.50 & 74.69 \\
\hline 7 & 74.48 & 74.37 & 74.52 & 74.66 & - & 74.47 & 74.59 & 74.47 & 74.47 & 74.53 & 74.47 & 74.67 \\
\hline 8 & 74.56 & 74.42 & 74.56 & 74.64 & 74.55 & 74.48 & 74.61 & 74.47 & 74.46 & 74.49 & 74.46 & 74.75 \\
\hline 9 & 74.57 & 74.38 & 74.63 & 74.64 & 74.52 & - & 74.59 & 74.50 & - & 74.48 & - & - \\
\hline 10 & 74.52 & 74.40 & - & 74.63 & 74.54 & 74.45 & 74.55 & 74.52 & 74.49 & 74.51 & - & 74.74 \\
\hline 11 & 74.44 & 74.46 & 74.64 & 74.61 & - & 74.45 & 74.51 & 74.52 & 74.52 & 74.52 & 74.60 & - \\
\hline 12 & 74.49 & 74.42 & 74.63 & 74.61 & - & 74.45 & 74.52 & 74.52 & 74.54 & - & 74.62 & 74.74 \\
\hline 13 & 74.51 & 74.43 & 74.61 & 74.61 & 74.54 & 74.43 & 74.53 & 74.61 & 74.55 & 74.42 & 74.62 & - \\
\hline 14 & 74.50 & 74.46 & 74.58 & 74.61 & 74.56 & 74.40 & 74.54 & 74.62 & 74.54 & 74.42 & 74.62 & 74.75 \\
\hline 15 & 74.51 & 74.43 & 74.56 & 74.61 & 74.64 & 74.41 & 74.53 & 74.61 & 74.57 & 74.45 & 74.61 & - \\
\hline 16 & 74.43 & 74.50 & 74.5 & 74.63 & 74.65 & 74.45 & 74.53 & 74.56 & 74.60 & 74.45 & 74.60 & 74.77 \\
\hline 17 & 74.36 & 74.55 & 74.57 & 74.64 & - & 74.44 & 74.53 & 74.51 & 74.61 & 74.45 & 74.59 & 74.70 \\
\hline 18 & 74.38 & 74.58 & 74.59 & 74.64 & - & 74.43 & - & 74.52 & 74.60 & 74.42 & 74.62 & 74.75 \\
\hline 19 & 74.39 & 74.59 & 74.61 & 74.66 & 74.57 & 74.42 & - & 74.51 & 74.59 & 74.42 & 74.61 & 74.80 \\
\hline 20 & - & 74.58 & 74.55 & 74.63 & 74.51 & 74.42 & 74.48 & 74.50 & 74.59 & - & 74.64 & 74.81 \\
\hline 21 & 74.37 & 74.62 & 74.54 & 74. & 74. & 74.43 & 74.46 & 74.47 & 74 & 74.39 & 74.63 & 74.79 \\
\hline 22 & 74.40 & 74.62 & 74.54 & 74.61 & 74.56 & 74.45 & - & 74.43 & 74.61 & 74.48 & 74.59 & 74.86 \\
\hline 23 & 74.42 & 74.64 & 74.57 & 74.66 & 74.59 & - & 74.44 & 74.41 & 50 & 74.47 & 74.61 & 74.92 \\
\hline 24 & 74.40 & 74.64 & 74.62 & 74.65 & 74.66 & 74.40 & 74.46 & 74.42 & 74.56 & 74.40 & 74.64 & $74.8 \mathrm{C}$ \\
\hline 25 & 74.36 & 74.65 & 74.58 & 74.64 & 74.66 & 74.39 & 74.50 & 74.45 & - & 74.40 & 74.70 & 74.8 \\
\hline 26 & 74.36 & 74.65 & 74.55 & 74. & 74. & 74.39 & 74.51 & 74.48 & 74.62 & & 74 & 74.78 \\
\hline 27 & 74.41 & 74.65 & 74.53 & 74.64 & 74.52 & - & 74.51 & 74.51 & - & 74 & 74.70 & 74.72 \\
\hline 28 & 74.40 & 74.62 & 74.59 & 74.63 & 74.52 & - & 74.50 & 74.50 & 74.60 & 74.33 & 74.66 & 74.68 \\
\hline 29 & 74.43 & 74.54 & 74.61 & 74.58 & 74.56 & - & 74.48 & 74.47 & 74.59 & 74.33 & 74.71 & 74.69 \\
\hline 30 & 74.42 & - & 74.59 & 74.55 & 74.61 & 74.49 & - & 74.46 & 74.57 & 74.37 & 74.75 & 74.80 \\
\hline 31 & 74.37 & - & 74.61 & - & - & - & - & 74.45 & - & 74.82 & - & 74.78 \\
\hline Mean & 74.45 & 74.50 & 74.57 & 74.62 & 74 & 74.45 & 74.53 & 74.50 & 74 & 74. & 74.60 & 74.75 \\
\hline $\operatorname{Max}$ & 74.60 & 74.65 & 74.64 & 74.66 & 74.66 & 74.55 & 74.61 & 74.62 & 74.62 & 74.82 & 74.75 & 74.92 \\
\hline Min & 74.36 & 74.33 & 74.50 & 74.55 & 74.51 & 74.39 & 74.44 & 74.41 & 74.44 & 74.33 & 74.46 & 74.64 \\
\hline
\end{tabular}




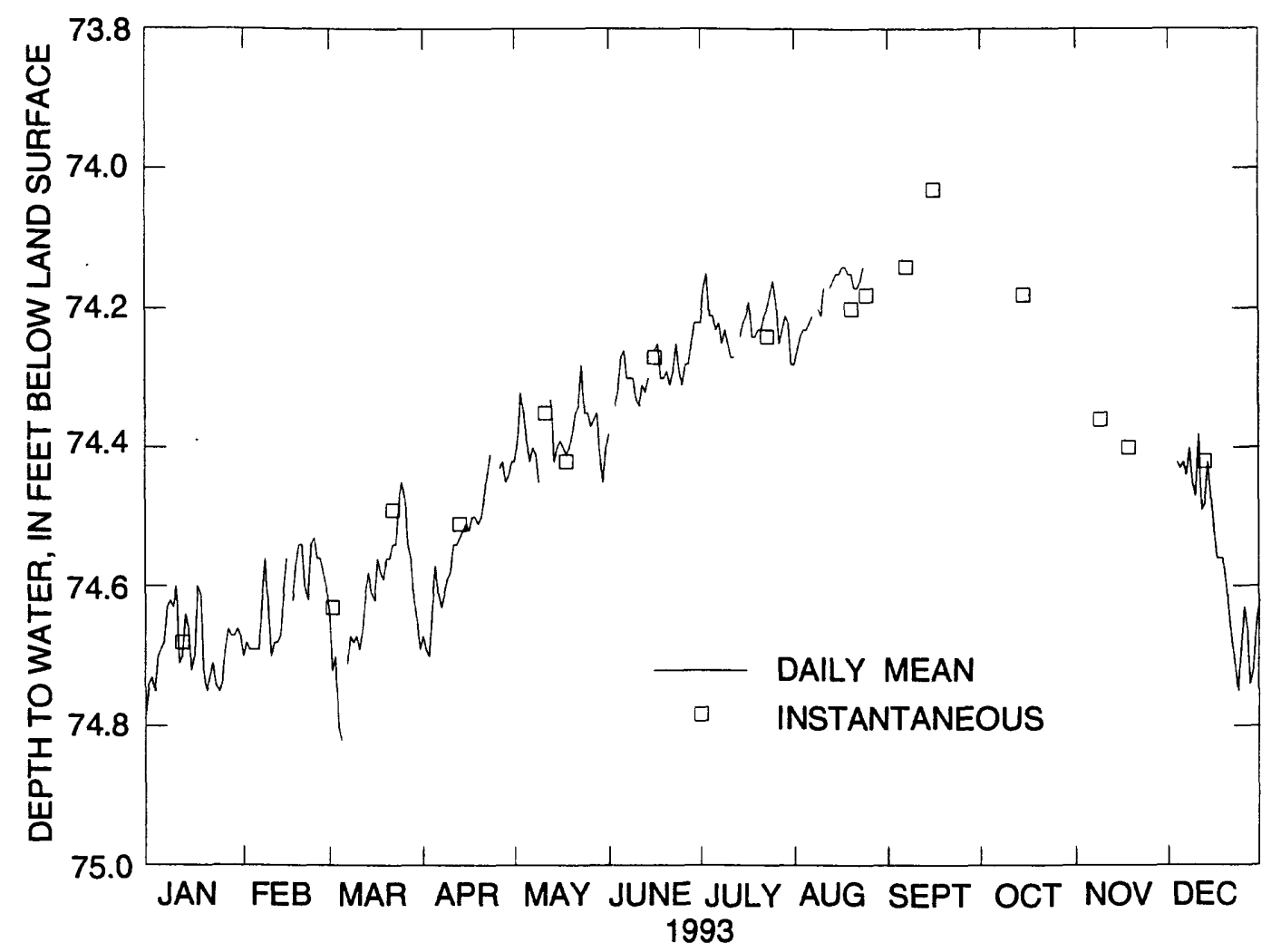

Figure 20. (G) Daily mean and instantaneous water levels, well CH-10B, Long Valley Caldera, Mono County, California, 1993. 
Table 35. Daily mean water levels, well CH-10B, Long Valley Caldera, Mono County, California, 1993

[Values are in feet below land surface. Max, maximum; Min, minimum; -, no data]

\begin{tabular}{|c|c|c|c|c|c|c|c|c|c|c|c|c|}
\hline Day & Jan & Feb & Mar & Apr & May & June & July & Aug & Sept & Oct & Nov & Dec \\
\hline 1 & 74.74 & 74.70 & 74.64 & 74.67 & 74.42 & 74.38 & 74.22 & 74.28 & - & - & - & - \\
\hline 2 & 74.73 & 74.68 & 74.72 & 74.69 & 74.39 & - & 74.17 & 74.26 & - & - & - & - \\
\hline 3 & 74.75 & 74.69 & 74.70 & 74.70 & 74.32 & 74.34 & 74.15 & 74.24 & - & - & - & - \\
\hline 4 & 74.70 & 74.69 & 74.80 & 74.63 & 74.35 & 74.32 & 74.21 & 74.23 & - & - & - & 74.41 \\
\hline 5 & 74.69 & 74.69 & 74.82 & 74.57 & 74.39 & 74.27 & 74.21 & 74.23 & - & - & - & 74.41 \\
\hline 6 & 74.68 & 74.69 & - & 74.61 & 74.42 & 74.26 & 74.23 & 74.22 & - & - & - & 74.40 \\
\hline 7 & 74.63 & 74.62 & 74.71 & 74.63 & 74.40 & 74.30 & 74.22 & 74.21 & - & - & - & 74.42 \\
\hline 8 & 74.62 & 74.56 & 74.67 & 74.61 & 74.41 & 74.30 & 74.25 & - & - & - & - & 74.38 \\
\hline 9 & 74.63 & 74.63 & 74.68 & 74.59 & 74.45 & 74.30 & 74.23 & 74.20 & - & - & - & 74.43 \\
\hline 10 & 74.60 & 74.70 & 74.67 & 74.58 & - & 74.33 & 74.25 & 74.21 & - & - & - & 74.44 \\
\hline 11 & 74.71 & 74.68 & 74.69 & 74.54 & - & 74.34 & 74.27 & 74.17 & - & - & - & 74.35 \\
\hline 12 & 74.70 & 74.68 & 74.66 & 74.54 & - & 74.31 & 74.27 & - & - & - & - & 74.46 \\
\hline 13 & 74.64 & 74.67 & 74.61 & 74.53 & 74.33 & 74.32 & 一 & 74.17 & - & - & - & 74.45 \\
\hline 14 & 74.66 & 74.61 & 74.58 & 74.52 & 74.42 & 74.30 & 74.24 & 74.16 & - & - & - & 74.39 \\
\hline 15 & 74.72 & 74.56 & 74.61 & 74.51 & 74.40 & 一 & 74.22 & 74.15 & - & - & - & 74.44 \\
\hline 16 & 74.70 & - & 74.62 & 74.52 & 74.39 & 74.26 & 74.21 & 74.15 & - & - & - & 74.49 \\
\hline 17 & 74.60 & 74.62 & 74.56 & 74.50 & 74.40 & 74.25 & 74.19 & 74.14 & - & - & - & 74.53 \\
\hline 18 & 74.61 & 74.57 & 74.58 & 74.50 & 74.41 & 74.30 & 74.24 & 74.14 & - & - & - & 74.53 \\
\hline 19 & 74.72 & 74.54 & 74.59 & 74.51 & 74.40 & 74.30 & 74.24 & 74.15 & - & - & - & 74.53 \\
\hline 20 & 74.75 & 74.54 & 74.56 & 74.50 & 74.38 & 74.29 & 74.23 & 74.15 & - & - & - & 74.56 \\
\hline 21 & 74.73 & 74.60 & 74.56 & 74.47 & 74.35 & 74.31 & 74.23 & 74.17 & - & - & - & 74.61 \\
\hline 22 & 74.71 & 74.62 & 74.54 & 74.44 & 74.34 & 74.29 & 74.21 & 74.17 & - & - & - & 74.65 \\
\hline 23 & 74.74 & 74.54 & 74.54 & 74.41 & 74.28 & 74.25 & 74.20 & 74.16 & - & - & - & 74.68 \\
\hline 24 & 74.75 & 74.53 & 74.48 & - & 74.35 & 74.29 & 74.18 & 74.14 & - & - & - & 74.72 \\
\hline 25 & 74.74 & 74.56 & 74.45 & - & 74.35 & 74.31 & 74.16 & - & - & - & - & 74.66 \\
\hline 26 & 74.69 & 74.56 & 74.47 & 74.43 & 74.37 & 74.28 & 74.19 & - & - & - & - & 74.60 \\
\hline 27 & 74.66 & 74.58 & 74.54 & 74.42 & 74.36 & 74.28 & 74.25 & - & - & - & - & 74.63 \\
\hline 28 & 74.67 & 74.60 & 74.56 & 74.45 & 74.35 & 74.25 & 74.23 & - & - & - & - & 74.71 \\
\hline 29 & 74.67 & - & 74.62 & 74.44 & 74.41 & 74.22 & 74.21 & - & - & - & - & 74.69 \\
\hline 30 & 74.66 & - & 74.65 & 74.42 & 74.45 & 74.22 & 74.22 & - & - & - & - & 74.62 \\
\hline 31 & 74.67 & - & 74.69 & - & 74.40 & - & 74.28 & - & - & - & - & 74.60 \\
\hline Mean & 74.69 & 74.62 & 74.62 & 74.53 & 74.38 & 74.29 & 74.22 & 74.19 & - & - & - & 74.53 \\
\hline Max & 74.75 & 74.70 & 74.82 & 74.70 & 74.45 & 74.38 & 74.28 & 74.28 & - & - & - & 74.72 \\
\hline Min & 74.60 & 74.53 & 74.45 & 74.41 & 74.28 & 74.22 & 74.15 & 74.14 & - & - & - & 74.35 \\
\hline
\end{tabular}


Table 36. Ground-water levels in selected wells, Long Valley Caldera, Mono County, California, 1987-93

[Water levels are in feet below land-surface datum. Readings above land surface indicated by "+". M, method of measurement: $S$ is steel tape, $V$ is calibrated electric sounder. $W$, status of well: $P$ is pumping; $R$ is recently pumped; $\mathrm{D}$ is dry; $\mathrm{O}$ is obstruction; $\mathrm{N}$ is nearby pumping; $\mathrm{T}$ is nearby recently pumped]

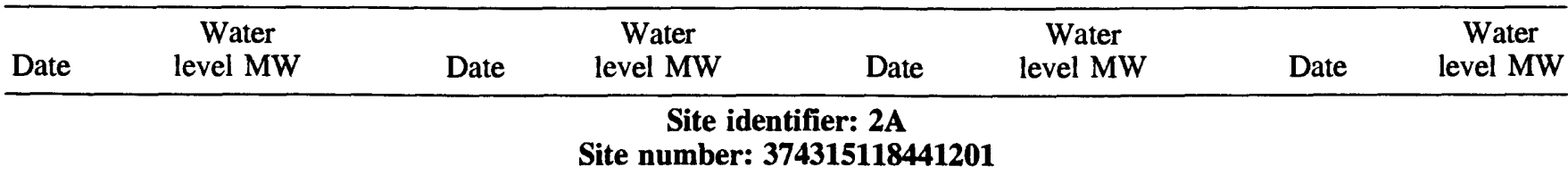

About 13.7 miles northeast of Mammoth Lakes. Drilled livestock well. Diameter 8 inches, depth 32 feet. Altitude of land-surface datum 6,922 feet. Water-level records available 1982-94.

\begin{tabular}{|c|c|c|c|c|c|c|c|c|c|}
\hline $05-30-87$ & \multicolumn{2}{|c|}{$5.59 \mathrm{~S}$} & $11-29-88$ & $3.80 \mathrm{~S}$ & & $11-14-90$ & $4.11 \mathrm{~S}$ & $07-23-92$ & $4.36 \mathrm{~S}$ \\
\hline $08-28-87$ & \multicolumn{2}{|c|}{$3.88 \mathrm{~S}$} & $04-26-89$ & $3.65 \mathrm{~S}$ & & $04-12-91$ & $3.55 \mathrm{~S}$ & $12-03-92$ & $4.16 \mathrm{~S}$ \\
\hline $11-14-87$ & \multicolumn{2}{|c|}{$3.64 \mathrm{~S}$} & $11-28-89$ & $3.95 \mathrm{~S}$ & & $07-17-91$ & $4.19 \mathrm{~S}$ & $05-17-93$ & $3.20 \mathrm{~S}$ \\
\hline $04-21-88$ & \multicolumn{2}{|c|}{$3.39 \mathrm{~S}$} & $04-23-90$ & $3.70 \mathrm{~S}$ & & $11-20-91$ & $4.13 \mathrm{~S}$ & $07-20-93$ & $2.64 \mathrm{SP}$ \\
\hline $07-27-88$ & \multicolumn{2}{|c|}{$4.51 \mathrm{~S}$} & $07-17-90$ & $4.27 \mathrm{~S}$ & & $04-08-92$ & $4.27 \mathrm{~S}$ & $11-10-93$ & $3.93 \mathrm{~S}$ \\
\hline Highest & 2.64 & $07-20-93$ & & & & & & & \\
\hline Lowest & 5.59 & $05-30-87$ & & & & & & & \\
\hline \multicolumn{2}{|c|}{$\begin{array}{l}\text { Period of record } \\
\text { Record begins }\end{array}$} & $\begin{array}{l}\text { Highest } \\
05-26-82\end{array}$ & $\begin{array}{l}2.64 \quad 07-20-93 \\
30 \text { Entries }\end{array}$ & Lowest & 5.59 & $05-30-87$ & & & \\
\hline
\end{tabular}

Site identifier: 27R1

Site number: $\mathbf{3 7 4 4 2 0 1 1 8 5 1 5 5 0 1}$

About 8.3 miles northeast of Mammoth Lakes and 2.6 miles southeast of Arcularius Ranch. Drilled livestock well. Diameter 6 inches, depth 44.3 feet. Altitude of land-surface datum 7,039.18 feet. Water-level records available 1982-94.

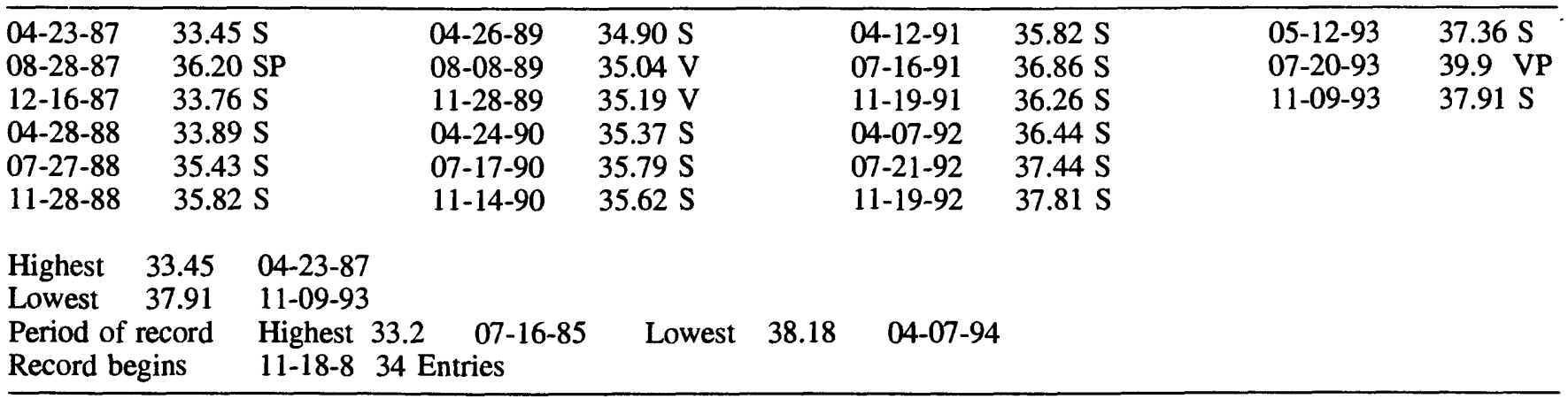

\section{Site identifier: $28 \mathrm{P1}$ \\ Site number: $\mathbf{3 7 4 4 2 0 1 1 8 5 3 2 5 0 1}$}

About 7.5 miles northeast of Mammoth Lakes and 1.4 miles southeast of Arcularius Ranch. Drilled livestock well. Diameter 6 inches, depth 125 feet. Altitude of land-surface datum 7,109.02 feet. Water-level records available 1983-94.

\begin{tabular}{llllllll}
\hline $04-23-87$ & $78.17 \mathrm{~S}$ & $04-26-89$ & $82.23 \mathrm{~V}$ & $04-12-91$ & $86.24 \mathrm{~V}$ & $05-12-93$ & $87.66 \mathrm{~V}$ \\
$08-28-87$ & $81.44 \mathrm{~S}$ & $08-08-89$ & $84.73 \mathrm{~V}$ & $07-16-91$ & $87.34 \mathrm{~V}$ & $07-20-93$ & $88.30 \mathrm{~V}$ \\
$12-16-87$ & $79.63 \mathrm{~S}$ & $11-28-89$ & $84.75 \mathrm{~V}$ & $11-19-91$ & $86.76 \mathrm{~V}$ & $11-09-93$ & $88.37 \mathrm{~V}$ \\
$04-28-88$ & $79.95 \mathrm{~V}$ & $04-25-90$ & $84.99 \mathrm{~V}$ & $04-07-92$ & $87.20 \mathrm{~S}$ & & \\
$07-27-88$ & $83.66 \mathrm{~S}$ & $07-17-90$ & $85.55 \mathrm{~V}$ & $07-21-92$ & $87.63 \mathrm{~V}$ & & \\
$11-28-88$ & $82.53 \mathrm{~S}$ & $11-14-90$ & $86.21 \mathrm{~V}$ & $11-19-92$ & $87.96 \mathrm{~V}$ & &
\end{tabular}

Highest $\quad 78.17 \quad 04-23-87$

Lowest $88.37 \quad 11-09-93$

Period of record Highest 75.28 $07-25-84 \quad$ Lowest $116.55 \quad 06-03-83$

Record begins 06-03-83 32 Entries 
Table 36. Ground-water levels in selected wells, Long Valley Caldera, Mono County, California, 1987-93-Continued

\begin{tabular}{|c|c|c|c|c|c|c|c|}
\hline Date & $\begin{array}{c}\text { Water } \\
\text { level MW }\end{array}$ & Date & $\begin{array}{c}\text { Water } \\
\text { level MW }\end{array}$ & Date & $\begin{array}{c}\text { Water } \\
\text { level MW }\end{array}$ & Date & $\begin{array}{c}\text { Water } \\
\text { level MW }\end{array}$ \\
\hline
\end{tabular}

Site identifier: 30N2

Site number: 374420118493301

About 7.8 miles northeast of Mammoth Lakes and 4.8 miles southeast of Arcularius Ranch. Drilled observation well. Diameter 8 inches, depth measured 31.8 feet in 1992. Altitude of land-surface datum 6,913.21 feet. Water-level records available 1972, 1982-94. Measurements from August 1989 through 1992 water year published as 003S029E19R001M.

\begin{tabular}{|c|c|c|c|c|c|c|c|c|c|}
\hline $04-23-87$ & $6.99 \mathrm{~s}$ & & $04-26-89$ & $8.20 \mathrm{~S}$ & & $04-12-91$ & $8.52 \mathrm{~S}$ & $05-12-93$ & $4.94 \mathrm{~S}$ \\
\hline $08-28-87$ & $8.55 \mathrm{~S}$ & & $08-08-89$ & $9.15 \mathrm{~S}$ & & $07-16-91$ & $8.65 \mathrm{~S}$ & $07-20-93$ & $7.44 \mathrm{~S}$ \\
\hline $12-16-87$ & $8.45 \mathrm{~S}$ & & $11-28-89$ & $9.12 \mathrm{~S}$ & & $11-19-91$ & $9.04 \mathrm{~S}$ & $11-09-93$ & $9.12 \mathrm{~S}$ \\
\hline $04-28-88$ & $7.63 \mathrm{~S}$ & & $04-24-90$ & $8.49 \mathrm{~S}$ & & $04-07-92$ & $8.77 \mathrm{~S}$ & & \\
\hline $07-27-88$ & $8.65 \mathrm{~S}$ & & $07-17-90$ & $9.00 \mathrm{~S}$ & & $07-21-92$ & $9.09 \mathrm{~S}$ & & \\
\hline $11-28-88$ & $8.82 \mathrm{~S}$ & & $11-14-90$ & $9.44 \mathrm{~S}$ & & $11-19-92$ & $9.15 \mathrm{~S}$ & & \\
\hline Highest & 4.94 & $05-12-93$ & & & & & & & \\
\hline Lowest & 9.44 & $11-14-90$ & & & & \multirow{3}{*}{\multicolumn{2}{|c|}{$11-14-90$}} & & \\
\hline \multirow{2}{*}{\multicolumn{2}{|c|}{$\begin{array}{l}\text { Period of record } \\
\text { Record begins }\end{array}$}} & Highest & $2.95 \quad 05-10-86$ & Lowest & 9.44 & & & & \\
\hline & & $05-11-72$ & 36 Entries & & & & & & \\
\hline
\end{tabular}

\section{Site identifier: 31P1}

Site number: 374332118491001

About 9.4 miles northeast of Mammoth Lakes and 5.4 miles southeast of Arcularius Ranch. Unused water-table well in alluvium of Quaternary age. Diameter 30 inches, depth 7.6 feet. Altitude of land-surface datum 6,914.01 feet. Water-level records available 1966, 1972-73, 1978-94.

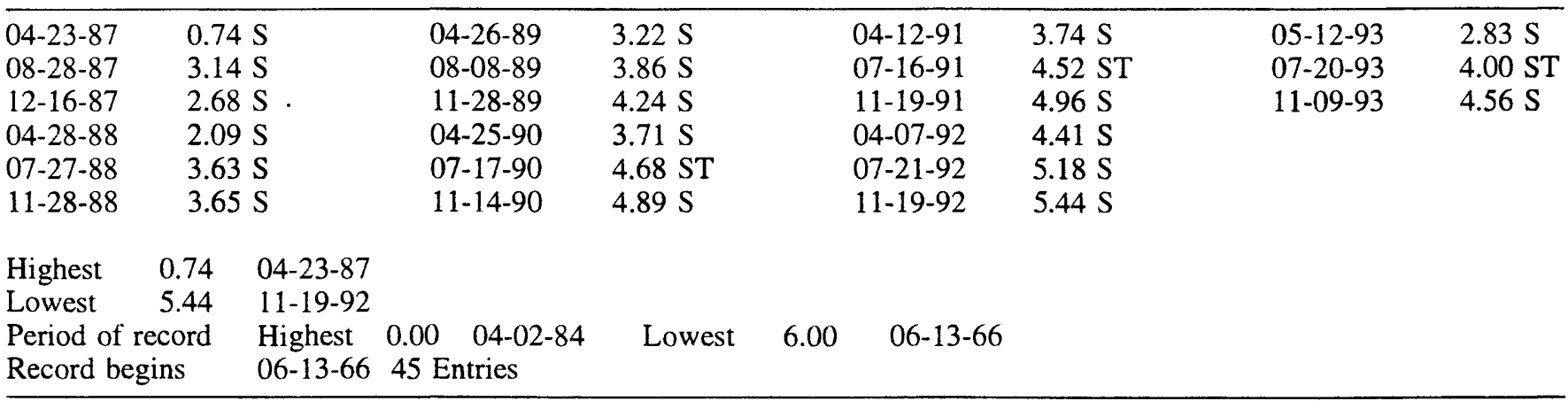

\section{Site identifier: 31P2}

Site number: 374332118491002

About 9.4 miles northeast of Mammoth Lakes and 5.4 miles southeast of Arcularius Ranch. Livestock well. Diameter 4 inches, depth unknown. Altitude of land-surface datum 6,915.7 feet. Water-level records available 1988-94.

\begin{tabular}{llllllll}
\hline $04-28-88$ & $2.09 \mathrm{~S}$ & $11-28-89$ & $4.10 \mathrm{~S}$ & $04-12-91$ & $3.62 \mathrm{~S}$ & $11-19-92$ & $5.33 \mathrm{~S}$ \\
$07-27-88$ & $3.41 \mathrm{~S}$ & $04-25-90$ & $3.58 \mathrm{~S}$ & $11-19-91$ & $4.83 \mathrm{~S}$ & $07-20-93$ & $9.70 \mathrm{SR}$ \\
$11-28-88$ & $3.71 \mathrm{~S}$ & $07-17-90$ & $4.63 \mathrm{SR}$ & $04-07-92$ & $4.30 \mathrm{~S}$ & $11-09-93$ & $4.44 \mathrm{~S}$ \\
$04-26-89$ & $3.17 \mathrm{~S}$ & $11-14-90$ & $4.76 \mathrm{~S}$ & $07-21-92$ & $5.05 \mathrm{~S}$ & &
\end{tabular}

$\begin{array}{lrlllll}\text { Highest } & 2.09 & 04-28-88 & & & & \\ \text { Lowest } & 9.70 & 07-20-93 & & & & \\ \text { Period of record } & \text { Highest } & 2.09 & 04-28-88 & \text { Lowest } & 9.70 & 07-20-93 \\ \text { Record begins } & 04-28-88 & 16 \text { Entries } & & & \end{array}$


Table 36. Ground-water levels in selected wells, Long Valley Caldera, Mono County, California, 1987-93-Continued

\begin{tabular}{|c|c|c|c|c|c|c|c|}
\hline Date & $\begin{array}{c}\text { Water } \\
\text { level MW }\end{array}$ & Date & $\begin{array}{c}\text { Water } \\
\text { level MW }\end{array}$ & Date & $\begin{array}{c}\text { Water } \\
\text { level MW }\end{array}$ & Date & $\begin{array}{c}\text { Water } \\
\text { level MW }\end{array}$ \\
\hline
\end{tabular}

Site identifier: $35 \mathrm{~N} 1$

Site number: $\mathbf{3 7 4 3 2 7 1 1 8 4 4 5 9 0 1}$

About 13 miles northeast of Mammoth Lakes. Drilled livestock well. Diameter unknown, depth 75 feet. Altitude of land-surface datum 6,914 feet. Water-level records available 1972, 1984-94.

\begin{tabular}{|c|c|c|c|c|c|c|c|c|c|}
\hline $05-30-87$ & \multicolumn{2}{|c|}{$1.77 \mathrm{~S}$} & $11-29-88$ & $3.03 \mathrm{~S}$ & & $11-14-90$ & $3.43 \mathrm{~S}$ & $07-23-92$ & $3.70 \mathrm{~S}$ \\
\hline $08-28-87$ & \multicolumn{2}{|c|}{$4.28 \mathrm{~S}$} & $04-26-89$ & $2.27 \mathrm{~S}$ & & $04-12-91$ & $1.12 \mathrm{~S}$ & $12-03-92$ & $3.05 \mathrm{~S}$ \\
\hline $11-14-87$ & \multicolumn{2}{|c|}{$2.61 \mathrm{~S}$} & $11-28-89$ & $3.03 \mathrm{~S}$ & & $07-17-91$ & $3.43 \mathrm{~S}$ & $05-17-93$ & $1.25 \mathrm{~S}$ \\
\hline $04-21-88$ & \multicolumn{2}{|c|}{$1.61 \mathrm{~S}$} & $04-23-90$ & $1.81 \mathrm{~S}$ & & $11-20-91$ & $3.22 \mathrm{~S}$ & $07-20-93$ & $2.95 \mathrm{~S}$ \\
\hline $07-27-88$ & \multicolumn{2}{|c|}{$4.17 \mathrm{~S}$} & $07-17-90$ & $3.81 \mathrm{~S}$ & & 04-08-92 & $1.80 \mathrm{~S}$ & $11-10-93$ & $2.70 \mathrm{~S}$ \\
\hline Highest & 1.12 & $04-12-91$ & & & & & & & \\
\hline Lowest & 4.28 & $08-28-87$ & & & & & & & \\
\hline \multicolumn{2}{|c|}{$\begin{array}{l}\text { Period of record } \\
\text { Record begins }\end{array}$} & $\begin{array}{l}\text { Highest } \\
05-10-72\end{array}$ & $\begin{array}{l}1.05 \quad 07-25-84 \\
30 \text { Entries }\end{array}$ & Lowest & 4.39 & $07-17-$ & & & \\
\hline
\end{tabular}

Site identifier: 36P1

Site number: 374335118434101

About 14.2 miles northeast of Mammoth Lakes. Unused livestock well. Diameter unknown, depth measured 72 feet in 1992. Altitude of land-surface datum 6,995 feet. Water-level records available 1986-94.

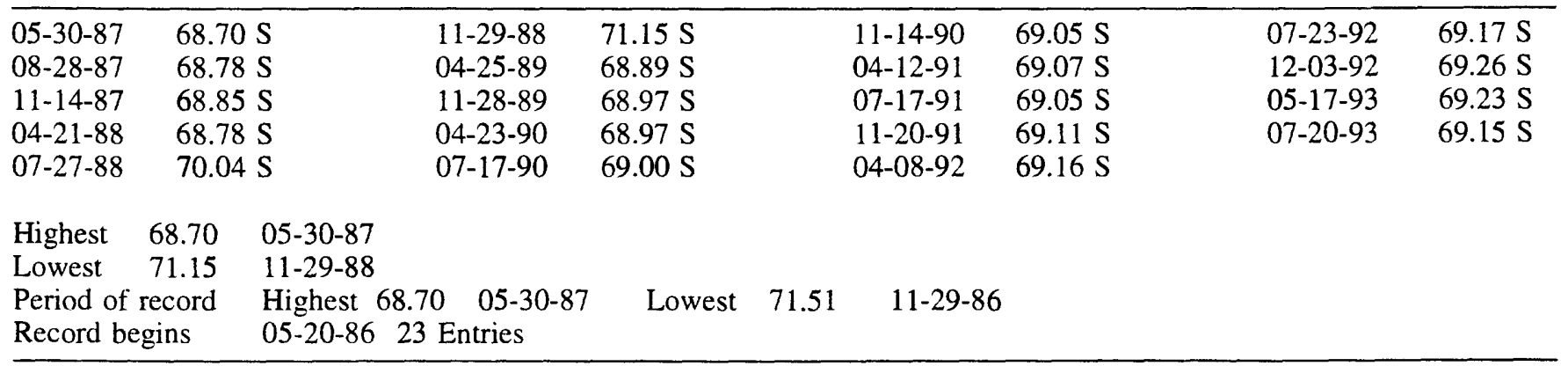

Site identifier: AP

Site number: 373737118501701

About 7 miles southeast of Mammoth Lakes at the airport. Drilled public-supply well. Diameter 10 inches, depth 70 feet, perforated 52 to 66 feet. Altitude of land-surface datum 7,120 feet. Water-level records available 1984-94.

\begin{tabular}{llllllll}
\hline $04-21-87$ & $27.10 \mathrm{~S}$ & $11-28-88$ & $28.34 \mathrm{~S}$ & $11-15-90$ & $29.25 \mathrm{SP}$ & $11-19-92$ & $30.10 \mathrm{SP}$ \\
$08-30-87$ & $25.88 \mathrm{~S}$ & $04-25-89$ & $28.97 \mathrm{~S}$ & $04-10-91$ & $29.84 \mathrm{SP}$ & $05-11-93$ & $27.48 \mathrm{SP}$ \\
$12-18-87$ & $27.66 \mathrm{~S}$ & $08-09-89$ & $28.40 \mathrm{SP}$ & $07-17-91$ & $28.08 \mathrm{~S}$ & $07-23-93$ & $26.40 \mathrm{~S}$ \\
$03-02-88$ & $28.89 \mathrm{~S}$ & $11-29-89$ & $29.02 \mathrm{SP}$ & $11-21-91$ & $29.02 \mathrm{~S}$ & $11-10-93$ & $27.22 \mathrm{~S}$ \\
$04-04-88$ & $28.90 \mathrm{~S}$ & $04-24-90$ & $29.54 \mathrm{SP}$ & $04-08-92$ & $29.91 \mathrm{SR}$ & & \\
$07-27-88$ & $26.89 \mathrm{~S}$ & $07-16-90$ & $29.24 \mathrm{~S}$ & $07-22-92$ & $29.51 \mathrm{SP}$ & &
\end{tabular}

\begin{tabular}{|c|c|c|c|}
\hline Highest & $08-30-87$ & & \\
\hline Lowest & $11-19-92$ & & \\
\hline $\begin{array}{l}\text { Period of record } \\
\text { Record begins }\end{array}$ & $\begin{array}{ll}\text { Highest } 19.24 \quad 08-30-86 \\
10-09-8 \quad 29 \text { Entries }\end{array}$ & Lowest 30.10 & $11-19-92$ \\
\hline
\end{tabular}


Table 36. Ground-water levels in selected wells, Long Valley Caldera, Mono County, California, 1987-93-Continued

\begin{tabular}{llccccc}
\hline Date & $\begin{array}{c}\text { Water } \\
\text { level MW }\end{array}$ & Date & $\begin{array}{c}\text { Water } \\
\text { level MW }\end{array}$ & Date & $\begin{array}{c}\text { Water } \\
\text { level MW }\end{array}$ & $\begin{array}{c}\text { Water } \\
\text { level MW }\end{array}$ \\
\hline
\end{tabular}

Site identifier: CD-2

Site number: $\mathbf{3 7 3 8 4 7 1 1 8 5 4 5 8 0 2}$

About 2.5 miles east of Mammoth Lakes and 0.1 mile northwest of Casa Diablo Hot Springs. Drilled irrigation well. Diameter 6 inches, depth 75 feet. Altitude of land-surface datum 7,316 feet. Water-level records available 1990-94.

\begin{tabular}{|c|c|c|c|c|c|c|c|}
\hline $08-29-90$ & $13.94 \mathrm{~S}$ & $06-21-91$ & $30.45 \mathrm{SR}$ & $04-22-92$ & $38.63 \mathrm{~S}$ & $03-26-93$ & $41.68 \mathrm{~S}$ \\
\hline $09-25-90$ & $22.75 \mathrm{~S}$ & $08-23-91$ & SO & $05-15-92$ & $42.36 \mathrm{~S}$ & $04-15-93$ & $31.56 \mathrm{~S}$ \\
\hline $10-16-90$ & $17.74 \mathrm{~S}$ & $09-19-91$ & $29.80 \mathrm{~S}$ & $06-09-92$ & $35.4 \mathrm{~S}$ & $05-13-93$ & $31.70 \mathrm{~S}$ \\
\hline $11-16-90$ & $23.47 \mathrm{SR}$ & $10-22-91$ & $25.25 \mathrm{~V}$ & $07-22-92$ & $37.22 \mathrm{~S}$ & $07-21-93$ & $40.11 \mathrm{~S}$ \\
\hline $12-18-90$ & $16.90 \mathrm{SN}$ & $11-20-91$ & $25.49 \mathrm{~V}$ & $09-17-92$ & $42.76 \mathrm{~S}$ & $09-07-93$ & $45.55 \mathrm{~S}$ \\
\hline $01-15-91$ & $15.29 \mathrm{~S}$ & $12-12-91$ & $29.10 \mathrm{~S}$ & $10-07-92$ & $44.50 \mathrm{~S}$ & $10-15-93$ & $43.72 \mathrm{~S}$ \\
\hline $02-15-91$ & $19.71 \mathrm{~S}$ & $01-15-92$ & $29.01 \mathrm{~S}$ & $11-19-92$ & $43.64 \mathrm{~S}$ & $11-11-93$ & $43.90 \mathrm{~S}$ \\
\hline $03-22-91$ & $16.38 \mathrm{~S}$ & $02-21-92$ & $29.07 \mathrm{~S}$ & $12-17-92$ & $42.87 \mathrm{SR}$ & & \\
\hline $04-10-91$ & $14.37 \mathrm{~S}$ & $03-15-92$ & $30.71 \mathrm{~S}$ & $01-14-93$ & $45.52 \mathrm{~S}$ & & \\
\hline $05-13-91$ & $18.10 \mathrm{~S}$ & $03-15-92$ & $30.71 \mathrm{~S}$ & $03-05-93$ & $46.46 \mathrm{~S}$ & & \\
\hline
\end{tabular}

Highest $\quad 13.94 \quad 08-29-90$

Lowest $\quad 46.46 \quad 03-05-93$

Period of record Highest $13.94 \quad 08-29-90 \quad$ Lowest $48.39 \quad 05-19-94$

Record begins $\quad 08-29-90 \quad 41$ Entries

Site identifier: $\mathrm{CH}-1$

Site number: 374045118491001

About 8.1 miles northeast of Mammoth Lakes and 0.7 mile southwest of Cashbaugh Ranch. Drilled observation well. Diameter 1.25 inches, depth 973 feet, perforated 470 to 475 feet. Altitude of land-surface datum 6,969.93 feet.

Water-level records available 1982-94.

\begin{tabular}{llllll}
\hline $04-19-87$ & $39.26 \mathrm{~S}$ & $12-07-8839.88 \mathrm{~S}$ & $07-19-9040.47 \mathrm{~S}$ & $12-17-91$ & $40.60 \mathrm{~S}$ \\
$08-28-87$ & $39.62 \mathrm{~S}$ & $01-11-8939.91 \mathrm{~S}$ & $08-21-9040.52 \mathrm{~S}$ & $01-18-92$ & $40.64 \mathrm{~S}$ \\
$10-18-87$ & $39.75 \mathrm{~S}$ & $02-16-8939.83 \mathrm{~S}$ & $09-24-9040.56 \mathrm{~S}$ & $02-21-92$ & $40.61 \mathrm{~S}$ \\
$01-04-88$ & $39.64 \mathrm{~S}$ & $03-29-8939.85 \mathrm{~S}$ & $10-15-9040.52 \mathrm{~S}$ & $03-14-92$ & $40.64 \mathrm{~S}$ \\
$02-04-88$ & $39.65 \mathrm{~S}$ & $04-26-8939.85 \mathrm{~S}$ & $11-14-9040.44 \mathrm{~S}$ & $04-07-92$ & $40.69 \mathrm{~S}$ \\
$03-04-88$ & $39.65 \mathrm{~S}$ & $06-08-8939.88 \mathrm{~S}$ & $11-18-9040.37 \mathrm{~S}$ & $04-10-92$ & $40.66 \mathrm{~S}$ \\
$04-04-88$ & $39.69 \mathrm{~S}$ & $07-23-8939.92 \mathrm{~S}$ & $12-18-9040.42 \mathrm{~S}$ & $05-26-92$ & $40.72 \mathrm{~S}$ \\
$04-28-88$ & $39.67 \mathrm{~S}$ & $08-09-8939.86 \mathrm{~S}$ & $01-18-9140.43 \mathrm{~S}$ & $06-10-92$ & $40.78 \mathrm{~S}$ \\
$05-03-88$ & $39.70 \mathrm{~S}$ & $11-28-8940.25 \mathrm{~S}$ & $02-20-9140.49 \mathrm{~S}$ & $07-22-92$ & $40.88 \mathrm{~S}$ \\
$06-07-88$ & $39.73 \mathrm{~S}$ & $12-12-8940.30 \mathrm{~S}$ & $05-14-9140.31 \mathrm{~S}$ & $09-16-92$ & $41.26 \mathrm{~S}$ \\
$07-06-88$ & $39.74 \mathrm{~S}$ & $01-09-9040.35 \mathrm{~S}$ & $06-20-9140.39 \mathrm{~S}$ & $10-06-92$ & $41.36 \mathrm{~S}$ \\
$07-27-88$ & $39.81 \mathrm{~S}$ & $02-06-9040.28 \mathrm{~S}$ & $07-17-9140.40 \mathrm{~S}$ & $11-18-92$ & $41.32 \mathrm{~S}$ \\
$08-03-88$ & $39.81 \mathrm{~S}$ & $03-07-9040.28 \mathrm{~S}$ & $07-24-9140.40 \mathrm{~S}$ & $12-03-92$ & $41.28 \mathrm{~S}$ \\
$09-02-88$ & $39.87 \mathrm{~S}$ & $04-23-9040.31 \mathrm{~S}$ & $08-22-9140.45 \mathrm{~S}$ & $05-12-93$ & $40.55 \mathrm{~S}$ \\
$09-29-88$ & $39.96 \mathrm{~S}$ & $05-30-9040.38 \mathrm{~S}$ & $09-25-9140.53 \mathrm{~S}$ & $07-23-93$ & $40.10 \mathrm{~S}$ \\
$11-07-88$ & $39.87 \mathrm{~S}$ & $06-28-9040.43 \mathrm{~S}$ & $11-15-9140.55 \mathrm{~S}$ & $11-09-93$ & $40.50 \mathrm{~S}$ \\
$11-28-88$ & $39.90 \mathrm{~S}$ & $07-17-9040.47 \mathrm{~S}$ & $11-19-9140.68 \mathrm{~S}$ & &
\end{tabular}

Highest $\quad 39.26 \quad 04-19-87$

Lowest $41.36 \quad 10-06-92$

Period of record Highest $33.67 \quad 08-03-82$ Lowest $41.36 \quad 10-06-92$

Record begins 08-03-82 106 Entries 
Table 36. Ground-water levels in selected wells, Long Valley Caldera, Mono County, California, 1987-93-Continued

\begin{tabular}{|c|c|c|c|c|c|c|c|}
\hline Date & $\begin{array}{c}\text { Water } \\
\text { level MW }\end{array}$ & Date & $\begin{array}{c}\text { Water } \\
\text { level MW }\end{array}$ & Date & $\begin{array}{c}\text { Water } \\
\text { level MW }\end{array}$ & Date & $\begin{array}{c}\text { Water } \\
\text { level MW }\end{array}$ \\
\hline
\end{tabular}

Site identifier: $\mathrm{CH}-10$

Site number: 373932118491501

About 7.8 miles east of Mammoth Lakes and 2 miles north of Whitmore Hot Springs. Drilled observation well.

Diameter 1.25 inches, depth 175 feet, perforated 115 to 120 feet. Altitude of land-surface datum 7,074.88 feet.

Water-level records available 1982-94.

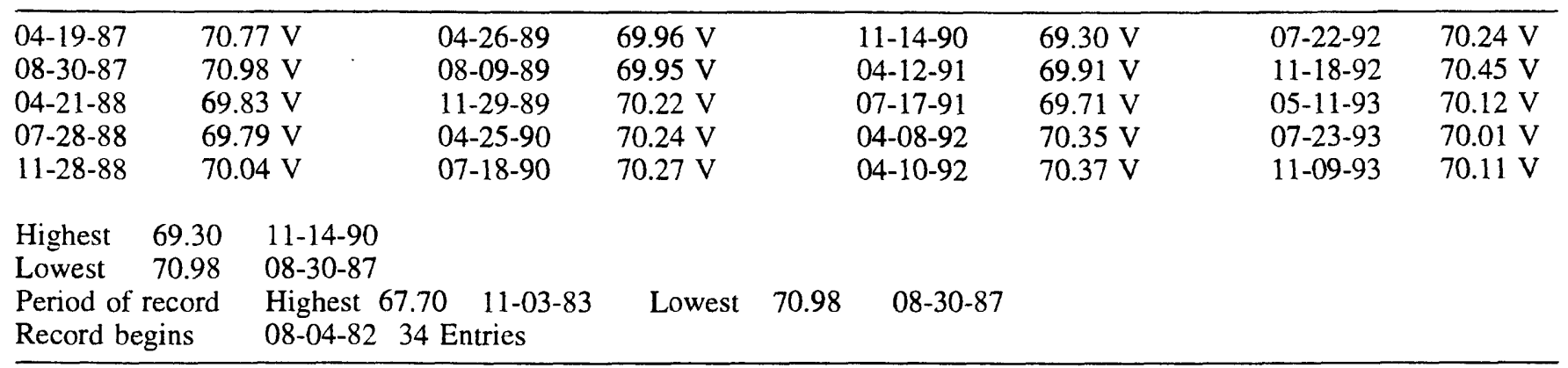

\section{Site identifier: $\mathrm{CH} \cdot 10 \mathrm{~A}$ \\ Site number: 373930118491601}

About 7.8 miles east of Mammoth Lakes and 2 miles north of Whitmore Hot Springs. Drilled observation well. Diameter 6 inches, depth 110 feet, perforated 90 to 110 feet. Altitude of land-surface datum 7,078.80 feet. Water-level records available 1983-94.

\begin{tabular}{llllllll}
\hline $04-19-87$ & $74.73 \mathrm{~V}$ & $04-26-89$ & $73.93 \mathrm{~V}$ & $04-12-91$ & $73.81 \mathrm{~V}$ & $05-11-93$ & $74.12 \mathrm{~V}$ \\
$08-30-87$ & $74.96 \mathrm{~V}$ & $08-09-89$ & $73.92 \mathrm{~S}$ & $07-17-91$ & $73.61 \mathrm{~V}$ & $07-23-93$ & $74.01 \mathrm{~V}$ \\
$12-15-87$ & $74.05 \mathrm{~V}$ & $11-29-89$ & $74.12 \mathrm{~V}$ & $04-08-92$ & $74.10 \mathrm{~V}$ & $11-09-93$ & $74.14 \mathrm{~V}$ \\
$04-21-88$ & $73.80 \mathrm{~V}$ & $04-25-90$ & $74.18 \mathrm{~V}$ & $04-10-92$ & $74.28 \mathrm{~V}$ & & \\
$07-28-88$ & $73.85 \mathrm{~V}$ & $07-18-90$ & $74.14 \mathrm{~V}$ & $07-22-92$ & $74.22 \mathrm{~V}$ & & \\
$11-28-88$ & $74.00 \mathrm{~V}$ & $11-14-90$ & $73.22 \mathrm{~V}$ & $11-18-92$ & $74.40 \mathrm{~V}$ & &
\end{tabular}

Highest $\quad 73.22 \quad 11-14-90$

Lowest $74.96 \quad 08-30-87$

Period of record Highest $69.8 \quad 09-28-83 \quad$ Lowest $74.96 \quad 08-30-87$

Record begins $\quad 08-10-8347$ Entries 
Table 36. Ground-water levels in selected wells, Long Valley Caldera, Mono County, California, 1987-93-Continued

\begin{tabular}{|c|c|c|c|c|c|c|c|}
\hline Date & $\begin{array}{c}\text { Water } \\
\text { level MW }\end{array}$ & Date & $\begin{array}{c}\text { Water } \\
\text { level MW }\end{array}$ & Date & $\begin{array}{c}\text { Water } \\
\text { level MW }\end{array}$ & Date & $\begin{array}{c}\text { Water } \\
\text { level MW }\end{array}$ \\
\hline
\end{tabular}

Site Identifier: CH-10B

Site Number: 373930118491602

About 7.8 miles east of Mammoth Lakes and 2 miles north of Whitmore Hot Springs. Drilled observation well.

Diameter 4 inches, depth 315 feet, open hole 300 to 315 feet. Altitude of land-surface datum 7,078.99 feet. Water-level records available 1983-94.

\begin{tabular}{llllllll}
\hline $02-10-87$ & $74.72 \mathrm{~V}$ & $04-26-89$ & $74.19 \mathrm{~V}$ & $02-15-91$ & $73.94 \mathrm{~V}$ & $06-12-92$ & $74.41 \mathrm{~V}$ \\
$04-19-87$ & $74.99 \mathrm{~V}$ & $05-16-89$ & $74.31 \mathrm{~V}$ & $02-20-91$ & $74.10 \mathrm{~V}$ & $07-22-92$ & $74.45 \mathrm{~V}$ \\
$05-27-87$ & $74.76 \mathrm{~V}$ & $06-08-89$ & $74.33 \mathrm{~V}$ & $04-12-91$ & $74.06 \mathrm{~V}$ & $09-16-92$ & $74.60 \mathrm{~V}$ \\
$05-28-87$ & $74.77 \mathrm{~V}$ & $07-23-89$ & $74.28 \mathrm{~V}$ & $05-13-91$ & $73.96 \mathrm{~V}$ & $10-06-92$ & $74.53 \mathrm{~V}$ \\
$06-18-87$ & $74.75 \mathrm{~V}$ & $08-09-89$ & $74.20 \mathrm{~V}$ & $05-15-91$ & $74.01 \mathrm{~V}$ & $11-18-92$ & $74.64 \mathrm{~V}$ \\
$07-28-87$ & $74.95 \mathrm{~V}$ & $09-19-89$ & $74.26 \mathrm{~V}$ & $06-14-91$ & $73.87 \mathrm{~V}$ & $12-15-92$ & $74.71 \mathrm{~V}$ \\
$08-25-87$ & $75.10 \mathrm{~V}$ & $10-16-89$ & $74.30 \mathrm{~V}$ & $06-19-91$ & $73.81 \mathrm{~V}$ & $01-12-93$ & $74.68 \mathrm{~V}$ \\
$10-18-87$ & $74.67 \mathrm{~V}$ & $11-21-89$ & $74.30 \mathrm{~V}$ & $07-17-91$ & $73.89 \mathrm{~V}$ & $03-02-93$ & $74.63 \mathrm{~V}$ \\
$11-14-87$ & $74.69 \mathrm{~V}$ & $11-29-89$ & $74.40 \mathrm{~V}$ & $07-24-91$ & $73.79 \mathrm{~V}$ & $03-22-93$ & $74.49 \mathrm{~V}$ \\
$12-15-87$ & $74.33 \mathrm{~V}$ & $12-20-89$ & $74.68 \mathrm{~V}$ & $08-07-91$ & $73.53 \mathrm{~V}$ & $04-13-93$ & $74.51 \mathrm{~V}$ \\
$03-03-88$ & $74.17 \mathrm{~V}$ & $01-09-90$ & $74.62 \mathrm{~V}$ & $08-08-91$ & $73.86 \mathrm{~V}$ & $05-11-93$ & $74.35 \mathrm{~V}$ \\
$04-22-88$ & $74.07 \mathrm{~V}$ & $02-22-90$ & $74.45 \mathrm{~V}$ & $08-09-91$ & $73.74 \mathrm{~V}$ & $06-16-93$ & $74.27 \mathrm{~V}$ \\
$06-01-88$ & $74.12 \mathrm{~V}$ & $03-20-90$ & $74.32 \mathrm{~V}$ & $08-22-91$ & $73.87 \mathrm{~V}$ & $07-23-93$ & $74.24 \mathrm{~V}$ \\
$07-12-88$ & $73.77 \mathrm{~V}$ & $04-25-90$ & $74.39 \mathrm{~V}$ & $09-18-91$ & $73.93 \mathrm{~V}$ & $08-20-93$ & $74.20 \mathrm{~V}$ \\
$07-28-88$ & $74.01 \mathrm{~V}$ & $05-24-90$ & $74.51 \mathrm{~V}$ & $09-25-91$ & $74.08 \mathrm{~V}$ & $08-25-93$ & $74.18 \mathrm{~V}$ \\
$08-12-88$ & $74.02 \mathrm{~V}$ & $06-13-90$ & $74.31 \mathrm{~V}$ & $10-22-91$ & $74.24 \mathrm{~V}$ & $09-07-93$ & $74.14 \mathrm{~V}$ \\
$09-22-88$ & $74.01 \mathrm{~V}$ & $08-21-90$ & $74.44 \mathrm{~V}$ & $12-12-91$ & $74.62 \mathrm{~V}$ & $09-08-93$ & $74.16 \mathrm{~V}$ \\
$10-21-88$ & $74.04 \mathrm{~V}$ & $08-27-90$ & $74.41 \mathrm{~V}$ & $12-17-91$ & $74.46 \mathrm{~V}$ & $09-16-93$ & $74.03 \mathrm{~V}$ \\
$11-28-88$ & $74.26 \mathrm{~V}$ & $09-25-90$ & $74.40 \mathrm{~V}$ & $01-18-92$ & $74.52 \mathrm{~V}$ & $10-15-93$ & $74.18 \mathrm{~V}$ \\
$12-19-88$ & $74.25 \mathrm{~V}$ & $10-17-90$ & $73.76 \mathrm{~V}$ & $02-21-92$ & $74.67 \mathrm{~V}$ & $11-09-93$ & $74.36 \mathrm{~V}$ \\
$01-10-89$ & $74.16 \mathrm{~V}$ & $11-14-90$ & $73.43 \mathrm{~V}$ & $03-13-92$ & $74.59 \mathrm{~V}$ & $11-10-93$ & $74.31 \mathrm{~V}$ \\
$02-17-89$ & $74.20 \mathrm{~V}$ & $12-13-90$ & $73.70 \mathrm{~V}$ & $04-08-92$ & $74.50 \mathrm{~V}$ & $11-18-93$ & $74.40 \mathrm{~V}$ \\
$03-20-89$ & $74.23 \mathrm{~V}$ & $12-18-90$ & $73.61 \mathrm{~V}$ & $04-10-92$ & $74.55 \mathrm{~V}$ & $12-13-93$ & $74.42 \mathrm{~V}$ \\
$04-12-89$ & $74.22 \mathrm{~V}$ & $01-15-91$ & $73.83 \mathrm{~V}$ & $04-21-92$ & $74.46 \mathrm{~V}$ & &
\end{tabular}

Highest $\quad 73.43 \quad 11-14-90$

Lowest $\quad 75.10 \quad 08-25-87$

Period of record Highest $71.72 \quad 09-28-83 \quad$ Lowest $75.10 \quad 08-25-87$

Record begins $\quad 08-10-83 \quad 134$ Entries

Site identifier: $\mathrm{CH}-3$

Site number: 373929118455405

About 11.2 miles east of Mammoth Lakes and 3.2 miles northeast of Whitmore Hot Springs. Drilled observation well. Diameter 1.25 inches, depth 160 feet. Altitude of land-surface datum 6,870 feet. Water-level records available 1982-94.

\begin{tabular}{llllllll}
\hline $04-24-87$ & $6.41 \mathrm{~S}$ & $04-26-89$ & $6.29 \mathrm{~S}$ & $04-11-91$ & $5.84 \mathrm{~S}$ & $05-14-93$ & $5.50 \mathrm{~S}$ \\
$08-28-87$ & $6.57 \mathrm{~S}$ & $08-08-89$ & $6.40 \mathrm{~S}$ & $07-17-91$ & $5.99 \mathrm{~S}$ & $07-20-93$ & $5.84 \mathrm{~S}$ \\
$12-18-87$ & $6.43 \mathrm{~S}$ & $11-28-89$ & $6.79 \mathrm{~S}$ & $11-20-91$ & $5.85 \mathrm{~S}$ & $11-10-93$ & $5.61 \mathrm{~S}$ \\
$04-21-88$ & $6.23 \mathrm{~S}$ & $04-24-90$ & $6.34 \mathrm{~S}$ & $04-08-92$ & $5.69 \mathrm{~S}$ & & \\
$07-27-88$ & $6.39 \mathrm{~S}$ & $07-17-90$ & $6.49 \mathrm{~S}$ & $07-23-92$ & $6.71 \mathrm{~S}$ & & \\
$11-29-88$ & $6.34 \mathrm{~S}$ & $11-15-90$ & $6.34 \mathrm{~S}$ & $11-20-92$ & $6.71 \mathrm{~S}$ &
\end{tabular}

$\begin{array}{lrlllll}\text { Highest } & 5.50 & 05-14-93 & & & & \\ \text { Lowest } & 6.79 & 11-28-89 & & & & \\ \text { Period of record } & \text { Highest } & 5.50 & 05-14-93 & \text { Lowest } & 7.73 & 06-02-83 \\ \text { Record begins } & 08-24-82 & 34 \text { Entries } & & & \end{array}$


Table 36. Ground-water levels in selected wells, Long Valley Caldera, Mono County, California, 1987-93-Continued

\begin{tabular}{|c|c|c|c|c|c|c|c|}
\hline Date & $\begin{array}{c}\text { Water } \\
\text { level MW }\end{array}$ & Date & $\begin{array}{c}\text { Water } \\
\text { level MW }\end{array}$ & Date & $\begin{array}{c}\text { Water } \\
\text { level MW }\end{array}$ & Date & $\begin{array}{c}\text { Water } \\
\text { level MW }\end{array}$ \\
\hline
\end{tabular}

Site identifier: $\mathrm{CH}-5$

Site number: 373759118474105

About 9.9 miles east of Mammoth Lakes and 1.6 miles northeast of Whitmore Hot Springs. Drilled observation well. Diameter 1.25 inches, depth 875 feet. Altitude of land-surface datum 6,918 feet. Water-level records available 1982 to current year.

\begin{tabular}{llllllll}
\hline $04-24-87$ & $43.73 \mathrm{~S}$ & $04-25-89$ & $44.75 \mathrm{~S}$ & $11-15-90$ & $45.37 \mathrm{~S}$ & $07-23-92$ & $45.48 \mathrm{~S}$ \\
$09-03-87$ & $44.11 \mathrm{~S}$ & $08-08-89$ & $44.46 \mathrm{~S}$ & $04-10-91$ & $45.32 \mathrm{~S}$ & $11-20-92$ & $45.96 \mathrm{~S}$ \\
$12-18-87$ & $44.51 \mathrm{~S}$ & $11-29-89$ & $45.20 \mathrm{~S}$ & $07-17-91$ & $44.94 \mathrm{~S}$ & $05-14-93$ & $45.00 \mathrm{~S}$ \\
$04-21-88$ & $44.53 \mathrm{~S}$ & $04-24-90$ & $45.55 \mathrm{~S}$ & $11-20-91$ & $45.17 \mathrm{~S}$ & $07-20-93$ & $44.49 \mathrm{~S}$ \\
$07-27-88$ & $44.33 \mathrm{~S}$ & $07-18-90$ & $45.43 \mathrm{~S}$ & $04-08-92$ & $45.37 \mathrm{~S}$ & $11-10-93$ & $44.76 \mathrm{~S}$ \\
$11-28-88$ & $44.78 \mathrm{~S}$ & & & & & &
\end{tabular}

Highest $\quad 43.73 \quad 04-24-87$

Lowest $45.96 \quad 11-20-92$

Period of record Highest $42.40 \quad 11-12-84 \quad$ Lowest $45.96 \quad 11-20-92$

Record begins 08-24-82 34 Entries

\section{Site identifier: $\mathrm{CH}-6$ \\ Site number: 374040118522501}

About 5.3 miles northeast of Mammoth Lakes in Little Antelope Valley. Drilled observation well. Diameter 1.25 inches, depth 677 feet, perforated 535 to 539 feet. Altitude of land-surface datum 7,248.23 feet. Water-level records available 1982-94.

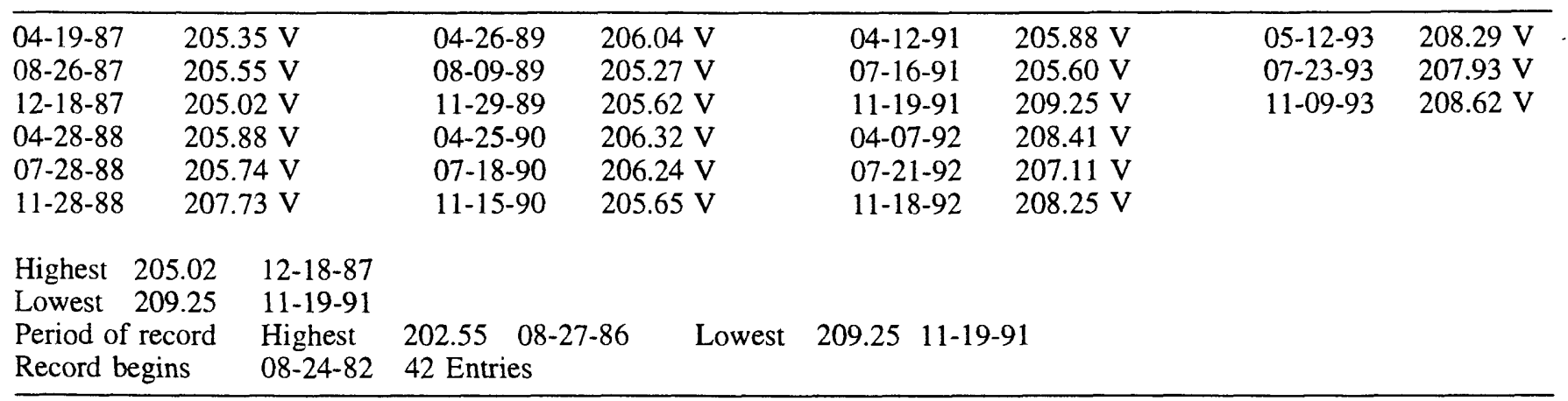

Site identifier: CH-7

Site number: $\mathbf{3 7 4 0 0 0 1 1 8 4 8 3 1 0 2}$

About 8.6 miles northeast of Mammoth Lakes and 1.2 miles south of Cashbaugh Ranch. Drilled observation well. Diameter 1.25 inches, depth 210 feet, perforated 198 to 203 feet. Altitude of land-surface datum 6,960 feet. Water-level records available 1982-94.

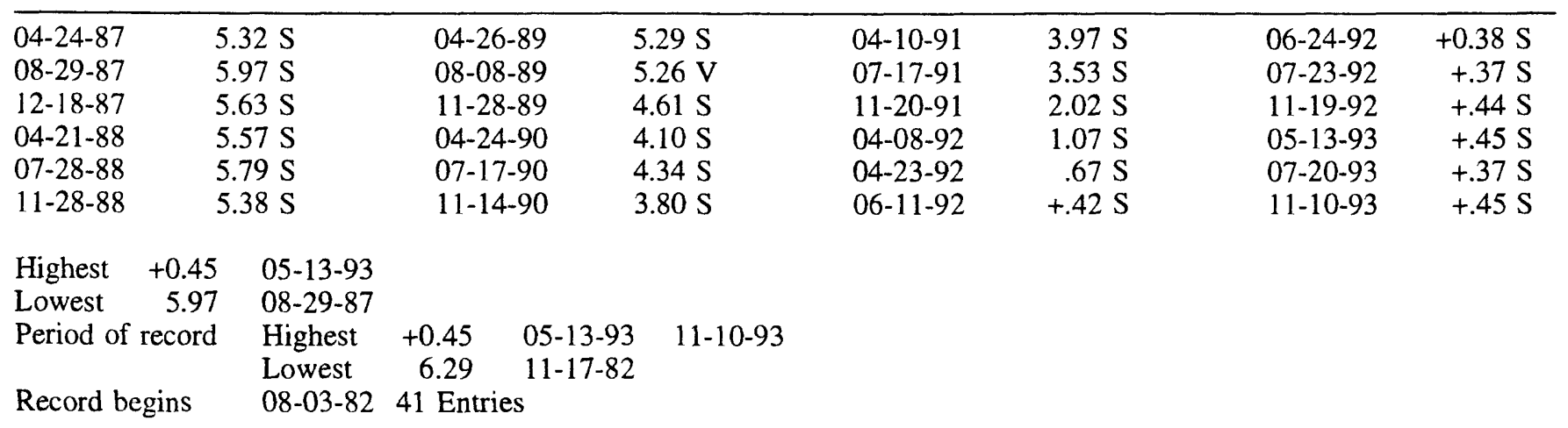


Table 36. Ground-water levels in selected wells, Long Valley Caldera, Mono County, California, 1987-93-Continued

\begin{tabular}{|c|c|c|c|c|c|c|c|}
\hline Date & $\begin{array}{c}\text { Water } \\
\text { level MW }\end{array}$ & Date & $\begin{array}{c}\text { Water } \\
\text { level MW }\end{array}$ & Date & $\begin{array}{c}\text { Water } \\
\text { level MW }\end{array}$ & Date & $\begin{array}{c}\text { Water } \\
\text { level MW }\end{array}$ \\
\hline
\end{tabular}

\section{Site identifier: CHURCH}

Site number: $\mathbf{3 7 3 7 0 7 1 1 8 4 9 1 7 0 1}$

About 8 miles southeast of Mammoth Lakes and 1 mile southwest of Whitmore Hot Springs. Drilled domestic well. Diameter 6 inches, depth 45.5 feet. Altitude of land-surface datum 7,035 feet. Water-level records available 1989-94.

\begin{tabular}{|c|c|c|c|c|c|c|c|c|c|}
\hline $04-25-89$ & \multicolumn{2}{|c|}{$8.25 \mathrm{~S}$} & $07-18-90$ & $7.78 \mathrm{~S}$ & & $11-20-91$ & $7.61 \mathrm{~S}$ & $05-14-93$ & $6.79 \mathrm{~S}$ \\
\hline 08-08-89 & \multicolumn{2}{|c|}{$7.83 \mathrm{~S}$} & $11-15-90$ & $8.29 \mathrm{~S}$ & & $04-08-92$ & $8.02 \mathrm{~S}$ & $07-20-93$ & $4.29 \mathrm{~S}$ \\
\hline $11-29-89$ & \multicolumn{2}{|c|}{$7.84 \mathrm{~S}$} & $04-10-91$ & $7.36 \mathrm{~S}$ & & $07-22-92$ & $8.54 \mathrm{~S}$ & $11-10-93$ & $6.82 \mathrm{~V}$ \\
\hline $04-24-90$ & \multicolumn{2}{|c|}{$8.32 \mathrm{~S}$} & $07-17-91$ & $4.15 \mathrm{~S}$ & & $11-19-92$ & $8.58 \mathrm{~S}$ & & \\
\hline Highest & 4.15 & $07-17-91$ & & & & & & & \\
\hline Lowest & 8.58 & $11-19-92$ & & & & & & & \\
\hline \multicolumn{2}{|c|}{$\begin{array}{l}\text { Period of record } \\
\text { Record begins }\end{array}$} & $\begin{array}{l}\text { Highest } \\
04-25-89\end{array}$ & $\begin{array}{l}4.1507-17-91 \\
16 \text { Entries }\end{array}$ & Lowest & 8.58 & $11-19-92$ & & & \\
\hline
\end{tabular}

\section{Site identifier: $\mathrm{CM}-2$ \\ Site number: 373906118522301}

About 4.9 miles east of Mammoth Lakes near Hot Creek Fish Hatchery. Drilled observation well. Diameter 4 inches, depth 152 feet. Altitude of land-surface datum 7,077.0 feet. Water-level records available 1985-94.

\begin{tabular}{llllllll}
\hline $04-19-87$ & $3.65 \mathrm{~S}$ & $12-19-89$ & $4.88 \mathrm{~S}$ & $07-16-91$ & $3.47 \mathrm{~S}$ & $07-07-92$ & $6.65 \mathrm{~S}$ \\
$09-03-87$ & $3.61 \mathrm{~S}$ & $01-08-90$ & $4.95 \mathrm{~S}$ & $08-27-91$ & $4.80 \mathrm{~S}$ & $07-23-92$ & $6.08 \mathrm{~S}$ \\
$04-19-88$ & $3.93 \mathrm{~S}$ & $02-21-90$ & $5.09 \mathrm{~S}$ & $11-19-91$ & $6.84 \mathrm{~S}$ & $08-03-92$ & $6.11 \mathrm{~S}$ \\
$07-28-88$ & $3.39 \mathrm{~S}$ & $03-20-90$ & $4.99 \mathrm{~S}$ & $12-17-91$ & $6.80 \mathrm{~S}$ & $09-16-92$ & $7.01 \mathrm{~S}$ \\
$04-25-89$ & $4.61 \mathrm{~S}$ & $04-25-90$ & $5.06 \mathrm{~S}$ & $03-14-92$ & $7.08 \mathrm{~S}$ & $11-18-92$ & $7.58 \mathrm{~S}$ \\
$08-09-89$ & $3.84 \mathrm{~S}$ & $05-24-90$ & $4.74 \mathrm{~S}$ & $04-07-92$ & $7.25 \mathrm{~S}$ & $05-13-93$ & $6.71 \mathrm{~S}$ \\
$09-20-89$ & $4.53 \mathrm{~S}$ & $07-18-90$ & $3.66 \mathrm{~S}$ & $05-14-92$ & $6.86 \mathrm{~S}$ & $07-22-93$ & $5.21 \mathrm{~S}$ \\
$10-18-89$ & $4.83 \mathrm{~S}$ & $11-15-90$ & $4.58 \mathrm{~S}$ & $06-10-92$ & $6.62 \mathrm{~S}$ & $11-09-93$ & $7.35 \mathrm{~S}$ \\
$11-21-89$ & $4.74 \mathrm{~S}$ & $04-12-91$ & $4.76 \mathrm{~S}$ & $06-24-92$ & $6.85 \mathrm{~S}$ & &
\end{tabular}

Highest $\quad 3.39 \quad 07-28-88$

Lowest $7.58 \quad 11-18-92$

Period of record Highest $\quad 0.25 \quad 08-27-86 \quad$ Lowest $7.58 \quad 11-18-92$

Record begins $04-23-85$ 41 Entries

\section{Site identifier: $\mathbf{C R}$}

Site number: 373816118532001

About 4 miles east of Mammoth Lakes. Drilled domestic well. Diameter 6 inches, depth 80 feet. Altitude of landsurface datum 7,161.76 feet. Water-level records available 1986-93.

\begin{tabular}{llllllll}
\hline $04-21-87$ & $17.87 \mathrm{~S}$ & $07-29-88$ & $26.60 \mathrm{~S}$ & $11-29-89$ & $19.08 \mathrm{~S}$ & $07-17-91$ & $15.64 \mathrm{~S}$ \\
$08-30-87$ & $20.15 \mathrm{~S}$ & $04-26-89$ & $17.77 \mathrm{~S}$ & $04-24-90$ & $17.09 \mathrm{~S}$ & $07-21-92$ & $22.71 \mathrm{SR}$ \\
$12-16-87$ & $21.88 \mathrm{~S}$ & $08-09-89$ & $17.84 \mathrm{~S}$ & $07-18-90$ & $15.95 \mathrm{~S}$ & $07-23-93$ & $16.73 \mathrm{~S}$
\end{tabular}

Highest $\quad 15.64 \quad 07-17-91$

Lowest $26.60 \quad 07-29-88$

Period of record Highest $10.40 \quad 05-21-86$ Lowest $26.60 \quad 07-29-88$

Record begins 05-21-86 15 Entries 
Table 36. Ground-water levels in selected wells, Long Valley Caldera, Mono County, California 1987-93-Continued

\begin{tabular}{|c|c|c|c|c|c|c|c|}
\hline Date & $\begin{array}{c}\text { Water } \\
\text { level MW }\end{array}$ & Date & $\begin{array}{c}\text { Water } \\
\text { level MW }\end{array}$ & Date & $\begin{array}{c}\text { Water } \\
\text { level MW }\end{array}$ & Date & $\begin{array}{c}\text { Water } \\
\text { level MW }\end{array}$ \\
\hline
\end{tabular}

Site identifier: CT

Site number: $\mathbf{3 7 4 5 1 1 1 1 8 5 8 5 8 0 1}$

About 7.5 miles north of Mammoth Lakes at the Caltrans Crestview Maintenance Station. Drilled commercial well. Diameter 10 inches, depth 490 feet. Altitude of land-surface datum 7,520 feet. Water-level records available 1990-94.

\begin{tabular}{llllllll}
\hline $08-02-90$ & $123.72 \mathrm{~V}$ & $07-12-91$ & $126.43 \mathrm{~V}$ & $11-20-91$ & $133.81 \mathrm{~V}$ & $11-19-92$ & $134.31 \mathrm{~V}$ \\
$12-14-90$ & $130.03 \mathrm{~V}$ & $07-16-91$ & $126.61 \mathrm{~V}$ & $04-07-92$ & $133.03 \mathrm{~V}$ & $05-12-93$ & $130.00 \mathrm{~V}$ \\
$04-11-91$ & $132.63 \mathrm{VR}$ & $08-09-91$ & $127.62 \mathrm{~V}$ & $07-20-92$ & $129.33 \mathrm{~S}$ & $11-09-93$ & $124.55 \mathrm{~V}$
\end{tabular}

Highest $123.72 \quad 08-02-90$

Lowest $134.31 \quad 11-19-92$

Period of record Highest $123.72 \quad$ 08-02-90 Lowest $134.31 \quad$ 11-19-92

Record begins $\quad 08-02-90 \quad 13$ Entries

Site identifier: CW-1

Site number: 373850118513601

About 5.6 miles east of Mammoth Lakes near Hot Creek Fish Hatchery. Drilled observation well. Diameter 18 inches, drilled depth 805 feet, open hole 223 to 805 feet. Altitude of land-surface datum 7,084.99 feet. Water-level records available 1973, 1982-94.

\begin{tabular}{rrrrrrrr}
\hline $04-19-87$ & $9.73 \mathrm{~V}$ & $04-25-90$ & $10.53 \mathrm{~V}$ & $11-19-91$ & $14.68 \mathrm{~V}$ & $05-14-92$ & $15.03 \mathrm{~V}$ \\
$04-19-88$ & $9.96 \mathrm{~V}$ & $07-18-90$ & $10.10 \mathrm{~V}$ & $12-17-91$ & $14.63 \mathrm{~V}$ & $07-23-92$ & $13.83 \mathrm{~V}$ \\
$07-28-88$ & $10.05 \mathrm{~V}$ & $11-15-90$ & $9.76 \mathrm{~V}$ & $01-16-92$ & $14.45 \mathrm{~V}$ & $11-18-92$ & $15.36 \mathrm{~V}$ \\
$04-25-89$ & $10.30 \mathrm{~V}$ & $01-17-91$ & $10.61 \mathrm{~V}$ & $03-11-92$ & $15.02 \mathrm{~V}$ & $05-12-93$ & $14.78 \mathrm{~V}$ \\
$08-09-89$ & $9.80 \mathrm{~V}$ & $04-12-91$ & $10.79 \mathrm{~V}$ & $04-09-92$ & $14.93 \mathrm{~V}$ & $07-22-93$ & $14.45 \mathrm{~V}$ \\
$11-21-89$ & $10.26 \mathrm{~V}$ & $07-16-91$ & $11.34 \mathrm{~V}$ & $04-17-92$ & $14.97 \mathrm{~V}$ & $11-09-93$ & $15.35 \mathrm{~V}$ \\
$01-09-90$ & $10.55 \mathrm{~S}$ & $08-27-91$ & $12.84 \mathrm{~V}$ & $04-23-92$ & $15.15 \mathrm{~V}$ & &
\end{tabular}

$\begin{array}{lrlllll}\text { Highest } & 9.73 & 04-19-87 & & & & \\ \text { Lowest } & 15.36 & 11-18-92 & & & & \\ \text { Period of record } & \text { Highest } & 6.77 & 08-13-83 & \text { Lowest } & 15.70 & 04-06-94 \\ \text { Record begins } & 08-01-73 & 44 \text { Entries }\end{array}$


Table 36. Ground-water levels in selected wells, Long Valley Caldera, Mono County, California, 1987-93-Continued

\begin{tabular}{|c|c|c|c|c|c|c|c|}
\hline Date & $\begin{array}{c}\text { Water } \\
\text { level MW }\end{array}$ & Date & $\begin{array}{c}\text { Water } \\
\text { level MW }\end{array}$ & Date & $\begin{array}{c}\text { Water } \\
\text { level MW }\end{array}$ & Date & $\begin{array}{c}\text { Water } \\
\text { level MW }\end{array}$ \\
\hline
\end{tabular}

About 5.7 miles east of Mammoth Lakes near Hot Creek Fish Hatchery. Drilled observation well. Diameter 13 inches, accessible depth 420 feet, depth of hole 917 feet. Altitude of land-surface datum 7,062.20 feet. Water-level records available 1986-94.

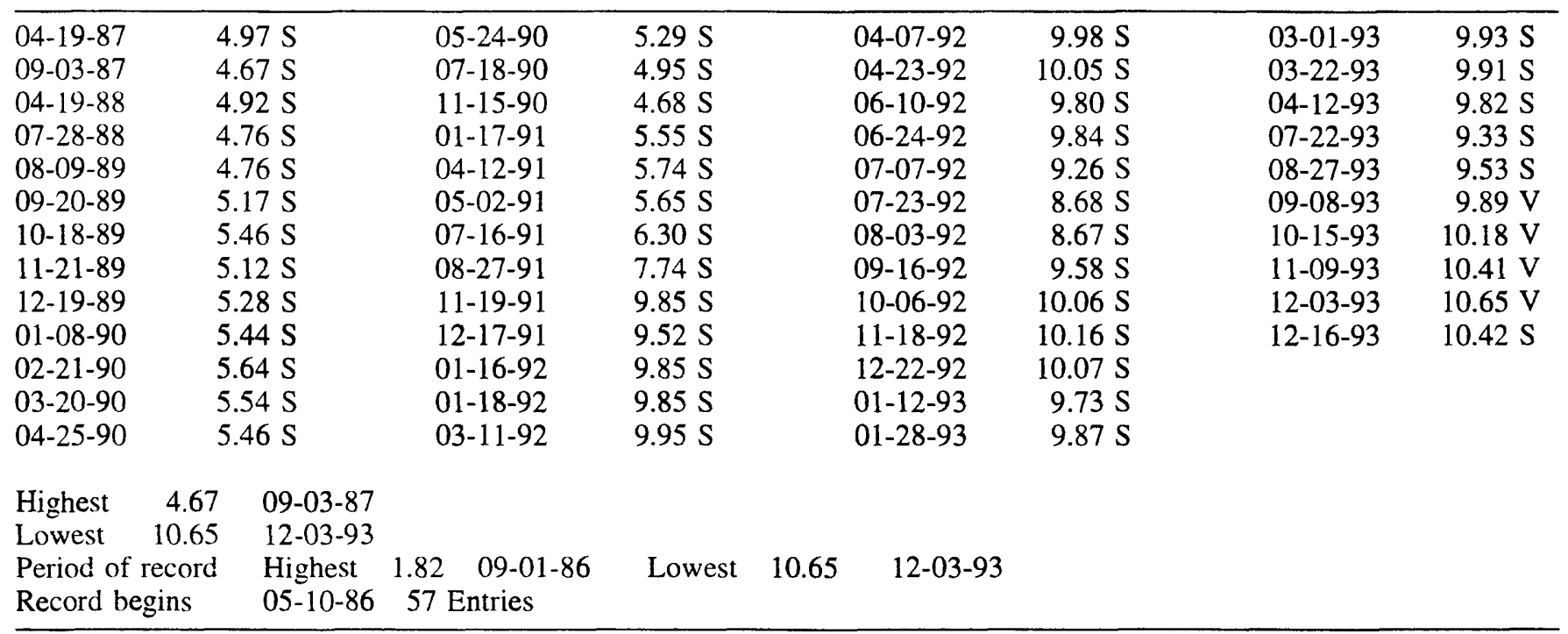

\section{Site number: $\mathbf{3 7 4 4 1 0 1 1 8 5 8 3 4 0 1}$}

About 6.3 miles north of Mammoth Lakes and 0.4 mile west of Highway 395. Drilled observation well. Diameter 1.25 inches, depth 633 feet. Altitude of land-surface datum 7,515.27 feet. Water-level records available 1982-92.

\begin{tabular}{llllllll}
\hline $05-30-87$ & $182.75 \mathrm{~V}$ & $03-30-89$ & $199.85 \mathrm{~V}$ & $07-18-90$ & $204.67 \mathrm{~V}$ & $11-19-91$ & $212.98 \mathrm{~V}$ \\
$08-30-87$ & $185.15 \mathrm{~V}$ & $04-26-89$ & $199.99 \mathrm{~V}$ & $11-13-90$ & $207.56 \mathrm{~V}$ & $04-07-92$ & $213.85 \mathrm{~V}$ \\
$04-22-88$ & $192.50 \mathrm{~V}$ & $08-08-89$ & $200.37 \mathrm{~V}$ & $04-11-91$ & $211.42 \mathrm{~V}$ & $04-07-92$ & $213.70 \mathrm{~S}$ \\
$07-27-88$ & $193.63 \mathrm{~V}$ & $01-08-90$ & $203.75 \mathrm{~V}$ & $07-16-91$ & $209.05 \mathrm{~V}$ & & \\
$11-23-88$ & $196.69 \mathrm{~V}$ & $04-27-90$ & $205.22 \mathrm{~V}$ & $08-26-91$ & $209.30 \mathrm{~V}$ & &
\end{tabular}

$\begin{array}{lrllll}\text { Highest } & 182.75 & 05-30-87 & & & \\ \text { Lowest } & 213.85 & 04-07-92 & & & \\ \text { Period of record } & \text { Highest 171.30 } & 07-20-83 & \text { Lowest } 213.85 & 04-07-92 \\ \text { Record begins } & 07-20-83 & \text { 25 Entries } & & \end{array}$


Table 36. Ground-water levels in selected wells, Long Valley Caldera, Mono County, California, 1987-93-Continued

\begin{tabular}{|c|c|c|c|c|c|c|c|}
\hline Date & $\begin{array}{c}\text { Water } \\
\text { level MW }\end{array}$ & Date & $\begin{array}{c}\text { Water } \\
\text { level MW }\end{array}$ & Date & $\begin{array}{c}\text { Water } \\
\text { level MW }\end{array}$ & Date & $\begin{array}{c}\text { Water } \\
\text { level MW }\end{array}$ \\
\hline
\end{tabular}

Site identifier: ESO

Site number: 373818118514502

About 5.5 miles east of Mammoth Lakes at elementary school. Drilled institutional well. Diameter 6 inches, depth 44.3 feet. Altitude of land-surface datum 7,090 feet. Water-level records available 1985-94.

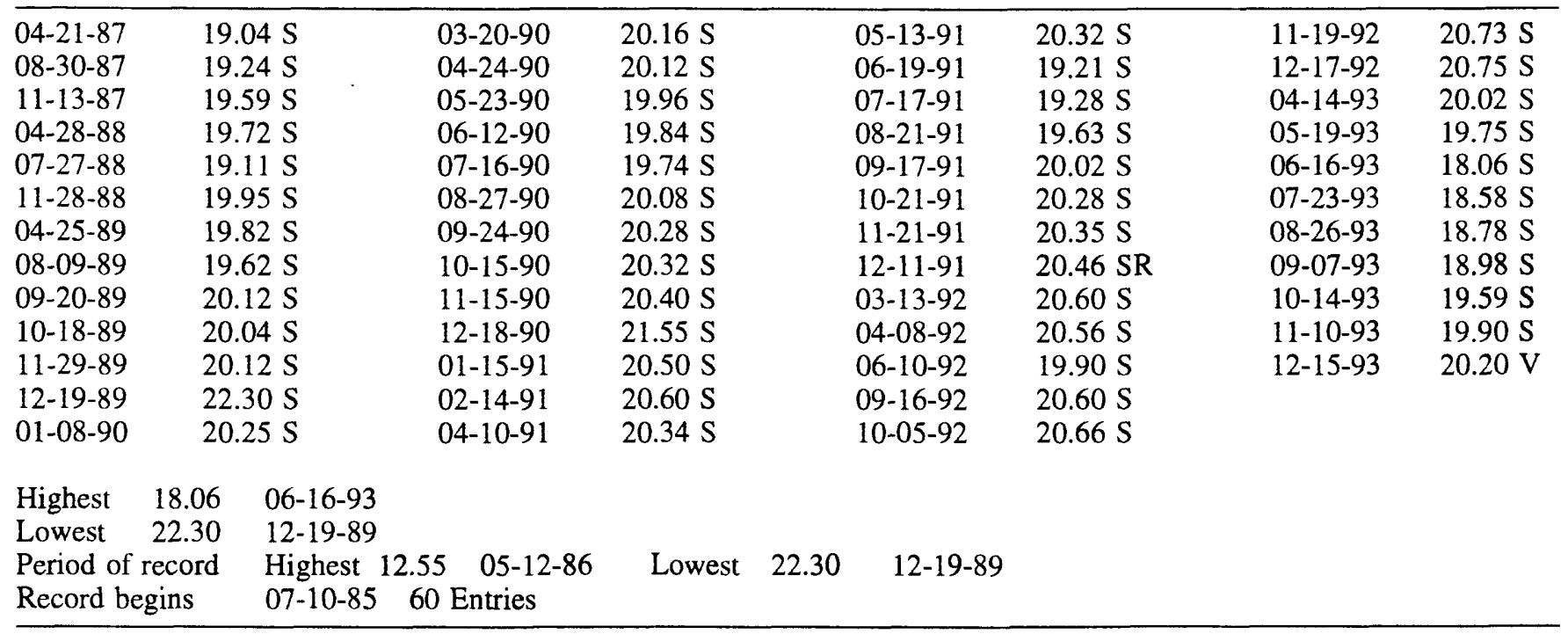

Site identifier: LAV-1

Site number: $\mathbf{3 7 4 0 5 1 1 1 8 5 2 3 3 0 1}$

About 5.3 miles northeast of Mammoth Lakes in Little Antelope Valley. Observation well. Diameter 30 inches, depth 14 feet. Altitude of land-surface datum 7,200 feet. Water-level records available 1972-73, 1982-94.

\begin{tabular}{llllllll}
\hline $08-26-87$ & $2.65 \mathrm{~S}$ & $11-29-89$ & $6.90 \mathrm{~S}$ & $07-16-91$ & $5.91 \mathrm{~S}$ & $05-12-93$ & $1.70 \mathrm{~S}$ \\
$07-28-88$ & $5.79 \mathrm{~S}$ & $04-25-90$ & $2.19 \mathrm{~S}$ & $11-19-91$ & $7.90 \mathrm{~S}$ & $07-23-93$ & $5.71 \mathrm{~S}$ \\
$11-28-88$ & $5.17 \mathrm{~S}$ & $07-18-90$ & $2.18 \mathrm{~S}$ & $04-07-92$ & $2.81 \mathrm{~S}$ & $11-10-93$ & $6.98 \mathrm{~S}$ \\
$04-26-89$ & $2.38 \mathrm{~S}$ & $11-15-90$ & $8.62 \mathrm{~S}$ & $07-21-92$ & $7.40 \mathrm{~S}$ & & \\
$08-09-89$ & $7.99 \mathrm{~S}$ & $04-12-91$ & $.61 \mathrm{~S}$ & $11-18-92$ & $9.10 \mathrm{~S}$ & &
\end{tabular}

$\begin{array}{lrlll}\text { Highest } & 0.61 & 04-12-91 & \\ \text { Lowest } 9.10 & 11-18-92 & \\ \text { Period of record } & \text { Highest }+1.35 & 05-10-86 & 08-27-86 \\ & \text { Lowest } & 9.10 & 11-18-92 & \\ \text { Record begins } & \text { 05-08-72 } & 31 \text { Entries }\end{array}$


Table 36. Ground-water levels in selected wells, Long Valley Caldera, Mono County, California, 1987-93-Continued

\begin{tabular}{|c|c|c|c|c|c|c|c|}
\hline Date & $\begin{array}{c}\text { Water } \\
\text { level MW }\end{array}$ & Date & $\begin{array}{c}\text { Water } \\
\text { level MW }\end{array}$ & Date & $\begin{array}{c}\text { Water } \\
\text { level MW }\end{array}$ & Date & $\begin{array}{c}\text { Water } \\
\text { level MW }\end{array}$ \\
\hline
\end{tabular}

\section{Site identifier: LKT}

Site number: 374300118554401

About 5.2 miles northeast of Mammoth Lakes and 1.3 miles east of Highway 395. Drilled observation well. Diameter 6 inches, depth 3,500 feet, open hole 500 to 3,500 feet. Obstruction in well at 980 feet. Altitude of land-surface datum 7,350 feet. Water-level records available 1984-94.

\begin{tabular}{llllllll}
\hline $01-05-87$ & $57.61 \mathrm{~S}$ & $09-02-88$ & $61.01 \mathrm{~S}$ & $08-21-90$ & $65.65 \mathrm{~S}$ & $09-17-92$ & $70.74 \mathrm{~S}$ \\
$01-29-87$ & $57.83 \mathrm{~S}$ & $09-29-88$ & $61.17 \mathrm{~S}$ & $09-24-90$ & $65.80 \mathrm{~S}$ & $10-06-92$ & $70.84 \mathrm{~S}$ \\
$02-24-87$ & $57.71 \mathrm{~S}$ & $11-07-88$ & $61.21 \mathrm{~S}$ & $10-15-90$ & $65.84 \mathrm{~S}$ & $11-18-92$ & $70.90 \mathrm{~S}$ \\
$03-19-87$ & $57.93 \mathrm{~S}$ & $11-23-88$ & $61.18 \mathrm{~S}$ & $11-14-90$ & $66.36 \mathrm{~S}$ & $12-22-92$ & $71.17 \mathrm{~S}$ \\
$04-09-87$ & $58.16 \mathrm{~S}$ & $12-07-88$ & $61.45 \mathrm{~S}$ & $11-18-90$ & $66.27 \mathrm{~S}$ & $01-27-93$ & $71.21 \mathrm{~S}$ \\
$04-23-87$ & $58.13 \mathrm{~S}$ & $01-11-89$ & $61.74 \mathrm{~S}$ & $12-17-90$ & $66.49 \mathrm{~S}$ & $03-03-93$ & $71.45 \mathrm{~S}$ \\
$05-07-87$ & $58.28 \mathrm{~S}$ & $02-16-89$ & $61.95 \mathrm{~S}$ & $01-19-91$ & $66.49 \mathrm{~S}$ & $03-11-93$ & $71.50 \mathrm{~S}$ \\
$06-04-87$ & $58.44 \mathrm{~S}$ & $03-30-89$ & $62.18 \mathrm{~S}$ & $02-20-91$ & $66.80 \mathrm{~S}$ & $03-23-93$ & $71.54 \mathrm{~S}$ \\
$06-19-87$ & $58.42 \mathrm{~S}$ & $04-26-89$ & $62.24 \mathrm{~S}$ & $05-14-91$ & $67.27 \mathrm{~S}$ & $04-15-93$ & $71.63 \mathrm{~S}$ \\
$07-07-87$ & $58.61 \mathrm{~S}$ & $06-08-89$ & $62.47 \mathrm{~S}$ & $06-21-91$ & $67.45 \mathrm{~S}$ & $05-12-93$ & $71.51 \mathrm{~S}$ \\
$08-07-87$ & $58.74 \mathrm{~S}$ & $07-23-89$ & $63.05 \mathrm{~S}$ & $07-16-91$ & $67.72 \mathrm{~S}$ & $06-16-93$ & $71.86 \mathrm{~S}$ \\
$09-04-87$ & $58.90 \mathrm{~S}$ & $08-09-89$ & $63.04 \mathrm{~S}$ & $07-24-91$ & $67.71 \mathrm{~S}$ & $07-20-93$ & $71.89 \mathrm{~S}$ \\
$12-16-87$ & $59.22 \mathrm{~S}$ & $09-29-89$ & $63.33 \mathrm{~S}$ & $08-21-91$ & $67.95 \mathrm{~S}$ & $08-26-93$ & $72.08 \mathrm{~S}$ \\
$01-04-88$ & $59.54 \mathrm{~S}$ & $10-31-89$ & $63.99 \mathrm{~S}$ & $09-25-91$ & $68.07 \mathrm{~S}$ & $09-17-93$ & $72.06 \mathrm{~S}$ \\
$02-03-88$ & $59.75 \mathrm{~S}$ & $11-28-89$ & $64.12 \mathrm{~S}$ & $11-19-91$ & $68.46 \mathrm{~S}$ & $10-14-93$ & $72.23 \mathrm{~V}$ \\
$03-08-88$ & $59.99 \mathrm{~S}$ & $12-12-89$ & $64.11 \mathrm{~S}$ & $12-17-91$ & $68.48 \mathrm{~S}$ & $10-22-93$ & $72.30 \mathrm{~S}$ \\
$04-05-88$ & $60.19 \mathrm{~S}$ & $01-11-90$ & $64.18 \mathrm{~S}$ & $01-18-92$ & $68.69 \mathrm{~S}$ & $12-01-93$ & $72.49 \mathrm{~S}$ \\
$04-22-88$ & $60.06 \mathrm{~S}$ & $02-07-90$ & $64.33 \mathrm{~S}$ & $03-13-92$ & $69.00 \mathrm{~V}$ & $12-02-93$ & $72.57 \mathrm{~S}$ \\
$05-03-88$ & $60.28 \mathrm{~S}$ & $03-07-90$ & $64.52 \mathrm{~S}$ & $04-07-92$ & $69.17 \mathrm{~S}$ & $12-03-93$ & $72.52 \mathrm{~S}$ \\
$06-07-88$ & $60.41 \mathrm{~S}$ & $04-23-90$ & $64.77 \mathrm{~S}$ & $04-10-92$ & $69.15 \mathrm{~S}$ & $12-15-93$ & $72.40 \mathrm{~S}$ \\
$07-06-88$ & $60.61 \mathrm{~S}$ & $05-30-90$ & $65.08 \mathrm{~S}$ & $06-11-92$ & $69.56 \mathrm{~S}$ & &. \\
$07-27-88$ & $60.82 \mathrm{~S}$ & $06-28-90$ & $65.23 \mathrm{~S}$ & $07-09-92$ & $70.86 \mathrm{~S}$ & $70.73 \mathrm{~S}$ &
\end{tabular}

\section{Highest $57.61 \quad 01-05-87$ Lowest $72.57 \quad 12-02-93$ \\ Period of record Highest $57.61 \quad 01-05-87 \quad$ Lowest $73.13 \quad 05-19-94$ Record begins 11-12-84 113 Entries}

\section{Site identifier: $\mathbf{L V}-15$ \\ Site number: 373913118551401}

About 2.4 miles northeast of Mammoth Lakes and 0.9 mile north of junction of Highways 395 and 203. Drilled observation well. Diameter 2 inches, depth 57 feet, depth measured 14 feet in 1992, perforated 55 to 57 feet. Altitude of land-surface datum 7,340 feet. Water-level records available 1982-94.

\begin{tabular}{llrrrrrr}
\hline $08-26-87$ & $0.19 \mathrm{~S}$ & $04-25-90$ & $6.35 \mathrm{~S}$ & $11-19-91$ & $13.05 \mathrm{~S}$ & $07-21-93$ & SD \\
$11-28-88$ & $1.98 \mathrm{~S}$ & $07-17-90$ & $7.20 \mathrm{~S}$ & $04-07-92$ & SD & $11-10-93$ & SD \\
$04-25-89$ & $2.14 \mathrm{~S}$ & $11-15-90$ & $8.45 \mathrm{~S}$ & $07-21-92$ & SD & & \\
$08-09-89$ & $4.37 \mathrm{~S}$ & $04-13-91$ & $10.12 \mathrm{~S}$ & $11-15-92$ & SD & & \\
$11-21-89$ & $5.35 \mathrm{~S}$ & $07-16-91$ & $10.96 \mathrm{~S}$ & $05-12-93$ & SD & &
\end{tabular}

Highest $\quad 0.19 \quad 08-26-87$

Lowest $\quad 13.05 \quad 11-19-91$

Period of record Highest $0.19 \quad 08-26-87$ Lowest $13.05 \quad 11-19-91$

Record begins 08-04-82 20 Entries 
Table 36. Ground-water levels in selected wells, Long Valley Caldera, Mono County, California, 1987-93-Continued

\begin{tabular}{|c|c|c|c|c|c|c|c|}
\hline Date & $\begin{array}{c}\text { Water } \\
\text { level MW }\end{array}$ & Date & $\begin{array}{c}\text { Water } \\
\text { level MW }\end{array}$ & Date & $\begin{array}{c}\text { Water } \\
\text { level MW }\end{array}$ & Date & $\begin{array}{c}\text { Water } \\
\text { level MW }\end{array}$ \\
\hline
\end{tabular}

Site identifier: $\mathrm{LV}-18$

Site number: $\mathbf{3 7 3 9 1 8 1 1 8 4 8 1 9 0 1}$

About 8.8 miles east of Mammoth Lakes and 1.9 miles northeast of Whitmore Hot Springs. Drilled observation well. Diameter 2 inches, depth 71 feet, perforated 69 to 71 feet. Altitude of land-surface datum 6,966.0 feet. Water-level records available $1972-73,1982-94$.

\begin{tabular}{|c|c|c|c|c|c|c|c|c|c|}
\hline $04-23-87$ & \multicolumn{2}{|c|}{$18.15 \mathrm{~S}$} & $04-26-89$ & $19.20 \mathrm{~S}$ & & $04-10-91$ & $19.54 \mathrm{~S}$ & $05-13-93$ & $17.50 \mathrm{~S}$ \\
\hline $08-29-87$ & \multicolumn{2}{|c|}{$18.61 \mathrm{~S}$} & $08-08-89$ & $19.45 \mathrm{~S}$ & & $07-17-91$ & $19.53 \mathrm{~S}$ & $07-20-93$ & $18.24 \mathrm{~S}$ \\
\hline $12-18-87$ & \multicolumn{2}{|c|}{$18.84 \mathrm{~S}$} & $11-22-89$ & $19.54 \mathrm{~S}$ & & $11-20-91$ & $19.73 \mathrm{~S}$ & $11-10-93$ & $18.76 \mathrm{~S}$ \\
\hline $04-21-88$ & \multicolumn{2}{|c|}{$19.26 \mathrm{~S}$} & $04-27-90$ & $18.77 \mathrm{~S}$ & & $04-08-92$ & $19.77 \mathrm{~S}$ & & \\
\hline $07-28-88$ & \multicolumn{2}{|c|}{$19.00 \mathrm{~S}$} & $07-17-90$ & $19.63 \mathrm{~S}$ & & $07-23-92$ & $19.98 \mathrm{~S}$ & & \\
\hline $11-28-88$ & \multicolumn{2}{|c|}{$19.16 \mathrm{~S}$} & $11-14-90$ & $20.16 \mathrm{~S}$ & & $11-19-92$ & $19.99 \mathrm{~S}$ & & \\
\hline Highest & 17.50 & $05-13-93$ & & & & & & & \\
\hline Lowest & 20.16 & $11-14-90$ & & & & & & & \\
\hline \multirow{2}{*}{\multicolumn{2}{|c|}{$\begin{array}{l}\text { Period of record } \\
\text { Record begins }\end{array}$}} & Highest 12.44 & $4 \quad 06-02-83$ & Lowest & 21.00 & $06-02-72$ & & & \\
\hline & & $06-02-72 \quad 39$ & Entries & & & & & & \\
\hline
\end{tabular}

Site identifier: LV-19

Site number: 373754118501701

About 7 miles southeast of Mammoth Lakes and 1.4 miles west of Whitmore Hot Springs. Drilled observation well. Diameter 2 inches, depth 98.6 feet, perforated 96.6 to 98.6 feet. Altitude of land-surface datum 7,090.40 feet.

Water-level records available 1972-73, 1986-94.

\begin{tabular}{llllllll}
\hline $04-21-87$ & $35.40 \mathrm{~S}$ & $04-24-90$ & $38.44 \mathrm{~S}$ & $07-17-91$ & $37.50 \mathrm{~S}$ & $12-23-92$ & $39.35 \mathrm{~S}$ \\
$08-30-87$ & $34.83 \mathrm{~S}$ & $05-23-90$ & $38.40 \mathrm{~S}$ & $08-22-91$ & $36.95 \mathrm{~S}$ & $01-13-93$ & $39.35 \mathrm{~S}$ \\
$11-13-87$ & $35.90 \mathrm{~S}$ & $06-12-90$ & $38.42 \mathrm{~S}$ & $09-18-91$ & $37.24 \mathrm{~S}$ & $03-23-93$ & $39.25 \mathrm{~S}$ \\
$04-21-88$ & $36.87 \mathrm{~S}$ & $07-16-90$ & $38.08 \mathrm{~S}$ & $10-21-91$ & $37.60 \mathrm{~S}$ & $04-13-93$ & $38.43 \mathrm{~S}$ \\
$07-27-88$ & $36.34 \mathrm{~S}$ & $08-27-90$ & $37.76 \mathrm{~S}$ & $11-21-91$ & $37.96 \mathrm{~S}$ & $05-11-93$ & $37.51 \mathrm{~S}$ \\
$11-28-88$ & $37.18 \mathrm{~S}$ & $09-24-90$ & $37.95 \mathrm{~S}$ & $12-11-91$ & $38.13 \mathrm{~S}$ & $06-16-93$ & $37.43 \mathrm{~S}$ \\
$04-25-89$ & $37.80 \mathrm{~S}$ & $10-15-90$ & $38.07 \mathrm{~S}$ & $01-15-92$ & $38.44 \mathrm{~S}$ & $07-23-93$ & $36.47 \mathrm{~S}$ \\
$08-09-89$ & $37.45 \mathrm{~S}$ & $11-15-90$ & $38.25 \mathrm{~S}$ & $02-20-92$ & $38.65 \mathrm{~S}$ & $08-26-93$ & $35.70 \mathrm{~S}$ \\
$09-20-89$ & $37.46 \mathrm{~S}$ & $12-17-90$ & $38.50 \mathrm{~S}$ & $03-10-92$ & $38.74 \mathrm{~S}$ & $09-07-93$ & $35.62 \mathrm{~S}$ \\
$10-18-89$ & $37.74 \mathrm{~S}$ & $01-15-91$ & $38.61 \mathrm{~S}$ & $04-08-92$ & $38.80 \mathrm{~S}$ & $10-14-93$ & $35.86 \mathrm{~S}$ \\
$11-22-89$ & $37.86 \mathrm{~S}$ & $02-14-91$ & $38.80 \mathrm{~S}$ & $06-10-92$ & $38.82 \mathrm{~S}$ & $11-10-93$ & $36.24 \mathrm{~S}$ \\
$12-19-89$ & $38.00 \mathrm{~S}$ & $03-20-91$ & $38.74 \mathrm{~S}$ & $07-22-92$ & $38.81 \mathrm{~S}$ & $12-15-93$ & $37.00 \mathrm{~V}$ \\
$01-08-90$ & $38.10 \mathrm{~S}$ & $04-10-91$ & $38.72 \mathrm{~S}$ & $09-16-92$ & $39.04 \mathrm{~S}$ & & \\
$02-21-90$ & $38.29 \mathrm{~S}$ & $05-14-91$ & $38.72 \mathrm{~S}$ & $10-05-92$ & $39.08 \mathrm{~S}$ & & \\
$03-20-90$ & $38.36 \mathrm{~S}$ & $06-19-91$ & $38.45 \mathrm{~S}$ & $11-19-92$ & $39.20 \mathrm{~S}$ & &
\end{tabular}

Highest $\quad 34.83 \quad 08-30-87$

Lowest $\quad 39.35 \quad 12-23-92 \quad 01-13-93$

Period of record Highest $33.00 \quad 11-13-86 \quad$ Lowest $39.35 \quad 12-23-92 \quad 01-13-93$

Record begins 06-07-72 66 Entries 
Table 36. Ground-water levels in selected wells, Long Valley Caldera, Mono County, California, 1987-93-Continued

\begin{tabular}{|c|c|c|c|c|c|c|c|}
\hline Date & $\begin{array}{c}\text { Water } \\
\text { level MW }\end{array}$ & Date & $\begin{array}{c}\text { Water } \\
\text { level MW }\end{array}$ & Date & $\begin{array}{c}\text { Water } \\
\text { level MW }\end{array}$ & Date & $\begin{array}{c}\text { Water } \\
\text { level MW }\end{array}$ \\
\hline
\end{tabular}

About 4.5 miles southeast of Mammoth Lakes. Drilled observation well. Diameter 2 inches, depth 44 feet, depth measured 41.5 feet in 1992, perforated 40 to 42 feet. Altitude of land-surface datum 7,167.39 feet. Water-level records available 1972-73, 1982-94.

\begin{tabular}{llllllll}
\hline $04-21-87$ & $37.13 \mathrm{~S}$ & $04-25-89$ & SD & $04-10-91$ & SD & $11-19-92$ & SD \\
$08-29-87$ & $38.17 \mathrm{~S}$ & $08-08-89$ & SD & $07-17-91$ & $38.86 \mathrm{~S}$ & $05-13-93$ & SD \\
$12-18-87$ & $40.27 \mathrm{~S}$ & $11-29-89$ & SD & $08-27-91$ & SD & $07-20-93$ & $32.57 \mathrm{~S}$ \\
$03-04-88$ & $40.98 \mathrm{~S}$ & $04-24-90$ & SD & $11-20-91$ & SD & $08-20-93$ & 34.54 S \\
$04-28-88$ & $41.63 \mathrm{~S}$ & $07-16-90$ & SD & $04-08-92$ & SD & $11-11-93$ & SD \\
$07-28-88$ & $39.46 \mathrm{~S}$ & $11-15-90$ & SD & $07-21-92$ & SD & &
\end{tabular}

Highest $\quad 32.57 \quad 07-20-93$

Lowest $\quad 41.63 \quad 04-28-88$

Period of record Highest $24.07 \quad 05-26-82 \quad$ Lowest $42.65 \quad 05-17-72$

Record begins $\quad 05-17-72 \quad 39$ Entries

Site identifier: LV-25

Site number: 374233118482701

About 8.9 miles northeast of Mammoth Lakes and 0.8 mile north of Cashbaugh Ranch. Drilled observation well.

Diameter 2 inches, depth 18 feet, depth measured 5.45 feet in 1992, perforated 16 to 18 feet. Altitude of land-surface datum 6.873 .81 feet. Water-level records available 1972-73, 1982-94.

\begin{tabular}{|c|c|c|c|c|c|c|c|c|c|}
\hline $07-23-87$ & 5.59 & & $04-26-89$ & $8.44 \mathrm{~S}$ & & $04-12-91$ & $1.20 \mathrm{~S}$ & $05-12-93$ & $2.06 \mathrm{~S}$ \\
\hline $08-28-87$ & 8.09 & & $08-08-89$ & $9.00 \mathrm{~S}$ & & $07-16-91$ & $3.23 \mathrm{~S}$ & $07-20-93$ & $2.36 \mathrm{~S}$ \\
\hline $12-16-87$ & 9.40 & & $11-21-89$ & $3.06 \mathrm{~S}$ & & $11-19-91$ & $3.75 \mathrm{~S}$ & $11-09-93$ & $4.40 \mathrm{~S}$ \\
\hline $04-28-88$ & 7.59 & & $04-25-90$ & $1.58 \mathrm{~S}$ & & $04-07-92$ & $1.96 \mathrm{~S}$ & & \\
\hline $07-27-88$ & 8.28 & & $07-17-90$ & $1.98 \mathrm{~S}$ & & $07-21-92$ & $2.65 \mathrm{~S}$ & & \\
\hline $11-28-88$ & 10.28 & & $11-14-90$ & $3.67 \mathrm{~S}$ & & $11-19-92$ & $4.05 \mathrm{~S}$ & & \\
\hline Highest & 1.20 & $04-12-91$ & & & & & & & \\
\hline Lowest & 10.28 & $11-28-88$ & & & & & & & \\
\hline \multirow{2}{*}{\multicolumn{2}{|c|}{$\begin{array}{l}\text { Period of record } \\
\text { Record begins }\end{array}$}} & Highest & $0.62 \quad 06-03-83$ & Lowest & 10.44 & \multirow{2}{*}{\multicolumn{2}{|c|}{$07-25-72$}} & & \\
\hline & & $07-25-72$ & 39 Entries & & & & & & \\
\hline
\end{tabular}

\section{Site identifier: $\mathbf{L V - 3}$}

Site number: 373759118474101

About 9.9 miles east of Mammoth Lakes and 1.6 miles northeast of Whitmore Hot Springs. Drilled observation well. Diameter 2 inches, depth 98 feet, perforated 96 to 98 feet. Altitude of land-surface datum 6,918 feet. Water-level records available 1972-73, 1982-85, 1988.

\begin{tabular}{llllll}
\hline $04-21-88$ & $44.51 \mathrm{~S}$ & $07-27-88$ & $45.52 \mathrm{~S}$ & $11-28-88$ & $46.14 \mathrm{~S}$
\end{tabular}

Highest $\quad 44.51 \quad 04-21-88$

Lowest $\quad 46.14 \quad 11-28-88$

Period of record Highest $44.32 \quad 09-11-73 \quad$ Lowest $46.14 \quad 11-28-88$

Record available from 05-17-72 to 11-28-88 11 Entries 
Table 36. Ground-water levels in selected wells, Long Valley Caldera, Mono County, California, 1987-93-Continued

\begin{tabular}{|c|c|c|c|c|c|c|c|}
\hline Date & $\begin{array}{c}\text { Water } \\
\text { level MW }\end{array}$ & Date & $\begin{array}{c}\text { Water } \\
\text { level MW }\end{array}$ & Date & $\begin{array}{c}\text { Water } \\
\text { level MW }\end{array}$ & Date & $\begin{array}{c}\text { Water } \\
\text { level MW }\end{array}$ \\
\hline
\end{tabular}

Site identifier: $\mathrm{LV}-\mathbf{3 0}$

Site number: 374109118484101

About 8.9 miles northeast of Mammoth Lakes and 0.1 mile north of Cashbaugh Ranch. Drilled observation well.

Diameter 2 inches, depth 9.1 feet, depth measured 5.9 feet in 1991, perforated 7.1 to 9.1 feet. Altitude of land-surface datum 6,895.62 feet. Water-level records available 1982-94.

\begin{tabular}{|c|c|c|c|c|c|c|c|c|c|}
\hline $04-23-87$ & \multicolumn{2}{|c|}{$3.03 \mathrm{~S}$} & $04-26-89$ & $1.69 \mathrm{~S}$ & & $04-12-91$ & $1.50 \mathrm{~S}$ & $05-12-93$ & $0.38 \mathrm{~S}$ \\
\hline $08-28-87$ & \multicolumn{2}{|c|}{$2.64 \mathrm{~S}$} & $08-08-89$ & $2.59 \mathrm{~S}$ & & $07-16-91$ & $.58 \mathrm{~S}$ & $07-20-93$ & $5.56 \mathrm{~S}$ \\
\hline $12-16-87$ & \multicolumn{2}{|c|}{$4.68 \mathrm{~S}$} & $11-21-89$ & $4.92 \mathrm{~S}$ & & $11-19-91$ & $4.94 \mathrm{~S}$ & $11-09-93$ & $4.11 \mathrm{~S}$ \\
\hline $04-28-88$ & \multicolumn{2}{|c|}{$.44 \mathrm{~S}$} & $04-24-90$ & $1.32 \mathrm{~S}$ & & $04-07-92$ & $1.05 \mathrm{~S}$ & & \\
\hline $07-27-88$ & \multicolumn{2}{|c|}{$1.18 \mathrm{~S}$} & $07-17-90$ & $1.06 \mathrm{~S}$ & & $07-21-92$ & $1.94 \mathrm{~S}$ & & \\
\hline $11-28-88$ & \multicolumn{2}{|c|}{$5.13 \mathrm{~S}$} & $11-14-90$ & $4.89 \mathrm{~S}$ & & $11-19-92$ & $5.42 \mathrm{~S}$ & & \\
\hline Highest & 0.38 & $05-12-93$ & & & & & & & \\
\hline Lowest & 5.56 & $07-20-93$ & & & & & & & \\
\hline \multirow{2}{*}{\multicolumn{2}{|c|}{$\begin{array}{l}\text { Period of record } \\
\text { Record begins }\end{array}$}} & Highest & $0.05 \quad 06-03-83$ & Lowest & 5.56 & $07-20-93$ & & & \\
\hline & & $10-20-72$ & 38 Entries & & & & & & \\
\hline
\end{tabular}

\section{Site identifier: $\mathrm{LV}-44$ \\ Site number: 373917118551402}

About 2.4 miles northeast of Mammoth Lakes and 1 mile north of junction of Highways 395 and 203. Drilled observation well. Diameter 2 inches, depth 79.7 feet, depth measured 55.2 feet in 1992, well point 77.7 to 79.7 feet. Altitude of land-surface datum 7,340 feet. Water-level records available 1973-94.

\begin{tabular}{lrllllll}
\hline $04-19-87$ & $+0.79 \mathrm{~S}$ & $11-28-88$ & $2.81 \mathrm{~S}$ & $11-15-90$ & $10.88 \mathrm{~S}$ & $07-21-92$ & $24.75 \mathrm{~S}$ \\
$08-26-87$ & $+.75 \mathrm{~S}$ & $04-25-89$ & $4.20 \mathrm{~S}$ & $04-10-91$ & $13.18 \mathrm{~S}$ & $11-19-92$ & $27.75 \mathrm{~S}$ \\
$12-16-87$ & $+.15 \mathrm{~S}$ & $08-09-89$ & $5.56 \mathrm{~S}$ & $07-16-91$ & $15.00 \mathrm{~S}$ & $05-12-93$ & $28.30 \mathrm{~S}$ \\
$04-28-88$ & $.16 \mathrm{~S}$ & $04-25-90$ & $8.65 \mathrm{~S}$ & $11-19-91$ & $18.45 \mathrm{~S}$ & $07-21-93$ & $30.10 \mathrm{~S}$ \\
$07-28-88$ & $1.12 \mathrm{~S}$ & $07-17-90$ & $9.45 \mathrm{~S}$ & $04-07-92$ & $22.21 \mathrm{~S}$ & $11-10-93$ & $32.55 \mathrm{~S}$
\end{tabular}

Highest $\quad+0.79 \quad 04-19-87$

Lowest $32.55 \quad 11-10-93$

Period of record Highest $+1.00 \quad 05-21-86 \quad$ Lowest $35.80 \quad 04-06-94$

Record begins 08-07-73 27 Entries

\section{Site identifier: $L V-45$}

Site number: 373914118472201

About 9.6 miles east of Mammoth Lakes and 2 miles northeast of Whitmore Hot Springs. Drilled observation well. Diameter 2 inches, depth 96 feet. Altitude of land-surface datum 6,985.38 feet. Water-level records available 1982-94.

\begin{tabular}{llllllll}
\hline $04-23-87$ & $30.69 \mathrm{~S}$ & $04-26-89$ & $34.43 \mathrm{~S}$ & $04-10-91$ & $37.84 \mathrm{~S}$ & $05-13-93$ & $40.05 \mathrm{~S}$ \\
$08-28-87$ & $31.61 \mathrm{~S}$ & $08-08-89$ & $34.45 \mathrm{~S}$ & $07-17-91$ & $38.53 \mathrm{~S}$ & $07-20-93$ & $40.09 \mathrm{~S}$ \\
$12-18-87$ & $33.29 \mathrm{~S}$ & $11-22-89$ & $34.61 \mathrm{~S}$ & $11-20-91$ & $39.07 \mathrm{~S}$ & $11-10-93$ & $40.10 \mathrm{~S}$ \\
$04-21-88$ & $34.31 \mathrm{~S}$ & $04-24-90$ & $35.13 \mathrm{~S}$ & $04-08-92$ & $39.45 \mathrm{~S}$ & & \\
$07-27-88$ & $34.36 \mathrm{~S}$ & $07-17-90$ & $35.75 \mathrm{~S}$ & $07-23-92$ & $39.59 \mathrm{~S}$ & & \\
$11-28-88$ & $34.40 \mathrm{~S}$ & $11-14-90$ & $36.83 \mathrm{~S}$ & $11-19-92$ & $39.88 \mathrm{~S}$ & &
\end{tabular}

Highest $\quad 30.69 \quad 04-23-87$

Lowest $40.10 \quad 11-10-93$

Period of record Highest $26.63 \quad 10-19-85 \quad$ Lowest $40.15 \quad 04-06-94$

Record begins $\quad 05-25-82 \quad 36$ Entries 
Table 36. Ground-water levels in selected wells, Long Valley Caldera, Mono County, California, 1987-93-Continued

\begin{tabular}{|c|c|c|c|c|c|c|c|}
\hline Date & $\begin{array}{c}\text { Water } \\
\text { level MW }\end{array}$ & Date & $\begin{array}{c}\text { Water } \\
\text { level MW }\end{array}$ & Date & $\begin{array}{c}\text { Water } \\
\text { level MW }\end{array}$ & Date & $\begin{array}{c}\text { Water } \\
\text { level MW }\end{array}$ \\
\hline
\end{tabular}

Site identifier: MW-1

Site number: $\mathbf{3 7 3 8 1 6 1 1 8 5 2 3 9 0 1}$

About 4.6 miles east of Mammoth Lakes near Hot Creek Fish Hatchery. Drilled observation well. Diameter 6 inches 0 to 40 feet, 2 inches 40 to 504 feet, depth 504 feet, perforated 450 to 504 feet. Altitude of land-surface datum 7046.43 feet. Water-level records available 1992-94.

\begin{tabular}{llllllll}
\hline $09-16-92$ & $34.49 \mathrm{~S}$ & $01-13-93$ & $39.67 \mathrm{~S}$ & $07-23-93$ & $26.08 \mathrm{~S}$ & $11-11-93$ & $32.50 \mathrm{~S}$ \\
$10-05-92$ & $34.72 \mathrm{~S}$ & $03-02-93$ & $38.92 \mathrm{~S}$ & $08-26-93$ & $24.68 \mathrm{~S}$ & $12-16-93$ & $34.31 \mathrm{~S}$ \\
$11-19-92$ & $36.92 \mathrm{~S}$ & $06-16-93$ & $27.61 \mathrm{~S}$ & $09-17-93$ & $29.58 \mathrm{~S}$ & &
\end{tabular}

Highest $24.68 \quad 08-26-93$

Lowest $39.67 \quad 01-13-93$

Period of record Highest $24.68 \quad 08-26-93 \quad$ Lowest $39.67 \quad 01-13-93$

Record begins $\quad 09-16-92 \quad 16$ Entries

Site identifier: MW-2

Site number: $\mathbf{3 7 3 8 4 1 1 1 8 5 1 2 9 0 2}$

About 5.6 miles east of Mammoth Lakes near Hot Creek Fish Hatchery. Drilled observation well. Diameter 5 inches 0 to 40 feet, 2 inches 40 to 255 feet, depth 255 feet, perforated 230 to 255 feet. Altitude of land-surface datum 7,057.08 feet. Water-level records available 1987-94.

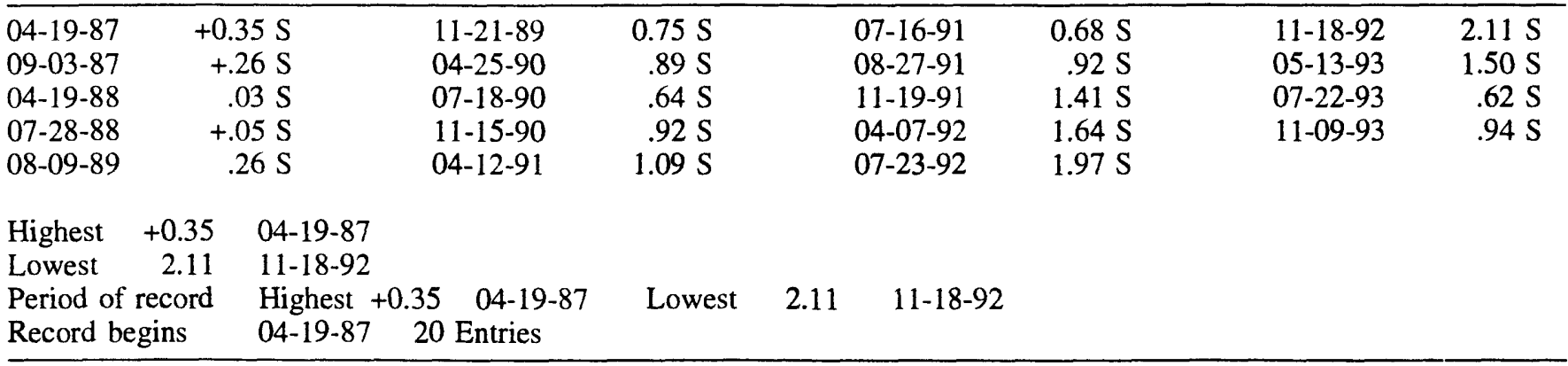

Site identifier: MW-4

Site number: $\mathbf{3 7 3 9 0 7 1 1 8 5 0 3 8 0 1}$

About 6.5 miles east of Mammoth Lakes near Hot Creek Fish Hatchery. Drilled observation well. Diameter 6 inches 0 to 80 feet, 2 inches 80 to 340 feet, depth 340 feet, perforated 320 to 340 feet. Altitude of land-surface datum 7,081.26 feet. Water-level records available 1986-94.

\begin{tabular}{llllllll}
\hline $07-28-88$ & $45.50 \mathrm{~S}$ & $07-18-90$ & $46.36 \mathrm{~S}$ & $11-19-91$ & $48.64 \mathrm{~S}$ & $07-22-93$ & $48.85 \mathrm{~S}$ \\
$11-28-88$ & $45.82 \mathrm{~S}$ & $11-15-90$ & $46.00 \mathrm{~S}$ & $04-07-92$ & $49.26 \mathrm{~S}$ & $11-09-93$ & $49.60 \mathrm{~S}$ \\
$08-09-89$ & $45.60 \mathrm{~S}$ & $04-12-91$ & $46.40 \mathrm{~S}$ & $07-23-92$ & $48.2 \mathrm{~S}$ & & \\
$11-29-89$ & $46.16 \mathrm{~S}$ & $07-16-91$ & $46.62 \mathrm{~S}$ & $11-18-92$ & $49.23 \mathrm{~S}$ & & \\
$04-25-90$ & $46.59 \mathrm{~S}$ & $08-27-91$ & $47.20 \mathrm{~S}$ & $05-13-93$ & $50.20 \mathrm{~S}$ & &
\end{tabular}

Highest $\quad 45.50 \quad 07-28-88$

Lowest $\quad 50.20 \quad 05-13-93$

Period of record Highest $43.60 \quad 08-12-86 \quad$ Lowest $50.20 \quad 05-13-93$

Record begins 05-09-86 21 Entries 
Table 36. Ground-water levels in selected wells, Long Valley Caldera, Mono County, California, 1987-93-Continued

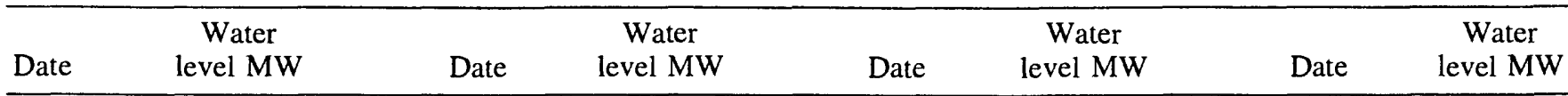

Site identifier: MW-5

Site number: $\mathbf{3 7 3 8 4 1 1 1 8 5 1 2 9 0 1}$

About 5.6 miles east of Mammoth Lakes near Hot Creek Fish Hatchery. Drilled observation well. Diameter 6 inches 0 to 50 feet, 2 inches 50 to 496 feet, depth 496 feet, perforated 460 to 496 feet. Altitude of land-surface datum 7,054.12 feet. Water-level records available 1986-94.

\begin{tabular}{|c|c|c|c|c|c|c|c|c|c|}
\hline $04-19-87$ & \multicolumn{2}{|c|}{$2.99 \mathrm{~S}$} & $01-08-90$ & $5.60 \mathrm{~S}$ & & $07-16-91$ & $5.30 \mathrm{~S}$ & $09-16-92$ & $7.08 \mathrm{~S}$ \\
\hline $07-28-88$ & \multicolumn{2}{|c|}{$3.82 \mathrm{~S}$} & $02-21-90$ & $5.78 \mathrm{~S}$ & & $08-27-91$ & $5.20 \mathrm{~S}$ & $11-18-92$ & $7.20 \mathrm{~S}$ \\
\hline $04-25-89$ & \multicolumn{2}{|c|}{$5.33 \mathrm{~S}$} & $03-20-90$ & $5.88 \mathrm{~S}$ & & $11-19-91$ & $5.92 \mathrm{~S}$ & $05-13-93$ & $6.31 \mathrm{~S}$ \\
\hline $08-09-89$ & \multicolumn{2}{|c|}{$4.89 \mathrm{~S}$} & $04-25-90$ & $5.98 \mathrm{~S}$ & & $04-07-92$ & $6.69 \mathrm{~S}$ & $07-22-93$ & $5.20 \mathrm{~S}$ \\
\hline $09-20-89$ & \multicolumn{2}{|c|}{$5.01 \mathrm{~S}$} & $05-24-90$ & $5.90 \mathrm{~S}$ & & $06-24-92$ & $6.63 \mathrm{~S}$ & $11-09-93$ & $4.94 \mathrm{~S}$ \\
\hline $10-18-89$ & \multicolumn{2}{|c|}{$5.20 \mathrm{~S}$} & $07-18-90$ & $4.61 \mathrm{~S}$ & & $07-07-92$ & $6.97 \mathrm{~S}$ & & \\
\hline $11-21-89$ & \multirow{2}{*}{\multicolumn{2}{|c|}{$\begin{array}{l}5.42 \mathrm{~S} \\
5.50 \mathrm{~S}\end{array}$}} & $11-15-90$ & $5.88 \mathrm{~S}$ & & $07-23-92$ & $6.96 \mathrm{~S}$ & & \\
\hline $12-19-89$ & & & $04-12-91$ & $6.32 \mathrm{~S}$ & & $08-03-92$ & $6.98 \mathrm{~S}$ & & \\
\hline Highest & 2.99 & $04-19-87$ & & & & & & & \\
\hline Lowest & 7.20 & $11-18-92$ & & & & & & & \\
\hline \multicolumn{2}{|c|}{$\begin{array}{l}\text { Period of record } \\
\text { Record begins }\end{array}$} & $\begin{array}{l}\text { Highest } \\
05-10-86\end{array}$ & $\begin{array}{l}0.2109-01-86 \\
33 \text { Entries }\end{array}$ & Lowest & 7.20 & $1-18-92$ & & & \\
\hline
\end{tabular}

\section{Site identifier: MWD 5 \\ Site number: $\mathbf{3 7 3 7 5 7 1 1 8 5 9 1 8 0 1}$}

In Old Mammoth near Mammoth Creek. Drilled unused well. Diameter 8 inches, depth 357.5 feet, perforated 59.8 to 112.7 and 132.7 to 357.5 feet. Altitude of land-surface datum 7,985.28 feet. Water-level records available 1988-94.

\begin{tabular}{|c|c|c|c|c|c|c|c|c|c|}
\hline 03-03-88 & \multicolumn{2}{|c|}{$0.58 \mathrm{~S}$} & $11-29-89$ & $3.41 \mathrm{~S}$ & & $07-18-91$ & $3.19 \mathrm{~S}$ & $11-19-92$ & $7.10 \mathrm{~S}$ \\
\hline $04-21-88$ & \multicolumn{2}{|c|}{$.22 \mathrm{~S}$} & $04-24-90$ & $2.45 \mathrm{~S}$ & & $08-27-91$ & $3.94 \mathrm{~S}$ & $05-14-93$ & $2.58 \mathrm{~S}$ \\
\hline $11-29-88$ & \multicolumn{2}{|c|}{$1.83 \mathrm{~S}$} & $08-02-90$ & $3.90 \mathrm{~S}$ & & $11-18-91$ & $4.54 \mathrm{~S}$ & $07-19-93$ & $3.32 \mathrm{~S}$ \\
\hline $04-25-89$ & \multicolumn{2}{|c|}{$.71 \mathrm{~S}$} & $11-16-90$ & $3.94 \mathrm{~S}$ & & $04-09-92$ & $3.77 \mathrm{~S}$ & $11-08-93$ & $3.40 \mathrm{~S}$ \\
\hline 08-08-89 & \multicolumn{2}{|c|}{$2.18 \mathrm{~S}$} & $04-11-91$ & $3.76 \mathrm{~S}$ & & $07-21-92$ & $4.28 \mathrm{~S}$ & $12-15-93$ & $4.59 \mathrm{~S}$ \\
\hline Highest & 0.22 & $04-21-88$ & & & & & & & \\
\hline Lowest & 7.10 & $11-19-92$ & & & & & & & \\
\hline \multirow{2}{*}{\multicolumn{2}{|c|}{$\begin{array}{l}\text { Period of record } \\
\text { Record begins }\end{array}$}} & Highest & $0.22 \quad 04-21-88$ & Lowest & 7.10 & $11-19-92$ & & & \\
\hline & & $03-03-88$ & 21 Entries & & & & & & \\
\hline
\end{tabular}

\section{Site identifier: MWD M-10 \\ Site number: 373719118585301}

About 0.8 mile south of Mammoth Lakes near the Snow Creek development. Drilled observation well. Diameter 4 inches, depth measured 27.2 feet. perforated 7 to 27 feet. Altitude of land-surface datum 7,939.63 feet. Water-level records available $1988-94$.

\begin{tabular}{|c|c|c|c|c|c|c|c|}
\hline $07-28-88$ & $13.27 \mathrm{~S}$ & $01-09-90$ & SD & $07-18-91$ & $\mathrm{SD}$ & $05-14-93$ & SD \\
\hline $10-16-88$ & $22.49 \mathrm{~S}$ & $02-20-90$ & $26.90 \mathrm{~S}$ & $08-27-91$ & SD & $07-19-93$ & SD \\
\hline $11-29-88$ & $26.98 \mathrm{~S}$ & $03-19-90$ & $27.01 \mathrm{~S}$ & $11-18-91$ & SD & $11-08-93$ & SD \\
\hline $10-18-89$ & SD & $04-24-90$ & $27.03 \mathrm{~S}$ & $04-09-92$ & $\mathrm{SD}$ & & \\
\hline $11-22-89$ & SD & $05-24-90$ & $27.14 \mathrm{~S}$ & $07-21-92$ & SD & & \\
\hline $12-20-89$ & SD & $11-16-90$ & SD & $11-19-92$ & SD & & \\
\hline
\end{tabular}

Highest $\quad 13.27 \quad 07-28-88$

Lowest $27.14 \quad 05-24-90$

Period of record Highest $13.27 \quad 07-28-88$ Lowest $27.14 \quad 05-24-90$

Record begins 07-28-88 22 Entries 
Table 36. Ground-water levels in setected wells, Long Valley Caldera, Mono County, California, 1987-93-Continued

\begin{tabular}{|c|c|c|c|c|c|c|c|}
\hline Date & $\begin{array}{c}\text { Water } \\
\text { level MW }\end{array}$ & Date & $\begin{array}{c}\text { Water } \\
\text { level MW }\end{array}$ & Date & $\begin{array}{c}\text { Water } \\
\text { level MW }\end{array}$ & Date & $\begin{array}{c}\text { Water } \\
\text { level MW }\end{array}$ \\
\hline
\end{tabular}

Site identifier: MWD M-11

Site number: 373705118585601

About 0.8 mile south of Mammoth Lakes near the Snow Creek development. Drilled observation well. Diameter 4 inches, depth 43 feet, perforated 5 to 43 feet. Altitude of land-surface datum 7,968.56 feet. Water-level records available 1988-94.

\begin{tabular}{|c|c|c|c|c|c|c|c|c|c|}
\hline $07-28-88$ & \multicolumn{2}{|c|}{$12.14 \mathrm{~S}$} & $12-20-89$ & $27.89 \mathrm{~S}$ & & $11-16-90$ & $28.82 \mathrm{~S}$ & $07-21-92$ & $22.75 \mathrm{~S}$ \\
\hline $10-16-88$ & \multicolumn{2}{|c|}{$19.18 \mathrm{~S}$} & $01-09-90$ & $29.10 \mathrm{~S}$ & & $06-16-91$ & $16.98 \mathrm{~V}$ & $11-19-92$ & $34.40 \mathrm{~S}$ \\
\hline $11-29-88$ & \multicolumn{2}{|c|}{$20.40 \mathrm{~S}$} & $02-20-90$ & $30.62 \mathrm{~S}$ & & $07-18-91$ & $19.10 \mathrm{~S}$ & $05-14-93$ & $22.34 \mathrm{~S}$ \\
\hline $04-25-89$ & \multicolumn{2}{|c|}{$21.75 \mathrm{~S}$} & $03-19-90$ & $30.85 \mathrm{~S}$ & & $08-27-91$ & $22.42 \mathrm{~S}$ & $07-19-93$ & $4.26 \mathrm{~S}$ \\
\hline $10-18-89$ & \multicolumn{2}{|c|}{$23.95 \mathrm{~S}$} & $04-24-90$ & $26.84 \mathrm{~S}$ & & $11-18-91$ & $30.46 \mathrm{~S}$ & $11-08-93$ & $23.39 \mathrm{~S}$ \\
\hline $11-22-89$ & \multicolumn{2}{|c|}{$26.19 \mathrm{~S}$} & $05-24-90$ & $27.05 \mathrm{~S}$ & & 04-09-92 & $33.67 \mathrm{~S}$ & & \\
\hline Highest & 4.26 & $07-19-93$ & & & & & & & \\
\hline Lowest & 34.40 & $11-19-92$ & & & & & & & \\
\hline \multirow{2}{*}{\multicolumn{2}{|c|}{ Period of record }} & Highest & $4.26 \quad 07-19-93$ & Lowest & 34.40 & $11-19-92$ & & & \\
\hline & & $07-28-88$ & 24 Entries & & & & & & \\
\hline
\end{tabular}

Site identifier: MWD M-12

Site number: $\mathbf{3 7 3 7 1 4 1 1 8 5 8 5 5 0 1}$

About 0.9 mile south of Mammoth Lakes near the Snow Creek development. Drilled observation well. Diameter 4 inches, depth measured 26.45 feet in 1991, original depth 27 feet, perforated 7 to 27 feet. Altitude of land-surface datum 7,952.92 feet. Water-level records available 1988-94.

\begin{tabular}{|c|c|c|c|c|c|c|c|c|}
\hline $10-16-88$ & $20.65 \mathrm{~S}$ & $01-09-90$ & 25.03 & $S$ & $04-11-91$ & SD & $11-19-92$ & SD \\
\hline $11-29-88$ & $21.32 \mathrm{~S}$ & $02-20-90$ & 25.63 & $S$ & $06-16-91$ & SD & $05-14-93$ & SD \\
\hline $04-25-89$ & $25.02 \mathrm{~S}$ & $03-19-90$ & 26.00 & $\mathrm{~S}$ & $07-18-91$ & SD & $07-19-93$ & $8.25 \mathrm{~S}$ \\
\hline $08-08-89$ & $18.83 \mathrm{~S}$ & $04-24-90$ & & SD & $08-27-91$ & SD & $11-08-93$ & $24.88 \mathrm{~S}$ \\
\hline $10-18-89$ & $23.35 \mathrm{~S}$ & $05-24-90$ & & SD & $11-18-91$ & SD & & \\
\hline $11-22-89$ & $24.17 \mathrm{~S}$ & $08-29-90$ & 24.61 & $\mathrm{~S}$ & $04-09-92$ & SD & & \\
\hline $12-20-89$ & $24.72 \mathrm{~S}$ & $11-16-90$ & & SD & $07-21-92$ & SD & & \\
\hline
\end{tabular}

Highest $\quad 8.25 \quad 07-19-93$

Lowest $26.00 \quad 03-19-90$

Period of record Highest $8.25 \quad 07-19-93 \quad$ Lowest $26.21 \quad 04-07-94$

Record begins 10-16-88 26 Entries 
Table 36. Ground-water levels in selected wells, Long Valley Caldera, Mono County, California 1987-93-Continued

\begin{tabular}{|c|c|c|c|c|c|c|c|}
\hline Date & $\begin{array}{c}\text { Water } \\
\text { level MW }\end{array}$ & Date & $\begin{array}{c}\text { Water } \\
\text { level MW }\end{array}$ & Date & $\begin{array}{c}\text { Water } \\
\text { level MW }\end{array}$ & Date & $\begin{array}{c}\text { Water } \\
\text { level MW }\end{array}$ \\
\hline
\end{tabular}

\section{Site identifier: MWD M-14 \\ Site number: 373739118582101}

About 0.3 mile south of Mammoth Lakes in the Snow Creek development. Drilled observation well. Diameter 4 inches, depth 501 feet, perforated 100 to 501 feet. Altitude of land-surface datum 7,877.79 feet. Water-level records available 1988-94.

\begin{tabular}{llllllll}
\hline $10-16-88$ & $237.00 \mathrm{~V}$ & $05-14-91$ & $347.18 \mathrm{~V}$ & $02-21-92$ & $353.84 \mathrm{~V}$ & $04-14-93$ & $360.12 \mathrm{~V}$ \\
$11-29-88$ & $252.32 \mathrm{~V}$ & $06-16-91$ & $348.22 \mathrm{~V}$ & $03-14-92$ & $352.95 \mathrm{~V}$ & $05-12-93$ & $359.45 \mathrm{~V}$ \\
$04-25-89$ & $287.73 \mathrm{~V}$ & $06-21-91$ & $347.74 \mathrm{~V}$ & $04-09-92$ & $354.91 \mathrm{~V}$ & $06-14-93$ & $357.18 \mathrm{~V}$ \\
$08-02-90$ & $335.25 \mathrm{~V}$ & $07-16-91$ & $347.56 \mathrm{~V}$ & $06-10-92$ & $356.75 \mathrm{~V}$ & $07-19-93$ & $348.93 \mathrm{~V}$ \\
$10-18-90$ & $339.55 \mathrm{~V}$ & $08-22-91$ & $346.72 \mathrm{~V}$ & $07-21-92$ & $356.50 \mathrm{~V}$ & $08-26-93$ & $343.00 \mathrm{~V}$ \\
$11-15-90$ & $340.50 \mathrm{~V}$ & $08-27-91$ & $346.69 \mathrm{~V}$ & $09-16-92$ & $356.80 \mathrm{~V}$ & $09-17-93$ & $345.22 \mathrm{~V}$ \\
$12-11-90$ & $341.20 \mathrm{~V}$ & $09-17-91$ & $347.91 \mathrm{~V}$ & $10-05-92$ & $357.88 \mathrm{~V}$ & $10-14-93$ & $347.97 \mathrm{~V}$ \\
$12-13-90$ & $340.95 \mathrm{~V}$ & $10-21-91$ & $349.57 \mathrm{~V}$ & $11-19-92$ & $357.10 \mathrm{~V}$ & $11-08-93$ & $349.30 \mathrm{~V}$ \\
$01-14-91$ & $340.56 \mathrm{~V}$ & $11-18-91$ & $350.25 \mathrm{~V}$ & $12-16-92$ & $357.55 \mathrm{~V}$ & $12-15-93$ & $350.64 \mathrm{~V}$ \\
$02-14-91$ & $343.82 \mathrm{~V}$ & $12-18-91$ & $350.65 \mathrm{~V}$ & $01-14-93$ & $357.58 \mathrm{~V}$ & & \\
$04-30-91$ & $346.88 \mathrm{~V}$ & $01-16-92$ & $352.09 \mathrm{~V}$ & $03-25-93$ & $359.10 \mathrm{~V}$ & &
\end{tabular}

Highest $237.00 \quad 10-16-88$

Lowest $360.12 \quad 04-14-93$

Period of record Highest $237.00 \quad 10-16-88 \quad$ Lowest $360.12 \quad 04-14-93$

Record begins 10-16-88 47 Entries

Site identifier: PD

Site number: 373732118490001

About 8.2 miles southeast of Mammoth Lakes at Mono County Probation Department Camp. Drilled institutional well.

Diameter 6 inches, depth 150 feet. Altitude of land-surface datum 7,014 feet. Water-level records available 1986-94.

\begin{tabular}{llllllll}
\hline $04-23-87$ & $24.53 \mathrm{~V}$ & $11-28-88$ & $25.02 \mathrm{~S}$ & $07-17-90$ & $29.00 \mathrm{SP}$ & $04-08-92$ & $25.60 \mathrm{VR}$ \\
$09-03-87$ & $24.62 \mathrm{~S}$ & $04-25-89$ & $29.45 \mathrm{~V}$ & $11-15-90$ & $24.35 \mathrm{VP}$ & $07-22-92$ & $24.78 \mathrm{~V}$ \\
$12-18-87$ & $24.75 \mathrm{~V}$ & $08-08-89$ & $27.04 \mathrm{VP}$ & $04-11-91$ & $24.11 \mathrm{VP}$ & $11-19-92$ & $24.91 \mathrm{~S}$ \\
$04-21-88$ & $24.63 \mathrm{~V}$ & $11-29-89$ & $26.14 \mathrm{VP}$ & $07-17-91$ & $23.98 \mathrm{~V}$ & $05-14-93$ & $24.53 \mathrm{SP}$ \\
$07-27-88$ & $28.59 \mathrm{~S}$ & $04-24-90$ & $28.12 \mathrm{VP}$ & $11-20-91$ & $25.69 \mathrm{~V}$ & $07-20-93$ & $24.09 \mathrm{SR}$
\end{tabular}

Highest $23.71 \quad 11-19-92$

Lowest $29.45 \quad 04-25-89$

Period of record Highest $21.69 \quad 08-30-86 \quad$ Lowest $29.45 \quad 04-25-89$

Record begins $05-21-86$ 24 Entries

\section{Site identifier: PLV-2 \\ Site number: 374249118584501}

About 4.7 miles north of Mammoth Lakes and 1.3 miles west of Highway 395. Drilled observation well. Diameter 1.25 inches, depth 2,330 feet, perforated 1,845 to 1,855 feet. Altitude of land-surface datum 7,734 feet. Water-level records available 1984-94.

\begin{tabular}{llllllll}
\hline $04-23-87$ & $425.23 \mathrm{~V}$ & $08-08-89$ & $422.02 \mathrm{~V}$ & $07-16-91$ & $438.76 \mathrm{~V}$ & $07-19-93$ & $437.20 \mathrm{~V}$ \\
$08-30-87$ & $418.55 \mathrm{~V}$ & $04-25-90$ & $430.34 \mathrm{~V}$ & $11-19-91$ & $440.80 \mathrm{~V}$ & $11-09-93$ & $438.35 \mathrm{~V}$ \\
$07-27-88$ & $413.14 \mathrm{~V}$ & $07-18-90$ & $431.78 \mathrm{~V}$ & $04-06-92$ & $444.63 \mathrm{~V}$ & & \\
$11-23-88$ & $417.39 \mathrm{~V}$ & $11-13-90$ & $435.88 \mathrm{~V}$ & $07-20-92$ & $444.06 \mathrm{~V}$ & & \\
$04-26-89$ & $424.61 \mathrm{~V}$ & $04-11-91$ & $439.44 \mathrm{~V}$ & $11-19-92$ & $445.70 \mathrm{~V}$ & &
\end{tabular}

Highest $\quad 413.14 \quad 07-27-88$

Lowest $445.70 \quad 11-19-92$

Period of record Highest $398.00 \quad 08-30-84 \quad$ Lowest $445.70 \quad 11-19-92$

Record begins $\quad 08-30-84 \quad 38$ Entries 
Table 36. Ground-water levels in selected wells, Long Valley Caldera, Mono County, California, 1987-93-Continued

\begin{tabular}{|c|c|c|c|c|c|c|c|}
\hline Date & $\begin{array}{c}\text { Water } \\
\text { level MW }\end{array}$ & Date & $\begin{array}{c}\text { Water } \\
\text { level MW }\end{array}$ & Date & $\begin{array}{c}\text { Water } \\
\text { level MW }\end{array}$ & Date & $\begin{array}{c}\text { Water } \\
\text { level MW }\end{array}$ \\
\hline
\end{tabular}

Site identifier: RDO-8

Site number: 373924118571201

About 0.8 mile northeast of Mammoth Lakes and 0.7 mile north of U.S. Forest Service ranger station. Drilled observation well. Diameter 2.38 inches, depth 1,304 feet, original depth of hole 2,346 feet, perforated 1,100 to 1,110 feet. Altitude of land-surface datum 7,788.4 feet. Water-level records available 1986-94.

\begin{tabular}{llllllll}
\hline $01-14-87$ & $437.98 \mathrm{~V}$ & $05-03-88$ & $441.98 \mathrm{~V}$ & $02-06-90$ & $439.95 \mathrm{~V}$ & $07-12-91$ & $438.36 \mathrm{~V}$ \\
$01-29-87$ & $438.10 \mathrm{~V}$ & $06-03-88$ & $442.73 \mathrm{~V}$ & $03-08-90$ & $439.60 \mathrm{~V}$ & $07-18-91$ & $436.12 \mathrm{~V}$ \\
$02-24-87$ & $437.47 \mathrm{~V}$ & $08-02-88$ & $444.20 \mathrm{~V}$ & $04-03-90$ & $440.45 \mathrm{~V}$ & $08-06-91$ & $435.09 \mathrm{~V}$ \\
$03-09-87$ & $438.03 \mathrm{~V}$ & $08-15-88$ & $443.90 \mathrm{~V}$ & $04-23-90$ & $440.30 \mathrm{~V}$ & $08-22-91$ & $433.28 \mathrm{~V}$ \\
$03-19-87$ & $437.93 \mathrm{~V}$ & $09-02-88$ & $443.90 \mathrm{~V}$ & $05-23-90$ & $440.13 \mathrm{~V}$ & $09-25-91$ & $431.36 \mathrm{~V}$ \\
$04-09-87$ & $438.23 \mathrm{~V}$ & $09-29-88$ & $444.55 \mathrm{~V}$ & $05-30-90$ & $439.88 \mathrm{~V}$ & $11-15-91$ & $430.22 \mathrm{~V}$ \\
$04-20-87$ & $441.46 \mathrm{~V}$ & $10-03-88$ & $444.45 \mathrm{~V}$ & $06-28-90$ & $440.20 \mathrm{~V}$ & $12-17-91$ & $430.87 \mathrm{~V}$ \\
$05-29-87$ & $438.22 \mathrm{~V}$ & $10-13-88$ & $444.60 \mathrm{~V}$ & $07-12-90$ & $440.88 \mathrm{~V}$ & $01-18-92$ & $431.70 \mathrm{~V}$ \\
$06-18-87$ & $438.65 \mathrm{~V}$ & $10-17-88$ & $444.80 \mathrm{~V}$ & $08-01-90$ & $441.24 \mathrm{~V}$ & $02-21-92$ & $432.30 \mathrm{~V}$ \\
$07-14-87$ & $438.97 \mathrm{~V}$ & $11-07-88$ & $444.65 \mathrm{~V}$ & $08-21-90$ & $439.84 \mathrm{~V}$ & $03-13-92$ & $432.13 \mathrm{~V}$ \\
$08-25-87$ & $439.10 \mathrm{~V}$ & $12-07-88$ & $445.25 \mathrm{~V}$ & $09-12-90$ & $440.08 \mathrm{~V}$ & $04-10-92$ & $433.05 \mathrm{~V}$ \\
$08-29-87$ & $439.35 \mathrm{~V}$ & $01-11-89$ & $446.30 \mathrm{~V}$ & $09-24-90$ & $440.28 \mathrm{~V}$ & $06-11-92$ & $432.65 \mathrm{~V}$ \\
$08-30-87$ & $439.45 \mathrm{~V}$ & $02-16-89$ & $446.60 \mathrm{~V}$ & $10-17-90$ & $440.99 \mathrm{~V}$ & $07-20-92$ & $434.28 \mathrm{~V}$ \\
$09-13-87$ & $439.17 \mathrm{~V}$ & $03-30-89$ & $447.35 \mathrm{~V}$ & $11-13-90$ & $441.27 \mathrm{~V}$ & $10-06-92$ & $432.70 \mathrm{~V}$ \\
$12-16-87$ & $440.82 \mathrm{~V}$ & $04-25-89$ & $447.55 \mathrm{~V}$ & $11-18-90$ & $441.11 \mathrm{~V}$ & $05-11-93$ & $436.14 \mathrm{~V}$ \\
$12-17-87$ & $439.95 \mathrm{~V}$ & $06-07-89$ & $448.40 \mathrm{~V}$ & $12-13-90$ & $441.58 \mathrm{~V}$ & $06-16-93$ & $436.05 \mathrm{~V}$ \\
$12-22-87$ & $440.61 \mathrm{~V}$ & $07-07-89$ & $448.33 \mathrm{~V}$ & $12-18-90$ & $441.47 \mathrm{~V}$ & $08-25-93$ & $435.27 \mathrm{~V}$ \\
$01-06-88$ & $440.80 \mathrm{~V}$ & $07-22-89$ & $447.80 \mathrm{~V}$ & $01-18-91$ & $437.68 \mathrm{~V}$ & $08-27-93$ & $435.45 \mathrm{~V}$ \\
$01-14-88$ & $440.72 \mathrm{~V}$ & $08-08-89$ & $447.91 \mathrm{~V}$ & $02-20-91$ & $438.27 \mathrm{~V}$ & $10-21-93$ & $435.52 \mathrm{~V}$ \\
$01-27-88$ & $440.95 \mathrm{~V}$ & $10-12-89$ & $444.00 \mathrm{~V}$ & $05-01-91$ & $439.41 \mathrm{~V}$ & $11-09-93$ & $435.30 \mathrm{~V}$ \\
$02-02-88$ & $440.80 \mathrm{~V}$ & $11-01-89$ & $443.32 \mathrm{~V}$ & $05-14-91$ & $439.83 \mathrm{~V}$ & $11-09-93$ & $435.30 \mathrm{~V}$ \\
$03-04-88$ & $441.45 \mathrm{~V}$ & $12-11-89$ & $441.75 \mathrm{~V}$ & $06-13-91$ & $441.24 \mathrm{~V}$ & $12-02-93$ & $435.60 \mathrm{~V}$ \\
$04-22-88$ & $441.85 \mathrm{~V}$ & $01-11-90$ & $441.28 \mathrm{~V}$ & $06-19-91$ & $441.31 \mathrm{~V}$ & &
\end{tabular}

Highest $430.22 \quad 11-15-91$

Lowest $448.40 \quad 06-07-89$

Period of record Highest $430.22 \quad 11-15-91 \quad$ Lowest $448.40 \quad 06-07-89$

Record begins 10-21-86 100 Entries 
Table 36. Ground-water levels in selected wells, Long Valley Caldera, Mono County, California, 1987-93-Continued

\begin{tabular}{|c|c|c|c|c|c|c|c|}
\hline Date & $\begin{array}{c}\text { Water } \\
\text { level MW }\end{array}$ & Date & $\begin{array}{c}\text { Water } \\
\text { level MW }\end{array}$ & Date & $\begin{array}{c}\text { Water } \\
\text { level MW }\end{array}$ & Date & $\begin{array}{c}\text { Water } \\
\text { level MW }\end{array}$ \\
\hline
\end{tabular}

Site identifier: SC-1

Site number: 373745118554001

About 2.3 miles southeast of Mammoth Lakes. Drilled observation well. Diameter 2.5 inches, depth of hole 132 feet, open hole 40 to 132 feet. Altitude of land-surface datum 7,469.84 feet. Water-level records available 1982-94.

\begin{tabular}{llllllll}
\hline $04-28-88$ & $111.09 \mathrm{~V}$ & $06-12-90$ & $112.73 \mathrm{~V}$ & $08-21-91$ & $113.74 \mathrm{~V}$ & $10-05-92$ & $119.09 \mathrm{~V}$ \\
$07-28-88$ & $108.90 \mathrm{~S}$ & $07-16-90$ & $113.02 \mathrm{~V}$ & $08-27-91$ & $114.09 \mathrm{~V}$ & $11-19-92$ & $119.15 \mathrm{~V}$ \\
$11-28-88$ & $111.55 \mathrm{~S}$ & $08-27-90$ & $114.52 \mathrm{~V}$ & $09-17-91$ & $115.38 \mathrm{~V}$ & $03-23-93$ & $120.46 \mathrm{~V}$ \\
$04-25-89$ & $111.55 \mathrm{~V}$ & $09-24-90$ & $114.44 \mathrm{~V}$ & $10-21-91$ & $116.72 \mathrm{~V}$ & $04-14-93$ & $114.00 \mathrm{~V}$ \\
$08-08-89$ & $112.03 \mathrm{~V}$ & $10-15-90$ & $114.30 \mathrm{~V}$ & $11-18-91$ & $116.91 \mathrm{~V}$ & $05-13-93$ & $113.52 \mathrm{~V}$ \\
$09-20-89$ & $113.10 \mathrm{~V}$ & $11-15-90$ & $114.47 \mathrm{~V}$ & $12-11-91$ & $116.10 \mathrm{~S}$ & $06-14-93$ & $108.70 \mathrm{~V}$ \\
$09-29-89$ & $110.45 \mathrm{~V}$ & $12-19-90$ & $114.07 \mathrm{~V}$ & $02-21-92$ & $119.12 \mathrm{~V}$ & $07-20-93$ & $104.68 \mathrm{~V}$ \\
$10-18-89$ & $113.40 \mathrm{~V}$ & $01-14-91$ & $113.82 \mathrm{~V}$ & $03-14-92$ & $119.57 \mathrm{~V}$ & $08-20-93$ & $109.95 \mathrm{~V}$ \\
$11-22-89$ & $113.57 \mathrm{~V}$ & $02-14-91$ & $114.62 \mathrm{~V}$ & $04-06-92$ & $119.51 \mathrm{~V}$ & $09-17-93$ & $113.10 \mathrm{~V}$ \\
$12-20-89$ & $113.30 \mathrm{~V}$ & $04-11-91$ & $115.56 \mathrm{~V}$ & $05-12-92$ & $115.87 \mathrm{~V}$ & $10-14-93$ & $115.05 \mathrm{~V}$ \\
$01-08-90$ & $113.86 \mathrm{~V}$ & $05-14-91$ & $114.89 \mathrm{~V}$ & $06-08-92$ & $113.81 \mathrm{~V}$ & $11-11-93$ & $116.60 \mathrm{~V}$ \\
$04-24-90$ & $114.58 \mathrm{~V}$ & $06-19-91$ & $110.22 \mathrm{~V}$ & $07-21-92$ & $116.40 \mathrm{~V}$ & $12-15-93$ & $117.96 \mathrm{~V}$ \\
$05-23-90$ & $113.34 \mathrm{~V}$ & $07-16-91$ & $111.02 \mathrm{~V}$ & $09-16-92$ & $118.61 \mathrm{~V}$ & &
\end{tabular}

Highest $\quad 104.68 \quad 07-20-93$

Lowest $120.46 \quad 03-23-93$

Period of record Highest $69.95 \quad 07-06-83 \quad$ Lowest $120.46 \quad 03-23-93$

Record begins $08-05-82 \quad 84$ Entries

Site identifier: SC-2

Site number: 373745118554002

About 2.3 miles southeast of Mammoth Lakes. Drilled observation well. Diameter 4 inches, depth of hole 230 feet, open hole 215 to 230 feet. Altitude of land-surface datum 7,470.65 feet. Water-level records available 1983-94.

\begin{tabular}{llllllll}
\hline $01-16-87$ & $117.14 \mathrm{~V}$ & $04-04-88$ & $125.61 \mathrm{~V}$ & $06-12-90$ & $143.56 \mathrm{~V}$ & $01-19-92$ & $156.48 \mathrm{~V}$ \\
$02-12-87$ & $117.75 \mathrm{~V}$ & $04-28-88$ & $126.09 \mathrm{~V}$ & $07-16-90$ & $144.56 \mathrm{~V}$ & $03-14-92$ & $157.07 \mathrm{~V}$ \\
$03-17-87$ & $118.27 \mathrm{~V}$ & $07-28-88$ & $127.99 \mathrm{~S}$ & $08-27-90$ & $145.79 \mathrm{~V}$ & $04-06-92$ & $157.40 \mathrm{~V}$ \\
$04-08-87$ & $118.69 \mathrm{~V}$ & $09-02-88$ & $129.21 \mathrm{~V}$ & $09-24-90$ & $146.42 \mathrm{~V}$ & $05-12-92$ & $157.70 \mathrm{~V}$ \\
$04-20-87$ & $118.92 \mathrm{~V}$ & $09-26-88$ & $129.91 \mathrm{~V}$ & $10-15-90$ & $146.80 \mathrm{~V}$ & $06-08-92$ & $157.60 \mathrm{~V}$ \\
$05-06-87$ & $118.91 \mathrm{~V}$ & $09-30-88$ & $130.00 \mathrm{~V}$ & $11-15-90$ & $147.51 \mathrm{~V}$ & $07-21-92$ & $158.68 \mathrm{~V}$ \\
$05-17-87$ & $118.27 \mathrm{~V}$ & $10-13-88$ & $130.22 \mathrm{~V}$ & $12-12-90$ & $147.68 \mathrm{~V}$ & $09-16-92$ & $159.97 \mathrm{~V}$ \\
$05-20-87$ & $118.36 \mathrm{~V}$ & $11-10-88$ & $130.91 \mathrm{~V}$ & $12-19-90$ & $147.59 \mathrm{~V}$ & $10-05-92$ & $160.27 \mathrm{~V}$ \\
$05-28-87$ & $118.57 \mathrm{~V}$ & $11-28-88$ & $131.63 \mathrm{~S}$ & $01-14-91$ & $148.85 \mathrm{~V}$ & $11-19-92$ & $160.67 \mathrm{~V}$ \\
$06-05-87$ & $118.74 \mathrm{~V}$ & $03-29-89$ & $133.10 \mathrm{~V}$ & $02-14-91$ & $149.36 \mathrm{~V}$ & $03-23-93$ & $162.35 \mathrm{~V}$ \\
$06-17-87$ & $118.68 \mathrm{~V}$ & $04-25-89$ & $133.12 \mathrm{~S}$ & $04-11-91$ & $150.30 \mathrm{~V}$ & $04-14-93$ & $162.26 \mathrm{~V}$ \\
$06-26-87$ & $119.00 \mathrm{~V}$ & $08-08-89$ & $135.90 \mathrm{~S}$ & $05-14-91$ & $151.06 \mathrm{~V}$ & $05-13-93$ & $161.74 \mathrm{~V}$ \\
$07-13-87$ & $119.37 \mathrm{~V}$ & $09-20-89$ & $137.54 \mathrm{~V}$ & $06-19-91$ & $150.50 \mathrm{~V}$ & $06-14-93$ & $159.82 \mathrm{~V}$ \\
$08-11-87$ & $119.93 \mathrm{~V}$ & $09-29-89$ & $137.54 \mathrm{~V}$ & $07-16-91$ & $151.07 \mathrm{~V}$ & $07-20-93$ & $158.17 \mathrm{~V}$ \\
$08-28-87$ & $120.46 \mathrm{~V}$ & $10-18-89$ & $137.78 \mathrm{~V}$ & $08-21-91$ & $152.36 \mathrm{~V}$ & $08-20-93$ & $159.02 \mathrm{~V}$ \\
$09-03-87$ & $120.46 \mathrm{~V}$ & $11-22-89$ & $138.86 \mathrm{~V}$ & $08-27-91$ & $152.36 \mathrm{~V}$ & $09-17-93$ & $160.04 \mathrm{~V}$ \\
$10-19-87$ & $121.50 \mathrm{~V}$ & $12-20-89$ & $139.55 \mathrm{~V}$ & $09-17-91$ & $153.28 \mathrm{~V}$ & $10-14-93$ & $160.92 \mathrm{~V}$ \\
$01-06-88$ & $123.29 \mathrm{~V}$ & $01-08-90$ & $140.00 \mathrm{~V}$ & $10-21-91$ & $154.16 \mathrm{~V}$ & $11-11-93$ & $161.29 \mathrm{~V}$ \\
$02-02-88$ & $123.85 \mathrm{~V}$ & $04-24-90$ & $142.60 \mathrm{~V}$ & $11-18-91$ & $155.20 \mathrm{~V}$ & $12-15-93$ & $161.97 \mathrm{~V}$ \\
$03-07-88$ & $124.93 \mathrm{~V}$ & $05-23-90$ & $143.09 \mathrm{~V}$ & $12-11-91$ & $155.34 \mathrm{~S}$ & &
\end{tabular}

Highest $117.14 \quad 01-16-87$

Lowest $162.35 \quad 03-23-93$

Period of record Highest $99.66 \quad 06-21-84 \quad$ Lowest $163.05 \quad 03-08-94$

Record begins $\quad 08-20-83 \quad 111$ Entries 
Table 36. Ground-water levels in selected wells, Long Valley Caldera, Mono County, California, 1987-93-Continued

\begin{tabular}{lcccccc}
\hline Date & $\begin{array}{c}\text { Water } \\
\text { level MW }\end{array}$ & Date & $\begin{array}{c}\text { Water } \\
\text { level MW }\end{array}$ & Date & $\begin{array}{c}\text { Water } \\
\text { level MW }\end{array}$ & $\begin{array}{c}\text { Water } \\
\text { level MW }\end{array}$ \\
\hline
\end{tabular}

\section{Site identifier: SNARL}

Site number: $\mathbf{3 7 3 6 5 4 1 1 8 4 9 5 4 0 1}$

About 7.6 miles southeast of Mammoth Lakes at the Sierra Nevada Aquatic Research Laboratory. Drilled commercial well. Diameter 6 inches. depth 70 feet, perforated 34 to 70 feet. Altitude of land-surface datum 7,095 feet. Water-level records available 1959-94.

\begin{tabular}{|c|c|c|c|c|c|c|c|c|c|}
\hline $07-27-88$ & \multicolumn{2}{|c|}{$27.48 \mathrm{~S}$} & $04-24-90$ & $33.24 \mathrm{~V}$ & & $11-20-91$ & $29.57 \mathrm{~V}$ & $07-20-93$ & $21.31 \mathrm{~S}$ \\
\hline $11-28-88$ & \multicolumn{2}{|c|}{$31.50 \mathrm{~S}$} & $07-18-90$ & $27.19 \mathrm{~V}$ & & $04-08-92$ & $32.55 \mathrm{~S}$ & $11-10-93$ & $28.85 \mathrm{~V}$ \\
\hline $04-25-89$ & \multicolumn{2}{|c|}{$30.99 \mathrm{~V}$} & $11-15-90$ & $31.88 \mathrm{~V}$ & & $07-22-92$ & $30.44 \mathrm{~V}$ & & \\
\hline $08-08-89$ & \multicolumn{2}{|c|}{$30.03 \mathrm{~V}$} & $04-10-91$ & $32.25 \mathrm{~V}$ & & $11-19-92$ & 33.07 VR & & \\
\hline $11-29-89$ & \multicolumn{2}{|c|}{$32.30 \mathrm{~V}$} & $07-17-91$ & $22.67 \mathrm{~V}$ & & $05-14-93$ & $29.51 \mathrm{~S}$ & & \\
\hline Highest & 21.31 & $07-20-93$ & & & & & & & \\
\hline Lowest & 33.24 & $04-24-90$ & & & & & & & \\
\hline \multirow{2}{*}{\multicolumn{2}{|c|}{$\begin{array}{l}\text { Period of record } \\
\text { Record begins }\end{array}$}} & Highest 21.31 & $1 \quad 07-20-93$ & Lowest & 33.69 & $04-06-94$ & & & \\
\hline & & $09-16-59$ & 0 Entries & & & & & & \\
\hline
\end{tabular}

Site identifier: SQ

Site number: $\mathbf{3 7 3 7 4 2 1 1 8 5 1 5 7 0 3}$

About 5.6 miles southeast of Mammoth Lakes. Drilled unused well. Diameter 8 inches, depth 125 feet, perforated 27 to 125 feet. Altitude of land-surface datum 7,102.22 feet. Water-level records available 1984-94.

\begin{tabular}{llllllll}
\hline $04-21-87$ & $16.09 \mathrm{~S}$ & $04-24-90$ & $20.24 \mathrm{~S}$ & $07-17-91$ & $17.92 \mathrm{~S}$ & $10-05-92$ & $21.93 \mathrm{~S}$ \\
$08-30-87$ & $16.58 \mathrm{~S}$ & $05-23-90$ & $19.90 \mathrm{~S}$ & $08-21-91$ & $18.66 \mathrm{~S}$ & $11-19-92$ & $22.17 \mathrm{~S}$ \\
$12-18-87$ & $18.04 \mathrm{~S}$ & $06-12-90$ & $19.70 \mathrm{~S}$ & $08-27-91$ & $18.84 \mathrm{~S}$ & $12-16-92$ & $22.34 \mathrm{~S}$ \\
$03-01-88$ & $18.18 \mathrm{~S}$ & $07-16-90$ & $19.50 \mathrm{~S}$ & $09-17-91$ & $20.81 \mathrm{~S}$ & $01-13-93$ & $21.80 \mathrm{~S}$ \\
$04-21-88$ & $18.39 \mathrm{~S}$ & $08-27-90$ & $19.92 \mathrm{~S}$ & $10-21-91$ & $20.48 \mathrm{~S}$ & $03-04-93$ & $21.63 \mathrm{~S}$ \\
$07-27-88$ & $17.00 \mathrm{~S}$ & $09-24-90$ & $20.53 \mathrm{~S}$ & $11-20-91$ & $20.95 \mathrm{~S}$ & $03-23-93$ & $21.54 \mathrm{~V}$ \\
$04-25-89$ & $19.09 \mathrm{~S}$ & $10-15-90$ & $20.78 \mathrm{~S}$ & $12-11-91$ & $21.18 \mathrm{~S}$ & $04-14-93$ & $20.28 \mathrm{~S}$ \\
$08-08-89$ & $18.59 \mathrm{~S}$ & $11-15-90$ & $21.12 \mathrm{~S}$ & $01-15-92$ & $21.45 \mathrm{~S}$ & $05-14-93$ & $19.90 \mathrm{~S}$ \\
$09-20-89$ & $19.55 \mathrm{~S}$ & $12-17-90$ & $21.48 \mathrm{~S}$ & $02-21-92$ & $21.58 \mathrm{~S}$ & $06-16-93$ & $17.22 \mathrm{~S}$ \\
$10-18-89$ & $19.84 \mathrm{~S}$ & $01-15-91$ & $21.42 \mathrm{~S}$ & $03-10-92$ & $21.79 \mathrm{~S}$ & $07-20-93$ & $15.18 \mathrm{~S}$ \\
$11-22-89$ & $19.95 \mathrm{~S}$ & $02-14-91$ & $21.42 \mathrm{~S}$ & $04-08-92$ & $21.65 \mathrm{~S}$ & $08-26-93$ & $15.48 \mathrm{~S}$ \\
$12-19-89$ & $20.19 \mathrm{~S}$ & $03-19-91$ & $21.32 \mathrm{~S}$ & $05-14-92$ & $21.56 \mathrm{~S}$ & $09-07-93$ & $16.35 \mathrm{~S}$ \\
$01-08-90$ & $20.38 \mathrm{~S}$ & $04-10-91$ & $21.07 \mathrm{~S}$ & $06-08-92$ & $19.96 \mathrm{~S}$ & $10-14-93$ & $18.53 \mathrm{~S}$ \\
$02-21-90$ & $20.55 \mathrm{~S}$ & $05-13-91$ & $21.10 \mathrm{~S}$ & $07-21-92$ & $20.76 \mathrm{~S}$ & $11-10-93$ & $19.64 \mathrm{~S}$ \\
$03-20-90$ & $20.41 \mathrm{~S}$ & $06-19-91$ & $18.08 \mathrm{~S}$ & $09-16-92$ & $21.72 \mathrm{~S}$ & $12-16-93$ & $20.54 \mathrm{~S}$
\end{tabular}

$\begin{array}{lrlllll}\text { Highest } & 15.18 & 07-20-93 & & & \\ \text { Lowest 22.34 } & 12-16-92 & & & \\ \text { Period of record } & \text { Highest } 10.55 & 06-08-86 & \text { Lowest } & 22.34 & 12-16-92 \\ \text { Record begins } & 07-17-84 \quad 72 \text { Entries }\end{array}$


Table 36. Ground-water levels in selected wells, Long Valley Caldera, Mono County, California, 1987-93-Continued

\begin{tabular}{|c|c|c|c|c|c|c|c|}
\hline Date & $\begin{array}{c}\text { Water } \\
\text { level MW }\end{array}$ & Date & $\begin{array}{c}\text { Water } \\
\text { level MW }\end{array}$ & Date & $\begin{array}{c}\text { Water } \\
\text { level MW }\end{array}$ & Date & $\begin{array}{c}\text { Water } \\
\text { level MW }\end{array}$ \\
\hline
\end{tabular}

Site identifier: SS-1

Site number: $\mathbf{3 7 3 8 1 8 1 1 8 5 3 3 2 0 1}$

About 3.9 miles east of Mammoth Lakes at the Mono County Sheriff Substation. Unused public-supply well. Diameter 8 inches, depth 75 feet, perforated 51 to 75 feet. Altitude of land-surface datum 7,180.61 feet. Water-level records available 1971-72, 1982-93.

\begin{tabular}{|c|c|c|c|c|c|c|c|c|c|}
\hline $04-21-87$ & \multicolumn{2}{|c|}{$28.38 \mathrm{~S}$} & $11-30-89$ & $31.31 \mathrm{~S}$ & & $07-17-91$ & $27.69 \mathrm{SP}$ & $05-14-93$ & $33.74 \mathrm{~S}$ \\
\hline $08-29-87$ & \multicolumn{2}{|c|}{$28.44 \mathrm{~S}$} & $04-24-90$ & $31.08 \mathrm{~S}$ & & $11-20-91$ & $36.48 \mathrm{~V}$ & $07-23-93$ & $23.76 \mathrm{~S}$ \\
\hline $07-28-88$ & \multicolumn{2}{|c|}{$27.93 \mathrm{~S}$} & $07-16-90$ & $29.78 \mathrm{~S}$ & & $04-08-92$ & $34.44 \mathrm{~S}$ & & \\
\hline $11-28-88$ & \multicolumn{2}{|c|}{$31.06 \mathrm{~S}$} & $11-15-90$ & $33.10 \mathrm{~S}$ & & $07-21-92$ & $34.08 \mathrm{VR}$ & & \\
\hline 08-09-89 & \multicolumn{2}{|c|}{$29.68 \mathrm{~S}$} & $04-10-91$ & $33.43 \mathrm{~S}$ & & $11-19-92$ & $39.77 \mathrm{~V}$ & & \\
\hline Highest & 23.76 & $07-23-93$ & & & & & & & \\
\hline Lowest & 39.77 & $11-19-92$ & & & & & & & \\
\hline \multirow{2}{*}{\multicolumn{2}{|c|}{$\begin{array}{l}\text { Period of record } \\
\text { Record begins }\end{array}$}} & Highest 16.53 & $3 \quad 05-21-86$ & Lowest & 39.77 & $11-19-92$ & & & \\
\hline & & $08-28-71$ & 30 Entries & & & & & & \\
\hline
\end{tabular}

\section{Site identifier: SS-2}

Site number: $\mathbf{3 7 3 8 1 8 1 1 8 5 3 3 2 0 2}$

About 3.9 miles east of Mammoth Lakes at the Mono County Sheriff Substation. Drilled public-supply well. Diameter 6 inches, depth 100 feet, perforated 34 to 60 and 80 to 100 feet. Altitude of land-surface datum 7,179.13 feet. Water-level records available 1978, 1984-94.

\begin{tabular}{|c|c|c|c|c|c|c|c|c|c|}
\hline $04-21-87$ & \multicolumn{2}{|c|}{$14.85 \mathrm{~S}$} & $04-26-89$ & $16.39 \mathrm{~V}$ & & $06-14-91$ & $14.91 \mathrm{~V}$ & $11-19-92$ & $21.60 \mathrm{~V}$ \\
\hline $08-29-87$ & \multicolumn{2}{|c|}{$8.98 \mathrm{~S}$} & $08-09-89$ & $15.70 \mathrm{~S}$ & & $07-17-91$ & $14.78 \mathrm{SN}$ & $05-14-93$ & $16.94 \mathrm{~S}$ \\
\hline $12-16-87$ & \multicolumn{2}{|c|}{$16.73 \mathrm{~S}$} & $11-29-89$ & 17.30 SR & & $08-27-91$ & $17.02 \mathrm{~V}$ & $07-23-93$ & $9.93 \mathrm{~S}$ \\
\hline $04-28-88$ & \multicolumn{2}{|c|}{$17.30 \mathrm{~S}$} & $04-24-90$ & $20.45 \mathrm{SR}$ & & $11-20-91$ & $20.00 \mathrm{~V}$ & $11-11-93$ & $18.85 \mathrm{~S}$ \\
\hline $07-28-88$ & \multicolumn{2}{|c|}{$14.68 \mathrm{~S}$} & $07-16-90$ & $15.15 \mathrm{~S}$ & & $04-08-92$ & $21.28 \mathrm{SP}$ & & \\
\hline $11-28-88$ & \multicolumn{2}{|c|}{$17.68 \mathrm{~S}$} & $11-15-90$ & $19.26 \mathrm{~S}$ & & $07-21-92$ & $17.95 \mathrm{VT}$ & & \\
\hline Highest & 8.98 & $08-29-87$ & & & & & & & \\
\hline Lowest & 21.60 & $11-19-92$ & & & & & & & \\
\hline \multirow{2}{*}{\multicolumn{2}{|c|}{$\begin{array}{l}\text { Period of record } \\
\text { Record begins }\end{array}$}} & Highest & $6.35 \quad 07-19-84$ & Lowest & 21.60 & $11-19-92$ & & & \\
\hline & & $10-17-78$ & 30 Entries & & & & & & \\
\hline
\end{tabular}




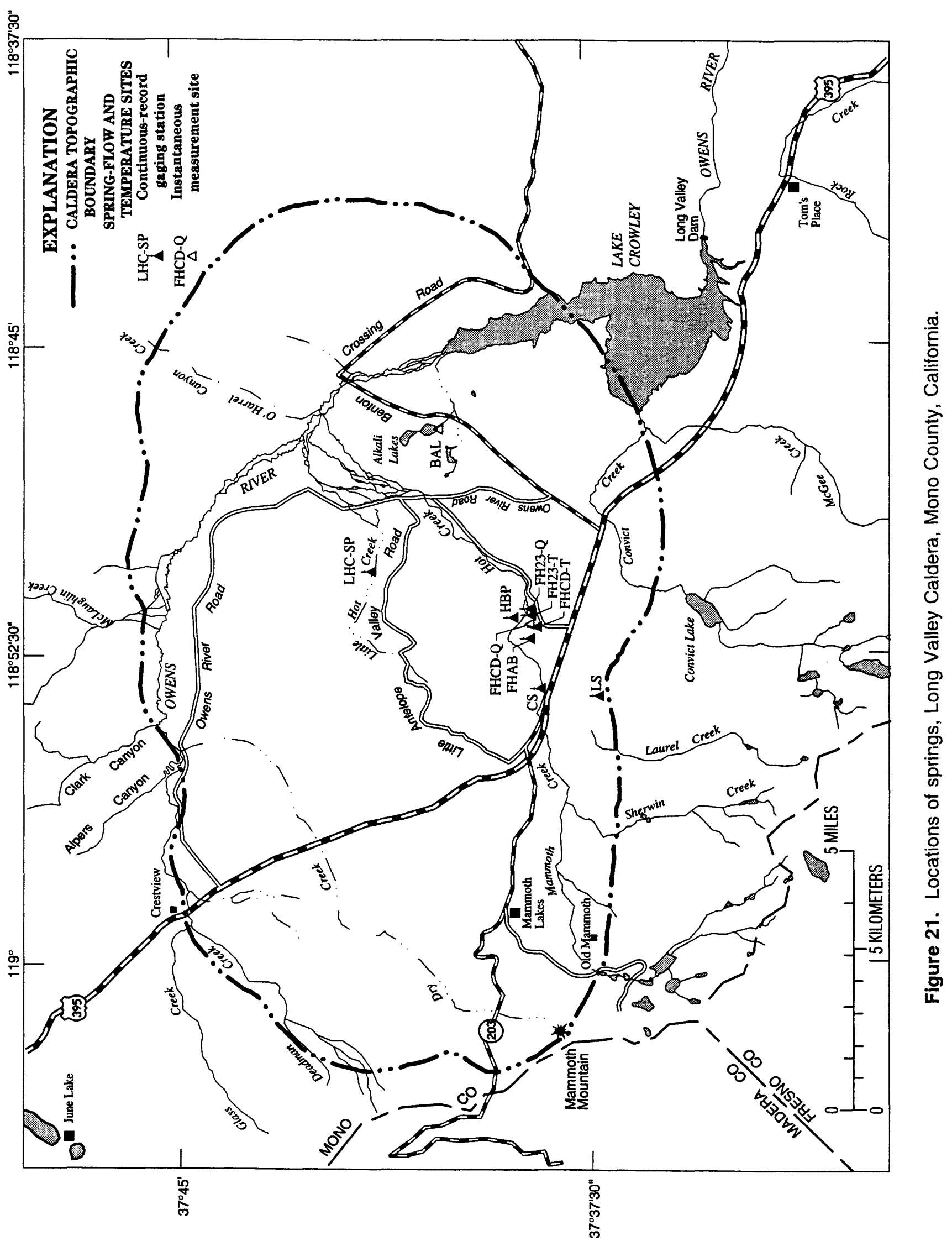




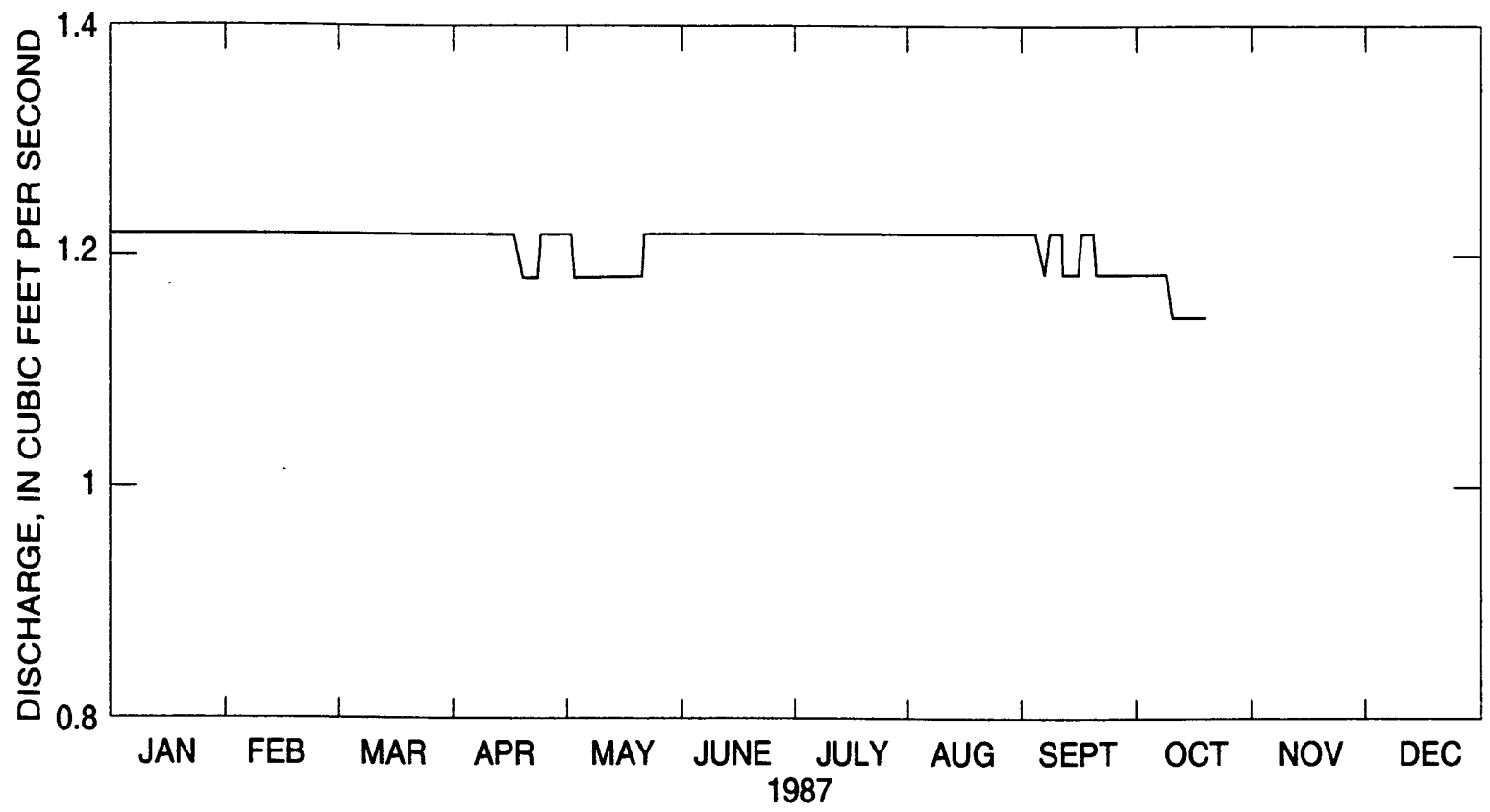

Figure 22. Daily mean discharge, Laurel Spring (LS), Long Valley Caldera, Mono County, California, 1987. 
Table 37. Daily mean discharge, Laurel Spring (LS), Long Valley Caldera, Mono County, California, 1987

[Values are in cubic feet per second.. Max, maximum; Min, minimum; -, no data]

\begin{tabular}{|c|c|c|c|c|c|c|c|c|c|c|c|c|}
\hline Day & Jan & Feb & Mar & Apr & May & June & July & Aug & Sept & Oct & Nov & Dec \\
\hline 1 & 1.22 & 1.22 & 1.22 & 1.22 & 1.22 & 1.22 & 1.22 & 1.22 & 1.22 & 1.18 & - & - \\
\hline 2 & 1.22 & 1.22 & 1.22 & 1.22 & 1.22 & 1.22 & 1.22 & 1.22 & 1.22 & 1.18 & - & - \\
\hline 3 & 1.22 & 1.22 & 1.22 & 1.22 & 1.22 & 1.22 & 1.22 & 1.22 & 1.22 & 1.18 & - & - \\
\hline 4 & 1.22 & 1.22 & 1.22 & 1.22 & 1.22 & 1.22 & 1.22 & 1.22 & 1.22 & 1.18 & - & - \\
\hline 5 & 1.22 & 1.22 & 1.22 & 1.22 & 1.22 & 1.22 & 1.22 & 1.22 & 1.22 & 1.18 & - & - \\
\hline 6 & 1.22 & 1.22 & 1.22 & 1.22 & 1.18 & 1.22 & 1.22 & 1.22 & 1.22 & 1.18 & - & - \\
\hline 7 & 1.22 & 1.22 & 1.22 & 1.22 & 1.18 & 1.22 & 1.22 & 1.22 & 1.22 & 1.18 & - & - \\
\hline 8 & 1.22 & 1.22 & 1.22 & 1.22 & 1.18 & 1.22 & 1.22 & 1.22 & 1.22 & 1.18 & - & - \\
\hline 9 & 1.22 & 1.22 & 1.22 & 1.22 & 1.18 & 1.22 & 1.22 & 1.22 & 1.18 & 1.18 & - & - \\
\hline 10 & 1.22 & 1.22 & 1.22 & 1.22 & 1.18 & 1.22 & 1.22 & 1.22 & 1.18 & 1.18 & - & - \\
\hline 11 & 1.22 & 1.22 & 1.22 & 1.22 & 1.18 & 1.22 & 1.22 & 1.22 & 1.22 & 1.18 & - & - \\
\hline 12 & 1.22 & 1.22 & 1.22 & 1.22 & 1.18 & 1.22 & 1.22 & 1.22 & 1.22 & 1.14 & - & - \\
\hline 13 & 1.22 & 1.22 & 1.22 & 1.22 & 1.18 & 1.22 & 1.22 & 1.22 & 1.22 & 1.14 & - & - \\
\hline 14 & 1.22 & 1.22 & 1.22 & 1.22 & 1.18 & 1.22 & 1.22 & 1.22 & 1.18 & 1.14 & - & - \\
\hline 15 & 1.22 & 1.22 & 1.22 & 1.22 & 1.18 & 1.22 & 1.22 & 1.22 & 1.18 & 1.14 & - & - \\
\hline 16 & 1.22 & 1.22 & 1.22 & 1.22 & 1.18 & 1.22 & 1.22 & 1.22 & 1.18 & 1.14 & - & - \\
\hline 17 & 1.22 & 1.22 & 1.22 & 1.22 & 1.18 & 1.22 & 1.22 & 1.22 & 1.18 & 1.14 & - & - \\
\hline 18 & 1.22 & 1.22 & 1.22 & 1.22 & 1.18 & 1.22 & 1.22 & 1.22 & 1.18 & 1.14 & - & - \\
\hline 19 & 1.22 & 1.22 & 1.22 & 1.22 & 1.18 & 1.22 & 1.22 & 1.22 & 1.22 & 1.14 & - & - \\
\hline 20 & 1.22 & 1.22 & 1.22 & 1.22 & 1.18 & 1.22 & 1.22 & 1.22 & 1.22 & 1.14 & - & - \\
\hline 21 & 1.22 & 1.22 & 1.22 & 1.22 & 1.18 & 1.22 & 1.22 & 1.22 & 1.22 & 1.14 & - & - \\
\hline 22 & 1.22 & 1.22 & 1.22 & 1.18 & 1.18 & 1.22 & 1.22 & 1.22 & 1.22 & 1.14 & - & - \\
\hline 23 & 1.22 & 1.22 & 1.22 & 1.18 & 1.18 & 1.22 & 1.22 & 1.22 & 1.18 & - & - & - \\
\hline 24 & 1.22 & 1.22 & 1.22 & 1.18 & 1.18 & 1.22 & 1.22 & 1.22 & 1.18 & - & - & - \\
\hline 25 & 1.22 & 1.22 & 1.22 & 1.18 & 1.22 & 1.22 & 1.22 & 1.22 & 1.18 & - & - & - \\
\hline 26 & 1.22 & 1.22 & 1.22 & 1.18 & 1.22 & 1.22 & 1.22 & 1.22 & 1.18 & - & - & - \\
\hline 27 & 1.22 & 1.22 & 1.22 & 1.22 & 1.22 & 1.22 & 1.22 & 1.22 & 1.18 & - & - & - \\
\hline 28 & 1.22 & 1.22 & 1.22 & 1.22 & 1.22 & 1.22 & 1.22 & 1.22 & 1.18 & - & - & - \\
\hline 29 & 1.22 & - & 1.22 & 1.22 & 1.22 & 1.22 & 1.22 & 1.22 & 1.18 & - & - & - \\
\hline 30 & 1.22 & - & 1.22 & 1.22 & 1.22 & 1.22 & 1.22 & 1.22 & 1.18 & - & - & - \\
\hline 31 & 1.22 & - & 1.22 & - & 1.22 & - & 1.22 & 1.22 & - & - & - & - \\
\hline Mean & 1.22 & 1.22 & 1.22 & 1.21 & 1.20 & 1.22 & 1.22 & 1.22 & 1.20 & 1.16 & - & - \\
\hline $\operatorname{Max}$ & 1.22 & 1.22 & 1.22 & 1.22 & 1.22 & 1.22 & 1.22 & 1.22 & 1.22 & 1.18 & - & - \\
\hline Min & 1.22 & 1.22 & 1.22 & 1.18 & 1.18 & 1.22 & 1.22 & 1.22 & 1.18 & 1.14 & - & - \\
\hline
\end{tabular}


Table 38. Miscellaneous instantaneous discharge measurements, Laurel Spring (LS), Long Valley Caldera, Mono County, California, 1991-92

[Measurements made with current meter. Discharge is in cubic feet per second]

\begin{tabular}{cc}
\hline Date & Discharge \\
\hline $01-19-91$ & 0.70 \\
$06-16-91$ & .82 \\
$07-13-91$ & .99 \\
$03-15-92$ & .87 \\
$04-23-92$ & .77 \\
$05-28-92$ & .68 \\
$06-12-92$ & .74 \\
$07-21-92$ & .58 \\
$08-14-92$ & .61 \\
$09-02-92$ & .56 \\
$09-27-92$ & .60 \\
\hline
\end{tabular}




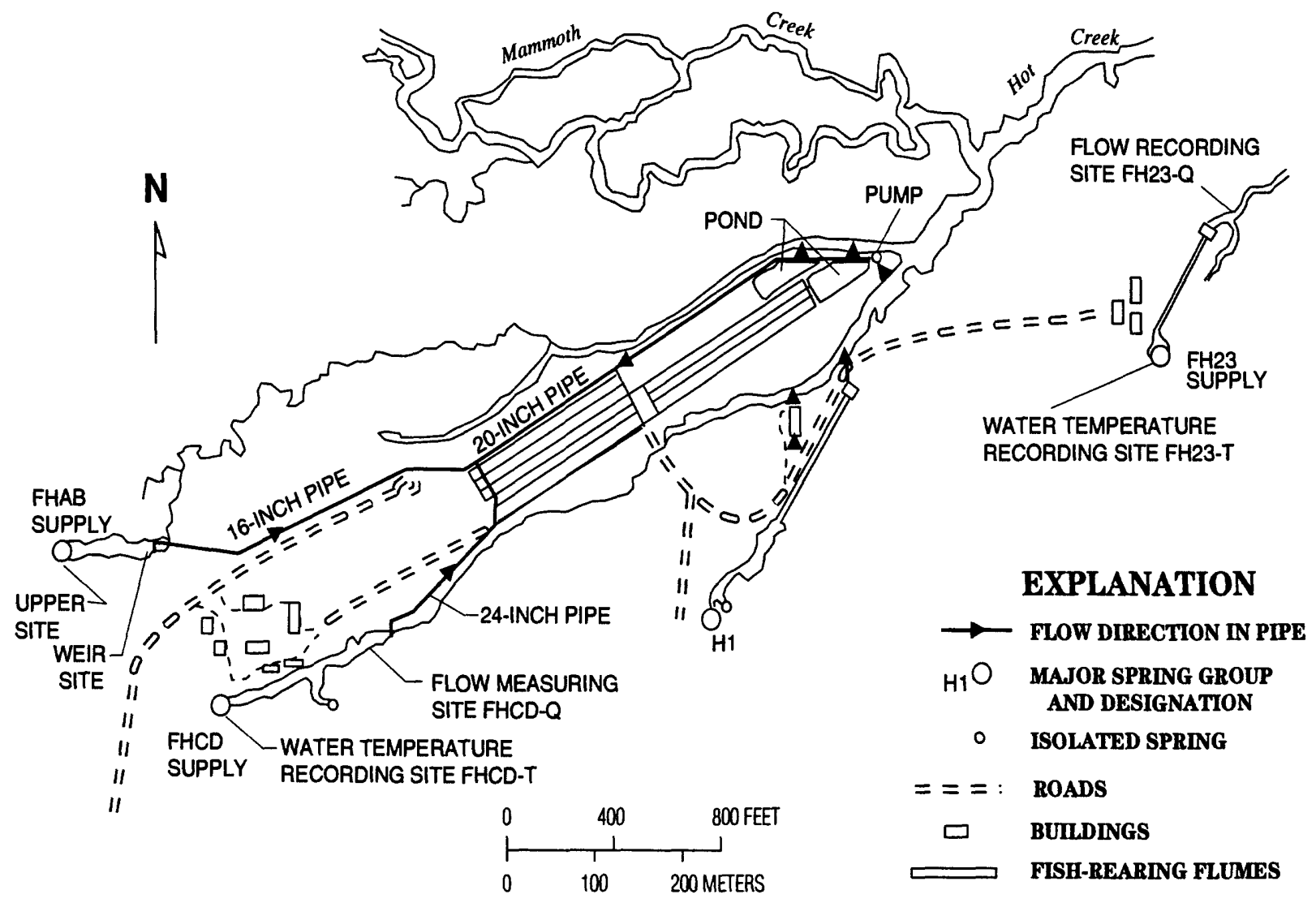

Figure 23. Locations of data-collection sites at Hot Creek Fish Hatchery, Long Valley Caldera, Mono County, California. 


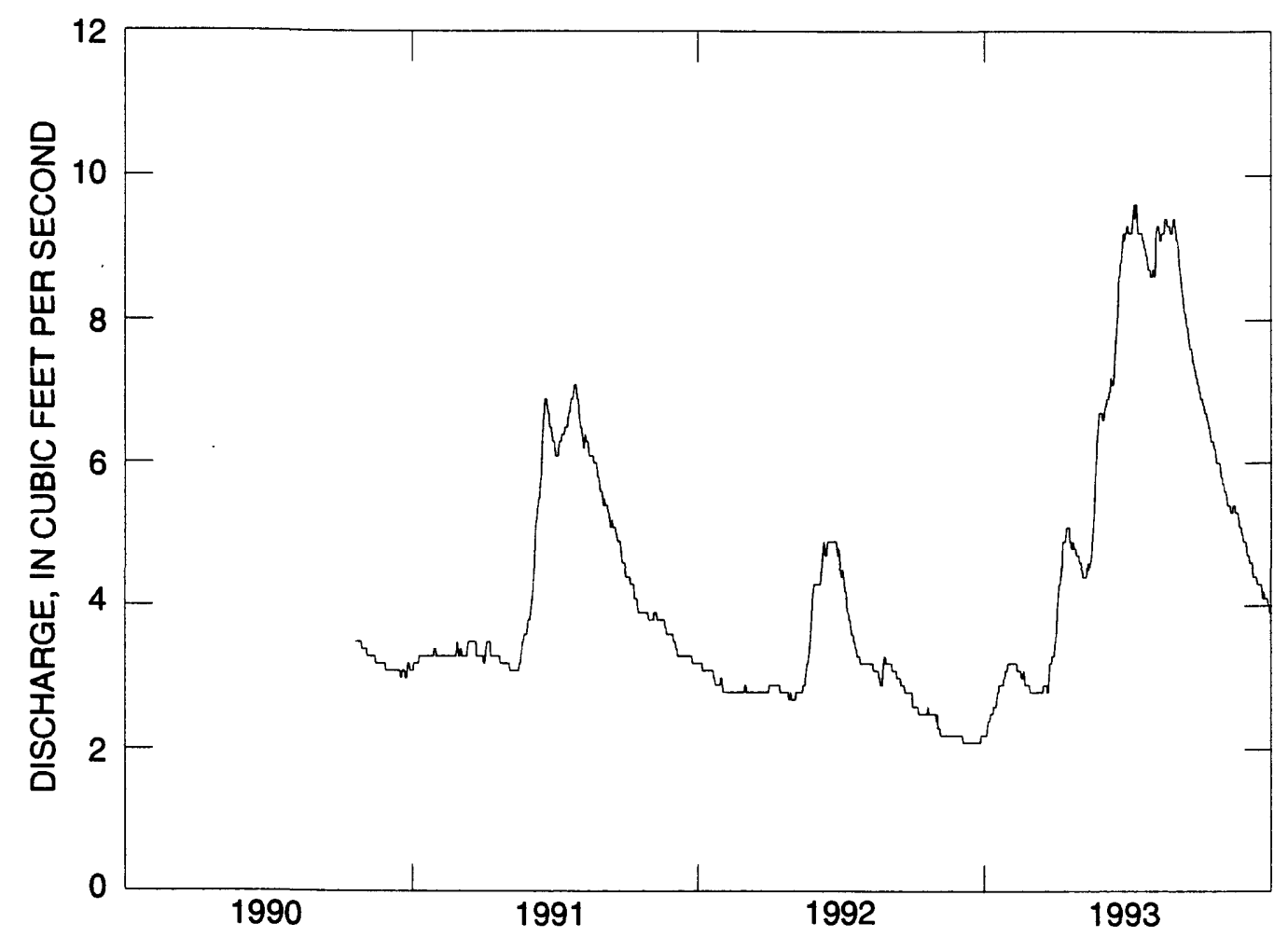

Figure 24. Daily mean discharge, Fish Hatchery Spring AB (FHAB), weir site, Long Valley Caldera, Mono County, California, 1990-93. 
Table 39. Daily mean discharge, Fish Hatchery Spring AB (FHAB), weir site, Long Valley Caldera, Mono County, California, 1990

[Values are in cubic feet per second. Max, maximum; Min, minimum; -, no data]

\begin{tabular}{|c|c|c|c|c|c|c|c|c|c|c|c|c|}
\hline Day & Jan & Feb & Mar & Apr & May & June & July & Aug & Sept & Oct & Nov & Dec \\
\hline 1 & - & - & - & - & - & - & - & - & - & - & 3.4 & 3.1 \\
\hline 2 & - & - & - & - & - & - & - & - & - & - & 3.4 & 3.1 \\
\hline 3 & - & - & - & - & - & - & - & - & - & - & 3.4 & 3.1 \\
\hline 4 & - & - & - & - & - & - & - & - & - & - & 3.3 & 3.1 \\
\hline 5 & - & - & - & - & - & - & - & - & - & - & 3.3 & 3.1 \\
\hline 6 & - & - & - & - & - & - & - & - & - & - & 3.3 & 3.1 \\
\hline 7 & - & - & - & - & - & - & - & - & - & - & 3.3 & 3.1 \\
\hline 8 & - & - & - & - & - & - & - & - & - & - & 3.3 & 3.1 \\
\hline 9 & - & - & - & - & - & - & - & - & - & - & 3.3 & 3.1 \\
\hline 10 & - & - & - & - & - & - & - & - & - & - & 3.3 & 3.1 \\
\hline 11 & - & - & - & - & - & - & - & - & - & - & 3.3 & 3.1 \\
\hline 12 & - & - & - & - & - & - & - & - & - & - & 3.3 & 3.1 \\
\hline 13 & - & - & - & - & - & - & - & - & - & - & 3.3 & 3.1 \\
\hline 14 & - & - & - & - & - & - & - & - & - & - & 3.3 & 3.1 \\
\hline 15 & - & - & - & - & - & - & - & - & - & - & 3.2 & 3.1 \\
\hline 16 & - & - & - & - & - & - & - & - & - & - & 3.2 & 3.1 \\
\hline 17 & - & - & - & - & - & - & - & - & - & - & 3.2 & 3.0 \\
\hline 18 & - & - & - & - & - & - & - & - & - & - & 3.2 & 3.0 \\
\hline 19 & - & - & - & - & - & - & - & - & - & 3.5 & 3.2 & 3.1 \\
\hline 20 & - & - & - & - & 一 & - & - & - & - & 3.5 & 3.2 & 3.1 \\
\hline 21 & - & - & - & - & - & - & - & - & - & 3.5 & 3.2 & 3.1 \\
\hline 22 & - & - & - & - & - & - & - & - & - & 3.5 & 3.2 & 3.1 \\
\hline 23 & - & - & - & - & - & - & - & - & - & 3.5 & 3.2 & 3.0 \\
\hline 24 & - & - & - & - & - & - & - & - & - & 3.5 & 3.2 & 3.0 \\
\hline 25 & - & - & - & - & - & - & - & - & - & 3.5 & 3.2 & 3.0 \\
\hline 26 & - & - & - & - & - & - & - & - & - & 3.5 & 3.2 & 3.2 \\
\hline 27 & - & - & - & - & - & - & - & - & - & 3.5 & 3.2 & 3.2 \\
\hline 28 & - & - & - & - & - & - & - & - & - & 3.4 & 3.1 & 3.2 \\
\hline 29 & - & - & - & - & - & - & - & - & - & 3.4 & 3.1 & 3.1 \\
\hline 30 & - & - & - & - & - & - & - & - & - & 3.4 & 3.1 & 3.1 \\
\hline 31 & - & - & - & - & - & - & - & - & - & 3.4 & - & 3.1 \\
\hline Mean & - & - & - & - & - & - & - & - & - & 3.47 & 3.25 & 3.09 \\
\hline $\operatorname{Max}$ & - & - & - & - & - & - & - & - & - & 3.5 & 3.4 & 3.2 \\
\hline Min & - & - & - & - & - & - & - & - & - & 3.4 & 3.1 & 3.0 \\
\hline
\end{tabular}




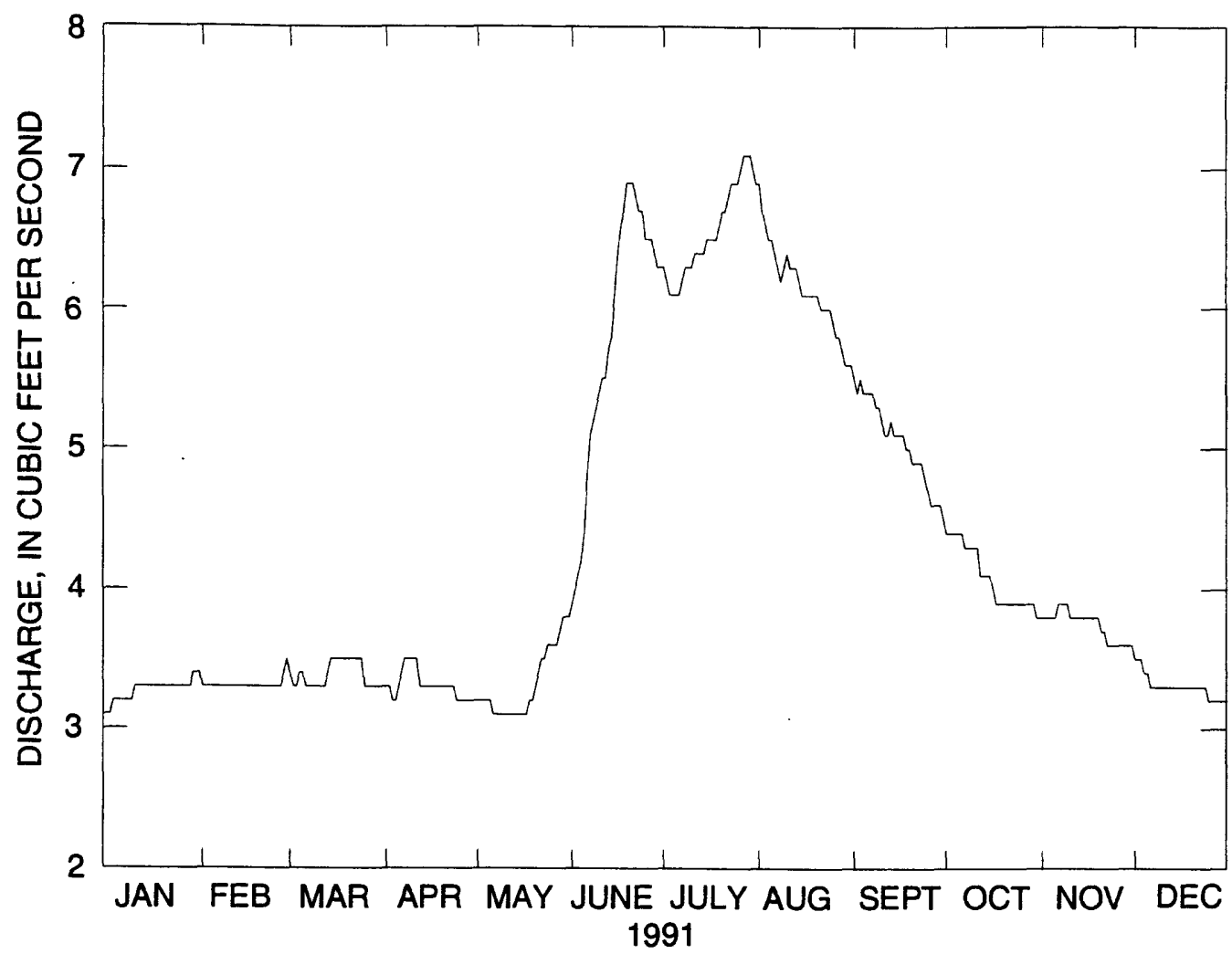

Figure 25. (A) Daily mean discharge, Fish Hatchery Spring AB (FHAB), weir site, Long Valley Caldera, Mono County, California, 1991. 
Table 40. Daily mean discharge, Fish Hatchery Spring AB (FHAB), weir site, Long Valley Caldera, Mono County, California, 1991

[Values are in cubic feet per second. Max, maximum; Min, minimum; -, no data]

\begin{tabular}{|c|c|c|c|c|c|c|c|c|c|c|c|c|}
\hline Day & Jan & Feb & Mar & Apr & May & June & July & Aug & Sept & Oct & Nov & Dec \\
\hline 1 & 3.1 & 3.3 & 3.4 & 3.3 & 3.2 & 3.9 & 6.3 & 6.9 & 5.5 & 4.4 & 3.8 & 3.5 \\
\hline 2 & 3.1 & 3.3 & 3.3 & 3.3 & 3.2 & 4.0 & 6.2 & 6.7 & 5.4 & 4.4 & 3.8 & 3.5 \\
\hline 3 & 3.2 & 3.3 & 3.3 & 3.2 & 3.2 & 4.1 & 6.1 & 6.6 & 5.5 & 4.4 & 3.8 & 3.5 \\
\hline 4 & 3.2 & 3.3 & 3.4 & 3.2 & 3.2 & 4.2 & 6.1 & 6.5 & 5.4 & 4.4 & 3.8 & 3.4 \\
\hline 5 & 3.2 & 3.3 & 3.4 & 3.3 & 3.2 & 4.4 & 6.1 & 6.5 & 5.4 & 4.4 & 3.8 & 3.4 \\
\hline 6 & 3.2 & 3.3 & 3.3 & 3.4 & 3.1 & 4.8 & 6.1 & 6.4 & 5.4 & 4.4 & 3.9 & 3.3 \\
\hline 7 & 3.2 & 3.3 & 3.3 & 3.5 & 3.1 & 5.1 & 6.2 & 6.3 & 5.4 & 4.3 & 3.9 & 3.3 \\
\hline 8 & 3.2 & 3.3 & 3.3 & 3.5 & 3.1 & 5.2 & 6.3 & 6.2 & 5.3 & 4.3 & 3.9 & 3.3 \\
\hline 9 & 3.2 & 3.3 & 3.3 & 3.5 & 3.1 & 5.3 & 6.3 & 6.3 & 5.3 & 4.3 & 3.9 & 3.3 \\
\hline 10 & 3.3 & 3.3 & 3.3 & 3.5 & 3.1 & 5.4 & 6.3 & 6.4 & 5.2 & 4.3 & 3.8 & 3.3 \\
\hline 11 & 3.3 & 3.3 & 3.3 & 3.5 & 3.1 & 5.5 & 6.4 & 6.3 & 5.1 & 4.3 & 3.8 & 3.3 \\
\hline 12 & 3.3 & 3.3 & 3.3 & 3.3 & 3.1 & 5.5 & 6.4 & 6.3 & 5.1 & 4.1 & 3.8 & 3.3 \\
\hline 13 & 3.3 & 3.3 & 3.4 & 3.3 & 3.1 & 5.7 & 6.4 & 6.3 & 5.2 & 4.1 & 3.8 & 3.3 \\
\hline 14 & 3.3 & 3.3 & 3.5 & 3.3 & 3.1 & 5.8 & 6.4 & 6.2 & 5.1 & 4.1 & 3.8 & 3.3 \\
\hline 15 & 3.3 & 3.3 & 3.5 & 3.3 & 3.1 & 6.1 & 6.5 & 6.1 & 5.1 & 4.1 & 3.8 & 3.3 \\
\hline 16 & 3.3 & 3.3 & 3.5 & 3.3 & 3.1 & 6.4 & 6.5 & 6.1 & 5.1 & 4.0 & 3.8 & 3.3 \\
\hline 17 & 3.3 & 3.3 & 3.5 & 3.3 & 3.1 & 6.6 & 6.5 & 6.1 & 5.1 & 3.9 & 3.8 & 3.3 \\
\hline 18 & 3.3 & 3.3 & 3.5 & 3.3 & 3.2 & 6.7 & 6.5 & 6.1 & 5.0 & 3.9 & 3.8 & 3.3 \\
\hline 19 & 3.3 & 3.3 & 3.5 & 3.3 & 3.2 & 6.9 & 6.6 & 6.1 & 5.0 & 3.9 & 3.8 & 3.3 \\
\hline 20 & 3.3 & 3.3 & 3.5 & 3.3 & 3.3 & 6.9 & 6.7 & 6.1 & 4.9 & 3.9 & 3.7 & 3.3 \\
\hline 21 & 3.3 & 3.3 & 3.5 & 3.3 & 3.4 & 6.9 & 6.7 & 6.0 & 4.9 & 3.9 & 3.7 & 3.3 \\
\hline 22 & 3.3 & 3.3 & 3.5 & 3.3 & 3.5 & 6.8 & 6.8 & 6.0 & 4.9 & 3.9 & 3.6 & 3.3 \\
\hline 23 & 3.3 & 3.3 & 3.5 & 3.3 & 3.5 & 6.7 & 6.9 & 6.0 & 4.9 & 3.9 & 3.6 & 3.3 \\
\hline 24 & 3.3 & 3.3 & 3.5 & 3.2 & 3.6 & 6.7 & 6.9 & 6.0 & 4.8 & 3.9 & 3.6 & 3.3 \\
\hline 25 & 3.3 & 3.3 & 3.3 & 3.2 & 3.6 & 6.5 & 6.9 & 5.9 & 4.7 & 3.9 & 3.6 & 3.2 \\
\hline 26 & 3.3 & 3.3 & 3.3 & 3.2 & 3.6 & 6.5 & 7.0 & 5.8 & 4.6 & 3.9 & 3.6 & 3.2 \\
\hline 27 & 3.3 & 3.4 & 3.3 & 3.2 & 3.6 & 6.5 & 7.1 & 5.8 & 4.6 & 3.9 & 3.6 & 3.2 \\
\hline 28 & 3.3 & 3.5 & 3.3 & 3.2 & 3.7 & 6.4 & 7.1 & 5.7 & 4.6 & 3.9 & 3.6 & 3.2 \\
\hline 29 & 3.4 & - & 3.3 & 3.2 & 3.8 & 6.3 & 7.1 & 5.6 & 4.6 & 3.9 & 3.6 & 3.2 \\
\hline 30 & 3.4 & - & 3.3 & 3.2 & 3.8 & 6.3 & 7.0 & 5.6 & 4.5 & 3.8 & 3.6 & 3.2 \\
\hline 31 & 3.4 & - & 3.3 & - & 3.8 & - & 6.9 & 5.6 & - & 3.8 & - & 3.2 \\
\hline Mean & 3.27 & 3.31 & 3.38 & 3.31 & 3.32 & 5.80 & 6.56 & 6.15 & 5.05 & 4.08 & 3.75 & 3.30 \\
\hline Max & 3.4 & 3.5 & 3.5 & 3.5 & 3.8 & 6.9 & 7.1 & 6.9 & 5.5 & 4.4 & 3.9 & 3.5 \\
\hline Min & 3.1 & 3.3 & 3.3 & 3.2 & 3.1 & 3.9 & 6.1 & 5.6 & 4.5 & 3.8 & 3.6 & 3.2 \\
\hline
\end{tabular}




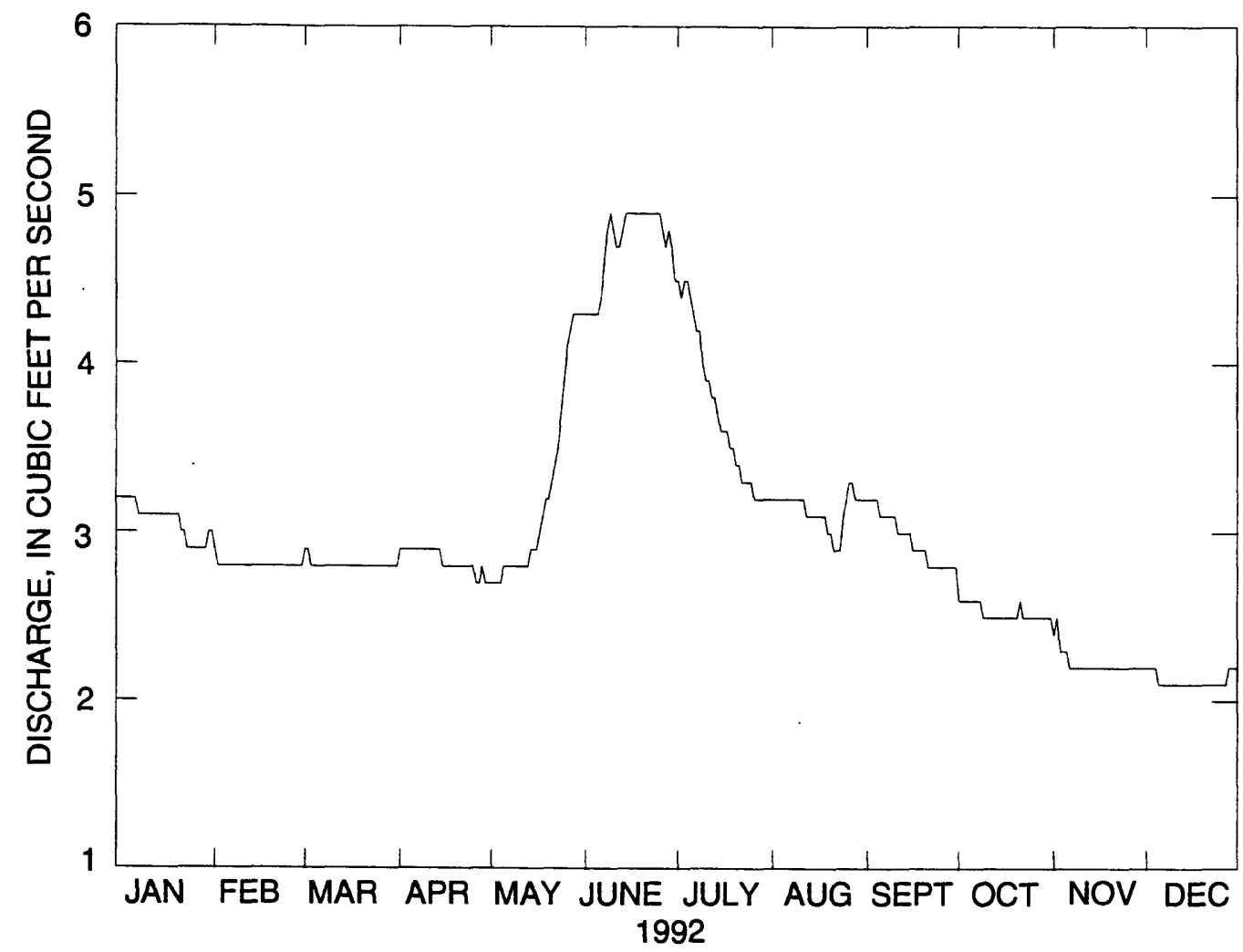

Figure 25. (B) Daily mean discharge, Fish Hatchery Spring AB (FHAB), weir site, Long Valley Caldera, Mono County, California, 1992 
Table 41. Daily mean discharge, Fish Hatchery Spring AB (FHAB), weir site, Long Valley Caldera, Mono County, California, 1992

[Values are in cubic feet per second. Max, maximum; Min, minimum; -, no data]

\begin{tabular}{|c|c|c|c|c|c|c|c|c|c|c|c|c|}
\hline Day & Jan & Feb & Mar & Apr & May & June & July & Aug & Sept & Oct & Nov & Dec \\
\hline 1 & 3.2 & 2.9 & 2.9 & 2.9 & 2.7 & 4.3 & 4.5 & 3.2 & 3.2 & 2.6 & 2.4 & 2.2 \\
\hline 2 & 3.2 & 2.8 & 2.9 & 2.9 & 2.7 & 4.3 & 4.4 & 3.2 & 3.2 & 2.6 & 2.5 & 2.2 \\
\hline 3 & 3.2 & 2.8 & 2.8 & 2.9 & 2.7 & 4.3 & 4.5 & 3.2 & 3.2 & 2.6 & 2.3 & 2.2 \\
\hline 4 & 3.2 & 2.8 & 2.8 & 2.9 & 2.7 & 4.3 & 4.5 & 3.2 & 3.2 & 2.6 & 2.3 & 2.2 \\
\hline 5 & 3.2 & 2.8 & 2.8 & 2.9 & 2.8 & 4.3 & 4.4 & 3.2 & 3.1 & 2.6 & 2.3 & 2.1 \\
\hline 6 & 3.2 & 2.8 & 2.8 & 2.9 & 2.8 & 4.4 & 4.3 & 3.2 & 3.1 & 2.6 & 2.2 & 2.1 \\
\hline 7 & 3.1 & 2.8 & 2.8 & 2.9 & 2.8 & 4.6 & 4.2 & 3.2 & 3.1 & 2.6 & 2.2 & 2.1 \\
\hline 8 & 3.1 & 2.8 & 2.8 & 2.9 & 2.8 & 4.8 & 4.2 & 3.2 & 3.1 & 2.6 & 2.2 & 2.1 \\
\hline 9 & 3.1 & 2.8 & 2.8 & 2.9 & 2.8 & 4.9 & 4.0 & 3.2 & 3.1 & 2.5 & 2.2 & 2.1 \\
\hline 10 & 3.1 & 2.8 & 2.8 & 2.9 & 2.8 & 4.8 & 3.9 & 3.2 & 3.1 & 2.5 & 2.2 & 2.1 \\
\hline 11 & 3.1 & 2.8 & 2.8 & 2.9 & 2.8 & 4.7 & 3.9 & 3.2 & 3.0 & 2.5 & 2.2 & 2.1 \\
\hline 12 & 3.1 & 2.8 & 2.8 & 2.9 & 2.8 & 4.7 & 3.8 & 3.1 & 3.0 & 2.5 & 2.2 & 2.1 \\
\hline 13 & 3.1 & 2.8 & 2.8 & 2.9 & 2.8 & 4.8 & 3.8 & 3.1 & 3.0 & 2.5 & 2.2 & 2.1 \\
\hline 14 & 3.1 & 2.8 & 2.8 & 2.9 & 2.9 & 4.9 & 3.7 & 3.1 & 3.0 & 2.5 & 2.2 & 2.1 \\
\hline 15 & 3.1 & 2.8 & 2.8 & 2.8 & 2.9 & 4.9 & 3.6 & 3.1 & 3.0 & 2.5 & 2.2 & 2.1 \\
\hline 16 & 3.1 & 2.8 & 2.8 & 2.8 & 2.9 & 4.9 & 3.6 & 3.1 & 2.9 & 2.5 & 2.2 & 2.1 \\
\hline 17 & 3.1 & 2.8 & 2.8 & 2.8 & 3.0 & 4.9 & 3.6 & 3.1 & 2.9 & 2.5 & 2.2 & 2.1 \\
\hline 18 & 3.1 & 2.8 & 2.8 & 2.8 & 3.1 & 4.9 & 3.5 & 3.1 & 2.9 & 2.5 & 2.2 & 2.1 \\
\hline 19 & 3.1 & 2.8 & 2.8 & 2.8 & 3.2 & 4.9 & 3.5 & 3.0 & 2.9 & 2.5 & 2.2 & 2.1 \\
\hline 20 & 3.1 & 2.8 & 2.8 & 2.8 & 3.2 & 4.9 & 3.4 & 3.0 & 2.9 & 2.5 & 2.2 & 2.1 \\
\hline 21 & 3.0 & 2.8 & 2.8 & 2.8 & 3.3 & 4.9 & 3.4 & 2.9 & 2.8 & 2.6 & 2.2 & 2.1 \\
\hline 22 & 3.0 & 2.8 & 2.8 & 2.8 & 3.4 & 4.9 & 3.3 & 2.9 & 2.8 & 2.5 & 2.2 & 2.1 \\
\hline 23 & 2.9 & 2.8 & 2.8 & 2.8 & 3.5 & 4.9 & 3.3 & 2.9 & 2.8 & 2.5 & 2.2 & 2.1 \\
\hline 24 & 2.9 & 2.8 & 2.8 & 2.8 & 3.7 & 4.9 & 3.3 & 3.1 & 2.8 & 2.5 & 2.2 & 2.1 \\
\hline 25 & 2.9 & 2.8 & 2.8 & 2.8 & 3.9 & 4.9 & 3.3 & 3.2 & 2.8 & 2.5 & 2.2 & 2.1 \\
\hline 26 & 2.9 & 2.8 & 2.8 & 2.7 & 4.1 & 4.8 & 3.2 & 3.3 & 2.8 & 2.5 & 2.2 & 2.1 \\
\hline 27 & 2.9 & 2.8 & 2.8 & 2.7 & 4.2 & 4.7 & 3.2 & 3.3 & 2.8 & 2.5 & 2.2 & 2.1 \\
\hline 28 & 2.9 & 2.8 & 2.8 & 2.8 & 4.3 & 4.8 & 3.2 & 3.2 & 2.8 & 2.5 & 2.2 & 2.2 \\
\hline 29 & 2.9 & 2.8 & 2.8 & 2.7 & 4.3 & 4.7 & 3.2 & 3.2 & 2.8 & 2.5 & 2.2 & 2.2 \\
\hline 30 & 3.0 & - & 2.8 & 2.7 & 4.3 & 4.5 & 3.2 & 3.2 & 2.8 & 2.5 & 2.2 & 2.2 \\
\hline 31 & 3.0 & - & 2.8 & - & 4.3 & - & 3.2 & 3.2 & - & 2.5 & - & 2.2 \\
\hline Mean & 3.06 & 2.80 & 2.81 & 2.83 & 3.24 & 4.72 & 3.71 & 3.14 & 2.96 & 2.53 & 2.23 & 2.13 \\
\hline Max & 3.2 & 2.9 & 2.9 & 2.9 & 4.3 & 4.9 & 4.5 & 3.3 & 3.2 & 2.6 & 2.5 & 2.2 \\
\hline Min & 2.9 & 2.8 & 2.8 & 2.7 & 2.7 & 4.3 & 3.2 & 2.9 & 2.8 & 2.5 & 2.2 & 2.1 \\
\hline
\end{tabular}




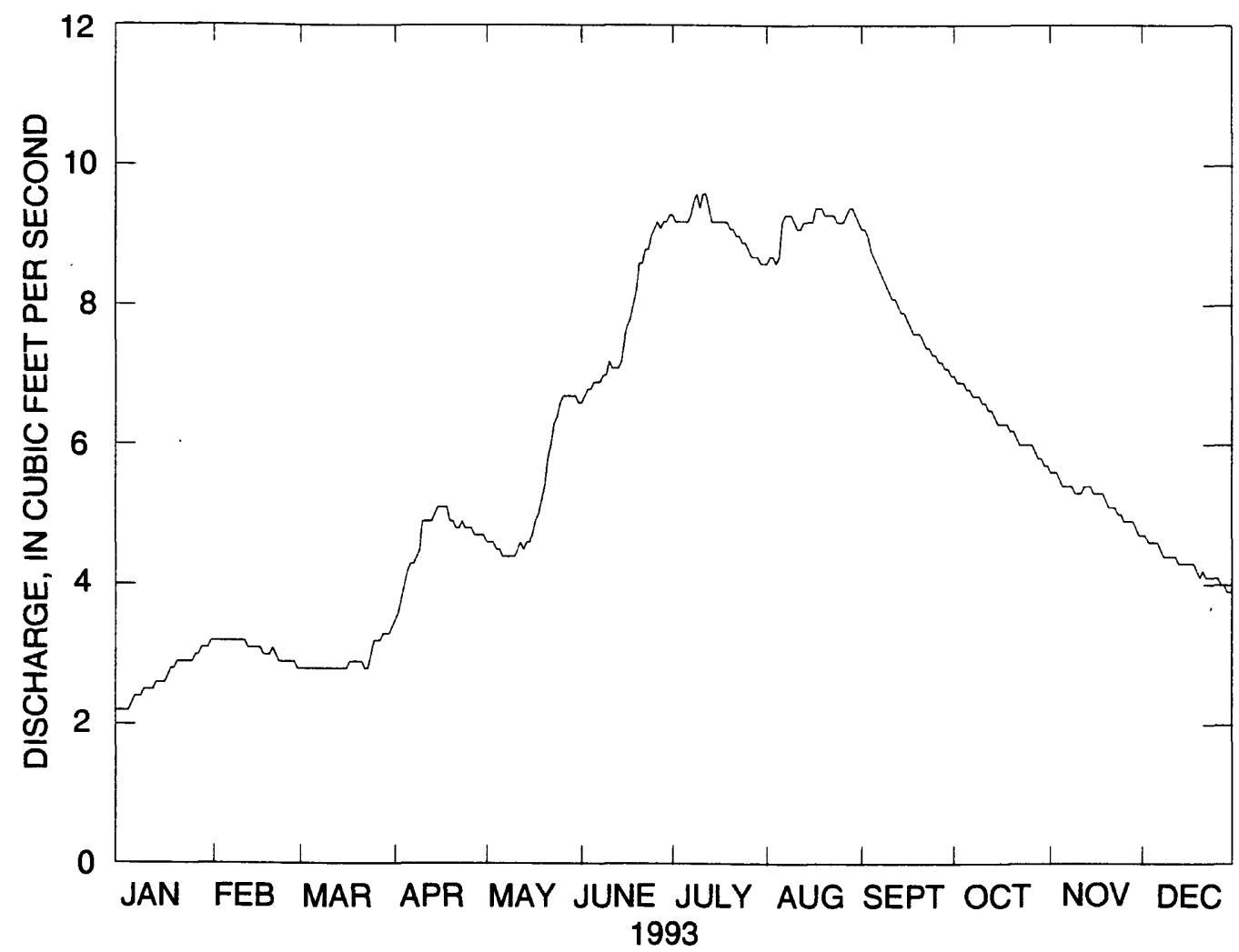

Figure 25. (C) Daily mean discharge, Fish Hatchery Spring AB (FHAB), weir site, Long Valley Caldera, Mono County, California, 1993. 
Table 42. Daily mean discharge, Fish Hatchery Spring AB (FHAB), weir site, Long Valley Caldera, Mono County, California, 1993

[Values are in cubic feet per second. Max, maximum; Min, minimum; -, no data]

\begin{tabular}{|c|c|c|c|c|c|c|c|c|c|c|c|c|}
\hline Day & Jan & Feb & Mar & Apr & May & June & July & Aug & Sept & Oct & Nov & Dec \\
\hline 1 & 2.2 & 3.2 & 2.8 & 3.5 & 4.6 & 6.6 & 9.3 & 8.6 & 9.1 & 7.0 & 5.6 & 4.7 \\
\hline 2 & 2.2 & 3.2 & 2.8 & 3.6 & 4.6 & 6.7 & 9.2 & 8.7 & 9.1 & 6.9 & 5.6 & 4.7 \\
\hline 3 & 2.2 & 3.2 & 2.8 & 3.8 & 4.6 & 6.8 & 9.2 & 8.7 & 9.0 & 6.9 & 5.6 & 4.6 \\
\hline 4 & 2.2 & 3.2 & 2.8 & 4.0 & 4.5 & 6.8 & 9.2 & 8.6 & 8.8 & 6.9 & 5.5 & 4.6 \\
\hline 5 & 2.3 & 3.2 & 2.8 & 4.2 & 4.5 & 6.9 & 9.2 & 8.7 & 8.7 & 6.8 & 5.4 & 4.6 \\
\hline 6 & 2.4 & 3.2 & 2.8 & 4.3 & 4.4 & 6.9 & 9.2 & 9.2 & 8.6 & 6.8 & 5.4 & 4.6 \\
\hline 7 & 2.4 & 3.2 & 2.8 & 4.3 & 4.4 & 6.9 & 9.3 & 9.3 & 8.5 & 6.7 & 5.4 & 4.5 \\
\hline 8 & 2.4 & 3.2 & 2.8 & 4.4 & 4.4 & 7.0 & 9.5 & 9.3 & 8.4 & 6.7 & 5.4 & 4.4 \\
\hline 9 & 2.5 & 3.2 & 2.8 & 4.5 & 4.4 & 7.0 & 9.6 & 9.3 & 8.3 & 6.7 & 5.3 & 4.4 \\
\hline 10 & 2.5 & 3.2 & 2.8 & 4.9 & 4.4 & 7.2 & 9.4 & 9.2 & 8.2 & 6.6 & 5.3 & 4.4 \\
\hline 11 & 2.5 & 3.2 & 2.8 & 4.9 & 4.5 & 7.1 & 9.6 & 9.1 & 8.1 & 6.6 & 5.3 & 4.4 \\
\hline 12 & 2.5 & 3.1 & 2.8 & 4.9 & 4.6 & 7.1 & 9.6 & 9.1 & 8.1 & 6.5 & 5.4 & 4.4 \\
\hline 13 & 2.6 & 3.1 & 2.8 & 4.9 & 4.5 & 7.1 & 9.4 & 9.2 & 8.0 & 6.5 & 5.4 & 4.3 \\
\hline 14 & 2.6 & 3.1 & 2.8 & 5.0 & 4.6 & 7.2 & 9.2 & 9.2 & 7.9 & 6.4 & 5.4 & 4.3 \\
\hline 15 & 2.6 & 3.1 & 2.8 & 5.1 & 4.6 & 7.5 & 9.2 & 9.2 & 7.9 & 6.3 & 5.3 & 4.3 \\
\hline 16 & 2.6 & 3.1 & 2.8 & 5.1 & 4.7 & 7.7 & 9.2 & 9.2 & 7.8 & 6.3 & 5.3 & 4.3 \\
\hline 17 & 2.7 & 3.0 & 2.9 & 5.1 & 4.9 & 7.8 & 9.2 & 9.4 & 7.7 & 6.3 & 5.3 & 4.3 \\
\hline 18 & 2.8 & 3.0 & 2.9 & 5.1 & 5.0 & 8.0 & 9.2 & 9.4 & 7.6 & 6.3 & 5.3 & 4.3 \\
\hline 19 & 2.8 & 3.0 & 2.9 & 4.9 & 5.2 & 8.2 & 9.2 & 9.4 & 7.6 & 6.2 & 5.2 & 4.2 \\
\hline 20 & 2.9 & 3.1 & 2.9 & 4.9 & 5.4 & 8.6 & 9.1 & 9.3 & 7.6 & 6.2 & 5.1 & 4.1 \\
\hline 21 & 2.9 & 3.0 & 2.9 & 4.8 & 5.8 & 8.6 & 9.1 & 9.3 & 7.5 & 6.1 & 5.1 & 4.2 \\
\hline 22 & 2.9 & 2.9 & 2.8 & 4.8 & 6.0 & 8.8 & 9.0 & 9.3 & 7.4 & 6.0 & 5.1 & 4.1 \\
\hline 23 & 2.9 & 2.9 & 2.8 & 4.9 & 6.3 & 8.8 & 9.0 & 9.3 & 7.4 & 6.0 & 5.0 & 4.1 \\
\hline 24 & 2.9 & 2.9 & 3.0 & 4.8 & 6.4 & 9.0 & 8.9 & 9.2 & 7.3 & 6.0 & 5.0 & 4.1 \\
\hline 25 & 2.9 & 2.9 & 3.2 & 4.8 & 6.6 & 9.1 & 8.9 & 9.2 & 7.3 & 6.0 & 4.9 & 4.1 \\
\hline 26 & 3.0 & 2.9 & 3.2 & 4.8 & 6.7 & 9.2 & 8.8 & 9.2 & 7.2 & 6.0 & 4.9 & 4.1 \\
\hline 27 & 3.0 & 2.9 & 3.2 & 4.7 & 6.7 & 9.1 & 8.7 & 9.3 & 7.2 & 5.9 & 4.9 & 4.0 \\
\hline 28 & 3.1 & 2.8 & 3.3 & 4.7 & 6.7 & 9.2 & 8.7 & 9.4 & 7.1 & 5.8 & 4.9 & 4.0 \\
\hline 29 & 3.1 & - & 3.3 & 4.7 & 6.7 & 9.2 & 8.7 & 9.4 & 7.1 & 5.8 & 4.8 & 3.9 \\
\hline 30 & 3.1 & - & 3.3 & 4.7 & 6.7 & 9.3 & 8.6 & 9.3 & 7.0 & 5.7 & 4.7 & 3.9 \\
\hline 31 & 3.2 & - & 3.4 & - & 6.6 & - & 8.6 & 9.2 & - & 5.7 & - & 3.9 \\
\hline Mean & 2.67 & 3.07 & 2.93 & 4.64 & 5.29 & 7.85 & 9.13 & 9.17 & 7.92 & 6.34 & 5.23 & 4.28 \\
\hline $\operatorname{Max}$ & 3.2 & 3.2 & 3.4 & 5.1 & 6.7 & 9.3 & 9.6 & 9.4 & 9.1 & 7.0 & 5.6 & 4.7 \\
\hline Min & 2.2 & 2.8 & 2.8 & 3.5 & 4.4 & 6.6 & 8.6 & 8.6 & 7.0 & 5.7 & 4.7 & 3.9 \\
\hline
\end{tabular}




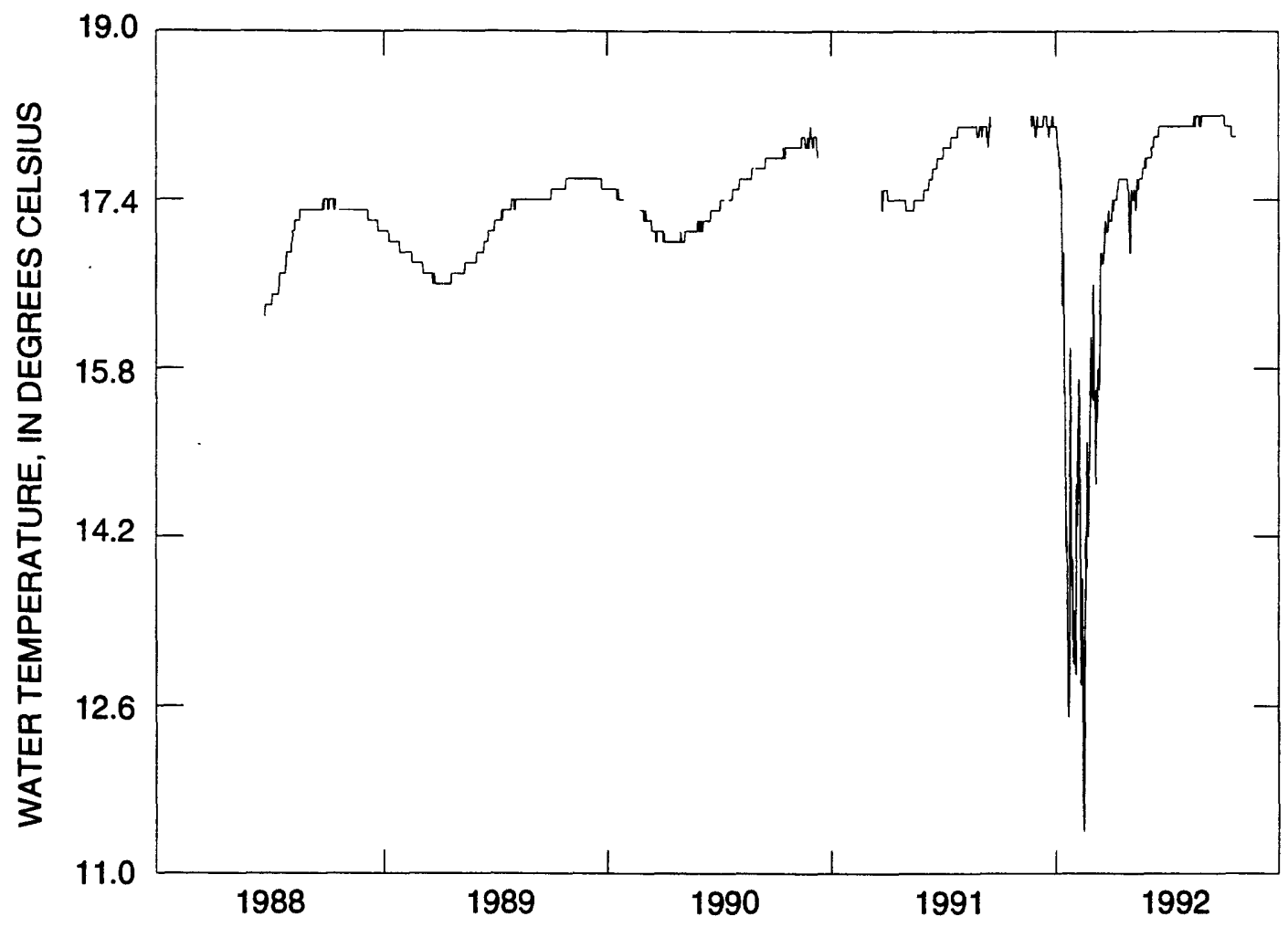

Figure 26. Daily mean water temperature, Fish Hatchery Spring AB (FHAB), upper site, Long Valley Caldera, Mono County, California, 1988-92.

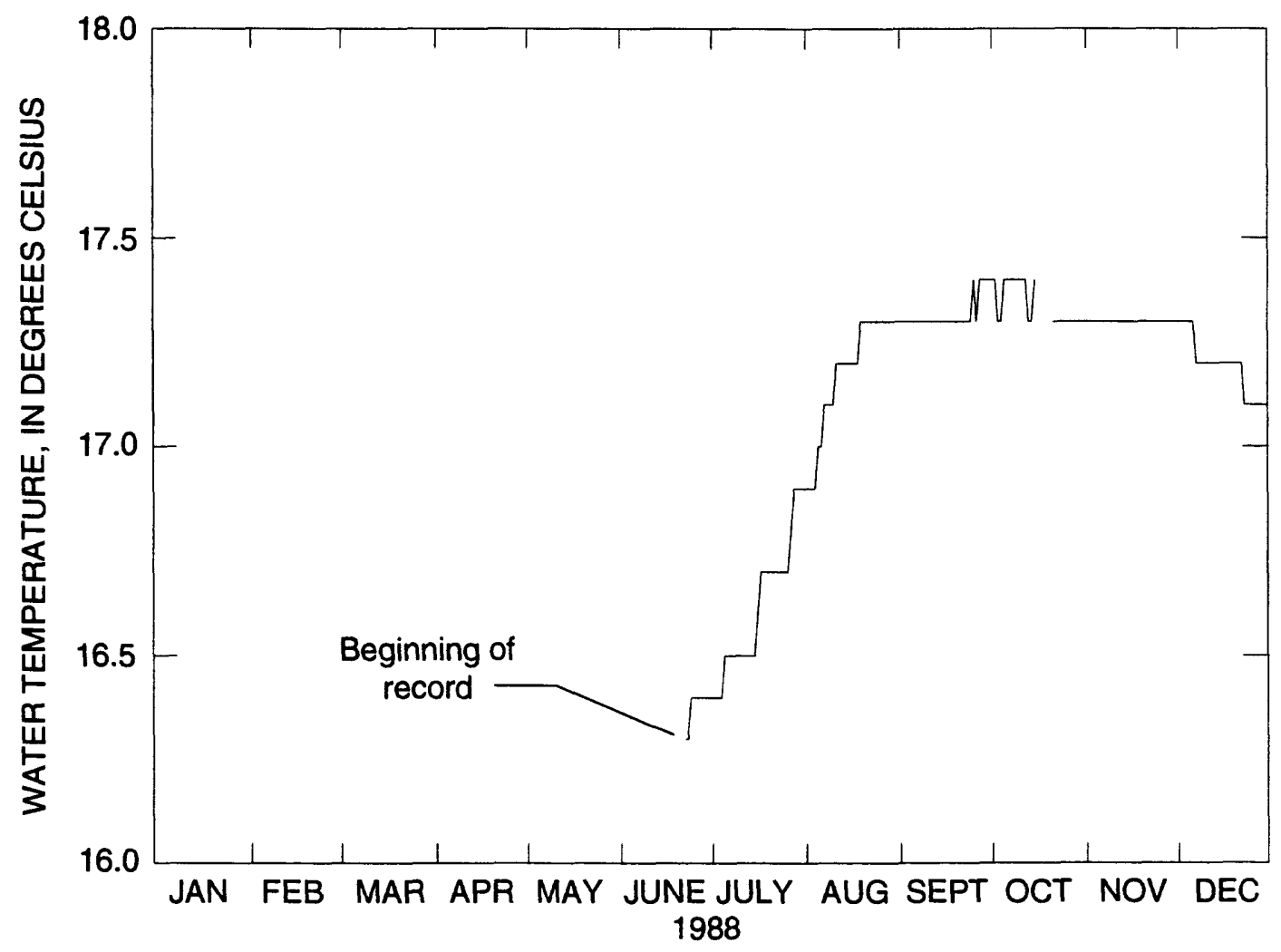

Figure 27. (A) Daily mean water temperature, Fish Hatchery Spring AB (FHAB), upper site, Long Valley Caldera, Mono County, California, 1988. 
Table 43. Daily mean water temperature, Fish Hatchery Spring AB (FHAB), upper site, Long Valley Caldera, Mono County, California, 1988

[Values are in degrees Celsius. Max, maximum; Min, minimum; -, no data]

\begin{tabular}{|c|c|c|c|c|c|c|c|c|c|c|c|c|}
\hline Day & Jan & Feb & Mar & Apr & May & June & July & Aug & Sept & Oct & Nov & Dec \\
\hline 1 & - & - & - & - & - & - & 16.4 & 16.9 & 17.3 & 17.4 & 17.3 & 17.3 \\
\hline 2 & - & - & - & - & - & - & 16.4 & 16.9 & 17.3 & 17.4 & 17.3 & 17.3 \\
\hline 3 & - & - & - & - & - & - & 16.4 & 16.9 & 17.3 & 17.3 & 17.3 & 17.3 \\
\hline 4 & - & - & - & - & - & - & 16.4 & 16.9 & 17.3 & 17.3 & 17.3 & 17.3 \\
\hline 5 & - & - & - & - & - & - & 16.5 & 17.0 & 17.3 & 17.4 & 17.3 & 17.3 \\
\hline 6 & - & - & - & - & - & - & 16.5 & 17.0 & 17.3 & 17.4 & 17.3 & 17.3 \\
\hline 7 & - & - & - & - & - & - & 16.5 & 17.1 & 17.3 & 17.4 & 17.3 & 17.2 \\
\hline 8 & - & - & - & - & - & - & 16.5 & 17.1 & 17.3 & 17.4 & 17.3 & 17.2 \\
\hline 9 & - & - & - & - & - & - & 16.5 & 17.1 & 17.3 & 17.4 & 17.3 & 17.2 \\
\hline 10 & - & - & - & - & - & - & 16.5 & 17.1 & 17.3 & 17.4 & 17.3 & 17.2 \\
\hline 11 & - & - & - & - & - & - & 16.5 & 17.2 & 17.3 & 17.4 & 17.3 & 17.2 \\
\hline 12 & - & - & - & - & - & - & 16.5 & 17.2 & 17.3 & 17.4 & 17.3 & 17.2 \\
\hline 13 & - & - & - & - & - & - & 16.5 & 17.2 & 17.3 & 17.3 & 17.3 & 17.2 \\
\hline 14 & - & - & - & - & - & - & 16.5 & 17.2 & 17.3 & 17.3 & 17.3 & 17.2 \\
\hline 15 & - & - & - & - & - & - & 16.5 & 17.2 & 17.3 & 17.4 & 17.3 & 17.2 \\
\hline 16 & - & - & - & - & - & - & 16.6 & 17.2 & 17.3 & - & 17.3 & 17.2 \\
\hline 17 & - & - & - & - & - & - & 16.7 & 17.2 & 17.3 & - & 17.3 & 17.2 \\
\hline 18 & - & - & - & - & - & - & 16.7 & 17.2 & 17.3 & - & 17.3 & 17.2 \\
\hline 19 & - & - & - & - & - & - & 16.7 & 17.3 & 17.3 & - & 17.3 & 17.2 \\
\hline 20 & - & - & - & - & - & - & 16.7 & 17.3 & 17.3 & - & 17.3 & 17.2 \\
\hline 21 & - & - & - & - & - & - & 16.7 & 17.3 & 17.3 & 17.3 & 17.3 & 17.2 \\
\hline 22 & - & - & - & - & - & 16.3 & 16.7 & 17.3 & 17.3 & 17.3 & 17.3 & 17.2 \\
\hline 23 & - & - & - & - & - & 16.3 & 16.7 & 17.3 & 17.3 & 17.3 & 17.3 & 17.1 \\
\hline 24 & - & - & - & - & - & 16.4 & 16.7 & 17.3 & 17.3 & 17.3 & 17.3 & 17.1 \\
\hline 25 & - & - & 一 & - & - & 16.4 & 16.7 & 17.3 & 17.4 & 17.3 & 17.3 & 17.1 \\
\hline 26 & - & - & - & - & - & 16.4 & 16.7 & 17.3 & 17.3 & 17.3 & 17.3 & 17.1 \\
\hline 27 & - & - & - & - & - & 16.4 & 16.8 & 17.3 & 17.4 & 17.3 & 17.3 & 17.1 \\
\hline 28 & - & - & - & - & - & 16.4 & 16.9 & 17.3 & 17.4 & 17.3 & 17.3 & 17.1 \\
\hline 29 & - & - & - & - & - & 16.4 & 16.9 & 17.3 & 17.4 & 17.3 & 17.3 & 17.1 \\
\hline 30 & - & - & - & - & - & 16.4 & 16.9 & 17.3 & 17.4 & 17.3 & 17.3 & 17.1 \\
\hline 31 & - & - & - & - & - & - & 16.9 & 17.3 & - & 17.3 & - & 17.1 \\
\hline Mean & - & - & - & - & - & 16.4 & 16.6 & 17.2 & 17.3 & 17.3 & 17.3 & 17.2 \\
\hline Max & - & - & - & - & - & 16.4 & 16.9 & 17.3 & 17.4 & 17.4 & 17.3 & 17.3 \\
\hline Min & - & - & - & - & - & 16.3 & 16.4 & 16.9 & 17.3 & 17.3 & 17.3 & 17.1 \\
\hline
\end{tabular}




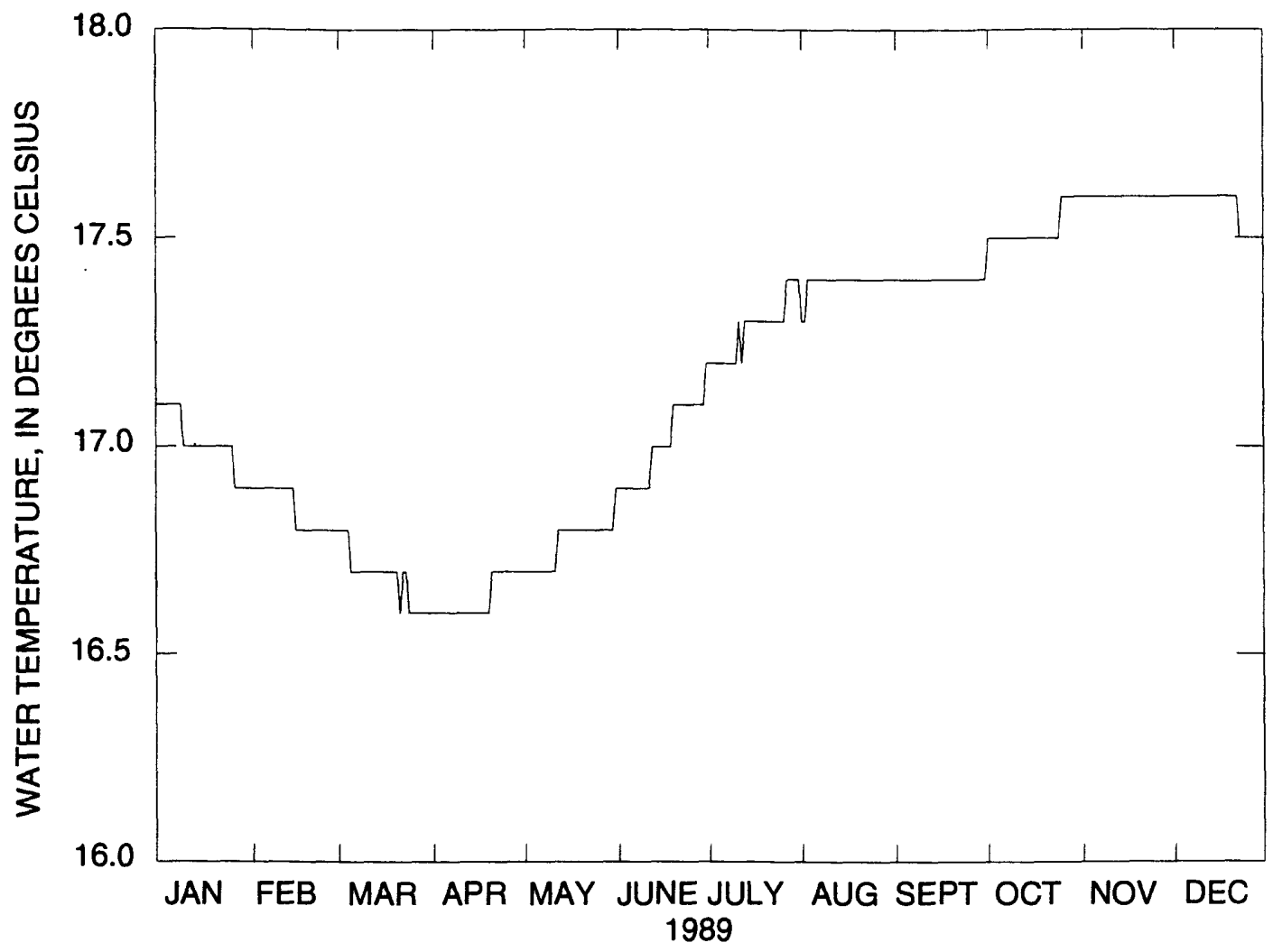

Figure 27. (B) Daily mean water temperature, Fish Hatchery Spring $A B$ (FHAB), upper site, Long Valley Caldera, Mono County, California, 1989. 
Table 44. Daily mean water temperature, Fish Hatchery Spring AB (FHAB), upper site, Long Valley Caldera, Mono County, California, 1989

[Values are in degrees Celsius. Max, maximum; Min, minimum; -, no data]

\begin{tabular}{|c|c|c|c|c|c|c|c|c|c|c|c|c|}
\hline Day & Jan & $\mathrm{Feb}$ & Mar & Apr & May & June & July & Aug & Sept & Oct & Nov & Dec \\
\hline 1 & 17.1 & 16.9 & 16.8 & 16.6 & 16.7 & 16.9 & 17.2 & 17.3 & 17.4 & 17.5 & 17.6 & 17.6 \\
\hline 2 & 17.1 & 16.9 & 16.8 & 16.6 & 16.7 & 16.9 & 17.2 & 17.3 & 17.4 & 17.5 & 17.6 & 17.6 \\
\hline 3 & 17.1 & 16.9 & 16.8 & 16.6 & 16.7 & 16.9 & 17.2 & 17.4 & 17.4 & 17.5 & 17.6 & 17.6 \\
\hline 4 & 17.1 & 16.9 & 16.8 & 16.6 & 16.7 & 16.9 & 17.2 & 17.4 & 17.4 & 17.5 & 17.6 & 17.6 \\
\hline 5 & 17.1 & 16.9 & 16.7 & 16.6 & 16.7 & 16.9 & 17.2 & 17.4 & 17.4 & 17.5 & 17.6 & 17.6 \\
\hline 6 & 17.1 & 16.9 & 16.7 & 16.6 & 16.7 & 16.9 & 17.2 & 17.4 & 17.4 & 17.5 & 17.6 & 17.6 \\
\hline 7 & 17.1 & 16.9 & 16.7 & 16.6 & 16.7 & 16.9 & 17.2 & 17.4 & 17.4 & 17.5 & 17.6 & 17.6 \\
\hline 8 & 17.1 & 16.9 & 16.7 & 16.6 & 16.7 & 16.9 & 17.2 & 17.4 & 17.4 & 17.5 & 17.6 & 17.6 \\
\hline 9 & 17.0 & 16.9 & 16.7 & 16.6 & 16.7 & 16.9 & 17.2 & 17.4 & 17.4 & 17.5 & 17.6 & 17.6 \\
\hline 10 & 17.0 & 16.9 & 16.7 & 16.6 & 16.7 & 16.9 & 17.2 & 17.4 & 17.4 & 17.5 & 17.6 & 17.6 \\
\hline 11 & 17.0 & 16.9 & 16.7 & 16.6 & 16.7 & 16.9 & 17.3 & 17.4 & 17.4 & 17.5 & 17.6 & 17.6 \\
\hline 12 & 17.0 & 16.9 & 16.7 & 16.6 & 16.8 & 17.0 & 17.2 & 17.4 & 17.4 & 17.5 & 17.6 & 17.6 \\
\hline 13 & 17.0 & 16.9 & 16.7 & 16.6 & 16.8 & 17.0 & 17.3 & 17.4 & 17.4 & 17.5 & 17.6 & 17.6 \\
\hline 14 & 17.0 & 16.9 & 16.7 & 16.6 & 16.8 & 17.0 & 17.3 & 17.4 & 17.4 & 17.5 & 17.6 & 17.6 \\
\hline 15 & 17.0 & 16.8 & 16.7 & 16.6 & 16.8 & 17.0 & 17.3 & 17.4 & 17.4 & 17.5 & 17.6 & 17.6 \\
\hline 16 & 17.0 & 16.8 & 16.7 & 16.6 & 16.8 & 17.0 & 17.3 & 17.4 & 17.4 & 17.5 & 17.6 & 17.6 \\
\hline 17 & 17.0 & 16.8 & 16.7 & 16.6 & 16.8 & 17.0 & 17.3 & 17.4 & 17.4 & 17.5 & 17.6 & 17.6 \\
\hline 18 & 17.0 & 16.8 & 16.7 & 16.6 & 16.8 & 17.0 & 17.3 & 17.4 & 17.4 & 17.5 & 17.6 & 17.6 \\
\hline 19 & 17.0 & 16.8 & 16.7 & 16.6 & 16.8 & 17.1 & 17.3 & 17.4 & 17.4 & 17.5 & 17.6 & 17.6 \\
\hline 20 & 17.0 & 16.8 & 16.7 & 16.7 & 16.8 & 17.1 & 17.3 & 17.4 & 17.4 & 17.5 & 17.6 & 17.6 \\
\hline 21 & 17.0 & 16.8 & 16.6 & 16.7 & 16.8 & 17.1 & 17.3 & 17.4 & 17.4 & 17.5 & 17.6 & 17.6 \\
\hline 22 & 17.0 & 16.8 & 16.7 & 16.7 & 16.8 & 17.1 & 17.3 & 17.4 & 17.4 & 17.5 & 17.6 & 17.6 \\
\hline 23 & 17.0 & 16.8 & 16.7 & 16.7 & 16.8 & 17.1 & 17.3 & 17.4 & 17.4 & 17.5 & 17.6 & 17.5 \\
\hline 24 & 17.0 & 16.8 & 16.6 & 16.7 & 16.8 & 17.1 & 17.3 & 17.4 & 17.4 & 17.5 & 17.6 & 17.5 \\
\hline 25 & 17.0 & 16.8 & 16.6 & 16.7 & 16.8 & 17.1 & 17.3 & 17.4 & 17.4 & 17.6 & 17.6 & 17.5 \\
\hline 26 & 16.9 & 16.8 & 16.6 & 16.7 & 16.8 & 17.1 & 17.3 & 17.4 & 17.4 & 17.6 & 17.6 & 17.5 \\
\hline 27 & 16.9 & 16.8 & 16.6 & 16.7 & 16.8 & 17.1 & 17.4 & 17.4 & 17.4 & 17.6 & 17.6 & 17.5 \\
\hline 28 & 16.9 & 16.8 & 16.6 & 16.7 & 16.8 & 17.1 & 17.4 & 17.4 & 17.4 & 17.6 & 17.6 & 17.5 \\
\hline 29 & 16.9 & - & 16.6 & 16.7 & 16.8 & 17.1 & 17.4 & 17.4 & 17.4 & 17.6 & 17.6 & 17.5 \\
\hline 30 & 16.9 & - & 16.6 & 16.7 & 16.8 & 17.2 & 17.4 & 17.4 & 17.4 & 17.6 & 17.6 & 17.5 \\
\hline 31 & 16.9 & - & 16.6 & - & 16.9 & - & 17.4 & 17.4 & - & 17.6 & - & 17.5 \\
\hline Mean & 17.0 & 16.8 & 16.7 & 16.6 & 16.8 & 17.0 & 17.3 & 17.4 & 17.4 & 17.5 & 17.6 & 17.6 \\
\hline Max & 17.1 & 16.9 & 16.8 & 16.7 & 16.9 & 17.2 & 17.4 & 17.4 & 17.4 & 17.6 & 17.6 & 17.6 \\
\hline Min & 16.9 & 16.8 & 16.6 & 16.6 & 16.7 & 16.9 & 17.2 & 17.3 & 17.4 & 17.5 & 17.6 & 17.5 \\
\hline
\end{tabular}




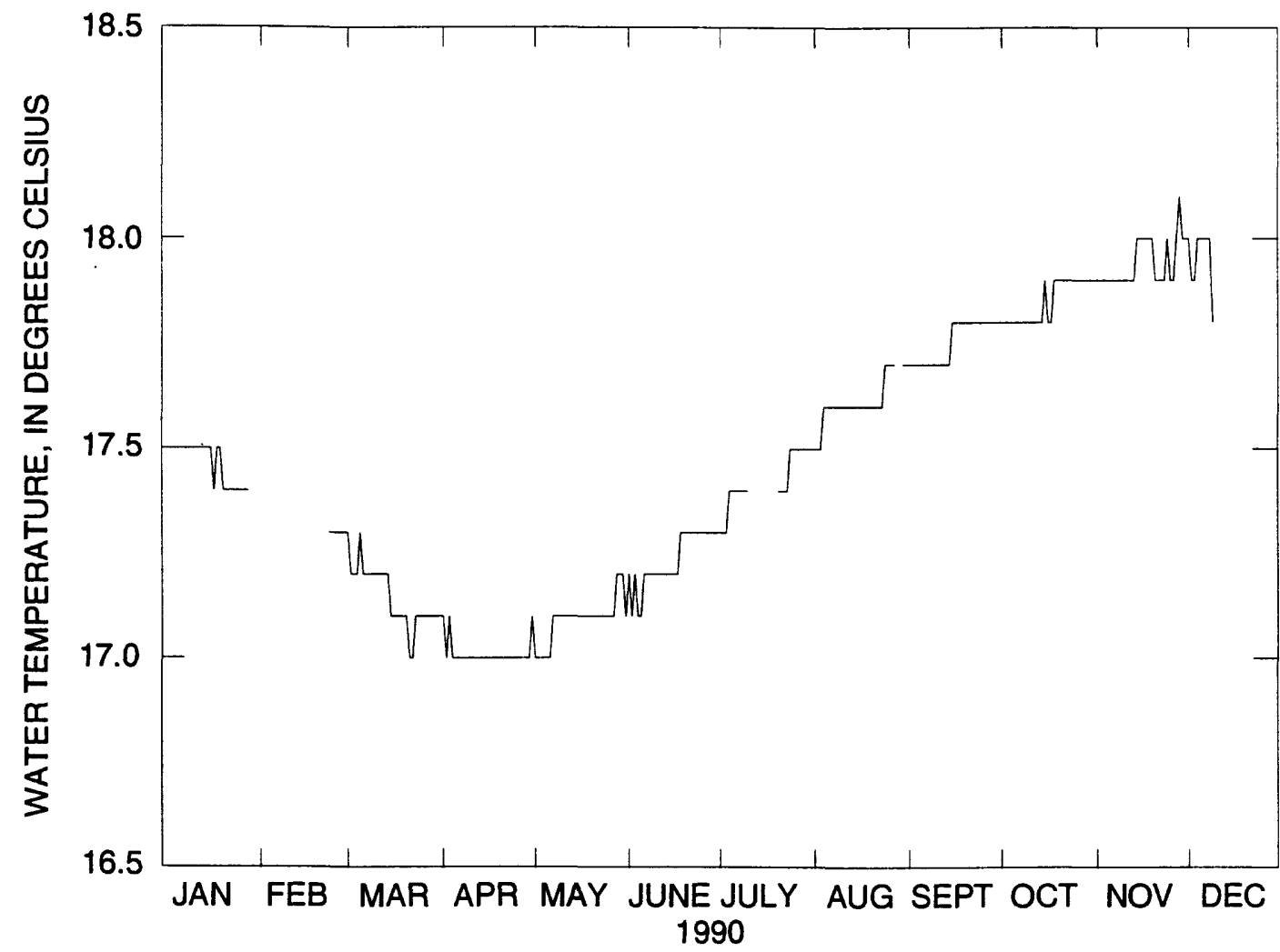

Figure 27. (C) Daily mean water temperature, Fish Hatchery Spring AB (FHAB), upper site, Long Valley Caldera, Mono County, California, 1990. 
Table 45. Daily mean water temperature, Fish Hatchery Spring AB (FHAB), upper site, Long Valley Caldera, Mono County, California, 1990

[Values are in degrees Celsius. Max, maximum; Min, minimum; -, no data]

\begin{tabular}{|c|c|c|c|c|c|c|c|c|c|c|c|c|}
\hline Day & Jan & Feb & Mar & Apr & May & June & July & Aug & Sept & Oct & Nov & Dec \\
\hline 1 & 17.5 & - & 17.3 & 17.1 & 17.0 & 17.2 & 17.3 & 17.5 & 17.7 & 17.8 & 17.9 & 18.0 \\
\hline 2 & 17.5 & - & 17.2 & 17.0 & 17.0 & 17.1 & 17.3 & 17.5 & 17.7 & 17.8 & 17.9 & 17.9 \\
\hline 3 & 17.5 & - & 17.2 & 17.1 & 17.0 & 17.2 & 17.3 & 17.5 & 17.7 & 17.8 & 17.9 & 17.9 \\
\hline 4 & 17.5 & - & 17.2 & 17.0 & 17.0 & 17.1 & 17.4 & 17.6 & 17.7 & 17.8 & 17.9 & 18.0 \\
\hline 5 & 17.5 & - & 17.3 & 17.0 & 17.0 & 17.1 & 17.4 & 17.6 & 17.7 & 17.8 & 17.9 & 18.0 \\
\hline 6 & 17.5 & - & 17.2 & 17.0 & 17.0 & 17.2 & 17.4 & 17.6 & 17.7 & 17.8 & 17.9 & 18.0 \\
\hline 7 & 17.5 & - & 17.2 & 17.0 & 17.1 & 17.2 & 17.4 & 17.6 & 17.7 & 17.8 & 17.9 & 18.0 \\
\hline 8 & 17.5 & - & 17.2 & 17.0 & 17.1 & 17.2 & 17.4 & 17.6 & 17.7 & 17.8 & 17.9 & 18.0 \\
\hline 9 & 17.5 & - & 17.2 & 17.0 & 17.1 & 17.2 & 17.4 & 17.6 & 17.7 & 17.8 & 17.9 & 17.8 \\
\hline 10 & 17.5 & - & 17.2 & 17.0 & 17.1 & 17.2 & 17.4 & 17.6 & 17.7 & 17.8 & 17.9 & - \\
\hline 11 & 17.5 & - & 17.2 & 17.0 & 17.1 & 17.2 & - & 17.6 & 17.7 & 17.8 & 17.9 & - \\
\hline 12 & 17.5 & - & 17.2 & 17.0 & 17.1 & 17.2 & - & 17.6 & 17.7 & 17.8 & 17.9 & - \\
\hline 13 & 17.5 & - & 17.2 & 17.0 & 17.1 & 17.2 & - & 17.6 & 17.7 & 17.8 & 17.9 & - \\
\hline 14 & 17.5 & - & 17.2 & 17.0 & 17.1 & 17.2 & - & 17.6 & 17.7 & 17.8 & 18.0 & - \\
\hline 15 & 17.5 & - & 17.1 & 17.0 & 17.1 & 17.2 & - & 17.6 & 17.8 & 17.9 & 18.0 & - \\
\hline 16 & 17.5 & - & 17.1 & 17.0 & 17.1 & 17.2 & - & 17.6 & 17.8 & 17.8 & 18.0 & - \\
\hline 17 & 17.4 & - & 17.1 & 17.0 & 17.1 & 17.2 & - & 17.6 & 17.8 & 17.8 & 18.0 & - \\
\hline 18 & 17.5 & - & 17.1 & 17.0 & 17.1 & 17.3 & - & 17.6 & 17.8 & 17.9 & 18.0 & - \\
\hline 19 & 17.5 & - & 17.1 & 17.0 & 17.1 & 17.3 & - & 17.6 & 17.8 & 17.9 & 18.0 & - \\
\hline 20 & 17.4 & - & 17.1 & 17.0 & 17.1 & 17.3 & 17.4 & 17.6 & 17.8 & 17.9 & 17.9 & - \\
\hline 21 & 17.4 & - & 17.0 & 17.0 & 17.1 & 17.3 & 17.4 & 17.6 & 17.8 & 17.9 & 17.9 & - \\
\hline 22 & 17.4 & - & 17.0 & 17.0 & 17.1 & 17.3 & 17.4 & 17.6 & 17.8 & 17.9 & 17.9 & - \\
\hline 23 & 17.4 & 17.3 & 17.1 & 17.0 & 17.1 & 17.3 & 17.4 & 17.6 & 17.8 & 17.9 & 17.9 & - \\
\hline 24 & 17.4 & 17.3 & 17.1 & 17.0 & 17.1 & 17.3 & 17.5 & 17.7 & 17.8 & 17.9 & 18.0 & - \\
\hline 25 & 17.4 & 17.3 & 17.1 & 17.0 & 17.1 & 17.3 & 17.5 & 17.7 & 17.8 & 17.9 & 17.9 & - \\
\hline 26 & 17.4 & 17.3 & 17.1 & 17.0 & 17.1 & 17.3 & 17.5 & 17.7 & 17.8 & 17.9 & 17.9 & - \\
\hline 27 & 17.4 & 17.3 & 17.1 & 17.0 & 17.1 & 17.3 & 17.5 & 17.7 & 17.8 & 17.9 & 18.0 & - \\
\hline 28 & 17.4 & 17.3 & 17.1 & 17.0 & 17.2 & 17.3 & 17.5 & - & 17.8 & 17.9 & 18.1 & - \\
\hline 29 & - & - & 17.1 & 17.0 & 17.2 & 17.3 & 17.5 & - & 17.8 & 17.9 & 18.0 & - \\
\hline 30 & - & - & 17.1 & 17.1 & 17.2 & 17.3 & 17.5 & 17.7 & 17.8 & 17.9 & 18.0 & - \\
\hline 31 & - & - & 17.1 & - & 17.1 & - & 17.5 & 17.7 & - & 17.9 & - & - \\
\hline Mean & 17.5 & 17.3 & 17.1 & 17.0 & 17.1 & 17.2 & 17.4 & 17.6 & 17.8 & 17.8 & 17.9 & 18.0 \\
\hline $\operatorname{Max}$ & 17.5 & 17.3 & 17.3 & 17.1 & 17.2 & 17.3 & 17.5 & 17.7 & 17.8 & 17.9 & 18.1 & 18.0 \\
\hline Min & 17.4 & 17.3 & 17.0 & 17.0 & 17.0 & 17.1 & 17.3 & 17.5 & 17.7 & 17.8 & 17.9 & 17.8 \\
\hline
\end{tabular}




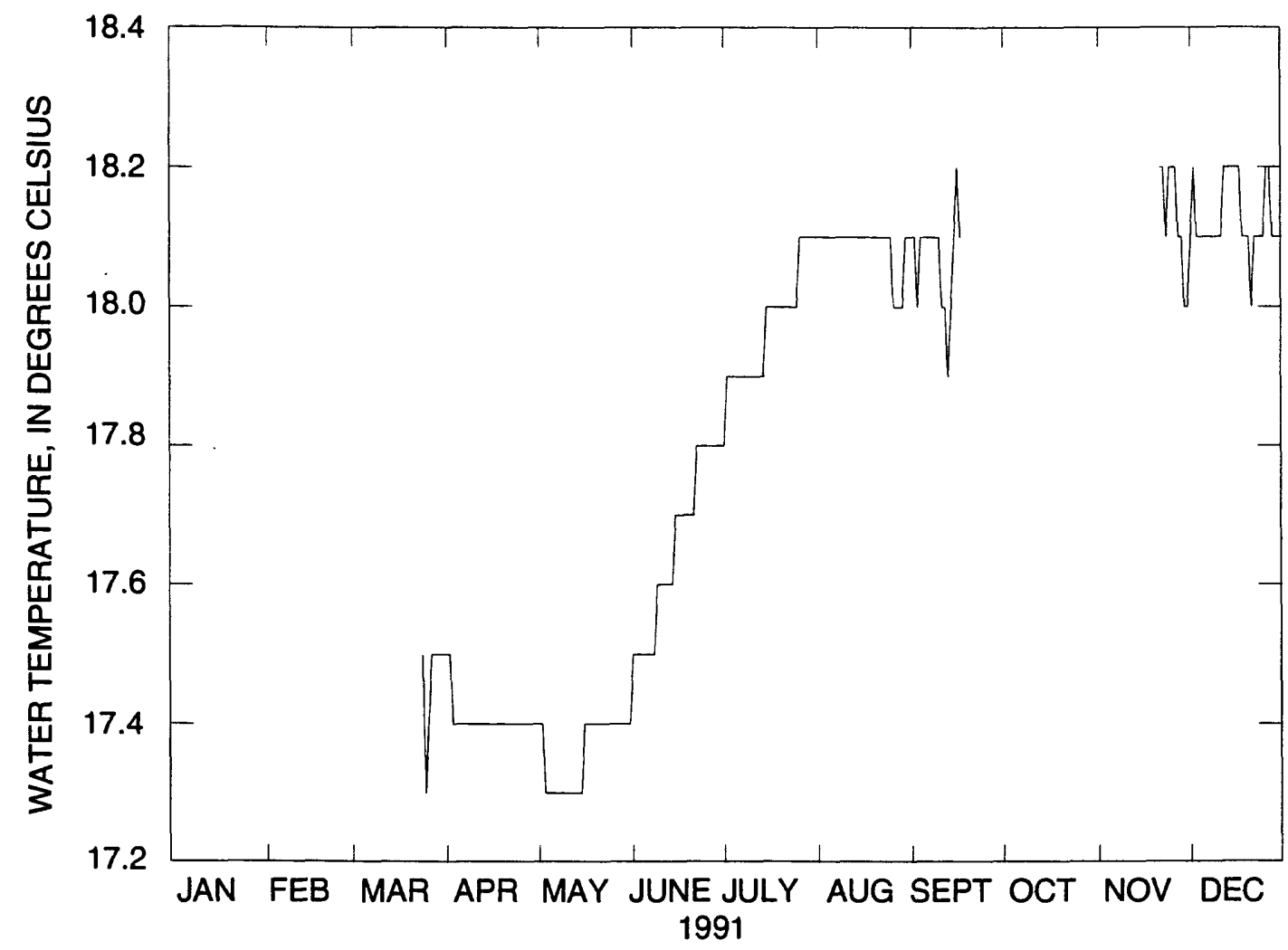

Figure 27. (D) Daily mean water temperature, Fish Hatchery Spring AB (FHAB), upper site, Long Valley Caldera, Mono County, California, 1991. 
Table 46. Daily mean water temperature, Fish Hatchery Spring AB (FHAB), upper site, Long Valley Caldera, Mono County, California, 1991

[Values are in degrees Celsius. Max, maximum: Min, minimum; -, no data]

\begin{tabular}{|c|c|c|c|c|c|c|c|c|c|c|c|c|}
\hline$\overline{\text { Day }}$ & Jan & Feb & Mar & Apr & May & June & July & Aug & Sept & Oct & Nov & Dec \\
\hline 1 & - & - & - & 17.5 & 17.4 & 17.5 & 17.8 & 18.1 & 18.1 & - & - & 18.1 \\
\hline 2 & - & - & - & 17.5 & 17.4 & 17.5 & 17.9 & 18.1 & 18.1 & - & - & 18.2 \\
\hline 3 & - & - & - & 17.4 & 17.3 & 17.5 & 17.9 & 18.1 & 18.0 & - & - & 18.1 \\
\hline 4 & - & - & - & 17.4 & 17.3 & 17.5 & 17.9 & 18.1 & 18.1 & - & - & 18.1 \\
\hline 5 & - & - & - & 17.4 & 17.3 & 17.5 & 17.9 & 18.1 & 18.1 & - & - & 18.1 \\
\hline 6 & - & - & - & 17.4 & 17.3 & 17.5 & 17.9 & 18.1 & 18.1 & - & - & 18.1 \\
\hline 7 & - & - & - & 17.4 & 17.3 & 17.5 & 17.9 & 18.1 & 18.1 & - & - & 18.1 \\
\hline 8 & - & - & - & 17.4 & 17.3 & 17.5 & 17.9 & 18.1 & 18.1 & - & - & 18.1 \\
\hline 9 & - & - & - & 17.4 & 17.3 & 17.6 & 17.9 & 18.1 & 18.1 & - & - & 18.1 \\
\hline 10 & - & - & - & 17.4 & 17.3 & 17.6 & 17.9 & 18.1 & 18.1 & - & - & 18.1 \\
\hline 11 & - & - & - & 17.4 & 17.3 & 17.6 & 17.9 & 18.1 & 18.0 & - & - & 18.1 \\
\hline 12 & - & - & - & 17.4 & 17.3 & 17.6 & 17.9 & 18.1 & 18.0 & - & - & 18.2 \\
\hline 13 & - & - & - & 17.4 & 17.3 & 17.6 & 17.9 & 18.1 & 17.9 & - & - & 18.2 \\
\hline 14 & - & - & - & 17.4 & 17.3 & 17.6 & 17.9 & 18.1 & 18.0 & - & - & 18.2 \\
\hline 15 & - & - & - & 17.4 & 17.3 & 17.7 & 18.0 & 18.1 & 18.1 & - & - & 18.2 \\
\hline 16 & - & - & - & 17.4 & 17.4 & 17.7 & 18.0 & 18.1 & 18.2 & - & - & 18.2 \\
\hline 17 & - & - & - & 17.4 & 17.4 & 17.7 & 18.0 & 18.1 & 18.1 & - & - & 18.2 \\
\hline 18 & - & - & - & 17.4 & 17.4 & 17.7 & 18.0 & 18.1 & - & - & - & 18.1 \\
\hline 19 & - & - & - & 17.4 & 17.4 & 17.7 & 18.0 & 18.1 & - & - & - & 18.1 \\
\hline 20 & - & - & - & 17.4 & 17.4 & 17.7 & 18.0 & 18.1 & - & - & - & 18.1 \\
\hline 21 & - & - & - & 17.4 & 17.4 & 17.7 & 18.0 & 18.1 & - & - & 18.2 & 18.0 \\
\hline 22 & - & - & - & 17.4 & 17.4 & 17.8 & 18.0 & 18.1 & - & - & 18.2 & 18.1 \\
\hline 23 & - & - & - & 17.4 & 17.4 & 17.8 & 18.0 & 18.1 & - & - & 18.1 & 18.1 \\
\hline 24 & - & - & 17.5 & 17.4 & 17.4 & 17.8 & 18.0 & 18.1 & - & - & 18.2 & 18.1 \\
\hline 25 & - & - & 17.3 & 17.4 & 17.4 & 17.8 & 18.0 & 18.1 & - & - & 18.2 & 18.1 \\
\hline 26 & - & - & 17.4 & 17.4 & 17.4 & 17.8 & 18.1 & 18.0 & - & - & 18.2 & 18.2 \\
\hline 27 & - & - & 17.5 & 17.4 & 17.4 & 17.8 & 18.1 & 18.0 & - & - & 18.1 & 18.2 \\
\hline 28 & - & - & 17.5 & 17.4 & 17.4 & 17.8 & 18.1 & 18.0 & - & - & 18.1 & 18.1 \\
\hline 29 & - & - & 17.5 & 17.4 & 17.4 & 17.8 & 18.1 & 18.0 & - & - & 18.0 & 18.1 \\
\hline 30 & - & - & 17.5 & 17.4 & 17.4 & 17.8 & 18.1 & 18.1 & - & - & 18.0 & 18.1 \\
\hline 31 & - & - & 17.5 & - & 17.4 & - & 18.1 & 18.1 & - & - & - & 18.1 \\
\hline Mean & - & - & 17.5 & 17.4 & 17.4 & 17.7 & 18.0 & 18.1 & 18.1 & - & 18.1 & 18.1 \\
\hline $\operatorname{Max}$ & - & - & 17.5 & 17.5 & 17.4 & 17.8 & 18.1 & 18.1 & 18.2 & - & 18.2 & 18.2 \\
\hline Min & - & - & 17.3 & 17.4 & 17.3 & 17.5 & 17.8 & 18.0 & 17.9 & - & 18.0 & 18.0 \\
\hline
\end{tabular}




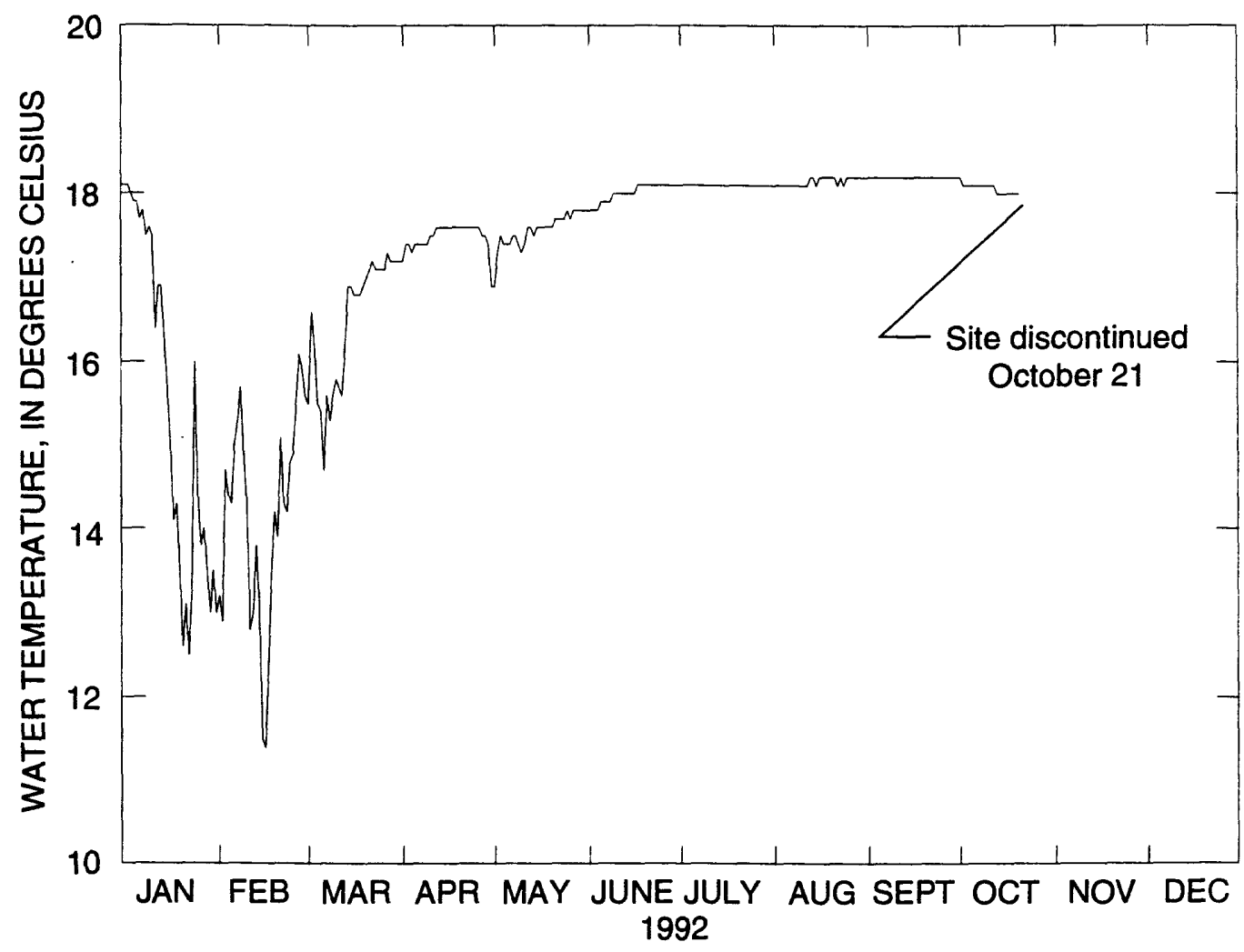

Figure 27. (E) Daily mean water temperature, Fish Hatchery Spring AB (FHAB), upper site, Long Valley Caldera, Mono County, California, 1992. 
Table 47. Daily mean water temperature, Fish Hatchery Spring AB (FHAB), upper site, Long Valley Caldera, Mono County, California, 1992

[Values are in degrees Celsius. Max, maximum; Min, minimum; -, no data]

\begin{tabular}{|c|c|c|c|c|c|c|c|c|c|c|c|c|}
\hline Day & Jan & Feb & Mar & Apr & May & June & July & Aug & Sept & Oct & Nov & Dec \\
\hline 1 & 18.1 & 13.2 & 15.5 & 17.2 & 16.9 & 17.8 & 18.1 & 18.1 & 18.2 & 18.2 & - & - \\
\hline 2 & 18.1 & 12.9 & 16.6 & 17.4 & 17.3 & 17.8 & 18.1 & 18.1 & 18.2 & 18.1 & - & - \\
\hline 3 & 18.0 & 14.7 & 16.2 & 17.4 & 17.5 & 17.8 & 18.1 & 18.1 & 18.2 & 18.1 & - & - \\
\hline 4 & 17.9 & 14.4 & 15.5 & 17.3 & 17.4 & 17.8 & 18.1 & 18.1 & 18.2 & 18.1 & 一 & - \\
\hline 5 & 17.9 & 14.3 & 15.4 & 17.4 & 17.4 & 17.9 & 18.1 & 18.1 & 18.2 & 18.1 & - & - \\
\hline 6 & 17.7 & 15.0 & 14.7 & 17.4 & 17.4 & 17.9 & 18.1 & 18.1 & 18.2 & 18.1 & - & - \\
\hline 7 & 17.8 & 15.3 & 15.6 & 17.4 & 17.5 & 17.9 & 18.1 & 18.1 & 18.2 & 18.1 & - & - \\
\hline 8 & 17.5 & 15.7 & 15.3 & 17.4 & 17.5 & 17.9 & 18.1 & 18.1 & 18.2 & 18.1 & - & - \\
\hline 9 & 17.6 & 14.9 & 15.6 & 17.4 & 17.4 & 18.0 & 18.1 & 18.1 & 18.2 & 18.1 & - & - \\
\hline 10 & 17.5 & 14.3 & 15.8 & 17.5 & 17.3 & 18.0 & 18.1 & 18.1 & 18.2 & 18.1 & - & - \\
\hline 11 & 16.4 & 12.8 & 15.7 & 17.5 & 17.4 & 18.0 & 18.1 & 18.1 & 18.2 & 18.1 & - & - \\
\hline 12 & 16.9 & 13.0 & 15.6 & 17.6 & 17.6 & 18.0 & 18.1 & 18.1 & 18.2 & 18.1 & - & - \\
\hline 13 & 16.9 & 13.8 & 16.2 & 17.6 & 17.6 & 18.0 & 18.1 & 18.2 & 18.2 & 18.0 & - & - \\
\hline 14 & 16.3 & 13.1 & 16.9 & 17.6 & 17.5 & 18.0 & 18.1 & 18.2 & 18.2 & 18.0 & - & - \\
\hline 15 & 15.7 & 11.5 & 16.9 & 17.6 & 17.6 & 18.0 & 18.1 & 18.1 & 18.2 & 18.0 & - & - \\
\hline 16 & 15.0 & 11.4 & 16.8 & 17.6 & 17.6 & 18.0 & 18.1 & 18.2 & 18.2 & 18.0 & - & - \\
\hline 17 & 14.1 & 12.4 & 16.8 & 17.6 & 17.6 & 18.1 & 18.1 & 18.2 & 18.2 & 18.0 & - & - \\
\hline 18 & 14.3 & 13.5 & 16.8 & 17.6 & 17.6 & 18.1 & 18.1 & 18.2 & 18.2 & 18.0 & - & - \\
\hline 19 & 13.5 & 14.2 & 16.9 & 17.6 & 17.6 & 18.1 & 18.1 & 18.2 & 18.2 & 18.0 & - & - \\
\hline 20 & 12.6 & 13.9 & 17.0 & 17.6 & 17.6 & 18.1 & 18.1 & 18.2 & 18.2 & 18.0 & - & - \\
\hline 21 & 13.1 & 15.1 & 17.1 & 17.6 & 17.7 & 18.1 & 18.1 & 18.2 & 18.2 & - & - & - \\
\hline 22 & 12.5 & 14.3 & 17.2 & 17.6 & 17.7 & 18.1 & 18.1 & 18.1 & 18.2 & - & $\leftarrow$ & - \\
\hline 23 & 13.3 & 14.2 & 17.1 & 17.6 & 17.7 & 18.1 & 18.1 & 18.2 & 18.2 & - & - & - \\
\hline 24 & 16.0 & 14.8 & 17.1 & 17.6 & 17.7 & 18.1 & 18.1 & 18.1 & 18.2 & - & - & - \\
\hline 25 & 14.4 & 14.9 & 17.1 & 17.6 & 17.8 & 18.1 & 18.1 & 18.2 & 18.2 & - & - & - \\
\hline 26 & 13.8 & 15.5 & 17.1 & 17.6 & 17.7 & 18.1 & 18.1 & 18.2 & 18.2 & - & - & - \\
\hline 27 & 14.0 & 16.1 & 17.3 & 17.5 & 17.8 & 18.1 & 18.1 & 18.2 & 18.2 & - & - & - \\
\hline 28 & 13.5 & 15.9 & 17.2 & 17.5 & 17.8 & 18.1 & 18.1 & 18.2 & 18.2 & - & - & - \\
\hline 29 & 13.0 & 15.6 & 17.2 & 17.4 & 17.8 & 18.1 & 18.1 & 18.2 & 18.2 & - & - & - \\
\hline 30 & 13.5 & - & 17.2 & 16.9 & 17.8 & 18.1 & 18.1 & 18.2 & 18.2 & - & - & - \\
\hline 31 & 13.0 & - & 17.2 & - & 17.8 & - & 18.1 & 18.2 & - & - & - & - \\
\hline Mean & 15.5 & 14.2 & 16.5 & 17.5 & 17.6 & 18.0 & 18.1 & 18.2 & 18.2 & 18.1 & - & - \\
\hline $\operatorname{Max}$ & 18.1 & 16.1 & 17.3 & 17.6 & 17.8 & 18.1 & 18.1 & 18.2 & 18.2 & 18.2 & - & - \\
\hline Min & 12.5 & 11.4 & 14.7 & 16.9 & 16.9 & 17.8 & 18.1 & 18.1 & 18.2 & 18.0 & - & - \\
\hline
\end{tabular}




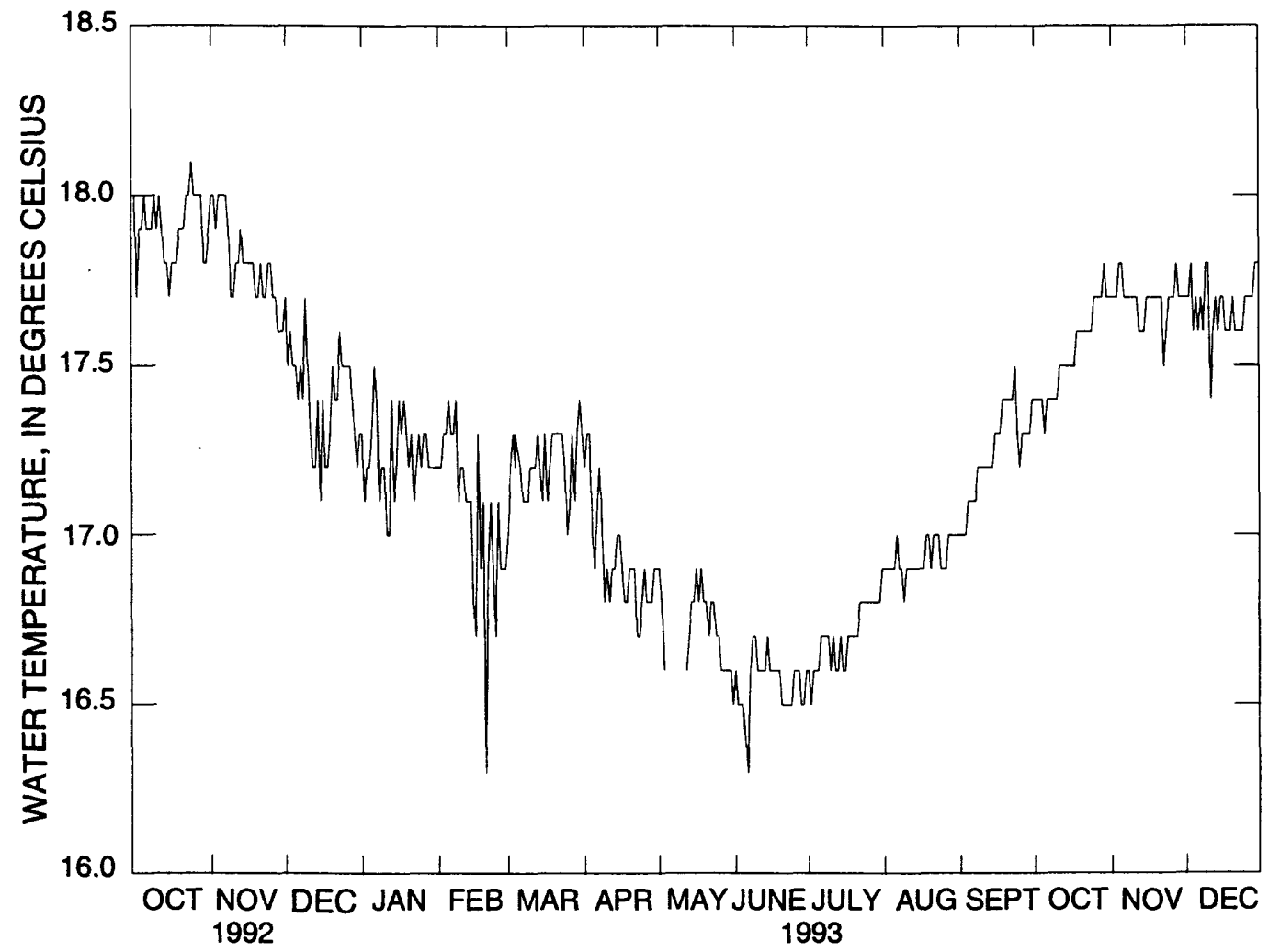

Figure 27. (F) Daily mean water temperature, Fish Hatchery Spring AB (FHAB), weir site, Long Valley Caldera, Mono County, California, October 1992 through December 1993. 
Table 48. Daily mean water temperature, Fish

Hatchery Spring $A B(F H A B)$, weir site, Long Valley

Caldera, Mono County, California, 1992

[Values are in degrees Celsius. Max, maximum; Min, minimum: -, no data]

\begin{tabular}{|c|c|c|c|}
\hline Day & Oct & Nov & Dec \\
\hline 1 & 18.0 & 18.0 & 17.7 \\
\hline 2 & 17.7 & 18.0 & 17.5 \\
\hline 3 & 17.9 & 17.9 & 17.6 \\
\hline 4 & 17.9 & 18.0 & 17.5 \\
\hline 5 & 18.0 & 18.0 & 17.5 \\
\hline 6 & 17.9 & 18.0 & 17.4 \\
\hline 7 & 17.9 & 18.0 & 17.5 \\
\hline 8 & 17.9 & 17.9 & 17.4 \\
\hline 9 & 18.0 & 17.7 & 17.7 \\
\hline 10 & 17.9 & 17.7 & 17.5 \\
\hline 11 & 18.0 & 17.8 & 17.3 \\
\hline 12 & 17.9 & 17.8 & 17.2 \\
\hline 13 & 17.8 & 17.9 & 17.2 \\
\hline 14 & 17.8 & 17.8 & 17.4 \\
\hline 15 & 17.7 & 17.8 & 17.1 \\
\hline 16 & 17.8 & 17.8 & 17.4 \\
\hline 17 & 17.8 & 17.8 & 17.2 \\
\hline 18 & 17.8 & 17.8 & 17.2 \\
\hline 19 & 17.9 & 17.7 & 17.3 \\
\hline 20 & 17.9 & 17.7 & 17.5 \\
\hline 21 & 17.9 & 17.8 & 17.4 \\
\hline 22 & 18.0 & 17.7 & 17.4 \\
\hline 23 & 18.0 & 17.7 & 17.6 \\
\hline 24 & 18.1 & 17.8 & 17.5 \\
\hline 25 & 18.0 & 17.8 & 17.5 \\
\hline 26 & 18.0 & 17.7 & 17.5 \\
\hline 27 & 18.0 & 17.7 & 17.5 \\
\hline 28 & 18.0 & 17.6 & 17.4 \\
\hline 29 & 17.8 & 17.6 & 17.3 \\
\hline 30 & 17.8 & 17.6 & 17.2 \\
\hline 31 & 17.9 & - & 17.3 \\
\hline Mean & 17.9 & 17.8 & 17.4 \\
\hline $\operatorname{Max}$ & 18.1 & 18.0 & 17.7 \\
\hline Min & 17.7 & 17.6 & 17.1 \\
\hline
\end{tabular}


Table 49. Daily mean water temperature, Fish Hatchery Spring AB (FHAB), weir site, Long Valley Caldera, Mono County, California, 1993

[Values are in degrees Celsius. Max, maximum; Min, minimum; -, no data]

\begin{tabular}{|c|c|c|c|c|c|c|c|c|c|c|c|c|}
\hline Day & Jan & Feb & Mar & Apr & May & June & July & Aug & Sept & Oct & Nov & Dec \\
\hline 1 & 17.3 & 17.2 & 17.0 & 17.2 & 16.9 & 16.6 & 16.6 & 16.9 & 17.0 & 17.4 & 17.7 & 17.7 \\
\hline 2 & 17.1 & 17.2 & 17.2 & 17.3 & 16.8 & 16.5 & 16.5 & 16.9 & 17.0 & 17.4 & 17.7 & 17.7 \\
\hline 3 & 17.2 & 17.3 & 17.3 & 17.3 & 16.6 & 16.5 & 16.6 & 16.9 & 17.0 & 17.4 & 17.7 & 17.8 \\
\hline 4 & 17.2 & 17.3 & 17.2 & 17.0 & - & 16.5 & 16.6 & 16.9 & 17.1 & 17.4 & 17.8 & 17.6 \\
\hline 5 & 17.3 & 17.4 & 17.3 & 16.9 & - & 16.4 & 16.6 & 16.9 & 17.1 & 17.3 & 17.8 & 17.7 \\
\hline 6 & 17.5 & 17.3 & 17.2 & 17.1 & - & 16.3 & 16.7 & 17.0 & 17.1 & 17.4 & 17.7 & 17.6 \\
\hline 7 & 17.4 & 17.3 & 17.1 & 17.2 & - & 16.6 & 16.7 & 16.9 & 17.1 & 17.4 & 17.7 & 17.7 \\
\hline 8 & 17.1 & 17.4 & 17.1 & 17.0 & - & 16.7 & 16.7 & 16.9 & 17.2 & 17.4 & 17.7 & 17.6 \\
\hline 9 & 17.2 & 17.1 & 17.1 & 16.8 & - & 16.7 & 16.7 & 16.8 & 17.2 & 17.4 & 17.7 & 17.8 \\
\hline 10 & 17.2 & 17.2 & 17.2 & 16.9 & - & 16.6 & 16.6 & 16.9 & 17.2 & 17.4 & 17.7 & 17.8 \\
\hline 11 & 17.0 & 17.2 & 17.2 & 16.8 & - & 16.6 & 16.7 & 16.9 & 17.2 & 17.5 & 17.7 & 17.4 \\
\hline 12 & 17.0 & 17.1 & 17.2 & 16.9 & 16.6 & 16.6 & 16.6 & 16.9 & 17.2 & 17.5 & 17.6 & 17.6 \\
\hline 13 & 17.4 & 17.1 & 17.3 & 16.9 & 16.7 & 16.6 & 16.6 & 16.9 & 17.2 & 17.5 & 17.6 & 17.7 \\
\hline 14 & 17.1 & 17.1 & 17.2 & 17.0 & 16.8 & 16.7 & 16.7 & 16.9 & 17.2 & 17.5 & 17.6 & 17.6 \\
\hline 15 & 17.2 & 16.8 & 17.1 & 17.0 & 16.8 & 16.6 & 16.6 & 16.9 & 17.3 & 17.5 & 17.7 & 17.7 \\
\hline 16 & 17.4 & 16.7 & 17.3 & 16.9 & 16.9 & 16.6 & 16.6 & 16.9 & 17.3 & 17.5 & 17.7 & 17.7 \\
\hline 17 & 17.3 & 17.3 & 17.1 & 16.8 & 16.8 & 16.6 & 16.7 & 16.9 & 17.3 & 17.5 & 17.7 & 17.6 \\
\hline 18 & 17.4 & 16.9 & 17.2 & 16.8 & 16.9 & 16.6 & 16.7 & 17.0 & 17.4 & 17.6 & 17.7 & 17.6 \\
\hline 19 & 17.3 & 17.1 & 17.3 & 16.9 & 16.8 & 16.6 & 16.7 & 17.0 & 17.4 & 17.6 & 17.7 & 17.6 \\
\hline 20 & 17.2 & 16.3 & 17.3 & 16.9 & 16.8 & 16.5 & 16.7 & 16.9 & 17.4 & 17.6 & 17.7 & 17.7 \\
\hline 21 & 17.3 & 16.9 & 17.3 & 16.9 & 16.7 & 16.5 & 16.7 & 17.0 & 17.4 & 17.6 & 17.7 & 17.6 \\
\hline 22 & 17.1 & 17.1 & 17.3 & 16.7 & 16.8 & 16.5 & 16.8 & 17.0 & 17.4 & 17.6 & 17.5 & 17.6 \\
\hline 23 & 17.2 & 16.9 & 17.3 & 16.7 & 16.8 & 16.5 & 16.8 & 17.0 & 17.5 & 17.6 & 17.6 & 17.6 \\
\hline 24 & 17.3 & 16.7 & 17.2 & 16.8 & 16.7 & 16.5 & 16.8 & 16.9 & 17.3 & 17.6 & 17.7 & 17.6 \\
\hline 25 & 17.2 & 17.1 & 17.0 & 16.9 & 16.7 & 16.6 & 16.8 & 16.9 & 17.2 & 17.7 & 17.7 & 17.7 \\
\hline 26 & 17.3 & 16.9 & 17.1 & 16.8 & 16.6 & 16.6 & 16.8 & 16.9 & 17.3 & 17.7 & 17.7 & 17.7 \\
\hline 27 & 17.3 & 16.9 & 17.3 & 16.8 & 16.6 & 16.6 & 16.8 & 17.0 & 17.3 & 17.7 & 17.8 & 17.7 \\
\hline 28 & 17.2 & 16.9 & 17.1 & 16.8 & 16.6 & 16.5 & 16.8 & 17.0 & 17.3 & 17.7 & 17.7 & 17.7 \\
\hline 29 & 17.2 & - & 17.3 & 16.9 & 16.6 & 16.5 & 16.8 & 17.0 & 17.3 & 17.8 & 17.7 & 17.8 \\
\hline 30 & 17.2 & - & 17.4 & 16.9 & 16.6 & 16.6 & 16.8 & 17.0 & 17.4 & 17.7 & 17.7 & 17.8 \\
\hline 31 & 17.2 & - & 17.3 & - & 16.5 & - & 16.9 & 17.0 & - & 17.7 & - & 17.8 \\
\hline Mean & 17.2 & 17.1 & 17.2 & 16.9 & 16.7 & 16.6 & 16.7 & 16.9 & 17.2 & 17.5 & 17.7 & 17.7 \\
\hline $\operatorname{Max}$ & 17.5 & 17.4 & 17.4 & 17.3 & 16.9 & 16.7 & 16.9 & 17.0 & 17.5 & 17.8 & 17.8 & 17.8 \\
\hline Min & 17.0 & 16.3 & 17.0 & 16.7 & 16.5 & 16.3 & 16.5 & 16.8 & 17.0 & 17.3 & 17.5 & 17.4 \\
\hline
\end{tabular}


Table 50. Instantaneous discharge measurements, Fish Hatchery Spring CD (FHCD-Q), Long Valley Caldera, Mono County, California, 1988-93

[Gage height is in feet relative to an arbitrary datum; discharge is in cubic feet per second]

\begin{tabular}{|c|c|c|c|c|c|}
\hline Date & $\begin{array}{l}\text { Gage } \\
\text { height }\end{array}$ & Discharge & Date & $\begin{array}{c}\text { Gage } \\
\text { height }\end{array}$ & Discharge \\
\hline $10-21-88$ & 1.35 & 7.37 & $06-19-91$ & 1.36 & 8.89 \\
\hline $11-22-88$ & 1.35 & 6.95 & $07-23-91$ & 1.35 & 9.65 \\
\hline \multirow[t]{2}{*}{$12-14-88$} & 1.35 & 8.23 & $08-22-91$ & 1.34 & 11.0 \\
\hline & & & $09-18-91$ & 1.34 & 9.51 \\
\hline $01-10-89$ & 1.35 & 8.21 & $10-23-91$ & 1.36 & 7.33 \\
\hline $02-16-89$ & 1.34 & 8.35 & & & \\
\hline $03-20-89$ & 1.35 & 8.07 & $01-14-92$ & 1.33 & 7.48 \\
\hline $04-12-89$ & 1.35 & 9.30 & $02-19-92$ & 1.31 & 7.52 \\
\hline $05-17-89$ & 1.36 & 7.96 & $03-12-92$ & 1.30 & 7.71 \\
\hline $06-28-89$ & 1.38 & 8.67 & $04-15-92$ & 1.31 & 7.52 \\
\hline $06-28-89$ & 1.38 & 9.16 & $05-13-92$ & 1.30 & 7.20 \\
\hline $07-20-89$ & 1.37 & 9.48 & $05-27-92$ & 1.34 & 7.26 \\
\hline $08-16-89$ & 1.36 & 9.36 & $06-09-92$ & 1.32 & 7.97 \\
\hline $10-19-89$ & 1.36 & 6.18 & $07-07-92$ & 1.33 & 8.03 \\
\hline $11-20-89$ & 1.34 & 6.00 & $08-04-92$ & 1.31 & 7.64 \\
\hline \multirow[t]{2}{*}{$12-20-89$} & 1.34 & 8.25 & $09-15-92$ & 1.31 & 7.02 \\
\hline & & & $10-20-92$ & 1.31 & 7.13 \\
\hline $01-08-90$ & 1.33 & 8.65 & $11-17-92$ & 1.30 & 6.59 \\
\hline $02-22-90$ & 1.34 & 7.05 & $12-16-92$ & 1.30 & 6.71 \\
\hline $03-21-90$ & 1.34 & 9.42 & & & \\
\hline $04-24-90$ & 1.33 & 7.75 & $01-26-93$ & 1.31 & 7.12 \\
\hline $05-24-90$ & 1.34 & 7.57 & $02-23-93$ & 1.29 & 7.34 \\
\hline $07-17-90$ & 1.35 & 8.82 & $03-24-93$ & 1.31 & 8.80 \\
\hline $08-29-90$ & 1.34 & 7.69 & $03-25-93$ & 1.30 & 8.49 \\
\hline $09-25-90$ & 1.34 & 8.08 & $04-13-93$ & 1.32 & 9.03 \\
\hline $10-16-90$ & 1.33 & 9.19 & $05-19-93$ & 1.32 & 8.20 \\
\hline $11-14-90$ & 1.32 & 9.17 & $06-15-93$ & 1.36 & 9.18 \\
\hline \multirow{2}{*}{$12-18-90$} & 1.32 & 8.53 & $08-18-93$ & 2.18 & 10.2 \\
\hline & & & $08-19-93$ & 2.18 & 10.4 \\
\hline $01-17-91$ & 1.32 & 7.69 & $09-08-93$ & 1.36 & 11.0 \\
\hline $02-14-91$ & 1.32 & 8.45 & $10-13-93$ & 1.35 & 10.4 \\
\hline $03-20-91$ & 1.31 & 8.03 & $11-17-93$ & 1.33 & 7.71 \\
\hline $04-16-91$ & 1.32 & 7.70 & $12-14-93$ & 1.31 & 8.12 \\
\hline $05-16-91$ & 1.32 & 6.80 & & & \\
\hline
\end{tabular}




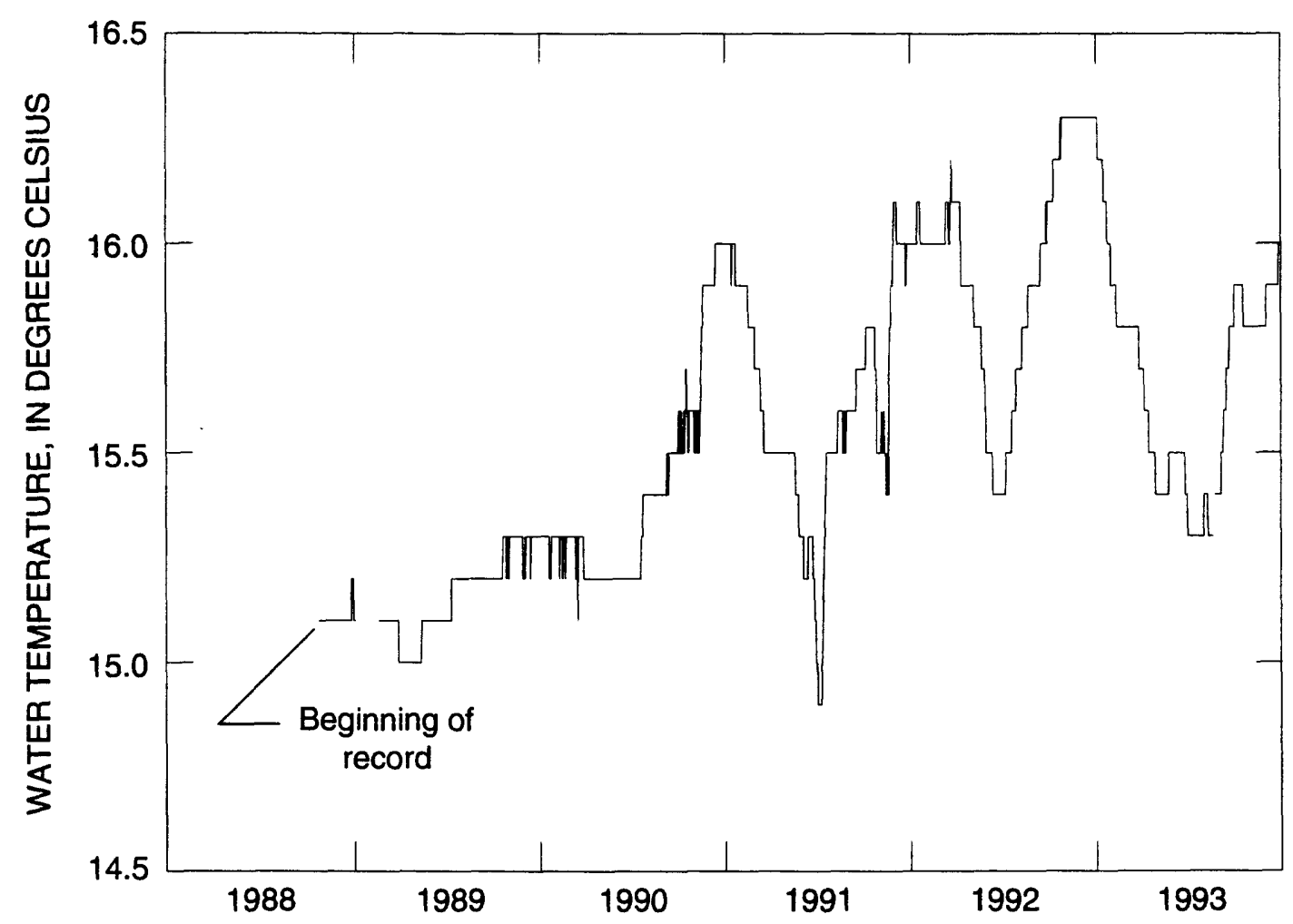

Figure 28. Daily mean water temperature, Fish Hatchery Spring CD (FHCD-T), Long Valley Caldera, Mono County, California, 1988-93.

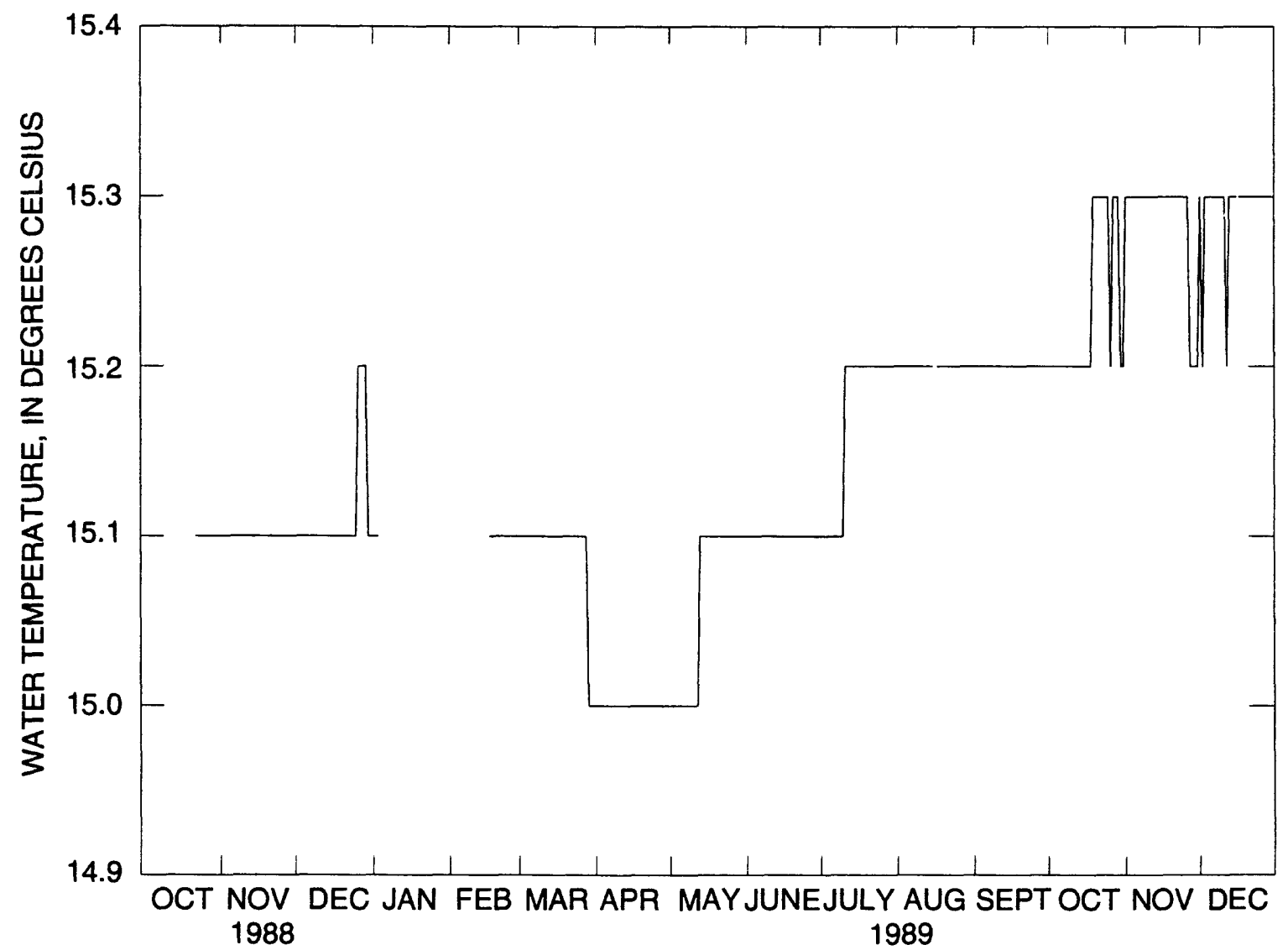

Figure 29. (A) Daily mean water temperature, Fish Hatchery Spring CD (FHCD-T), Long Valley Caldera, Mono County, California, October 1988 through December 1989. 
Table 51. Daily mean water temperature, Fish Hatchery Spring CD (FHCD-T), Long Valley Caldera, Mono County, California, 1988

[Values are in degrees Celsius. Max, maximum; Min, minimum; -, no data]

\begin{tabular}{|c|c|c|c|c|c|c|c|c|c|c|c|c|}
\hline Day & Jan & Feb & Mar & Apr & May & June & July & Aug & Sept & Oct & Nov & Dec \\
\hline 1 & - & - & - & - & - & - & - & - & - & - & 15.1 & 15.1 \\
\hline 2 & - & - & - & - & - & - & - & - & - & - & 15.1 & 15.1 \\
\hline 3 & - & - & - & - & - & - & - & - & - & - & 15.1 & 15.1 \\
\hline 4 & - & - & - & - & - & - & - & - & - & - & 15.1 & 15.1 \\
\hline 5 & - & - & - & - & - & - & - & - & - & - & 15.1 & 15.1 \\
\hline 6 & - & - & - & - & - & - & - & - & - & - & 15.1 & 15.1 \\
\hline 7 & - & - & - & - & - & - & - & - & - & - & 15.1 & 15.1 \\
\hline 8 & - & - & - & - & - & - & - & - & - & - & 15.1 & 15.1 \\
\hline 9 & - & - & - & - & - & - & - & - & - & - & 15.1 & 15.1 \\
\hline 10 & - & - & - & - & - & - & - & - & - & - & 15.1 & 15.1 \\
\hline 11 & - & - & - & - & - & - & - & - & - & - & 15.1 & 15.1 \\
\hline 12 & - & - & - & - & - & - & - & - & - & - & 15.1 & 15.1 \\
\hline 13 & - & - & - & - & - & - & - & - & - & - & 15.1 & 15.1 \\
\hline 14 & - & - & - & - & - & - & - & - & - & - & 15.1 & 15.1 \\
\hline 15 & - & - & - & - & - & - & - & - & - & - & 15.1 & 15.1 \\
\hline 16 & - & - & - & - & - & - & - & - & - & - & 15.1 & 15.1 \\
\hline 17 & - & - & - & - & - & - & - & - & - & - & 15.1 & 15.1 \\
\hline 18 & - & - & - & - & - & - & - & - & - & - & 15.1 & 15.1 \\
\hline 19 & - & - & - & - & - & - & - & - & - & - & 15.1 & 15.1 \\
\hline 20 & - & - & - & - & - & - & - & - & - & - & 15.1 & 15.1 \\
\hline 21 & - & - & - & - & - & - & - & - & - & - & 15.1 & 15.1 \\
\hline 22 & - & - & - & - & - & - & - & - & - & 15.1 & 15.1 & 15.1 \\
\hline 23 & - & - & - & - & - & - & - & - & - & 15.1 & 15.1 & 15.1 \\
\hline 24 & - & - & - & - & - & - & - & - & - & 15.1 & 15.1 & 15.1 \\
\hline 25 & - & - & - & - & - & - & - & - & - & 15.1 & 15.1 & 15.1 \\
\hline 26 & - & - & - & - & - & - & - & - & - & 15.1 & 15.1 & 15.2 \\
\hline 27 & - & - & - & - & - & - & - & - & - & 15.1 & 15.1 & 15.2 \\
\hline 28 & - & - & - & - & - & - & - & - & - & 15.1 & 15.1 & 15.2 \\
\hline 29 & - & - & - & - & - & - & - & - & - & 15.1 & 15.1 & 15.2 \\
\hline 30 & - & - & - & - & - & - & - & - & - & 15.1 & 15.1 & 15.1 \\
\hline 31 & - & - & - & - & - & - & - & - & - & 15.1 & - & 15.1 \\
\hline Mean & - & - & - & - & - & - & - & - & - & 15.1 & 15.1 & 15.1 \\
\hline $\operatorname{Max}$ & - & - & - & - & - & - & - & - & - & 15.1 & 15.1 & 15.2 \\
\hline Min & - & - & - & - & - & - & - & - & - & 15.1 & 15.1 & 15.1 \\
\hline
\end{tabular}


Table 52. Daily mean water temperature, Fish Hatchery Spring CD (FHCD-T), Long Valley Caldera, Mono County, California, 1989

[Values are in degrees Celsius. Max, maximum; Min, minimum; -, no data]

\begin{tabular}{|c|c|c|c|c|c|c|c|c|c|c|c|c|}
\hline Day & Jan & Feb & Mar & Apr & May & June & July & Aug & Sept & Oct & Nov & Dec \\
\hline 1 & 15.1 & - & 15.1 & 15.0 & 15.0 & 15.1 & 15.1 & 15.2 & 15.2 & 15.2 & 15.3 & 15.3 \\
\hline 2 & 15.1 & - & 15.1 & 15.0 & 15.0 & 15.1 & 15.1 & 15.2 & 15.2 & 15.2 & 15.3 & 15.2 \\
\hline 3 & 15.1 & - & 15.1 & 15.0 & 15.0 & 15.1 & 15.1 & 15.2 & 15.2 & 15.2 & 15.3 & 15.3 \\
\hline 4 & - & - & 15.1 & 15.0 & 15.0 & 15.1 & 15.1 & 15.2 & 15.2 & 15.2 & 15.3 & 15.3 \\
\hline 5 & - & - & 15.1 & 15.0 & 15.0 & 15.1 & 15.1 & 15.2 & 15.2 & 15.2 & 15.3 & 15.3 \\
\hline 6 & - & - & 15.1 & 15.0 & 15.0 & 15.1 & 15.1 & 15.2 & 15.2 & 15.2 & 15.3 & 15.3 \\
\hline 7 & - & - & 15.1 & 15.0 & 15.0 & 15.1 & 15.1 & 15.2 & 15.2 & 15.2 & 15.3 & 15.3 \\
\hline 8 & - & - & 15.1 & 15.0 & 15.0 & 15.1 & 15.1 & 15.2 & 15.2 & 15.2 & 15.3 & 15.3 \\
\hline 9 & - & - & 15.1 & 15.0 & 15.0 & 15.1 & 15.1 & 15.2 & 15.2 & 15.2 & 15.3 & 15.3 \\
\hline 10 & - & - & 15.1 & 15.0 & 15.0 & 15.1 & 15.1 & 15.2 & 15.2 & 15.2 & 15.3 & 15.3 \\
\hline 11 & - & - & 15.1 & 15.0 & 15.0 & 15.1 & 15.2 & 15.2 & 15.2 & 15.2 & 15.3 & 15.3 \\
\hline 12 & - & - & 15.1 & 15.0 & 15.0 & 15.1 & 15.2 & 15.2 & 15.2 & 15.2 & 15.3 & 15.2 \\
\hline 13 & - & - & 15.1 & 15.0 & 15.1 & 15.1 & 15.2 & 15.2 & 15.2 & 15.2 & 15.3 & 15.3 \\
\hline 14 & - & - & 15.1 & 15.0 & 15.1 & 15.1 & 15.2 & 15.2 & 15.2 & 15.2 & 15.3 & 15.3 \\
\hline 15 & - & - & 15.1 & 15.0 & 15.1 & 15.1 & 15.2 & 15.2 & 15.2 & 15.2 & 15.3 & 15.3 \\
\hline 16 & - & - & 15.1 & 15.0 & 15.1 & 15.1 & 15.2 & - & 15.2 & 15.2 & 15.3 & 15.3 \\
\hline 17 & - & 15.1 & 15.1 & 15.0 & 15.1 & 15.1 & 15.2 & 15.2 & 15.2 & 15.2 & 15.3 & 15.3 \\
\hline 18 & - & 15.1 & 15.1 & 15.0 & 15.1 & 15.1 & 15.2 & 15.2 & 15.2 & 15.2 & 15.3 & 15.3 \\
\hline 19 & - & 15.1 & 15.1 & 15.0 & 15.1 & 15.1 & 15.2 & 15.2 & 15.2 & 15.3 & 15.3 & 15.3 \\
\hline 20 & - & 15.1 & 15.1 & 15.0 & 15.1 & 15.1 & 15.2 & 15.2 & 15.2 & 15.3 & 15.3 & 15.3 \\
\hline 21 & - & 15.1 & 15.1 & 15.0 & 15.1 & 15.1 & 15.2 & 15.2 & 15.2 & 15.3 & 15.3 & 15.3 \\
\hline 22 & - & 15.1 & 15.1 & 15.0 & 15.1 & 15.1 & 15.2 & 15.2 & 15.2 & 15.3 & 15.3 & 15.3 \\
\hline 23 & - & 15.1 & 15.1 & 15.0 & 15.1 & 15.1 & 15.2 & 15.2 & 15.2 & 15.3 & 15.3 & 15.3 \\
\hline 24 & - & 15.1 & 15.1 & 15.0 & 15.1 & 15.1 & 15.2 & 15.2 & 15.2 & 15.3 & 15.3 & 15.3 \\
\hline 25 & - & 15.1 & 15.1 & 15.0 & 15.1 & 15.1 & 15.2 & 15.2 & 15.2 & 15.3 & 15.3 & 15.3 \\
\hline 26 & - & 15.1 & 15.1 & 15.0 & 15.1 & 15.1 & 15.2 & 15.2 & 15.2 & 15.2 & 15.3 & 15.3 \\
\hline 27 & - & 15.1 & 15.1 & 15.0 & 15.1 & 15.1 & 15.2 & 15.2 & 15.2 & 15.3 & 15.2 & 15.3 \\
\hline 28 & - & 15.1 & 15.1 & 15.0 & 15.1 & 15.1 & 15.2 & 15.2 & 15.2 & 15.3 & 15.2 & 15.3 \\
\hline 29 & - & - & 15.0 & 15.0 & 15.1 & 15.1 & 15.2 & 15.2 & 15.2 & 15.3 & 15.2 & 15.3 \\
\hline 30 & - & - & 15.0 & 15.0 & 15.1 & 15.1 & 15.2 & 15.2 & 15.2 & 15.2 & 15.2 & 15.3 \\
\hline 31 & - & - & 15.0 & - & 15.1 & - & 15.2 & 15.2 & - & 15.2 & - & 15.3 \\
\hline Mean & 15.1 & 15.1 & 15.1 & 15.0 & 15.1 & 15.1 & 15.2 & 15.2 & 15.2 & 15.2 & 15.3 & 15.3 \\
\hline $\operatorname{Max}$ & 15.1 & 15.1 & 15.1 & 15.0 & 15.1 & 15.1 & 15.2 & 15.2 & 15.2 & 15.3 & 15.3 & 15.3 \\
\hline Min & 15.1 & 15.1 & 15.0 & 15.0 & 15.0 & 15.1 & 15.1 & 15.2 & 15.2 & 15.2 & 15.2 & 15.2 \\
\hline
\end{tabular}


This page intentionally left blank. 


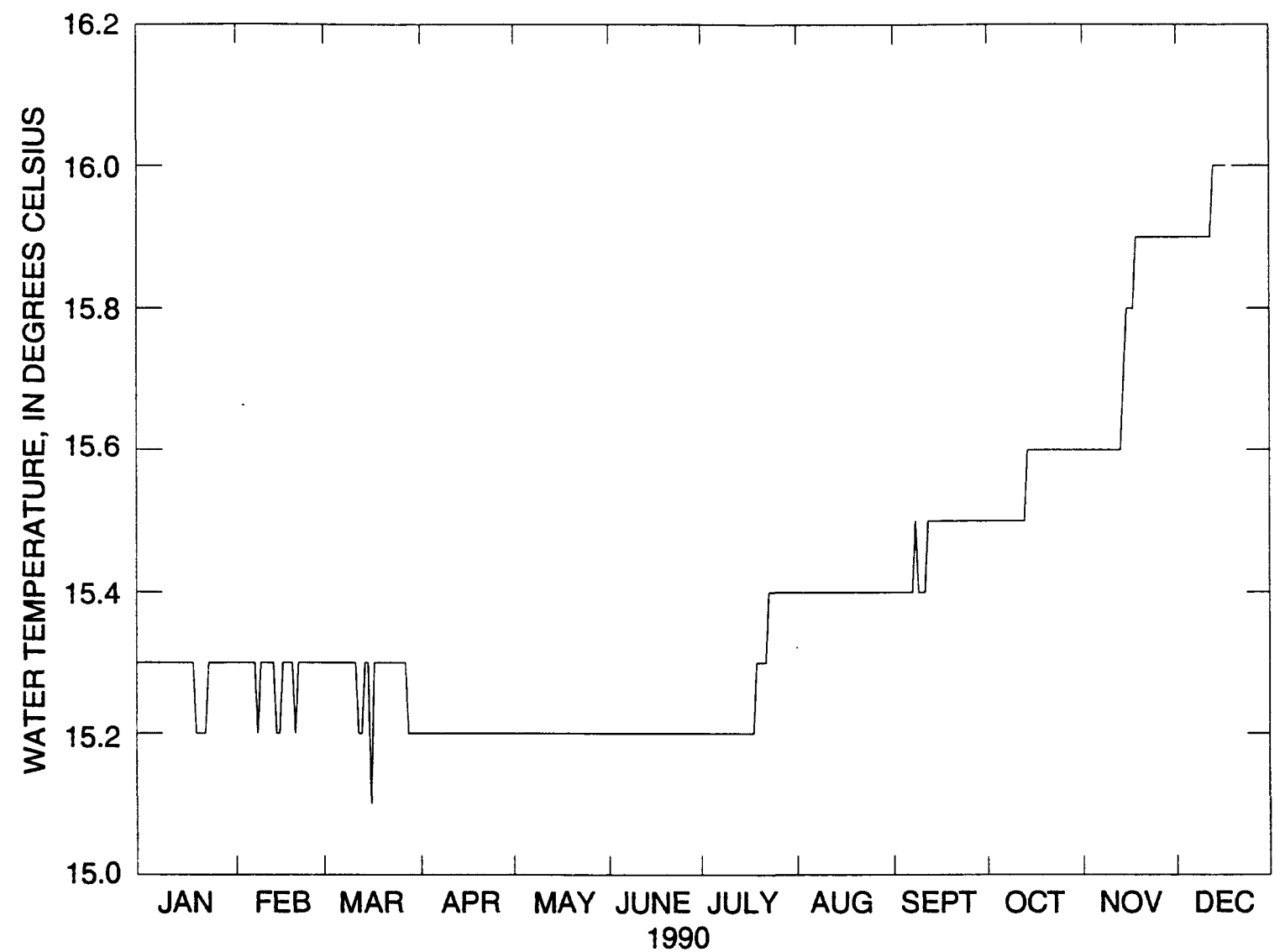

Figure 29. (B) Daily mean water temperature, Fish Hatchery Spring CD (FHCD-T), Long Valley Caldera, Mono County, California, 1990. 
Table 53. Daily mean water temperature, Fish Hatchery Spring CD (FHCD-T), Long Valley Caldera, Mono County, California, 1990

[Values are in degrees Celsius. Max, maximum; Min, minimum; -, no data]

\begin{tabular}{|c|c|c|c|c|c|c|c|c|c|c|c|c|}
\hline Day & Jan & Feb & Mar & Apr & May & June & July & Aug & Sept & Oct & Nov & $\mathrm{Dec}$ \\
\hline 1 & 15.3 & 15.3 & 15.3 & 15.2 & 15.2 & 15.2 & 15.2 & 15.4 & 15.4 & 15.5 & 15.6 & 15.9 \\
\hline 2 & 15.3 & 15.3 & 15.3 & 15.2 & 15.2 & 15.2 & 15.2 & 15.4 & 15.4 & 15.5 & 15.6 & 15.9 \\
\hline 3 & 15.3 & 15.3 & 15.3 & 15.2 & 15.2 & 15.2 & 15.2 & 15.4 & 15.4 & 15.5 & 15.6 & 15.9 \\
\hline 4 & 15.3 & 15.3 & 15.3 & 15.2 & 15.2 & 15.2 & 15.2 & 15.4 & 15.4 & 15.5 & 15.6 & 15.9 \\
\hline 5 & 15.3 & 15.3 & 15.3 & 15.2 & 15.2 & 15.2 & 15.2 & 15.4 & 15.4 & 15.5 & 15.6 & 15.9 \\
\hline 6 & 15.3 & 15.3 & 15.3 & 15.2 & 15.2 & 15.2 & 15.2 & 15.4 & 15.4 & 15.5 & 15.6 & 15.9 \\
\hline 7 & 15.3 & 15.3 & 15.3 & 15.2 & 15.2 & 15.2 & 15.2 & 15.4 & 15.4 & 15.5 & 15.6 & 15.9 \\
\hline 8 & 15.3 & 15.2 & 15.3 & 15.2 & 15.2 & 15.2 & 15.2 & 15.4 & 15.5 & 15.5 & 15.6 & 15.9 \\
\hline 9 & 15.3 & 15.3 & 15.3 & 15.2 & 15.2 & 15.2 & 15.2 & 15.4 & 15.4 & 15.5 & 15.6 & 15.9 \\
\hline 10 & 15.3 & 15.3 & 15.3 & 15.2 & 15.2 & 15.2 & 15.2 & 15.4 & 15.4 & 15.5 & 15.6 & 15.9 \\
\hline 11 & 15.3 & 15.3 & 15.3 & 15.2 & 15.2 & 15.2 & 15.2 & 15.4 & 15.4 & 15.5 & 15.6 & 15.9 \\
\hline 12 & 15.3 & 15.3 & 15.2 & 15.2 & 15.2 & 15.2 & 15.2 & 15.4 & 15.5 & 15.5 & 15.6 & 15.9 \\
\hline 13 & 15.3 & 15.3 & 15.2 & 15.2 & 15.2 & 15.2 & 15.2 & 15.4 & 15.5 & 15.5 & 15.6 & 16.0 \\
\hline 14 & 15.3 & 15.2 & 15.3 & 15.2 & 15.2 & 15.2 & 15.2 & 15.4 & 15.5 & 15.6 & 15.7 & 16.0 \\
\hline 15 & 15.3 & 15.2 & 15.3 & 15.2 & 15.2 & 15.2 & 15.2 & 15.4 & 15.5 & 15.6 & 15.8 & 16.0 \\
\hline 16 & 15.3 & 15.3 & 15.1 & 15.2 & 15.2 & 15.2 & 15.2 & 15.4 & 15.5 & 15.6 & 15.8 & 16.0 \\
\hline 17 & 15.3 & 15.3 & 15.3 & 15.2 & 15.2 & 15.2 & 15.2 & 15.4 & 15.5 & 15.6 & 15.8 & 16.0 \\
\hline 18 & 15.3 & 15.3 & 15.3 & 15.2 & 15.2 & 15.2 & 15.2 & 15.4 & 15.5 & 15.6 & 15.9 & - \\
\hline 19 & 15.2 & 15.3 & 15.3 & 15.2 & 15.2 & 15.2 & 15.3 & 15.4 & 15.5 & 15.6 & 15.9 & 16.0 \\
\hline 20 & 15.2 & 15.2 & 15.3 & 15.2 & 15.2 & 15.2 & 15.3 & 15.4 & 15.5 & 15.6 & 15.9 & 16.0 \\
\hline 21 & 15.2 & 15.3 & 15.3 & 15.2 & 15.2 & 15.2 & 15.3 & 15.4 & 15.5 & 15.6 & 15.9 & 16.0 \\
\hline 22 & 15.2 & 15.3 & 15.3 & 15.2 & 15.2 & 15.2 & 15.3 & 15.4 & 15.5 & 15.6 & 15.9 & 16.0 \\
\hline 23 & 15.3 & 15.3 & 15.3 & 15.2 & 15.2 & 15.2 & 15.4 & 15.4 & 15.5 & 15.6 & 15.9 & 16.0 \\
\hline 24 & 15.3 & 15.3 & 15.3 & 15.2 & 15.2 & 15.2 & 15.4 & 15.4 & 15.5 & 15.6 & 15.9 & 16.0 \\
\hline 25 & 15.3 & 15.3 & 15.3 & 15.2 & 15.2 & 15.2 & 15.4 & 15.4 & 15.5 & 15.6 & 15.9 & 16.0 \\
\hline 26 & 15.3 & 15.3 & 15.3 & 15.2 & 15.2 & 15.2 & 15.4 & 15.4 & 15.5 & 15.6 & 15.9 & 16.0 \\
\hline 27 & 15.3 & 15.3 & 15.3 & 15.2 & 15.2 & 15.2 & 15.4 & 15.4 & 15.5 & 15.6 & 15.9 & 16.0 \\
\hline 28 & 15.3 & 15.3 & 15.2 & 15.2 & 15.2 & 15.2 & 15.4 & 15.4 & 15.5 & 15.6 & 15.9 & 16.0 \\
\hline 29 & 15.3 & - & 15.2 & 15.2 & 15.2 & 15.2 & 15.4 & 15.4 & 15.5 & 15.6 & 15.9 & 16.0 \\
\hline 30 & 15.3 & - & 15.2 & 15.2 & 15.2 & 15.2 & 15.4 & 15.4 & 15.5 & 15.6 & 15.9 & 16.0 \\
\hline 31 & 15.3 & - & 15.2 & - & 15.2 & - & 15.4 & 15.4 & - & 15.6 & - & 16.0 \\
\hline Mean & 15.3 & 15.3 & 15.3 & 15.2 & 15.2 & 15.2 & 15.3 & 15.4 & 15.5 & 15.6 & 15.8 & 16.0 \\
\hline $\operatorname{Max}$ & 15.3 & 15.3 & 15.3 & 15.2 & 15.2 & 15.2 & 15.4 & 15.4 & 15.5 & 15.6 & 15.9 & 16.0 \\
\hline Min & 15.2 & 15.2 & 15.1 & 15.2 & 15.2 & 15.2 & 15.2 & 15.4 & 15.4 & 15.5 & 15.6 & 15.9 \\
\hline
\end{tabular}




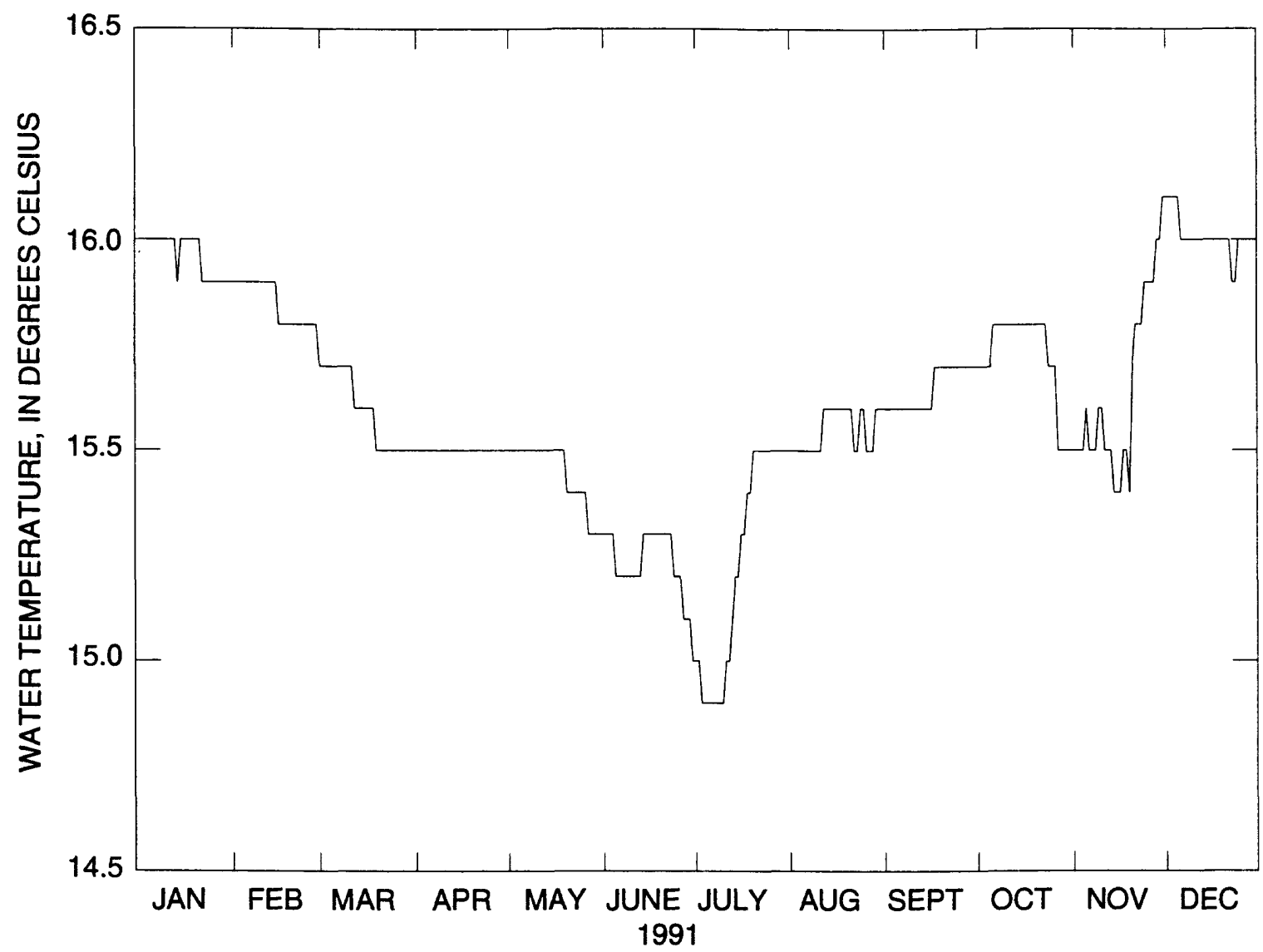

Figure 29. (C) Daily mean water temperature, Fish Hatchery Spring CD (FHCD-T), Long Valley Caldera, Mono County, California, 1991. 
Table 54. Daily mean water temperature, Fish Hatchery Spring CD (FHCD-T), Long Valley Caldera, Mono County, California, 1991

[Values are in degrees Celsius. Max, maximum; Min, minimum; - , no data]

\begin{tabular}{|c|c|c|c|c|c|c|c|c|c|c|c|c|}
\hline Day & Jan & Feb & Mar & Apr & May & June & July & Aug & Sept & Oct & Nov & Dec \\
\hline $\begin{array}{l}1 \\
2 \\
3 \\
4 \\
5\end{array}$ & $\begin{array}{l}16.0 \\
16.0 \\
16.0 \\
16.0 \\
16.0\end{array}$ & $\begin{array}{l}15.9 \\
15.9 \\
15.9 \\
15.9 \\
15.9\end{array}$ & $\begin{array}{l}15.7 \\
15.7 \\
15.7 \\
15.7 \\
15.7\end{array}$ & $\begin{array}{l}15.5 \\
15.5 \\
15.5 \\
15.5 \\
15.5\end{array}$ & $\begin{array}{l}15.5 \\
15.5 \\
15.5 \\
15.5 \\
15.5\end{array}$ & $\begin{array}{l}15.3 \\
15.3 \\
15.3 \\
15.3 \\
15.2\end{array}$ & $\begin{array}{l}15.0 \\
15.0 \\
14.9 \\
14.9 \\
14.9\end{array}$ & $\begin{array}{l}15.5 \\
15.5 \\
15.5 \\
15.5 \\
15.5\end{array}$ & $\begin{array}{l}15.6 \\
15.6 \\
15.6 \\
15.6 \\
15.6\end{array}$ & $\begin{array}{l}15.7 \\
15.7 \\
15.7 \\
15.7 \\
15.7\end{array}$ & $\begin{array}{l}15.5 \\
15.5 \\
15.5 \\
15.5 \\
15.6\end{array}$ & $\begin{array}{l}16.1 \\
16.1 \\
16.1 \\
16.1 \\
16.1\end{array}$ \\
\hline $\begin{array}{r}6 \\
7 \\
8 \\
9 \\
10\end{array}$ & $\begin{array}{l}16.0 \\
16.0 \\
16.0 \\
16.0 \\
16.0\end{array}$ & $\begin{array}{l}15.9 \\
15.9 \\
15.9 \\
15.9 \\
15.9\end{array}$ & $\begin{array}{l}15.7 \\
15.7 \\
15.7 \\
15.7 \\
15.7\end{array}$ & $\begin{array}{l}15.5 \\
15.5 \\
15.5 \\
15.5 \\
15.5\end{array}$ & $\begin{array}{l}15.5 \\
15.5 \\
15.5 \\
15.5 \\
15.5\end{array}$ & $\begin{array}{l}15.2 \\
15.2 \\
15.2 \\
15.2 \\
15.2\end{array}$ & $\begin{array}{l}14.9 \\
14.9 \\
14.9 \\
14.9 \\
14.9\end{array}$ & $\begin{array}{l}15.5 \\
15.5 \\
15.5 \\
15.5 \\
15.5\end{array}$ & $\begin{array}{l}15.6 \\
15.6 \\
15.6 \\
15.6 \\
15.6\end{array}$ & $\begin{array}{l}15.8 \\
15.8 \\
15.8 \\
15.8 \\
15.8\end{array}$ & $\begin{array}{l}15.5 \\
15.5 \\
15.5 \\
15.6 \\
15.6\end{array}$ & $\begin{array}{l}16.0 \\
16.0 \\
16.0 \\
16.0 \\
16.0\end{array}$ \\
\hline $\begin{array}{l}11 \\
12 \\
13 \\
14 \\
15\end{array}$ & $\begin{array}{l}16.0 \\
16.0 \\
16.0 \\
15.9 \\
16.0\end{array}$ & $\begin{array}{l}15.9 \\
15.9 \\
15.9 \\
15.9 \\
15.9\end{array}$ & $\begin{array}{l}15.7 \\
15.6 \\
15.6 \\
15.6 \\
15.6\end{array}$ & $\begin{array}{l}15.5 \\
15.5 \\
15.5 \\
15.5 \\
15.5\end{array}$ & $\begin{array}{l}15.5 \\
15.5 \\
15.5 \\
15.5 \\
15.5\end{array}$ & $\begin{array}{l}15.2 \\
15.2 \\
15.2 \\
15.3 \\
15.3\end{array}$ & $\begin{array}{l}15.0 \\
15.0 \\
15.1 \\
15.2 \\
15.2\end{array}$ & $\begin{array}{l}15.5 \\
15.6 \\
15.6 \\
15.6 \\
15.6\end{array}$ & $\begin{array}{l}15.6 \\
15.6 \\
15.6 \\
15.6 \\
15.6\end{array}$ & $\begin{array}{l}15.8 \\
15.8 \\
15.8 \\
15.8 \\
15.8\end{array}$ & $\begin{array}{l}15.5 \\
15.5 \\
15.5 \\
15.4 \\
15.4\end{array}$ & $\begin{array}{l}16.0 \\
16.0 \\
16.0 \\
16.0 \\
16.0\end{array}$ \\
\hline $\begin{array}{l}16 \\
17 \\
18 \\
19 \\
20\end{array}$ & $\begin{array}{l}16.0 \\
16.0 \\
16.0 \\
16.0 \\
16.0\end{array}$ & $\begin{array}{l}15.8 \\
15.8 \\
15.8 \\
15.8 \\
15.8\end{array}$ & $\begin{array}{l}15.6 \\
15.6 \\
15.6 \\
15.5 \\
15.5\end{array}$ & $\begin{array}{l}15.5 \\
15.5 \\
15.5 \\
15.5 \\
15.5\end{array}$ & $\begin{array}{l}15.5 \\
15.5 \\
15.5 \\
15.5 \\
15.4\end{array}$ & $\begin{array}{l}15.3 \\
15.3 \\
15.3 \\
15.3 \\
15.3\end{array}$ & $\begin{array}{l}15.3 \\
15.3 \\
15.4 \\
15.4 \\
15.5\end{array}$ & $\begin{array}{l}15.6 \\
15.6 \\
15.6 \\
15.6 \\
15.6\end{array}$ & $\begin{array}{l}15.6 \\
15.7 \\
15.7 \\
15.7 \\
15.7\end{array}$ & $\begin{array}{l}15.8 \\
15.8 \\
15.8 \\
15.8 \\
15.8\end{array}$ & $\begin{array}{l}15.4 \\
15.5 \\
15.5 \\
15.4 \\
15.7\end{array}$ & $\begin{array}{l}16.0 \\
16.0 \\
16.0 \\
16.0 \\
16.0\end{array}$ \\
\hline $\begin{array}{l}21 \\
22 \\
23 \\
24 \\
25\end{array}$ & $\begin{array}{l}16.0 \\
15.9 \\
15.9 \\
15.9 \\
15.9\end{array}$ & $\begin{array}{l}15.8 \\
15.8 \\
15.8 \\
15.8 \\
15.8\end{array}$ & $\begin{array}{l}15.5 \\
15.5 \\
15.5 \\
15.5 \\
15.5\end{array}$ & $\begin{array}{l}15.5 \\
15.5 \\
15.5 \\
15.5 \\
15.5\end{array}$ & $\begin{array}{l}15.4 \\
15.4 \\
15.4 \\
15.4 \\
15.4\end{array}$ & $\begin{array}{l}15.3 \\
15.3 \\
15.3 \\
15.2 \\
15.2\end{array}$ & $\begin{array}{l}15.5 \\
15.5 \\
15.5 \\
15.5 \\
15.5\end{array}$ & $\begin{array}{l}15.6 \\
15.5 \\
15.5 \\
15.6 \\
15.6\end{array}$ & $\begin{array}{l}15.7 \\
15.7 \\
15.7 \\
15.7 \\
15.7\end{array}$ & $\begin{array}{l}15.8 \\
15.8 \\
15.8 \\
15.7 \\
15.7\end{array}$ & $\begin{array}{l}15.8 \\
15.8 \\
15.8 \\
15.9 \\
15.9\end{array}$ & $\begin{array}{l}16.0 \\
16.0 \\
15.9 \\
15.9 \\
16.0\end{array}$ \\
\hline $\begin{array}{l}26 \\
27 \\
28 \\
29 \\
30 \\
31\end{array}$ & $\begin{array}{l}15.9 \\
15.9 \\
15.9 \\
15.9 \\
15.9 \\
15.9\end{array}$ & $\begin{array}{l}15.8 \\
15.8 \\
15.8 \\
- \\
- \\
-\end{array}$ & $\begin{array}{l}15.5 \\
15.5 \\
15.5 \\
15.5 \\
15.5 \\
15.5\end{array}$ & $\begin{array}{l}15.5 \\
15.5 \\
15.5 \\
15.5 \\
15.5 \\
-\end{array}$ & $\begin{array}{l}15.4 \\
15.3 \\
15.3 \\
15.3 \\
15.3 \\
15.3\end{array}$ & $\begin{array}{l}15.2 \\
15.1 \\
15.1 \\
15.1 \\
15.0 \\
-\end{array}$ & $\begin{array}{l}15.5 \\
15.5 \\
15.5 \\
15.5 \\
15.5 \\
15.5\end{array}$ & $\begin{array}{l}15.5 \\
15.5 \\
15.5 \\
15.6 \\
15.6 \\
15.6\end{array}$ & $\begin{array}{l}15.7 \\
15.7 \\
15.7 \\
15.7 \\
15.7 \\
-\end{array}$ & $\begin{array}{l}15.7 \\
15.5 \\
15.5 \\
15.5 \\
15.5 \\
15.5\end{array}$ & $\begin{array}{l}15.9 \\
15.9 \\
16.0 \\
16.0 \\
16.1 \\
-\end{array}$ & $\begin{array}{l}16.0 \\
16.0 \\
16.0 \\
16.0 \\
16.0 \\
16.0\end{array}$ \\
\hline $\begin{array}{l}\text { Mean } \\
\text { Max } \\
\text { Min }\end{array}$ & $\begin{array}{l}16.0 \\
16.0 \\
15.9\end{array}$ & $\begin{array}{l}15.9 \\
15.9 \\
15.8\end{array}$ & $\begin{array}{l}15.6 \\
15.7 \\
15.5\end{array}$ & $\begin{array}{l}15.5 \\
15.5 \\
15.5\end{array}$ & $\begin{array}{l}15.4 \\
15.5 \\
15.3\end{array}$ & $\begin{array}{l}15.2 \\
15.3 \\
15.0\end{array}$ & $\begin{array}{l}15.2 \\
15.5 \\
14.9\end{array}$ & $\begin{array}{l}15.5 \\
15.6 \\
15.5\end{array}$ & $\begin{array}{l}15.6 \\
15.7 \\
15.6\end{array}$ & $\begin{array}{l}15.7 \\
15.8 \\
15.5\end{array}$ & $\begin{array}{l}15.6 \\
16.1 \\
15.4\end{array}$ & $\begin{array}{l}16.0 \\
16.1 \\
15.9\end{array}$ \\
\hline
\end{tabular}




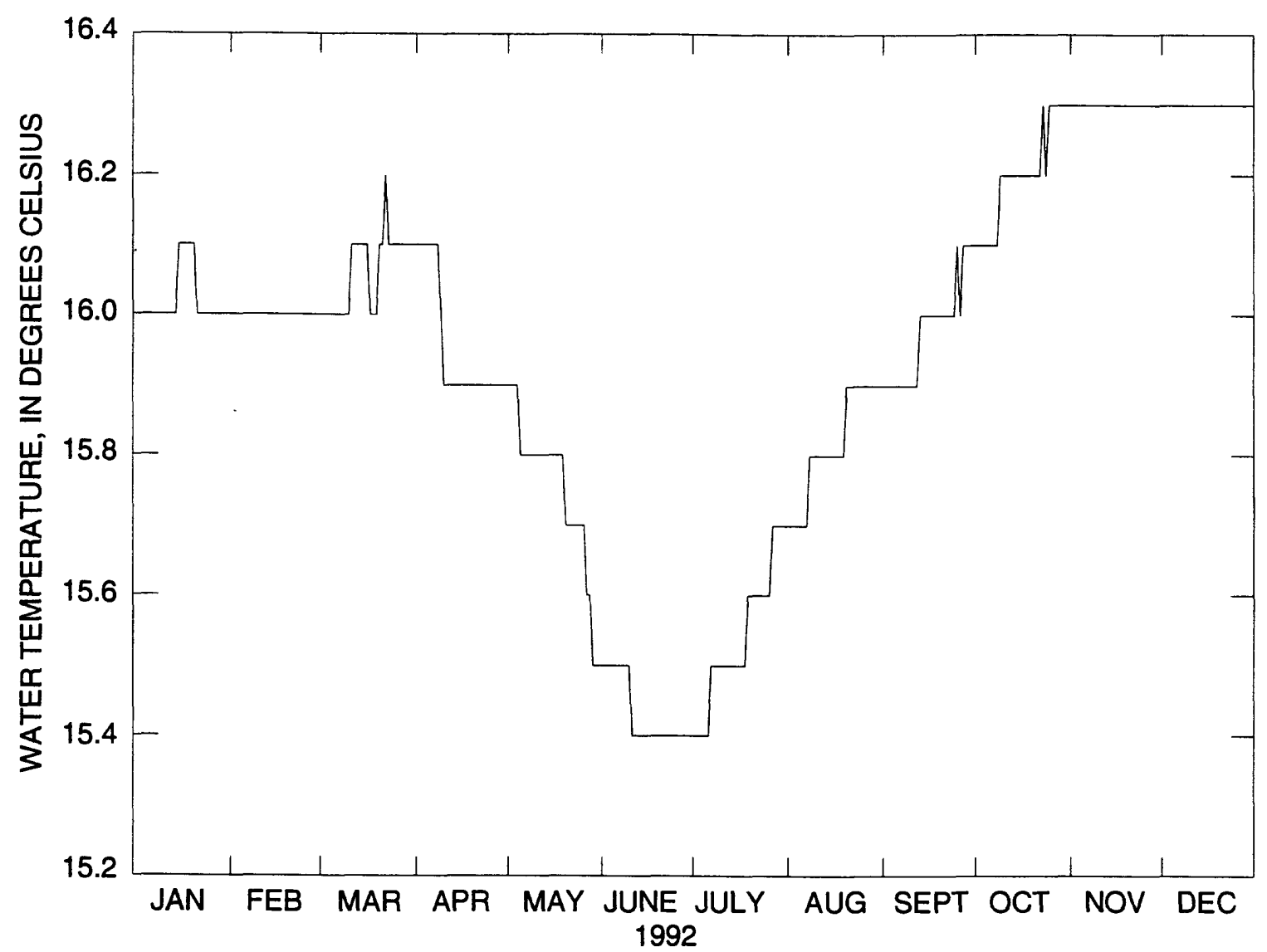

Figure 29. (D) Daily mean water temperature, Fish Hatchery Spring CD (FHCD-T), Long Valley Caidera, Mono County, California, 1992. 
Table 55. Daily mean water temperature, Fish Hatchery Spring CD (FHCD-T), Long Valley Caldera, Mono County, California, 1992

[Values are in degrees Celsius. Max, maximum; Min, minimum; -, no data]

\begin{tabular}{|c|c|c|c|c|c|c|c|c|c|c|c|c|}
\hline Day & Jan & Feb & Mar & Apr & May & June & July & Aug & Sept & Oct & Nov & Dec \\
\hline 1 & 16.0 & 16.0 & 16.0 & 16.1 & 15.9 & 15.5 & 15.4 & 15.7 & 15.9 & 16.1 & 16.3 & 16.3 \\
\hline 2 & 16.0 & 16.0 & 16.0 & 16.1 & 15.9 & 15.5 & 15.4 & 15.7 & 15.9 & 16.1 & 16.3 & 16.3 \\
\hline 3 & 16.0 & 16.0 & 16.0 & 16.1 & 15.9 & 15.5 & 15.4 & 15.7 & 15.9 & 16.1 & 16.3 & 16.3 \\
\hline 4 & 16.0 & 16.0 & 16.0 & 16.1 & 15.9 & 15.5 & 15.4 & 15.7 & 15.9 & 16.1 & 16.3 & 16.3 \\
\hline 5 & 16.0 & 16.0 & 16.0 & 16.1 & 15.8 & 15.5 & 15.4 & 15.7 & 15.9 & 16.1 & 16.3 & 16.3 \\
\hline 6 & 16.0 & 16.0 & 16.0 & 16.1 & 15.8 & 15.5 & 15.4 & 15.7 & 15.9 & 16.1 & 16.3 & 16.3 \\
\hline 7 & 16.0 & 16.0 & 16.0 & 16.1 & 15.8 & 15.5 & 15.5 & 15.7 & 15.9 & 16.1 & 16.3 & 16.3 \\
\hline 8 & 16.0 & 16.0 & 16.0 & 16.1 & 15.8 & 15.5 & 15.5 & 15.8 & 15.9 & 16.1 & 16.3 & 16.3 \\
\hline 9 & 16.0 & 16.0 & 16.0 & 16.0 & 15.8 & 15.5 & 15.5 & 15.8 & 15.9 & 16.2 & 16.3 & 16.3 \\
\hline 10 & 16.0 & 16.0 & 16.0 & 15.9 & 15.8 & 15.5 & 15.5 & 15.8 & 15.9 & 16.2 & 16.3 & 16.3 \\
\hline 11 & 16.0 & 16.0 & 16.1 & 15.9 & 15.8 & 15.4 & 15.5 & 15.8 & 15.9 & 16.2 & 16.3 & 16.3 \\
\hline 12 & 16.0 & 16.0 & 16.1 & 15.9 & 15.8 & 15.4 & 15.5 & 15.8 & 15.9 & 16.2 & 16.3 & 16.3 \\
\hline 13 & 16.0 & 16.0 & 16.1 & 15.9 & 15.8 & 15.4 & 15.5 & 15.8 & 16.0 & 16.2 & 16.3 & 16.3 \\
\hline 14 & 16.0 & 16.0 & 16.1 & 15.9 & 15.8 & 15.4 & 15.5 & 15.8 & 16.0 & 16.2 & 16.3 & 16.3 \\
\hline 15 & 16.1 & 16.0 & 16.1 & 15.9 & 15.8 & 15.4 & 15.5 & 15.8 & 16.0 & 16.2 & 16.3 & 16.3 \\
\hline 16 & 16.1 & 16.0 & 16.1 & 15.9 & 15.8 & 15.4 & 15.5 & 15.8 & 16.0 & 16.2 & 16.3 & 16.3 \\
\hline 17 & 16.1 & 16.0 & 16.0 & 15.9 & 15.8 & 15.4 & 15.5 & 15.8 & 16.0 & 16.2 & 16.3 & 16.3 \\
\hline 18 & 16.1 & 16.0 & 16.0 & 15.9 & 15.8 & 15.4 & 15.5 & 15.8 & 16.0 & 16.2 & 16.3 & 16.3 \\
\hline 19 & 16.1 & 16.0 & 16.0 & 15.9 & 15.8 & 15.4 & 15.6 & 15.8 & 16.0 & 16.2 & 16.3 & 16.3 \\
\hline 20 & 16.1 & 16.0 & 16.1 & 15.9 & 15.7 & 15.4 & 15.6 & 15.9 & 16.0 & 16.2 & 16.3 & 16.3 \\
\hline 21 & 16.0 & 16.0 & 16.1 & 15.9 & 15.7 & 15.4 & 15.6 & 15.9 & 16.0 & 16.2 & 16.3 & 16.3 \\
\hline 22 & 16.0 & 16.0 & 16.2 & 15.9 & 15.7 & 15.4 & 15.6 & 15.9 & 16.0 & 16.2 & 16.3 & 16.3 \\
\hline 23 & 16.0 & 16.0 & 16.1 & 15.9 & 15.7 & 15.4 & 15.6 & 15.9 & 16.0 & 16.3 & 16.3 & 16.3 \\
\hline 24 & 16.0 & 16.0 & 16.1 & 15.9 & 15.7 & 15.4 & 15.6 & 15.9 & 16.0 & 16.2 & 16.3 & 16.3 \\
\hline 25 & 16.0 & 16.0 & 16.1 & 15.9 & 15.7 & 15.4 & 15.6 & 15.9 & 16.1 & 16.3 & 16.3 & 16.3 \\
\hline 26 & 16.0 & 16.0 & 16.1 & 15.9 & 15.7 & 15.4 & 15.6 & 15.9 & 16.0 & 16.3 & 16.3 & 16.3 \\
\hline 27 & 16.0 & 16.0 & 16.1 & 15.9 & 15.6 & 15.4 & 15.7 & 15.9 & 16.1 & 16.3 & 16.3 & 16.3 \\
\hline 28 & 16.0 & 16.0 & 16.1 & 15.9 & 15.6 & 15.4 & 15.7 & 15.9 & 16.1 & 16.3 & 16.3 & 16.3 \\
\hline 29 & 16.0 & 16.0 & 16.1 & 15.9 & 15.5 & 15.4 & 15.7 & 15.9 & 16.1 & 16.3 & 16.3 & 16.3 \\
\hline 30 & 16.0 & - & 16.1 & 15.9 & 15.5 & 15.4 & 15.7 & 15.9 & 16.1 & 16.3 & 16.3 & 16.3 \\
\hline 31 & 16.0 & 一 & 16.1 & - & 15.5 & - & 15.7 & 15.9 & - & 16.3 & - & 16.3 \\
\hline Mean & 16.0 & 16.0 & 16.1 & 16.0 & 15.7 & 15.4 & 15.5 & 15.8 & 16.0 & 16.2 & 16.3 & 16.3 \\
\hline Max & 16.1 & 16.0 & 16.2 & 16.1 & 15.9 & 15.5 & 15.7 & 15.9 & 16.1 & 16.3 & 16.3 & 16.3 \\
\hline Min & 16.0 & 16.0 & 16.0 & 15.9 & 15.5 & 15.4 & 15.4 & 15.7 & 15.9 & 16.1 & 16.3 & 16.3 \\
\hline
\end{tabular}




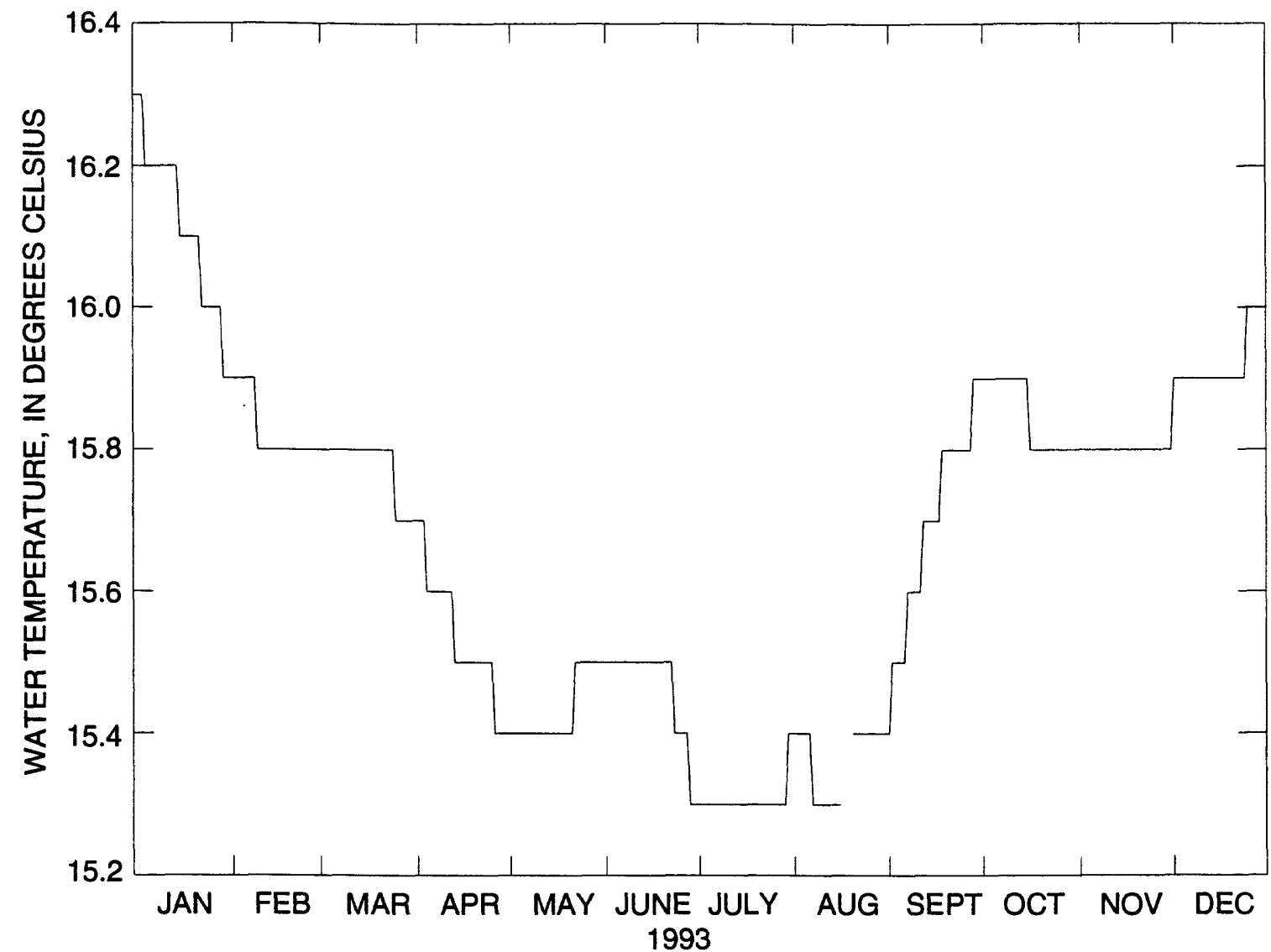

Figure 29. (E) Daily mean water temperature, Fish Hatchery Spring CD (FHCD-T), Long Valley Caldera, Mono County, California, 1993. 
Table 56. Daily mean water temperature, Fish Hatchery Spring CD (FHCD-T), Long Valley Caldera, Mono County, California, 1993

[Values are in degrees Celsius. Max, maximum; Min, minimum; -, no data]

\begin{tabular}{|c|c|c|c|c|c|c|c|c|c|c|c|c|}
\hline Day & Jan & Feb & Mar & Apr & May & June & July & Aug & Sept & Oct & Nov & Dec \\
\hline 1 & 16.3 & 15.9 & 15.8 & 15.7 & 15.4 & 15.5 & 15.3 & 15.4 & 15.4 & 15.9 & 15.8 & 15.9 \\
\hline 2 & 16.3 & 15.9 & 15.8 & 15.7 & 15.4 & 15.5 & 15.3 & 15.4 & 15.5 & 15.9 & 15.8 & 15.9 \\
\hline 3 & 16.3 & 15.9 & 15.8 & 15.7 & 15.4 & 15.5 & 15.3 & 15.4 & 15.5 & 15.9 & 15.8 & 15.9 \\
\hline 4 & 16.2 & 15.9 & 15.8 & 15.6 & 15.4 & 15.5 & 15.3 & 15.4 & 15.5 & 15.9 & 15.8 & 15.9 \\
\hline 5 & 16.2 & 15.9 & 15.8 & 15.6 & 15.4 & 15.5 & 15.3 & 15.4 & 15.5 & 15.9 & 15.8 & 15.9 \\
\hline 6 & 16.2 & 15.9 & 15.8 & 15.6 & 15.4 & 15.5 & 15.3 & 15.4 & 15.5 & 15.9 & 15.8 & 15.9 \\
\hline 7 & 16.2 & 15.9 & 15.8 & 15.6 & 15.4 & 15.5 & 15.3 & 15.3 & 15.6 & 15.9 & 15.8 & 15.9 \\
\hline 8 & 16.2 & 15.9 & 15.8 & 15.6 & 15.4 & 15.5 & 15.3 & 15.3 & 15.6 & 15.9 & 15.8 & 15.9 \\
\hline 9 & 16.2 & 15.8 & 15.8 & 15.6 & 15.4 & 15.5 & 15.3 & 15.3 & 15.6 & 15.9 & 15.8 & 15.9 \\
\hline 10 & 16.2 & 15.8 & 15.8 & 15.6 & 15.4 & 15.5 & 15.3 & 15.3 & 15.6 & 15.9 & 15.8 & 15.9 \\
\hline 11 & 16.2 & 15.8 & 15.8 & 15.6 & 15.4 & 15.5 & 15.3 & 15.3 & 15.6 & 15.9 & 15.8 & 15.9 \\
\hline 12 & 16.2 & 15.8 & 15.8 & 15.6 & 15.4 & 15.5 & 15.3 & 15.3 & 15.7 & 15.9 & 15.8 & 15.9 \\
\hline 13 & 16.2 & 15.8 & 15.8 & 15.5 & 15.4 & 15.5 & 15.3 & 15.3 & 15.7 & 15.9 & 15.8 & 15.9 \\
\hline 14 & 16.2 & 15.8 & 15.8 & 15.5 & 15.4 & 15.5 & 15.3 & 15.3 & 15.7 & 15.9 & 15.8 & 15.9 \\
\hline 15 & 16.1 & 15.8 & 15.8 & 15.5 & 15.4 & 15.5 & 15.3 & 15.3 & 15.7 & 15.9 & 15.8 & 15.9 \\
\hline 16 & 16.1 & 15.8 & 15.8 & 15.5 & 15.4 & 15.5 & 15.3 & 15.3 & 15.7 & 15.8 & 15.8 & 15.9 \\
\hline 17 & 16.1 & 15.8 & 15.8 & 15.5 & 15.4 & 15.5 & 15.3 & - & 15.7 & 15.8 & 15.8 & 15.9 \\
\hline 18 & 16.1 & 15.8 & 15.8 & 15.5 & 15.4 & 15.5 & 15.3 & - & 15.8 & 15.8 & 15.8 & 15.9 \\
\hline 19 & 16.1 & 15.8 & 15.8 & 15.5 & 15.4 & 15.5 & 15.3 & - & 15.8 & 15.8 & 15.8 & 15.9 \\
\hline 20 & 16.1 & 15.8 & 15.8 & 15.5 & 15.4 & 15.5 & 15.3 & 15.4 & 15.8 & 15.8 & 15.8 & 15.9 \\
\hline 21 & 16.1 & 15.8 & 15.8 & 15.5 & 15.4 & 15.5 & 15.3 & 15.4 & 15.8 & 15.8 & 15.8 & 15.9 \\
\hline 22 & 16.0 & 15.8 & 15.8 & 15.5 & 15.5 & 15.5 & 15.3 & 15.4 & 15.8 & 15.8 & 15.8 & 15.9 \\
\hline 23 & 16.0 & 15.8 & 15.8 & 15.5 & 15.5 & 15.4 & 15.3 & 15.4 & 15.8 & 15.8 & 15.8 & 15.9 \\
\hline 24 & 16.0 & 15.8 & 15.8 & 15.5 & 15.5 & 15.4 & 15.3 & 15.4 & 15.8 & 15.8 & 15.8 & 15.9 \\
\hline 25 & 16.0 & 15.8 & 15.7 & 15.5 & 15.5 & 15.4 & 15.3 & 15.4 & 15.8 & 15.8 & 15.8 & 16.0 \\
\hline 26 & 16.0 & 15.8 & 15.7 & 15.4 & 15.5 & 15.4 & 15.3 & 15.4 & 15.8 & 15.8 & 15.8 & 16.0 \\
\hline 27 & 16.0 & 15.8 & 15.7 & 15.4 & 15.5 & 15.4 & 15.3 & 15.4 & 15.8 & 15.8 & 15.8 & 16.0 \\
\hline 28 & 16.0 & 15.8 & 15.7 & 15.4 & 15.5 & 15.3 & 15.3 & 15.4 & 15.9 & 15.8 & 15.8 & 16.0 \\
\hline 29 & 15.9 & - & 15.7 & 15.4 & 15.5 & 15.3 & 15.3 & 15.4 & 15.9 & 15.8 & 15.8 & 16.0 \\
\hline 30 & 15.9 & - & 15.7 & 15.4 & 15.5 & 15.3 & 15.4 & 15.4 & 15.9 & 15.8 & 15.8 & 16.0 \\
\hline 31 & 15.9 & - & 15.7 & - & 15.5 & - & 15.4 & 15.4 & - & 15.8 & - & 16.0 \\
\hline Mean & 16.1 & 15.8 & 15.8 & 15.5 & 15.4 & 15.5 & 15.3 & 15.4 & 15.7 & 15.8 & 15.8 & 15.9 \\
\hline $\operatorname{Max}$ & 16.3 & 15.9 & 15.8 & 15.7 & 15.5 & 15.5 & 15.4 & 15.4 & 15.9 & 15.9 & 15.8 & 16.0 \\
\hline Min & 15.9 & 15.8 & 15.7 & 15.4 & 15.4 & 15.3 & 15.3 & 15.3 & 15.4 & 15.8 & 15.8 & 15.9 \\
\hline
\end{tabular}




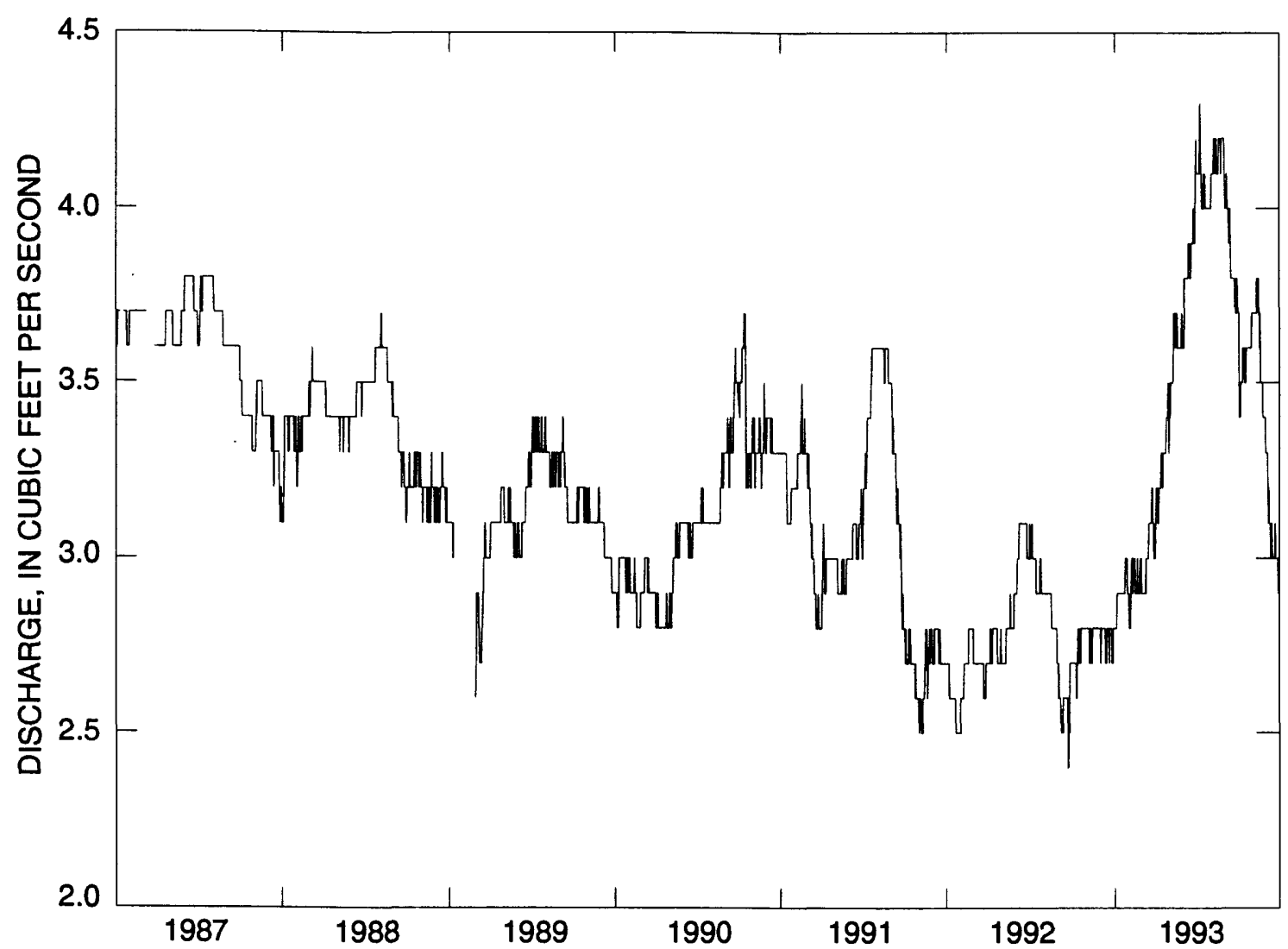

Figure 30. Daily mean discharge, Fish Hatchery Spring 23 (FH23-Q), Long Valley Caldera, Mono County, California, 1987-93.

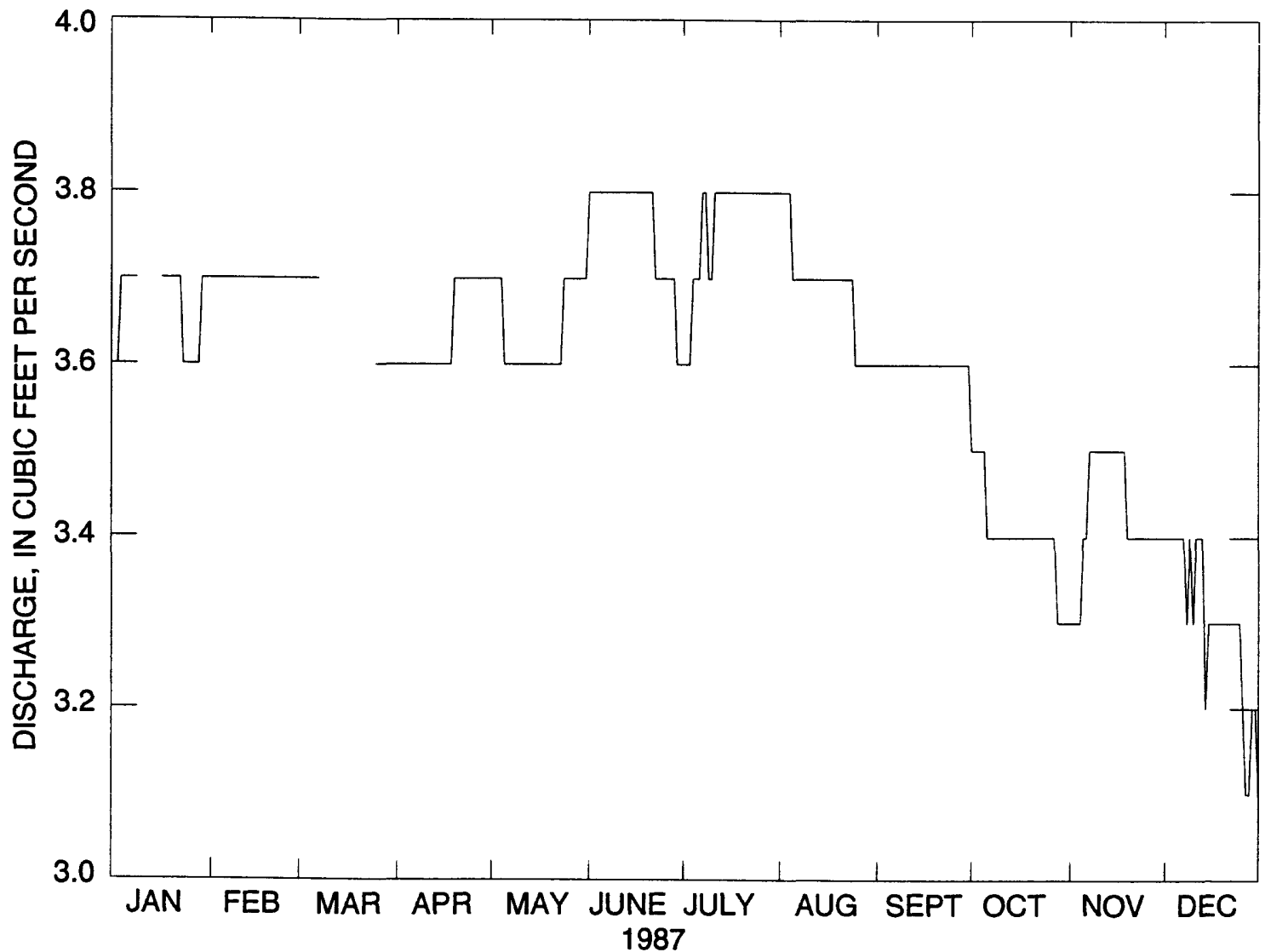

Figure 31. (A) Daily mean discharge, Fish Hatchery Spring 23 (FH23-Q), Long Valley Caldera, Mono County, California, 1987. 
Table 57. Daily mean discharge, Fish Hatchery Spring 23 (FH23-Q), Long Valley Caldera, Mono County, California, 1987

[Values are in cubic feet per second. Max, maximum; Min, minimum; - , no data]

\begin{tabular}{|c|c|c|c|c|c|c|c|c|c|c|c|c|}
\hline Day & Jan & Feb & Mar & Apr & May & June & July & Aug & Sept & Oct & Nov & $\mathrm{Dec}$ \\
\hline 1 & 3.6 & 3.7 & 3.7 & 3.6 & 3.7 & 3.8 & 3.6 & 3.8 & 3.6 & 3.5 & 3.3 & 3.4 \\
\hline 2 & 3.6 & 3.7 & 3.7 & 3.6 & 3.7 & 3.8 & 3.6 & 3.8 & 3.6 & 3.5 & 3.3 & 3.4 \\
\hline 3 & 3.7 & 3.7 & 3.7 & 3.6 & 3.7 & 3.8 & 3.6 & 3.8 & 3.6 & 3.5 & 3.3 & 3.4 \\
\hline 4 & 3.7 & 3.7 & 3.7 & 3.6 & 3.7 & 3.8 & 3.7 & 3.8 & 3.6 & 3.5 & 3.3 & 3.4 \\
\hline 5 & 3.7 & 3.7 & 3.7 & 3.6 & 3.6 & 3.8 & 3.7 & 3.7 & 3.6 & 3.5 & 3.4 & 3.4 \\
\hline 6 & 3.7 & 3.7 & 3.7 & 3.6 & 3.6 & 3.8 & 3.7 & 3.7 & 3.6 & 3.4 & 3.4 & 3.4 \\
\hline 7 & 3.7 & 3.7 & 3.7 & 3.6 & 3.6 & 3.8 & 3.8 & 3.7 & 3.6 & 3.4 & 3.5 & 3.4 \\
\hline 8 & 3.7 & 3.7 & - & 3.6 & 3.6 & 3.8 & 3.8 & 3.7 & 3.6 & 3.4 & 3.5 & 3.3 \\
\hline 9 & - & 3.7 & - & 3.6 & 3.6 & 3.8 & 3.7 & 3.7 & 3.6 & 3.4 & 3.5 & 3.4 \\
\hline 10 & - & 3.7 & - & 3.6 & 3.6 & 3.8 & 3.7 & 3.7 & 3.6 & 3.4 & 3.5 & 3.3 \\
\hline 11 & - & 3.7 & - & 3.6 & 3.6 & 3.8 & 3.8 & 3.7 & 3.6 & 3.4 & 3.5 & 3.4 \\
\hline 12 & - & 3.7 & - & 3.6 & 3.6 & 3.8 & 3.8 & 3.7 & 3.6 & 3.4 & 3.5 & 3.4 \\
\hline 13 & 3.7 & 3.7 & - & 3.6 & 3.6 & 3.8 & 3.8 & 3.7 & 3.6 & 3.4 & 3.5 & 3.4 \\
\hline 14 & - & 3.7 & - & 3.6 & 3.6 & 3.8 & 3.8 & 3.7 & 3.6 & 3.4 & 3.5 & 3.2 \\
\hline 15 & - & 3.7 & - & 3.6 & 3.6 & 3.8 & 3.8 & 3.7 & 3.6 & 3.4 & 3.5 & 3.3 \\
\hline 16 & 3.7 & 3.7 & - & 3.6 & 3.6 & 3.8 & 3.8 & 3.7 & 3.6 & 3.4 & 3.5 & 3.3 \\
\hline 17 & 3.7 & 3.7 & - & 3.6 & 3.6 & 3.8 & 3.8 & 3.7 & 3.6 & 3.4 & 3.5 & 3.3 \\
\hline 18 & 3.7 & 3.7 & - & 3.6 & 3.6 & 3.8 & 3.8 & 3.7 & 3.6 & 3.4 & 3.5 & 3.3 \\
\hline 19 & 3.7 & 3.7 & - & 3.7 & 3.6 & 3.8 & 3.8 & 3.7 & 3.6 & 3.4 & 3.4 & 3.3 \\
\hline 20 & 3.7 & 3.7 & - & 3.7 & 3.6 & 3.8 & 3.8 & 3.7 & 3.6 & 3.4 & 3.4 & 3.3 \\
\hline 21 & 3.7 & 3.7 & - & 3.7 & 3.6 & 3.8 & 3.8 & 3.7 & 3.6 & 3.4 & 3.4 & 3.3 \\
\hline 22 & 3.7 & 3.7 & - & 3.7 & 3.6 & 3.7 & 3.8 & 3.7 & 3.6 & 3.4 & 3.4 & 3.3 \\
\hline 23 & 3.6 & 3.7 & - & 3.7 & 3.6 & 3.7 & 3.8 & 3.7 & 3.6 & 3.4 & 3.4 & 3.3 \\
\hline 24 & 3.6 & 3.7 & - & 3.7 & 3.7 & 3.7 & 3.8 & 3.7 & 3.6 & 3.4 & 3.4 & 3.3 \\
\hline 25 & 3.6 & 3.7 & 3.6 & 3.7 & 3.7 & 3.7 & 3.8 & 3.6 & 3.6 & 3.4 & 3.4 & 3.3 \\
\hline 26 & 3.6 & 3.7 & 3.6 & 3.7 & 3.7 & 3.7 & 3.8 & 3.6 & 3.6 & 3.4 & 3.4 & 3.2 \\
\hline 27 & 3.6 & 3.7 & 3.6 & 3.7 & 3.7 & 3.7 & 3.8 & 3.6 & 3.6 & 3.4 & 3.4 & 3.1 \\
\hline 28 & 3.6 & 3.7 & 3.6 & 3.7 & 3.7 & 3.7 & 3.8 & 3.6 & 3.6 & 3.3 & 3.4 & 3.1 \\
\hline 29 & 3.7 & - & 3.6 & 3.7 & 3.7 & 3.6 & 3.8 & 3.6 & 3.6 & 3.3 & 3.4 & 3.2 \\
\hline 30 & 3.7 & - & 3.6 & 3.7 & 3.7 & 3.6 & 3.8 & 3.6 & 3.6 & 3.3 & 3.4 & 3.2 \\
\hline 31 & 3.7 & - & 3.6 & - & 3.7 & - & 3.8 & 3.6 & - & 3.3 & - & 3.1 \\
\hline Mean & 3.67 & 3.70 & 3.65 & 3.64 & 3.64 & 3.76 & 3.76 & 3.69 & 3.60 & 3.40 & 3.43 & 3.30 \\
\hline $\operatorname{Max}$ & 3.7 & 3.7 & 3.7 & 3.7 & 3.7 & 3.8 & 3.8 & 3.8 & 3.6 & 3.5 & 3.5 & 3.4 \\
\hline Min & 3.6 & 3.7 & 3.6 & 3.6 & 3.6 & 3.6 & 3.6 & 3.6 & 3.6 & 3.3 & 3.3 & 3.1 \\
\hline
\end{tabular}




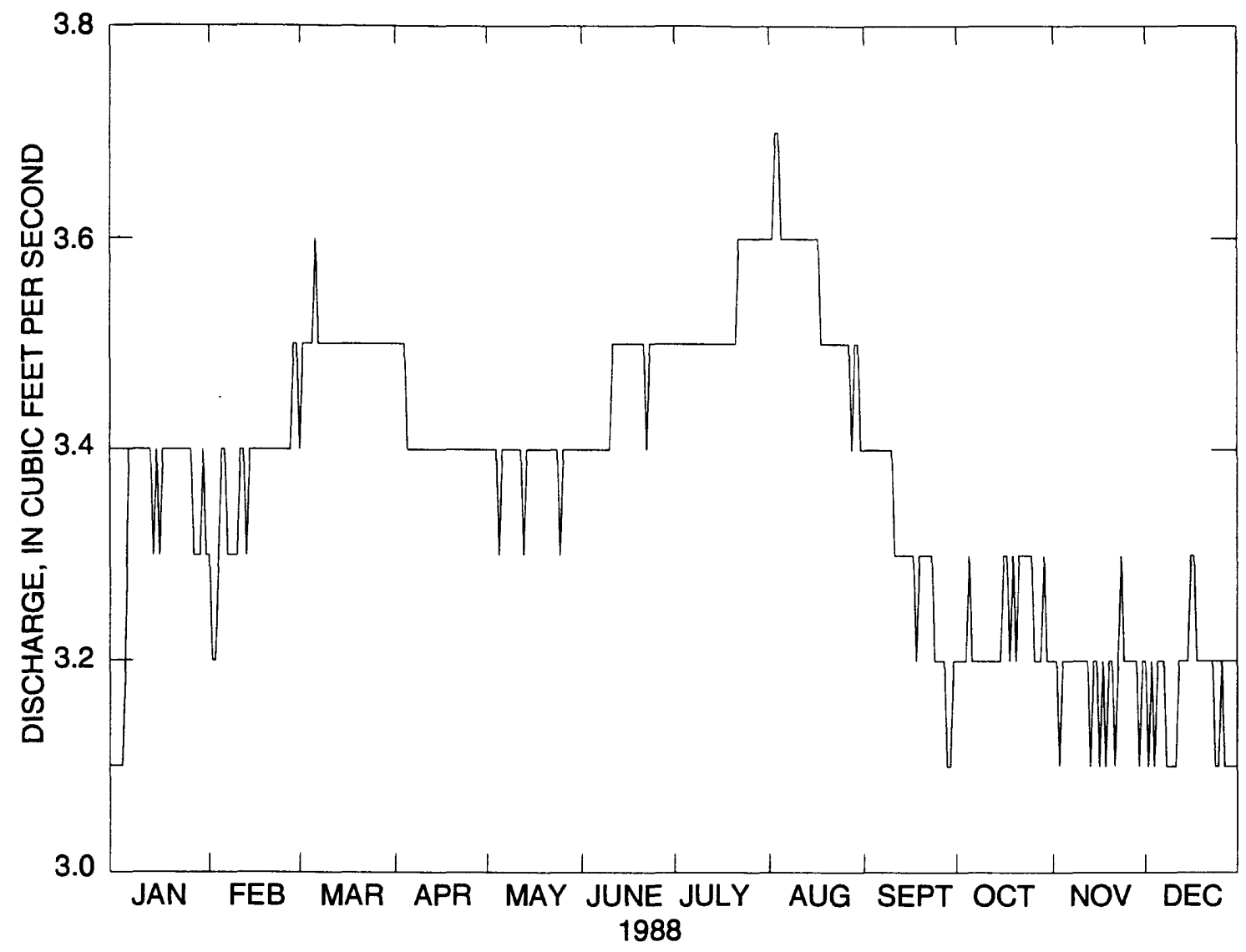

Figure 31. (B) Daily mean discharge, Fish Hatchery Spring 23 (FH23-Q), Long Valley Caldera, Mono County, California, 1988. 
Table 58. Daily mean discharge, Fish Hatchery Spring 23 (FH23-Q), Long Valley Caldera, Mono County, California, 1988

[Values are in cubic feet per second. e, estimated; Max, maximum; Min, minimum; - , no data]

\begin{tabular}{|c|c|c|c|c|c|c|c|c|c|c|c|c|}
\hline Day & Jan & Feb & Mar & Ap̣r & May & June & July & Aug & Sept & Oct & Nov & Dec \\
\hline 1 & 3.1 & 3.3 & 3.4 & 3.5 & 3.4 & 3.4 & 3.5 & 3.6 & 3.4 & 3.2 & 3.2 & 3.2 \\
\hline 2 & 3.1 & 3.2 & 3.5 & 3.5 & 3.4 & 3.4 & 3.5 & 3.6 & 3.4 & 3.2 & 3.2 & 3.1 \\
\hline 3 & 3.1 & 3.2 & 3.5 & 3.5 & 3.4 & 3.4 & 3.5 & 3.7 & 3.4 & 3.2 & 3.1 & 3.2 \\
\hline 4 & 3.1 & 3.3 & 3.5 & 3.5 & 3.4 & 3.4 & 3.5 & 3.7 & 3.4 & 3.2 & 3.2 & 3.1 \\
\hline 5 & 3.2 & 3.4 & 3.5 & 3.4 & 3.3 & 3.4 & 3.5 & 3.6 & 3.4 & 3.3 & 3.2 & 3.2 \\
\hline 6 & 3.4 & 3.4 & 3.6 & 3.4 & 3.4 & 3.4 & 3.5 & 3.6 & 3.4 & 3.2 & 3.2 & 3.2 \\
\hline 7 & 3.4 & 3.3 & e3.5 & 3.4 & 3.4 & 3.4 & 3.5 & 3.6 & 3.4 & 3.2 & 3.2 & 3.2 \\
\hline 8 & 3.4 & 3.3 & e3.5 & 3.4 & 3.4 & 3.4 & 3.5 & 3.6 & 3.4 & 3.2 & 3.2 & 3.1 \\
\hline 9 & 3.4 & 3.3 & e3.5 & 3.4 & 3.4 & 3.4 & 3.5 & 3.6 & 3.4 & 3.2 & 3.2 & 3.1 \\
\hline 10 & 3.4 & 3.3 & e3.5 & 3.4 & 3.4 & 3.4 & 3.5 & 3.6 & 3.4 & 3.2 & 3.2 & 3.1 \\
\hline 11 & 3.4 & 3.4 & e3.5 & 3.4 & 3.4 & 3.5 & 3.5 & 3.6 & 3.3 & 3.2 & 3.2 & 3.1 \\
\hline 12 & 3.4 & 3.4 & e3.5 & 3.4 & 3.4 & 3.5 & 3.5 & 3.6 & 3.3 & 3.2 & 3.2 & 3.2 \\
\hline 13 & 3.4 & 3.3 & e3.5 & 3.4 & 3.3 & 3.5 & 3.5 & 3.6 & 3.3 & 3.2 & 3.1 & 3.2 \\
\hline 14 & 3.3 & 3.4 & e3.5 & 3.4 & 3.4 & 3.5 & 3.5 & 3.6 & 3.3 & 3.2 & 3.2 & 3.2 \\
\hline 15 & 3.4 & 3.4 & e3.5 & 3.4 & 3.4 & 3.5 & 3.5 & 3.6 & 3.3 & 3.2 & 3.2 & 3.2 \\
\hline 16 & 3.3 & 3.4 & e3.5 & 3.4 & 3.4 & 3.5 & 3.5 & 3.6 & 3.3 & 3.3 & 3.1 & 3.3 \\
\hline 17 & 3.4 & 3.4 & e3.5 & 3.4 & 3.4 & 3.5 & 3.5 & 3.6 & 3.3 & 3.3 & 3.2 & 3.3 \\
\hline 18 & 3.4 & 3.4 & 3.5 & 3.4 & 3.4 & 3.5 & 3.5 & 3.5 & 3.2 & 3.2 & 3.1 & 3.2 \\
\hline 19 & 3.4 & 3.4 & 3.5 & 3.4 & 3.4 & 3.5 & 3.5 & 3.5 & 3.3 & 3.3 & 3.2 & 3.2 \\
\hline 20 & e3.4 & 3.4 & 3.5 & 3.4 & 3.4 & 3.5 & 3.5 & 3.5 & 3.3 & 3.2 & 3.2 & 3.2 \\
\hline 21 & e3.4 & 3.4 & 3.5 & 3.4 & 3.4 & 3.5 & 3.5 & 3.5 & 3.3 & 3.3 & 3.1 & 3.2 \\
\hline 22 & e3.4 & 3.4 & 3.5 & 3.4 & 3.4 & 3.4 & 3.6 & 3.5 & 3.3 & 3.3 & 3.2 & 3.2 \\
\hline 23 & e3.4 & 3.4 & 3.5 & 3.4 & 3.4 & 3.5 & 3.6 & 3.5 & 3.3 & 3.3 & 3.3 & 3.2 \\
\hline 24 & e3.4 & 3.4 & 3.5 & 3.4 & 3.4 & 3.5 & 3.6 & 3.5 & 3.2 & 3.3 & 3.2 & 3.1 \\
\hline 25 & e 3.4 & 3.4 & 3.5 & 3.4 & 3.3 & 3.5 & 3.6 & 3.5 & 3.2 & 3.3 & 3.2 & 3.1 \\
\hline 26 & 3.4 & 3.4 & 3.5 & 3.4 & 3.4 & 3.5 & 3.6 & 3.5 & 3.2 & 3.2 & 3.2 & 3.2 \\
\hline 27 & 3.3 & 3.4 & 3.5 & 3.4 & 3.4 & 3.5 & 3.6 & 3.5 & 3.2 & 3.2 & 3.2 & 3.1 \\
\hline 28 & 3.3 & 3.5 & 3.5 & 3.4 & 3.4 & 3.5 & 3.6 & 3.4 & 3.1 & 3.2 & 3.2 & 3.1 \\
\hline 29 & 3.3 & 3.5 & 3.5 & 3.4 & 3.4 & 3.5 & 3.6 & 3.5 & 3.1 & 3.3 & 3.1 & 3.1 \\
\hline 30 & 3.4 & - & 3.5 & 3.4 & 3.4 & 3.5 & 3.6 & 3.5 & 3.2 & 3.2 & 3.2 & 3.1 \\
\hline 31 & 3.3 & - & 3.5 & - & 3.4 & - & 3.6 & 3.4 & - & 3.2 & - & 3.1 \\
\hline Mean & 3.34 & 3.37 & 3.50 & 3.41 & 3.39 & 3.46 & 3.53 & 3.55 & 3.30 & 3.23 & 3.18 & 3.16 \\
\hline $\operatorname{Max}$ & 3.4 & 3.5 & 3.6 & 3.5 & 3.4 & 3.5 & 3.6 & 3.7 & 3.4 & 3.3 & 3.3 & 3.3 \\
\hline Min & 3.1 & 3.2 & 3.4 & 3.4 & 3.3 & 3.4 & 3.5 & 3.4 & 3.1 & 3.2 & 3.1 & 3.1 \\
\hline
\end{tabular}




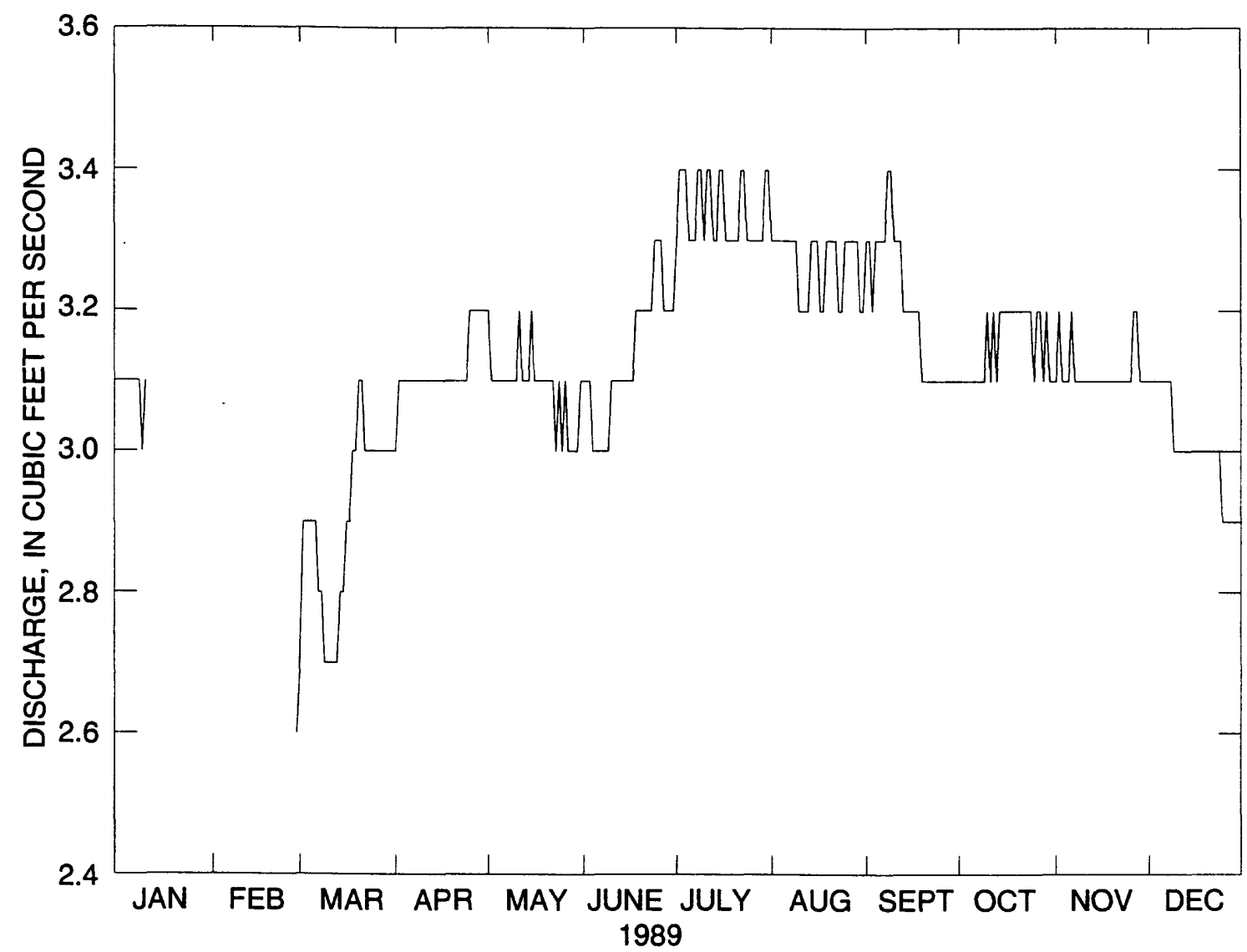

Figure 31. (C) Daily mean discharge, Fish Hatchery Spring 23 (FH23-Q), Long Valley Caidera, Mono County, California, 1989. 
Table 59. Daily mean discharge, Fish Hatchery Spring 23 (FH23-Q), Long Valley Caldera, Mono County, California, 1989

[Values are in cubic feet per second. e, estimated; Max, maximum; Min, minimum; - , no data]

\begin{tabular}{|c|c|c|c|c|c|c|c|c|c|c|c|c|}
\hline Day & Jan & Feb & Mar & Apr & May & June & July & Aug & Sept & Oct & Nov & Dec \\
\hline 1 & 3.1 & - & 2.7 & 3.0 & 3.2 & 3.1 & 3.3 & 3.3 & 3.3 & 3.1 & 3.1 & e3.1 \\
\hline 2 & 3.1 & - & 2.9 & 3.1 & 3.1 & 3.1 & 3.4 & 3.3 & 3.3 & 3.1 & 3.2 & e3.1 \\
\hline 3 & 3.1 & - & 2.9 & 3.1 & 3.1 & 3.1 & 3.4 & 3.3 & 3.2 & 3.1 & 3.1 & e3.1 \\
\hline 4 & 3.1 & - & 2.9 & 3.1 & 3.1 & 3.0 & 3.4 & 3.3 & 3.3 & 3.1 & 3.1 & e3.1 \\
\hline 5 & 3.1 & - & 2.9 & 3.1 & 3.1 & 3.0 & 3.3 & 3.3 & 3.3 & 3.1 & 3.1 & e3.1 \\
\hline 6 & 3.1 & - & 2.9 & 3.1 & 3.1 & 3.0 & 3.3 & 3.3 & 3.3 & 3.1 & 3.2 & e3.1 \\
\hline 7 & 3.1 & - & 2.8 & 3.1 & 3.1 & 3.0 & 3.3 & 3.3 & 3.3 & 3.1 & 3.1 & e3.1 \\
\hline 8 & 3.1 & - & 2.8 & 3.1 & 3.1 & 3.0 & 3.4 & 3.3 & 3.4 & 3.1 & 3.1 & e3.1 \\
\hline 9 & 3.0 & - & 2.7 & 3.1 & 3.1 & 3.0 & 3.4 & 3.3 & 3.4 & 3.1 & 3.1 & e3.0 \\
\hline 10 & 3.1 & - & 2.7 & 3.1 & 3.1 & 3.1 & 3.3 & 3.2 & 3.3 & 3.2 & 3.1 & e3.0 \\
\hline 11 & - & - & 2.7 & 3.1 & 3.2 & 3.1 & 3.4 & 3.2 & 3.3 & 3.1 & 3.1 & e3.0 \\
\hline 12 & - & - & 2.7 & 3.1 & 3.1 & 3.1 & 3.4 & 3.2 & 3.3 & 3.2 & 3.1 & e3.0 \\
\hline 13 & - & - & 2.7 & 3.1 & 3.1 & 3.1 & 3.3 & 3.2 & 3.2 & 3.1 & 3.1 & e3.0 \\
\hline 14 & - & - & 2.8 & 3.1 & 3.1 & 3.1 & 3.3 & 3.3 & 3.2 & 3.2 & 3.1 & e3.0 \\
\hline 15 & - & - & 2.8 & 3.1 & 3.2 & 3.1 & 3.4 & 3.3 & 3.2 & 3.2 & 3.1 & e3.0 \\
\hline 16 & - & - & 2.9 & 3.1 & 3.1 & 3.1 & 3.4 & 3.3 & 3.2 & 3.2 & 3.1 & e3.0 \\
\hline 17 & - & - & 2.9 & 3.1 & 3.1 & 3.1 & 3.3 & 3.2 & 3.2 & 3.2 & 3.1 & e3.0 \\
\hline 18 & - & - & 3.0 & 3.1 & 3.1 & 3.2 & 3.3 & 3.2 & 3.2 & 3.2 & 3.1 & e3.0 \\
\hline 19 & - & - & 3.0 & 3.1 & 3.1 & 3.2 & 3.3 & 3.3 & 3.1 & 3.2 & 3.1 & e3.0 \\
\hline 20 & - & - & 3.1 & 3.1 & 3.1 & 3.2 & 3.3 & 3.3 & 3.1 & 3.2 & 3.1 & 3.0 \\
\hline 21 & - & - & 3.1 & 3.1 & 3.1 & 3.2 & 3.3 & 3.3 & 3.1 & 3.2 & 3.1 & 3.0 \\
\hline 22 & - & - & 3.0 & 3.1 & 3.1 & 3.2 & 3.4 & 3.3 & 3.1 & 3.2 & 3.1 & 3.0 \\
\hline 23 & - & - & 3.0 & 3.1 & 3.0 & 3.2 & 3.4 & 3.2 & 3.1 & 3.2 & 3.1 & 3.0 \\
\hline 24 & - & - & 3.0 & 3.1 & 3.1 & 3.3 & 3.3 & 3.2 & 3.1 & 3.2 & 3.1 & 3.0 \\
\hline 25 & - & - & 3.0 & 3.2 & 3.0 & 3.3 & 3.3 & 3.3 & 3.1 & 3.1 & 3.1 & 2.9 \\
\hline 26 & - & - & 3.0 & 3.2 & 3.1 & 3.3 & 3.3 & 3.3 & 3.1 & 3.2 & 3.2 & 2.9 \\
\hline 27 & - & - & 3.0 & 3.2 & 3.0 & 3.2 & 3.3 & 3.3 & 3.1 & 3.2 & 3.2 & 2.9 \\
\hline 28 & - & 2.6 & 3.0 & 3.2 & 3.0 & 3.2 & 3.3 & 3.3 & 3.1 & 3.1 & e3.1 & 2.9 \\
\hline 29 & - & - & 3.0 & 3.2 & 3.0 & 3.2 & 3.3 & 3.3 & 3.1 & 3.2 & e3.1 & 2.9 \\
\hline 30 & - & - & 3.0 & 3.2 & 3.0 & 3.2 & 3.4 & 3.2 & 3.1 & 3.1 & e3.1 & 2.9 \\
\hline 31 & - & - & 3.0 & - & 3.1 & - & 3.4 & 3.2 & - & 3.1 & - & 2.9 \\
\hline Mean & 3.09 & 2.60 & 2.90 & 3.12 & 3.09 & 3.13 & 3.34 & 3.27 & 3.20 & 3.15 & 3.11 & 3.00 \\
\hline Max & 3.1 & 2.6 & 3.1 & 3.2 & 3.2 & 3.3 & 3.4 & 3.3 & 3.4 & 3.2 & 3.2 & 3.1 \\
\hline Min & 3.0 & 2.6 & 2.7 & 3.0 & 3.0 & 3.0 & 3.3 & 3.2 & 3.1 & 3.1 & 3.1 & 2.9 \\
\hline
\end{tabular}




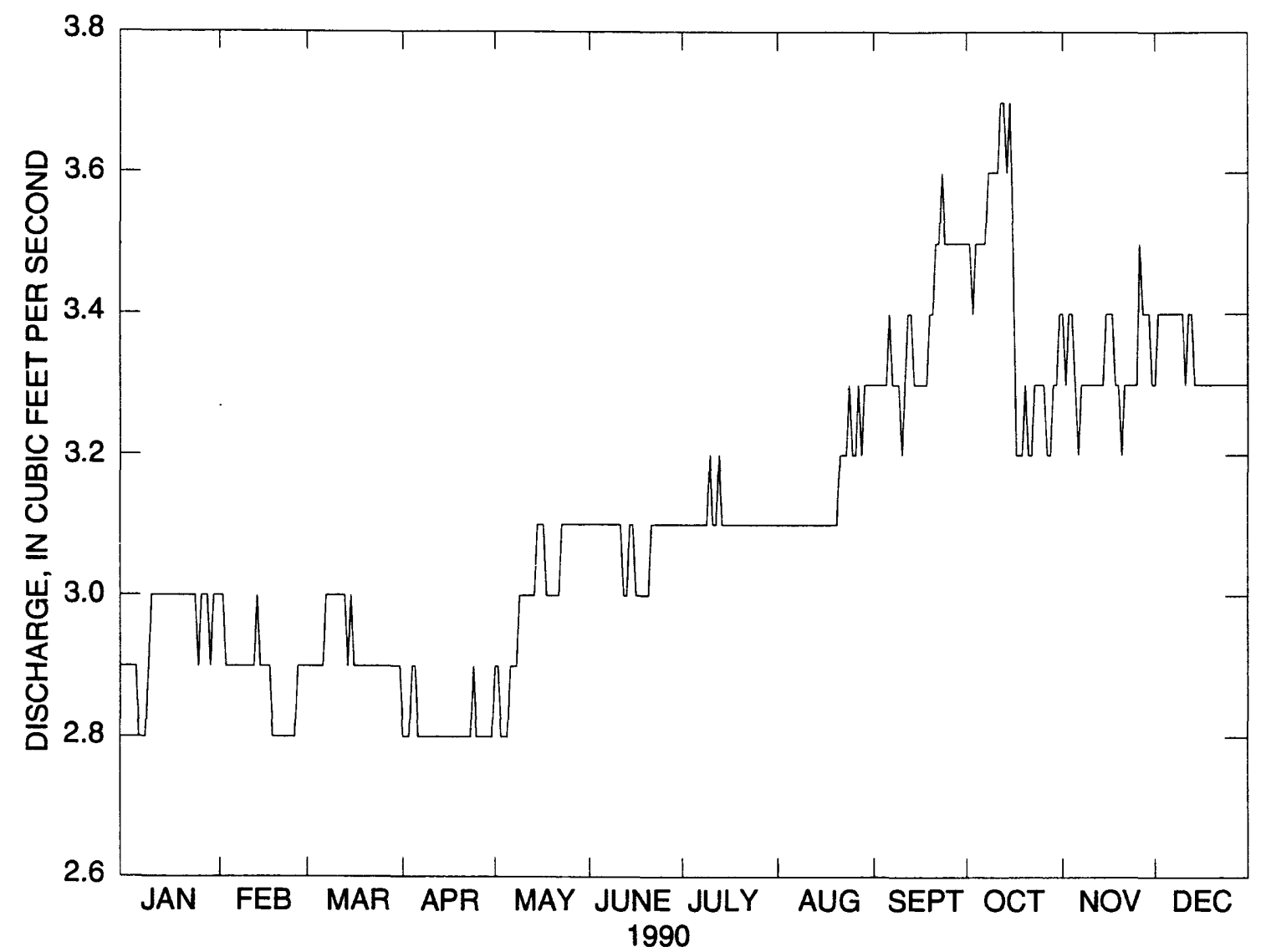

Figure 31. (D) Daily mean discharge, Fish Hatchery Spring 23 (FH23-Q), Long Valley Caldera, Mono County, California, 1990. 
Table 60. Daily mean discharge, Fish Hatchery Spring $23(\mathrm{FH} 23-\mathrm{Q})$, Long Valley Caldera, Mono County,

California, 1990

[Values are in cubic feet per second. e. estimated; Max, maximum; Min, minimum; -, no data]

\begin{tabular}{|c|c|c|c|c|c|c|c|c|c|c|c|c|}
\hline Day & Jan & Feb & Mar & Apr & May & June & July & Aug & Sept & Oct & Nov & $\mathrm{Dec}$ \\
\hline 1 & 2.9 & 3.0 & 2.9 & 2.8 & 2.9 & 3.1 & 3.1 & 3.1 & 3.3 & 3.5 & 3.4 & 3.3 \\
\hline 2 & 2.9 & 3.0 & 2.9 & 2.8 & 2.9 & 3.1 & 3.1 & 3.1 & 3.3 & 3.5 & 3.3 & 3.4 \\
\hline 3 & 2.9 & 2.9 & 2.9 & 2.8 & 2.8 & 3.1 & 3.1 & 3.1 & 3.3 & 3.4 & 3.4 & 3.4 \\
\hline 4 & 2.9 & 2.9 & 2.9 & 2.9 & 2.8 & 3.1 & 3.1 & 3.1 & 3.3 & 3.5 & 3.4 & 3.4 \\
\hline 5 & 2.9 & 2.9 & 2.9 & 2.9 & 2.8 & 3.1 & 3.1 & 3.1 & 3.3 & 3.5 & 3.3 & 3.4 \\
\hline 6 & 2.8 & 2.9 & 2.9 & 2.8 & 2.9 & 3.1 & 3.1 & 3.1 & 3.4 & 3.5 & 3.2 & 3.4 \\
\hline 7 & 2.8 & 2.9 & 3.0 & 2.8 & 2.9 & 3.1 & 3.1 & 3.1 & 3.3 & 3.5 & 3.3 & 3.4 \\
\hline 8 & 2.8 & 2.9 & 3.0 & 2.8 & 2.9 & 3.1 & 3.1 & 3.1 & 3.3 & 3.6 & 3.3 & 3.4 \\
\hline 9 & 2.9 & 2.9 & 3.0 & 2.8 & 3.0 & 3.1 & 3.1 & 3.1 & 3.3 & 3.6 & 3.3 & 3.4 \\
\hline 10 & 3.0 & 2.9 & 3.0 & 2.8 & 3.0 & 3.1 & 3.2 & 3.1 & 3.2 & 3.6 & 3.3 & 3.4 \\
\hline 11 & 3.0 & 2.9 & 3.0 & 2.8 & 3.0 & 3.1 & 3.1 & 3.1 & 3.3 & 3.6 & 3.3 & 3.3 \\
\hline 12 & 3.0 & 2.9 & 3.0 & 2.8 & 3.0 & 3.0 & 3.1 & 3.1 & 3.4 & 3.7 & 3.3 & 3.4 \\
\hline 13 & 3.0 & 3.0 & 3.0 & 2.8 & 3.0 & 3.0 & 3.2 & 3.1 & 3.4 & 3.7 & 3.3 & 3.4 \\
\hline 14 & 3.0 & 2.9 & 2.9 & 2.8 & 3.0 & 3.1 & 3.1 & 3.1 & 3.3 & 3.6 & 3.3 & 3.3 \\
\hline 15 & 3.0 & 2.9 & 3.0 & 2.8 & 3.1 & 3.1 & 3.1 & 3.1 & 3.3 & 3.7 & 3.4 & 3.3 \\
\hline 16 & 3.0 & 2.9 & 2.9 & 2.8 & 3.1 & 3.0 & 3.1 & 3.1 & 3.3 & 3.5 & 3.4 & 3.3 \\
\hline 17 & 3.0 & 2.9 & 2.9 & 2.8 & 3.1 & 3.0 & 3.1 & 3.1 & 3.3 & 3.2 & 3.4 & 3.3 \\
\hline 18 & 3.0 & 2.8 & 2.9 & 2.8 & 3.0 & 3.0 & 3.1 & 3.1 & 3.3 & 3.2 & 3.3 & 3.3 \\
\hline 19 & 3.0 & 2.8 & 2.9 & 2.8 & 3.0 & 3.0 & 3.1 & 3.1 & 3.4 & 3.2 & 3.3 & e3.3 \\
\hline 20 & 3.0 & 2.8 & 2.9 & 2.8 & 3.0 & 3.0 & 3.1 & 3.1 & 3.4 & 3.3 & 3.2 & e3.3 \\
\hline 21 & 3.0 & 2.8 & 2.9 & 2.8 & 3.0 & 3.1 & 3.1 & 3.2 & 3.5 & 3.2 & 3.3 & e3.3 \\
\hline 22 & 3.0 & 2.8 & 2.9 & 2.8 & 3.0 & 3.1 & 3.1 & 3.2 & 3.5 & 3.2 & 3.3 & e3.3 \\
\hline 23 & 3.0 & 2.8 & 2.9 & 2.8 & 3.1 & 3.1 & 3.1 & 3.2 & 3.6 & 3.3 & 3.3 & e3.3 \\
\hline 24 & 3.0 & 2.8 & 2.9 & 2.9 & 3.1 & 3.1 & 3.1 & 3.3 & 3.5 & 3.3 & 3.3 & e3.3 \\
\hline 25 & 2.9 & 2.8 & 2.9 & 2.8 & 3.1 & 3.1 & 3.1 & 3.2 & 3.5 & 3.3 & 3.3 & e3.3 \\
\hline 26 & 3.0 & 2.9 & 2.9 & 2.8 & 3.1 & 3.1 & 3.1 & 3.2 & 3.5 & 3.3 & 3.5 & e3.3 \\
\hline 27 & 3.0 & 2.9 & 2.9 & 2.8 & 3.1 & 3.1 & 3.1 & 3.3 & 3.5 & 3.2 & 3.4 & e3.3 \\
\hline 28 & 3.0 & 2.9 & 2.9 & 2.8 & 3.1 & 3.1 & 3.1 & 3.2 & 3.5 & 3.2 & 3.4 & e3.3 \\
\hline 29 & 2.9 & - & 2.9 & 2.8 & 3.1 & 3.1 & 3.1 & 3.3 & 3.5 & 3.3 & 3.4 & e3.3 \\
\hline 30 & 3.0 & - & 2.9 & 2.8 & 3.1 & 3.1 & 3.1 & 3.3 & 3.5 & 3.3 & 3.3 & e3.3 \\
\hline 31 & 3.0 & - & 2.9 & - & 3.1 & - & 3.1 & 3.3 & - & 3.4 & - & e3.3 \\
\hline Mean & 2.95 & 2.88 & 2.93 & 2.81 & 3.00 & 3.08 & 3.11 & 3.15 & 3.38 & 3.42 & 3.33 & 3.34 \\
\hline Max & 3.0 & 3.0 & 3.0 & 2.9 & 3.1 & 3.1 & 3.2 & 3.3 & 3.6 & 3.7 & 3.5 & 3.4 \\
\hline Min & 2.8 & 2.8 & 2.9 & 2.8 & 2.8 & 3.0 & 3.1 & 3.1 & 3.2 & 3.2 & 3.2 & 3.3 \\
\hline
\end{tabular}




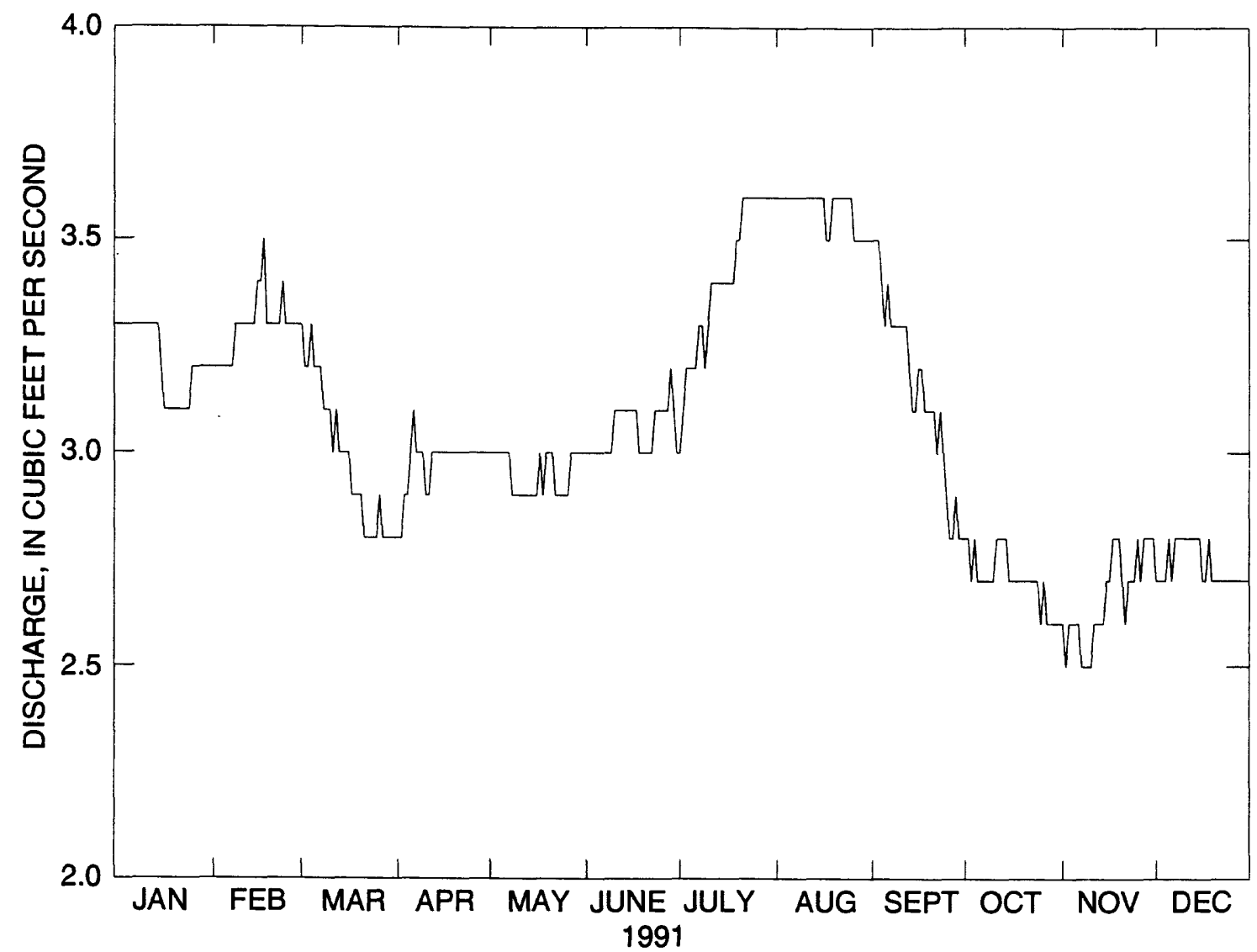

Figure 31. (E) Daily mean discharge, Fish Hatchery Spring 23 (FH23-Q), Long Valley Caldera, Mono County, California, 1991. 
Table 61. Daily mean discharge, Fish Hatchery Spring 23 (FH23-Q), Long Valley Caldera, Mono County, California, 1991

[Values are in cubic feet per second. e, estimated; Max, maximum; Min, minimum; - , no data]

\begin{tabular}{|c|c|c|c|c|c|c|c|c|c|c|c|c|}
\hline Day & Jan & Feb & Mar & Apr & May & June & July & Aug & Sept & Oct & Nov & Dec \\
\hline 1 & e3.3 & $\mathrm{e} 3.2$ & 3.3 & 2.8 & 3.0 & 3.0 & 3.0 & 3.6 & 3.5 & 2.8 & 2.6 & 2.7 \\
\hline 2 & e3.3 & e3.2 & 3.2 & 2.8 & 3.0 & 3.0 & 3.1 & 3.6 & 3.5 & 2.8 & 2.5 & 2.7 \\
\hline 3 & e 3.3 & $\mathrm{e} 3.2$ & 3.2 & 2.9 & 3.0 & 3.0 & 3.2 & 3.6 & 3.5 & 2.7 & 2.6 & 2.7 \\
\hline 4 & e3.3 & e3.2 & 3.3 & 2.9 & 3.0 & 3.0 & 3.2 & 3.6 & 3.4 & 2.8 & 2.6 & 2.7 \\
\hline 5 & e3.3 & e3.2 & 3.2 & 3.0 & 3.0 & 3.0 & 3.2 & 3.6 & 3.3 & 2.7 & 2.6 & 2.8 \\
\hline 6 & e3.3 & $\mathrm{e} 3.2$ & 3.2 & 3.1 & 3.0 & 3.0 & 3.2 & 3.6 & 3.4 & 2.7 & 2.6 & 2.7 \\
\hline 7 & e3.3 & e3.2 & 3.2 & 3.0 & 3.0 & 3.0 & 3.3 & 3.6 & 3.3 & 2.7 & 2.5 & 2.8 \\
\hline 8 & e3.3 & e3.3 & 3.1 & 3.0 & 2.9 & 3.0 & 3.3 & 3.6 & 3.3 & 2.7 & 2.5 & 2.8 \\
\hline 9 & e 3.3 & e3.3 & 3.1 & 3.0 & 2.9 & 3.0 & 3.2 & 3.6 & 3.3 & 2.7 & 2.5 & 2.8 \\
\hline 10 & e3.3 & e3.3 & 3.1 & 2.9 & 2.9 & 3.1 & 3.3 & 3.6 & 3.3 & 2.7 & 2.5 & 2.8 \\
\hline 11 & e3.3 & e3.3 & 3.0 & 2.9 & 2.9 & 3.1 & 3.4 & 3.6 & 3.3 & 2.8 & 2.6 & 2.8 \\
\hline 12 & e3.3 & e3.3 & 3.1 & 3.0 & 2.9 & 3.1 & 3.4 & 3.6 & 3.3 & 2.8 & 2.6 & 2.8 \\
\hline 13 & e3.3 & e3.3 & 3.0 & 3.0 & 2.9 & 3.1 & 3.4 & 3.6 & 3.2 & 2.8 & 2.6 & 2.8 \\
\hline 14 & e3.3 & e3.3 & 3.0 & 3.0 & 2.9 & 3.1 & 3.4 & 3.6 & 3.1 & 2.8 & 2.6 & 2.8 \\
\hline 15 & e 3.2 & 3.4 & 3.0 & 3.0 & 2.9 & 3.1 & 3.4 & 3.6 & 3.1 & 2.7 & 2.7 & 2.8 \\
\hline 16 & e3.1 & 3.4 & 3.0 & 3.0 & 2.9 & 3.1 & 3.4 & 3.6 & 3.2 & 2.7 & 2.7 & 2.7 \\
\hline 17 & e3.1 & 3.5 & 2.9 & 3.0 & 3.0 & 3.1 & 3.4 & 3.5 & 3.2 & 2.7 & 2.8 & 2.7 \\
\hline 18 & e3.1 & 3.3 & 2.9 & 3.0 & 2.9 & e 3.0 & 3.4 & 3.5 & 3.1 & 2.7 & 2.8 & 2.8 \\
\hline 19 & e3.1 & 3.3 & 2.9 & 3.0 & 3.0 & e3.0 & 3.5 & 3.6 & 3.1 & 2.7 & 2.8 & 2.7 \\
\hline 20 & $\mathrm{e} 3.1$ & 3.3 & 2.9 & 3.0 & 3.0 & e 3.0 & 3.5 & 3.6 & 3.1 & 2.7 & 2.7 & 2.7 \\
\hline 21 & e3.1 & 3.3 & 2.8 & 3.0 & 3.0 & 3.0 & 3.6 & 3.6 & 3.1 & 2.7 & 2.6 & 2.7 \\
\hline 22 & e3.1 & 3.3 & 2.8 & 3.0 & 2.9 & 3.0 & 3.6 & 3.6 & 3.0 & 2.7 & 2.7 & 2.7 \\
\hline 23 & e3.1 & 3.4 & 2.8 & 3.0 & 2.9 & 3.1 & 3.6 & 3.6 & 3.1 & 2.7 & 2.7 & 2.7 \\
\hline 24 & e3.1 & 3.3 & 2.8 & 3.0 & 2.9 & 3.1 & 3.6 & 3.6 & 3.0 & 2.7 & 2.7 & 2.7 \\
\hline 25 & $\mathrm{e} 3.2$ & 3.3 & 2.8 & 3.0 & 2.9 & 3.1 & 3.6 & 3.6 & 2.9 & 2.6 & 2.8 & 2.7 \\
\hline 26 & e 3.2 & 3.3 & 2.9 & 3.0 & 2.9 & 3.1 & 3.6 & 3.5 & 2.8 & 2.7 & 2.7 & 2.7 \\
\hline 27 & e3.2 & 3.3 & 2.8 & 3.0 & 3.0 & 3.1 & 3.6 & 3.5 & 2.8 & 2.6 & 2.8 & 2.7 \\
\hline 28 & e3.2 & 3.3 & 2.8 & 3.0 & 3.0 & 3.2 & 3.6 & 3.5 & 2.9 & 2.6 & 2.8 & 2.7 \\
\hline 29 & e3.2 & - & 2.8 & 3.0 & 3.0 & 3.1 & 3.6 & 3.5 & 2.8 & 2.6 & 2.8 & 2.7 \\
\hline 30 & e3.2 & - & 2.8 & 3.0 & 3.0 & 3.0 & 3.6 & 3.5 & 2.8 & 2.6 & 2.8 & 2.7 \\
\hline 31 & e 3.2 & - & 2.8 & - & 3.0 & - & 3.6 & 3.5 & - & 2.6 & - & 2.7 \\
\hline Mean & 3.22 & 3.29 & 2.98 & 2.98 & 2.95 & 3.05 & 3.41 & 3.57 & 3.16 & 2.70 & 2.66 & 2.74 \\
\hline $\operatorname{Max}$ & 3.3 & 3.5 & 3.3 & 3.1 & 3.0 & 3.2 & 3.6 & 3.6 & 3.5 & 2.8 & 2.8 & 2.8 \\
\hline Min & 3.1 & 3.2 & 2.8 & 2.8 & 2.9 & 3.0 & 3.0 & 3.5 & 2.8 & 2.6 & 2.5 & 2.7 \\
\hline
\end{tabular}




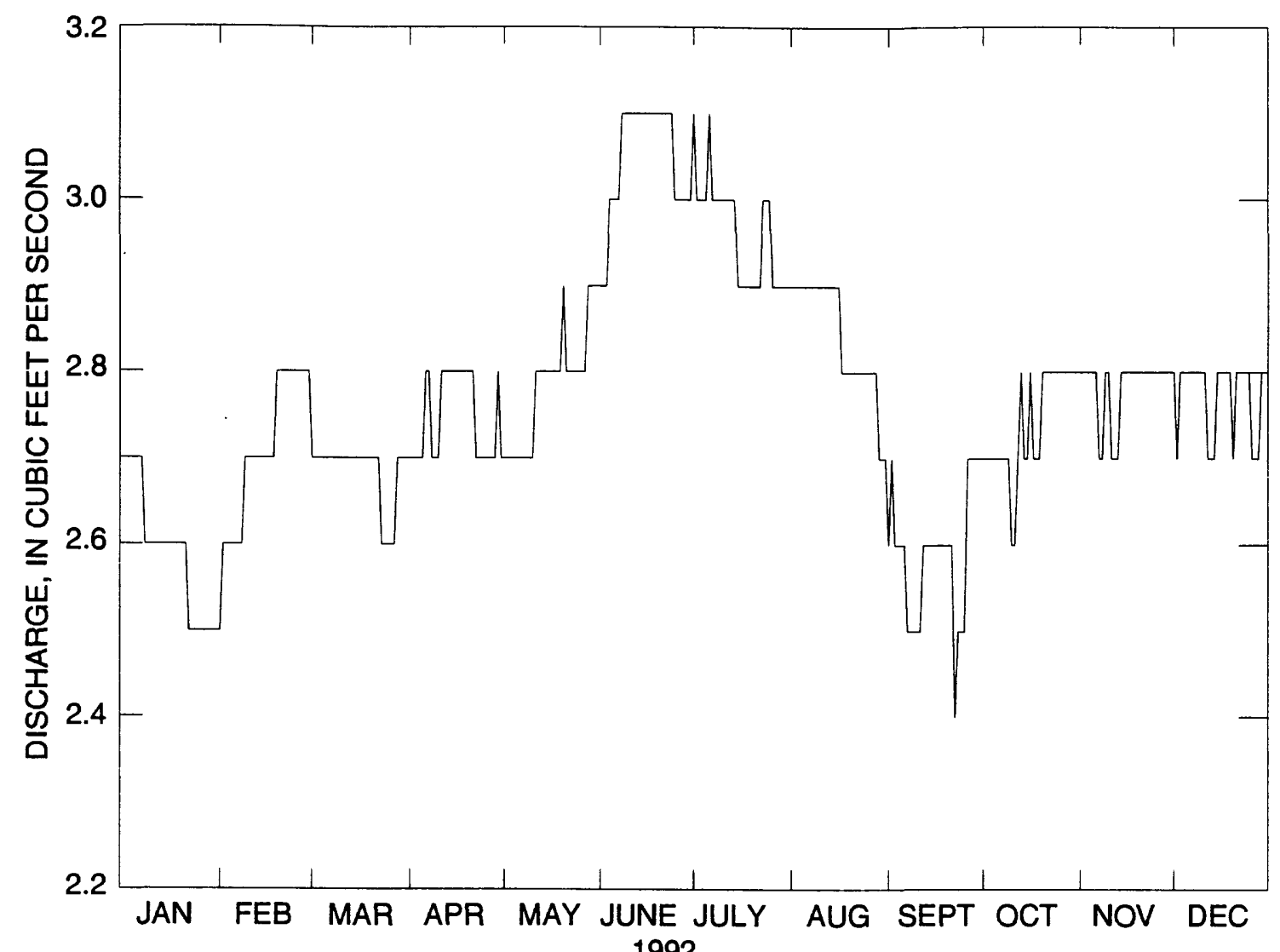

Figure 31. (F) Daily mean discharge, Fish Hatchery Spring 23 (FH23-Q), Long Valley Caldera, Mono County, California, 1992. 
Table 62. Daily mean discharge, Fish Hatchery Spring 23 (FH23-Q), Long Valley Caldera, Mono County, California, 1992

[Values are in cubic feet per second. Max, maximum; Min, minimum; -, no data]

\begin{tabular}{|c|c|c|c|c|c|c|c|c|c|c|c|c|}
\hline Day & Jan & $\mathrm{Feb}$ & Mar & Apr & May & June & July & Aug & Sept & Oct & Nov & Dec \\
\hline 1 & 2.7 & 2.5 & 2.7 & 2.7 & 2.7 & 2.9 & 3.1 & 2.9 & 2.6 & 2.7 & 2.8 & 2.8 \\
\hline 2 & 2.7 & 2.6 & 2.7 & 2.7 & 2.7 & 2.9 & 3.0 & 2.9 & 2.7 & 2.7 & 2.8 & 2.7 \\
\hline 3 & 2.7 & 2.6 & 2.7 & 2.7 & 2.7 & 2.9 & 3.0 & 2.9 & 2.6 & 2.7 & 2.8 & 2.8 \\
\hline 4 & 2.7 & 2.6 & 2.7 & 2.7 & 2.7 & 3.0 & 3.0 & 2.9 & 2.6 & 2.7 & 2.8 & 2.8 \\
\hline 5 & 2.7 & 2.6 & 2.7 & 2.7 & 2.7 & 3.0 & 3.0 & 2.9 & 2.6 & 2.7 & 2.8 & 2.8 \\
\hline 6 & 2.7 & 2.6 & 2.7 & 2.8 & 2.7 & 3.0 & 3.1 & 2.9 & 2.6 & 2.7 & 2.8 & 2.8 \\
\hline 7 & 2.7 & 2.6 & 2.7 & 2.8 & 2.7 & 3.0 & 3.0 & 2.9 & 2.5 & 2.7 & 2.7 & 2.8 \\
\hline 8 & 2.6 & 2.6 & 2.7 & 2.7 & 2.7 & 3.1 & 3.0 & 2.9 & 2.5 & 2.7 & 2.7 & 2.8 \\
\hline 9 & 2.6 & 2.7 & 2.7 & 2.7 & 2.7 & 3.1 & 3.0 & 2.9 & 2.5 & 2.7 & 2.8 & 2.8 \\
\hline 10 & 2.6 & 2.7 & 2.7 & 2.7 & 2.7 & 3.1 & 3.0 & 2.9 & 2.5 & 2.6 & 2.8 & 2.8 \\
\hline 11 & 2.6 & 2.7 & 2.7 & 2.8 & 2.8 & 3.1 & 3.0 & 2.9 & 2.5 & 2.6 & 2.7 & 2.8 \\
\hline 12 & 2.6 & 2.7 & 2.7 & 2.8 & 2.8 & 3.1 & 3.0 & 2.9 & 2.6 & 2.7 & 2.7 & 2.7 \\
\hline 13 & 2.6 & 2.7 & 2.7 & 2.8 & 2.8 & 3.1 & 3.0 & 2.9 & 2.6 & 2.8 & 2.7 & 2.7 \\
\hline 14 & 2.6 & 2.7 & 2.7 & 2.8 & 2.8 & 3.1 & 3.0 & 2.9 & 2.6 & 2.7 & 2.8 & 2.7 \\
\hline 15 & 2.6 & 2.7 & 2.7 & 2.8 & 2.8 & 3.1 & 2.9 & 2.9 & 2.6 & 2.7 & 2.8 & 2.8 \\
\hline 16 & 2.6 & 2.7 & 2.7 & 2.8 & 2.8 & 3.1 & 2.9 & 2.9 & 2.6 & 2.8 & 2.8 & 2.8 \\
\hline 17 & 2.6 & 2.7 & 2.7 & 2.8 & 2.8 & 3.1 & 2.9 & 2.8 & 2.6 & 2.7 & 2.8 & 2.8 \\
\hline 18 & 2.6 & 2.7 & 2.7 & 2.8 & 2.8 & 3.1 & 2.9 & 2.8 & 2.6 & 2.7 & 2.8 & 2.8 \\
\hline 19 & 2.6 & 2.8 & 2.7 & 2.8 & 2.8 & 3.1 & 2.9 & 2.8 & 2.6 & 2.7 & 2.8 & 2.8 \\
\hline 20 & 2.6 & 2.8 & 2.7 & 2.8 & 2.9 & 3.1 & 2.9 & 2.8 & 2.6 & 2.8 & 2.8 & 2.7 \\
\hline 21 & 2.6 & 2.8 & 2.7 & 2.8 & 2.8 & 3.1 & 2.9 & 2.8 & 2.6 & 2.8 & 2.8 & 2.8 \\
\hline 22 & 2.5 & 2.8 & 2.7 & 2.7 & 2.8 & 3.1 & 2.9 & 2.8 & 2.4 & 2.8 & 2.8 & 2.8 \\
\hline 23 & 2.5 & 2.8 & 2.6 & 2.7 & 2.8 & 3.1 & 3.0 & 2.8 & 2.5 & 2.8 & 2.8 & 2.8 \\
\hline 24 & 2.5 & 2.8 & 2.6 & 2.7 & 2.8 & 3.1 & 3.0 & 2.8 & 2.5 & 2.8 & 2.8 & 2.8 \\
\hline 25 & 2.5 & 2.8 & 2.6 & 2.7 & 2.8 & 3.0 & 3.0 & 2.8 & 2.5 & 2.8 & 2.8 & 2.8 \\
\hline 26 & 2.5 & 2.8 & 2.6 & 2.7 & 2.8 & 3.0 & 2.9 & 2.8 & 2.7 & 2.8 & 2.8 & 2.7 \\
\hline 27 & 2.5 & 2.8 & 2.6 & 2.7 & 2.8 & 3.0 & 2.9 & 2.8 & 2.7 & 2.8 & 2.8 & 2.7 \\
\hline 28 & 2.5 & 2.8 & 2.7 & 2.7 & 2.9 & 3.0 & 2.9 & 2.8 & 2.7 & 2.8 & 2.8 & 2.7 \\
\hline 29 & 2.5 & 2.8 & 2.7 & 2.8 & 2.9 & 3.0 & 2.9 & 2.7 & 2.7 & 2.8 & 2.8 & 2.8 \\
\hline 30 & 2.5 & - & 2.7 & 2.7 & 2.9 & 3.0 & 2.9 & 2.7 & 2.7 & 2.8 & 2.8 & 2.8 \\
\hline 31 & 2.5 & - & 2.7 & - & 2.9 & - & 2.9 & 2.7 & - & 2.8 & - & 2.8 \\
\hline Mean & 2.59 & 2.71 & 2.68 & 2.75 & 2.78 & 3.05 & 2.96 & 2.84 & 2.59 & 2.74 & 2.78 & 2.77 \\
\hline Max & 2.7 & 2.8 & 2.7 & 2.8 & 2.9 & 3.1 & 3.1 & 2.9 & 2.7 & 2.8 & 2.8 & 2.8 \\
\hline Min & 2.5 & 2.5 & 2.6 & 2.7 & 2.7 & 2.9 & 2.9 & 2.7 & 2.4 & 2.6 & 2.7 & 2.7 \\
\hline
\end{tabular}




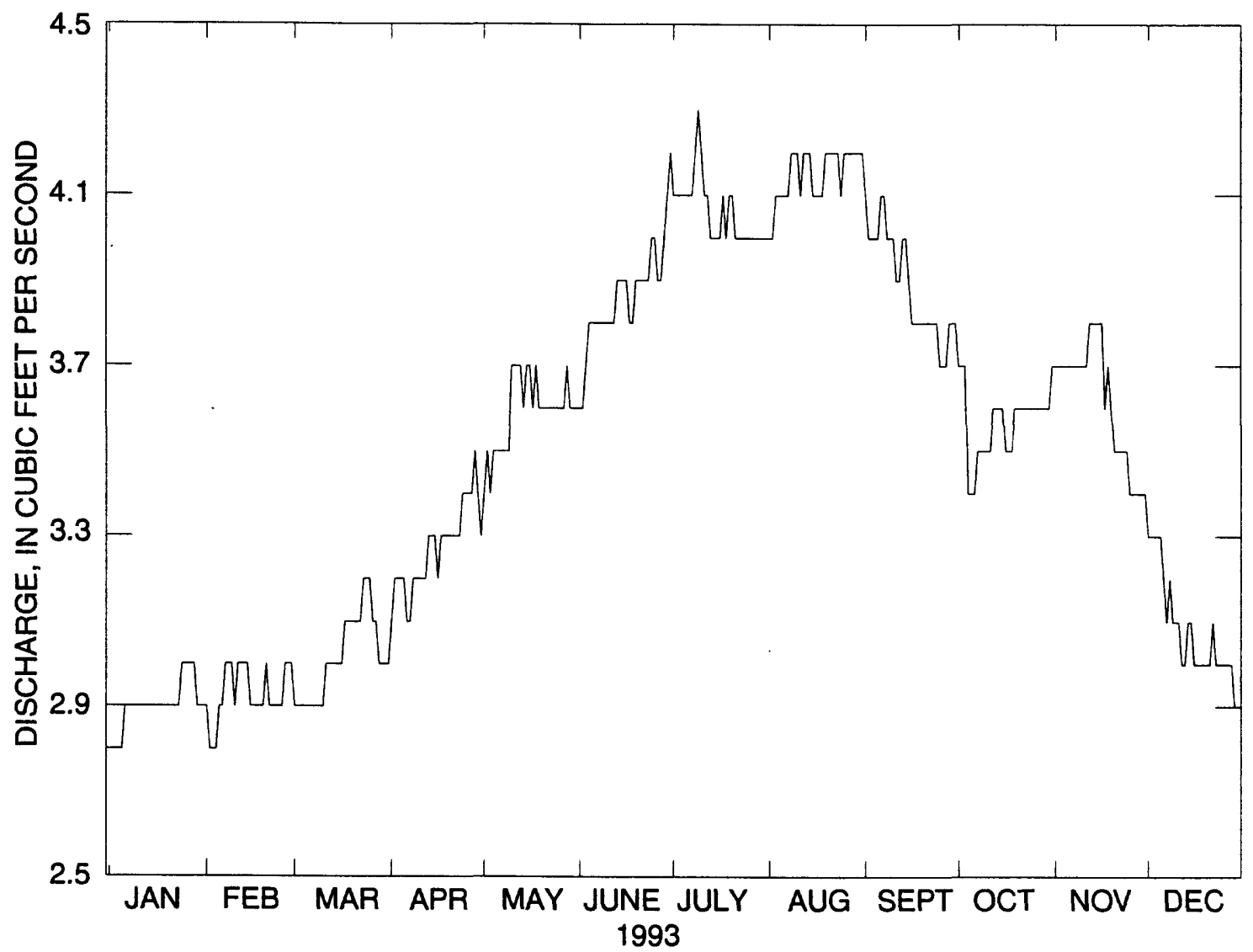

Figure 31. (G) Daily mean discharge, Fish Hatchery Spring 23 (FH23-Q), Long Valley Caldera, Mono County, California, 1993. 
Table 63. Daily mean discharge, Fish Hatchery Spring 23 (FH23-Q), Long Valley Caldera, Mono County, California, 1993

[Values are in cubic feet per second. Max, maximum; Min, minimum; -, no data]

\begin{tabular}{|c|c|c|c|c|c|c|c|c|c|c|c|c|}
\hline Day & Jan & Feb & Mar & Apr & May & June & July & Aug & Sept & Oct & Nov & Dec \\
\hline 1 & 2.8 & 2.9 & 2.9 & 3.1 & 3.4 & 3.6 & 4.1 & 4.0 & 4.1 & 3.7 & 3.7 & 3.3 \\
\hline 2 & 2.8 & 2.8 & 2.9 & 3.2 & 3.5 & 3.6 & 4.1 & 4.0 & 4.0 & 3.7 & 3.7 & 3.3 \\
\hline 3 & 2.8 & 2.8 & 2.9 & 3.2 & 3.4 & 3.7 & 4.1 & 4.1 & 4.0 & 3.7 & 3.7 & 3.3 \\
\hline 4 & 2.8 & 2.8 & 2.9 & 3.2 & 3.5 & 3.8 & 4.1 & 4.1 & 4.0 & 3.4 & 3.7 & 3.3 \\
\hline 5 & 2.8 & 2.9 & 2.9 & 3.2 & 3.5 & 3.8 & 4.1 & 4.1 & 4.0 & 3.4 & 3.7 & 3.3 \\
\hline 6 & 2.9 & 2.9 & 2.9 & 3.1 & 3.5 & 3.8 & 4.1 & 4.1 & 4.1 & 3.4 & 3.7 & 3.2 \\
\hline 7 & 2.9 & 3.0 & 2.9 & 3.1 & 3.5 & 3.8 & 4.1 & 4.1 & 4.1 & 3.5 & 3.7 & 3.1 \\
\hline 8 & 2.9 & 3.0 & 2.9 & 3.2 & 3.5 & 3.8 & 4.2 & 4.2 & 4.0 & 3.5 & 3.7 & 3.2 \\
\hline 9 & 2.9 & 3.0 & 2.9 & 3.2 & 3.5 & 3.8 & 4.3 & 4.2 & 4.0 & 3.5 & 3.7 & 3.1 \\
\hline 10 & 2.9 & 2.9 & 2.9 & 3.2 & 3.7 & 3.8 & 4.2 & 4.2 & 4.0 & 3.5 & 3.7 & 3.1 \\
\hline 11 & 2.9 & 3.0 & 3.0 & 3.2 & 3.7 & 3.8 & 4.1 & 4.1 & 3.9 & 3.5 & 3.7 & 3.1 \\
\hline 12 & 2.9 & 3.0 & 3.0 & 3.2 & 3.7 & 3.8 & 4.1 & 4.2 & 3.9 & 3.6 & 3.8 & 3.0 \\
\hline 13 & 2.9 & 3.0 & 3.0 & 3.3 & 3.7 & 3.9 & 4.0 & 4.2 & 4.0 & 3.6 & 3.8 & 3.0 \\
\hline 14 & 2.9 & 3.0 & 3.0 & 3.3 & 3.6 & 3.9 & 4.0 & 4.2 & 4.0 & 3.6 & 3.8 & 3.1 \\
\hline 15 & 2.9 & 2.9 & 3.0 & 3.3 & 3.7 & 3.9 & 4.0 & 4.1 & 3.9 & 3.6 & 3.8 & 3.1 \\
\hline 16 & 2.9 & 2.9 & 3.0 & 3.2 & 3.7 & 3.9 & 4.0 & 4.1 & 3.8 & 3.5 & 3.8 & 3.0 \\
\hline 17 & 2.9 & 2.9 & 3.1 & 3.3 & 3.6 & 3.8 & 4.1 & 4.1 & 3.8 & 3.5 & 3.6 & 3.0 \\
\hline 18 & 2.9 & 2.9 & 3.1 & 3.3 & 3.7 & 3.8 & 4.0 & 4.1 & 3.8 & 3.5 & 3.7 & 3.0 \\
\hline 19 & 2.9 & 2.9 & 3.1 & 3.3 & 3.6 & 3.9 & 4.1 & 4.2 & 3.8 & 3.6 & 3.6 & 3.0 \\
\hline 20 & 2.9 & 3.0 & 3.1 & 3.3 & 3.6 & 3.9 & 4.1 & 4.2 & 3.8 & 3.6 & 3.5 & 3.0 \\
\hline 21 & 2.9 & 2.9 & 3.1 & 3.3 & 3.6 & 3.9 & 4.0 & 4.2 & 3.8 & 3.6 & 3.5 & 3.0 \\
\hline 22 & 2.9 & 2.9 & 3.1 & 3.3 & 3.6 & 3.9 & 4.0 & 4.2 & 3.8 & 3.6 & 3.5 & 3.1 \\
\hline 23 & 2.9 & 2.9 & 3.2 & 3.3 & 3.6 & 3.9 & 4.0 & 4.2 & 3.8 & 3.6 & 3.5 & 3.0 \\
\hline 24 & 3.0 & 2.9 & 3.2 & 3.4 & 3.6 & 4.0 & 4.0 & 4.1 & 3.8 & 3.6 & 3.5 & 3.0 \\
\hline 25 & 3.0 & 2.9 & 3.2 & 3.4 & 3.6 & 4.0 & 4.0 & 4.2 & 3.7 & 3.6 & 3.4 & 3.0 \\
\hline 26 & 3.0 & 3.0 & 3.1 & 3.4 & 3.6 & 3.9 & 4.0 & 4.2 & 3.7 & 3.6 & 3.4 & 3.0 \\
\hline 27 & 3.0 & 3.0 & 3.1 & 3.4 & 3.6 & 3.9 & 4.0 & 4.2 & 3.7 & 3.6 & 3.4 & 3.0 \\
\hline 28 & 3.0 & 3.0 & 3.0 & 3.5 & 3.7 & 4.0 & 4.0 & 4.2 & 3.8 & 3.6 & 3.4 & 3.0 \\
\hline 29 & 2.9 & - & 3.0 & 3.4 & 3.6 & 4.1 & 4.0 & 4.2 & 3.8 & 3.6 & 3.4 & 2.9 \\
\hline 30 & 2.9 & - & 3.0 & 3.3 & 3.6 & 4.2 & 4.0 & 4.2 & 3.8 & 3.6 & 3.4 & 2.9 \\
\hline 31 & 2.9 & - & 3.0 & - & 3.6 & - & 4.0 & 4.2 & - & 3.7 & - & 2.9 \\
\hline Mean & 2.90 & 2.93 & 3.01 & 3.27 & 3.59 & 3.86 & 4.06 & 4.15 & 3.89 & 3.57 & 3.62 & 3.07 \\
\hline $\operatorname{Max}$ & 3.0 & 3.0 & 3.2 & 3.5 & 3.7 & 4.2 & 4.3 & 4.2 & 4.1 & 3.7 & 3.8 & 3.3 \\
\hline Min & 2.8 & 2.8 & 2.9 & 3.1 & 3.4 & 3.6 & 4.0 & 4.0 & 3.7 & 3.4 & 3.4 & 2.9 \\
\hline
\end{tabular}


This page intentionally left blank. 


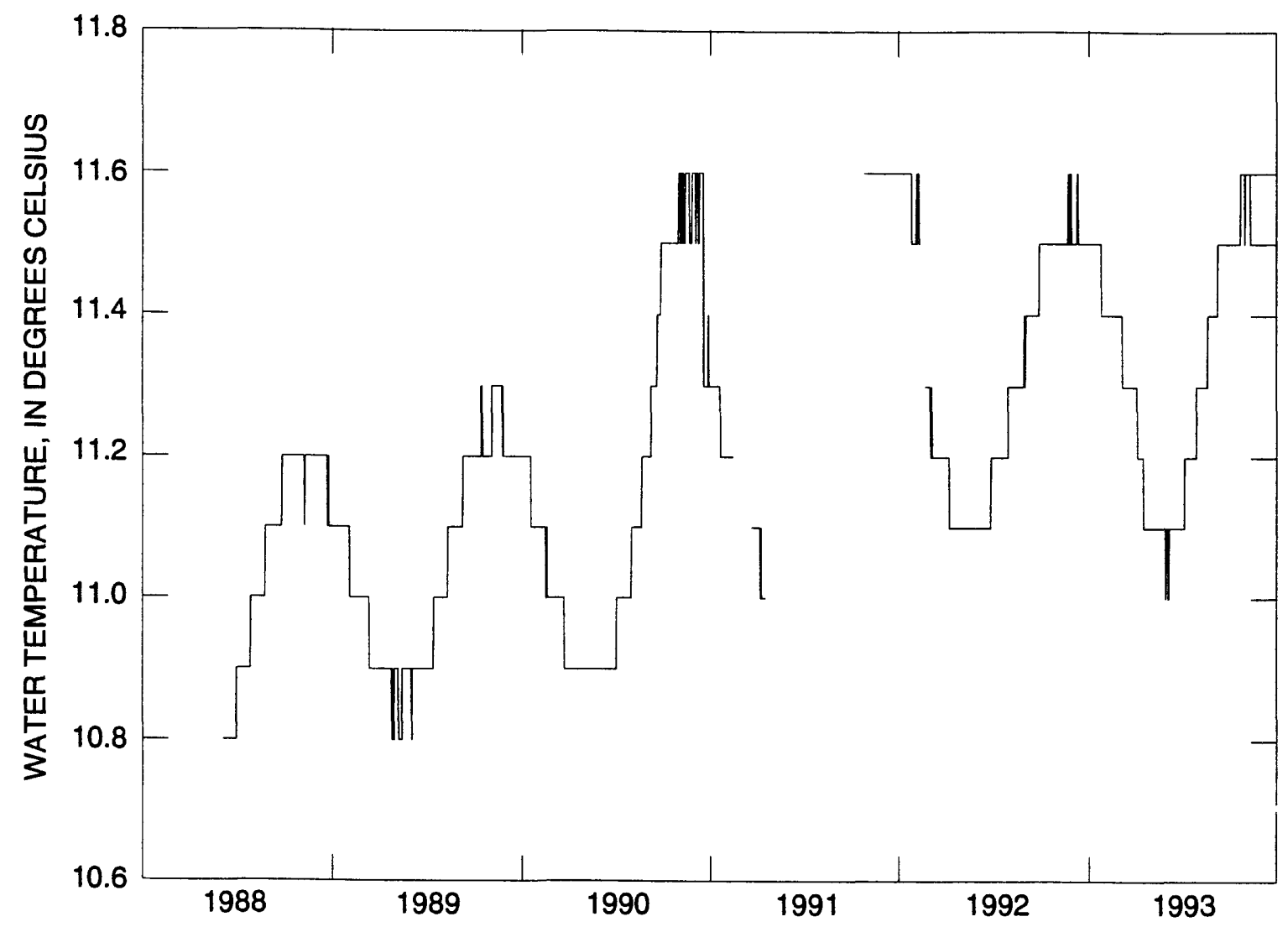

Figure 32. Daily mean water temperature, Fish Hatchery Spring 23 (FH23-T), Long Valley Caldera, Mono County, California, 1988-93. 


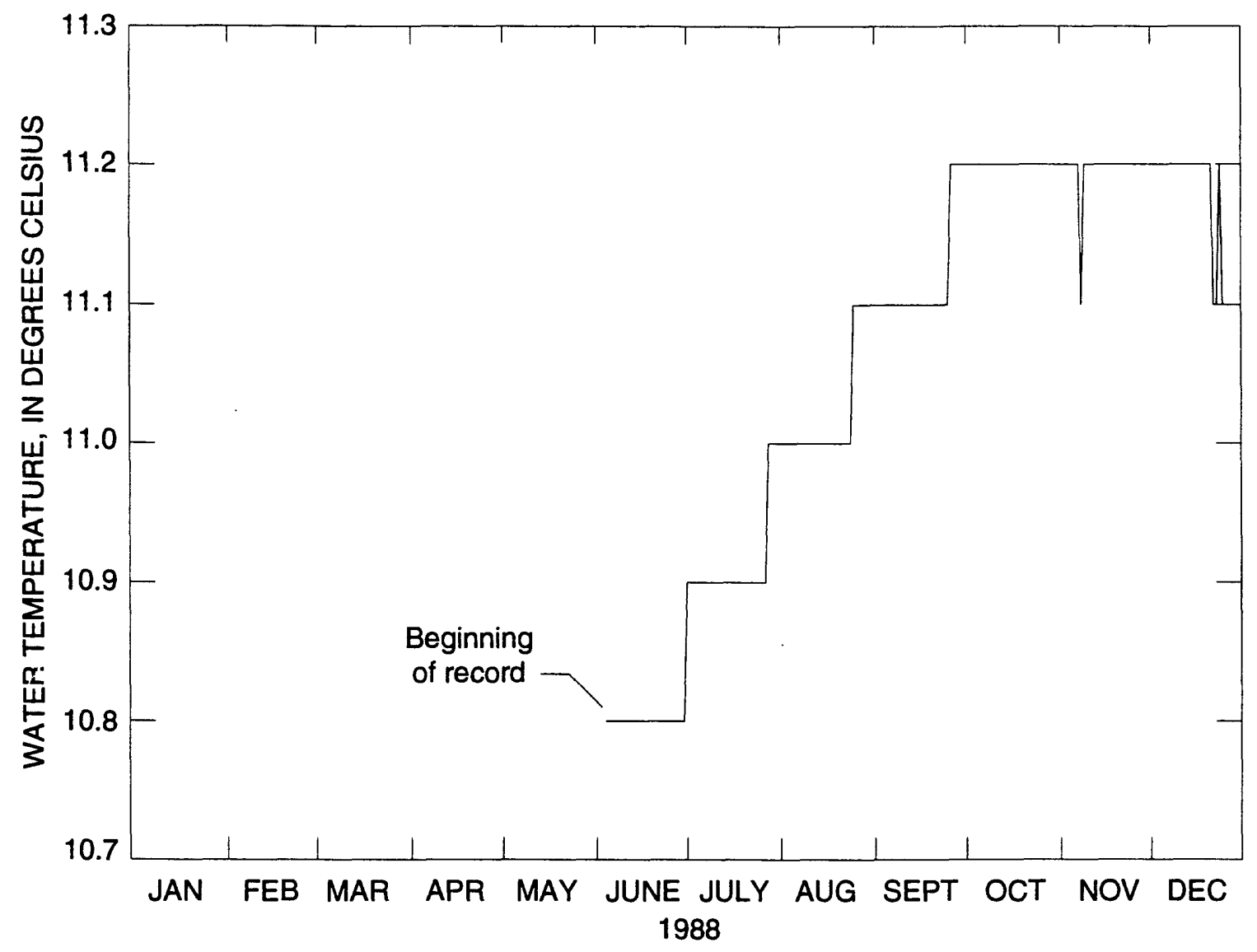

Figure 33. (A) Daily mean water temperature, Fish Hatchery Spring 23 (FH23-T), Long Valley Caldera, Mono County, California, . 1988. 
Table 64. Daily mean water temperature, Fish Hatchery Spring 23 (FH23-T), Long Valley Caldera, Mono County, California, 1988

[Values are in degrees Celsius. Max, maximum; Min, minimum; - , no data]

\begin{tabular}{|c|c|c|c|c|c|c|c|c|c|c|c|c|}
\hline Day & Jan & Feb & Mar & Apr & May & June & July & Aug & Sept & Oct & Nov & Dec \\
\hline 1 & - & - & - & - & - & - & 10.9 & 11.0 & 11.1 & 11.2 & 11.2 & 11.2 \\
\hline 2 & - & - & - & - & - & - & 10.9 & 11.0 & 11.1 & 11.2 & 11.2 & 11.2 \\
\hline 3 & - & - & - & - & - & - & 10.9 & 11.0 & 11.1 & 11.2 & 11.2 & 11.2 \\
\hline 4 & - & - & - & - & - & 10.8 & 10.9 & 11.0 & 11.1 & 11.2 & 11.2 & 11.2 \\
\hline 5 & - & - & - & - & - & 10.8 & 10.9 & 11.0 & 11.1 & 11.2 & 11.2 & 11.2 \\
\hline 6 & - & - & - & - & - & 10.8 & 10.9 & 11.0 & 11.1 & 11.2 & 11.2 & 11.2 \\
\hline 7 & - & - & - & - & - & 10.8 & 10.9 & 11.0 & 11.1 & 11.2 & 11.2 & 11.2 \\
\hline 8 & - & - & - & - & - & 10.8 & 10.9 & 11.0 & 11.1 & 11.2 & 11.1 & 11.2 \\
\hline 9 & - & - & - & - & - & 10.8 & 10.9 & 11.0 & 11.1 & 11.2 & 11.2 & 11.2 \\
\hline 10 & - & - & - & - & - & 10.8 & 10.9 & 11.0 & 11.1 & 11.2 & 11.2 & 11.2 \\
\hline 11 & - & - & - & - & - & 10.8 & 10.9 & 11.0 & 11.1 & 11.2 & 11.2 & 11.2 \\
\hline 12 & - & - & - & - & - & 10.8 & 10.9 & 11.0 & 11.1 & 11.2 & 11.2 & 11.2 \\
\hline 13 & - & - & - & - & - & 10.8 & 10.9 & 11.0 & 11.1 & 11.2 & 11.2 & 11.2 \\
\hline 14 & - & - & - & - & - & 10.8 & 10.9 & 11.0 & 11.1 & 11.2 & 11.2 & 11.2 \\
\hline 15 & - & - & - & - & - & 10.8 & 10.9 & 11.0 & 11.1 & 11.2 & 11.2 & 11.2 \\
\hline 16 & - & - & - & - & - & 10.8 & 10.9 & 11.0 & 11.1 & 11.2 & 11.2 & 11.2 \\
\hline 17 & - & - & - & - & - & 10.8 & 10.9 & 11.0 & 11.1 & 11.2 & 11.2 & 11.2 \\
\hline 18 & - & - & - & - & - & 10.8 & 10.9 & 11.0 & 11.1 & 11.2 & 11.2 & 11.2 \\
\hline 19 & - & - & - & - & - & 10.8 & 10.9 & 11.0 & 11.1 & 11.2 & 11.2 & 11.2 \\
\hline 20 & - & - & - & - & - & 10.8 & 10.9 & 11.0 & 11.1 & 11.2 & 11.2 & 11.2 \\
\hline 21 & - & - & - & - & - & 10.8 & 10.9 & 11.0 & 11.1 & 11.2 & 11.2 & 11.2 \\
\hline 22 & - & - & - & - & - & 10.8 & 10.9 & 11.0 & 11.1 & 11.2 & 11.2 & 11.1 \\
\hline 23 & - & - & - & - & - & 10.8 & 10.9 & 11.0 & 11.1 & 11.2 & 11.2 & 11.1 \\
\hline 24 & - & - & - & - & - & 10.8 & 10.9 & 11.0 & 11.1 & 11.2 & 11.2 & 11.2 \\
\hline 25 & - & - & - & - & - & 10.8 & 10.9 & 11.1 & 11.1 & 11.2 & 11.2 & 11.1 \\
\hline 26 & - & - & - & - & - & 10.8 & 10.9 & 11.1 & 11.2 & 11.2 & 11.2 & 11.1 \\
\hline 27 & - & - & - & - & - & 10.8 & 10.9 & 11.1 & 11.2 & 11.2 & 11.2 & 11.1 \\
\hline 28 & - & - & - & - & - & 10.8 & 11.0 & 11.1 & 11.2 & 11.2 & 11.2 & 11.1 \\
\hline 29 & - & - & - & - & - & 10.8 & 11.0 & 11.1 & 11.2 & 11.2 & 11.2 & 11.1 \\
\hline 30 & - & - & - & - & - & 10.8 & 11.0 & 11.1 & 11.2 & 11.2 & 11.2 & 11.1 \\
\hline 31 & - & - & - & - & - & - & 11.0 & 11.1 & - & 11.2 & - & 11.1 \\
\hline Mean & - & - & - & - & - & 10.8 & 10.9 & 11.0 & 11.1 & 11.2 & 11.2 & 11.2 \\
\hline Max & - & - & - & - & - & 10.8 & 11.0 & 11.1 & 11.2 & 11.2 & 11.2 & 11.2 \\
\hline Min & - & - & - & - & - & 10.8 & 10.9 & 11.0 & 11.1 & 11.2 & 11.1 & 11.1 \\
\hline
\end{tabular}




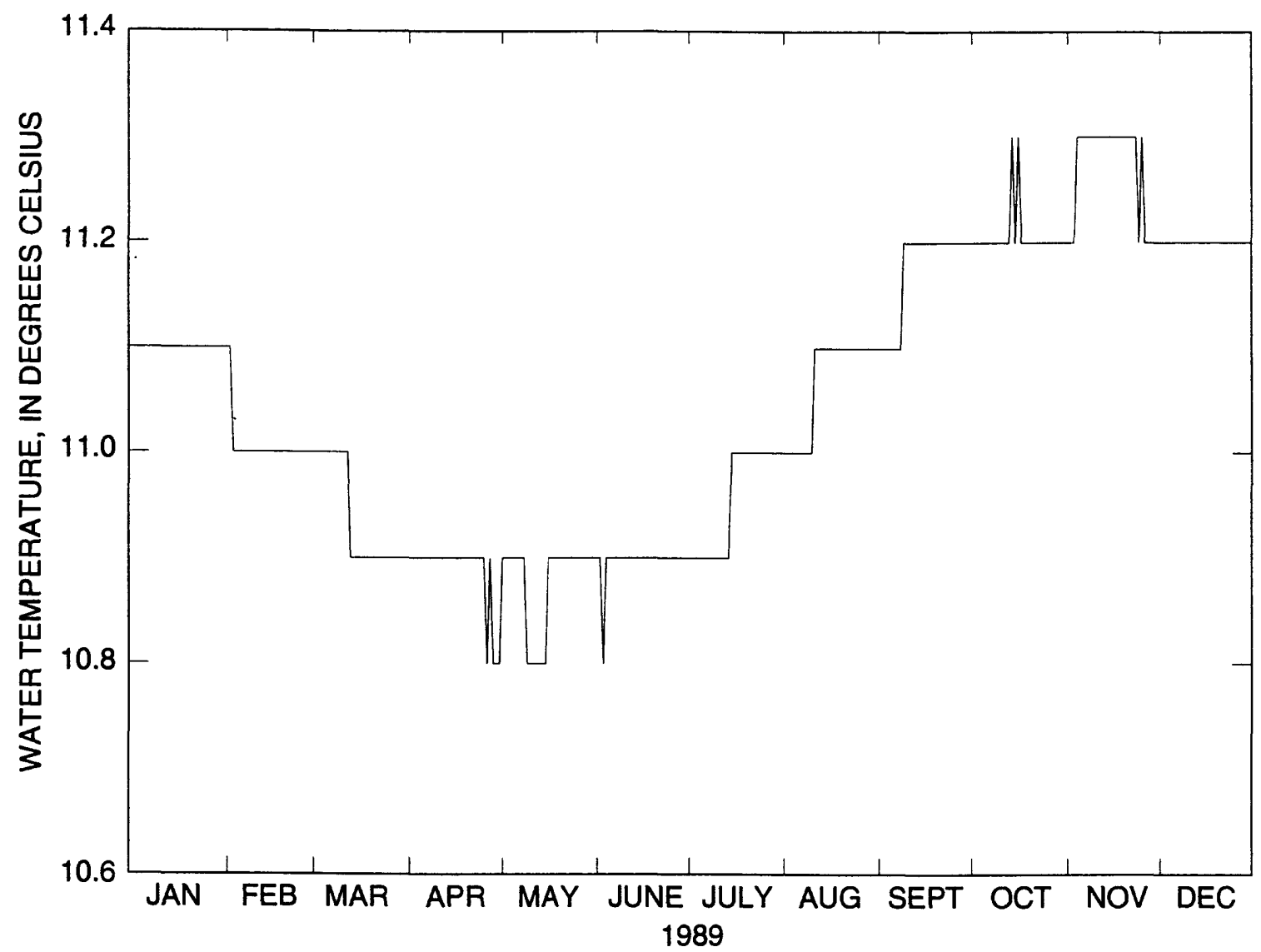

Figure 33. (B) Daily mean water temperature, Fish Hatchery Spring 23 (FH23-T), Long Valley Caldera, Mono County, California, 1989. 
Table 65. Daily mean water temperature, Fish Hatchery Spring 23 (FH23-T), Long Valley Caldera, Mono County, California, 1989

[Values are in degrees Celsius. Max, maximum; Min, minimum; -, no data]

\begin{tabular}{|c|c|c|c|c|c|c|c|c|c|c|c|c|}
\hline Day & Jan & Feb & Mar & Apr & May & June & July & Aug & Sept & Oct & Nov & Dec \\
\hline 1 & 11.1 & 11.1 & 11.0 & 10.9 & 10.9 & 10.9 & 10.9 & 11.0 & 11.1 & 11.2 & 11.2 & 11.2 \\
\hline 2 & 11.1 & 11.1 & 11.0 & 10.9 & 10.9 & 10.9 & 10.9 & 11.0 & 11.1 & 11.2 & 11.2 & 11.2 \\
\hline 3 & 11.1 & 11.0 & 11.0 & 10.9 & 10.9 & 10.8 & 10.9 & 11.0 & 11.1 & 11.2 & 11.2 & 11.2 \\
\hline 4 & 11.1 & 11.0 & 11.0 & 10.9 & 10.9 & 10.9 & 10.9 & 11.0 & 11.1 & 11.2 & 11.3 & 11.2 \\
\hline 5 & 11.1 & 11.0 & 11.0 & 10.9 & 10.9 & 10.9 & 10.9 & 11.0 & 11.1 & 11.2 & 11.3 & 11.2 \\
\hline 6 & 11.1 & 11.0 & 11.0 & 10.9 & 10.9 & 10.9 & 10.9 & 11.0 & 11.1 & 11.2 & 11.3 & 11.2 \\
\hline 7 & 11.1 & 11.0 & 11.0 & 10.9 & 10.9 & 10.9 & 10.9 & 11.0 & 11.1 & 11.2 & 11.3 & 11.2 \\
\hline 8 & 11.1 & 11.0 & 11.0 & 10.9 & 10.9 & 10.9 & 10.9 & 11.0 & 11.1 & 11.2 & 11.3 & 11.2 \\
\hline 9 & 11.1 & 11.0 & 11.0 & 10.9 & 10.8 & 10.9 & 10.9 & 11.0 & 11.2 & 11.2 & 11.3 & 11.2 \\
\hline 10 & 11.1 & 11.0 & 11.0 & 10.9 & 10.8 & 10.9 & 10.9 & 11.0 & 11.2 & 11.2 & 11.3 & 11.2 \\
\hline 11 & 11.1 & 11.0 & 11.0 & 10.9 & 10.8 & 10.9 & 10.9 & 11.1 & 11.2 & 11.2 & 11.3 & 11.2 \\
\hline 12 & 11.1 & 11.0 & 11.0 & 10.9 & 10.8 & 10.9 & 10.9 & 11.1 & 11.2 & 11.2 & 11.3 & 11.2 \\
\hline 13 & 11.1 & 11.0 & 10.9 & 10.9 & 10.8 & 10.9 & 10.9 & 11.1 & 11.2 & 11.2 & 11.3 & 11.2 \\
\hline 14 & 11.1 & 11.0 & 10.9 & 10.9 & 10.8 & 10.9 & 10.9 & 11.1 & 11.2 & 11.3 & 11.3 & 11.2 \\
\hline 15 & 11.1 & 11.0 & 10.9 & 10.9 & 10.8 & 10.9 & 11.0 & 11.1 & 11.2 & 11.2 & 11.3 & 11.2 \\
\hline 16 & 11.1 & 11.0 & 10.9 & 10.9 & 10.9 & 10.9 & 11.0 & 11.1 & 11.2 & 11.3 & 11.3 & 11.2 \\
\hline 17 & 11.1 & 11.0 & 10.9 & 10.9 & 10.9 & 10.9 & 11.0 & 11.1 & 11.2 & 11.2 & 11.3 & 11.2 \\
\hline 18 & 11.1 & 11.0 & 10.9 & 10.9 & 10.9 & 10.9 & 11.0 & 11.1 & 11.2 & 11.2 & 11.3 & 11.2 \\
\hline 19 & 11.1 & 11.0 & 10.9 & 10.9 & 10.9 & 10.9 & 11.0 & 11.1 & 11.2 & 11.2 & 11.3 & 11.2 \\
\hline 20 & 11.1 & 11.0 & 10.9 & 10.9 & 10.9 & 10.9 & 11.0 & 11.1 & 11.2 & 11.2 & 11.3 & 11.2 \\
\hline 21 & 11.1 & 11.0 & 10.9 & 10.9 & 10.9 & 10.9 & 11.0 & 11.1 & 11.2 & 11.2 & 11.3 & 11.2 \\
\hline 22 & 11.1 & 11.0 & 10.9 & 10.9 & 10.9 & 10.9 & 11.0 & 11.1 & 11.2 & 11.2 & 11.3 & 11.2 \\
\hline 23 & 11.1 & 11.0 & 10.9 & 10.9 & 10.9 & 10.9 & 11.0 & 11.1 & 11.2 & 11.2 & 11.3 & 11.2 \\
\hline 24 & 11.1 & 11.0 & 10.9 & 10.9 & 10.9 & 10.9 & 11.0 & 11.1 & 11.2 & 11.2 & 11.2 & 11.2 \\
\hline 25 & 11.1 & 11.0 & 10.9 & 10.9 & 10.9 & 10.9 & 11.0 & 11.1 & 11.2 & 11.2 & 11.3 & 11.2 \\
\hline 26 & 11.1 & 11.0 & 10.9 & 10.8 & 10.9 & 10.9 & 11.0 & 11.1 & 11.2 & 11.2 & 11.2 & 11.2 \\
\hline 27 & 11.1 & 11.0 & 10.9 & 10.9 & 10.9 & 10.9 & 11.0 & 11.1 & 11.2 & 11.2 & 11.2 & 11.2 \\
\hline 28 & 11.1 & 11.0 & 10.9 & 10.8 & 10.9 & 10.9 & 11.0 & 11.1 & 11.2 & 11.2 & 11.2 & 11.2 \\
\hline 29 & 11.1 & - & 10.9 & 10.8 & 10.9 & 10.9 & 11.0 & 11.1 & 11.2 & 11.2 & 11.2 & 11.2 \\
\hline 30 & 11.1 & - & 10.9 & 10.8 & 10.9 & 10.9 & 11.0 & 11.1 & 11.2 & 11.2 & 11.2 & 11.2 \\
\hline 31 & 11.1 & - & 10.9 & - & 10.9 & - & 11.0 & 11.1 & - & 11.2 & - & 11.2 \\
\hline Mean & 11.1 & 11.0 & 10.9 & 10.9 & 10.9 & 10.9 & 11.0 & 11.1 & 11.2 & 11.2 & 11.3 & 11.2 \\
\hline $\operatorname{Max}$ & 11.1 & 11.1 & 11.0 & 10.9 & 10.9 & 10.9 & 11.0 & 11.1 & 11.2 & 11.3 & 11.3 & 11.2 \\
\hline Min & 11.1 & 11.0 & 10.9 & 10.8 & 10.8 & 10.8 & 10.9 & 11.0 & 11.1 & 11.2 & 11.2 & 11.2 \\
\hline
\end{tabular}




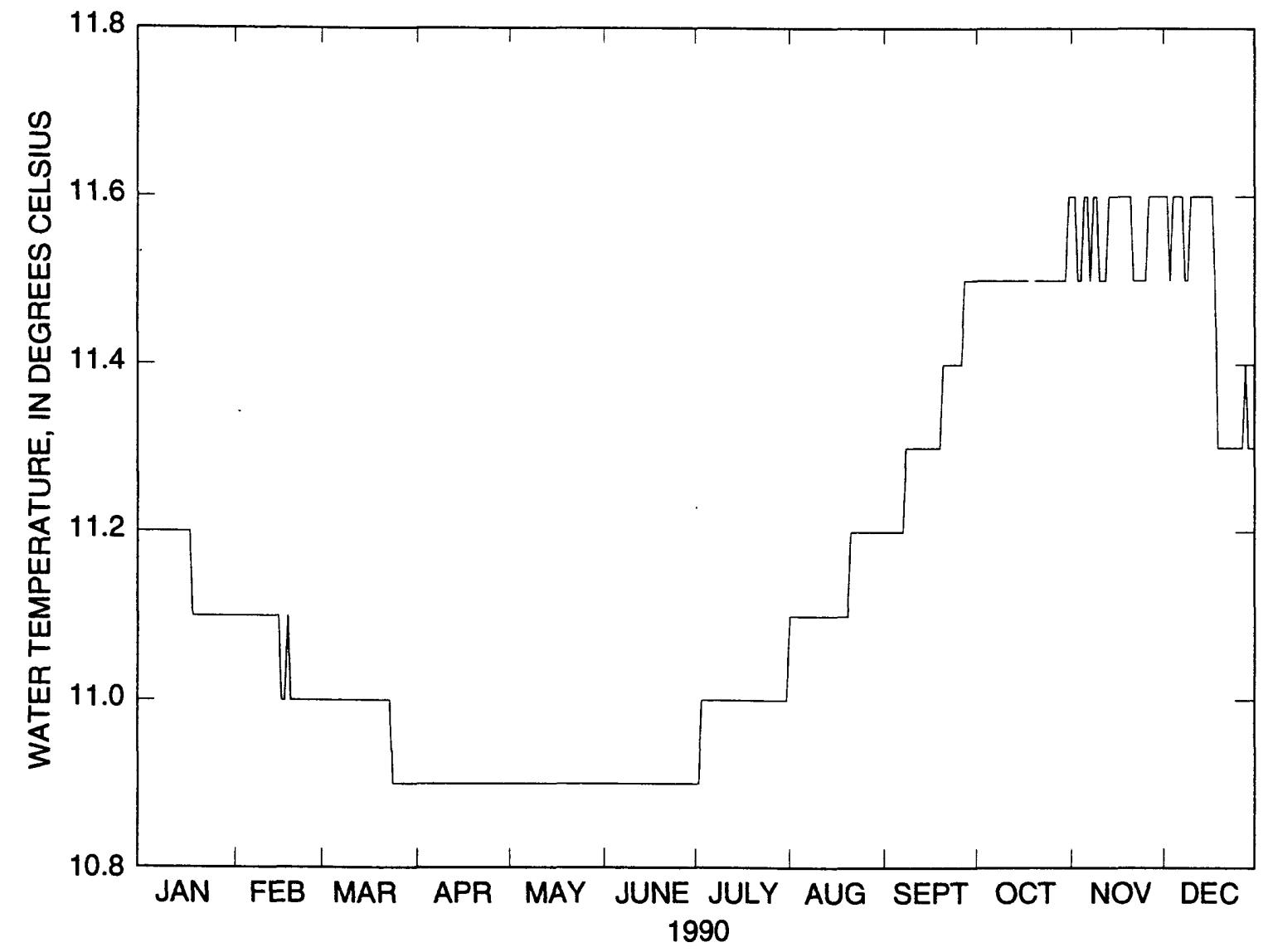

Figure 33. (C) Daily mean water temperature, Fish Hatchery Spring 23 (FH23-T), Long Valley Caldera, Mono County, California, 1990. 
Table 66. Daily mean water temperature, Fish Hatchery Spring 23 (FH23-T), Long Valley Caldera, Mono County, California, 1990

[Values are in degrees Celsius. Max, maximum; Min, minimum; -, no data]

\begin{tabular}{|c|c|c|c|c|c|c|c|c|c|c|c|c|}
\hline Day & Jan & Feb & Mar & Apr & May & June & July & Aug & Sept & Oct & Nov & Dec \\
\hline 1 & 11.2 & 11.1 & 11.0 & 10.9 & 10.9 & 10.9 & 10.9 & 11.1 & 11.2 & 11.5 & 11.6 & 11.6 \\
\hline 2 & 11.2 & 11.1 & 11.0 & 10.9 & 10.9 & 10.9 & 10.9 & 11.1 & 11.2 & 11.5 & 11.6 & 11.6 \\
\hline 3 & 11.2 & 11.1 & 11.0 & 10.9 & 10.9 & 10.9 & 11.0 & 11.1 & 11.2 & 11.5 & 11.5 & 11.5 \\
\hline 4 & 11.2 & 11.1 & 11.0 & 10.9 & 10.9 & 10.9 & 11.0 & 11.1 & 11.2 & 11.5 & 11.5 & 11.6 \\
\hline 5 & 11.2 & 11.1 & 11.0 & 10.9 & 10.9 & 10.9 & 11.0 & 11.1 & 11.2 & 11.5 & 11.6 & 11.6 \\
\hline 6 & 11.2 & 11.1 & 11.0 & 10.9 & 10.9 & 10.9 & 11.0 & 11.1 & 11.2 & 11.5 & 11.6 & 11.6 \\
\hline 7 & 11.2 & 11.1 & 11.0 & 10.9 & 10.9 & 10.9 & 11.0 & 11.1 & 11.2 & 11.5 & 11.5 & 11.6 \\
\hline 8 & 11.2 & 11.1 & 11.0 & 10.9 & 10.9 & 10.9 & 11.0 & 11.1 & 11.3 & 11.5 & 11.6 & 11.5 \\
\hline 9 & 11.2 & 11.1 & 11.0 & 10.9 & 10.9 & 10.9 & 11.0 & 11.1 & 11.3 & 11.5 & 11.6 & 11.5 \\
\hline 10 & 11.2 & 11.1 & 11.0 & 10.9 & 10.9 & 10.9 & 11.0 & 11.1 & 11.3 & 11.5 & 11.5 & 11.6 \\
\hline 11 & 11.2 & 11.1 & 11.0 & 10.9 & 10.9 & 10.9 & 11.0 & 11.1 & 11.3 & 11.5 & 11.5 & 11.6 \\
\hline 12 & 11.2 & 11.1 & 11.0 & 10.9 & 10.9 & 10.9 & 11.0 & 11.1 & 11.3 & 11.5 & 11.5 & 11.6 \\
\hline 13 & 11.2 & 11.1 & 11.0 & 10.9 & 10.9 & 10.9 & 11.0 & 11.1 & 11.3 & 11.5 & 11.6 & 11.6 \\
\hline 14 & 11.2 & 11.1 & 11.0 & 10.9 & 10.9 & 10.9 & 11.0 & 11.1 & 11.3 & 11.5 & 11.6 & 11.6 \\
\hline 15 & 11.2 & 11.1 & 11.0 & 10.9 & 10.9 & 10.9 & 11.0 & 11.1 & 11.3 & 11.5 & 11.6 & 11.6 \\
\hline 16 & 11.2 & 11.0 & 11.0 & 10.9 & 10.9 & 10.9 & 11.0 & 11.1 & 11.3 & 11.5 & 11.6 & 11.6 \\
\hline 17 & 11.2 & 11.0 & 11.0 & 10.9 & 10.9 & 10.9 & 11.0 & 11.1 & 11.3 & 11.5 & 11.6 & 11.6 \\
\hline 18 & 11.1 & 11.1 & 11.0 & 10.9 & 10.9 & 10.9 & 11.0 & 11.1 & 11.3 & 11.5 & 11.6 & 11.5 \\
\hline 19 & 11.1 & 11.0 & 11.0 & 10.9 & 10.9 & 10.9 & 11.0 & 11.1 & 11.3 & - & 11.6 & 11.3 \\
\hline 20 & 11.1 & 11.0 & 11.0 & 10.9 & 10.9 & 10.9 & 11.0 & 11.1 & 11.4 & 11.5 & 11.6 & 11.3 \\
\hline 21 & 11.1 & 11.0 & 11.0 & 10.9 & 10.9 & 10.9 & 11.0 & 11.2 & 11.4 & 11.5 & 11.5 & 11.3 \\
\hline 22 & 11.1 & 11.0 & 11.0 & 10.9 & 10.9 & 10.9 & 11.0 & 11.2 & 11.4 & 11.5 & 11.5 & 11.3 \\
\hline 23 & 11.1 & 11.0 & 11.0 & 10.9 & 10.9 & 10.9 & 11.0 & 11.2 & 11.4 & 11.5 & 11.5 & 11.3 \\
\hline 24 & 11.1 & 11.0 & 10.9 & 10.9 & 10.9 & 10.9 & 11.0 & 11.2 & 11.4 & 11.5 & 11.5 & 11.3 \\
\hline 25 & 11.1 & 11.0 & 10.9 & 10.9 & 10.9 & 10.9 & 11.0 & 11.2 & 11.4 & 11.5 & 11.5 & 11.3 \\
\hline 26 & 11.1 & 11.0 & 10.9 & 10.9 & 10.9 & 10.9 & 11.0 & 11.2 & 11.4 & 11.5 & 11.6 & 11.3 \\
\hline 27 & 11.1 & 11.0 & 10.9 & 10.9 & 10.9 & 10.9 & 11.0 & 11.2 & 11.5 & 11.5 & 11.6 & 11.3 \\
\hline 28 & 11.1 & 11.0 & 10.9 & 10.9 & 10.9 & 10.9 & 11.0 & 11.2 & 11.5 & 11.5 & 11.6 & 11.4 \\
\hline 29 & 11.1 & - & 10.9 & 10.9 & 10.9 & 10.9 & 11.0 & 11.2 & 11.5 & 11.5 & 11.6 & 11.3 \\
\hline 30 & 11.1 & - & 10.9 & 10.9 & 10.9 & 10.9 & 11.0 & 11.2 & 11.5 & 11.5 & 11.6 & 11.3 \\
\hline 31 & 11.1 & - & 10.9 & - & 10.9 & - & 11.0 & 11.2 & - & 11.6 & - & 11.3 \\
\hline Mean & 11.2 & 11.1 & 11.0 & 10.9 & 10.9 & 10.9 & 11.0 & 11.1 & 11.3 & 11.5 & 11.6 & 11.5 \\
\hline $\operatorname{Max}$ & 11.2 & 11.1 & 11.0 & 10.9 & 10.9 & 10.9 & 11.0 & 11.2 & 11.5 & 11.6 & 11.6 & 11.6 \\
\hline Min & 11.1 & 11.0 & 10.9 & 10.9 & 10.9 & 10.9 & 10.9 & 11.1 & 11.2 & 11.5 & 11.5 & 11.3 \\
\hline
\end{tabular}




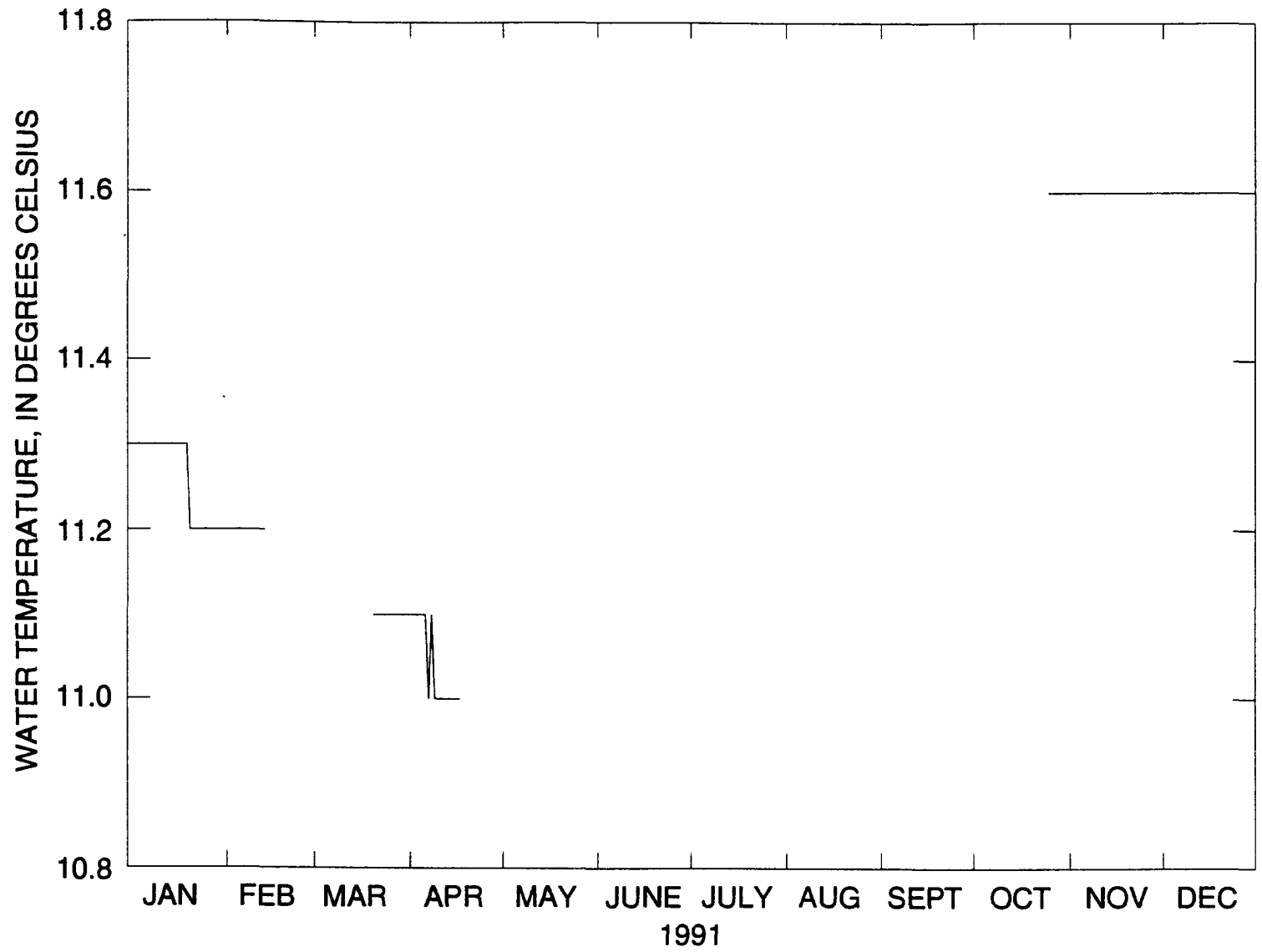

Figure 33. (D) Daily mean water temperature, Fish Hatchery Spring 23 (FH23-T), Long Valley Caldera, Mono County, California, 1991. 
Table 67. Daily mean water temperature, Fish Hatchery Spring 23 (FH23-T), Long Valley Caldera, Mono County, California, 1991

[Values are in degrees Celsius. Max, maximum; Min, minimum; -, no data]

\begin{tabular}{|c|c|c|c|c|c|c|c|c|c|c|c|c|}
\hline Day & Jan & Feb & Mar & Apr & May & June & July & Aug & Sept & Oct & Nov & Dec \\
\hline 1 & 11.3 & 11.2 & - & 11.1 & - & - & - & - & - & - & 11.6 & 11.6 \\
\hline 2 & 11.3 & 11.2 & - & 11.1 & - & - & - & - & - & - & 11.6 & 11.6 \\
\hline 3 & 11.3 & 11.2 & - & 11.1 & - & - & - & - & - & - & 11.6 & 11.6 \\
\hline 4 & 11.3 & 11.2 & - & 11.1 & - & - & - & - & - & - & 11.6 & 11.6 \\
\hline 5 & 11.3 & 11.2 & - & 11.1 & - & - & - & - & - & - & 11.6 & 11.6 \\
\hline 6 & 11.3 & 11.2 & - & 11.1 & - & - & - & - & - & - & 11.6 & 11.6 \\
\hline 7 & 11.3 & 11.2 & - & 11.0 & - & - & - & - & - & - & 11.6 & 11.6 \\
\hline 8 & 11.3 & 11.2 & - & 11.1 & - & - & - & - & - & - & 11.6 & 11.6 \\
\hline 9 & 11.3 & 11.2 & - & 11.0 & - & - & - & - & - & - & 11.6 & 11.6 \\
\hline 10 & 11.3 & 11.2 & - & 11.0 & - & - & - & - & - & - & 11.6 & 11.6 \\
\hline 11 & 11.3 & 11.2 & - & 11.0 & - & - & - & - & - & - & 11.6 & 11.6 \\
\hline 12 & 11.3 & 11.2 & - & 11.0 & - & - & - & - & - & - & 11.6 & 11.6 \\
\hline 13 & 11.3 & 11.2 & - & 11.0 & - & - & - & - & - & - & 11.6 & 11.6 \\
\hline 14 & 11.3 & - & - & 11.0 & - & - & - & - & - & - & 11.6 & 11.6 \\
\hline 15 & 11.3 & - & - & 11.0 & - & - & - & - & - & - & 11.6 & 11.6 \\
\hline 16 & 11.3 & - & - & 11.0 & - & - & - & - & - & - & 11.6 & 11.6 \\
\hline 17 & 11.3 & - & - & 11.0 & - & - & - & - & - & - & 11.6 & 11.6 \\
\hline 18 & 11.3 & - & - & - & - & - & - & - & - & - & 11.6 & 11.6 \\
\hline 19 & 11.3 & - & - & - & - & - & - & - & - & - & 11.6 & 11.6 \\
\hline 20 & 11.2 & - & 11.1 & - & - & - & - & - & - & - & 11.6 & 11.6 \\
\hline 21 & 11.2 & - & 11.1 & - & - & - & - & - & - & - & 11.6 & 11.6 \\
\hline 22 & 11.2 & - & 11.1 & - & - & - & - & - & - & - & 11.6 & 11.6 \\
\hline 23 & 11.2 & - & 11.1 & - & - & - & - & - & - & - & 11.6 & 11.6 \\
\hline 24 & 11.2 & - & 11.1 & - & - & - & - & - & - & - & 11.6 & 11.6 \\
\hline 25 & 11.2 & - & 11.1 & - & - & - & - & - & - & 11.6 & 11.6 & 11.6 \\
\hline 26 & 11.2 & - & 11.1 & - & - & - & - & - & - & 11.6 & 11.6 & 11.6 \\
\hline 27 & 11.2 & - & 11.1 & - & - & - & - & - & - & 11.6 & 11.6 & 11.6 \\
\hline 28 & 11.2 & - & 11.1 & - & - & - & - & - & - & 11.6 & 11.6 & 11.6 \\
\hline 29 & 11.2 & - & 11.1 & - & - & - & - & - & - & 11.6 & 11.6 & 11.6 \\
\hline 30 & 11.2 & - & 11.1 & - & - & - & - & - & - & 11.6 & 11.6 & 11.6 \\
\hline 31 & 11.2 & - & 11.1 & - & - & - & - & - & - & 11.6 & - & 11.6 \\
\hline Mean & 11.3 & 11.2 & 11.1 & 11.0 & - & - & - & - & - & 11.6 & 11.6 & 11.6 \\
\hline Max & 11.3 & 11.2 & 11.1 & 11.1 & - & - & - & - & - & 11.6 & 11.6 & 11.6 \\
\hline Min & 11.2 & 11.2 & 11.1 & 11.0 & - & - & - & - & - & 11.6 & 11.6 & 11.6 \\
\hline
\end{tabular}




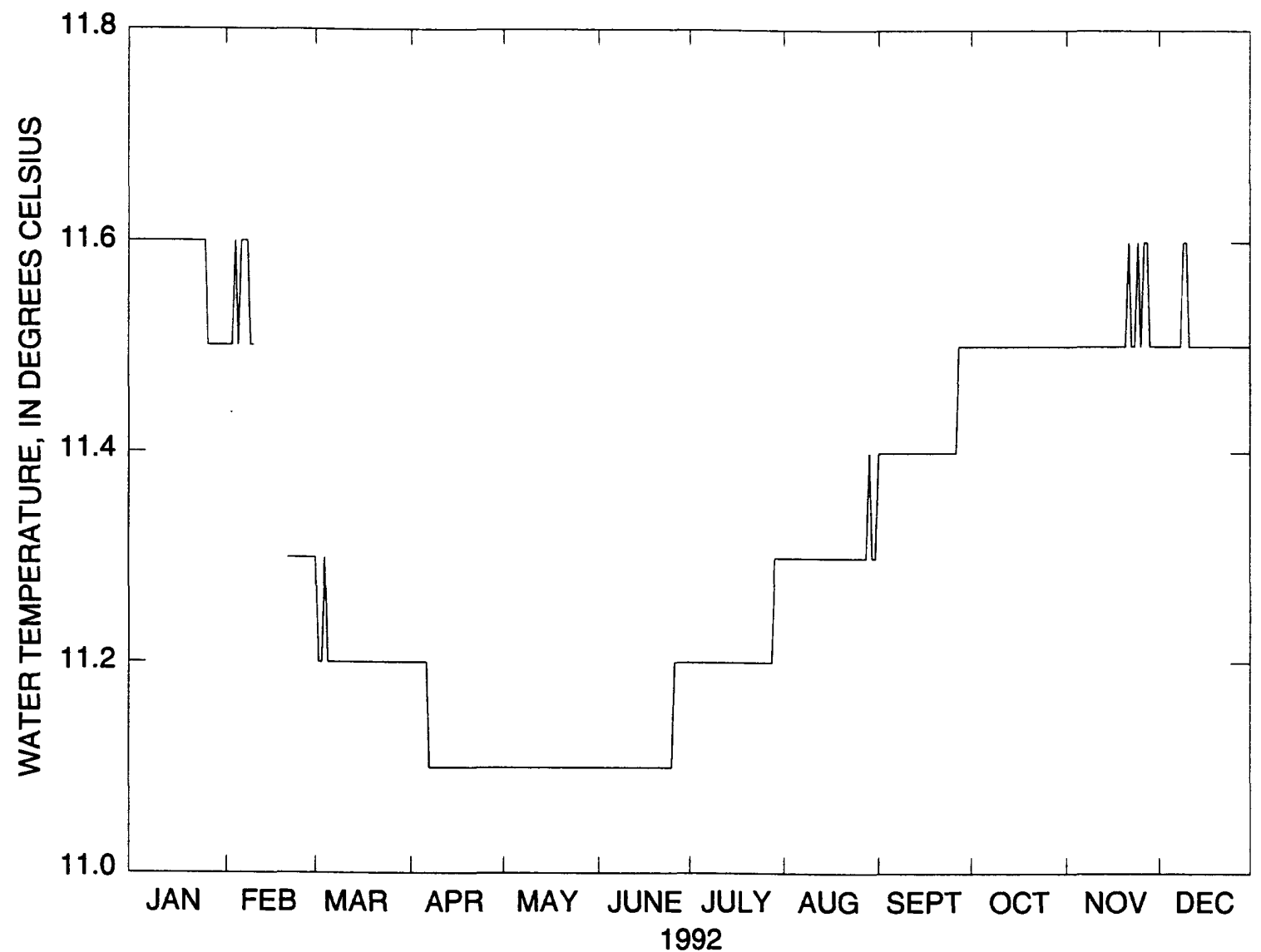

Figure 33. (E) Daily mean water temperature, Fish Hatchery Spring 23 (FH23-T), Long Valley Caldera, Mono County, California, 1992. 
Table 68. Daily mean water temperature, Fish Hatchery Spring 23 (FH23-T), Long Valley Caldera, Mono County, California, 1992

[Values are in degrees Celsius. Max, maximum; Min, minimum; - , no data]

\begin{tabular}{|c|c|c|c|c|c|c|c|c|c|c|c|c|}
\hline Day & Jan & Feb & Mar & Apr & May & June & July & Aug & Sept & Oct & Nov & Dec \\
\hline 1 & 11.6 & 11.5 & 11.3 & 11.2 & 11.1 & 11.1 & 11.2 & 11.3 & 11.4 & 11.5 & 11.5 & 11.5 \\
\hline 2 & 11.6 & 11.5 & 11.2 & 11.2 & 11.1 & 11.1 & 11.2 & 11.3 & 11.4 & 11.5 & 11.5 & 11.5 \\
\hline 3 & 11.6 & 11.5 & 11.2 & 11.2 & 11.1 & 11.1 & 11.2 & 11.3 & 11.4 & 11.5 & 11.5 & 11.5 \\
\hline 4 & 11.6 & 11.6 & 11.3 & 11.2 & 11.1 & 11.1 & 11.2 & 11.3 & 11.4 & 11.5 & 11.5 & 11.5 \\
\hline 5 & 11.6 & 11.5 & 11.2 & 11.2 & 11.1 & 11.1 & 11.2 & 11.3 & 11.4 & 11.5 & 11.5 & 11.5 \\
\hline 6 & 11.6 & 11.6 & 11.2 & 11.2 & 11.1 & 11.1 & 11.2 & 11.3 & 11.4 & 11.5 & 11.5 & 11.5 \\
\hline 7 & 11.6 & 11.6 & 11.2 & 11.1 & 11.1 & 11.1 & 11.2 & 11.3 & 11.4 & 11.5 & 11.5 & 11.5 \\
\hline 8 & 11.6 & 11.6 & 11.2 & 11.1 & 11.1 & 11.1 & 11.2 & 11.3 & 11.4 & 11.5 & 11.5 & 11.5 \\
\hline 9 & 11.6 & 11.5 & 11.2 & 11.1 & 11.1 & 11.1 & 11.2 & 11.3 & 11.4 & 11.5 & 11.5 & 11.6 \\
\hline 10 & 11.6 & 11.5 & 11.2 & 11.1 & 11.1 & 11.1 & 11.2 & 11.3 & 11.4 & 11.5 & 11.5 & 11.6 \\
\hline 11 & 11.6 & - & 11.2 & 11.1 & 11.1 & 11.1 & 11.2 & 11.3 & 11.4 & 11.5 & 11.5 & 11.5 \\
\hline 12 & 11.6 & - & 11.2 & 11.1 & 11.1 & 11.1 & 11.2 & 11.3 & 11.4 & 11.5 & 11.5 & 11.5 \\
\hline 13 & 11.6 & - & 11.2 & 11.1 & 11.1 & 11.1 & 11.2 & 11.3 & 11.4 & 11.5 & 11.5 & 11.5 \\
\hline 14 & 11.6 & - & 11.2 & 11.1 & 11.1 & 11.1 & 11.2 & 11.3 & 11.4 & 11.5 & 11.5 & 11.5 \\
\hline 15 & 11.6 & 一 & 11.2 & 11.1 & 11.1 & 11.1 & 11.2 & 11.3 & 11.4 & 11.5 & 11.5 & 11.5 \\
\hline 16 & 11.6 & - & 11.2 & 11.1 & 11.1 & 11.1 & 11.2 & 11.3 & 11.4 & 11.5 & 11.5 & 11.5 \\
\hline 17 & 11.6 & - & 11.2 & 11.1 & 11.1 & 11.1 & 11.2 & 11.3 & 11.4 & 11.5 & 11.5 & 11.5 \\
\hline 18 & 11.6 & - & 11.2 & 11.1 & 11.1 & 11.1 & 11.2 & 11.3 & 11.4 & 11.5 & 11.5 & 11.5 \\
\hline 19 & 11.6 & - & 11.2 & 11.1 & 11.1 & 11.1 & 11.2 & 11.3 & 11.4 & 11.5 & 11.5 & 11.5 \\
\hline 20 & 11.6 & - & 11.2 & 11.1 & 11.1 & 11.1 & 11.2 & 11.3 & 11.4 & 11.5 & 11.5 & 11.5 \\
\hline 21 & 11.6 & 11.3 & 11.2 & 11.1 & 11.1 & 11.1 & 11.2 & 11.3 & 11.4 & 11.5 & 11.6 & 11.5 \\
\hline 22 & 11.6 & 11.3 & 11.2 & 11.1 & 11.1 & 11.1 & 11.2 & 11.3 & 11.4 & 11.5 & 11.5 & 11.5 \\
\hline 23 & 11.6 & 11.3 & 11.2 & 11.1 & 11.1 & 11.1 & 11.2 & 11.3 & 11.4 & 11.5 & 11.5 & 11.5 \\
\hline 24 & 11.6 & 11.3 & 11.2 & 11.1 & 11.1 & 11.1 & 11.2 & 11.3 & 11.4 & 11.5 & 11.6 & 11.5 \\
\hline 25 & 11.6 & 11.3 & 11.2 & 11.1 & 11.1 & 11.1 & 11.2 & 11.3 & 11.4 & 11.5 & 11.5 & 11.5 \\
\hline 26 & 11.5 & 11.3 & 11.2 & 11.1 & 11.1 & 11.2 & 11.2 & 11.3 & 11.4 & 11.5 & 11.6 & 11.5 \\
\hline 27 & 11.5 & 11.3 & 11.2 & 11.1 & 11.1 & 11.2 & 11.2 & 11.3 & 11.5 & 11.5 & 11.6 & 11.5 \\
\hline 28 & 11.5 & 11.3 & 11.2 & 11.1 & 11.1 & 11.2 & 11.2 & 11.3 & 11.5 & 11.5 & 11.5 & 11.5 \\
\hline 29 & 11.5 & 11.3 & 11.2 & 11.1 & 11.1 & 11.2 & 11.3 & 11.4 & 11.5 & 11.5 & 11.5 & 11.5 \\
\hline 30 & 11.5 & - & 11.2 & 11.1 & 11.1 & 11.2 & 11.3 & 11.3 & 11.5 & 11.5 & 11.5 & 11.5 \\
\hline 31 & 11.5 & - & 11.2 & - & 11.1 & - & 11.3 & 11.3 & - & 11.5 & - & 11.5 \\
\hline Mean & 11.6 & 11.4 & 11.2 & 11.1 & 11.1 & 11.1 & 11.2 & 11.3 & 11.4 & 11.5 & 11.5 & 11.5 \\
\hline Max & 11.6 & 11.6 & 11.3 & 11.2 & 11.1 & 11.2 & 11.3 & 11.4 & 11.5 & 11.5 & 11.6 & 11.6 \\
\hline Min & 11.5 & 11.3 & 11.2 & 11.1 & 11.1 & 11.1 & 11.2 & 11.3 & 11.4 & 11.5 & 11.5 & 11.5 \\
\hline
\end{tabular}




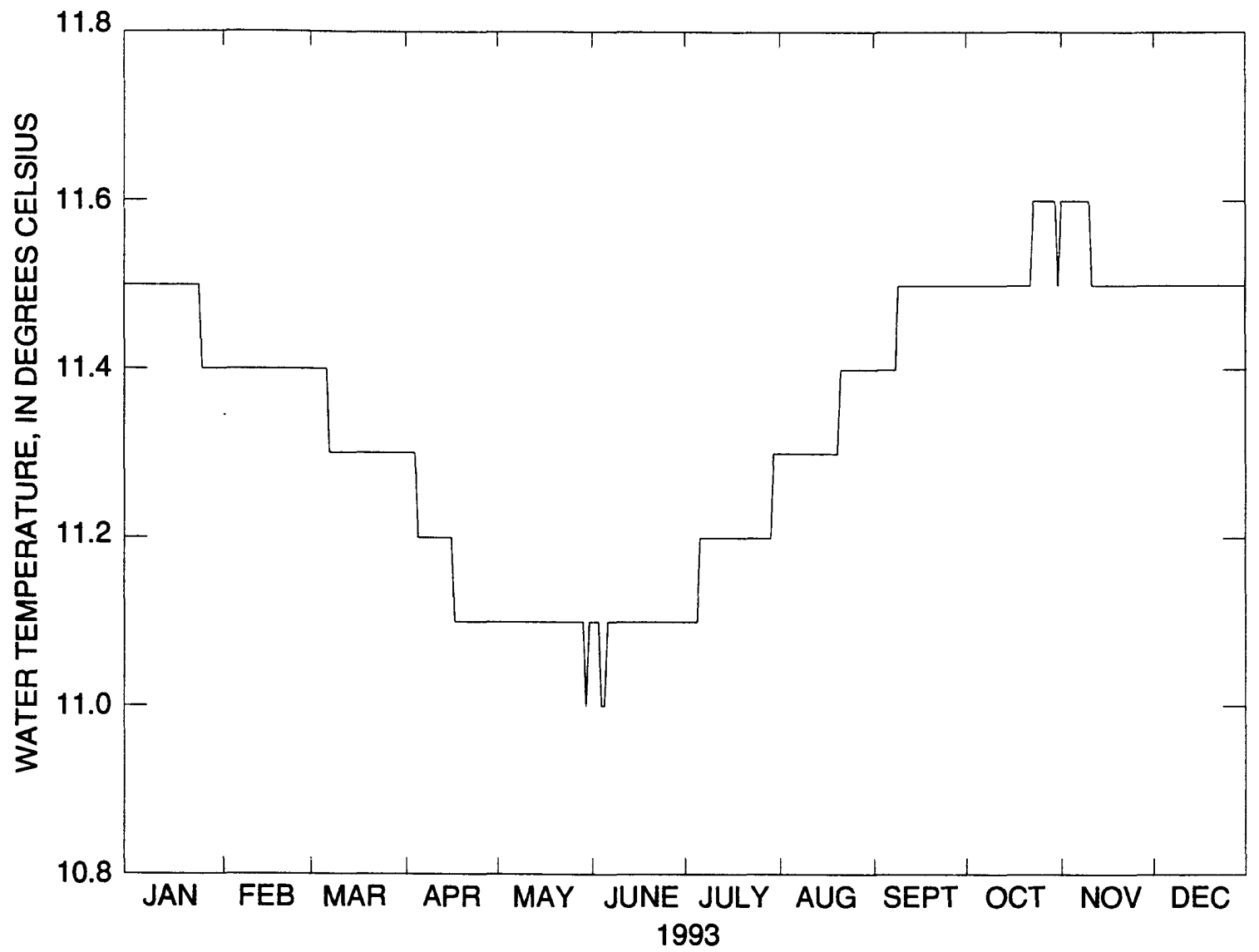

Figure 33. (F) Daily mean water temperature, Fish Hatchery Spring 23 (FH23-T), Long Valley Caldera, Mono County, California, 1993.

180

Hydrologic Data for Long Valley Caldera, Mono County, California, 1987-93 
Table 69. Daily mean water temperature, Fish Hatchery Spring 23 (FH23-T), Long Valley Caldera, Mono County, California, 1993

[Values are in degrees Celsius. Max, maximum: Min, minimum; -, no data]

\begin{tabular}{|c|c|c|c|c|c|c|c|c|c|c|c|c|}
\hline Day & Jan & Feb & Mar & Apr & May & June & July & Aug & Sept & Oct & Nov & Dec \\
\hline 1 & 11.5 & 11.4 & 11.4 & 11.3 & 11.1 & 11.1 & 11.1 & 11.3 & 11.4 & 11.5 & 11.6 & 11.5 \\
\hline 2 & 11.5 & 11.4 & 11.4 & 11.3 & 11.1 & 11.1 & 11.1 & 11.3 & 11.4 & 11.5 & 11.6 & 11.5 \\
\hline 3 & 11.5 & 11.4 & 11.4 & 11.3 & 11.1 & 11.1 & 11.1 & 11.3 & 11.4 & 11.5 & 11.6 & 11.5 \\
\hline 4 & 11.5 & 11.4 & 11.4 & 11.3 & 11.1 & 11.0 & 11.1 & 11.3 & 11.4 & 11.5 & 11.6 & 11.5 \\
\hline 5 & 11.5 & 11.4 & 11.4 & 11.2 & 11.1 & 11.0 & 11.1 & 11.3 & 11.4 & 11.5 & 11.6 & 11.5 \\
\hline 6 & 11.5 & 11.4 & 11.4 & 11.2 & 11.1 & 11.1 & 11.2 & 11.3 & 11.4 & 11.5 & 11.6 & 11.5 \\
\hline 7 & 11.5 & 11.4 & 11.3 & 11.2 & 11.1 & 11.1 & 11.2 & 11.3 & 11.4 & 11.5 & 11.6 & 11.5 \\
\hline 8 & 11.5 & 11.4 & 11.3 & 11.2 & 11.1 & 11.1 & 11.2 & 11.3 & 11.4 & 11.5 & 11.6 & 11.5 \\
\hline 9 & 11.5 & 11.4 & 11.3 & 11.2 & 11.1 & 11.1 & 11.2 & 11.3 & 11.5 & 11.5 & 11.6 & 11.5 \\
\hline 10 & 11.5 & 11.4 & 11.3 & 11.2 & 11.1 & 11.1 & 11.2 & 11.3 & 11.5 & 11.5 & 11.6 & 11.5 \\
\hline 11 & 11.5 & 11.4 & 11.3 & 11.2 & 11.1 & 11.1 & 11.2 & 11.3 & 11.5 & 11.5 & 11.5 & 11.5 \\
\hline 12 & 11.5 & 11.4 & 11.3 & 11.2 & 11.1 & 11.1 & 11.2 & 11.3 & 11.5 & 11.5 & 11.5 & 11.5 \\
\hline 13 & 11.5 & 11.4 & 11.3 & 11.2 & 11.1 & 11.1 & 11.2 & 11.3 & 11.5 & 11.5 & 11.5 & 11.5 \\
\hline 14 & 11.5 & 11.4 & 11.3 & 11.2 & 11.1 & 11.1 & 11.2 & 11.3 & 11.5 & 11.5 & 11.5 & 11.5 \\
\hline 15 & 11.5 & 11.4 & 11.3 & 11.2 & 11.1 & 11.1 & 11.2 & 11.3 & 11.5 & 11.5 & 11.5 & 11.5 \\
\hline 16 & 11.5 & 11.4 & 11.3 & 11.2 & 11.1 & 11.1 & 11.2 & 11.3 & 11.5 & 11.5 & 11.5 & 11.5 \\
\hline 17 & 11.5 & 11.4 & 11.3 & 11.1 & 11.1 & 11.1 & 11.2 & 11.3 & 11.5 & 11.5 & 11.5 & 11.5 \\
\hline 18 & 11.5 & 11.4 & 11.3 & 11.1 & 11.1 & 11.1 & 11.2 & 11.3 & 11.5 & 11.5 & 11.5 & 11.5 \\
\hline 19 & 11.5 & 11.4 & 11.3 & 11.1 & 11.1 & 11.1 & 11.2 & 11.3 & 11.5 & 11.5 & 11.5 & 11.5 \\
\hline 20 & 11.5 & 11.4 & 11.3 & 11.1 & 11.1 & 11.1 & 11.2 & 11.3 & 11.5 & 11.5 & 11.5 & 11.5 \\
\hline 21 & 11.5 & 11.4 & 11.3 & 11.1 & 11.1 & 11.1 & 11.2 & 11.4 & 11.5 & 11.5 & 11.5 & 11.5 \\
\hline 22 & 11.5 & 11.4 & 11.3 & 11.1 & 11.1 & 11.1 & 11.2 & 11.4 & 11.5 & 11.5 & 11.5 & 11.5 \\
\hline 23 & 11.5 & 11.4 & 11.3 & 11.1 & 11.1 & 11.1 & 11.2 & 11.4 & 11.5 & 11.6 & 11.5 & 11.5 \\
\hline 24 & 11.5 & 11.4 & 11.3 & 11.1 & 11.1 & 11.1 & 11.2 & 11.4 & 11.5 & 11.6 & 11.5 & 11.5 \\
\hline 25 & 11.4 & 11.4 & 11.3 & 11.1 & 11.1 & 11.1 & 11.2 & 11.4 & 11.5 & 11.6 & 11.5 & 11.5 \\
\hline 26 & 11.4 & 11.4 & 11.3 & 11.1 & 11.1 & 11.1 & 11.2 & 11.4 & 11.5 & 11.6 & 11.5 & 11.5 \\
\hline 27 & 11.4 & 11.4 & 11.3 & 11.1 & 11.1 & 11.1 & 11.2 & 11.4 & 11.5 & 11.6 & 11.5 & 11.5 \\
\hline 28 & 11.4 & 11.4 & 11.3 & 11.1 & 11.1 & 11.1 & 11.2 & 11.4 & 11.5 & 11.6 & 11.5 & 11.5 \\
\hline 29 & 11.4 & - & 11.3 & 11.1 & 11.1 & 11.1 & 11.2 & 11.4 & 11.5 & 11.6 & 11.5 & 11.5 \\
\hline 30 & 11.4 & - & 11.3 & 11.1 & 11.0 & 11.1 & 11.3 & 11.4 & 11.5 & 11.6 & 11.5 & 11.5 \\
\hline 31 & 11.4 & - & 11.3 & - & 11.1 & - & 11.3 & 11.4 & - & 11.5 & - & 11.5 \\
\hline Mean & 11.5 & 11.4 & 11.3 & 11.2 & 11.1 & 11.1 & 11.2 & 11.3 & 11.5 & 11.5 & 11.5 & 11.5 \\
\hline $\operatorname{Max}$ & 11.5 & 11.4 & 11.4 & 11.3 & 11.1 & 11.1 & 11.3 & 11.4 & 11.5 & 11.6 & 11.6 & 11.5 \\
\hline Min & 11.4 & 11.4 & 11.3 & 11.1 & 11.0 & 11.0 & 11.1 & 11.3 & 11.4 & 11.5 & 11.5 & 11.5 \\
\hline
\end{tabular}




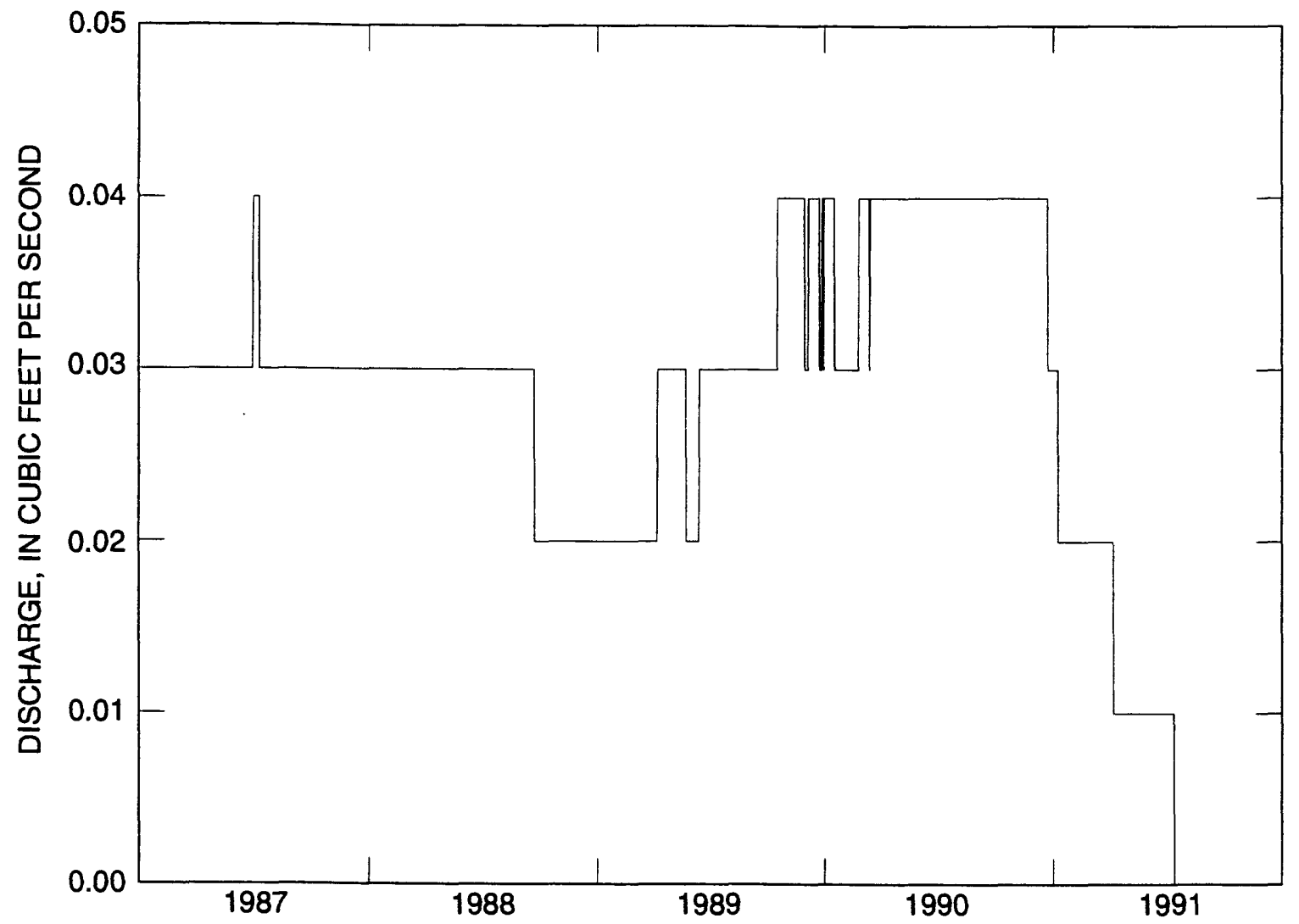

Figure 34. Daily mean discharge, Colton Spring (CS), Long Valley Caldera, Mono County, California, 1987-91. 
Table 70. Daily mean discharge, Colton Spring (CS), Long Valley Caldera, Mono County, California, 1987

[Values are in cubic feet per second. Max, maximum; Min, minimum; -, no data]

\begin{tabular}{|c|c|c|c|c|c|c|c|c|c|c|c|c|}
\hline Day & Jan & Feb & Mar & Apr & May & June & July & Aug & Sept & Oct & Nov & Dec \\
\hline 1 & 0.03 & 0.03 & 0.03 & 0.03 & 0.03 & 0.03 & 0.03 & 0.03 & 0.03 & 0.03 & 0.03 & 0.03 \\
\hline 2 & .03 & .03 & .03 & .03 & .03 & .03 & .04 & .03 & .03 & .03 & .03 & .03 \\
\hline 3 & .03 & .03 & .03 & .03 & .03 & .03 & .04 & .03 & .03 & .03 & .03 & .03 \\
\hline 4 & .03 & .03 & .03 & .03 & .03 & .03 & .04 & .03 & .03 & .03 & .03 & .03 \\
\hline 5 & .03 & .03 & .03 & .03 & .03 & .03 & .04 & .03 & .03 & .03 & .03 & .03 \\
\hline 6 & .03 & .03 & .03 & .03 & .03 & .03 & .04 & .03 & .03 & .03 & .03 & .03 \\
\hline 7 & .03 & .03 & .03 & .03 & .03 & .03 & .04 & .03 & .03 & .03 & .03 & .03 \\
\hline 8 & .03 & .03 & .03 & .03 & .03 & .03 & .04 & .03 & .03 & .03 & .03 & .03 \\
\hline 9 & .03 & .03 & .03 & .03 & .03 & .03 & .04 & .03 & .03 & .03 & .03 & .03 \\
\hline 10 & .03 & .03 & .03 & .03 & .03 & .03 & .04 & .03 & .03 & .03 & .03 & .03 \\
\hline 11 & .03 & .03 & .03 & .03 & .03 & .03 & .04 & .03 & .03 & .03 & .03 & .03 \\
\hline 12 & .03 & .03 & .03 & .03 & .03 & .03 & .03 & .03 & .03 & .03 & .03 & .03 \\
\hline 13 & .03 & .03 & .03 & .03 & .03 & .03 & .03 & .03 & .03 & .03 & .03 & .03 \\
\hline 14 & .03 & .03 & .03 & .03 & .03 & .03 & .03 & .03 & .03 & .03 & .03 & .03 \\
\hline 15 & .03 & .03 & .03 & .03 & .03 & .03 & .03 & .03 & .03 & .03 & .03 & .03 \\
\hline 16 & .03 & .03 & .03 & .03 & .03 & .03 & .03 & .03 & .03 & .03 & .03 & .03 \\
\hline 17 & .03 & .03 & .03 & .03 & .03 & .03 & .03 & .03 & .03 & .03 & .03 & .03 \\
\hline 18 & .03 & .03 & .03 & .03 & .03 & .03 & .03 & .03 & .03 & .03 & .03 & .03 \\
\hline 19 & .03 & .03 & .03 & .03 & .03 & .03 & .03 & .03 & .03 & .03 & .03 & .03 \\
\hline 20 & .03 & .03 & .03 & .03 & .03 & .03 & .03 & .03 & .03 & .03 & .03 & .03 \\
\hline 21 & .03 & .03 & .03 & .03 & .03 & .03 & .03 & .03 & .03 & .03 & .03 & .03 \\
\hline 22 & .03 & .03 & .03 & .03 & .03 & .03 & .03 & .03 & .03 & .03 & .03 & .03 \\
\hline 23 & .03 & .03 & .03 & .03 & .03 & .03 & .03 & .03 & .03 & .03 & .03 & .03 \\
\hline 24 & .03 & .03 & .03 & .03 & .03 & .03 & .03 & .03 & .03 & .03 & .03 & .03 \\
\hline 25 & .03 & .03 & .03 & .03 & .03 & .03 & .03 & .03 & .03 & .03 & .03 & .03 \\
\hline 26 & .03 & .03 & .03 & .03 & .03 & .03 & .03 & .03 & .03 & .03 & .03 & .03 \\
\hline 27 & .03 & .03 & .03 & .03 & .03 & .03 & .03 & .03 & .03 & .03 & .03 & .03 \\
\hline 28 & .03 & .03 & .03 & .03 & .03 & .03 & .03 & .03 & .03 & .03 & .03 & .03 \\
\hline 29 & .03 & - & .03 & .03 & .03 & .03 & .03 & .03 & .03 & .03 & .03 & .03 \\
\hline 30 & .03 & - & .03 & .03 & .03 & .03 & .03 & .03 & .03 & .03 & .03 & .03 \\
\hline 31 & .03 & - & .03 & - & .03 & - & .03 & .03 & - & .03 & - & .03 \\
\hline Mean & .030 & .030 & .030 & .030 & .030 & .030 & .033 & .030 & .030 & .030 & .030 & .030 \\
\hline $\operatorname{Max}$ & .03 & .03 & .03 & .03 & .03 & .03 & .04 & .03 & .03 & .03 & .03 & .03 \\
\hline Min & .03 & .03 & .03 & .03 & .03 & .03 & .03 & .03 & .03 & .03 & .03 & .03 \\
\hline
\end{tabular}


Table 71. Daily mean discharge, Colton Spring (CS), Long Valley Caldera, Mono County, California, 1988

[Values are in cubic feet per second. Max, maximum; Min, minimum; -, no data]

\begin{tabular}{|c|c|c|c|c|c|c|c|c|c|c|c|c|}
\hline Day & Jan & Feb & Mar & Apr & May & June & July & Aug & Sept & Oct & Nov & Dec \\
\hline 1 & 0.03 & 0.03 & 0.03 & 0.03 & 0.03 & 0.03 & 0.03 & 0.03 & 0.03 & 0.02 & 0.02 & 0.02 \\
\hline 2 & .03 & .03 & .03 & .03 & .03 & .03 & .03 & .03 & .03 & .02 & .02 & .02 \\
\hline 3 & .03 & .03 & .03 & .03 & .03 & .03 & .03 & .03 & .03 & .02 & .02 & .02 \\
\hline 4 & .03 & .03 & .03 & .03 & .03 & .03 & .03 & .03 & .03 & .02 & .02 & .02 \\
\hline 5 & .03 & .03 & .03 & .03 & .03 & .03 & .03 & .03 & .03 & .02 & .02 & .02 \\
\hline 6 & .03 & .03 & .03 & .03 & .03 & .03 & .03 & .03 & .03 & .02 & .02 & .02 \\
\hline 7 & .03 & .03 & .03 & .03 & .03 & .03 & .03 & .03 & .03 & .02 & .02 & .02 \\
\hline 8 & .03 & .03 & .03 & .03 & .03 & .03 & .03 & .03 & .03 & .02 & .02 & .02 \\
\hline 9 & .03 & .03 & .03 & .03 & .03 & .03 & .03 & .03 & .03 & .02 & .02 & .02 \\
\hline 10 & .03 & .03 & .03 & .03 & .03 & .03 & .03 & .03 & .03 & .02 & .02 & .02 \\
\hline 11 & .03 & .03 & .03 & .03 & .03 & .03 & .03 & .03 & .03 & .02 & .02 & .02 \\
\hline 12 & .03 & .03 & .03 & .03 & .03 & .03 & .03 & .03 & .03 & .02 & .02 & .02 \\
\hline 13 & .03 & .03 & .03 & .03 & .03 & .03 & .03 & .03 & .03 & .02 & .02 & .02 \\
\hline 14 & .03 & .03 & .03 & .03 & .03 & .03 & .03 & .03 & .03 & .02 & .02 & .02 \\
\hline 15 & .03 & .03 & .03 & .03 & .03 & .03 & .03 & .03 & .03 & .02 & .02 & .02 \\
\hline 16 & .03 & .03 & .03 & .03 & .03 & .03 & .03 & .03 & .03 & .02 & .02 & .02 \\
\hline 17 & .03 & .03 & .03 & .03 & .03 & .03 & .03 & .03 & .03 & .02 & .02 & .02 \\
\hline 18 & .03 & .03 & .03 & .03 & .03 & .03 & .03 & .03 & .03 & .02 & .02 & .02 \\
\hline 19 & .03 & .03 & .03 & .03 & .03 & .03 & .03 & .03 & .03 & .02 & .02 & .02 \\
\hline 20 & .03 & .03 & .03 & .03 & .03 & .03 & .03 & .03 & .03 & .02 & .02 & .02 \\
\hline 21 & .03 & .03 & .03 & .03 & .03 & .03 & .03 & .03 & .03 & .02 & .02 & .02 \\
\hline 22 & .03 & .03 & .03 & .03 & .03 & .03 & .03 & .03 & .02 & .02 & .02 & .02 \\
\hline 23 & .03 & .03 & .03 & .03 & .03 & .03 & .03 & .03 & .02 & .02 & .02 & .02 \\
\hline 24 & .03 & .03 & .03 & .03 & .03 & .03 & .03 & .03 & .02 & .02 & .02 & .02 \\
\hline 25 & .03 & .03 & .03 & .03 & .03 & .03 & .03 & .03 & .02 & .02 & .02 & .02 \\
\hline 26 & .03 & .03 & .03 & .03 & .03 & .03 & .03 & .03 & .02 & .02 & .02 & .02 \\
\hline 27 & .03 & .03 & .03 & .03 & .03 & .03 & .03 & .03 & .02 & .02 & .02 & .02 \\
\hline 28 & .03 & .03 & .03 & .03 & .03 & .03 & .03 & .03 & .02 & .02 & .02 & .02 \\
\hline 29 & .03 & .03 & .03 & .03 & .03 & .03 & .03 & .03 & .02 & .02 & .02 & .02 \\
\hline 30 & .03 & - & .03 & .03 & .03 & .03 & .03 & .03 & .02 & .02 & .02 & .02 \\
\hline 31 & .03 & - & .03 & - & .03 & - & .03 & .03 & - & .02 & - & .02 \\
\hline Mean & .030 & .030 & .030 & .030 & .030 & .030 & .030 & .030 & .027 & .020 & .020 & .020 \\
\hline $\operatorname{Max}$ & .03 & .03 & .03 & .03 & .03 & .03 & .03 & .03 & .03 & .02 & .02 & .02 \\
\hline Min & .03 & .03 & .03 & .03 & .03 & .03 & .03 & .03 & .02 & .02 & .02 & .02 \\
\hline
\end{tabular}


Table 72. Daily mean discharge, Colton Spring (CS), Long Valley Caldera, Mono County, California, 1989

[Values are in cubic feet per second. Max, maximum; Min, minimum; - , no data]

\begin{tabular}{|c|c|c|c|c|c|c|c|c|c|c|c|c|}
\hline Day & Jan & Feb & Mar & Apr & May & June & July & Aug & Sept & Oct & Nov & Dec \\
\hline 1 & 0.02 & 0.02 & 0.02 & 0.02 & 0.03 & 0.02 & 0.03 & 0.03 & 0.03 & 0.03 & 0.04 & 0.03 \\
\hline 2 & .02 & .02 & .02 & .02 & .03 & .02 & .03 & .03 & .03 & .03 & .04 & .03 \\
\hline 3 & .02 & .02 & .02 & .02 & .03 & .02 & .03 & .03 & .03 & .03 & .04 & .03 \\
\hline 4 & .02 & .02 & .02 & .02 & .03 & .02 & .03 & .03 & .03 & .03 & .04 & .03 \\
\hline 5 & .02 & .02 & .02 & .02 & .03 & .02 & .03 & .03 & .03 & .03 & .04 & .03 \\
\hline 6 & .02 & .02 & .02 & .02 & .03 & .02 & .03 & .03 & .03 & .03 & .04 & .04 \\
\hline 7 & .02 & .02 & .02 & .03 & .03 & .02 & .03 & .03 & .03 & .03 & .04 & .04 \\
\hline 8 & .02 & .02 & .02 & .03 & .03 & .02 & .03 & .03 & .03 & .03 & .04 & .04 \\
\hline 9 & .02 & .02 & .02 & .03 & .03 & .02 & .03 & .03 & .03 & .03 & .04 & .04 \\
\hline 10 & .02 & .02 & .02 & .03 & .03 & .02 & .03 & .03 & .03 & .03 & .04 & .04 \\
\hline 11 & .02 & .02 & .02 & .03 & .03 & .02 & .03 & .03 & .03 & .03 & .04 & .04 \\
\hline 12 & .02 & .02 & .02 & .03 & .03 & .02 & .03 & .03 & .03 & .03 & .04 & .04 \\
\hline 13 & .02 & .02 & .02 & .03 & .03 & .03 & .03 & .03 & .03 & .03 & .04 & .04 \\
\hline 14 & .02 & .02 & .02 & .03 & .03 & .03 & .03 & .03 & .03 & .03 & .04 & .04 \\
\hline 15 & .02 & .02 & .02 & .03 & .03 & .03 & .03 & .03 & .03 & .03 & .04 & .04 \\
\hline 16 & .02 & .02 & .02 & .03 & .03 & .03 & .03 & .03 & .03 & .03 & .04 & .04 \\
\hline 17 & .02 & .02 & .02 & .03 & .03 & .03 & .03 & .03 & .03 & .04 & .04 & .04 \\
\hline 18 & .02 & .02 & .02 & .03 & .03 & .03 & .03 & .03 & .03 & .04 & .04 & .04 \\
\hline 19 & .02 & .02 & .02 & .03 & .03 & .03 & .03 & .03 & .03 & .04 & .04 & .04 \\
\hline 20 & .02 & .02 & .02 & .03 & .03 & .03 & .03 & .03 & .03 & .04 & .04 & .04 \\
\hline 21 & .02 & .02 & .02 & .03 & .03 & .03 & .03 & .03 & .03 & .04 & .04 & .04 \\
\hline 22 & .02 & .02 & .02 & .03 & .03 & .03 & .03 & .03 & .03 & .04 & .04 & .04 \\
\hline 23 & .02 & .02 & .02 & .03 & .02 & .03 & .03 & .03 & .03 & .04 & .04 & .04 \\
\hline 24 & .02 & .02 & .02 & .03 & .02 & .03 & .03 & .03 & .03 & .04 & .04 & .03 \\
\hline 25 & .02 & .02 & .02 & .03 & .02 & .03 & .03 & .03 & .03 & .04 & .04 & .03 \\
\hline 26 & .02 & .02 & .02 & .03 & .02 & .03 & .03 & .03 & .03 & .04 & .04 & .03 \\
\hline 27 & .02 & .02 & .02 & .03 & .02 & .03 & .03 & .03 & .03 & .04 & .04 & .03 \\
\hline 28 & .02 & .02 & .02 & .03 & .02 & .03 & .03 & .03 & .03 & .04 & .04 & .04 \\
\hline 29 & .02 & - & .02 & .03 & .02 & .03 & .03 & .03 & .03 & .04 & .04 & .04 \\
\hline 30 & .02 & - & .02 & .03 & .02 & .03 & .03 & .03 & .03 & .04 & .03 & .03 \\
\hline 31 & .02 & - & .02 & - & .02 & - & .03 & .03 & - & .04 & - & .04 \\
\hline Mean & .020 & .020 & .020 & .028 & .027 & .026 & .030 & .030 & .030 & .035 & .040 & .037 \\
\hline $\operatorname{Max}$ & .02 & .02 & .02 & .03 & .03 & .03 & .03 & .03 & .03 & .04 & .04 & .04 \\
\hline Min & .02 & .02 & .02 & .02 & .02 & .02 & .03 & .03 & .03 & .03 & .03 & .03 \\
\hline
\end{tabular}


Table 73. Daily mean discharge, Colton Spring (CS), Long Valley Caldera, Mono County, California, 1990 [Values are in cubic feet per second. Max, maximum; Min, minimum; -, no data]

\begin{tabular}{|c|c|c|c|c|c|c|c|c|c|c|c|c|}
\hline Day & Jan & Feb & Mar & Apr & May & June & July & Aug & Sept & Oct & Nov & Dec \\
\hline 1 & 0.04 & 0.03 & 0.04 & 0.04 & 0.04 & 0.04 & 0.04 & 0.04 & 0.04 & 0.04 & 0.04 & 0.04 \\
\hline 2 & .04 & .03 & .04 & .04 & .04 & .04 & .04 & .04 & .04 & .04 & .04 & .04 \\
\hline 3 & .04 & .03 & .04 & .04 & .04 & .04 & .04 & .04 & .04 & .04 & .04 & .04 \\
\hline 4 & .04 & .03 & .04 & .04 & .04 & .04 & .04 & .04 & .04 & .04 & .04 & .04 \\
\hline 5 & .04 & .03 & .04 & .04 & .04 & .04 & .04 & .04 & .04 & .04 & .04 & .04 \\
\hline 6 & .04 & .03 & .04 & .04 & .04 & .04 & .04 & .04 & .04 & .04 & .04 & .04 \\
\hline 7 & .04 & .03 & .04 & .04 & .04 & .04 & .04 & .04 & .04 & .04 & .04 & .04 \\
\hline 8 & .04 & .03 & .04 & .04 & .04 & .04 & .04 & .04 & .04 & .04 & .04 & .04 \\
\hline 9 & .04 & .03 & .04 & .04 & .04 & .04 & .04 & .04 & .04 & .04 & .04 & .04 \\
\hline 10 & .04 & .03 & .04 & .04 & .04 & .04 & .04 & .04 & .04 & .04 & .04 & .04 \\
\hline 11 & .04 & .03 & .04 & .04 & .04 & .04 & .04 & .04 & .04 & .04 & .04 & .04 \\
\hline 12 & .04 & .03 & .04 & .04 & .04 & .04 & .04 & .04 & .04 & .04 & .04 & .04 \\
\hline 13 & .04 & .03 & .04 & .04 & .04 & .04 & .04 & .04 & .04 & .04 & .04 & .04 \\
\hline 14 & .04 & .03 & .03 & .04 & .04 & .04 & .04 & .04 & .04 & .04 & .04 & .04 \\
\hline 15 & .04 & .03 & .04 & .04 & .04 & .04 & .04 & .04 & .04 & .04 & .04 & .04 \\
\hline 16 & .04 & .03 & .04 & .04 & .04 & .04 & .04 & .04 & .04 & .04 & .04 & .04 \\
\hline 17 & .03 & .03 & .04 & .04 & .04 & .04 & .04 & .04 & .04 & .04 & .04 & .04 \\
\hline 18 & .03 & .03 & .04 & .04 & .04 & .04 & .04 & .04 & .04 & .04 & .04 & .04 \\
\hline 19 & .03 & .03 & .04 & .04 & .04 & .04 & .04 & .04 & .04 & .04 & .04 & .04 \\
\hline 20 & .03 & .03 & .04 & .04 & .04 & .04 & .04 & .04 & .04 & .04 & .04 & .04 \\
\hline 21 & .03 & .03 & .04 & .04 & .04 & .04 & .04 & .04 & .04 & .04 & .04 & .04 \\
\hline 22 & .03 & .03 & .04 & .04 & .04 & .04 & .04 & .04 & .04 & .04 & .04 & .04 \\
\hline 23 & .03 & .03 & .04 & .04 & .04 & .04 & .04 & .04 & .04 & .04 & .04 & .03 \\
\hline 24 & .03 & .03 & .04 & .04 & .04 & .04 & .04 & .04 & .04 & .04 & .04 & .03 \\
\hline 25 & .03 & .04 & .04 & .04 & .04 & .04 & .04 & .04 & .04 & .04 & .04 & .03 \\
\hline 26 & .03 & .04 & .04 & .04 & .04 & .04 & .04 & .04 & .04 & .04 & .04 & .03 \\
\hline 27 & .03 & .04 & .04 & .04 & .04 & .04 & .04 & .04 & .04 & .04 & .04 & .03 \\
\hline 28 & .03 & .04 & .04 & .04 & .04 & .04 & .04 & .04 & .04 & .04 & .04 & .03 \\
\hline 29 & .03 & - & .04 & .04 & .04 & .04 & .04 & .04 & .04 & .04 & .04 & .03 \\
\hline 30 & .03 & - & .04 & .04 & .04 & .04 & .04 & .04 & .04 & .04 & .04 & .03 \\
\hline 31 & .03 & - & .04 & - & .04 & - & .04 & .04 & - & .04 & - & .03 \\
\hline Mean & .035 & .031 & .040 & .040 & .040 & .040 & .040 & .040 & .040 & .040 & .040 & .037 \\
\hline $\operatorname{Max}$ & .04 & .04 & .04 & .04 & .04 & .04 & .04 & .04 & .04 & .04 & .04 & .04 \\
\hline Min & .03 & .03 & .03 & .04 & .04 & .04 & .04 & .04 & .04 & .04 & .04 & .03 \\
\hline
\end{tabular}


Table 74. Daily mean discharge, Colton Spring (CS), Long Valley Caldera, Mono County, California, 1991

[Values are in cubic feet per second. e, estimated; Max. maximum; Min, minimum; - , no data]

\begin{tabular}{|c|c|c|c|c|c|c|c|c|c|c|c|c|}
\hline Day & Jan & Feb & Mar & Apr & May & June & July & Aug & Sept & Oct & Nov & Dec \\
\hline 1 & 0.03 & 0.02 & 0.02 & 0.02 & 0.01 & 0.01 & 0.01 & 0 & 0 & - & - & - \\
\hline 2 & .03 & .02 & .02 & .02 & .01 & .01 & .01 & 0 & 0 & - & - & - \\
\hline 3 & .03 & .02 & .02 & .02 & .01 & .01 & .01 & 0 & 0 & - & - & - \\
\hline 4 & .03 & .02 & .02 & .02 & .01 & .01 & .01 & 0 & 0 & - & - & - \\
\hline 5 & .03 & .02 & .02 & .02 & .01 & .01 & .01 & 0 & 0 & - & - & - \\
\hline 6 & .03 & .02 & .02 & .01 & .01 & .01 & .01 & 0 & 0 & - & - & - \\
\hline 7 & .03 & .02 & .02 & .01 & .01 & .01 & .01 & 0 & 0 & - & - & - \\
\hline 8 & .03 & .02 & .02 & .01 & .01 & .01 & .01 & 0 & 0 & - & - & - \\
\hline 9 & .02 & .02 & .02 & .01 & .01 & .01 & .01 & 0 & 0 & - & - & - \\
\hline 10 & .02 & .02 & .02 & .01 & .01 & .01 & .01 & 0 & 0 & - & - & - \\
\hline 11 & .02 & .02 & .02 & .01 & .01 & .01 & .01 & 0 & 0 & - & - & - \\
\hline 12 & .02 & .02 & .02 & .01 & .01 & .01 & 0 & 0 & 0 & - & - & - \\
\hline 13 & .02 & .02 & .02 & .01 & .01 & .01 & 0 & 0 & 0 & - & - & - \\
\hline 14 & .02 & .02 & .02 & .01 & e.01 & .01 & 0 & 0 & 0 & - & - & - \\
\hline 15 & .02 & .02 & .02 & .01 & e. 01 & .01 & 0 & 0 & 0 & - & - & - \\
\hline 16 & .02 & .02 & .02 & .01 & .01 & .01 & 0 & 0 & 0 & - & - & - \\
\hline 17 & .02 & .02 & .02 & .01 & .01 & .01 & 0 & 0 & 0 & - & - & - \\
\hline 18 & .02 & .02 & .02 & .01 & .01 & .01 & 0 & 0 & 0 & - & - & - \\
\hline 19 & .02 & .02 & .02 & .01 & .01 & .01 & 0 & 0 & 0 & - & - & - \\
\hline 20 & .02 & .02 & .02 & .01 & .01 & .01 & 0 & 0 & 0 & - & - & - \\
\hline 21 & .02 & .02 & .02 & .01 & .01 & .01 & 0 & 0 & 0 & - & - & - \\
\hline 22 & .02 & .02 & .02 & .01 & .01 & .01 & 0 & 0 & 0 & - & - & - \\
\hline 23 & .02 & .02 & .02 & .01 & .01 & .01 & 0 & 0 & 0 & - & - & - \\
\hline 24 & .02 & .02 & .02 & .01 & .01 & .01 & 0 & 0 & 0 & - & - & - \\
\hline 25 & .02 & .02 & .02 & .01 & .01 & .01 & 0 & 0 & 0 & - & - & - \\
\hline 26 & .02 & .02 & .02 & .01 & .01 & .01 & 0 & 0 & 0 & - & - & - \\
\hline 27 & .02 & .02 & .02 & .01 & .01 & .01 & 0 & 0 & 0 & - & - & - \\
\hline 28 & .02 & .02 & .02 & .01 & .01 & .01 & 0 & 0 & 0 & - & - & - \\
\hline 29 & .02 & - & .02 & .01 & .01 & .01 & 0 & 0 & 0 & - & - & - \\
\hline 30 & .02 & - & .02 & .01 & .01 & .01 & 0 & 0 & 0 & - & - & - \\
\hline 31 & .02 & - & .02 & - & .01 & - & 0 & 0 & - & - & - & - \\
\hline Mean & .023 & .020 & .020 & .012 & .010 & .010 & .004 & 0 & 0 & - & - & - \\
\hline Max & .03 & .02 & .02 & .02 & .01 & .01 & .01 & 0 & 0 & - & - & - \\
\hline Min & .02 & .02 & .02 & .01 & .01 & .01 & 0 & 0 & 0 & - & - & - \\
\hline
\end{tabular}


This page intentionally left blank. 


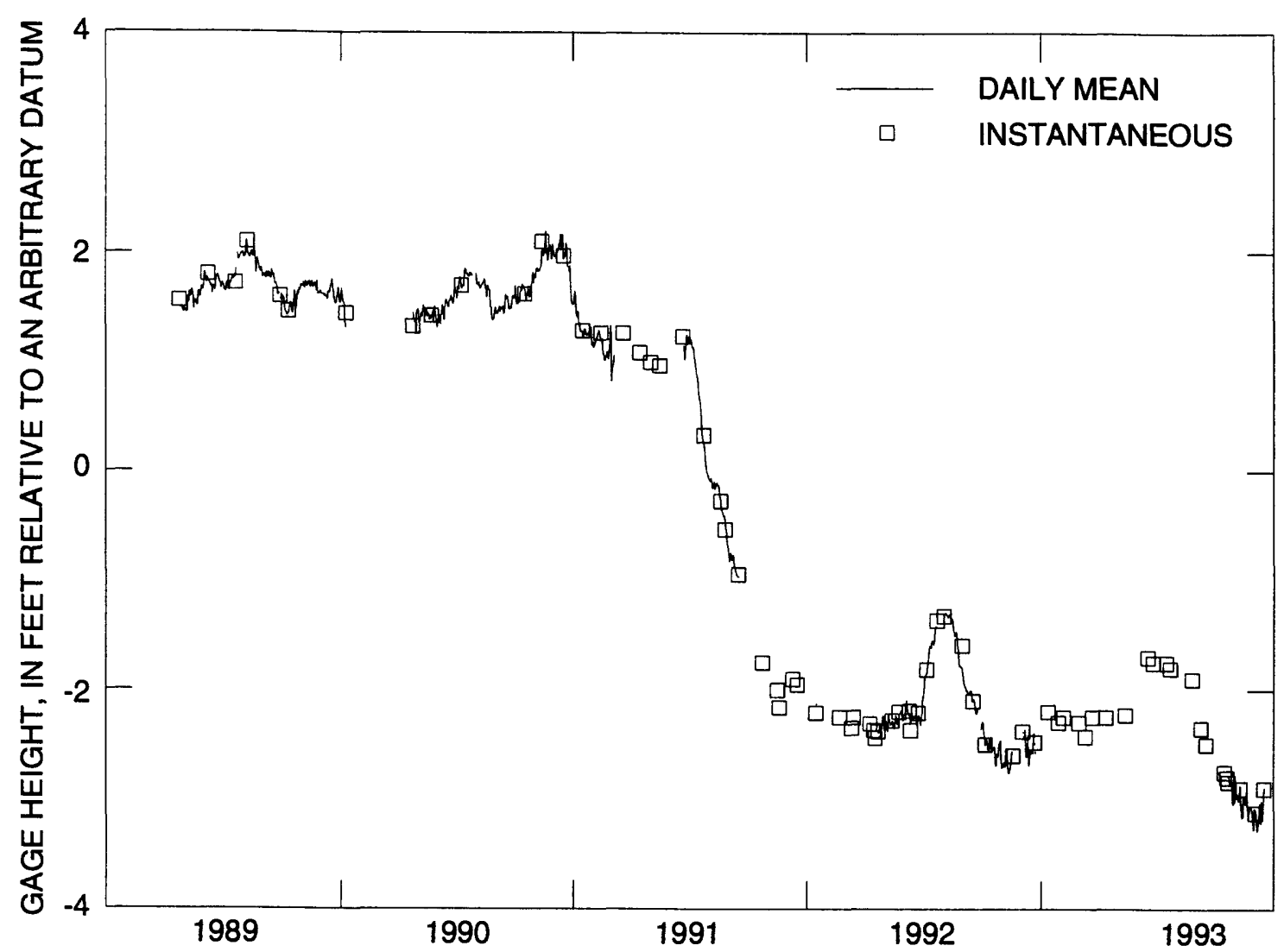

Figure 35. Daily mean and instantaneous measured gage heights, Hot Bubbling Pool (HBP), Long Valley Caldera, Mono County, California, 1989-93. 


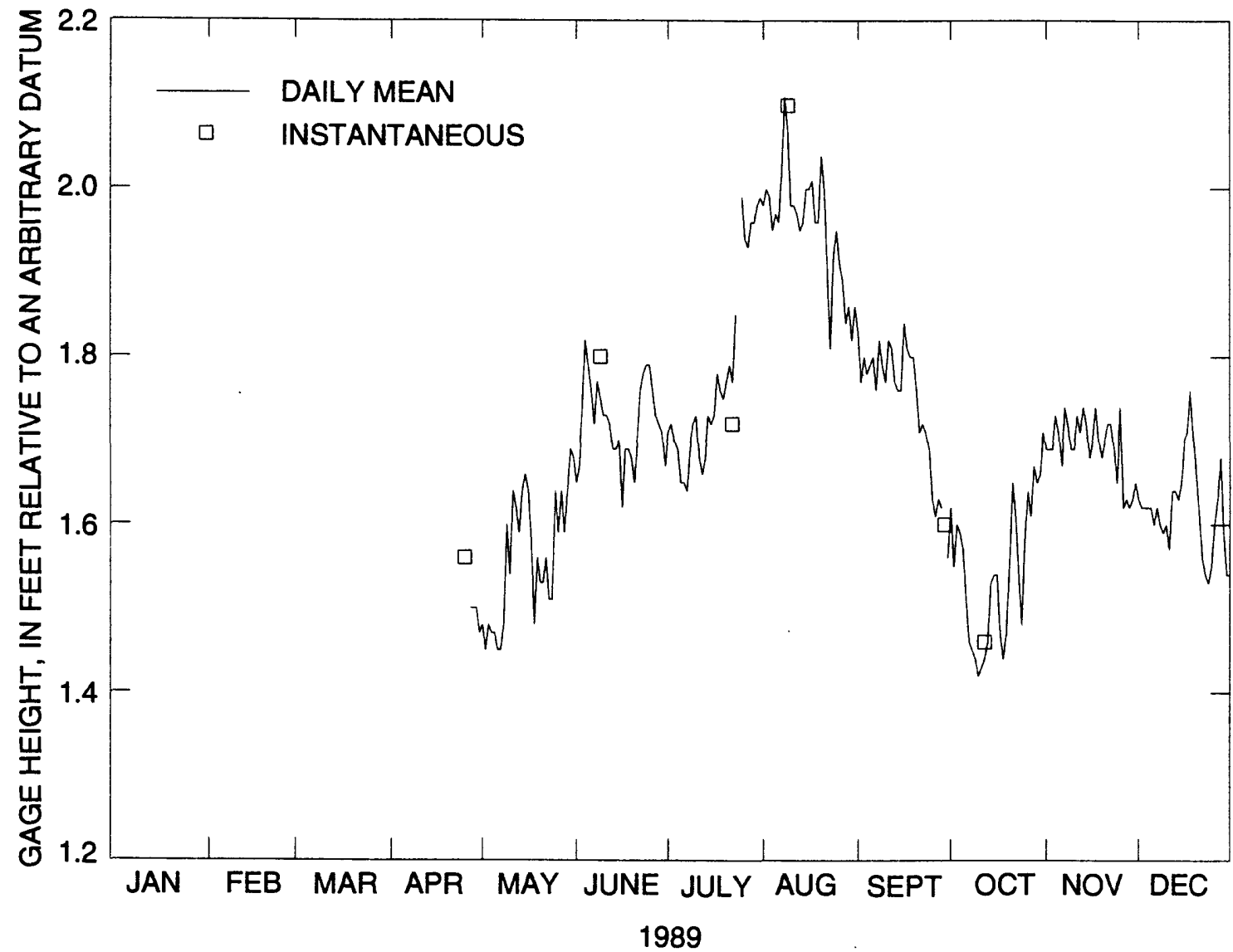

Figure 36. (A) Daily mean and instantaneous measured gage heights, Hot Bubbling Pool (HBP), Long Valley Caldera, Mono County, California, 1989. 
Table 75. Daily mean gage height, Hot Bubbling Pool (HBP), Long Valley Caldera, Mono County, California, 1989

[Values are in feet relative to an arbitrary datum. Max, maximum; Min, minimum; - , no data]

\begin{tabular}{|c|c|c|c|c|c|c|c|c|c|c|c|c|}
\hline Day & Jan & Feb & Mar & Apr & May & June & July & Aug & Sept & Oct & Nov & Dec \\
\hline 1 & - & - & - & - & 1.48 & 1.65 & 1.71 & 1.98 & 1.83 & 1.62 & 1.69 & 1.63 \\
\hline 2 & - & - & - & - & 1.45 & 1.67 & 1.72 & 2.00 & 1.77 & 1.55 & 1.69 & 1.62 \\
\hline 3 & - & - & - & - & 1.48 & 1.74 & 1.70 & 1.99 & 1.80 & 1.60 & 1.69 & 1.62 \\
\hline 4 & - & - & - & - & 1.47 & 1.82 & 1.69 & 1.95 & 1.78 & 1.59 & 1.73 & 1.62 \\
\hline 5 & - & - & - & - & 1.47 & 1.79 & 1.65 & 1.97 & 1.79 & 1.57 & 1.71 & 1.62 \\
\hline 6 & - & - & - & - & 1.45 & 1.76 & 1.65 & 1.96 & 1.80 & 1.51 & 1.67 & 1.60 \\
\hline 7 & - & - & - & - & 1.45 & 1.72 & 1.64 & 2.02 & 1.76 & 1.46 & 1.74 & 1.62 \\
\hline 8 & - & - & - & - & 1.48 & 1.77 & 1.69 & 2.11 & 1.82 & 1.45 & 1.72 & 1.60 \\
\hline 9 & - & - & - & - & 1.60 & 1.75 & 1.72 & 2.07 & 1.79 & 1.44 & 1.69 & 1.59 \\
\hline 10 & - & - & - & - & 1.54 & 1.73 & 1.73 & 1.98 & 1.77 & 1.42 & 1.69 & 1.60 \\
\hline 11 & - & - & - & - & 1.64 & 1.73 & 1.68 & 1.98 & 1.82 & 1.43 & 1.73 & 1.57 \\
\hline 12 & - & - & - & - & 1.62 & 1.72 & 1.66 & 1.97 & 1.81 & 1.44 & 1.71 & 1.64 \\
\hline 13 & - & - & - & - & 1.59 & 1.69 & 1.68 & 1.95 & 1.77 & 1.46 & 1.74 & 1.64 \\
\hline 14 & - & - & - & - & 1.64 & 1.69 & 1.73 & 1.96 & 1.76 & 1.53 & 1.72 & 1.63 \\
\hline 15 & - & - & - & - & 1.66 & 1.70 & 1.72 & 2.00 & 1.76 & 1.54 & 1.68 & 1.65 \\
\hline 16 & - & - & - & - & 1.64 & 1.62 & 1.73 & 2.00 & 1.84 & 1.54 & 1.70 & 1.70 \\
\hline 17 & - & - & - & - & 1.58 & 1.69 & 1.78 & 2.01 & 1.81 & 1.47 & 1.74 & 1.71 \\
\hline 18 & - & - & - & - & 1.48 & 1.69 & 1.76 & 1.96 & 1.80 & 1.44 & 1.70 & 1.76 \\
\hline 19 & - & - & - & - & 1.56 & 1.68 & 1.75 & 1.96 & 1.80 & 1.47 & 1.68 & 1.71 \\
\hline 20 & - & - & - & - & 1.53 & 1.65 & 1.77 & 2.04 & 1.76 & 1.55 & 1.70 & 1.66 \\
\hline 21 & - & - & - & - & 1.53 & 1.70 & 1.79 & 2.00 & 1.71 & 1.65 & 1.72 & 1.61 \\
\hline 22 & - & - & - & - & 1.56 & 1.76 & 1.77 & 1.90 & 1.72 & 1.61 & 1.72 & 1.56 \\
\hline 23 & - & - & - & - & 1.51 & 1.78 & 1.85 & 1.81 & 1.71 & 1.54 & 1.69 & 1.54 \\
\hline 24 & - & - & - & - & 1.51 & 1.79 & - & 1.92 & 1.69 & 1.48 & 1.65 & 1.53 \\
\hline 25 & - & - & - & - & 1.64 & 1.79 & 1.99 & 1.95 & 1.63 & 1.59 & 1.74 & 1.55 \\
\hline 26 & - & - & - & - & 1.59 & 1.76 & 1.94 & 1.91 & 1.61 & 1.64 & 1.62 & 1.60 \\
\hline 27 & - & - & - & 1.50 & 1.64 & 1.73 & 1.93 & 1.89 & 1.63 & 1.61 & 1.63 & 1.63 \\
\hline 28 & - & - & - & 1.50 & 1.59 & 1.72 & 1.96 & 1.84 & 1.62 & 1.67 & 1.62 & 1.68 \\
\hline 29 & - & - & - & 1.50 & 1.64 & 1.71 & 1.96 & 1.86 & - & 1.65 & 1.63 & 1.59 \\
\hline 30 & - & - & - & 1.47 & 1.69 & 1.67 & 1.98 & 1.82 & 1.56 & 1.66 & 1.65 & 1.54 \\
\hline 31 & - & - & - & - & 1.68 & - & 1.99 & 1.86 & - & 1.71 & - & 1.54 \\
\hline Mean & - & - & - & 1.49 & 1.56 & 1.72 & 1.78 & 1.96 & 1.75 & 1.54 & 1.69 & 1.62 \\
\hline Max & - & - & - & 1.50 & 1.69 & 1.82 & 1.99 & 2.11 & 1.84 & 1.71 & 1.74 & 1.76 \\
\hline Min & - & - & - & 1.47 & 1.45 & 1.62 & 1.64 & 1.81 & 1.56 & 1.42 & 1.62 & 1.53 \\
\hline
\end{tabular}




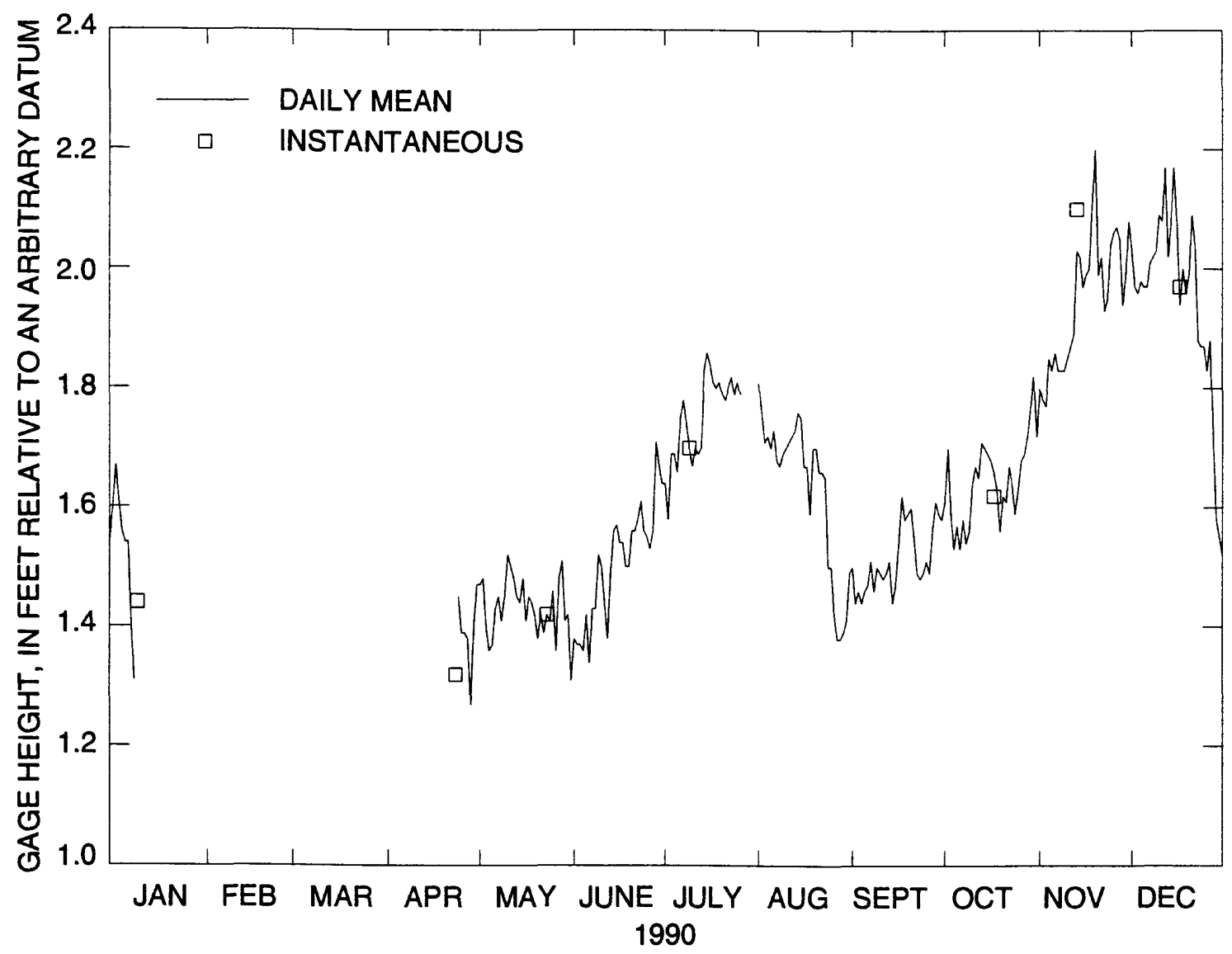

Figure 36. (B) Daily mean and instantaneous measured gage heights, Hot Bubbling Pool (HBP), Long Valley Caldera, Mono County, California, 1990. 
Table 76. Daily mean gage height, Hot Bubbling Pool (HBP), Long Valley Caldera, Mono County, California, 1990

[Values are in feet relative to an arbitrary datum. Max, maximum; Min, minimum; -, no data]

\begin{tabular}{|c|c|c|c|c|c|c|c|c|c|c|c|c|}
\hline Day & Jan & Feb & Mar & Apr & May & June & July & Aug & Sept & Oct & Nov & Dec \\
\hline 1 & 1.60 & - & - & - & 1.47 & 1.38 & 1.64 & 1.81 & 1.50 & 1.61 & 1.80 & 2.03 \\
\hline 2 & 1.67 & - & - & - & 1.48 & 1.37 & 1.58 & 1.76 & 1.44 & 1.70 & 1.78 & 1.97 \\
\hline 3 & 1.61 & - & - & - & 1.40 & 1.37 & 1.69 & 1.71 & 1.46 & 1.59 & 1.77 & 1.96 \\
\hline 4 & 1.56 & - & - & - & 1.36 & 1.36 & 1.69 & 1.72 & 1.44 & 1.53 & 1.85 & 1.98 \\
\hline 5 & 1.54 & - & - & - & 1.37 & 1.42 & 1.66 & 1.70 & 1.46 & 1.57 & 1.83 & 1.97 \\
\hline 6 & 1.54 & - & - & - & 1.43 & 1.34 & 1.75 & 1.73 & 1.47 & 1.53 & 1.86 & 1.97 \\
\hline 7 & 1.40 & - & - & - & 1.45 & 1.43 & 1.78 & 1.68 & 1.51 & 1.58 & 1.83 & 2.01 \\
\hline 8 & 1.31 & - & - & - & 1.41 & 1.43 & 1.74 & 1.67 & 1.46 & 1.54 & 1.83 & 2.02 \\
\hline 9 & - & - & - & - & 1.45 & 1.52 & 1.70 & 1.69 & 1.50 & 1.56 & 1.83 & 2.03 \\
\hline 10 & - & - & - & - & 1.52 & 1.50 & 1.67 & 1.70 & 1.49 & 1.64 & 1.85 & 2.09 \\
\hline 11 & - & - & - & - & 1.50 & 1.44 & 1.70 & 1.71 & 1.48 & 1.67 & 1.87 & 2.08 \\
\hline 12 & - & - & - & - & 1.48 & 1.38 & 1.69 & 1.72 & 1.49 & 1.65 & 1.89 & 2.17 \\
\hline 13 & - & - & - & - & 1.45 & 1.49 & 1.70 & 1.73 & 1.51 & 1.71 & 2.03 & 2.02 \\
\hline 14 & - & - & - & - & 1.44 & 1.56 & 1.83 & 1.76 & 1.44 & 1.70 & 2.02 & 2.08 \\
\hline 15 & - & - & - & - & 1.48 & 1.57 & 1.86 & 1.75 & 1.47 & 1.69 & 1.97 & 2.17 \\
\hline 16 & - & - & - & - & 1.41 & 1.54 & 1.84 & 1.67 & 1.54 & 1.68 & 1.99 & 2.08 \\
\hline 17 & - & - & - & - & 1.45 & 1.54 & 1.81 & 1.67 & 1.62 & 1.66 & 2.00 & 1.94 \\
\hline 18 & - & - & - & - & 1.44 & 1.50 & 1.80 & 1.59 & 1.58 & 1.63 & 2.11 & 2.00 \\
\hline 19 & - & - & - & - & 1.42 & 1.50 & 1.81 & 1.70 & 1.59 & 1.56 & 2.20 & 1.96 \\
\hline 20 & - & - & - & - & 1.38 & 1.56 & 1.79 & 1.70 & 1.60 & 1.62 & 1.99 & 1.99 \\
\hline 21 & - & - & - & - & 1.42 & 1.56 & 1.78 & 1.66 & 1.55 & 1.61 & 2.02 & 2.09 \\
\hline 22 & - & - & - & - & 1.39 & 1.58 & 1.80 & 1.66 & 1.49 & 1.67 & 1.93 & 2.04 \\
\hline 23 & - & - & - & - & 1.42 & 1.61 & 1.82 & 1.65 & 1.48 & 1.64 & 1.95 & 1.88 \\
\hline 24 & - & - & - & 1.45 & 1.41 & 1.56 & 1.79 & 1.50 & 1.49 & 1.59 & 2.04 & 1.87 \\
\hline 25 & - & - & - & 1.39 & 1.46 & 1.55 & 1.81 & 1.50 & 1.51 & 1.63 & 2.06 & 1.87 \\
\hline 26 & - & - & - & 1.39 & 1.36 & 1.53 & 1.79 & 1.42 & 1.49 & 1.68 & 2.07 & 1.83 \\
\hline 27 & - & - & - & 1.38 & 1.48 & 1.56 & - & 1.38 & 1.56 & 1.69 & 2.05 & 1.88 \\
\hline 28 & - & - & - & 1.27 & 1.51 & 1.71 & - & 1.38 & 1.61 & 1.72 & 1.94 & 1.71 \\
\hline 29 & - & - & - & 1.40 & 1.41 & 1.67 & - & 1.39 & 1.59 & 1.77 & 1.99 & 1.58 \\
\hline 30 & - & - & - & 1.47 & 1.42 & 1.64 & - & 1.41 & 1.58 & 1.82 & 2.08 & 1.55 \\
\hline 31 & - & - & - & - & 1.31 & - & - & 1.49 & - & 1.72 & - & 1.52 \\
\hline Mean & 1.53 & - & - & 1.39 & 1.43 & 1.51 & 1.75 & 1.63 & 1.51 & 1.64 & 1.95 & 1.95 \\
\hline Max & 1.67 & 一 & - & 1.47 & 1.52 & 1.71 & 1.86 & 1.81 & 1.62 & 1.82 & 2.20 & 2.17 \\
\hline Min & 1.31 & - & - & 1.27 & 1.31 & 1.34 & 1.58 & 1.38 & 1.44 & 1.53 & 1.77 & 1.52 \\
\hline
\end{tabular}




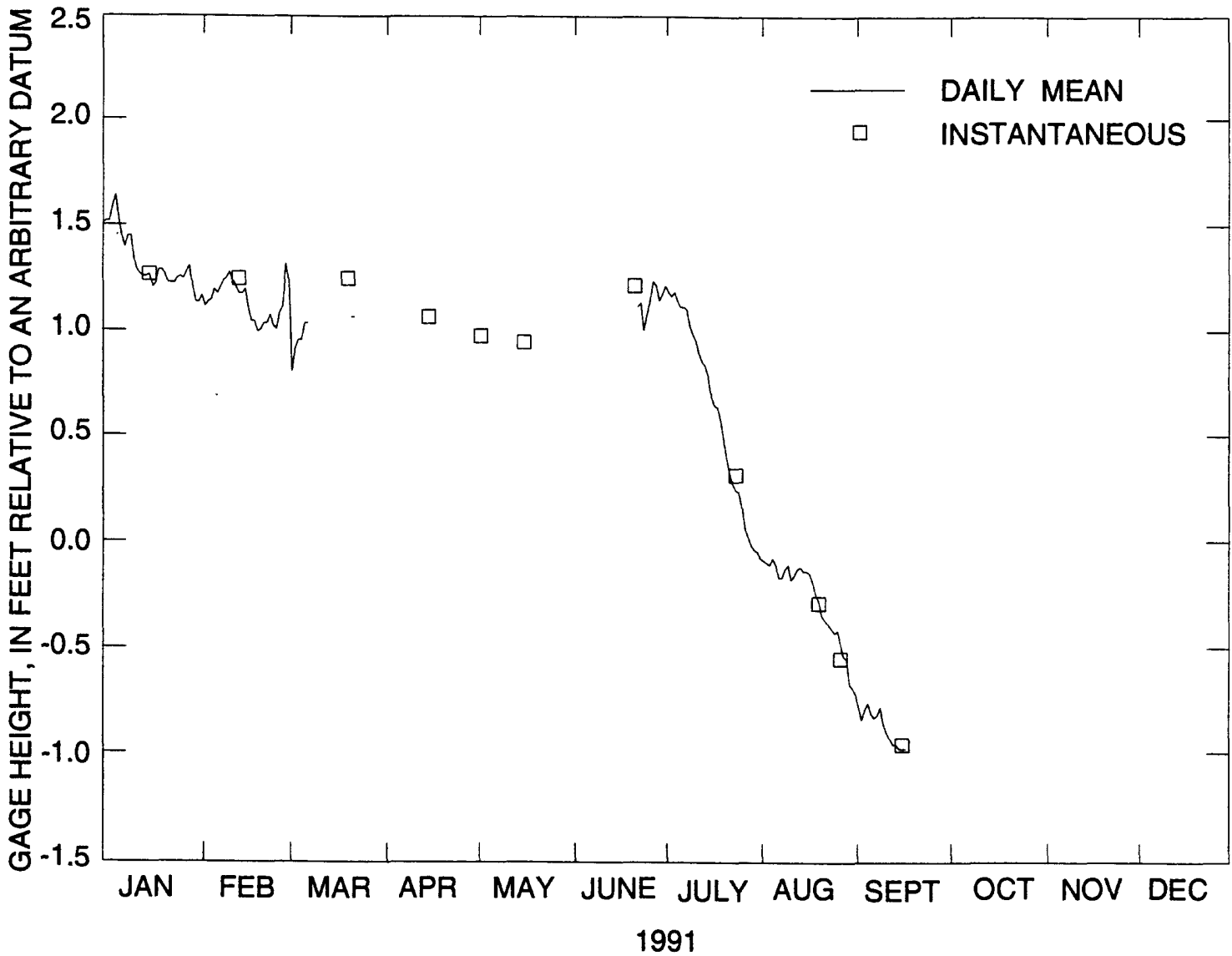

Figure 36. (C) Daily mean and instantaneous measured gage heights, Hot Bubbling Pool (HBP), Long Valley Caldera, Mono County, California, 1991. 
Table 77. Daily mean gage height, Hot Bubbling Pool (HBP), Long Valley Caldera, Mono County, California, 1991

[Values are in feet relative to an arbitrary datum. Max, maximum; Min, minimum; -, no data]

\begin{tabular}{|c|c|c|c|c|c|c|c|c|c|c|c|c|}
\hline Day & Jan & Feb & Mar & Apr & May & June & July & Aug & Sept & Oct & Nov & Dec \\
\hline 1 & 1.53 & 1.18 & 1.25 & - & - & - & 1.23 & -0.06 & -0.70 & - & - & - \\
\hline 2 & 1.53 & 1.13 & 0.82 & - & - & - & 1.20 & -.07 & -.76 & - & - & - \\
\hline 3 & 1.60 & 1.15 & 0.93 & - & - & - & 1.18 & -.08 & -.82 & - & - & - \\
\hline 4 & 1.65 & 1.16 & 0.97 & - & - & - & 1.20 & -.09 & -.77 & - & - & - \\
\hline 5 & 1.54 & 1.21 & 0.97 & - & - & - & 1.16 & -.06 & -.74 & - & - & - \\
\hline 6 & 1.46 & 1.19 & 1.05 & - & - & - & 1.13 & -.09 & -.79 & - & - & - \\
\hline 7 & 1.41 & 1.22 & 1.05 & - & - & - & 1.13 & -.15 & -.81 & - & - & - \\
\hline 8 & 1.46 & 1.25 & - & - & - & - & 1.12 & -.15 & -.80 & - & - & - \\
\hline 9 & 1.46 & 1.26 & - & - & - & - & 1.04 & -.11 & -.76 & - & - & - \\
\hline 10 & 1.35 & 1.29 & - & - & - & - & 1.00 & -.09 & -.84 & - & - & - \\
\hline 11 & 1.30 & 1.25 & - & - & - & - & .97 & -.16 & -.88 & - & - & - \\
\hline 12 & 1.28 & 1.22 & - & - & - & - & .91 & -.14 & -.91 & - & - & - \\
\hline 13 & 1.27 & 1.19 & - & - & - & - & .87 & -.11 & -.94 & - & - & - \\
\hline 14 & 1.27 & 1.19 & - & - & - & - & .85 & -.10 & -.94 & - & - & - \\
\hline 15 & 1.28 & 1.21 & - & - & - & - & .80 & -.12 & -.96 & - & - & - \\
\hline 16 & 1.22 & 1.12 & - & - & - & - & .70 & -.12 & -.96 & - & - & - \\
\hline 17 & 1.24 & 1.06 & - & - & - & - & .66 & -.13 & -.96 & - & - & - \\
\hline 18 & 1.30 & 1.06 & - & - & - & - & .65 & -.17 & - & - & - & - \\
\hline 19 & 1.30 & 1.01 & - & - & - & - & .59 & -.23 & - & - & - & - \\
\hline 20 & 1.28 & 1.02 & - & - & - & - & .50 & -.26 & - & - & - & - \\
\hline 21 & 1.24 & 1.05 & 1.08 & - & - & - & .41 & -.33 & - & - & - & - \\
\hline 22 & 1.24 & 1.05 & 1.08 & - & - & 1.13 & .34 & -.35 & - & - & - & - \\
\hline 23 & 1.24 & 1.09 & - & - & - & 1.15 & .29 & -.37 & - & - & - & - \\
\hline 24 & 1.26 & 1.04 & - & - & - & 1.02 & .26 & -.39 & - & - & - & - \\
\hline 25 & 1.27 & 1.02 & - & - & - & 1.09 & .25 & -.41 & - & - & - & - \\
\hline 26 & 1.26 & 1.10 & - & - & - & 1.16 & .18 & -.40 & - & - & - & - \\
\hline 27 & 1.29 & 1.13 & - & - & - & 1.25 & .08 & -.46 & - & - & - & - \\
\hline 28 & 1.32 & 1.33 & - & - & - & 1.23 & .04 & -.52 & - & - & - & - \\
\hline 29 & 1.22 & - & - & - & - & 1.16 & 0.00 & -.53 & - & - & - & - \\
\hline 30 & 1.15 & - & - & - & - & 1.19 & -.02 & -.65 & - & - & - & - \\
\hline 31 & 1.15 & - & - & - & - & - & -.03 & -.67 & - & - & - & - \\
\hline Mean & 1.33 & 1.15 & 1.02 & - & - & 1.15 & .67 & -.24 & -.84 & - & - & - \\
\hline $\operatorname{Max}$ & 1.65 & 1.33 & 1.25 & - & - & 1.25 & 1.23 & -.06 & -.70 & - & - & - \\
\hline Min & 1.15 & 1.01 & 0.82 & - & - & 1.02 & -.03 & -.67 & -.96 & - & - & - \\
\hline
\end{tabular}




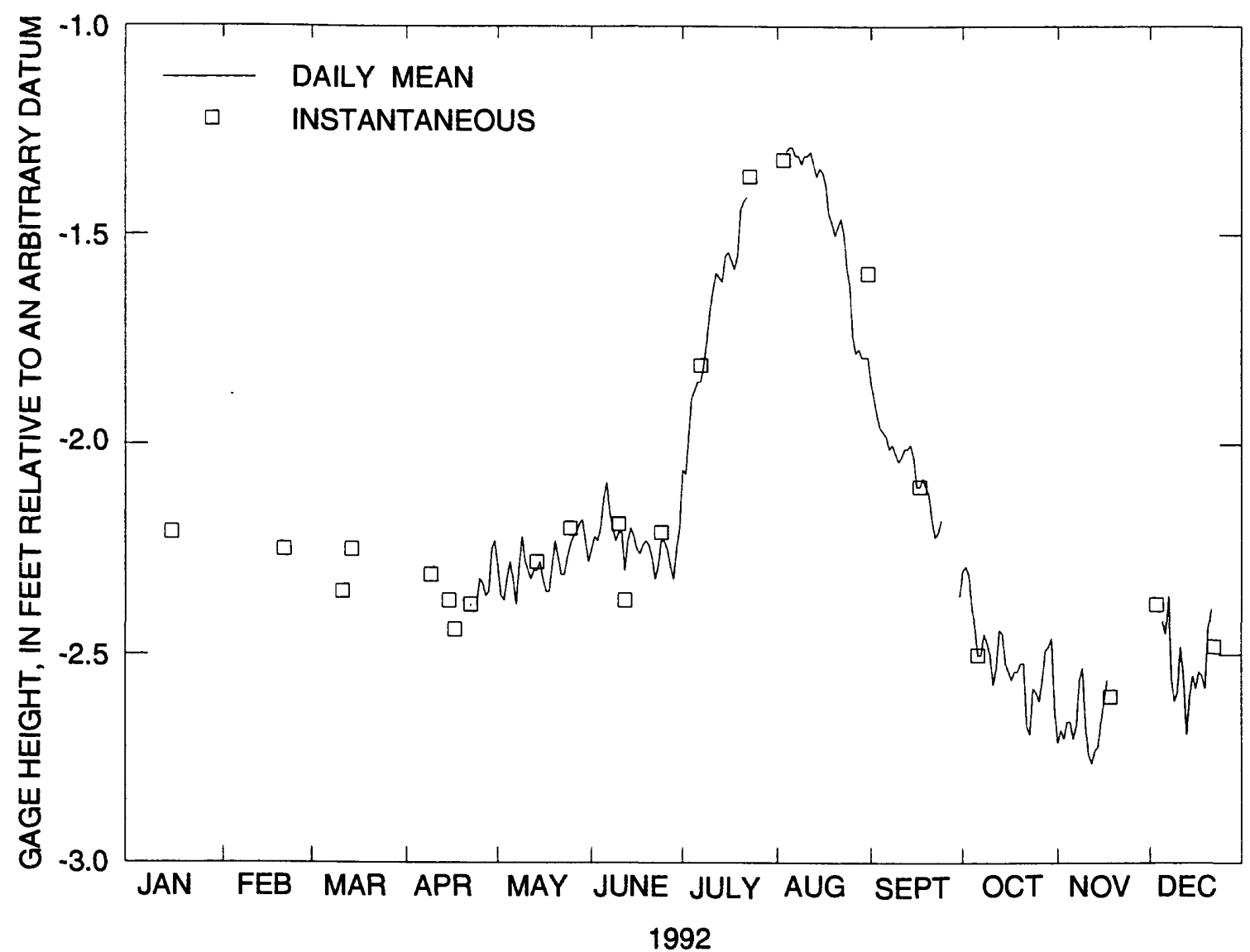

Figure 36. (D) Daily mean and instantaneous measured gage heights, Hot Bubbling Pool (HBP), Long Valley Caldera, Mono County, California, 1992. 
Table 78. Daily mean gage height, Hot Bubbling Pool (HBP), Long Valley Caldera, Mono County, California, 1992

[Values are in feet relative to an arbitrary datum. Max, maximum; Min, minimum; -, no data]

\begin{tabular}{|c|c|c|c|c|c|c|c|c|c|c|c|c|}
\hline Day & Jan & Feb & Mar & Apr & May & June & July & Aug & Sept & Oct & Nov & Dec \\
\hline 1 & - & - & - & - & -2.29 & -2.25 & -2.06 & - & -1.85 & -2.30 & -2.71 & - \\
\hline 2 & - & - & - & - & -2.36 & -2.22 & -2.07 & - & -1.89 & -2.29 & -2.68 & - \\
\hline 3 & - & - & - & - & -2.37 & -2.23 & -1.98 & - & -1.93 & -2.31 & -2.70 & - \\
\hline 4 & - & - & - & - & -2.32 & -2.20 & -1.89 & -1.30 & -1.96 & -2.39 & -2.66 & - \\
\hline 5 & - & - & - & - & -2.28 & -2.13 & -1.87 & -1.29 & -1.97 & -2.43 & -2.66 & -2.42 \\
\hline 6 & - & - & - & - & -2.32 & -2.09 & -1.85 & -1.29 & -1.98 & -2.50 & -2.70 & -2.45 \\
\hline 7 & - & - & - & - & -2.38 & -2.16 & -1.85 & -1.31 & -2.01 & -2.50 & -2.67 & -2.36 \\
\hline 8 & - & - & - & - & -2.29 & -2.20 & -1.81 & -1.31 & -2.00 & -2.45 & -2.56 & -2.56 \\
\hline 9 & - & - & - & - & -2.22 & -2.23 & -1.75 & -1.33 & -2.02 & -2.47 & -2.53 & -2.61 \\
\hline 10 & - & - & - & - & -2.28 & -2.21 & -1.68 & -1.31 & -2.04 & -2.50 & -2.67 & -2.59 \\
\hline 11 & - & - & - & - & -2.30 & -2.21 & -1.63 & -1.31 & -2.03 & -2.57 & -2.74 & -2.48 \\
\hline 12 & - & - & - & - & -2.32 & -2.30 & -1.59 & -1.30 & -2.01 & -2.53 & -2.76 & -2.55 \\
\hline 13 & - & - & - & - & -2.30 & -2.23 & -1.60 & -1.33 & -2.01 & -2.44 & -2.73 & -2.69 \\
\hline 14 & - & - & - & - & -2.30 & -2.20 & -1.61 & -1.36 & -2.00 & -2.45 & -2.72 & -2.60 \\
\hline 15 & - & - & - & - & -2.28 & -2.22 & -1.55 & -1.34 & -2.03 & -2.52 & -2.66 & -2.55 \\
\hline 16 & - & - & - & - & -2.32 & -2.25 & -1.54 & -1.35 & -2.10 & -2.54 & -2.61 & -2.58 \\
\hline 17 & - & - & - & - & -2.35 & -2.26 & -1.56 & -1.38 & -2.10 & -2.56 & -2.56 & -2.54 \\
\hline 18 & - & - & - & - & -2.35 & -2.24 & -1.58 & -1.45 & -2.08 & -2.54 & - & -2.55 \\
\hline 19 & - & - & - & - & -2.29 & -2.23 & -1.55 & -1.47 & -2.10 & -2.54 & - & -2.58 \\
\hline 20 & - & - & - & - & -2.23 & -2.24 & -1.44 & -1.50 & -2.12 & -2.52 & - & -2.44 \\
\hline 21 & - & - & - & - & -2.27 & -2.27 & -1.42 & -1.48 & -2.18 & -2.52 & - & -2.39 \\
\hline 22 & - & - & - & - & -2.31 & -2.32 & -1.41 & -1.46 & -2.22 & -2.67 & - & - \\
\hline 23 & - & - & - & - & -2.31 & -2.29 & - & -1.50 & -2.21 & -2.69 & - & - \\
\hline 24 & - & - & - & -2.38 & -2.27 & -2.23 & - & -1.58 & -2.18 & -2.58 & - & - \\
\hline 25 & - & - & - & -2.32 & -2.24 & -2.23 & - & -1.62 & - & -2.59 & - & - \\
\hline 26 & - & - & - & -2.33 & -2.22 & -2.25 & - & -1.74 & - & -2.61 & - & - \\
\hline 27 & - & - & - & -2.36 & -2.21 & -2.29 & - & -1.78 & - & -2.56 & - & - \\
\hline 28 & - & - & - & -2.35 & -2.19 & -2.32 & - & -1.77 & - & -2.49 & - & - \\
\hline 29 & - & - & - & -2.25 & -2.18 & -2.25 & - & -1.79 & - & -2.48 & - & - \\
\hline 30 & - & - & - & -2.23 & -2.23 & -2.20 & - & -1.79 & -2.36 & -2.46 & - & - \\
\hline 31 & - & - & - & - & -2.28 & - & - & -1.79 & - & -2.63 & - & - \\
\hline Mean & - & - & - & -2.32 & -2.29 & -2.23 & -1.70 & -1.47 & -2.06 & -2.50 & -2.67 & -2.53 \\
\hline Max & - & - & - & -2.23 & -2.18 & -2.09 & -1.41 & -1.29 & -1.85 & -2.29 & -2.53 & -2.36 \\
\hline Min & - & - & - & -2.38 & -2.38 & -2.32 & -2.07 & -1.79 & -2.36 & -2.69 & -2.76 & -2.69 \\
\hline
\end{tabular}




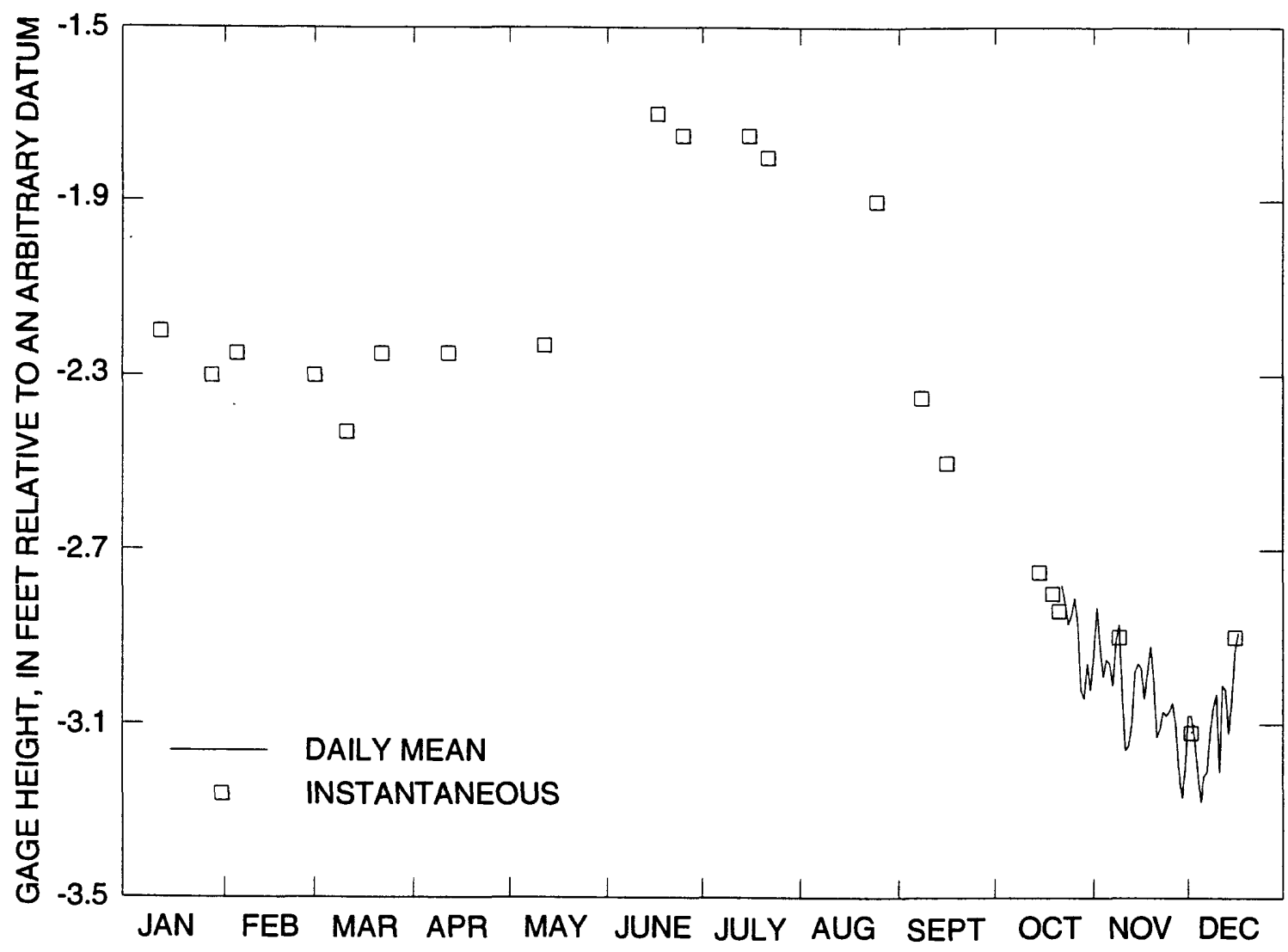

1993

Figure 36. (E) Daily mean and instantaneous measured gage heights, Hot Bubbling Pool (HBP), Long Valley Caldera, Mono County, California, 1993. 
Table 79. Daily mean gage height, Hot Bubbling Pool (HBP), Long Valley Caldera, Mono County, California, 1993

[Values are in feet relative to an arbitrary datum. Max, maximum; Min, minimum; - , no data]

\begin{tabular}{|c|c|c|c|c|c|c|c|c|c|c|c|c|}
\hline Day & Jan & Feb & Mar & Apr & May & June & July & Aug & Sept & Oct & Nov & Dec \\
\hline 1 & - & - & - & - & - & - & - & - & - & - & -2.94 & -3.08 \\
\hline 2 & - & - & - & - & - & - & - & - & - & - & -2.83 & -3.08 \\
\hline 3 & - & - & - & - & - & - & - & - & - & - & -2.92 & -3.13 \\
\hline 4 & - & - & - & - & - & - & - & - & - & - & -2.99 & -3.20 \\
\hline 5 & - & - & - & - & - & - & - & - & - & - & -2.95 & -3.28 \\
\hline 6 & - & - & - & - & - & - & - & - & - & - & -2.96 & -3.22 \\
\hline 7 & - & - & - & - & - & - & - & - & - & - & -3.01 & -3.21 \\
\hline 8 & - & - & - & - & - & - & - & - & - & - & -2.91 & -3.12 \\
\hline 9 & - & - & - & - & - & - & - & - & - & - & -2.87 & -3.06 \\
\hline 10 & - & - & - & - & - & - & - & - & - & - & -3.03 & -3.03 \\
\hline 11 & - & - & - & - & - & - & - & - & - & - & -3.16 & -3.21 \\
\hline 12 & - & - & - & - & - & - & - & - & - & - & -3.15 & -3.01 \\
\hline 13 & - & - & - & - & - & - & - & - & - & - & -3.10 & -3.02 \\
\hline 14 & - & - & - & - & - & - & - & - & - & - & -2.98 & -3.12 \\
\hline 15 & - & - & - & - & - & - & - & - & - & - & -2.96 & -3.04 \\
\hline 16 & - & - & - & - & - & - & - & - & - & - & -2.97 & -2.93 \\
\hline 17 & - & - & - & - & - & - & - & - & - & - & -3.04 & -2.89 \\
\hline 18 & - & - & - & - & - & - & - & - & - & - & -2.98 & - \\
\hline 19 & - & - & - & - & - & - & - & - & - & - & -2.92 & - \\
\hline 20 & - & - & - & - & - & - & - & - & - & - & -3.00 & - \\
\hline 21 & - & - & - & - & - & - & - & - & - & - & -3.13 & - \\
\hline 22 & - & - & - & - & - & - & - & - & - & -2.78 & -3.11 & - \\
\hline 23 & - & - & - & - & - & - & - & - & - & -2.82 & -3.07 & - \\
\hline 24 & - & - & - & - & - & - & - & - & - & -2.87 & -3.08 & - \\
\hline 25 & - & - & - & - & - & - & - & - & - & -2.85 & -3.07 & - \\
\hline 26 & - & - & - & - & - & - & - & - & - & -2.81 & -3.05 & - \\
\hline 27 & - & - & - & - & - & - & - & - & - & -2.87 & -3.10 & - \\
\hline 28 & - & - & - & - & - & - & - & - & - & -3.02 & -3.20 & - \\
\hline 29 & - & - & - & - & - & - & - & - & - & -3.04 & -3.27 & - \\
\hline 30 & - & - & - & - & - & - & - & - & - & -2.96 & -3.21 & - \\
\hline 31 & - & - & - & - & - & - & - & - & - & -3.02 & - & - \\
\hline Mean & - & - & - & - & - & - & - & - & - & -2.90 & -3.03 & -3.10 \\
\hline $\operatorname{Max}$ & - & - & - & - & - & - & - & - & - & -2.78 & -2.83 & -2.89 \\
\hline Min & - & - & - & - & - & - & - & - & - & -3.04 & -3.27 & -3.28 \\
\hline
\end{tabular}




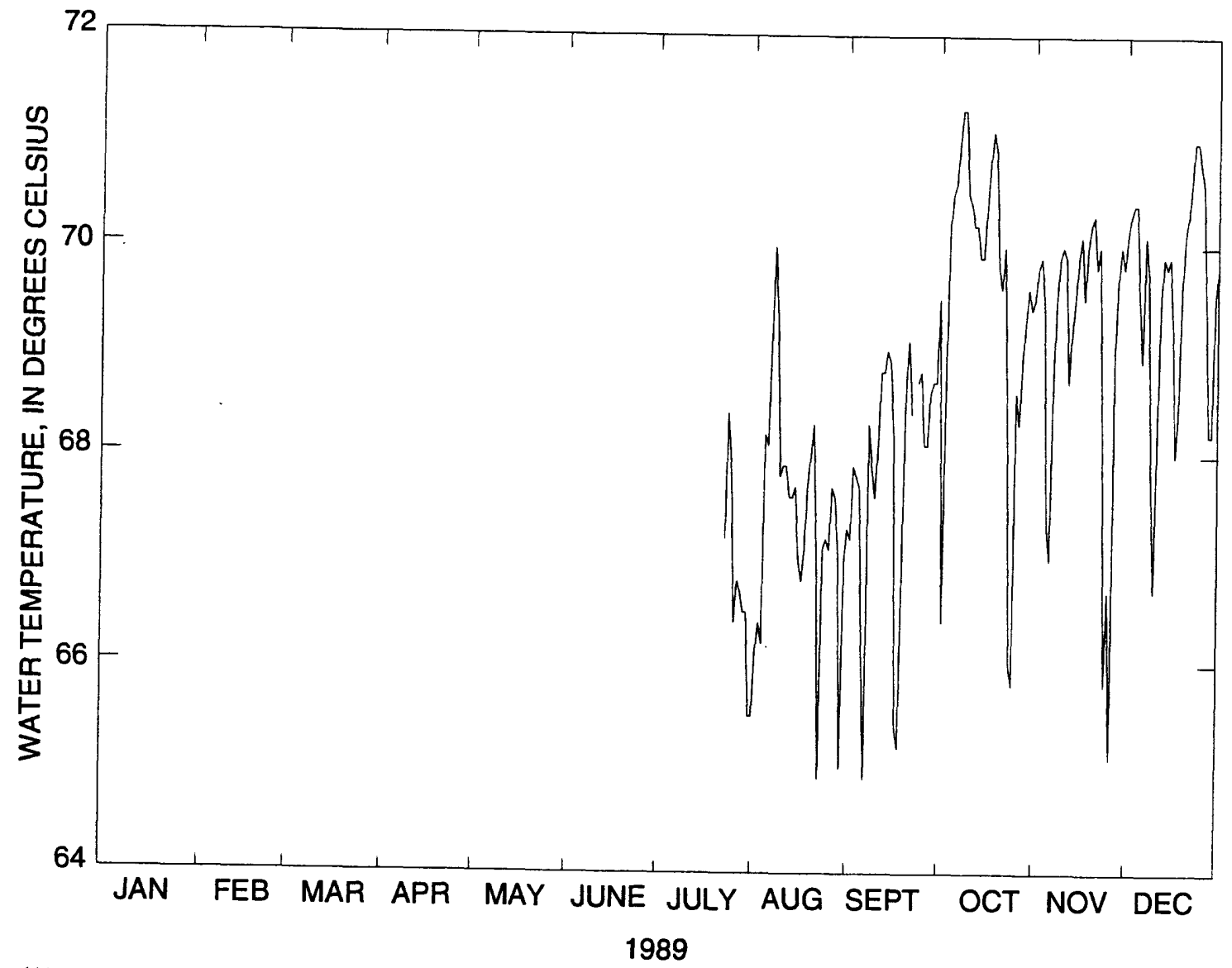

Figure 37. (A) Daily mean water temperature, Hot Bubbling Pool (HBP), Long Valley Caldera, Mono County, California, 1989. 
Table 80. Daily mean water temperature, Hot Bubbling Pool (HBP), Long Valley Caldera, Mono County, California, 1989

[Values are in degrees Celsius. Max, maximum; Min, minimum; -, no data]

\begin{tabular}{|c|c|c|c|c|c|c|c|c|c|c|c|c|}
\hline Day & Jan & Feb & Mar & Apr & May & June & July & Aug & Sept & Oct & Nov & Dec \\
\hline 1 & - & - & - & - & - & - & - & 65.5 & 67.3 & 69.5 & 69.5 & 70.1 \\
\hline 2 & - & - & - & - & - & - & - & 66.1 & 67.2 & 66.4 & 69.8 & 70.3 \\
\hline 3 & - & - & - & - & - & - & - & 66.4 & 67.9 & 68.7 & 69.9 & 70.4 \\
\hline 4 & - & - & - & - & - & - & - & 66.2 & 67.8 & 70.2 & 69.5 & 70.4 \\
\hline 5 & - & - & - & - & - & - & - & 68.2 & 67.7 & 70.5 & 67.4 & 69.5 \\
\hline 6 & - & - & - & - & - & - & - & 68.1 & 66.1 & 70.6 & 67.0 & 68.9 \\
\hline 7 & - & - & - & - & - & - & - & 69.1 & 64.9 & 71.0 & 68.7 & 70.1 \\
\hline 8 & - & - & - & - & - & - & - & 70.0 & 68.3 & 71.3 & 69.5 & 69.7 \\
\hline 9 & - & - & - & - & - & - & - & 69.3 & 67.9 & 71.3 & 69.9 & 67.8 \\
\hline 10 & - & - & - & - & - & - & - & 67.8 & 67.6 & 70.5 & 70.0 & 66.7 \\
\hline 11 & - & - & - & - & - & - & - & 67.9 & 68.1 & 70.4 & 69.9 & 68.2 \\
\hline 12 & - & - & - & - & - & - & - & 67.9 & 68.8 & 70.2 & 68.7 & 69.5 \\
\hline 13 & - & - & - & - & - & - & - & 67.6 & 68.8 & 70.2 & 69.2 & 69.9 \\
\hline 14 & - & - & - & - & - & - & - & 67.6 & 69.0 & 69.9 & 69.5 & 69.8 \\
\hline 15 & - & - & - & - & - & - & - & 67.7 & 68.9 & 69.9 & 69.9 & 69.9 \\
\hline 16 & - & - & - & - & - & - & - & 67.0 & 68.2 & 70.4 & 70.1 & 69.2 \\
\hline 17 & - & - & - & - & - & - & - & 66.8 & 65.4 & 70.8 & 69.5 & 68.0 \\
\hline 18 & - & - & - & - & - & - & - & 67.1 & 65.2 & 71.1 & 70.0 & 68.4 \\
\hline 19 & - & - & - & - & - & - & - & 67.7 & 67.1 & 70.9 & 70.2 & 69.6 \\
\hline 20 & - & - & - & - & - & - & - & 68.0 & 68.7 & 69.8 & 70.3 & 70.1 \\
\hline 21 & - & - & - & - & - & - & - & 68.3 & 69.1 & 69.6 & 69.8 & 70.3 \\
\hline 22 & - & - & - & - & - & - & - & 67.1 & 68.4 & 70.0 & 70.0 & 70.6 \\
\hline 23 & - & - & - & - & - & - & 67.2 & 64.9 & - & 68.4 & 68.3 & 71.0 \\
\hline 24 & - & - & - & - & - & - & 68.4 & 67.1 & 68.7 & 66.0 & 65.8 & 71.0 \\
\hline 25 & - & - & - & - & - & - & 67.9 & 67.2 & 68.8 & 65.8 & 66.7 & 70.8 \\
\hline 26 & - & - & - & - & - & - & 66.4 & 67.1 & 68.1 & 68.6 & 65.1 & 70.6 \\
\hline 27 & - & - & - & - & - & - & 66.8 & 67.7 & 68.1 & 68.3 & 69.0 & 69.5 \\
\hline 28 & - & - & - & - & - & - & 66.7 & 67.6 & 68.6 & 68.9 & 69.7 & 68.2 \\
\hline 29 & - & - & - & - & - & - & 66.5 & 67.1 & 68.7 & 69.3 & 70.0 & 68.2 \\
\hline 30 & - & - & - & - & - & - & 66.5 & 65.0 & 68.7 & 69.6 & 69.8 & 69.5 \\
\hline 31 & - & - & - & - & - & - & 65.5 & 67.0 & - & 69.4 & - & 69.8 \\
\hline Mean & - & - & - & - & - & - & 66.9 & 67.4 & 67.9 & 69.6 & 69.1 & 69.5 \\
\hline Max & - & - & - & - & - & - & 68.4 & 70.0 & 69.1 & 71.3 & 70.3 & 71.0 \\
\hline Min & - & - & - & - & - & - & 65.5 & 64.9 & 64.9 & 65.8 & 65.1 & 66.7 \\
\hline
\end{tabular}




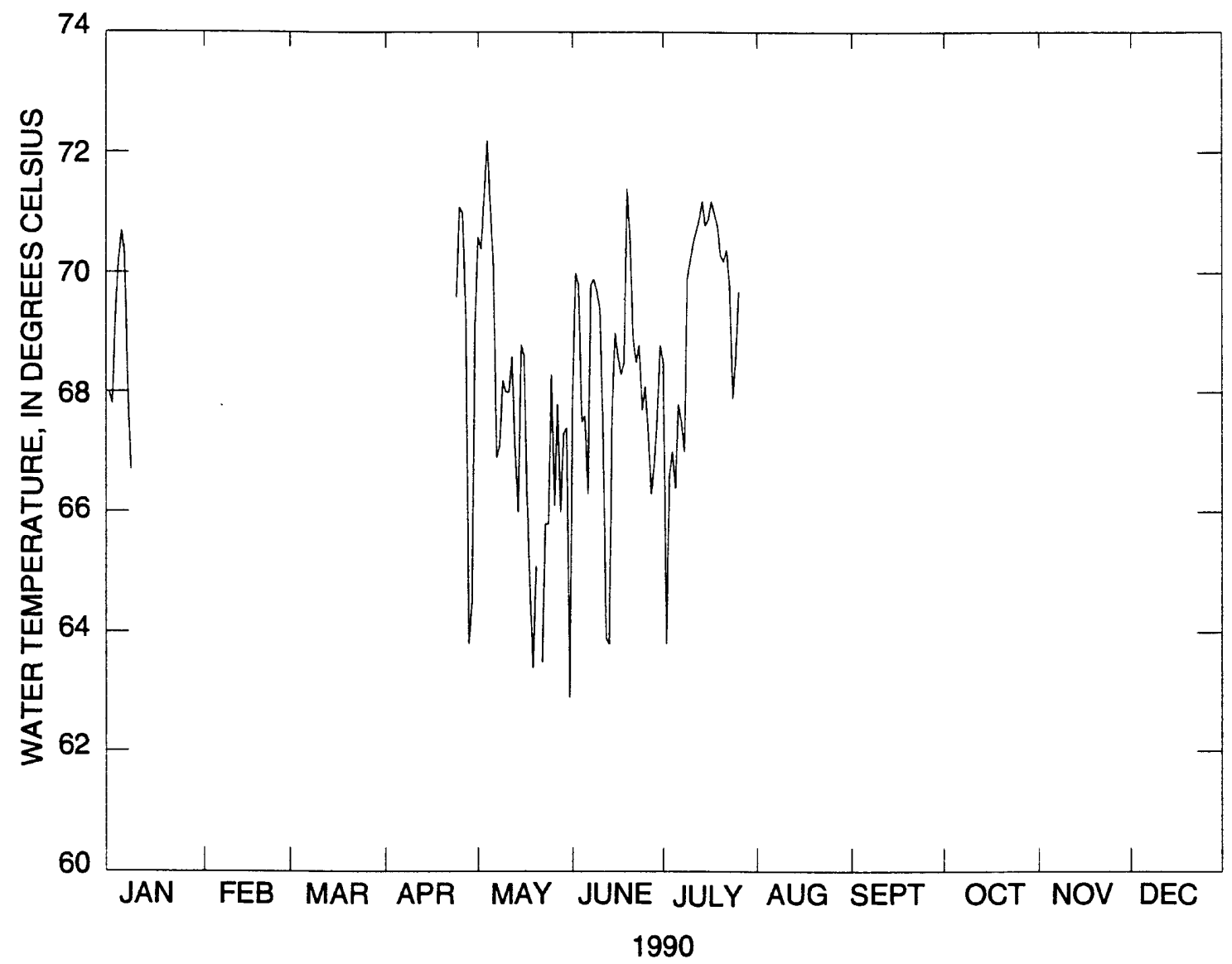

Figure 37. (B) Daily mean water temperature, Hot Bubbling Pool (HBP), Long Valley Caldera, Mono County, California, 1990. 
Table 81. Daily mean water temperature, Hot Bubbling Pool (HBP), Long Valley Caldera, Mono County, California, 1990

[Values are in degrees Celsius. Max, maximum; Min. minimum; -, no data]

\begin{tabular}{|c|c|c|c|c|c|c|c|c|c|c|c|c|}
\hline Day & Jan & Feb & Mar & Apr & May & June & July & Aug & Sept & Oct & Nov & Dec \\
\hline 1 & 68.0 & - & - & - & 70.6 & 68.0 & 68.5 & - & - & - & - & - \\
\hline 2 & 67.8 & - & - & - & 70.4 & 70.0 & 63.8 & - & - & - & - & - \\
\hline 3 & 69.3 & - & - & - & 71.3 & 69.8 & 66.6 & - & - & - & - & - \\
\hline 4 & 70.2 & - & - & - & 72.2 & 67.5 & 67.0 & - & - & - & - & - \\
\hline 5 & 70.7 & - & - & - & 71.1 & 67.6 & 66.4 & - & - & - & - & - \\
\hline 6 & 70.3 & - & - & - & 70.1 & 66.3 & 67.8 & - & - & - & - & - \\
\hline 7 & 68.1 & - & - & - & 66.9 & 69.8 & 67.5 & - & - & - & - & - \\
\hline 8 & 66.7 & - & - & - & 67.1 & 69.9 & 67.0 & - & - & - & - & - \\
\hline 9 & - & - & - & - & 68.2 & 69.7 & 69.9 & - & - & - & - & - \\
\hline 10 & - & - & - & - & 68.0 & 69.4 & 70.2 & - & - & - & - & - \\
\hline 11 & - & - & - & - & 68.0 & 67.3 & 70.5 & - & - & - & - & - \\
\hline 12 & - & - & - & - & 68.6 & 63.9 & 70.7 & - & - & - & - & - \\
\hline 13 & - & - & - & - & 67.0 & 63.8 & 70.9 & - & - & - & - & - \\
\hline 14 & - & - & - & - & 66.0 & 67.7 & 71.2 & - & - & - & - & - \\
\hline 15 & - & - & - & - & 68.8 & 69.0 & 70.8 & - & - & - & - & - \\
\hline 16 & - & - & - & - & 68.6 & 68.6 & 70.9 & - & - & - & - & - \\
\hline 17 & - & - & - & - & 66.3 & 68.3 & 71.2 & - & - & - & - & - \\
\hline 18 & - & - & - & - & 64.7 & 68.5 & 71.0 & - & - & - & - & - \\
\hline 19 & - & - & - & - & 63.4 & 71.4 & 70.8 & - & - & - & - & - \\
\hline 20 & - & - & - & - & 65.1 & 70.6 & 70.3 & - & - & - & - & - \\
\hline 21 & - & - & - & - & - & 68.9 & 70.2 & - & - & - & - & - \\
\hline 22 & - & - & - & - & 63.5 & 68.5 & 70.4 & - & - & - & - & - \\
\hline 23 & - & - & - & - & 65.8 & 68.8 & 69.8 & - & - & - & - & - \\
\hline 24 & - & - & - & 69.6 & 65.8 & 67.7 & 67.9 & - & - & - & - & - \\
\hline 25 & - & - & - & 71.1 & 68.3 & 68.1 & 68.6 & - & - & - & - & - \\
\hline 26 & - & - & - & 71.0 & 66.1 & 67.3 & 69.7 & - & - & - & - & - \\
\hline 27 & - & - & - & 69.4 & 67.8 & 66.3 & - & - & - & - & - & - \\
\hline 28 & - & - & - & 63.8 & 66.0 & 66.8 & - & - & - & - & - & - \\
\hline 29 & - & - & - & 64.5 & 67.3 & 67.6 & - & - & - & - & - & - \\
\hline 30 & - & - & - & 69.1 & 67.4 & 68.8 & - & - & - & - & - & - \\
\hline 31 & - & - & - & - & 62.9 & - & - & - & - & - & - & - \\
\hline Mean & 68.9 & - & - & 68.4 & 67.4 & 68.2 & 69.2 & - & - & - & - & - \\
\hline $\operatorname{Max}$ & 70.7 & - & - & 71.1 & 72.2 & 71.4 & 71.2 & - & - & - & - & - \\
\hline Min & 66.7 & - & - & 63.8 & 62.9 & 63.8 & 63.8 & - & - & - & - & - \\
\hline
\end{tabular}




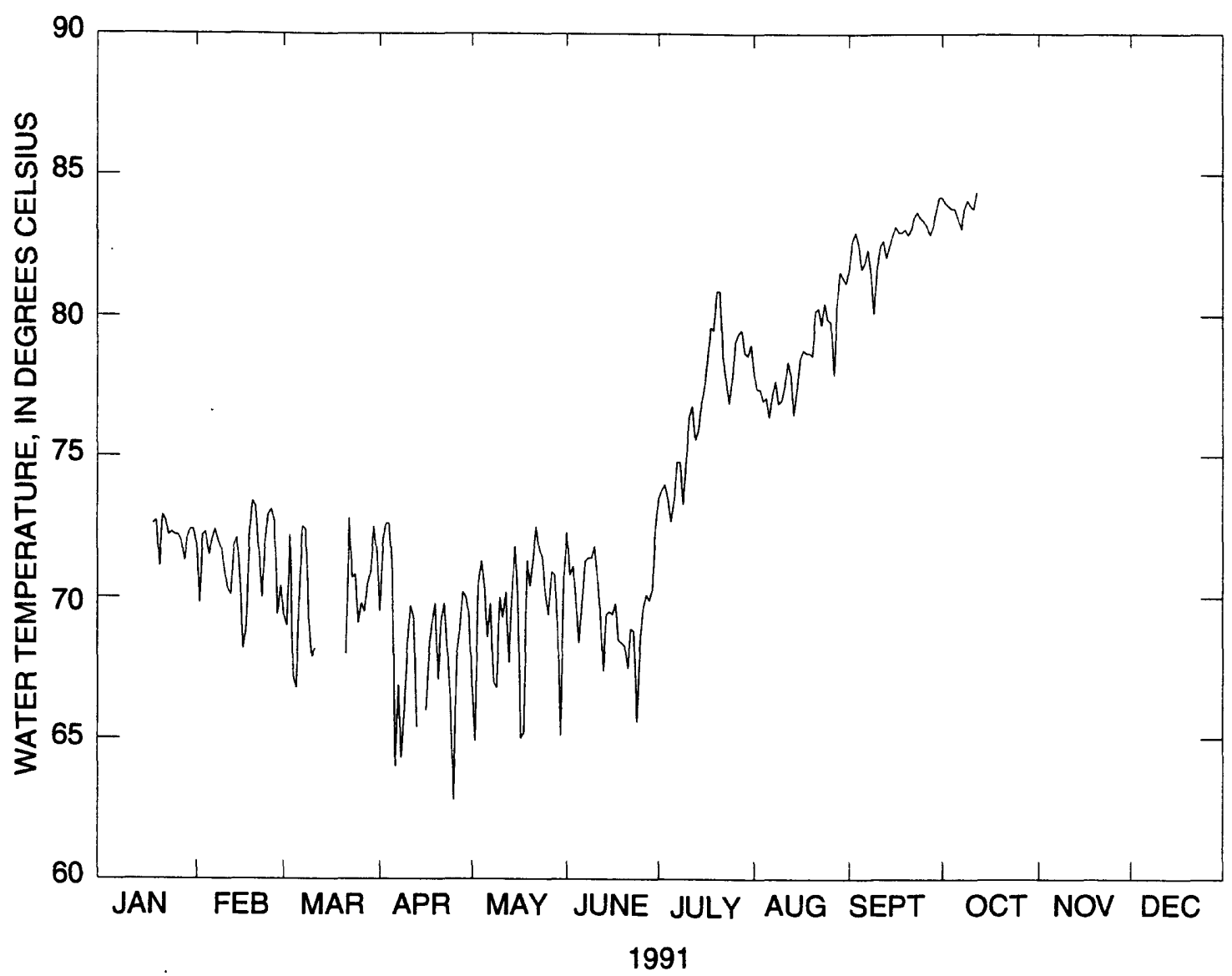

Figure 37. (C) Daily mean water temperature, Hot Bubbling Pool (HBP), Long Valley Caldera, Mono County, California, 1991. 
Table 82. Daily mean water temperature, Hot Bubbling Pool (HBP), Long Valley Caldera, Mono County, California, 1991

[Values are in degrees Celsius. Max, maximum; Min, minimum; -, no data]

\begin{tabular}{|c|c|c|c|c|c|c|c|c|c|c|c|c|}
\hline Day & Jan & Feb & Mar & Apr & May & June & July & Aug & Sept & Oct & Nov & Dec \\
\hline 1 & - & 71.9 & 69.4 & 69.5 & 66.9 & 72.3 & 73.5 & 78.0 & 81.7 & 84.2 & - & - \\
\hline 2 & - & 69.8 & 69.0 & 72.0 & 64.9 & 70.8 & 73.8 & 77.4 & 82.7 & 84.0 & - & - \\
\hline 3 & - & 72.2 & 72.2 & 72.6 & 70.5 & 71.1 & 74.0 & 77.4 & 83.0 & 83.9 & - & - \\
\hline 4 & - & 72.3 & 67.2 & 72.6 & 71.3 & 69.9 & 73.5 & 77.0 & 82.6 & 83.8 & - & - \\
\hline 5 & - & 71.5 & 66.8 & 71.0 & 70.4 & 68.4 & 72.7 & 77.1 & 81.7 & 83.8 & - & - \\
\hline 6 & - & 72.0 & 70.2 & 64.0 & 68.6 & 69.8 & 73.4 & 76.4 & 81.9 & 83.5 & - & - \\
\hline 7 & - & 72.4 & 72.5 & 66.9 & 69.8 & 71.3 & 74.8 & 77.2 & 82.4 & 83.1 & - & - \\
\hline 8 & - & 72.0 & 72.4 & 64.3 & 67.0 & 71.4 & 74.8 & 77.7 & 81.6 & 83.8 & - & - \\
\hline 9 & - & 71.7 & 69.3 & 66.1 & 66.8 & 71.4 & 73.3 & 76.9 & 80.1 & 84.1 & - & - \\
\hline 10 & - & 70.9 & 67.9 & 68.4 & 70.0 & 71.8 & 74.9 & 77.0 & 81.7 & 83.9 & - & - \\
\hline 11 & - & 70.3 & 68.2 & 69.7 & 69.3 & 70.7 & 76.4 & 77.5 & 82.5 & 83.8 & - & - \\
\hline 12 & - & 70.1 & - & 69.3 & 70.2 & 69.5 & 76.8 & 78.4 & 82.7 & 84.4 & - & - \\
\hline 13 & - & 71.8 & - & 65.4 & 67.7 & 67.4 & 75.6 & 77.9 & 82.1 & - & - & - \\
\hline 14 & - & 72.1 & - & - & 70.2 & 69.4 & 75.9 & 76.5 & 82.5 & - & - & - \\
\hline 15 & - & 70.6 & - & - & 71.8 & 69.5 & 76.9 & 77.4 & 82.9 & - & - & - \\
\hline 16 & - & 68.2 & - & 66.0 & 70.1 & 69.4 & 77.5 & 78.5 & 83.2 & - & - & - \\
\hline 17 & - & 68.9 & - & 68.1 & 65.0 & 69.8 & 78.6 & 78.8 & 83.0 & - & - & - \\
\hline 18 & 72.6 & 72.2 & - & 69.1 & 65.2 & 68.5 & 79.6 & 78.7 & 83.0 & - & - & - \\
\hline 19 & 72.7 & 73.4 & - & 69.8 & 71.3 & 68.4 & 79.5 & 78.7 & 83.1 & - & - & - \\
\hline 20 & 71.1 & 73.2 & - & 67.1 & 70.4 & 68.3 & 80.9 & 78.6 & 82.9 & - & - & - \\
\hline 21 & 72.9 & 71.7 & 68.0 & 69.2 & 71.4 & 67.5 & 80.9 & 80.2 & 83.1 & - & - & - \\
\hline 22 & 72.7 & 70.0 & 72.8 & 69.8 & 72.5 & 68.9 & 78.5 & 80.3 & 83.5 & - & - & - \\
\hline 23 & 72.2 & 71.9 & 70.7 & 68.2 & 71.7 & 68.8 & 77.7 & 79.7 & 83.7 & - & - & - \\
\hline 24 & 72.3 & 72.9 & 70.8 & 66.3 & 71.4 & 65.6 & 76.9 & 80.5 & 83.5 & - & - & - \\
\hline 25 & 72.2 & 73.1 & 69.1 & 62.8 & 70.1 & 68.3 & 77.8 & 79.9 & 83.4 & - & - & - \\
\hline 26 & 72.2 & 72.7 & 69.8 & 67.9 & 69.4 & 69.6 & 79.1 & 79.8 & 83.2 & - & - & - \\
\hline 27 & 72.0 & 69.4 & 69.5 & 68.8 & 70.9 & 70.1 & 79.4 & 77.9 & 82.9 & - & - & - \\
\hline 28 & 71.3 & 70.4 & 70.5 & 70.2 & 70.8 & 69.9 & 79.5 & 80.4 & 83.2 & - & - & - \\
\hline 29 & 72.1 & - & 70.9 & 70.0 & 69.1 & 70.3 & 78.7 & 81.6 & 83.7 & - & - & - \\
\hline 30 & 72.4 & - & 72.5 & 69.4 & 65.1 & 72.5 & 78.6 & 81.4 & 84.2 & - & - & - \\
\hline 31 & 72.4 & - & 71.6 & - & 70.7 & - & 79.0 & 81.2 & - & - & - & - \\
\hline Mean & 72.2 & 71.4 & 70.1 & 68.4 & 69.4 & 69.7 & 76.9 & 78.6 & 82.7 & 83.9 & - & - \\
\hline Max & 72.9 & 73.4 & 72.8 & 72.6 & 72.5 & 72.5 & 80.9 & 81.6 & 84.2 & 84.4 & - & - \\
\hline Min & 71.1 & 68.2 & 66.8 & 62.8 & 64.9 & 65.6 & 72.7 & 76.4 & 80.1 & 83.1 & - & - \\
\hline
\end{tabular}




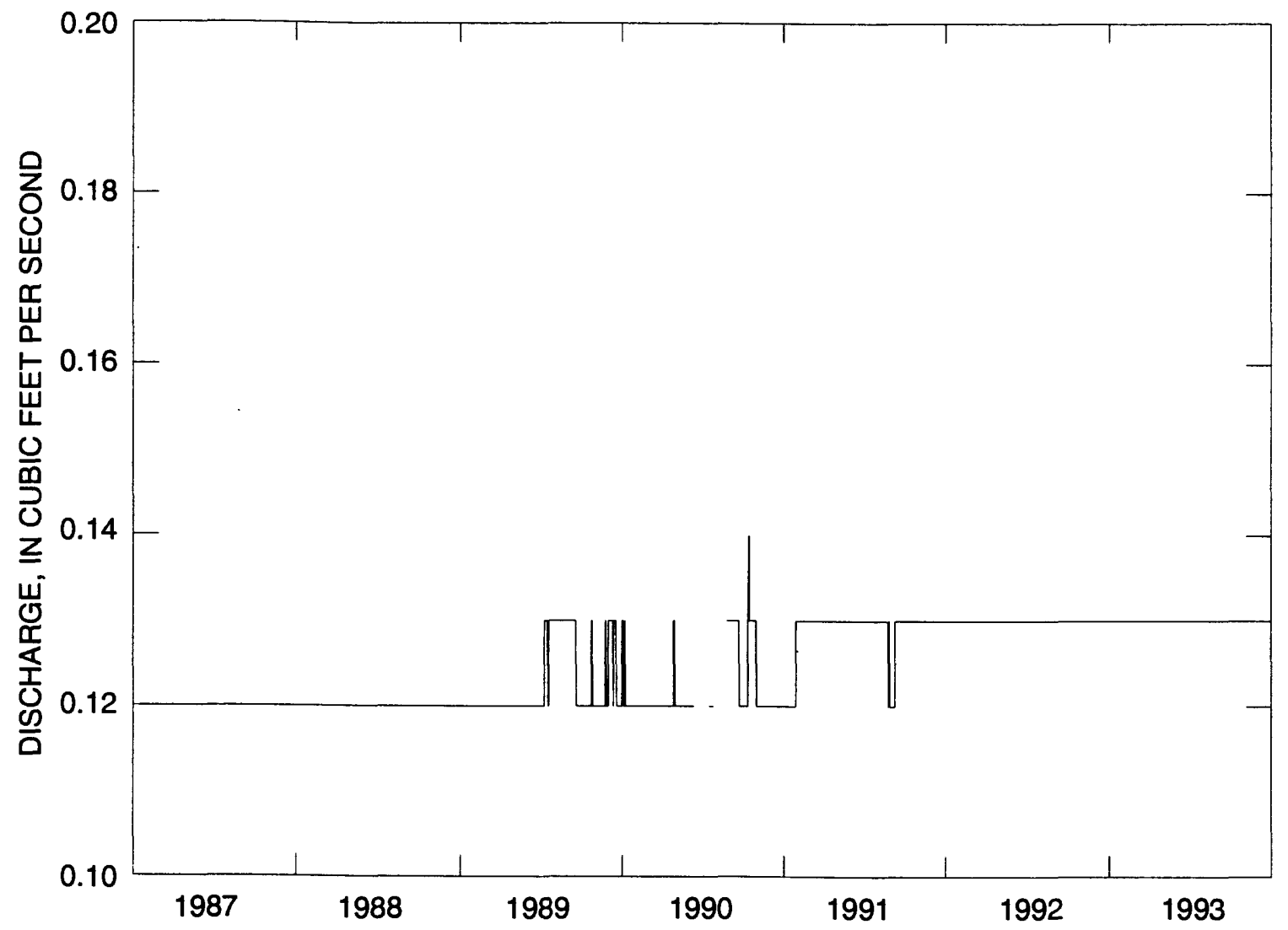

Figure 38. Daily mean discharge, Little Hot Creek Spring (LHC-SP), Long Valley Caldera, Mono County, California, 1987-93. 
Table 83. Monthly mean discharge, Little Hot Creek Spring (LHC-SP), Long Valley Caldera, Mono County, California, 1987-93

[Values are in cubic feet per second]

\begin{tabular}{ccccccccccccc}
\hline Year & Jan & Feb & Mar & Apr & May & June & July & Aug & Sept & Oct & Nov & Dec \\
\hline 1987 & 0.12 & .12 & 0.12 & 0.12 & 0.12 & 0.12 & 0.12 & 0.12 & 0.12 & 0.12 & 0.12 & 0.12 \\
1988 & .12 & .12 & .12 & .12 & .12 & .12 & .12 & .12 & .12 & .12 & .12 & .12 \\
1989 & .12 & .12 & .12 & .12 & .12 & .12 & .13 & .13 & .13 & .12 & .12 & .13 \\
1990 & .12 & .12 & .12 & .12 & .12 & .12 & .12 & .13 & .13 & .13 & .12 & .12 \\
1991 & .12 & .13 & .13 & .13 & .13 & .13 & .13 & .13 & .13 & .13 & .13 & .13 \\
1992 & .13 & .13 & .13 & .13 & .13 & .13 & .13 & .13 & .13 & .13 & .13 & .13 \\
1993 & .13 & .13 & .13 & .13 & .13 & .13 & .13 & .13 & .13 & .13 & .13 & .13 \\
\hline
\end{tabular}


Table 84. Miscellaneous discharge and water temperature, Big Alkali Lake Spring (BAL), Long Valley Caldera, Mono County, California, 1988-93

[Discharge is in cubic feet per second; water temperature is in degrees Celsius. - no data]

\begin{tabular}{|c|c|c|}
\hline Date & Discharge & $\begin{array}{c}\text { Water } \\
\text { temperature }\end{array}$ \\
\hline $06-22-88$ & 2.12 & 57.5 \\
\hline $07-14-88$ & 1.78 & 57.6 \\
\hline $08-11-88$ & 1.15 & 58.4 \\
\hline $09-14-88$ & 1.15 & 58.2 \\
\hline $10-17-88$ & 1.45 & 58.1 \\
\hline $11-22-88$ & 2.31 & 57.7 \\
\hline $12-13-88$ & 2.50 & 56.2 \\
\hline $01-12-89$ & 1.44 & 57.4 \\
\hline $02-16-89$ & 2.12 & 57.2 \\
\hline $03-20-89$ & 2.12 & 57.0 \\
\hline $04-10-89$ & 1.95 & 57.2 \\
\hline $05-17-89$ & 1.95 & 57.4 \\
\hline $06-26-89$ & 2.12 & 57.6 \\
\hline $07-17-89$ & 1.61 & 57.7 \\
\hline $08-17-89$ & 1.95 & 57.9 \\
\hline $09-20-89$ & 2.12 & 57.6 \\
\hline $10-16-89$ & 2.49 & 57.3 \\
\hline $11-22-89$ & 2.49 & 57.4 \\
\hline $12-19-89$ & 1.95 & 57.4 \\
\hline $01-10-90$ & 2.69 & 57.1 \\
\hline $02-20-90$ & 3.09 & 56.5 \\
\hline $03-21-90$ & 2.69 & 56.8 \\
\hline $04-25-90$ & 2.69 & 56.7 \\
\hline $05-23-90$ & 2.49 & 57.0 \\
\hline $07-17-90$ & 2.32 & - \\
\hline $05-01-91$ & 1.64 & 56.4 \\
\hline $06-16-91$ & 1.64 & - \\
\hline $04-23-92$ & 1.53 & 56.6 \\
\hline $08-14-92$ & 1.44 & 57.8 \\
\hline $07-14-93$ & 1.94 & 57.0 \\
\hline $12-16-93$ & 2.31 & 57.4 \\
\hline
\end{tabular}




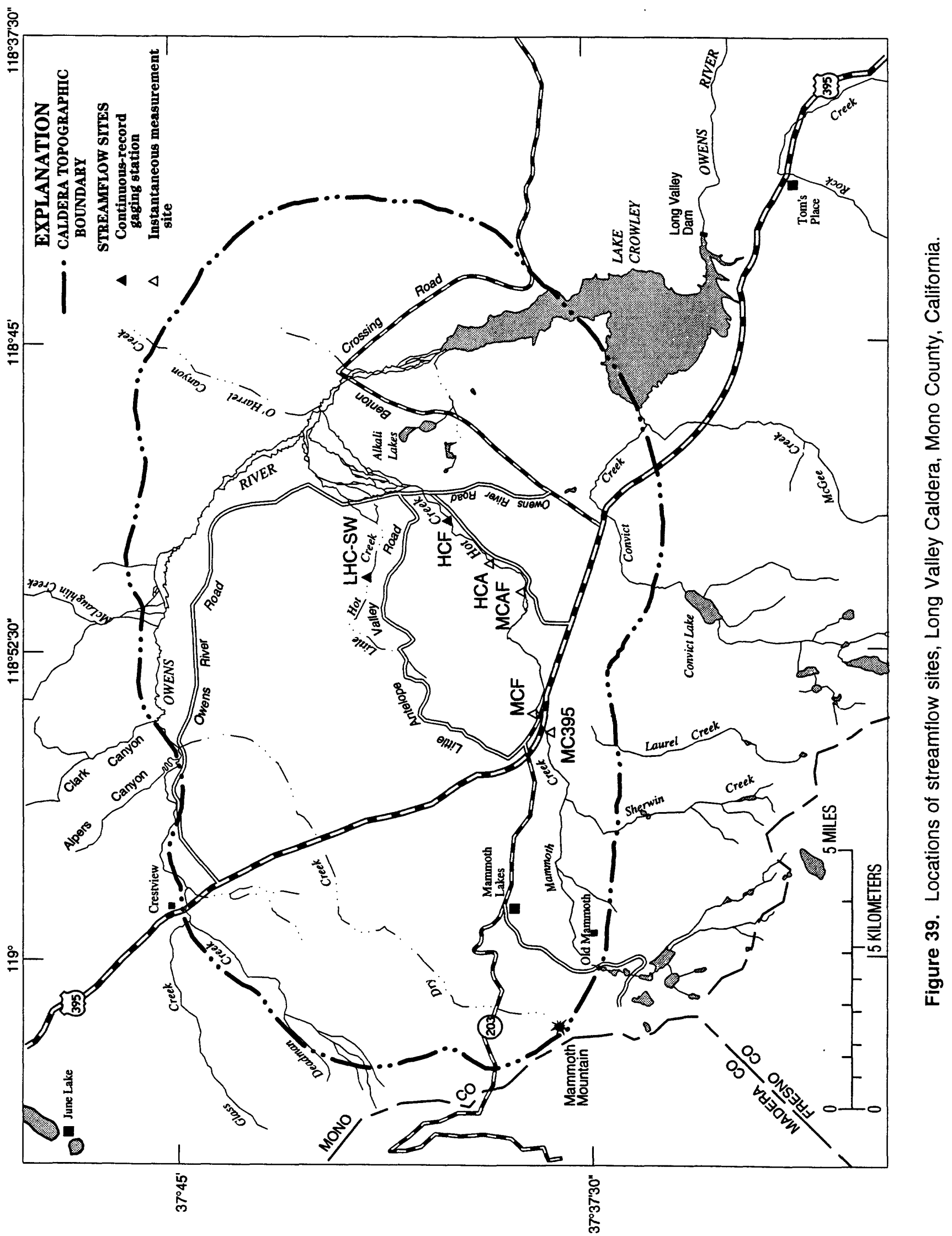


Table 85. Instantaneous discharge measurements, Mammoth Creek at Highway 395 (MC395), Long Valley Caldera, Mono County, California, 1988-93

[Discharge is in cubic feet per second]

\begin{tabular}{cc}
\hline Date & Discharge \\
\hline $07-13-88$ & 12.9 \\
$10-18-88$ & 3.48 \\
$01-11-89$ & 1.04 \\
$04-11-89$ & 9.14 \\
$07-18-89$ & 8.97 \\
$10-17-89$ & \\
$01-09-90$ & 3.90 \\
$04-25-90$ & 4.32 \\
$07-17-90$ & 10.4 \\
$10-16-90$ & 7.74 \\
$01-17-91$ & 2.03 \\
$04-16-91$ & 3.24 \\
$07-22-91$ & 2.36 \\
$10-22-91$ & 14.4 \\
$01-13-92$ & 3.59 \\
$04-14-92$ & 4.36 \\
$07-06-92$ & \\
$10-19-92$ & 3.74 \\
$04-12-93$ & 10.3 \\
$07-12-93$ & 4.14 \\
$10-13-93$ & 7.88 \\
\hline
\end{tabular}

Table 87. Instantaneous discharge measurements, Mammoth Creek above Hot Creek Fish Hatchery (MCAF), Long Valley Caldera, Mono County, California, 1988-93

[Discharge is in cubic feet per second]

\begin{tabular}{cc}
\hline Date & Discharge \\
\hline $07-13-88$ & 5.81 \\
$10-19-88$ & 2.09 \\
$01-11-89$ & Frozen solid \\
$04-11-89$ & 8.74 \\
$07-18-89$ & 5.27 \\
$10-18-89$ & \\
$01-09-90$ & 2.09 \\
$04-25-90$ & 5.32 \\
$07-17-90$ & 3.49 \\
$10-16-90$ & 3.28 \\
& 1.30 \\
$01-17-91$ & \\
$04-16-91$ & 1.25 \\
$07-22-91$ & 3.09 \\
$10-22-91$ & 2.33 \\
$01-13-92$ & 2.78 \\
$04-14-92$ & 4.57 \\
$07-06-92$ & \\
$10-19-92$ & 4.16 \\
$01-25-93$ & 5.03 \\
$04-12-93$ & 2.24 \\
$07-13-93$ & 3.40 \\
$10-13-93$ & 8.72 \\
\hline
\end{tabular}

Table 86. Instantaneous discharge measurements and gage heights, Mammoth Creek Flume (MCF), Long Valley Caldera, Mono County, California, 1988-90

[Discharge is in cubic feet per second; gage height is in feet relative to an arbitrary datum]

\begin{tabular}{ccc}
\hline Date & Discharge & Gage height \\
\hline $07-13-88$ & 12.0 & 0.87 \\
$10-19-88$ & 3.08 & .29 \\
$01-11-89$ & 3.59 & .34 \\
$04-11-89$ & 8.30 & .64 \\
$07-18-89$ & 7.77 & .45 \\
& & \\
$10-17-89$ & 3.04 & .28 \\
$01-09-90$ & 4.51 & .43 \\
$04-25-90$ & 8.82 & .72 \\
$07-12-90$ & 7.01 & .56 \\
\hline
\end{tabular}


Table 88. Instantaneous discharge measurements, Hot Creek above Gorge Geyser (HCA), Long Valley Caldera, Mono County, California, 1988-93

[Gage height is in feet relative to an arbitrary datum; discharge is in cubic feet per second. - , no data]

\begin{tabular}{|c|c|c|c|c|c|}
\hline Date & Gage height & Discharge & Date & Gage height & Discharge \\
\hline $06-10-88$ & - & 52.2 & $01-17-91$ & 0.61 & 23.2 \\
\hline $06-20-88$ & - & 55.2 & $02-15-91$ & .56 & 23.7 \\
\hline $07-07-88$ & - & 42.1 & $03-21-91$ & .58 & 28.7 \\
\hline $07-12-88$ & - & 43.6 & $04-17-91$ & .62 & 27.7 \\
\hline 08-03-88 & 0.86 & 35.0 & $05-16-91$ & .72 & 33.9 \\
\hline $08-10-88$ & .86 & 36.3 & $06-21-91$ & .95 & 62.3 \\
\hline $09-06-88$ & .88 & 31.8 & $07-23-91$ & .79 & 30.7 \\
\hline $09-13-88$ & .89 & 30.5 & $08-22-91$ & .90 & 32.9 \\
\hline $10-19-88$ & .90 & 31.2 & $09-18-91$ & .93 & 30.3 \\
\hline $11-21-88$ & .90 & 31.1 & $10-23-91$ & .90 & 26.4 \\
\hline $12-14-88$ & .87 & 27.4 & $12-12-91$ & .86 & 26.6 \\
\hline 01-09-89 & .89 & 28.9 & $01-14-92$ & .70 & 25.8 \\
\hline $02-15-89$ & .85 & 31.4 & $02-20-92$ & .61 & 27.0 \\
\hline $03-21-89$ & .81 & 30.9 & $03-12-92$ & .65 & 25.7 \\
\hline $04-12-89$ & .86 & 35.6 & $04-16-92$ & .74 & 26.6 \\
\hline $05-16-89$ & .96 & 50.2 & $05-13-92$ & .88 & 41.8 \\
\hline $06-27-89$ & .93 & 47.8 & $06-09-92$ & .81 & 31.3 \\
\hline $07-19-89$ & .96 & 36.2 & $07-08-92$ & 1.00 & 31.6 \\
\hline $08-15-89$ & .95 & 33.6 & $08-04-92$ & .82 & 26.7 \\
\hline $09-19-89$ & 1.00 & 31.0 & $09-15-92$ & .74 & 24.3 \\
\hline $10-17-89$ & .92 & 28.7 & $10-21-92$ & .72 & 22.6 \\
\hline $11-21-89$ & .88 & 29.8 & $11-17-92$ & .72 & 25.8 \\
\hline $12-20-89$ & .84 & 29.5 & $12-23-92$ & .67 & 24.4 \\
\hline $01-10-90$ & .81 & 29.5 & $01-27-93$ & .64 & 24.8 \\
\hline $02-21-90$ & .70 & 27.9 & $03-02-93$ & .62 & 26.0 \\
\hline 03-20-90 & .72 & 29.7 & $03-24-93$ & .81 & 50.6 \\
\hline $04-24-90$ & .74 & 27.1 & $04-13-93$ & .80 & 35.5 \\
\hline $05-25-90$ & .80 & 35.9 & $05-20-93$ & 1.37 & 102 \\
\hline $06-11-90$ & .94 & 52.6 & $06-15-93$ & 1.45 & 137 \\
\hline $07-18-90$ & .90 & 30.1 & $07-14-93$ & 1.32 & 97.2 \\
\hline $08-28-90$ & .87 & 29.3 & $08-19-93$ & 1.10 & 54.6 \\
\hline $09-26-90$ & .87 & 28.7 & $09-08-93$ & 1.12 & 51.8 \\
\hline $10-17-90$ & .84 & 26.1 & $10-14-93$ & 1.04 & 39.6 \\
\hline $11-15-90$ & .80 & 26.2 & $11-18-93$ & .89 & 35.6 \\
\hline $12-19-90$ & .75 & 25.0 & $12-14-93$ & .81 & 31.5 \\
\hline
\end{tabular}


This page intentionally left blank. 


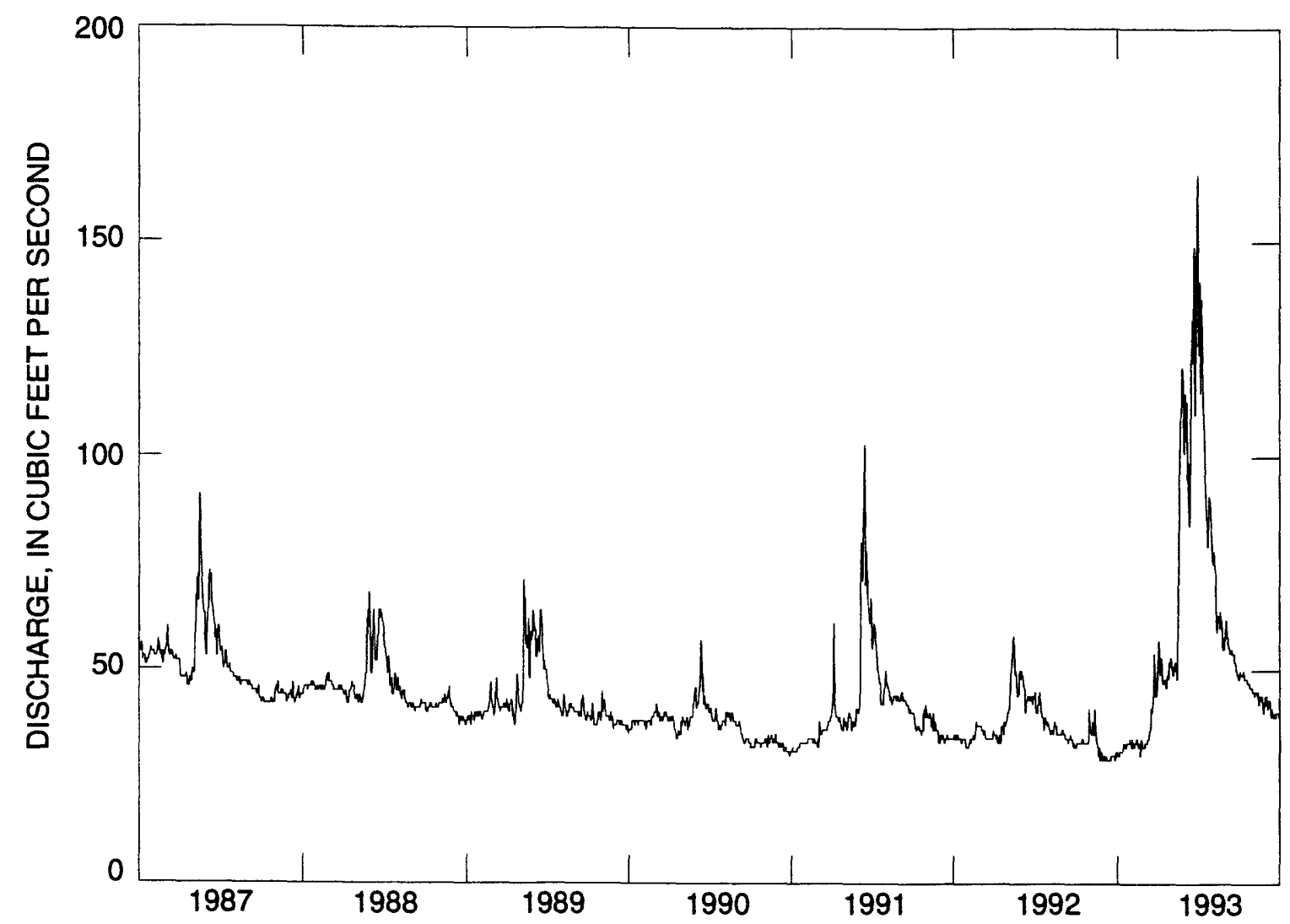

Figure 40. Daily mean discharge, Hot Creek Flume (HCF), Long Valley Caldera, Mono County, California, 1987-93. 


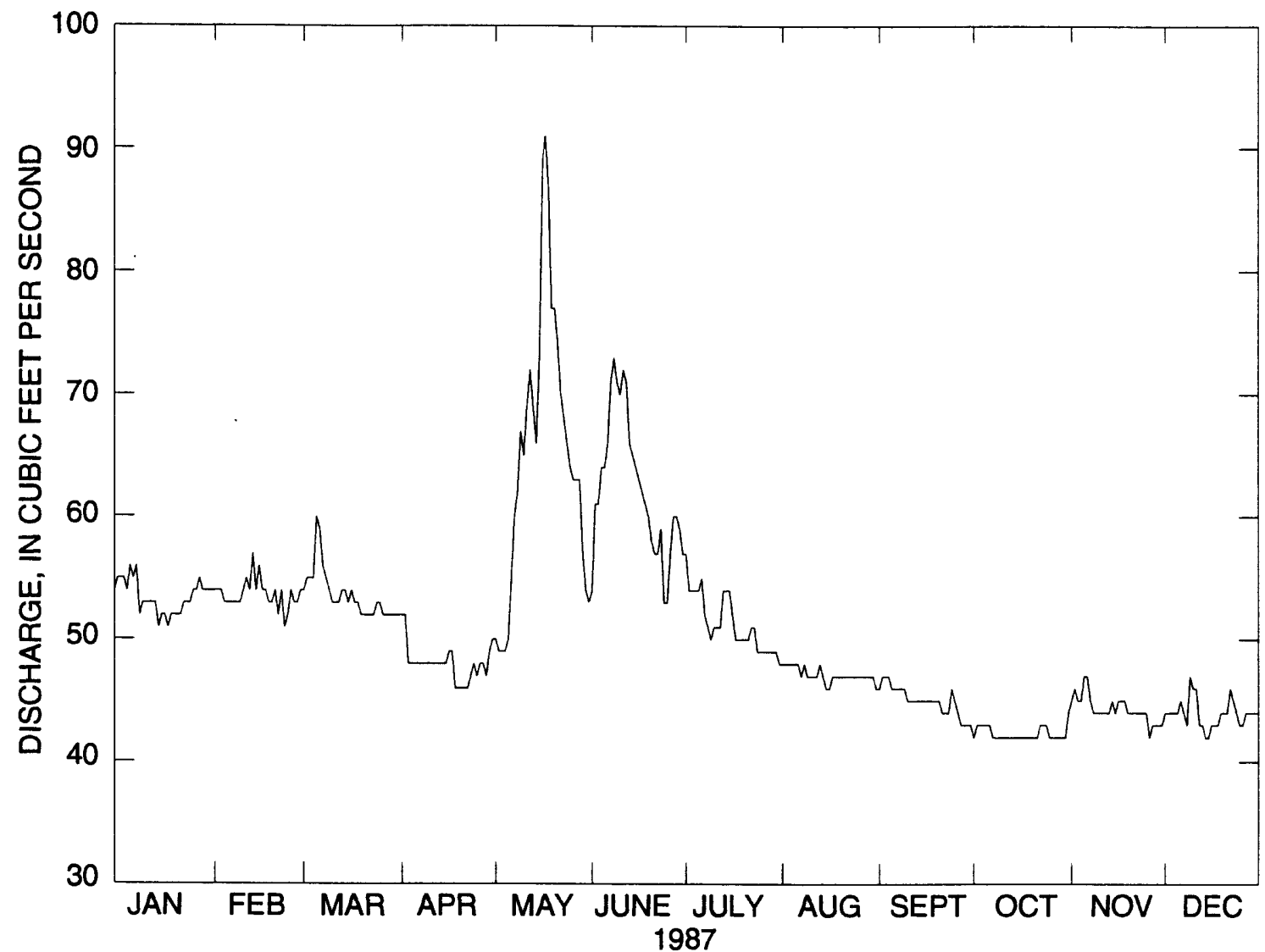

Figure 41. (A) Daily mean discharge, Hot Creek Flume (HCF), Long Valley Caldera, Mono County, California, 1987. 
Table 89. Daily mean discharge, Hot Creek Flume (HCF), Long Valley Caldera, Mono County, California, 1987 [Values are in cubic feet per second. Max, maximum; Min, minimum; -, no data]

\begin{tabular}{|c|c|c|c|c|c|c|c|c|c|c|c|c|}
\hline Day & Jan & Feb & Mar & Apr & May & June & July & Aug & Sept & Oct & Nov & Dec \\
\hline 1 & 55 & 54 & 54 & 52 & 50 & 54 & 57 & 48 & 46 & 42 & 45 & 44 \\
\hline 2 & 55 & 54 & 55 & 52 & 49 & 61 & 54 & 48 & 47 & 43 & 46 & 44 \\
\hline 3 & 55 & 54 & 55 & 48 & 49 & 61 & 54 & 48 & 47 & 43 & 45 & 44 \\
\hline 4 & 54 & 53 & 55 & 48 & 49 & 64 & 54 & 48 & 47 & 43 & 45 & 44 \\
\hline 5 & 56 & 53 & 60 & 48 & 50 & 64 & 54 & 48 & 46 & 43 & 47 & 44 \\
\hline 6 & 55 & 53 & 59 & 48 & 55 & 66 & 55 & 48 & 46 & 43 & 47 & 45 \\
\hline 7 & 56 & 53 & 56 & 48 & 60 & 71 & 52 & 47 & 46 & 42 & 45 & 44 \\
\hline 8 & 52 & 53 & 55 & 48 & 62 & 73 & 51 & 48 & 46 & 42 & 44 & 43 \\
\hline 9 & 53 & 53 & 54 & 48 & 67 & 71 & 50 & 47 & 46 & 42 & 44 & 47 \\
\hline 10 & 53 & 54 & 53 & 48 & 65 & 70 & 51 & 47 & 45 & 42 & 44 & 46 \\
\hline 11 & 53 & 55 & 53 & 48 & 69 & 72 & 51 & 47 & 45 & 42 & 44 & 46 \\
\hline 12 & 53 & 54 & 53 & 48 & 72 & 71 & 51 & 47 & 45 & 42 & 44 & 43 \\
\hline 13 & 53 & 57 & 54 & 48 & 69 & 66 & 54 & 48 & 45 & 42 & 44 & 43 \\
\hline 14 & 51 & 54 & 54 & 48 & 66 & 65 & 54 & 47 & 45 & 42 & 45 & 42 \\
\hline 15 & 52 & 56 & 53 & 48 & 73 & 64 & 54 & 46 & 45 & 42 & 44 & 42 \\
\hline 16 & 52 & 54 & 54 & 49 & 89 & 63 & 52 & 46 & 45 & 42 & 45 & 43 \\
\hline 17 & 51 & 54 & 53 & 49 & 91 & 62 & 50 & 47 & 45 & 42 & 45 & 43 \\
\hline 18 & 52 & 53 & 53 & 46 & 87 & 61 & 50 & 47 & 45 & 42 & 45 & 43 \\
\hline 19 & 52 & 53 & 52 & 46 & 77 & 60 & 50 & 47 & 45 & 42 & 44 & 44 \\
\hline 20 & 52 & 54 & 52 & 46 & 77 & 58 & 50 & 47 & 45 & 42 & 44 & 44 \\
\hline 21 & 52 & 52 & 52 & 46 & 74 & 57 & 50 & 47 & 44 & 42 & 44 & 44 \\
\hline 22 & 53 & 54 & 52 & 46 & 70 & 57 & 51 & 47 & 44 & 43 & 44 & 46 \\
\hline 23 & 53 & 51 & 52 & 47 & 68 & 59 & 51 & 47 & 44 & 43 & 44 & 45 \\
\hline 24 & 53 & 52 & 53 & 48 & 66 & 53 & 49 & 47 & 46 & 43 & 44 & 44 \\
\hline 25 & 54 & 54 & 53 & 47 & 64 & 53 & 49 & 47 & 45 & 42 & 44 & 43 \\
\hline 26 & 54 & 53 & 52 & 48 & 63 & 57 & 49 & 47 & 44 & 42 & 42 & 43 \\
\hline 27 & 55 & 53 & 52 & 48 & 63 & 60 & 49 & 47 & 43 & 42 & 43 & 44 \\
\hline 28 & 54 & 54 & 52 & 47 & 63 & 60 & 49 & 47 & 43 & 42 & 43 & 44 \\
\hline 29 & 54 & - & 52 & 49 & 57 & 59 & 49 & 47 & 43 & 42 & 43 & 44 \\
\hline 30 & 54 & - & 52 & 50 & 54 & 57 & 49 & 47 & 43 & 42 & 43 & 44 \\
\hline 31 & 54 & - & 52 & - & 53 & - & 48 & 46 & - & 44 & - & 44 \\
\hline Mean & 53.4 & 53.6 & 53.6 & 48.0 & 65.2 & 62.3 & 51.3 & 47.2 & 45.0 & 42.3 & 44.3 & 44.0 \\
\hline $\operatorname{Max}$ & 56 & 57 & 60 & 52 & 91 & 73 & 57 & 48 & 47 & 44 & 47 & 47 \\
\hline Min & 51 & 51 & 52 & 46 & 49 & 53 & 48 & 46 & 43 & 42 & 42 & 42 \\
\hline
\end{tabular}




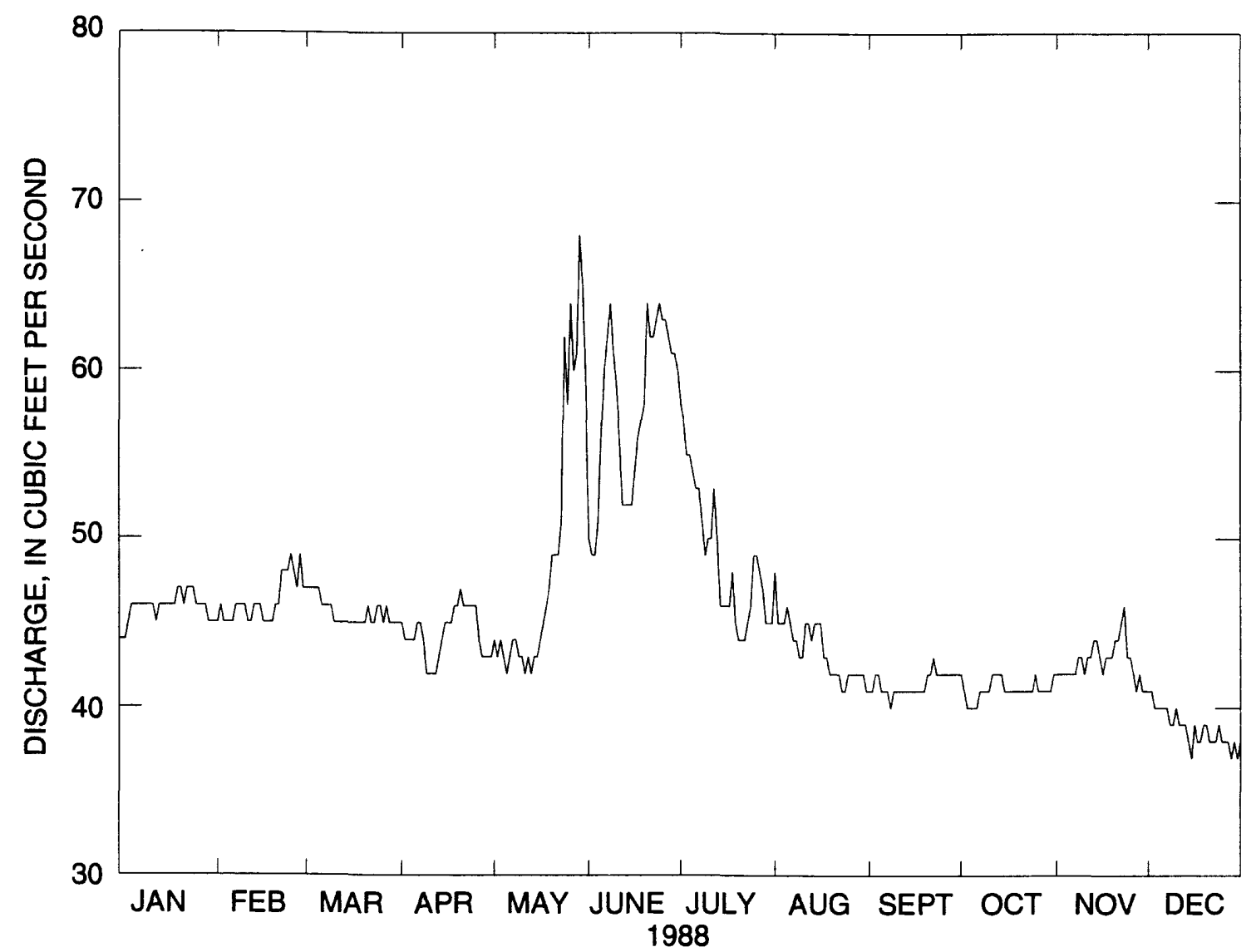

Figure 41. (B) Daily mean discharge, Hot Creek Flume (HCF), Long Valley Caldera, Mono County, California, 1988. 
Table 90. Daily mean discharge, Hot Creek Flume (HCF), Long Valley Caldera, Mono County, California, 1988 [Values are in cubic feet per second. Max, maximum; Min, minimum; - , no data]

\begin{tabular}{|c|c|c|c|c|c|c|c|c|c|c|c|c|}
\hline Day & Jan & Feb & Mar & Apr & May & June & July & Aug & Sept & Oct & Nov & Dec \\
\hline 1 & 44 & 45 & 47 & 45 & 44 & 50 & 58 & 48 & 41 & 42 & 42 & 41 \\
\hline 2 & 44 & 46 & 47 & 44 & 43 & 49 & 57 & 45 & 41 & 41 & 42 & 41 \\
\hline 3 & 45 & 45 & 47 & 44 & 44 & 49 & 55 & 45 & 42 & 40 & 42 & 40 \\
\hline 4 & 46 & 45 & 47 & 44 & 43 & 51 & 55 & 45 & 42 & 40 & 42 & 40 \\
\hline 5 & 46 & 45 & 47 & 44 & 42 & 56 & 54 & 46 & 41 & 40 & 42 & 40 \\
\hline 6 & 46 & 45 & 46 & 45 & 43 & 60 & 53 & 45 & 41 & 40 & 42 & 40 \\
\hline 7 & 46 & 46 & 46 & 45 & 44 & 62 & 53 & 44 & 41 & 41 & 42 & 40 \\
\hline 8 & 46 & 46 & 46 & 44 & 44 & 64 & 51 & 44 & 40 & 41 & 43 & 39 \\
\hline 9 & 46 & 46 & 46 & 42 & 43 & 61 & 49 & 43 & 41 & 41 & 43 & 39 \\
\hline 10 & 46 & 46 & 45 & 42 & 43 & 59 & 50 & 43 & 41 & 41 & 42 & 40 \\
\hline 11 & 46 & 45 & 45 & 42 & 42 & 56 & 50 & 45 & 41 & 42 & 43 & 39 \\
\hline 12 & 45 & 45 & 45 & 42 & 43 & 52 & 53 & 45 & 41 & 42 & 43 & 39 \\
\hline 13 & 46 & 46 & 45 & 43 & 42 & 52 & 50 & 44 & 41 & 42 & 44 & 39 \\
\hline 14 & 46 & 46 & 45 & 44 & 43 & 52 & 46 & 45 & 41 & 42 & 44 & 38 \\
\hline 15 & 46 & 46 & 45 & 45 & 43 & 52 & 46 & 45 & 41 & 41 & 43 & 37 \\
\hline 16 & 46 & 45 & 45 & 45 & 44 & 54 & 46 & 45 & 41 & 41 & 42 & 39 \\
\hline 17 & 46 & 45 & 45 & 45 & 45 & 56 & 46 & 43 & 41 & 41 & 43 & 38 \\
\hline 18 & 46 & 45 & 45 & 46 & 46 & 57 & 48 & 43 & 41 & 41 & 43 & 38 \\
\hline 19 & 47 & 45 & 45 & 46 & 47 & 58 & 45 & 42 & 41 & 41 & 43 & 39 \\
\hline 20 & 47 & 46 & 45 & 47 & 49 & 64 & 44 & 42 & 42 & 41 & 44 & 39 \\
\hline 21 & 46 & 46 & 46 & 46 & 49 & 62 & 44 & 42 & 42 & 41 & 44 & 38 \\
\hline 22 & 47 & 48 & 45 & 46 & 49 & 62 & 44 & 42 & 43 & 41 & 45 & 38 \\
\hline 23 & 47 & 48 & 45 & 46 & 51 & 63 & 45 & 41 & 42 & 41 & 46 & 38 \\
\hline 24 & 47 & 48 & 46 & 46 & 62 & 64 & 46 & 41 & 42 & 41 & 43 & 39 \\
\hline 25 & 46 & 49 & 46 & 46 & 58 & 63 & 49 & 42 & 42 & 42 & 43 & 38 \\
\hline 26 & 46 & 48 & 45 & 44 & 64 & 63 & 49 & 42 & 42 & 41 & 42 & 38 \\
\hline 27 & 46 & 47 & 46 & 43 & 60 & 62 & 48 & 42 & 42 & 41 & 41 & 38 \\
\hline 28 & 46 & 49 & 45 & 43 & 61 & 61 & 47 & 42 & 42 & 41 & 42 & 37 \\
\hline 29 & 45 & 47 & 45 & 43 & 68 & 61 & 45 & 42 & 42 & 41 & 41 & 38 \\
\hline 30 & 45 & - & 45 & 43 & 65 & 60 & 45 & 42 & 42 & 41 & 41 & 37 \\
\hline 31 & 45 & - & 45 & - & 59 & - & 45 & 41 & - & 42 & - & 38 \\
\hline Mean & 45.9 & 46.2 & 45.6 & 44.3 & 49.1 & 57.8 & 48.9 & 43.4 & 41.4 & 41.1 & 42.7 & 38.8 \\
\hline $\operatorname{Max}$ & 47 & 49 & 47 & 47 & 68 & 64 & 58 & 48 & 43 & 42 & 46 & 41 \\
\hline Min & 44 & 45 & 45 & 42 & 42 & 49 & 44 & 41 & 40 & 40 & 41 & 37 \\
\hline
\end{tabular}




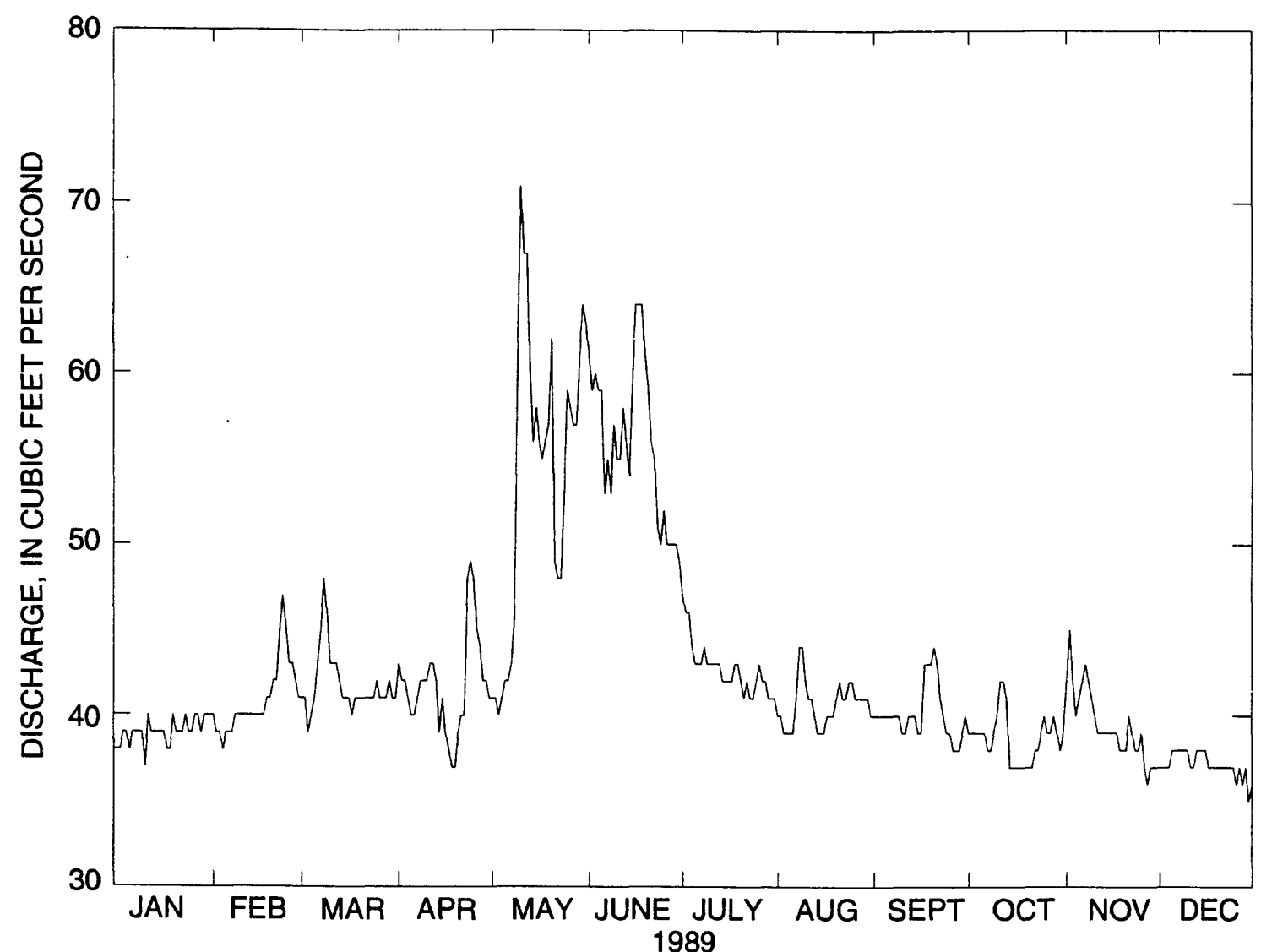

Figure 41. (C) Daily mean discharge, Hot Creek Flume (HCF), Long Valley Caldera, Mono County, California, 1989. 
Table 91. Daily mean discharge, Hot Creek Flume (HCF), Long Valley Caldera, Mono County, California, 1989 [Values are in cubic feet per second. Max, maximum; Min, minimum; -, no data]

\begin{tabular}{|c|c|c|c|c|c|c|c|c|c|c|c|c|}
\hline Day & Jan & Feb & Mar & Apr & May & June & July & Aug & Sept & Oct & Nov & Dec \\
\hline 1 & 38 & 40 & 41 & 43 & 41 & 61 & 47 & 40 & 40 & 39 & 42 & 37 \\
\hline 2 & 38 & 39 & 41 & 42 & 41 & 59 & 46 & 40 & 40 & 39 & 45 & 37 \\
\hline 3 & 39 & 39 & 39 & 42 & 40 & 60 & 46 & 39 & 40 & 39 & 42 & 37 \\
\hline 4 & 39 & 38 & 40 & 41 & 41 & 59 & 44 & 39 & 40 & 39 & 40 & 37 \\
\hline 5 & 38 & 39 & 41 & 40 & 42 & 59 & 43 & 39 & 40 & 39 & 41 & 38 \\
\hline 6 & 39 & 39 & 43 & 40 & 42 & 53 & 43 & 39 & 40 & 39 & 42 & 38 \\
\hline 7 & 39 & 39 & 45 & 41 & 43 & 55 & 43 & 41 & 40 & 38 & 43 & 38 \\
\hline 8 & 39 & 40 & 48 & 42 & 46 & 53 & 44 & 44 & 40 & 38 & 42 & 38 \\
\hline 9 & 39 & 40 & 46 & 42 & 61 & 57 & 43 & 44 & 40 & 39 & 41 & 38 \\
\hline 10 & 37 & 40 & 43 & 42 & 71 & 55 & 43 & 42 & 39 & 40 & 40 & 38 \\
\hline 11 & 40 & 40 & 43 & 43 & 67 & 55 & 43 & 41 & 39 & 42 & 39 & 37 \\
\hline 12 & 39 & 40 & 43 & 43 & 67 & 58 & 43 & 41 & 40 & 42 & 39 & 37 \\
\hline 13 & 39 & 40 & 42 & 42 & 60 & 56 & 43 & 40 & 40 & 41 & 39 & 38 \\
\hline 14 & 39 & 40 & 41 & 39 & 56 & 54 & 42 & 39 & 40 & 37 & 39 & 38 \\
\hline 15 & 39 & 40 & 41 & 41 & 58 & 60 & 42 & 39 & 39 & 37 & 39 & 38 \\
\hline 16 & 39 & 40 & 41 & 39 & 56 & 64 & 42 & 39 & 39 & 37 & 39 & 38 \\
\hline 17 & 38 & 40 & 40 & 38 & 55 & 64 & 42 & 40 & 43 & 37 & 39 & 37 \\
\hline 18 & 38 & 41 & 41 & 37 & 56 & 64 & 43 & 40 & 43 & 37 & 38 & 37 \\
\hline 19 & 40 & 41 & 41 & 37 & 57 & 61 & 43 & 40 & 43 & 37 & 38 & 37 \\
\hline 20 & 39 & 42 & 41 & 39 & 62 & 59 & 42 & 41 & 44 & 37 & 38 & 37 \\
\hline 21 & 39 & 42 & 41 & 40 & 49 & 56 & 41 & 42 & 43 & 37 & 40 & 37 \\
\hline 22 & 39 & 45 & 41 & 40 & 48 & 55 & 42 & 41 & 41 & 38 & 39 & 37 \\
\hline 23 & 40 & 47 & 41 & 48 & 48 & 51 & 41 & 41 & 40 & 38 & 38 & 37 \\
\hline 24 & 39 & 45 & 41 & 49 & 53 & 50 & 41 & 42 & 39 & 39 & 38 & 37 \\
\hline 25 & 39 & 43 & 42 & 48 & 59 & 52 & 42 & 42 & 39 & 40 & 39 & 37 \\
\hline 26 & 40 & 43 & 41 & 45 & 58 & 50 & 43 & 41 & 38 & 39 & 37 & 36 \\
\hline 27 & 40 & 42 & 41 & 44 & 57 & 50 & 42 & 41 & 38 & 39 & 36 & 37 \\
\hline 28 & 39 & 41 & 41 & 42 & 57 & 50 & 42 & 41 & 38 & 40 & 37 & 36 \\
\hline 29 & 40 & - & 42 & 42 & 61 & 50 & 41 & 41 & 39 & 39 & 37 & 37 \\
\hline 30 & 40 & - & 41 & 41 & 64 & 49 & 41 & 41 & 40 & 38 & 37 & 35 \\
\hline 31 & 40 & - & 41 & - & 63 & - & 41 & 40 & - & 39 & - & 36 \\
\hline Mean & 39.0 & 40.9 & 41.7 & 41.7 & 54.2 & 56.0 & 42.7 & 40.6 & 40.1 & 38.7 & 39.4 & 37.2 \\
\hline Max & 40 & 47 & 48 & 49 & 71 & 64 & 47 & 44 & 44 & 42 & 45 & 38 \\
\hline Min & 37 & 38 & 39 & 37 & 40 & 49 & 41 & 39 & 38 & 37 & 36 & 35 \\
\hline
\end{tabular}




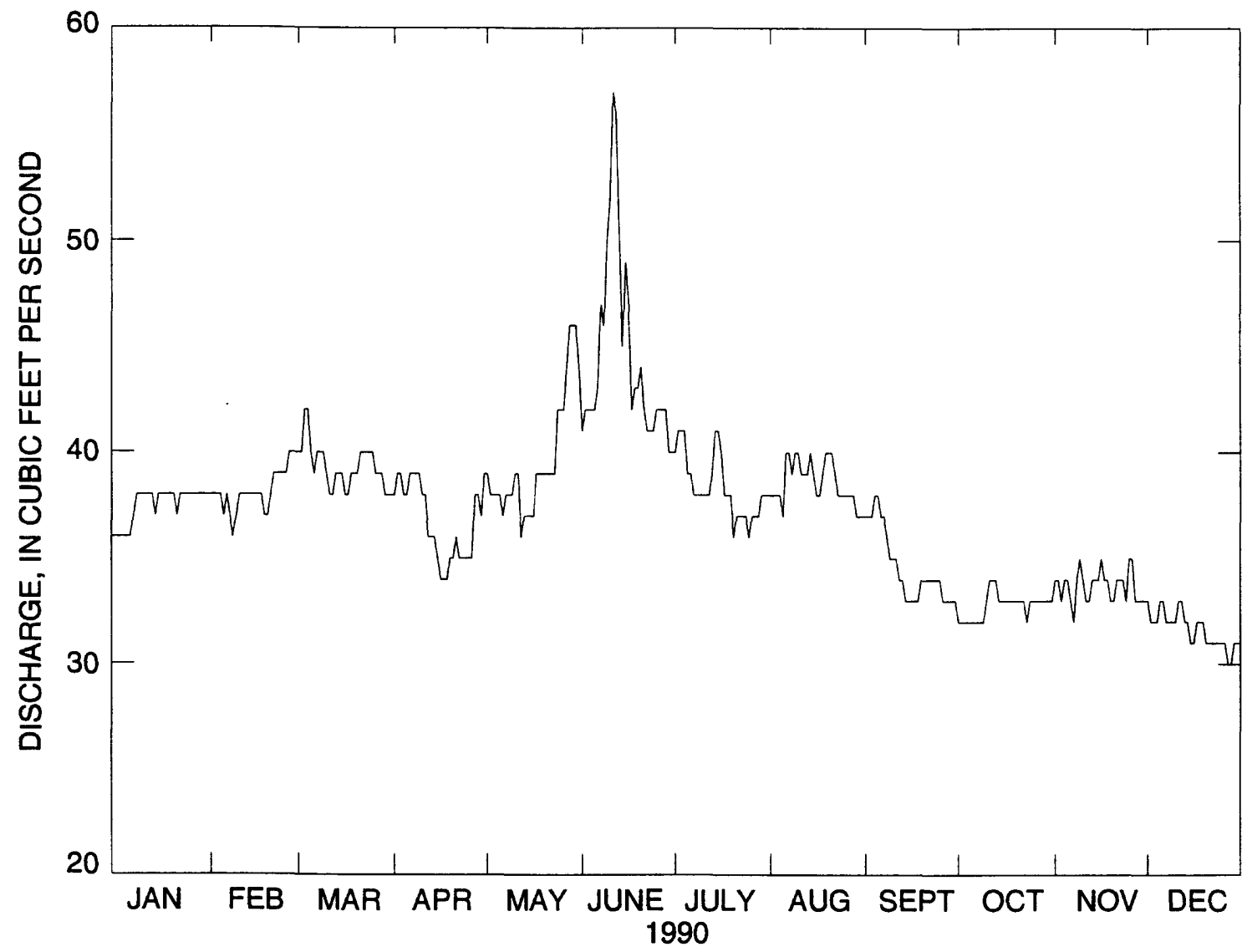

Figure 41. (D) Daily mean discharge, Hot Creek Flume (HCF), Long Valley Caldera, Mono County, California, 1990. 
Table 92. Daily mean discharge, Hot Creek Flume (HCF), Long Valley Caldera, Mono County, California, 1990 [Values are in cubic feet per second. Max, maximum; Min, minimum; - , no data]

\begin{tabular}{|c|c|c|c|c|c|c|c|c|c|c|c|c|}
\hline Day & Jan & Feb & Mar & Apr & May & June & July & Aug & Sept & Oct & Nov & Dec \\
\hline 1 & 36 & 38 & 40 & 38 & 39 & 41 & 40 & 38 & 37 & 32 & 34 & 33 \\
\hline 2 & 36 & 38 & 40 & 39 & 38 & 42 & 41 & 38 & 37 & 32 & 34 & 32 \\
\hline 3 & 36 & 38 & 42 & 39 & 38 & 42 & 41 & 38 & 37 & 32 & 33 & 32 \\
\hline 4 & 36 & 38 & 42 & 38 & 38 & 42 & 41 & 38 & 38 & 32 & 34 & 32 \\
\hline 5 & 36 & 37 & 40 & 38 & 38 & 42 & 39 & 37 & 38 & 32 & 34 & 33 \\
\hline 6 & 36 & 38 & 39 & 39 & 37 & 43 & 39 & 40 & 37 & 32 & 33 & 33 \\
\hline 7 & 37 & 37 & 40 & 39 & 38 & 47 & 38 & 40 & 37 & 32 & 32 & 32 \\
\hline 8 & 38 & 36 & 40 & 39 & 38 & 46 & 38 & 39 & 36 & 32 & 34 & 32 \\
\hline 9 & 38 & 37 & 40 & 39 & 38 & 50 & 38 & 40 & 35 & 32 & 35 & 32 \\
\hline 10 & 38 & 38 & 39 & 38 & 39 & 52 & 38 & 40 & 35 & 33 & 34 & 32 \\
\hline 11 & 38 & 38 & 38 & 38 & 39 & 57 & 38 & 39 & 35 & 34 & 33 & 33 \\
\hline 12 & 38 & 38 & 38 & 36 & 36 & 56 & 38 & 39 & 34 & 34 & 33 & 33 \\
\hline 13 & 38 & 38 & 39 & 36 & 37 & 50 & 39 & 39 & 34 & 34 & 34 & 32 \\
\hline 14 & 37 & 38 & 39 & 36 & 37 & 45 & 41 & 40 & 33 & 33 & 34 & 32 \\
\hline 15 & 38 & 38 & 39 & 35 & 37 & 49 & 41 & 39 & 33 & 33 & 34 & 31 \\
\hline 16 & 38 & 38 & 38 & 34 & 37 & 47 & 40 & 38 & 33 & 33 & 35 & 31 \\
\hline 17 & 38 & 38 & 38 & 34 & 39 & 42 & 38 & 38 & 33 & 33 & 34 & 32 \\
\hline 18 & 38 & 37 & 39 & 34 & 39 & 43 & 38 & 39 & 33 & 33 & 34 & 32 \\
\hline 19 & 38 & 37 & 39 & 35 & 39 & 43 & 38 & 40 & 34 & 33 & 33 & 32 \\
\hline 20 & 38 & 38 & 39 & 35 & 39 & 44 & 36 & 40 & 34 & 33 & 33 & 31 \\
\hline 21 & 37 & 39 & 40 & 36 & 39 & 42 & 37 & 40 & 34 & 33 & 34 & 31 \\
\hline 22 & 38 & 39 & 40 & 35 & 39 & 41 & 37 & 39 & 34 & 33 & 34 & 31 \\
\hline 23 & 38 & 39 & 40 & 35 & 39 & 41 & 37 & 38 & 34 & 32 & 34 & 31 \\
\hline 24 & 38 & 39 & 40 & 35 & 42 & 41 & 37 & 38 & 34 & 33 & 33 & 31 \\
\hline 25 & 38 & 39 & 40 & 35 & 42 & 42 & 36 & 38 & 34 & 33 & 35 & 31 \\
\hline 26 & 38 & 40 & 39 & 35 & 42 & 42 & 37 & 38 & 33 & 33 & 35 & 31 \\
\hline 27 & 38 & 40 & 39 & 38 & 44 & 42 & 37 & 38 & 33 & 33 & 33 & 30 \\
\hline 28 & 38 & 40 & 39 & 38 & 46 & 42 & 37 & 38 & 33 & 33 & 33 & 30 \\
\hline 29 & 38 & - & 38 & 37 & 46 & 40 & 38 & 37 & 33 & 33 & 33 & 31 \\
\hline 30 & 38 & - & 38 & 39 & 46 & 40 & 38 & 37 & 33 & 33 & 33 & 31 \\
\hline 31 & 38 & - & 38 & - & 44 & - & 38 & 37 & - & 33 & - & 31 \\
\hline Mean & 37.5 & 38.1 & 39.3 & 36.7 & 39.6 & 44.5 & 38.4 & 38.6 & 34.6 & 32.8 & 33.7 & 31.6 \\
\hline $\operatorname{Max}$ & 38 & 40 & 42 & 39 & 46 & 57 & 41 & 40 & 38 & 34 & 35 & 33 \\
\hline Min & 36 & 36 & 38 & 34 & 36 & 40 & 36 & 37 & 33 & 32 & 32 & 30 \\
\hline
\end{tabular}




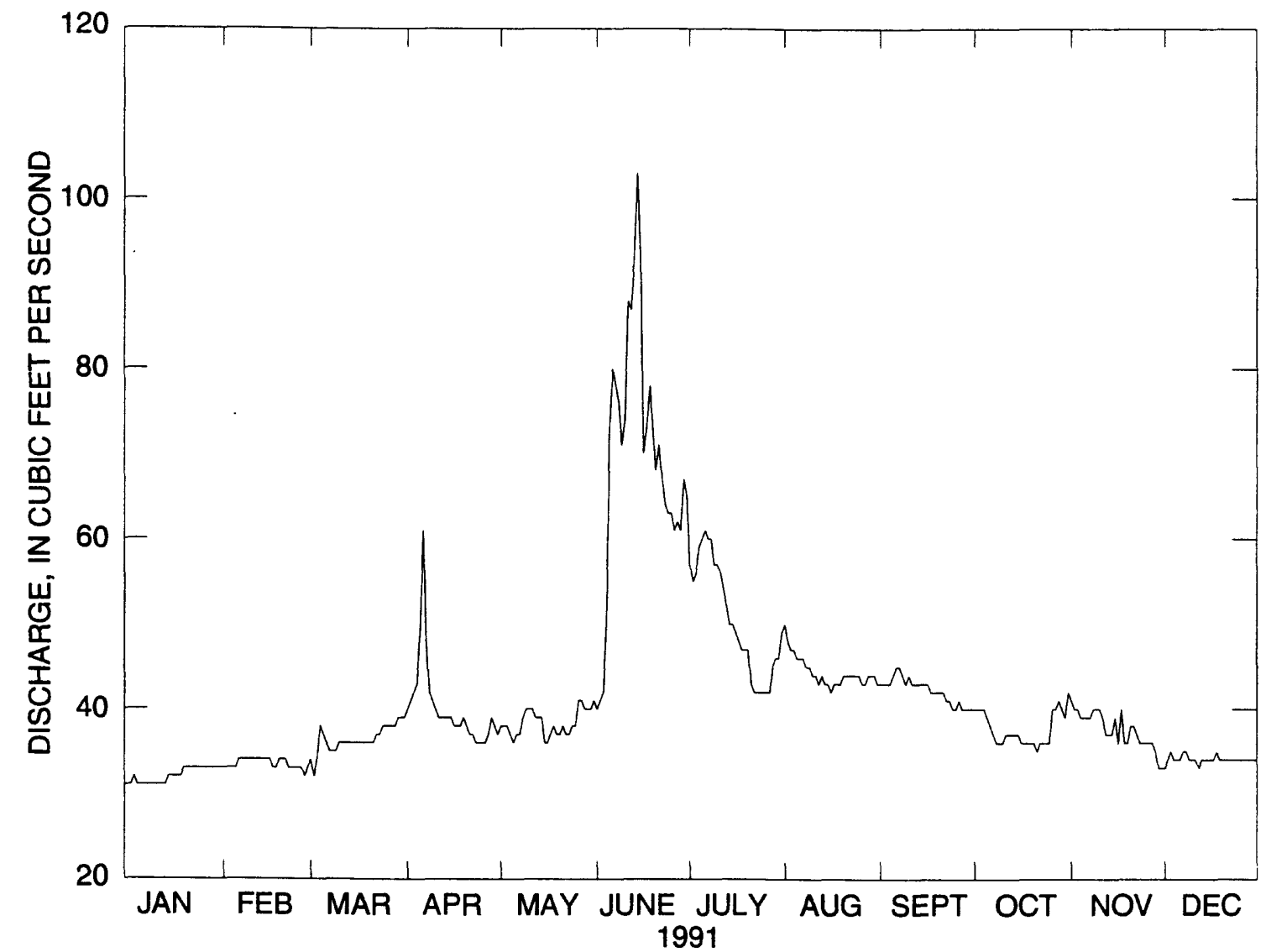

Figure 41. (E) Daily mean discharge, Hot Creek Flume (HCF), Long Valley Caldera, Mono County, California, 1991. 
Table 93. Daily mean discharge, Hot Creek Flume (HCF), Long Valley Caldera, Mono County, California 1991 [Values are in cubic feet per second. e, estimated; Max, maximum; Min, minimum; -, no data]

\begin{tabular}{|c|c|c|c|c|c|c|c|c|c|c|c|c|}
\hline Day & Jan & Feb & Mar & Apr & May & June & July & Aug & Sept & Oct & Nov & Dec \\
\hline 1 & 31 & 33 & 34 & 40 & 38 & 40 & 57 & 50 & 43 & 40 & 41 & 33 \\
\hline 2 & 31 & 33 & 32 & 41 & 38 & 41 & 55 & 48 & 43 & 40 & 40 & 34 \\
\hline 3 & 32 & 33 & 34 & 42 & 38 & 42 & 56 & 47 & 43 & 40 & 40 & 35 \\
\hline 4 & 31 & 33 & 38 & 43 & 37 & 50 & 59 & 47 & 43 & 40 & 39 & 34 \\
\hline 5 & 31 & 33 & 37 & 49 & 36 & 73 & 60 & 46 & 44 & 39 & 39 & 34 \\
\hline 6 & 31 & 34 & 36 & 61 & 37 & 80 & 61 & 46 & 45 & 38 & 39 & 34 \\
\hline 7 & 31 & 34 & 35 & 48 & 37 & 78 & 60 & 46 & 45 & 37 & 39 & 35 \\
\hline 8 & 31 & 34 & 35 & 42 & 39 & 76 & 60 & 45 & 44 & 36 & 40 & 35 \\
\hline 9 & 31 & 34 & 35 & 41 & 40 & 71 & 57 & 45 & 43 & 36 & 40 & 34 \\
\hline 10 & 31 & 34 & 36 & 40 & 40 & 74 & 57 & 44 & 44 & 36 & 40 & 34 \\
\hline 11 & 31 & 34 & 36 & 39 & 40 & 88 & 56 & 44 & 43 & 37 & 39 & 34 \\
\hline 12 & 31 & 34 & 36 & 39 & 39 & 87 & 54 & 43 & 43 & 37 & 37 & 33 \\
\hline 13 & 31 & 34 & 36 & 39 & 39 & 93 & 52 & 44 & 43 & 37 & 37 & 34 \\
\hline 14 & 32 & 34 & 36 & 39 & 39 & 103 & 50 & 43 & 43 & 37 & 37 & 34 \\
\hline 15 & 32 & 34 & 36 & 39 & 36 & 94 & 50 & 43 & 43 & 37 & 39 & 34 \\
\hline 16 & 32 & 34 & 36 & 38 & 36 & 70 & 49 & 42 & 43 & 36 & 36 & 34 \\
\hline 17 & 32 & 33 & 36 & 38 & 37 & 73 & 48 & 43 & 42 & 36 & 40 & 34 \\
\hline 18 & 32 & 33 & 36 & 38 & 38 & 78 & 47 & 43 & 42 & 36 & 36 & 35 \\
\hline 19 & 33 & 34 & 36 & 39 & 37 & e73 & 47 & 43 & 42 & 36 & 36 & 34 \\
\hline 20 & 33 & 34 & 36 & 38 & 37 & 68 & 47 & 44 & 42 & 36 & 38 & 34 \\
\hline 21 & 33 & 34 & 36 & 37 & 38 & 71 & 43 & 44 & 42 & 35 & 38 & 34 \\
\hline 22 & 33 & 33 & 37 & 37 & 37 & 67 & 42 & 44 & 41 & 36 & 37 & 34 \\
\hline 23 & 33 & 33 & 37 & 36 & 37 & 64 & 42 & 44 & 41 & 36 & 36 & 34 \\
\hline 24 & 33 & 33 & 38 & 36 & 38 & 63 & 42 & 44 & 40 & 36 & 36 & 34 \\
\hline 25 & 33 & 33 & 38 & 36 & 38 & 63 & 42 & 44 & 40 & 36 & 36 & 34 \\
\hline 26 & 33 & 33 & 38 & 36 & 41 & 61 & 42 & 43 & 41 & 40 & 36 & 34 \\
\hline 27 & 33 & 32 & 38 & 37 & 41 & 62 & 42 & 43 & 40 & 40 & 36 & 34 \\
\hline 28 & 33 & 33 & 38 & 39 & 40 & 61 & 45 & 44 & 40 & 41 & 35 & 34 \\
\hline 29 & 33 & - & 39 & 38 & 40 & 67 & 46 & 44 & 40 & 40 & 33 & 34 \\
\hline 30 & 33 & - & 39 & 37 & 40 & 65 & 46 & 44 & 40 & 39 & 33 & 34 \\
\hline 31 & 33 & - & 39 & - & 41 & - & 49 & 43 & - & 42 & - & 34 \\
\hline Mean & 32.0 & 33.5 & 36.4 & 40.1 & 38.4 & 69.9 & 50.4 & 44.4 & 42.3 & 37.7 & 37.6 & 34.1 \\
\hline Max & 33 & 34 & 39 & 61 & 41 & 103 & 61 & 50 & 45 & 42 & 41 & 35 \\
\hline Min & 31 & 32 & 32 & 36 & 36 & 40 & 42 & 42 & 40 & 35 & 33 & 33 \\
\hline
\end{tabular}




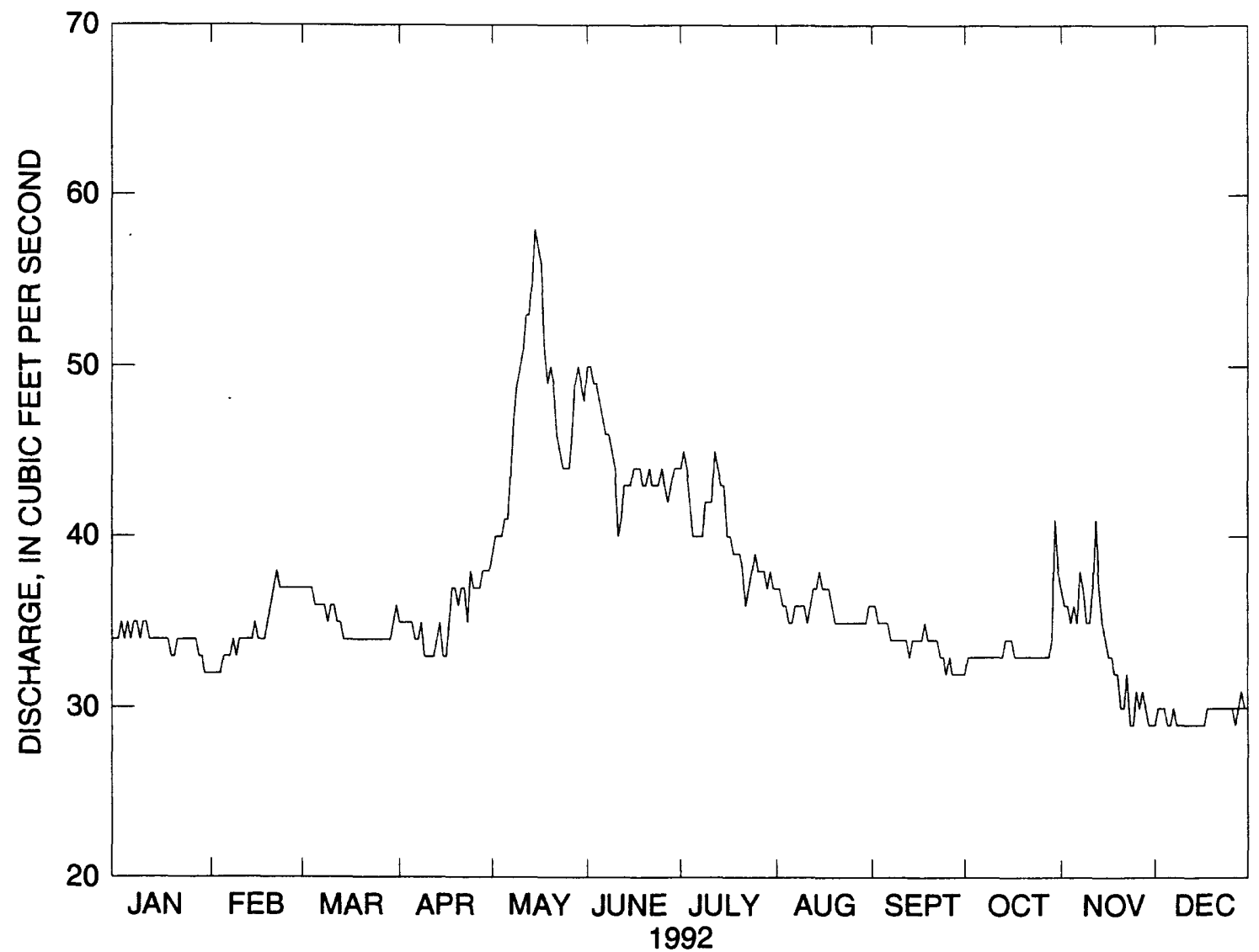

Figure 41. (F) Daily mean discharge, Hot Creek Flume (HCF), Long Valley Caldera, Mono County, California, 1992. 
Table 94. Daily mean discharge, Hot Creek Flume (HCF), Long Valley Caldera, Mono County, California, 1992 [Values are in cubic feet per second. Max, maximum; Min, minimum; -, no data]

\begin{tabular}{|c|c|c|c|c|c|c|c|c|c|c|c|c|}
\hline Day & $\operatorname{Jan}$ & Feb & Mar & Apr & May & June & July & Aug & Sept & Oct & Nov & Dec \\
\hline 1 & 34 & 32 & 37 & 35 & 39 & 50 & 44 & 37 & 36 & 32 & 37 & 29 \\
\hline 2 & 34 & 32 & 37 & 35 & 40 & 50 & 45 & 37 & 36 & 33 & 36 & 30 \\
\hline 3 & 35 & 32 & 37 & 35 & 40 & 49 & 44 & 36 & 35 & 33 & 36 & 30 \\
\hline 4 & 34 & 32 & 37 & 35 & 40 & 49 & 42 & 36 & 35 & 33 & 35 & 30 \\
\hline 5 & 35 & 33 & 36 & 35 & 41 & 48 & 40 & 35 & 35 & 33 & 36 & 29 \\
\hline 6 & 34 & 33 & 36 & 34 & 41 & 47 & 40 & 35 & 35 & 33 & 35 & 29 \\
\hline 7 & 35 & 33 & 36 & 34 & 44 & 46 & 40 & 36 & 34 & 33 & 38 & 30 \\
\hline 8 & 35 & 34 & 36 & 35 & 47 & 46 & 40 & 36 & 34 & 33 & 37 & 29 \\
\hline 9 & 34 & 33 & 35 & 33 & 49 & 45 & 42 & 36 & 34 & 33 & 35 & 29 \\
\hline 10 & 35 & 34 & 36 & 33 & 50 & 44 & 42 & 36 & 34 & 33 & 35 & 29 \\
\hline 11 & 35 & 34 & 36 & 33 & 51 & 40 & 42 & 35 & 34 & 33 & 37 & 29 \\
\hline 12 & 34 & 34 & 35 & 33 & 53 & 41 & 45 & 36 & 34 & 33 & 41 & 29 \\
\hline 13 & 34 & 34 & 35 & 34 & 53 & 43 & 44 & 37 & 33 & 33 & 37 & 29 \\
\hline 14 & 34 & 34 & 34 & 35 & 55 & 43 & 43 & 37 & 34 & 34 & 35 & 29 \\
\hline 15 & 34 & 35 & 34 & 33 & 58 & 43 & 43 & 38 & 34 & 34 & 34 & 29 \\
\hline 16 & 34 & 34 & 34 & 33 & 57 & 44 & 40 & 37 & 34 & 34 & 33 & 29 \\
\hline 17 & 34 & 34 & 34 & 35 & 56 & 44 & 40 & 37 & 34 & 33 & 33 & 29 \\
\hline 18 & 34 & 34 & 34 & 37 & 51 & 44 & 39 & 37 & 35 & 33 & 32 & 30 \\
\hline 19 & 33 & 35 & 34 & 37 & 49 & 43 & 39 & 36 & 34 & 33 & 32 & 30 \\
\hline 20 & 33 & 36 & 34 & 36 & 50 & 43 & 39 & 35 & 34 & 33 & 30 & 30 \\
\hline 21 & 34 & 37 & 34 & 37 & 49 & 44 & 38 & 35 & 34 & 33 & 30 & 30 \\
\hline 22 & 34 & 38 & 34 & 37 & 46 & 43 & 36 & 35 & 34 & 33 & 32 & 30 \\
\hline 23 & 34 & 37 & 34 & 35 & 45 & 43 & 37 & 35 & 33 & 33 & 29 & 30 \\
\hline 24 & 34 & 37 & 34 & 38 & 44 & 43 & 38 & 35 & 33 & 33 & 29 & 30 \\
\hline 25 & 34 & 37 & 34 & 37 & 44 & 44 & 39 & 35 & 32 & 33 & 31 & 30 \\
\hline 26 & 34 & 37 & 34 & 37 & 44 & 43 & 38 & 35 & 33 & 33 & 30 & 30 \\
\hline 27 & 34 & 37 & 34 & 37 & 46 & 42 & 38 & 35 & 32 & 33 & 31 & 29 \\
\hline 28 & 33 & 37 & 34 & 38 & 49 & 43 & 38 & 35 & 32 & 33 & 30 & 30 \\
\hline 29 & 33 & 37 & 34 & 38 & 50 & 44 & 37 & 35 & 32 & 34 & 29 & 31 \\
\hline 30 & 32 & - & 35 & 38 & 49 & 44 & 38 & 35 & 32 & 41 & 29 & 30 \\
\hline 31 & 32 & - & 36 & - & 48 & - & 37 & 36 & - & 38 & - & 30 \\
\hline Mean & 33.9 & 34.7 & 35.0 & 35.4 & 47.7 & 44.5 & 40.2 & 35.8 & 33.8 & 33.5 & 33.5 & 29.6 \\
\hline Max & 35 & 38 & 37 & 38 & 58 & 50 & 45 & 38 & 36 & 41 & 41 & 31 \\
\hline Min & 32 & 32 & 34 & 33 & 39 & 40 & 36 & 35 & 32 & 32 & 29 & 29 \\
\hline
\end{tabular}




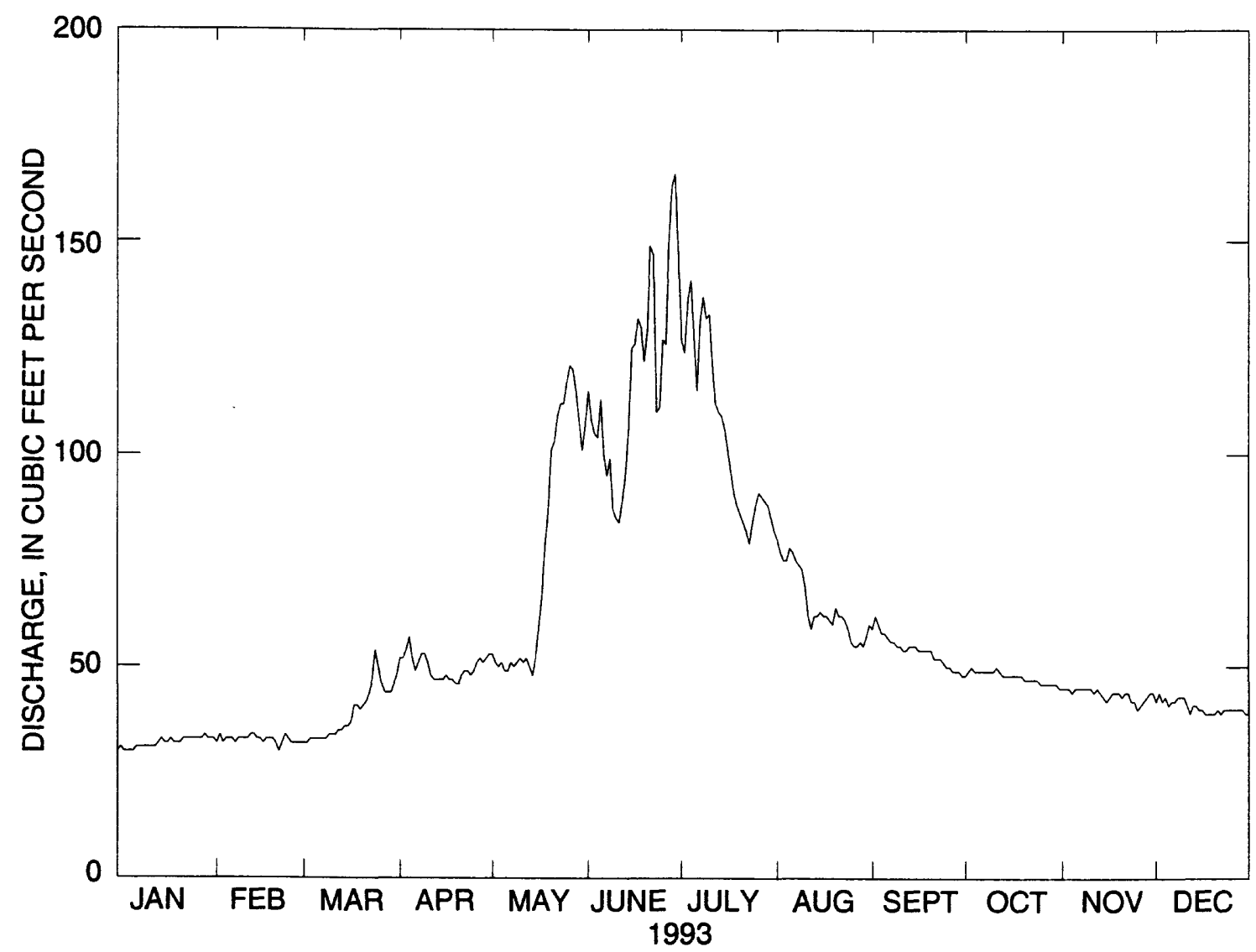

Figure 41. (G) Daily mean discharge, Hot Creek Flume (HCF), Long Valley Caldera, Mono County, California, 1993. 
Table 95. Daily mean discharge, Hot Creek Flume (HCF), Long Valley Caldera, Mono County, California, 1993

[Values are in cubic feet per second. Max, maximum; Min, minimum; -, no data]

\begin{tabular}{|c|c|c|c|c|c|c|c|c|c|c|c|c|}
\hline Day & Jan & Feb & Mar & Apr & May & June & July & Aug & Sept & Oct & Nov & Dec \\
\hline 1 & 31 & 32 & 32 & 52 & 53 & 115 & 127 & 80 & 59 & 48 & 45 & 42 \\
\hline 2 & 30 & 34 & 32 & 52 & 51 & 108 & 124 & 77 & 62 & 49 & 45 & 44 \\
\hline 3 & 30 & 32 & 33 & 54 & 50 & 105 & 136 & 75 & 60 & 50 & 45 & 42 \\
\hline 4 & 30 & 33 & 33 & 57 & 51 & 104 & 141 & 75 & 58 & 49 & 44 & 43 \\
\hline 5 & 30 & 33 & 33 & 52 & 49 & 113 & 128 & 78 & 58 & 49 & 45 & 41 \\
\hline 6 & 31 & 33 & 33 & 49 & 49 & 100 & 115 & 77 & 57 & 49 & 45 & 42 \\
\hline 7 & 31 & 32 & 33 & 51 & 51 & 95 & 131 & 75 & 56 & 49 & 45 & 42 \\
\hline 8 & 31 & 33 & 33 & 53 & 50 & 99 & 137 & 74 & 56 & 49 & 45 & 43 \\
\hline 9 & 31 & 33 & 34 & 53 & 51 & 87 & 132 & 73 & 55 & 49 & 45 & 43 \\
\hline 10 & 31 & 33 & 34 & 51 & 52 & 85 & 133 & 69 & 55 & 49 & 45 & 43 \\
\hline 11 & 31 & 33 & 34 & 48 & 51 & 84 & 121 & 62 & 54 & 50 & 44 & 41 \\
\hline 12 & 31 & 34 & 35 & 47 & 52 & 89 & 112 & 59 & 54 & 49 & 45 & 39 \\
\hline 13 & 32 & 34 & 35 & 47 & 50 & 95 & 110 & 62 & 55 & 48 & 44 & 41 \\
\hline 14 & 33 & 33 & 36 & 47 & 48 & 106 & 109 & 62 & 55 & 48 & 43 & 41 \\
\hline 15 & 32 & 33 & 36 & 47 & 52 & 125 & 106 & 63 & 55 & 48 & 42 & 40 \\
\hline 16 & 32 & 32 & 37 & 48 & 59 & 126 & 101 & 62 & 54 & 48 & 43 & 40 \\
\hline 17 & 33 & 33 & 41 & 47 & 66 & 132 & 96 & 62 & 54 & 48 & 44 & 39 \\
\hline 18 & 32 & 33 & 41 & 47 & 78 & 130 & 91 & 61 & 54 & 48 & 44 & 39 \\
\hline 19 & 32 & 33 & 40 & 46 & 86 & 122 & 88 & 60 & 54 & 48 & 44 & 39 \\
\hline 20 & 32 & 32 & 41 & 46 & 101 & 129 & 86 & 64 & 54 & 47 & 43 & 39 \\
\hline 21 & 33 & 30 & 42 & 48 & 103 & 149 & 84 & 62 & 52 & 47 & 44 & 40 \\
\hline 22 & 33 & 32 & 44 & 49 & 109 & 147 & 82 & 62 & 52 & 47 & 44 & 39 \\
\hline 23 & 33 & 34 & 47 & 49 & 112 & 110 & 79 & 61 & 52 & 47 & 42 & 40 \\
\hline 24 & 33 & 33 & 54 & 48 & 112 & 111 & 84 & 59 & 51 & 47 & 42 & 40 \\
\hline 25 & 33 & 32 & 50 & 49 & 117 & 127 & 88 & 56 & 50 & 46 & 40 & 40 \\
\hline 26 & 33 & 32 & 46 & 51 & 121 & 126 & 91 & 55 & 50 & 46 & 41 & 40 \\
\hline 27 & 33 & 32 & 44 & 52 & 120 & 149 & 90 & 55 & 49 & 46 & 42 & 40 \\
\hline 28 & 34 & 32 & 44 & 51 & 115 & 163 & 89 & 56 & 49 & 46 & 43 & 40 \\
\hline 29 & 33 & - & 44 & 52 & 108 & 166 & 88 & 55 & 49 & 46 & 44 & 40 \\
\hline 30 & 33 & - & 46 & 53 & 101 & 149 & 85 & 57 & 48 & 46 & 44 & 39 \\
\hline 31 & 33 & - & 48 & - & 107 & - & 82 & 60 & - & 45 & - & 39 \\
\hline Mean & 31.9 & 32.7 & 39.2 & 49.9 & 76.6 & 118 & 105 & 64.8 & 54.0 & 47.8 & 43.7 & 40.6 \\
\hline Max & 34 & 34 & 54 & 57 & 121 & 166 & 141 & 80 & 62 & 50 & 45 & 44 \\
\hline Min & 30 & 30 & 32 & 46 & 48 & 84 & 79 & 55 & 48 & 45 & 40 & 39 \\
\hline
\end{tabular}




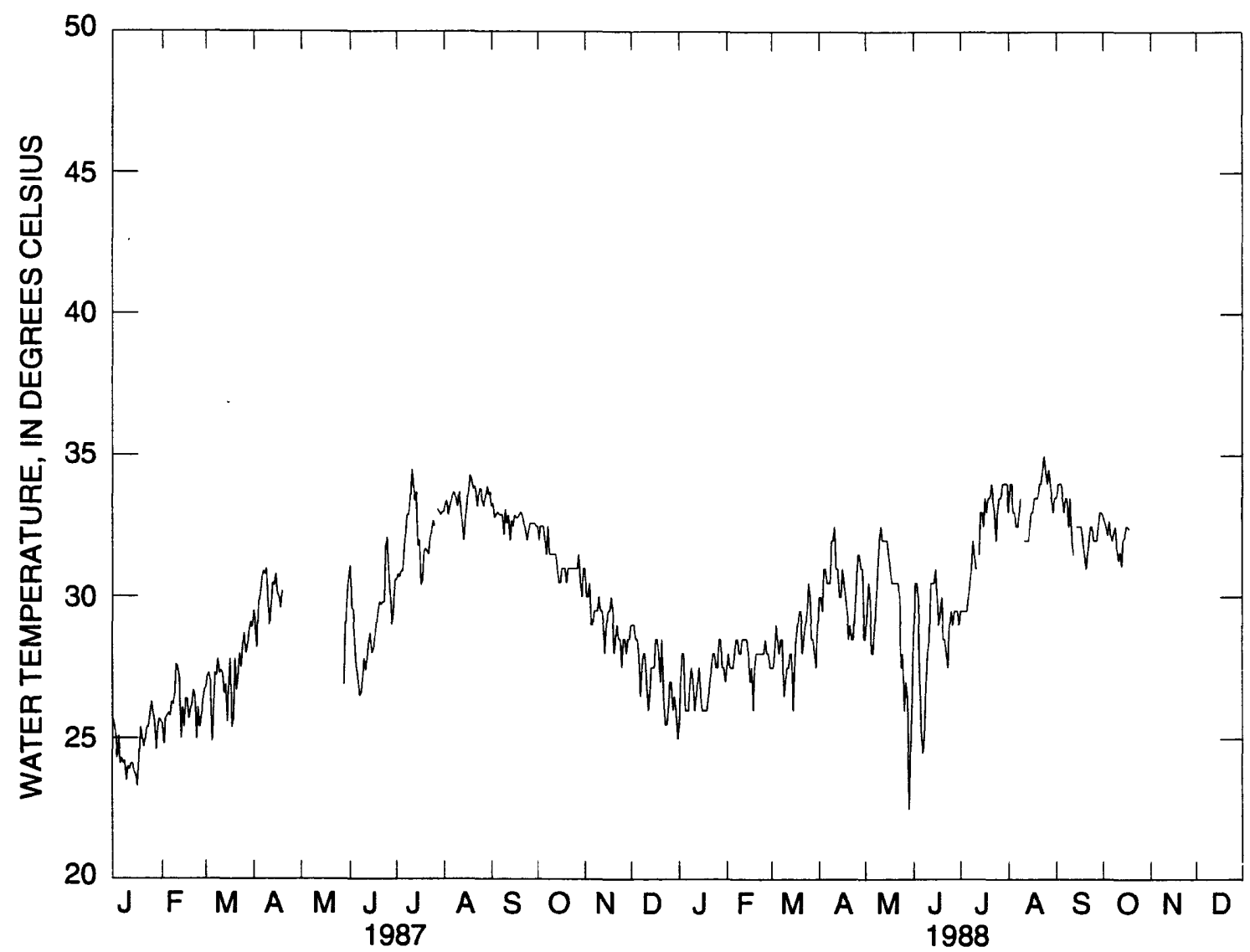

Figure 42. Daily mean water temperature, Hot Creek Flume (HCF), Long Valley Caldera, Mono County, California, 1987-88. 
Table 96. Daily mean water temperature, Hot Creek Flume (HCF), Long Valley Caldera, Mono County, California, 1987

[Values are in degrees Celsius. Max, maximum; Min. minimum; -, no data]

\begin{tabular}{|c|c|c|c|c|c|c|c|c|c|c|c|c|}
\hline Day & Jan & Feb & Mar & Apr & May & June & July & Aug & Sept & Oct & Nov & Dec \\
\hline 1 & 25.5 & 25.5 & 26.8 & 29.5 & - & 31.1 & 30.6 & 33.0 & 33.2 & 32.5 & 31.0 & 29.0 \\
\hline 2 & 25.2 & 24.8 & 27.2 & 29.0 & - & 29.6 & 30.8 & 33.3 & 33.3 & 32.0 & 30.0 & 29.0 \\
\hline 3 & 24.3 & 25.7 & 27.3 & 28.2 & - & 29.5 & 30.7 & 33.4 & 32.8 & 32.5 & 30.0 & 29.0 \\
\hline 4 & 25.1 & 25.8 & 27.0 & 29.8 & - & 28.3 & 30.9 & 32.9 & 32.9 & 32.5 & 30.5 & 28.5 \\
\hline 5 & 24.1 & 25.9 & 24.9 & 30.0 & - & 27.5 & 30.9 & 33.2 & 33.0 & 32.5 & 29.0 & 28.5 \\
\hline 6 & 24.3 & 25.8 & 25.8 & 30.6 & - & 27.1 & 31.7 & 33.4 & 32.9 & 32.0 & 29.0 & 28.0 \\
\hline 7 & 24.1 & 26.3 & 27.3 & 30.9 & - & 26.5 & 32.3 & 33.7 & 32.9 & 31.5 & 29.5 & 26.5 \\
\hline 8 & 24.2 & 26.2 & 27.2 & 30.8 & - & 26.6 & 32.9 & 33.6 & 32.9 & 32.5 & 29.5 & 27.5 \\
\hline 9 & 23.5 & 26.6 & 27.8 & 31.0 & - & 27.1 & 32.9 & 33.5 & 32.2 & 31.5 & 29.5 & 28.0 \\
\hline 10 & 24.0 & 27.6 & 27.3 & 29.8 & - & 27.8 & 33.5 & 33.2 & 33.1 & 31.5 & 30.0 & 28.0 \\
\hline 11 & 23.9 & 27.5 & 27.4 & 29.0 & - & 27.4 & 34.5 & 33.7 & 32.6 & 31.5 & 29.5 & 27.0 \\
\hline 12 & 24.1 & 27.1 & 27.3 & 29.7 & - & 27.8 & 33.9 & 33.2 & 32.9 & 31.5 & 29.5 & 26.0 \\
\hline 13 & 24.1 & 25.0 & 26.6 & 30.5 & - & 28.4 & 33.4 & 32.6 & 32.0 & 31.5 & 29.0 & 26.5 \\
\hline 14 & 23.8 & 26.1 & 26.9 & 30.4 & - & 28.7 & 33.7 & 32.0 & 32.7 & 31.0 & 28.0 & 27.5 \\
\hline 15 & 23.7 & 25.4 & 25.6 & 30.8 & - & 28.0 & 31.8 & 32.7 & 32.5 & 30.5 & 29.0 & 27.5 \\
\hline 16 & 23.3 & 26.4 & 27.0 & 30.1 & - & 28.1 & 32.0 & 33.2 & 32.9 & 30.5 & 29.5 & 27.5 \\
\hline 17 & 24.3 & 26.4 & 27.8 & 30.0 & - & 28.5 & 30.4 & 33.8 & 32.8 & 31.0 & 29.5 & 28.5 \\
\hline 18 & 25.4 & 25.7 & 25.4 & 29.6 & - & 29.0 & 30.6 & 34.3 & 32.8 & 31.0 & 30.0 & 28.5 \\
\hline 19 & 25.0 & 26.0 & 25.7 & 30.2 & - & 29.5 & 31.6 & 34.1 & 32.9 & 31.0 & 29.5 & 28.0 \\
\hline 20 & 24.7 & 26.2 & 27.8 & - & - & 29.8 & 31.7 & 33.8 & 33.0 & 30.5 & 28.0 & 27.0 \\
\hline 21 & 25.0 & 26.7 & 26.7 & - & - & 29.7 & 31.6 & 33.9 & 32.9 & 31.0 & 28.5 & 28.5 \\
\hline 22 & 25.4 & 26.5 & 27.2 & - & - & 29.8 & 31.5 & 33.6 & 32.6 & 31.0 & 29.0 & 26.5 \\
\hline 23 & 25.4 & 25.0 & 28.0 & - & - & 29.8 & 32.1 & 33.2 & 32.4 & 31.0 & 28.5 & 25.5 \\
\hline 24 & 25.9 & 26.1 & 27.5 & - & - & 31.8 & 32.3 & 33.8 & 32.0 & 31.0 & 28.5 & 25.5 \\
\hline 25 & 26.3 & 25.4 & 28.3 & - & - & 32.1 & 32.7 & 33.8 & 32.3 & 31.0 & 27.5 & 26.0 \\
\hline 26 & 25.9 & 25.7 & 28.7 & - & - & 30.8 & 32.5 & 33.4 & 32.6 & 31.0 & 28.5 & 27.0 \\
\hline 27 & 25.6 & 26.3 & 28.0 & - & - & 30.0 & - & 33.2 & 32.6 & 31.0 & 28.5 & 27.0 \\
\hline 28 & 24.6 & 26.7 & 28.2 & - & 26.9 & 29.0 & 33.1 & 33.5 & 32.6 & 31.5 & 28.0 & 26.0 \\
\hline 29 & 25.4 & - & 28.7 & - & 28.9 & 29.6 & 33.0 & 33.9 & 32.6 & 30.5 & 28.5 & 26.5 \\
\hline 30 & 25.7 & - & 29.1 & - & 30.1 & 30.6 & 32.9 & 33.6 & 32.5 & 30.0 & 28.5 & 26.0 \\
\hline 31 & 25.6 & - & 28.9 & - & 30.7 & - & 33.0 & 33.7 & - & 31.0 & - & 25.0 \\
\hline Mean & 24.8 & 26.1 & 27.3 & 30.0 & 29.2 & 29.0 & 32.2 & 33.4 & 32.7 & 31.3 & 29.1 & 27.3 \\
\hline Max & 26.3 & 27.6 & 29.1 & 31.0 & 30.7 & 32.1 & 34.5 & 34.3 & 33.3 & 32.5 & 31.0 & 29.0 \\
\hline Min & 23.3 & 24.8 & 24.9 & 28.2 & 26.9 & 26.5 & 30.4 & 32.0 & 32.0 & 30.0 & 27.5 & 25.0 \\
\hline
\end{tabular}


This page intentionally left blank. 
Table 97. Daily mean water temperature, Hot Creek Flume (HCF), Long Valley Caldera, Mono County,

California, 1988

[Values are in degrees Celsius. Max. maximum; Min. minimum; -, no data]

\begin{tabular}{|c|c|c|c|c|c|c|c|c|c|c|c|c|}
\hline Day & Jan & Feb & Mar & Apr & May & June & July & Aug & Sept & Oct & Nov & Dec \\
\hline 1 & 25.5 & 27.5 & 27.5 & 30.0 & 28.5 & 28.5 & 29.5 & 33.0 & 33.5 & 32.9 & - & - \\
\hline 2 & 27.0 & 28.0 & 27.5 & 30.0 & 29.5 & 30.5 & 29.5 & 34.0 & 34.0 & 32.7 & - & - \\
\hline 3 & 28.0 & 27.5 & 28.0 & 29.5 & 30.5 & 30.5 & 29.5 & 34.0 & 34.0 & 32.5 & - & - \\
\hline 4 & 28.0 & 27.5 & 29.0 & 31.0 & 30.0 & 30.0 & 29.5 & 33.0 & 34.0 & 32.2 & - & - \\
\hline 5 & 26.0 & 27.5 & 28.5 & 31.0 & 28.0 & 27.0 & 29.5 & 33.0 & 33.5 & 32.7 & - & - \\
\hline 6 & 26.0 & 28.0 & 28.0 & 30.5 & 28.0 & 25.0 & 30.0 & 32.5 & 33.0 & 32.2 & - & - \\
\hline 7 & 26.0 & 28.5 & 28.5 & 30.5 & 29.0 & 24.5 & 30.5 & 32.5 & 33.5 & 32.0 & - & - \\
\hline 8 & 27.0 & 28.5 & 28.5 & 30.5 & 30.0 & 25.5 & 31.0 & 33.0 & 33.5 & 32.3 & - & - \\
\hline 9 & 27.5 & 28.0 & 26.5 & 32.0 & 31.0 & 27.0 & 32.0 & 33.5 & 32.5 & 32.5 & - & - \\
\hline 10 & 27.0 & 28.0 & 27.0 & 32.0 & 32.0 & 28.0 & 31.5 & - & 33.5 & 31.7 & - & - \\
\hline 11 & 26.0 & 28.5 & 27.5 & 32.5 & 32.5 & 28.5 & 31.0 & 32.0 & 32.0 & 31.3 & - & - \\
\hline 12 & 26.5 & 28.5 & 27.5 & 31.0 & 32.0 & 30.5 & - & 32.0 & 31.5 & 31.6 & - & - \\
\hline 13 & 27.0 & 28.5 & 28.0 & 31.0 & 32.0 & 30.5 & 31.5 & 32.0 & - & 31.1 & - & - \\
\hline 14 & 27.5 & 28.5 & 28.0 & 30.0 & 32.0 & 30.5 & 33.0 & 32.0 & 32.5 & 32.0 & - & - \\
\hline 15 & 26.5 & 28.0 & 26.0 & 30.0 & 32.0 & 31.0 & 33.0 & 32.5 & 32.5 & 32.1 & - & - \\
\hline 16 & 26.0 & 27.0 & 27.5 & 31.0 & 31.5 & 30.0 & 32.5 & 33.0 & 32.5 & 32.5 & - & - \\
\hline 17 & 26.0 & 27.5 & 28.5 & 30.5 & 31.0 & 29.0 & 33.5 & 33.0 & 32.5 & 32.5 & - & - \\
\hline 18 & 26.0 & 26.0 & 29.0 & 30.0 & 30.5 & 29.5 & 33.0 & 33.5 & 32.0 & 32.4 & - & - \\
\hline 19 & 26.0 & 27.5 & 29.5 & 29.5 & 30.5 & 30.0 & 33.5 & 33.5 & 31.5 & - & - & - \\
\hline 20 & 26.5 & 28.0 & 29.5 & 28.5 & 30.5 & 28.5 & 33.5 & 33.5 & 31.0 & - & - & - \\
\hline 21 & 27.0 & 28.0 & 28.0 & 29.0 & 30.5 & 28.5 & 34.0 & 34.0 & 31.5 & - & - & - \\
\hline 22 & 27.5 & 28.0 & 28.5 & 28.5 & 30.5 & 28.0 & 33.5 & 34.0 & 32.0 & - & - & - \\
\hline 23 & 28.0 & 28.0 & 29.0 & 28.5 & 30.0 & 27.5 & 33.0 & 34.5 & 32.5 & - & - & - \\
\hline 24 & 28.0 & 28.0 & 29.5 & 29.5 & 27.5 & 29.0 & 32.0 & 35.0 & 32.5 & - & - & - \\
\hline 25 & 27.5 & 28.0 & 30.5 & 30.5 & 28.0 & 29.5 & 33.0 & 34.5 & 32.0 & - & - & - \\
\hline 26 & 27.5 & 28.5 & 30.0 & 31.5 & 26.0 & 29.0 & 33.5 & 34.0 & 32.0 & - & - & - \\
\hline 27 & 28.5 & 28.0 & 28.5 & 31.5 & 27.0 & 29.5 & 33.5 & 34.5 & 32.0 & - & - & - \\
\hline 28 & 28.5 & 28.0 & 28.5 & 31.0 & 26.5 & 29.5 & 34.0 & 34.0 & 32.5 & - & - & - \\
\hline 29 & 27.5 & 27.5 & 28.0 & 31.0 & 22.5 & 29.5 & 34.0 & 33.5 & 33.0 & - & - & - \\
\hline 30 & 27.5 & - & 27.5 & 28.5 & 24.5 & 29.0 & 34.0 & 33.0 & 33.0 & - & - & - \\
\hline 31 & 27.0 & - & 29.0 & - & 26.5 & - & 34.0 & 33.5 & - & - & - & - \\
\hline Mean & 27.0 & 27.9 & 28.3 & 30.3 & 29.4 & 28.8 & 32.2 & 33.3 & 32.6 & 32.2 & - & - \\
\hline $\operatorname{Max}$ & 28.5 & 28.5 & 30.5 & 32.5 & 32.5 & 31.0 & 34.0 & 35.0 & 34.0 & 32.9 & - & - \\
\hline Min & 25.5 & 26.0 & 26.0 & 28.5 & 22.5 & 24.5 & 29.5 & 32.0 & 31.0 & 31.1 & - & - \\
\hline
\end{tabular}




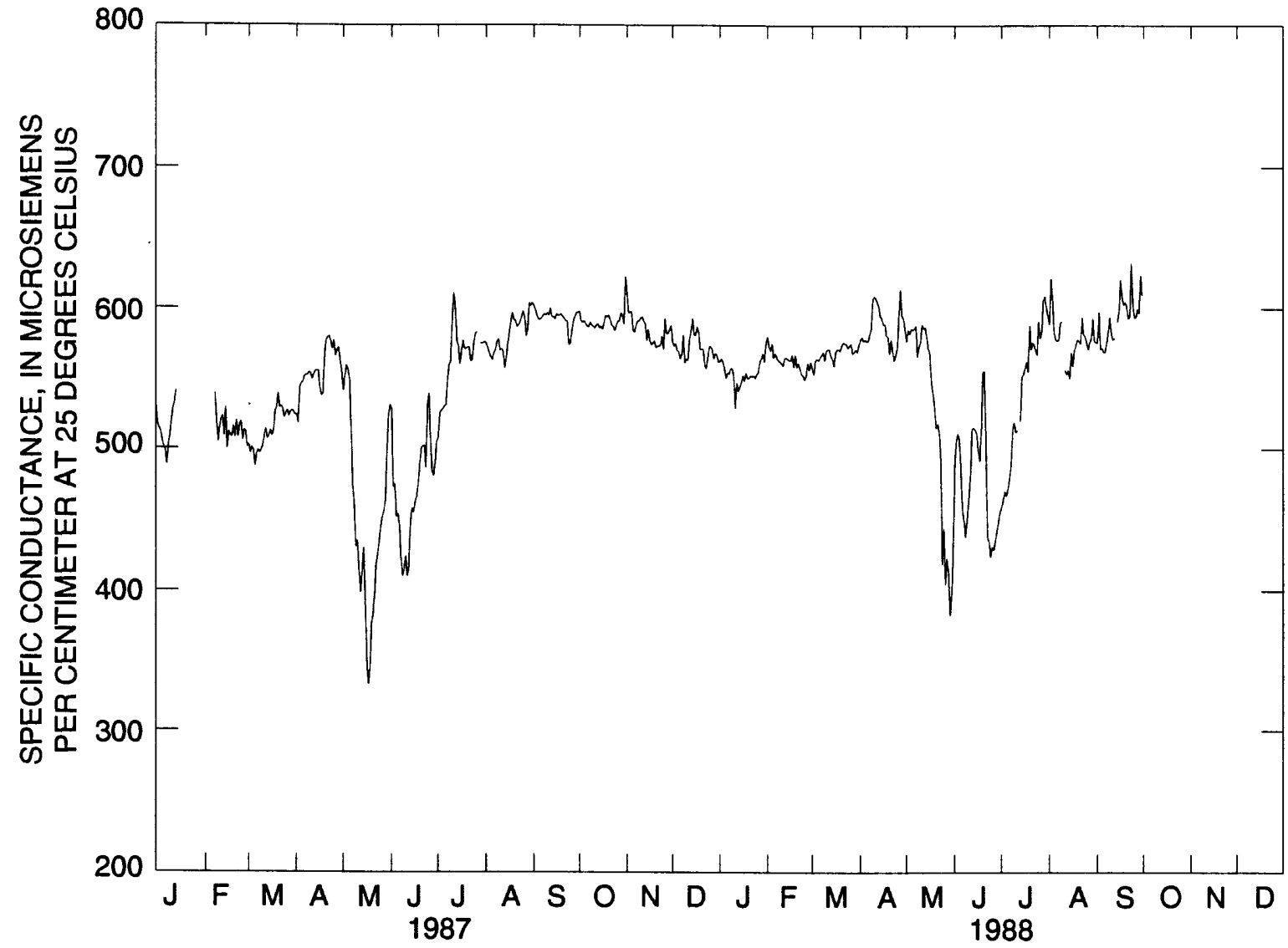

Figure 43. Daily mean specific conductance, Hot Creek Flume (HCF), Long Valley Caldera, Mono County, California, 1987-88. 
Table 98. Daily mean specific conductance, Hot Creek Flume (HCF), Long Valley Caldera, Mono County, California, 1987

[Values are in microsiemens per centimeter at 25 degrees Celsius. Max, maximum; Min, minimum; -, no data]

\begin{tabular}{|c|c|c|c|c|c|c|c|c|c|c|c|c|}
\hline Day & Jan & Feb & Mar & Apr & May & June & July & Aug & Sept & Oct & Nov & Dec \\
\hline 1 & 518 & - & 503 & 524 & 541 & 528 & 508 & 575 & 602 & 598 & 613 & 576 \\
\hline 2 & 514 & - & 497 & 518 & 551 & 473 & 525 & 572 & 599 & 591 & 597 & 573 \\
\hline 3 & 512 & - & 501 & 543 & 559 & 475 & 527 & 568 & 595 & 590 & 597 & 575 \\
\hline 4 & 506 & - & 499 & 546 & 556 & 452 & 528 & 565 & 592 & 591 & 598 & 570 \\
\hline 5 & 501 & 一 & 488 & 547 & 549 & 454 & 530 & 563 & 592 & 589 & 584 & 569 \\
\hline 6 & 499 & - & 496 & 551 & 512 & 446 & 531 & 568 & 593 & 587 & 583 & 564 \\
\hline 7 & 489 & 539 & 499 & 552 & 474 & 423 & 549 & 570 & 595 & 587 & 588 & 567 \\
\hline 8 & 502 & 520 & 497 & 552 & 460 & 410 & 559 & 576 & 596 & 590 & 591 & 581 \\
\hline 9 & 509 & 505 & 499 & 554 & 431 & 415 & 562 & 578 & 595 & 588 & 591 & 561 \\
\hline 10 & 520 & 515 & 502 & 553 & 435 & 424 & 592 & 570 & 597 & 587 & 593 & 564 \\
\hline 11 & 528 & 521 & 509 & 549 & 415 & 410 & 611 & 571 & 595 & 586 & 594 & 563 \\
\hline 12 & 533 & 523 & 514 & 552 & 399 & 416 & 602 & 569 & 600 & 588 & 591 & 578 \\
\hline 13 & 541 & 509 & 507 & 555 & 413 & 447 & 577 & 557 & 594 & 589 & 589 & 584 \\
\hline 14 & 539 & 529 & 509 & 555 & 430 & 458 & 573 & 564 & 594 & 586 & 577 & 593 \\
\hline 15 & - & 500 & 513 & 555 & 396 & 455 & 560 & 574 & 592 & 587 & 585 & 581 \\
\hline 16 & - & 512 & 509 & 543 & 351 & 462 & 569 & 583 & 596 & 585 & 577 & 581 \\
\hline 17 & - & 509 & 512 & 537 & 333 & 466 & 577 & 591 & 595 & 590 & 574 & 587 \\
\hline 18 & - & 508 & 526 & 539 & 343 & 475 & 571 & 597 & 595 & 595 & 575 & 583 \\
\hline 19 & - & 516 & 529 & 566 & 376 & 489 & 571 & 592 & 596 & 594 & 577 & 571 \\
\hline 20 & 一 & 508 & 539 & 577 & 383 & 500 & 572 & 591 & 594 & 595 & 572 & 571 \\
\hline 21 & - & 520 & 529 & 579 & 398 & 502 & 572 & 587 & 593 & 592 & 573 & 571 \\
\hline 22 & - & 508 & 530 & 580 & 419 & 502 & 562 & 588 & 591 & 588 & 573 & 559 \\
\hline 23 & - & 517 & 528 & 576 & 426 & 487 & 563 & 590 & 591 & 587 & 574 & 557 \\
\hline 24 & - & 519 & 522 & 571 & 435 & 531 & 575 & 594 & 574 & 584 & 580 & 565 \\
\hline 25 & - & 506 & 525 & 577 & 446 & 539 & 582 & 598 & 575 & 588 & 571 & 573 \\
\hline 26 & - & 513 & 527 & 566 & 452 & 507 & 583 & 594 & 584 & 591 & 593 & 573 \\
\hline 27 & - & 512 & 523 & 570 & 456 & 484 & - & 580 & 591 & 591 & 582 & 571 \\
\hline 28 & - & 501 & 526 & 572 & 464 & 481 & 575 & 585 & 594 & 597 & 582 & 564 \\
\hline 29 & - & - & 527 & 563 & 500 & 490 & 575 & 604 & 597 & 595 & 585 & 568 \\
\hline 30 & - & - & 526 & 554 & 524 & 505 & 575 & 602 & 597 & 589 & 588 & 567 \\
\hline 31 & - & - & 524 & - & 531 & - & 576 & 604 & - & 623 & - & 562 \\
\hline Mean & $515^{1}$ & $514^{1}$ & 514 & 556 & 450 & 470 & 564 & 581 & 593 & 591 & 585 & 572 \\
\hline Max & 541 & 539 & 539 & 580 & 559 & 539 & 611 & 604 & 602 & 623 & 613 & 593 \\
\hline Min & 489 & 500 & 488 & 518 & 333 & 410 & 508 & 557 & 574 & 584 & 571 & 557 \\
\hline
\end{tabular}

${ }^{1}$ Incomplete Record 
Table 99. Daily mean specific conductance, Hot Creek Flume (HCF), Long Valley Caldera, Mono County, California, 1988

[Values are in microsiemens per centimeter at 25 degrees Celsius. Max, maximum; Min, minimum; -, no data]

\begin{tabular}{|c|c|c|c|c|c|c|c|c|c|c|c|c|}
\hline Day & Jan & Feb & Mar & Apr & May & June & July & Aug & Sept & Oct & Nov & Dec \\
\hline 1 & 562 & 580 & 557 & 578 & 577 & 488 & 459 & 589 & 576 & - & - & - \\
\hline 2 & 564 & 573 & 553 & 580 & 585 & 506 & 464 & 622 & 598 & - & - & - \\
\hline 3 & 562 & 570 & 563 & 577 & 582 & 511 & 470 & 607 & 571 & - & - & - \\
\hline 4 & 557 & 575 & 564 & 578 & 586 & 507 & 467 & 583 & 572 & - & - & - \\
\hline 5 & 550 & 565 & 563 & 577 & 585 & 484 & 472 & 578 & 569 & - & - & - \\
\hline 6 & 554 & 568 & 565 & 577 & 586 & 457 & 479 & 577 & 569 & - & - & - \\
\hline 7 & 553 & 565 & 567 & 582 & 588 & 449 & 489 & 578 & 576 & - & - & - \\
\hline 8 & 557 & 563 & 569 & 586 & 566 & 438 & 514 & 590 & 584 & - & - & - \\
\hline 9 & 557 & 562 & 563 & 607 & 573 & 452 & 519 & 591 & 594 & - & - & - \\
\hline 10 & 552 & 561 & 570 & 609 & 576 & 465 & 512 & - & 582 & - & - & - \\
\hline 11 & 529 & 559 & 571 & 608 & 589 & 480 & 513 & 556 & 578 & - & - & - \\
\hline 12 & 547 & 564 & 571 & 605 & 586 & 514 & - & 553 & 579 & - & - & - \\
\hline 13 & 541 & 565 & 566 & 602 & 587 & 515 & 520 & 556 & - & - & - & - \\
\hline 14 & 544 & 564 & 564 & 595 & 580 & 514 & 551 & 550 & 591 & - & - & - \\
\hline 15 & 547 & 563 & 559 & 593 & 572 & 511 & 553 & 569 & 598 & - & - & - \\
\hline 16 & 552 & 563 & 569 & 589 & 568 & 499 & 557 & 559 & 621 & - & - & - \\
\hline 17 & 548 & 567 & 571 & 589 & 548 & 492 & 562 & 570 & 609 & - & - & - \\
\hline 18 & 554 & 558 & 571 & 581 & 540 & 514 & 555 & 574 & 603 & - & - & - \\
\hline 19 & 550 & 567 & 570 & 580 & 531 & 555 & 588 & 578 & 605 & - & - & - \\
\hline 20 & 550 & 558 & 574 & 568 & 515 & 555 & 571 & 577 & 602 & - & - & - \\
\hline 21 & 552 & 561 & 576 & 578 & 518 & 499 & 576 & 575 & 593 & - & - & - \\
\hline 22 & 551 & 557 & 575 & 570 & 512 & 438 & 574 & 594 & 595 & - & - & - \\
\hline 23 & 552 & 553 & 575 & 563 & 494 & 435 & 570 & 582 & 633 & - & - & - \\
\hline 24 & 550 & 552 & 572 & 567 & 419 & 424 & 567 & 580 & 604 & - & - & - \\
\hline 25 & 553 & 549 & 574 & 572 & 444 & 431 & 591 & 576 & 594 & - & - & - \\
\hline 26 & 554 & 552 & 575 & 596 & 405 & 429 & 579 & 571 & 594 & - & - & - \\
\hline 27 & 562 & 561 & 568 & 614 & 423 & 435 & 583 & 576 & 600 & - & - & - \\
\hline 28 & 564 & 556 & 569 & 595 & 414 & 442 & 606 & 579 & 597 & - & - & - \\
\hline 29 & 568 & 562 & 571 & 593 & 383 & 449 & 609 & 593 & 624 & - & - & - \\
\hline 30 & 562 & - & 569 & 585 & 395 & 456 & 601 & 577 & 610 & - & - & - \\
\hline 31 & 575 & - & 574 & - & 427 & - & 595 & 576 & - & - & - & - \\
\hline Mean & 554 & 563 & 568 & 586 & 524 & 478 & 542 & 578 & 594 & - & - & - \\
\hline $\operatorname{Max}$ & 575 & 580 & 576 & 614 & 589 & 555 & 609 & 622 & 633 & - & - & - \\
\hline Min & 529 & 549 & 553 & 563 & 383 & 424 & 459 & 550 & 569 & - & - & - \\
\hline
\end{tabular}




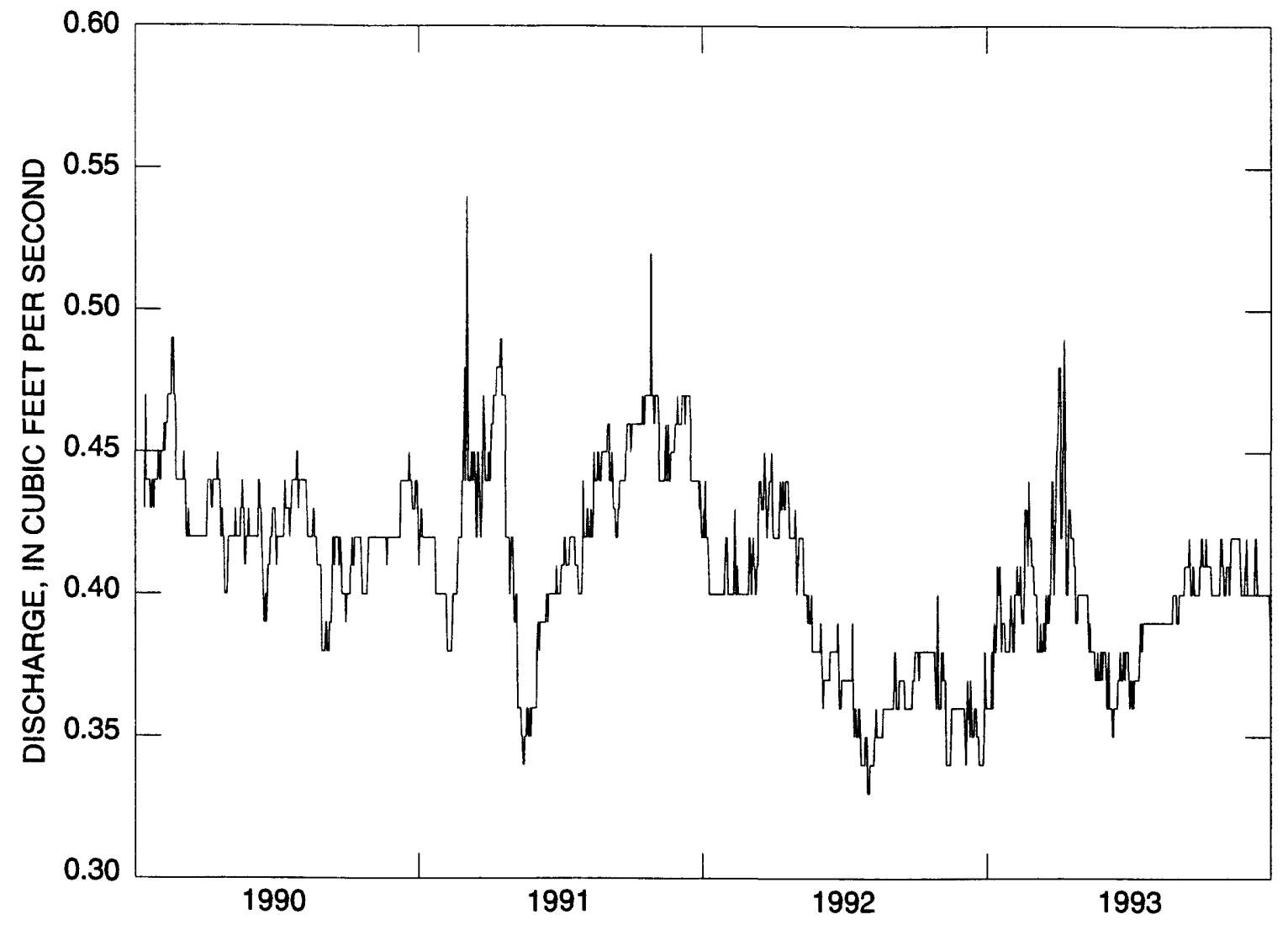

Figure 44. Daily mean discharge, Little Hot Creek downstream from Little Hot Creek Spring (LHC-SW), Long Valley Caldera, Mono County, California, 1990-93. 


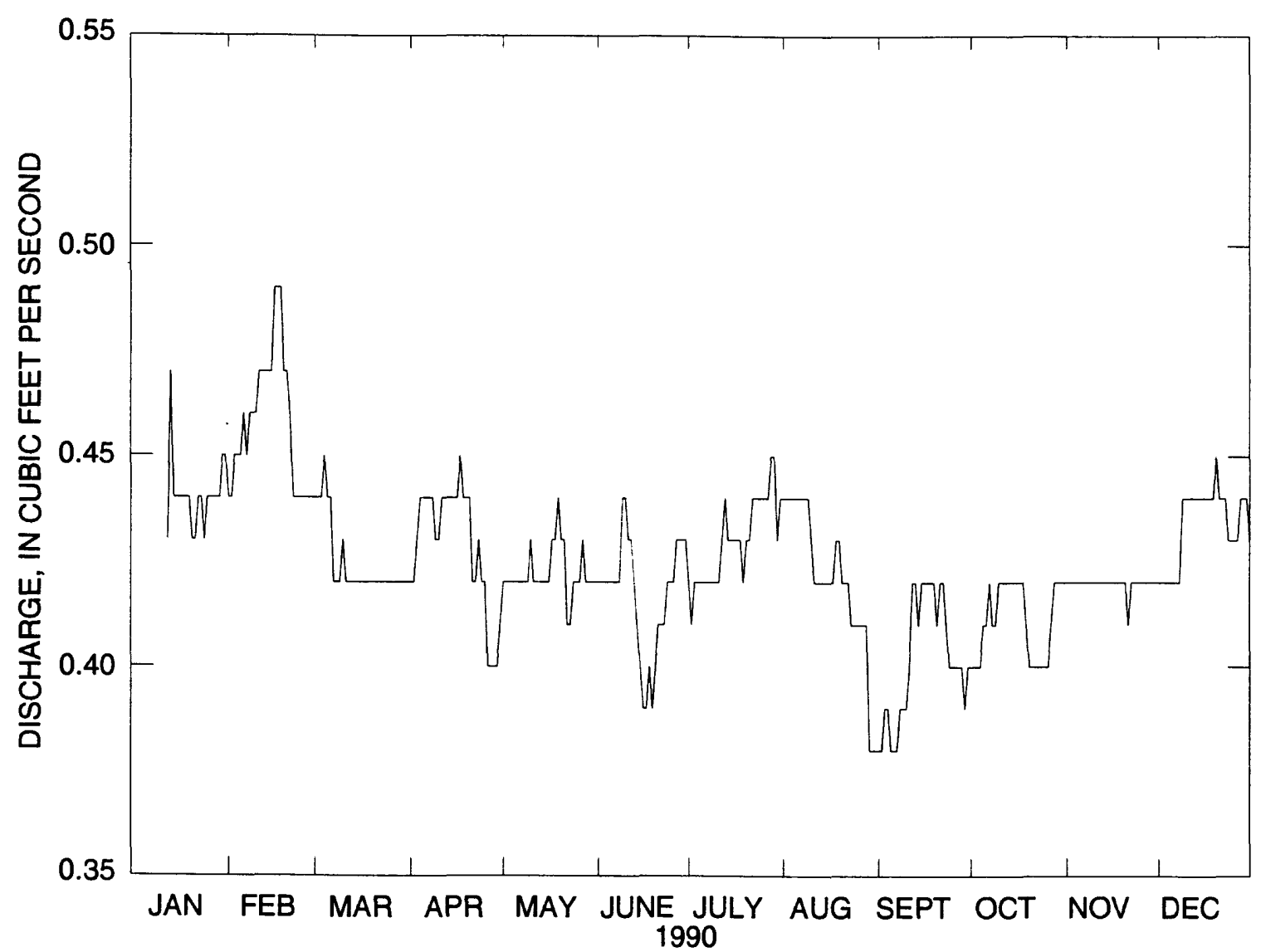

Figure 45. (A) Daily mean discharge, Little Hot Creek downstream from Little Hot Creek Spring (LHC-SW), Long Valley Caldera, Mono County, California, 1990. 
Table 100. Daily mean discharge, Little Hot Creek downstream of Little Hot Creek Spring (LHC-SW), Long Valley Caldera, Mono County, California, 1990

[Values are in cubic feet per second. e, estimated; Max, maximum; Min, minimum; -, no data]

\begin{tabular}{|c|c|c|c|c|c|c|c|c|c|c|c|c|}
\hline Day & Jan & Feb & Mar & Apr & May & June & July & Aug & Sept & Oct & Nov & Dec \\
\hline 1 & - & 0.44 & 0.44 & 0.42 & 0.42 & 0.42 & 0.42 & 0.44 & 0.38 & 0.40 & 0.42 & 0.42 \\
\hline 2 & - & .44 & .44 & .42 & .42 & .42 & .41 & .44 & .38 & .40 & .42 & .42 \\
\hline 3 & - & .45 & .44 & .43 & .42 & .42 & .42 & .44 & .39 & .40 & .42 & .42 \\
\hline 4 & - & .45 & .45 & .44 & .42 & .42 & .42 & .44 & .39 & .40 & .42 & .42 \\
\hline 5 & - & .45 & .44 & .44 & .42 & .42 & .42 & .44 & .38 & .41 & .42 & .42 \\
\hline 6 & - & .46 & .44 & .44 & .42 & .42 & .42 & .44 & .38 & .41 & .42 & .42 \\
\hline 7 & - & .45 & .42 & .44 & .42 & .42 & .42 & .44 & .38 & .42 & .42 & .42 \\
\hline 8 & - & .46 & .42 & .44 & .42 & .42 & .42 & .44 & .39 & .41 & .42 & .42 \\
\hline 9 & - & .46 & .42 & .43 & .42 & .44 & .42 & .44 & .39 & .41 & .42 & .44 \\
\hline 10 & - & .46 & .43 & .43 & .43 & .44 & .42 & .43 & .39 & .42 & .42 & .44 \\
\hline 11 & - & .47 & .42 & .44 & .42 & .43 & .42 & .42 & .40 & .42 & .42 & .44 \\
\hline 12 & 0.43 & .47 & .42 & .44 & .42 & e.43 & .43 & .42 & .42 & .42 & .42 & .44 \\
\hline 13 & .47 & .47 & .42 & .44 & .42 & e. 42 & .44 & .42 & .42 & .42 & .42 & .44 \\
\hline 14 & .44 & .47 & .42 & .44 & .42 & e.41 & .43 & .42 & .41 & .42 & .42 & .44 \\
\hline 15 & .44 & .47 & .42 & .44 & .42 & e. 40 & .43 & .42 & .42 & .42 & .42 & .44 \\
\hline 16 & .44 & .49 & .42 & .44 & .42 & .39 & .43 & .42 & .42 & .42 & .42 & .44 \\
\hline 17 & .44 & .49 & .42 & .45 & .43 & .39 & .43 & .42 & .42 & .42 & .42 & e.44 \\
\hline 18 & .44 & .49 & .42 & .44 & .43 & .40 & .43 & .43 & .42 & .42 & .42 & e.44 \\
\hline 19 & .44 & .47 & .42 & .44 & .44 & .39 & .42 & .43 & .42 & .41 & .42 & e.44 \\
\hline 20 & .43 & .47 & .42 & .44 & .43 & .40 & .43 & .42 & .41 & .40 & .42 & .45 \\
\hline 21 & .43 & .46 & .42 & .42 & .43 & .41 & .43 & .42 & .42 & .40 & .41 & .44 \\
\hline 22 & .44 & .44 & .42 & .42 & .41 & .41 & .44 & .42 & .42 & .40 & .42 & .44 \\
\hline 23 & .44 & .44 & .42 & .43 & e.41 & .41 & .44 & .41 & .41 & .40 & .42 & .44 \\
\hline 24 & .43 & .44 & .42 & .42 & e. 42 & .42 & .44 & .41 & .40 & .40 & .42 & .43 \\
\hline 25 & .44 & .44 & .42 & .42 & .42 & .42 & .44 & .41 & .40 & .40 & .42 & .43 \\
\hline 26 & .44 & .44 & .42 & .40 & .42 & .42 & .44 & .41 & .40 & .40 & .42 & .43 \\
\hline 27 & .44 & .44 & .42 & .40 & .43 & .43 & .44 & .41 & .40 & .41 & .42 & .43 \\
\hline 28 & .44 & .44 & .42 & .40 & .42 & .43 & .45 & .41 & .40 & .42 & .42 & .44 \\
\hline 29 & .44 & - & .42 & .40 & .42 & .43 & .45 & .38 & .39 & .42 & .42 & .44 \\
\hline 30 & .45 & - & .42 & .41 & .42 & .43 & .43 & .38 & .40 & .42 & .42 & .44 \\
\hline 31 & .45 & - & .42 & - & .42 & - & .44 & .38 & - & .42 & - & .43 \\
\hline Mean & .44 & .46 & .42 & .43 & .42 & .42 & .43 & .42 & .40 & .41 & .42 & .43 \\
\hline Max & .47 & .49 & .45 & .45 & .44 & .44 & .45 & .44 & .42 & .42 & .42 & .45 \\
\hline Min & .43 & .44 & .42 & .40 & .41 & .39 & .41 & .38 & .38 & .40 & .41 & .42 \\
\hline
\end{tabular}




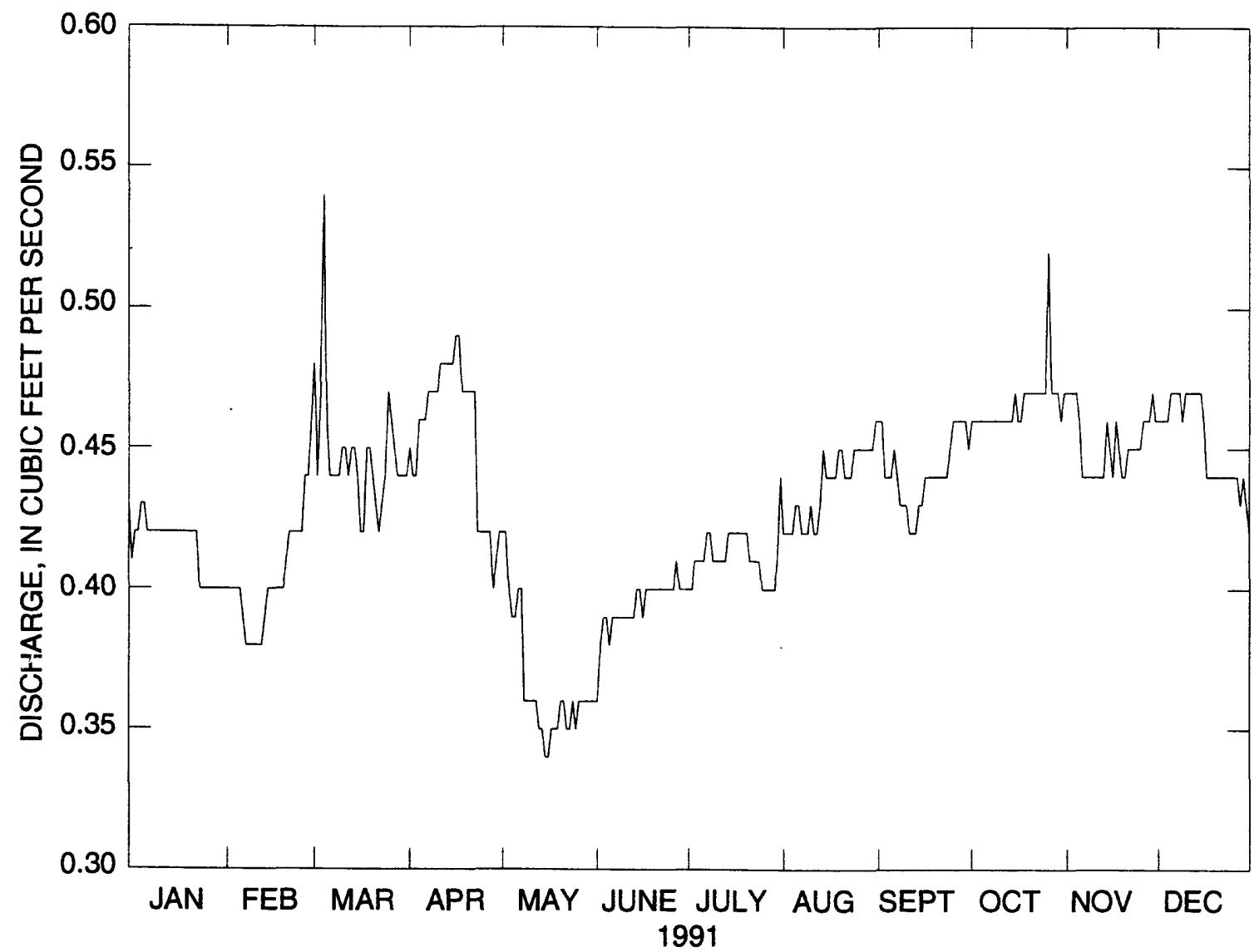

Figure 45. (B) Daily mean discharge, Little Hot Creek downstream from Little Hot Creek Spring (LHC-SW), Long Valley Caldera, Mono County, California, 1991. 
Table 101. Daily mean discharge, Little Hot Creek downstream of Little Hot Creek Spring (LHC-SW), Long Valley Caldera, Mono County, California, 1991

[Values are in cubic feet per second. Max, maximum; Min, minimum; -, no data]

\begin{tabular}{|c|c|c|c|c|c|c|c|c|c|c|c|c|}
\hline Day & Jan & Feb & Mar & Apr & May & June & July & Aug & Sept & Oct & Nov & Dec \\
\hline 1 & 0.41 & 0.40 & 0.48 & 0.45 & 0.42 & 0.36 & 0.40 & 0.42 & 0.46 & 0.46 & 0.47 & 0.46 \\
\hline 2 & .42 & .40 & .44 & .44 & .42 & .38 & .40 & .42 & .46 & .46 & .47 & .46 \\
\hline 3 & .42 & .40 & .47 & .44 & .40 & .39 & .41 & .42 & .44 & .46 & .47 & .46 \\
\hline 4 & .43 & .40 & .54 & .46 & .39 & .39 & .41 & .42 & .44 & .46 & .47 & .46 \\
\hline 5 & .43 & .40 & .46 & .46 & .39 & .38 & .41 & .43 & .44 & .46 & .46 & .47 \\
\hline 6 & .42 & .39 & .44 & .46 & .40 & .39 & .41 & .43 & .45 & .46 & .44 & .47 \\
\hline 7 & .42 & .38 & .44 & .47 & .40 & .39 & .42 & .42 & .44 & .46 & .44 & .47 \\
\hline 8 & .42 & .38 & .44 & .47 & .36 & .39 & .42 & .42 & .43 & .46 & .44 & .47 \\
\hline 9 & .42 & .38 & .44 & .47 & .36 & .39 & .41 & .42 & .43 & .46 & .44 & .46 \\
\hline 10 & .42 & .38 & .45 & .47 & .36 & .39 & .41 & .43 & .43 & .46 & .44 & .47 \\
\hline 11 & .42 & .38 & .45 & .48 & .36 & .39 & .41 & .42 & .42 & .46 & .44 & .47 \\
\hline 12 & .42 & .38 & .44 & .48 & .36 & .39 & .41 & .42 & .42 & .46 & .44 & .47 \\
\hline 13 & .42 & .39 & .45 & .48 & .35 & .39 & .41 & .43 & .42 & .46 & .44 & .47 \\
\hline 14 & .42 & .40 & .45 & .48 & .35 & .40 & .42 & .45 & .43 & .46 & .46 & .47 \\
\hline 15 & .42 & .40 & .44 & .48 & .34 & .40 & .42 & .44 & .43 & .47 & .45 & .47 \\
\hline 16 & .42 & .40 & .42 & .49 & .34 & .39 & .42 & .44 & .44 & .46 & .44 & .46 \\
\hline 17 & .42 & .40 & .42 & .49 & .35 & .40 & .42 & .44 & .44 & .46 & .46 & .44 \\
\hline 18 & .42 & .40 & .45 & .47 & .35 & .40 & .42 & .44 & .44 & .47 & .45 & .44 \\
\hline 19 & .42 & .40 & .45 & .47 & .35 & .40 & .42 & .45 & .44 & .47 & .44 & .44 \\
\hline 20 & .42 & .41 & .44 & .47 & .36 & .40 & .42 & .45 & .44 & .47 & .44 & .44 \\
\hline 21 & .42 & .42 & .43 & .47 & .36 & .40 & .41 & .44 & .44 & .47 & .45 & .44 \\
\hline 22 & .42 & .42 & .42 & .47 & .35 & .40 & .41 & .44 & .44 & .47 & .45 & .44 \\
\hline 23 & .40 & .42 & .43 & .42 & .35 & .40 & .41 & .44 & .44 & .47 & .45 & .44 \\
\hline 24 & .40 & .42 & .44 & .42 & .36 & .40 & .41 & .45 & .45 & .47 & .45 & .44 \\
\hline 25 & .40 & .42 & .47 & .42 & .35 & .40 & .40 & .45 & .46 & .47 & .45 & .44 \\
\hline 26 & .40 & .44 & .46 & .42 & .36 & .40 & .40 & .45 & .46 & .52 & .46 & .44 \\
\hline 27 & .40 & .44 & .45 & .42 & .36 & .41 & .40 & .45 & .46 & .47 & .46 & .44 \\
\hline 28 & .40 & .46 & .44 & .40 & .36 & .40 & .40 & .45 & .46 & .47 & .46 & .43 \\
\hline 29 & .40 & - & .44 & .41 & .36 & .40 & .40 & .45 & .46 & .47 & .47 & .44 \\
\hline 30 & .40 & - & .44 & .42 & .36 & .40 & .41 & .45 & .45 & .46 & .46 & .43 \\
\hline 31 & .40 & - & .44 & - & .36 & - & .44 & .46 & - & .47 & - & .42 \\
\hline Mean & .41 & .40 & .45 & .45 & .37 & .39 & .41 & .44 & .44 & .47 & .45 & .45 \\
\hline $\operatorname{Max}$ & .43 & .46 & .54 & .49 & .42 & .41 & .44 & .46 & .46 & .52 & .47 & .47 \\
\hline Min & .40 & .38 & .42 & .40 & .34 & .36 & .40 & .42 & .42 & .46 & .44 & .42 \\
\hline
\end{tabular}




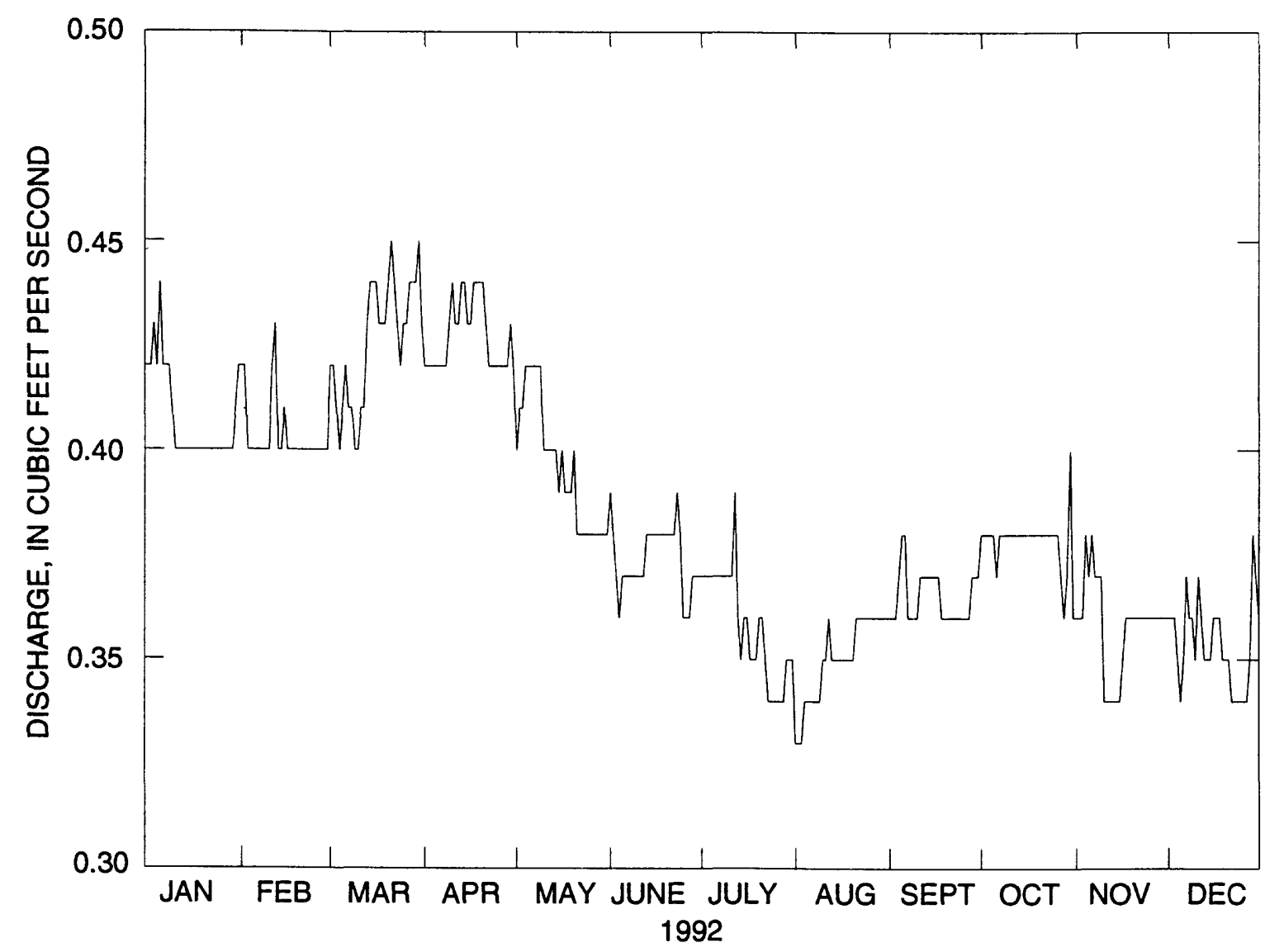

Figure 45. (C) Daily mean discharge, Little Hot Creek downstream from Little Hot Creek Spring (LHC-SW), Long Valley Caldera, Mono County, California, 1992. 
Table 102. Daily mean discharge, Little Hot Creek downstream of Little Hot Creek Spring (LHC-SW), Long Valley Caldera, Mono County, California, 1992

[Values are in cubic feet per second. Max, maximum; Min, minimum; -, no data]

\begin{tabular}{|c|c|c|c|c|c|c|c|c|c|c|c|c|}
\hline Day & Jan & Feb & Mar & Apr & May & June & July & Aug & Sept & Oct & Nov & Dec \\
\hline 1 & 0.42 & 0.42 & 0.42 & 0.42 & 0.40 & 0.39 & 0.37 & 0.33 & 0.36 & 0.38 & 0.36 & 0.36 \\
\hline 2 & .42 & .42 & .42 & .42 & .41 & .38 & .37 & .33 & .36 & .38 & .36 & .36 \\
\hline 3 & .43 & .40 & .41 & .42 & .41 & .37 & .37 & .33 & .36 & .38 & .36 & .36 \\
\hline 4 & .42 & .40 & .40 & .42 & .42 & .36 & .37 & .34 & .37 & .38 & .38 & .35 \\
\hline 5 & .44 & .40 & .41 & .42 & .42 & .37 & .37 & .34 & .38 & .38 & .37 & .34 \\
\hline 6 & .42 & .40 & .42 & .42 & .42 & .37 & .37 & .34 & .38 & .37 & .38 & .35 \\
\hline 7 & .42 & .40 & .41 & .42 & .42 & .37 & .37 & .34 & .36 & .38 & .37 & .37 \\
\hline 8 & .42 & .40 & .41 & .42 & .42 & .37 & .37 & .34 & .36 & .38 & .37 & .36 \\
\hline 9 & .41 & .40 & .40 & .43 & .42 & .37 & .37 & .34 & .36 & .38 & .37 & .36 \\
\hline 10 & .40 & .40 & .40 & .44 & .40 & .37 & .37 & .35 & .36 & .38 & .34 & .35 \\
\hline 11 & .40 & .42 & .41 & .43 & .40 & .37 & .37 & .35 & .37 & .38 & .34 & .37 \\
\hline 12 & .40 & .43 & .41 & .43 & .40 & .37 & .39 & .36 & .37 & .38 & .34 & .36 \\
\hline 13 & .40 & .40 & .43 & .44 & .40 & .38 & .36 & .35 & .37 & .38 & .34 & .35 \\
\hline 14 & .40 & .40 & .44 & .44 & .40 & .38 & .35 & .35 & .37 & .38 & .34 & .35 \\
\hline 15 & .40 & .41 & .44 & .43 & .39 & .38 & .36 & .35 & .37 & .38 & .34 & .35 \\
\hline 16 & .40 & .40 & .44 & .43 & .40 & .38 & .36 & .35 & .37 & .38 & .35 & .36 \\
\hline 17 & .40 & .40 & .43 & .44 & .39 & .38 & .35 & .35 & .37 & .38 & .36 & .36 \\
\hline 18 & .40 & .40 & .43 & .44 & .39 & .38 & .35 & .35 & .36 & .38 & .36 & .36 \\
\hline 19 & .40 & .40 & .43 & .44 & .39 & .38 & .35 & .35 & .36 & .38 & .36 & .35 \\
\hline 20 & .40 & .40 & .44 & .44 & .40 & .38 & .36 & .35 & .36 & .38 & .36 & .35 \\
\hline 21 & .40 & .40 & .45 & .43 & .38 & .38 & .36 & .36 & .36 & .38 & .36 & .35 \\
\hline 22 & .40 & .40 & .44 & .42 & .38 & .38 & .35 & .36 & .36 & .38 & .36 & .34 \\
\hline 23 & .40 & .40 & .43 & .42 & .38 & .39 & .34 & .36 & .36 & .38 & .36 & .34 \\
\hline 24 & .40 & .40 & .42 & .42 & .38 & .38 & .34 & .36 & .36 & .38 & .36 & .34 \\
\hline 25 & .40 & .40 & .43 & .42 & .38 & .36 & .34 & .36 & .36 & .38 & .36 & .34 \\
\hline 26 & .40 & .40 & .43 & .42 & .38 & .36 & .34 & .36 & .36 & .38 & .36 & .34 \\
\hline 27 & .40 & .40 & .44 & .42 & .38 & .36 & .34 & .36 & .36 & .37 & .36 & .34 \\
\hline 28 & .40 & .40 & .44 & .42 & .38 & .37 & .34 & .36 & .37 & .36 & .36 & .35 \\
\hline 29 & .40 & .40 & .44 & .43 & .38 & .37 & .35 & .36 & .37 & .37 & .36 & .38 \\
\hline 30 & .41 & - & .45 & .42 & .38 & .37 & .35 & .36 & .37 & .40 & .36 & .37 \\
\hline 31 & .42 & - & .43 & - & .38 & - & .35 & .36 & - & .36 & - & .36 \\
\hline Mean & .41 & .40 & .43 & .43 & .40 & .37 & .36 & .35 & .36 & .38 & .36 & .35 \\
\hline Max & .44 & .43 & .45 & .44 & .42 & .39 & .39 & .36 & .38 & .40 & .38 & .38 \\
\hline Min & .40 & .40 & .40 & .42 & .38 & .36 & .34 & .33 & .36 & .36 & .34 & .34 \\
\hline
\end{tabular}




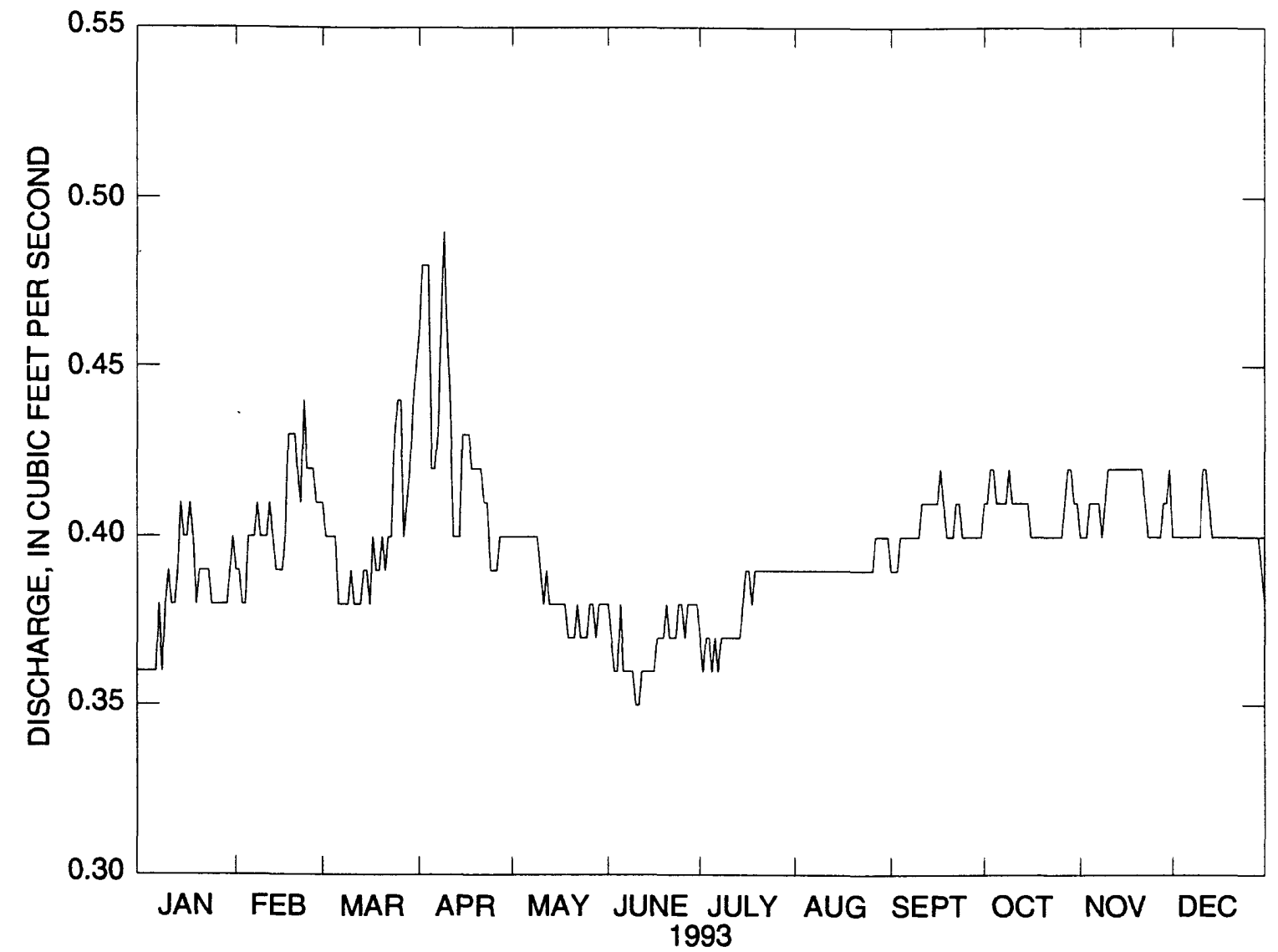

Figure 45. (D) Daily mean discharge, Little Hot Creek downstream from Little Hot Creek Spring (LHC-SW), Long Valley Caldera, Mono County, California, 1993. 
Table 103. Daily mean discharge, Little Hot Creek downstream of Little Hot Creek Spring (LHC-SW), Long Valley Caldera, Mono County, California, 1993

[Values are in cubic feet per second. Max, maximum: Min, minimum; -, no data]

\begin{tabular}{|c|c|c|c|c|c|c|c|c|c|c|c|c|}
\hline Day & Jan & Feb & Mar & Apr & May & June & July & Aug & Sept & Oct & Nov & Dec \\
\hline 1 & 0.36 & 0.39 & 0.41 & 0.46 & 0.40 & 0.38 & 0.37 & 0.39 & 0.39 & 0.41 & 0.40 & 0.40 \\
\hline 2 & .36 & .39 & .40 & .48 & .40 & .37 & .36 & .39 & .39 & .41 & .40 & .40 \\
\hline 3 & .36 & .38 & .40 & .48 & .40 & .36 & .37 & .39 & .39 & .42 & .40 & .40 \\
\hline 4 & .36 & .38 & .40 & .48 & .40 & .36 & .37 & .39 & .40 & .42 & .41 & .40 \\
\hline 5 & .36 & .40 & .40 & .42 & .40 & .38 & .36 & .39 & .40 & .41 & .41 & .40 \\
\hline 6 & .36 & .40 & .38 & .42 & .40 & .36 & .37 & .39 & .40 & .41 & .41 & .40 \\
\hline 7 & .38 & .40 & .38 & .43 & .40 & .36 & .36 & .39 & .40 & .41 & .41 & .40 \\
\hline 8 & .36 & .41 & .38 & .46 & .40 & .36 & .37 & .39 & .40 & .41 & .40 & .40 \\
\hline 9 & .38 & .40 & .38 & .49 & .40 & .36 & .37 & .39 & .40 & .42 & .41 & .40 \\
\hline 10 & .39 & .40 & .39 & .46 & .39 & .35 & .37 & .39 & .40 & .41 & .42 & .40 \\
\hline 11 & .38 & .40 & .38 & .44 & .38 & .35 & .37 & .39 & .41 & .41 & .42 & .42 \\
\hline 12 & .38 & .41 & .38 & .40 & .39 & .36 & .37 & .39 & .41 & .41 & .42 & .42 \\
\hline 13 & .39 & .40 & .38 & .40 & .38 & .36 & .37 & .39 & .41 & .41 & .42 & .41 \\
\hline 14 & .41 & .39 & .39 & .40 & .38 & .36 & .37 & .39 & .41 & .41 & .42 & .40 \\
\hline 15 & .40 & .39 & .39 & .43 & .38 & .36 & .38 & .39 & .41 & .41 & .42 & .40 \\
\hline 16 & .40 & .39 & .38 & .43 & .38 & .36 & .39 & .39 & .41 & .40 & .42 & .40 \\
\hline 17 & .41 & .40 & .40 & .43 & .38 & .37 & .39 & .39 & .42 & .40 & .42 & .40 \\
\hline 18 & .40 & .43 & .39 & .42 & .38 & .37 & .38 & .39 & .41 & .40 & .42 & .40 \\
\hline 19 & .38 & .43 & .39 & .42 & .37 & .37 & .39 & .39 & .40 & .40 & .42 & .40 \\
\hline 20 & .39 & .43 & .40 & .42 & .37 & .38 & .39 & .39 & .40 & .40 & .42 & .40 \\
\hline 21 & .39 & .42 & .39 & .42 & .37 & .37 & .39 & .39 & .40 & .40 & .42 & .40 \\
\hline 22 & .39 & .41 & .40 & .41 & .38 & .37 & .39 & .39 & .41 & .40 & .41 & .40 \\
\hline 23 & .39 & .44 & .40 & .41 & .37 & .37 & .39 & .39 & .41 & .40 & .40 & .40 \\
\hline 24 & .38 & .42 & .43 & .39 & .37 & .38 & .39 & .39 & .40 & .40 & .40 & .40 \\
\hline 25 & .38 & .42 & .44 & .39 & .37 & .38 & .39 & .39 & .40 & .40 & .40 & .40 \\
\hline 26 & .38 & .42 & .44 & .39 & .38 & .37 & .39 & .39 & .40 & .40 & .40 & .40 \\
\hline 27 & .38 & .41 & .40 & .40 & .38 & .38 & .39 & .40 & .40 & .41 & .40 & .40 \\
\hline 28 & .38 & .41 & .41 & .40 & .37 & .38 & .39 & .40 & .40 & .42 & .41 & .40 \\
\hline 29 & .38 & - & .42 & .40 & .38 & .38 & .39 & .40 & .40 & .42 & .41 & .40 \\
\hline 30 & .39 & - & .44 & .40 & .38 & .38 & .39 & .40 & .40 & .41 & .42 & .39 \\
\hline 31 & .40 & - & .45 & - & .38 & - & .39 & .40 & - & .41 & - & .38 \\
\hline Mean & .38 & .41 & .40 & .43 & .38 & .37 & .38 & .39 & .40 & .41 & .41 & .40 \\
\hline $\operatorname{Max}$ & .41 & .44 & .45 & .49 & .40 & .38 & .39 & .40 & .42 & .42 & .42 & .42 \\
\hline Min & .36 & .38 & .38 & .39 & .37 & .35 & .36 & .39 & .39 & .40 & .40 & .38 \\
\hline
\end{tabular}




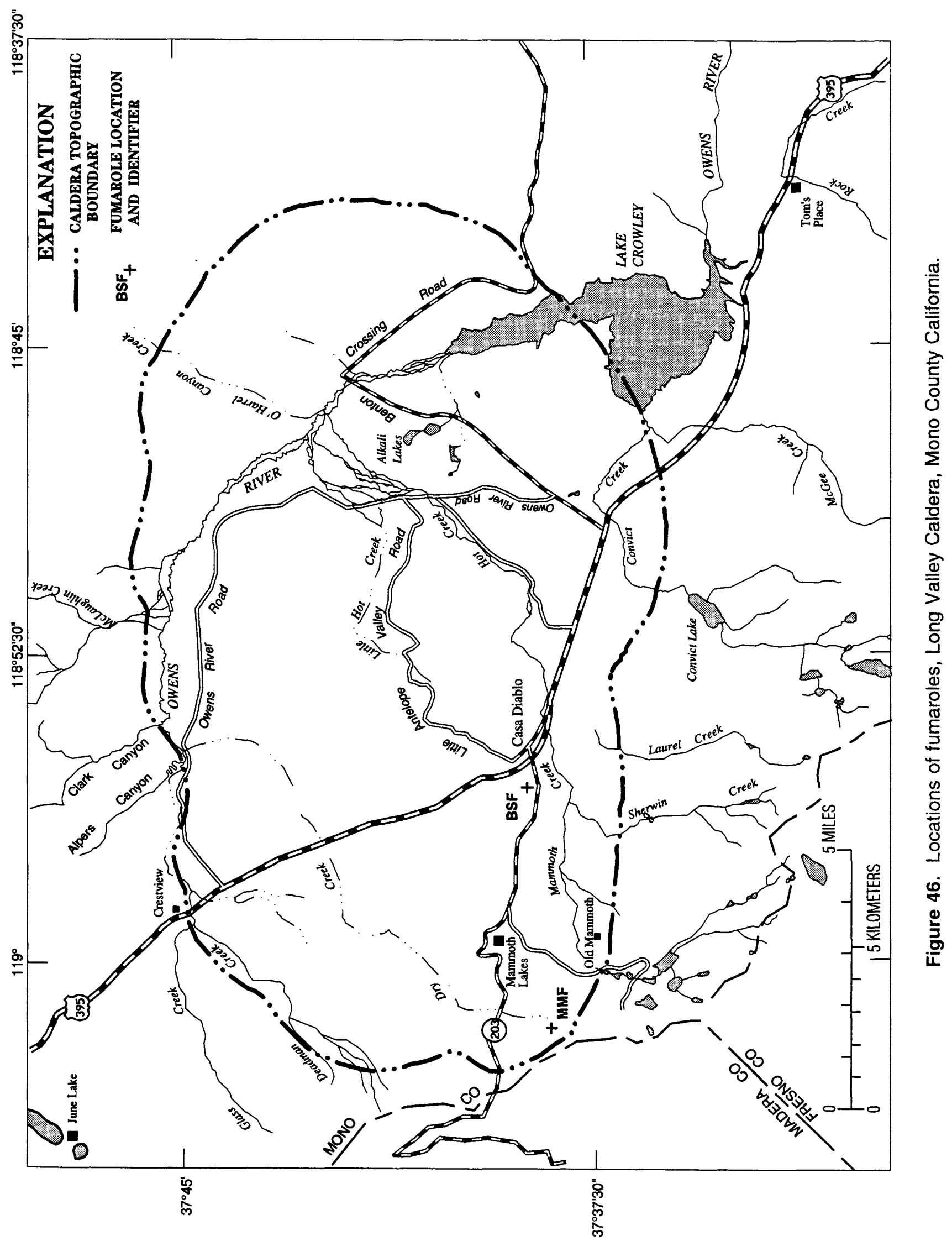




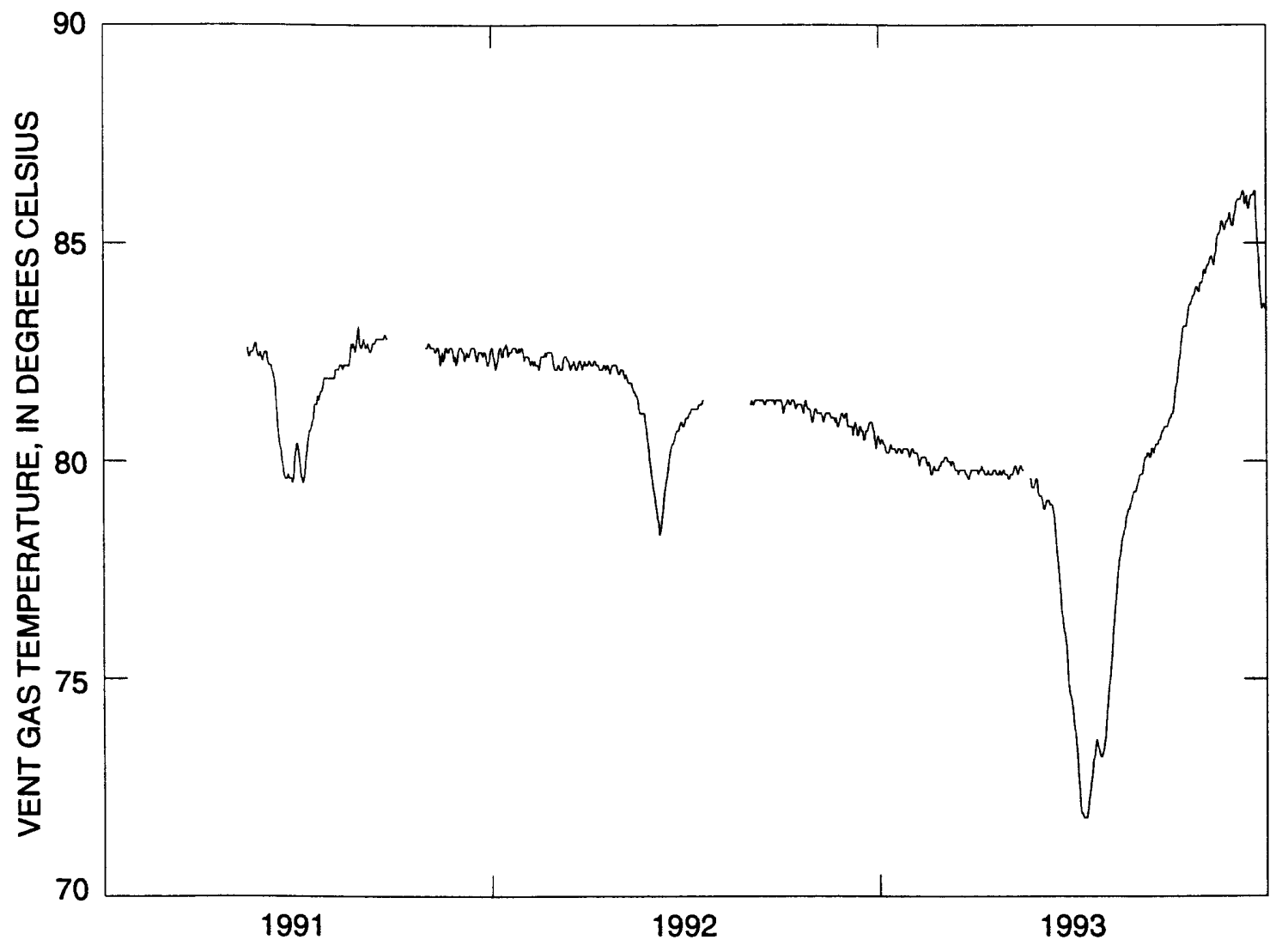

Figure 47. Daily mean vent gas temperature, Mammoth Mountain Fumarole (MMF), Long Valley Caldera, Mono County, California, 1991-93.

Figure $47 \quad 245$ 


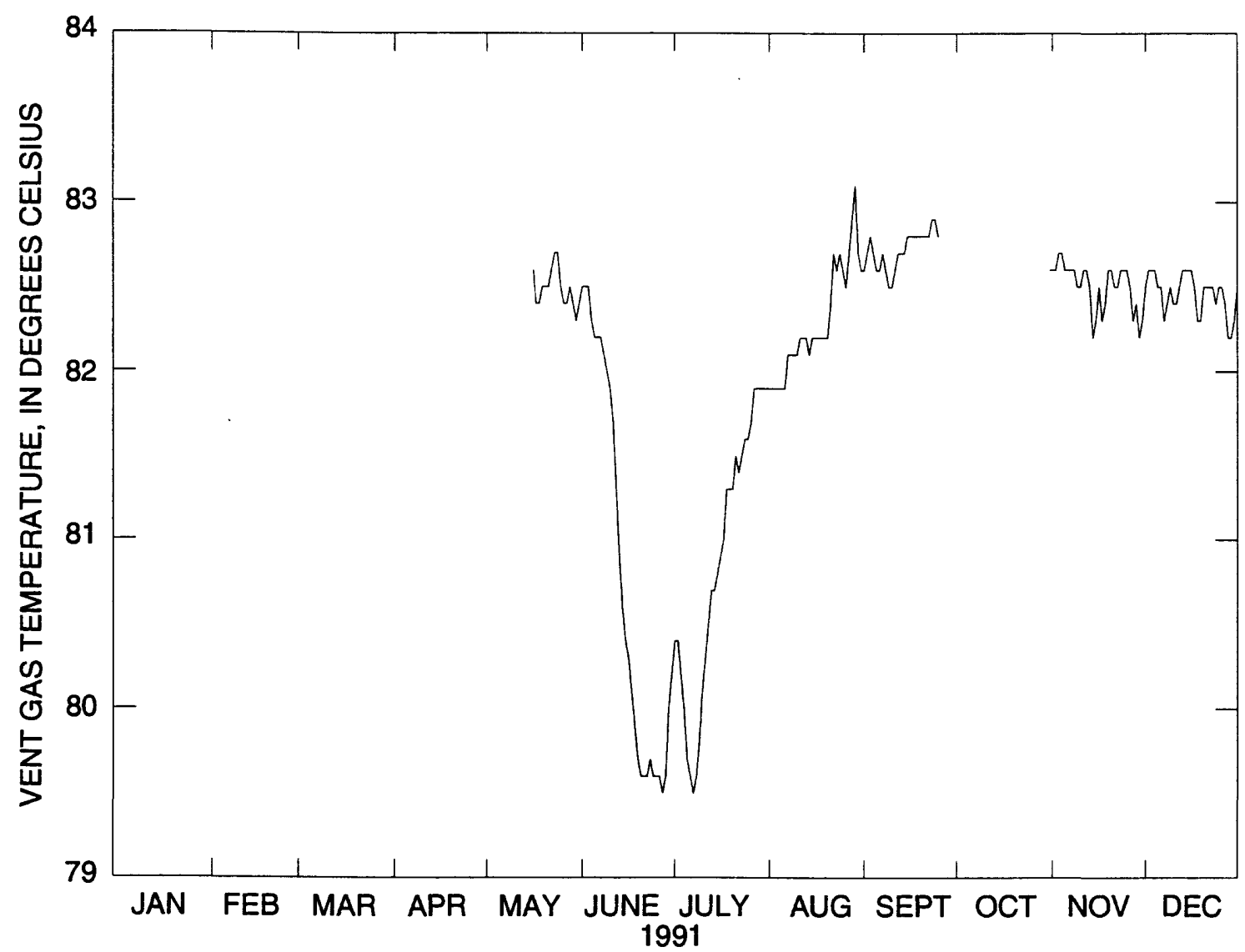

Figure 48. (A) Daily mean vent gas temperature, Mammoth Mountain Fumarole (MMF), Long Valley Caldera, Mono County, California, 1991. 
Table 104. Daily mean vent gas temperature, Mammoth Mountain Fumarole (MMF), Long Valley Caldera, Mono County, California, 1991

[Values are in degrees Celsius. Max. maximum: Min, minimum; -, no data]

\begin{tabular}{|c|c|c|c|c|c|c|c|c|c|c|c|c|}
\hline Day & Jan & Feb & Mar & Apr & May & June & July & Aug & Sept & Oct & Nov & Dec \\
\hline 1 & - & - & - & - & - & 82.5 & 80.4 & 81.9 & 82.6 & - & 82.6 & 82.5 \\
\hline 2 & - & - & - & - & - & 82.5 & 80.4 & 81.9 & 82.7 & - & 82.6 & 82.6 \\
\hline 3 & - & - & - & - & - & 82.5 & 80.2 & 81.9 & 82.8 & - & 82.7 & 82.6 \\
\hline 4 & - & - & - & - & - & 82.3 & 80.0 & 81.9 & 82.7 & - & 82.7 & 82.6 \\
\hline 5 & - & - & - & - & - & 82.2 & 79.7 & 81.9 & 82.6 & - & 82.6 & 82.5 \\
\hline 6 & - & - & - & - & - & 82.2 & 79.6 & 81.9 & 82.6 & - & 82.6 & 82.5 \\
\hline 7 & - & - & - & - & - & 82.2 & 79.5 & 82.1 & 82.7 & - & 82.6 & 82.3 \\
\hline 8 & - & - & - & - & - & 82.1 & 79.6 & 82.1 & 82.6 & - & 82.6 & 82.4 \\
\hline 9 & - & - & - & - & - & 82.0 & 79.8 & 82.1 & 82.5 & - & 82.5 & 82.5 \\
\hline 10 & - & - & - & - & - & 81.9 & 80.1 & 82.1 & 82.5 & - & 82.5 & 82.4 \\
\hline 11 & - & - & - & - & - & 81.7 & 80.3 & 82.2 & 82.6 & - & 82.6 & 82.4 \\
\hline 12 & - & - & - & - & - & 81.3 & 80.5 & 82.2 & 82.7 & - & 82.6 & 82.5 \\
\hline 13 & - & - & - & - & - & 80.9 & 80.7 & 82.2 & 82.7 & - & 82.5 & 82.6 \\
\hline 14 & - & - & - & - & - & 80.6 & 80.7 & 82.1 & 82.7 & - & 82.2 & 82.6 \\
\hline 15 & - & - & - & - & - & 80.4 & 80.8 & 82.2 & 82.8 & - & 82.3 & 82.6 \\
\hline 16 & - & - & - & - & 82.6 & 80.3 & 80.9 & 82.2 & 82.8 & - & 82.5 & 82.6 \\
\hline 17 & - & - & - & - & 82.4 & 80.1 & 81.0 & 82.2 & 82.8 & - & 82.3 & 82.5 \\
\hline 18 & - & - & - & - & 82.4 & 79.9 & 81.3 & 82.2 & 82.8 & - & 82.4 & 82.3 \\
\hline 19 & - & - & - & - & 82.5 & 79.7 & 81.3 & 82.2 & 82.8 & - & 82.6 & 82.3 \\
\hline 20 & - & - & - & - & 82.5 & 79.6 & 81.3 & 82.2 & 82.8 & - & 82.6 & 82.5 \\
\hline 21 & - & - & - & - & 82.5 & 79.6 & 81.5 & 82.4 & 82.8 & - & 82.5 & 82.5 \\
\hline 22 & - & - & - & - & 82.6 & 79.6 & 81.4 & 82.7 & 82.8 & - & 82.5 & 82.5 \\
\hline 23 & - & - & - & - & 82.7 & 79.7 & 81.5 & 82.6 & 82.9 & - & 82.6 & 82.5 \\
\hline 24 & - & - & - & - & 82.7 & 79.6 & 81.6 & 82.7 & 82.9 & - & 82.6 & 82.4 \\
\hline 25 & - & - & - & - & 82.5 & 79.6 & 81.6 & 82.6 & 82.8 & - & 82.6 & 82.5 \\
\hline 26 & - & - & - & - & 82.4 & 79.6 & 81.7 & 82.5 & - & - & 82.5 & 82.5 \\
\hline 27 & - & - & - & - & 82.4 & 79.5 & 81.9 & 82.7 & - & - & 82.3 & 82.4 \\
\hline 28 & - & - & - & - & 82.5 & 79.6 & 81.9 & 82.9 & - & - & 82.4 & 82.2 \\
\hline 29 & - & - & - & - & 82.4 & 80.0 & 81.9 & 83.1 & - & - & 82.2 & 82.2 \\
\hline 30 & - & - & - & - & 82.3 & 80.2 & 81.9 & 82.7 & - & - & 82.3 & 82.3 \\
\hline 31 & - & - & - & - & 82.4 & - & 81.9 & 82.6 & - & 82.6 & - & 82.5 \\
\hline Mean & - & - & - & - & 82.5 & 80.8 & 80.9 & 82.3 & 82.7 & 82.6 & 82.5 & 82.5 \\
\hline Max & - & - & - & - & 82.7 & 82.5 & 81.9 & 83.1 & 82.9 & 82.6 & 82.7 & 82.6 \\
\hline Min & - & - & - & - & 82.3 & 79.5 & 79.5 & 81.9 & 82.5 & 82.6 & 82.2 & 82.2 \\
\hline
\end{tabular}




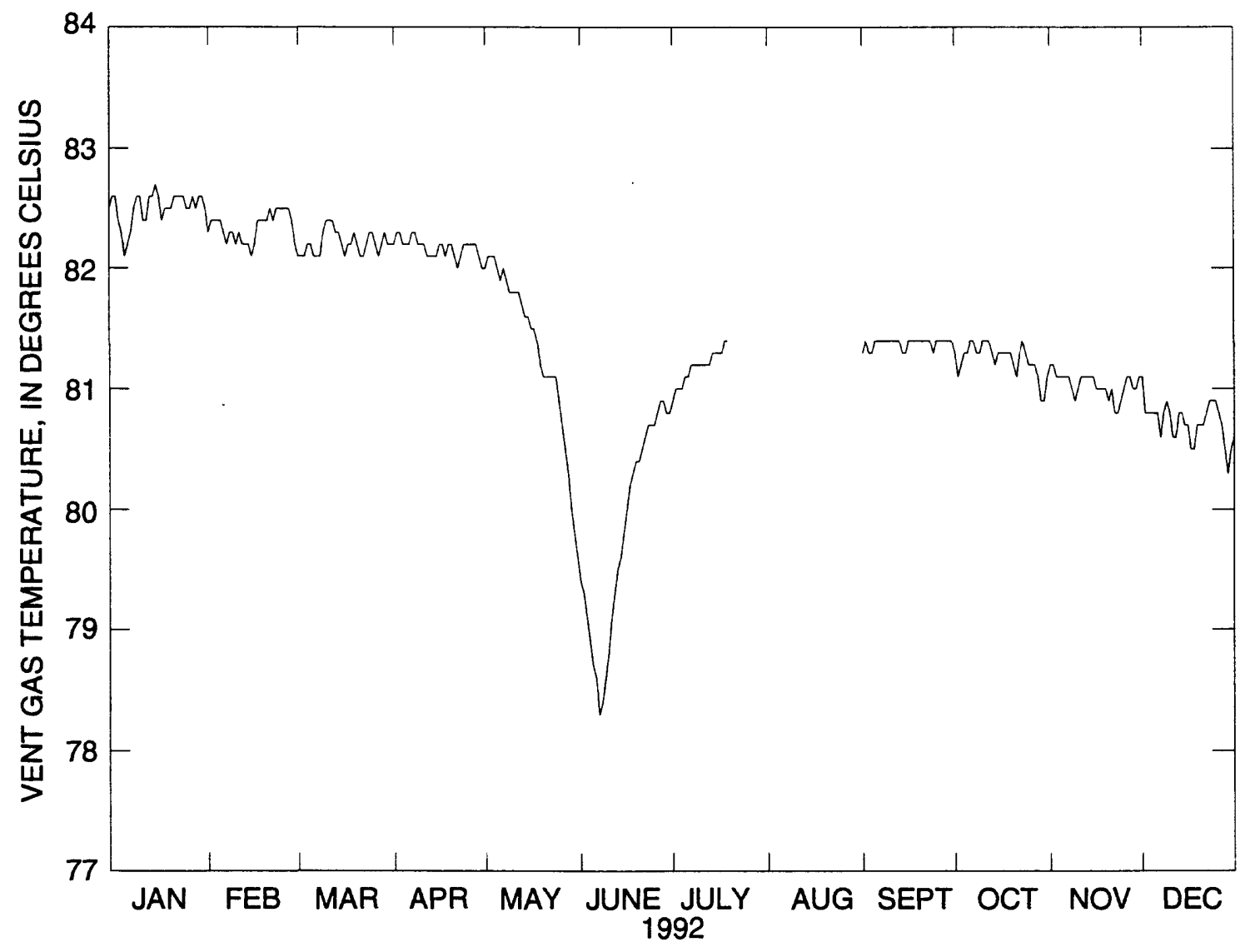

Figure 48. (B) Daily mean vent gas temperature, Mammoth Mountain Fumarole (MMF), Long Valley Caldera, Mono County, California, 1992. 
Table 105. Daily mean vent gas temperature, Mammoth Mountain Fumarole (MMF), Long Valley Caldera, Mono County, California, 1992

[Values are in degrees Celsius. Max, maximum; Min, minimum; -, no data]

\begin{tabular}{|c|c|c|c|c|c|c|c|c|c|c|c|c|}
\hline Day & Jan & Feb & Mar & Apr & May & June & July & Aug & Sept & Oct & Nov & Dec \\
\hline 1 & 82.6 & 82.3 & 82.1 & 82.2 & 82.0 & 79.4 & 80.9 & - & 81.3 & 81.3 & 81.2 & $81.1^{\prime}$ \\
\hline 2 & 82.6 & 82.4 & 82.1 & 82.3 & 82.1 & 79.3 & 81.0 & - & 81.4 & 81.1 & 81.2 & 80.8 \\
\hline 3 & 82.4 & 82.4 & 82.1 & 82.3 & 82.1 & 79.1 & 81.0 & - & 81.3 & 81.2 & 81.1 & 80.8 \\
\hline 4 & 82.3 & 82.4 & 82.2 & 82.2 & 82.1 & 78.9 & 81.0 & - & 81.3 & 81.3 & 81.1 & 80.8 \\
\hline 5 & 82.1 & 82.4 & 82.2 & 82.2 & 82.0 & 78.7 & 81.1 & - & 81.4 & 81.3 & 81.1 & 80.8 \\
\hline 6 & 82.2 & 82.3 & 82.1 & 82.2 & 81.9 & 78.6 & 81.1 & - & 81.4 & 81.4 & 81.1 & 80.8 \\
\hline 7 & 82.3 & 82.2 & 82.1 & 82.3 & 82.0 & 78.3 & 81.2 & - & 81.4 & 81.4 & 81.1 & 80.6 \\
\hline 8 & 82.5 & 82.3 & 82.1 & 82.3 & 81.9 & 78.4 & 81.2 & - & 81.4 & 81.3 & 81.0 & 80.8 \\
\hline 9 & 82.6 & 82.3 & 82.3 & 82.2 & 81.8 & 78.6 & 81.2 & - & 81.4 & 81.3 & 80.9 & 80.9 \\
\hline 10 & 82.6 & 82.2 & 82.4 & 82.2 & 81.8 & 78.8 & 81.2 & - & 81.4 & 81.4 & 81.0 & 80.8 \\
\hline 11 & 82.4 & 82.3 & 82.4 & 82.2 & 81.8 & 79.1 & 81.2 & - & 81.4 & 81.4 & 81.1 & 80.6 \\
\hline 12 & 82.4 & 82.2 & 82.4 & 82.1 & 81.8 & 79.3 & 81.2 & - & 81.4 & 81.4 & 81.1 & 80.6 \\
\hline 13 & 82.6 & 82.2 & 82.3 & 82.1 & 81.7 & 79.5 & 81.2 & - & 81.4 & 81.3 & 81.1 & 80.8 \\
\hline 14 & 82.6 & 82.2 & 82.3 & 82.1 & 81.6 & 79.6 & 81.3 & - & 81.3 & 81.2 & 81.1 & 80.8 \\
\hline 15 & 82.7 & 82.1 & 82.2 & 82.1 & 81.6 & 79.8 & 81.3 & - & 81.3 & 81.3 & 81.1 & 80.7 \\
\hline 16 & 82.6 & 82.2 & 82.1 & 82.2 & 81.5 & 80.0 & 81.3 & - & 81.4 & 81.3 & 81.0 & 80.7 \\
\hline 17 & 82.4 & 82.4 & 82.2 & 82.2 & 81.5 & 80.2 & 81.3 & - & 81.4 & 81.3 & 81.0 & 80.5 \\
\hline 18 & 82.5 & 82.4 & 82.2 & 82.1 & 81.4 & 80.3 & 81.4 & - & 81.4 & 81.3 & 81.0 & 80.5 \\
\hline 19 & 82.5 & 82.4 & 82.3 & 82.2 & 81.2 & 80.4 & 81.4 & - & 81.4 & 81.3 & 81.0 & 80.7 \\
\hline 20 & 82.5 & 82.4 & 82.2 & 82.2 & 81.1 & 80.4 & - & - & 81.4 & 81.2 & 80.9 & 80.7 \\
\hline 21 & 82.6 & 82.5 & 82.1 & 82.1 & 81.1 & 80.5 & - & - & 81.4 & 81.1 & 81.0 & 80.7 \\
\hline 22 & 82.6 & 82.4 & 82.1 & 82.0 & 81.1 & 80.6 & - & - & 81.4 & 81.3 & 80.8 & 80.8 \\
\hline 23 & 82.6 & 82.5 & 82.2 & 82.1 & 81.1 & 80.7 & - & - & 81.4 & 81.4 & 80.8 & 80.9 \\
\hline 24 & 82.6 & 82.5 & 82.3 & 82.2 & 81.1 & 80.7 & - & - & 81.3 & 81.3 & 80.9 & 80.9 \\
\hline 25 & 82.5 & 82.5 & 82.3 & 82.2 & 80.9 & 80.7 & - & - & 81.4 & 81.2 & 81.0 & 80.9 \\
\hline 26 & 82.5 & 82.5 & 82.2 & 82.2 & 80.7 & 80.8 & - & - & 81.4 & 81.2 & 81.1 & 80.8 \\
\hline 27 & 82.6 & 82.5 & 82.1 & 82.2 & 80.5 & 80.9 & - & - & 81.4 & 81.2 & 81.1 & 80.7 \\
\hline 28 & 82.5 & 82.4 & 82.2 & 82.2 & 80.3 & 80.9 & - & - & 81.4 & 81.1 & 81.0 & 80.5 \\
\hline 29 & 82.6 & 82.2 & 82.3 & 82.1 & 80.0 & 80.8 & - & - & 81.4 & 80.9 & 81.0 & 80.3 \\
\hline 30 & 82.6 & - & 82.2 & 82.0 & 79.8 & 80.8 & - & - & 81.4 & 80.9 & 81.1 & 80.5 \\
\hline 31 & 82.5 & - & 82.2 & - & 79.6 & - & - & - & - & 81.1 & - & 80.6 \\
\hline Mean & 82.5 & 82.3 & 82.2 & 82.2 & 81.3 & 79.8 & 81.2 & - & 81.4 & 81.2 & 81.0 & 80.7 \\
\hline $\operatorname{Max}$ & 82.7 & 82.5 & 82.4 & 82.3 & 82.1 & 80.9 & 81.4 & - & 81.4 & 81.4 & 81.2 & 81.1 \\
\hline Min & 82.1 & 82.1 & 82.1 & 82.0 & 79.6 & 78.3 & 80.9 & - & 81.3 & 80.9 & 80.8 & 80.3 \\
\hline
\end{tabular}




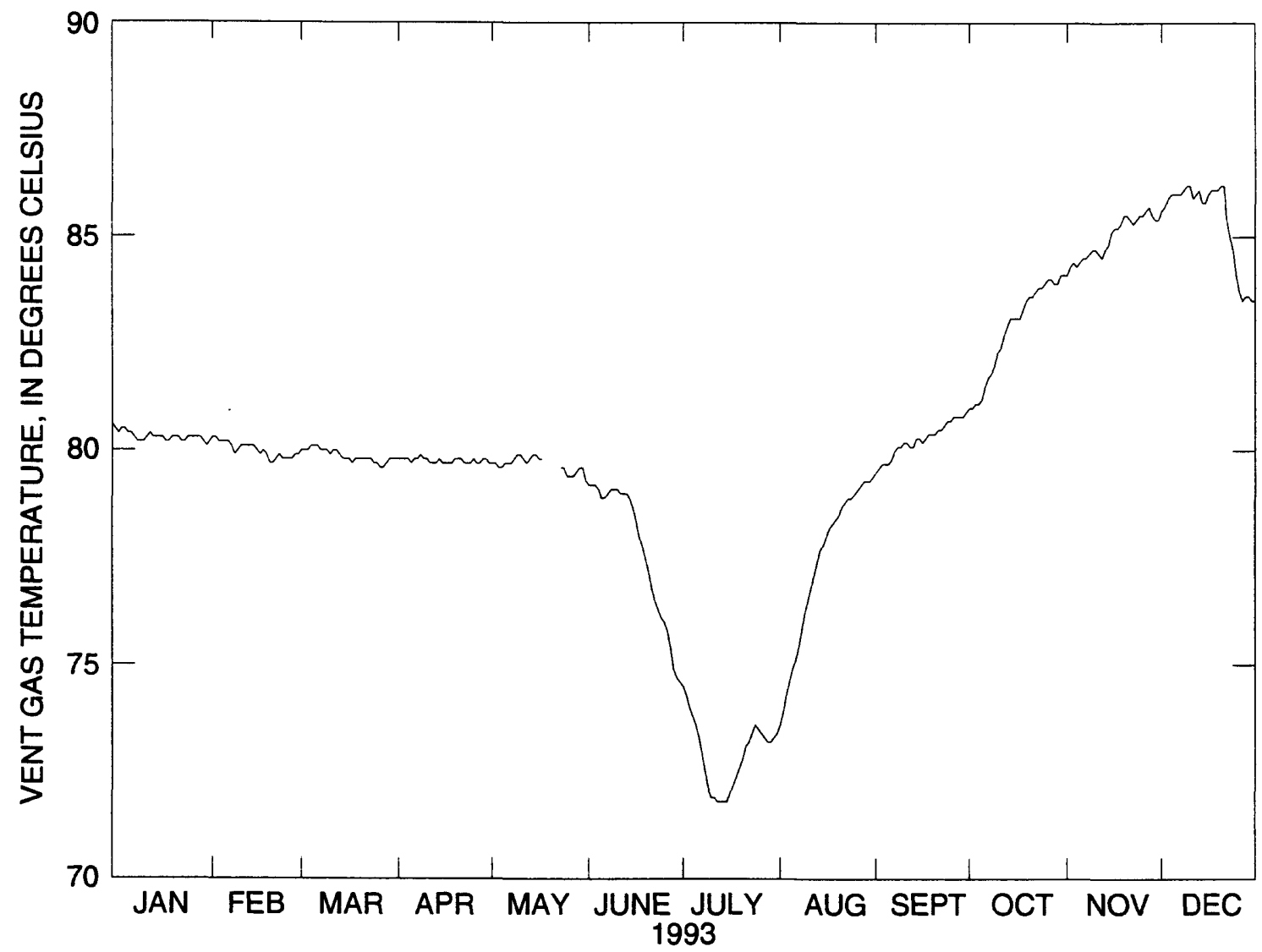

Figure 48. (C) Daily mean vent gas temperature, Mammoth Mountain Fumarole (MMF), Long Valley Caldera, Mono County, California, 1993. 
Table 106. Daily mean vent gas temperature, Mammoth Mountain Fumarole (MMF), Long Valley Caldera, Mono County, California, 1993

[Values are in degrees Celsius. Max, maximum; Min, minimum; -, no data]

\begin{tabular}{|c|c|c|c|c|c|c|c|c|c|c|c|c|}
\hline Day & Jan & Feb & Mar & Apr & May & June & July & Aug & Sept & Oct & Nov & Dec \\
\hline 1 & 80.5 & 80.3 & 80.0 & 79.8 & 79.7 & 79.2 & 74.5 & 73.6 & 79.5 & 81.0 & 84.1 & 85.6 \\
\hline 2 & 80.4 & 80.3 & 80.0 & 79.8 & 79.7 & 79.2 & 74.3 & 73.9 & 79.6 & 81.0 & 84.3 & 85.7 \\
\hline 3 & 80.5 & 80.2 & 80.0 & 79.8 & 79.6 & 79.2 & 74.0 & 74.3 & 79.7 & 81.1 & 84.4 & 85.9 \\
\hline 4 & 80.5 & 80.2 & 80.1 & 79.8 & 79.6 & 79.1 & 73.8 & 74.6 & 79.7 & 81.1 & 84.3 & 86.0 \\
\hline 5 & 80.4 & 80.2 & 80.1 & 79.7 & 79.7 & 78.9 & 73.6 & 74.9 & 79.7 & 81.2 & 84.4 & 86.0 \\
\hline 6 & 80.4 & 80.2 & 80.1 & 79.8 & 79.7 & 78.9 & 73.3 & 75.1 & 79.8 & 81.5 & 84.5 & 86.0 \\
\hline 7 & 80.3 & 80.1 & 80.0 & 79.8 & 79.7 & 79.0 & 72.9 & 75.4 & 80.0 & 81.7 & 84.5 & 86.0 \\
\hline 8 & 80.2 & 79.9 & 80.0 & 79.9 & 79.8 & 79.1 & 72.5 & 75.8 & 80.1 & 81.8 & 84.6 & 86.1 \\
\hline 9 & 80.2 & 80.0 & 80.0 & 79.8 & 79.9 & 79.1 & 72.1 & 76.2 & 80.1 & 82.0 & 84.7 & 86.2 \\
\hline 10 & 80.2 & 80.1 & 79.9 & 79.8 & 79.9 & 79.1 & 71.9 & 76.5 & 80.2 & 82.3 & 84.7 & 86.2 \\
\hline 11 & 80.3 & 80.1 & 80.0 & 79.7 & 79.8 & 79.0 & 71.9 & 76.8 & 80.2 & 82.4 & 84.6 & 85.9 \\
\hline 12 & 80.4 & 80.1 & 80.0 & 79.7 & 79.7 & 79.0 & 71.8 & 77.1 & 80.1 & 82.7 & 84.5 & 86.0 \\
\hline 13 & 80.3 & 80.1 & 79.9 & 79.7 & 79.8 & 79.0 & 71.8 & 77.4 & 80.1 & 82.9 & 84.7 & 86.1 \\
\hline 14 & 80.3 & 80.1 & 79.8 & 79.8 & 79.9 & 78.9 & 71.8 & 77.7 & 80.3 & 83.1 & 84.8 & 85.8 \\
\hline 15 & 80.3 & 80.0 & 79.8 & 79.7 & 79.9 & 78.7 & 71.8 & 77.8 & 80.3 & 83.1 & 85.1 & 85.8 \\
\hline 16 & 80.3 & 79.9 & 79.8 & 79.7 & 79.8 & 78.4 & 72.0 & 78.0 & 80.2 & 83.1 & 85.2 & 86.0 \\
\hline 17 & 80.2 & 80.0 & 79.7 & 79.7 & 79.8 & 78.0 & 72.2 & 78.2 & 80.3 & 83.1 & 85.2 & 86.1 \\
\hline 18 & 80.2 & 79.9 & 79.8 & 79.7 & - & 77.8 & 72.4 & 78.3 & 80.4 & 83.3 & 85.3 & 86.1 \\
\hline 19 & 80.3 & 79.7 & 79.8 & 79.8 & - & 77.5 & 72.6 & 78.4 & 80.4 & 83.5 & 85.5 & 86.1 \\
\hline 20 & 80.3 & 79.7 & 79.8 & 79.8 & - & 77.2 & 72.8 & 78.5 & 80.4 & 83.6 & 85.5 & 86.2 \\
\hline 21 & 80.3 & 79.8 & 79.8 & 79.8 & - & 76.8 & 73.1 & 78.7 & 80.5 & 83.6 & 85.4 & 86.2 \\
\hline 22 & 80.2 & 79.9 & 79.8 & 79.7 & - & 76.5 & 73.2 & 78.8 & 80.5 & 83.7 & 85.3 & 85.4 \\
\hline 23 & 80.2 & 79.8 & 79.8 & 79.7 & 79.6 & 76.3 & 73.4 & 78.9 & 80.6 & 83.8 & 85.4 & 85.0 \\
\hline 24 & 80.3 & 79.8 & 79.7 & 79.7 & 79.6 & 76.1 & 73.6 & 78.9 & 80.7 & 83.8 & 85.5 & 84.7 \\
\hline 25 & 80.3 & 79.8 & 79.7 & 79.8 & 79.4 & 76.0 & 73.5 & 79.0 & 80.7 & 83.9 & 85.5 & 84.1 \\
\hline 26 & 80.3 & 79.8 & 79.6 & 79.7 & 79.4 & 75.8 & 73.4 & 79.1 & 80.8 & 84.0 & 85.6 & 83.7 \\
\hline 27 & 80.3 & 79.9 & 79.6 & 79.7 & 79.4 & 75.4 & 73.3 & 79.2 & 80.8 & 84.0 & 85.7 & 83.5 \\
\hline 28 & 80.3 & 79.9 & 79.7 & 79.8 & 79.5 & 74.9 & 73.2 & 79.3 & 80.8 & 83.9 & 85.5 & 83.6 \\
\hline 29 & 80.2 & - & 79.8 & 79.8 & 79.6 & 74.7 & 73.2 & 79.3 & 80.8 & 83.9 & 85.4 & 83.6 \\
\hline 30 & 80.1 & - & 79.8 & 79.7 & 79.6 & 74.6 & 73.3 & 79.3 & 80.9 & 84.1 & 85.4 & 83.5 \\
\hline 31 & 80.2 & - & 79.8 & - & 79.3 & - & 73.4 & 79.4 & - & 84.1 & - & 83.5 \\
\hline Mean & 80.3 & 80.0 & 79.9 & 79.8 & 79.7 & 77.7 & 72.9 & 77.4 & 80.3 & 82.8 & 85.0 & 85.4 \\
\hline Max & 80.5 & 80.3 & 80.1 & 79.9 & 79.9 & 79.2 & 74.5 & 79.4 & 80.9 & 84.1 & 85.7 & 86.2 \\
\hline Min & 80.1 & 79.7 & 79.6 & 79.7 & 79.3 & 74.6 & 71.8 & 73.6 & 79.5 & 81.0 & 84.1 & 83.5 \\
\hline
\end{tabular}




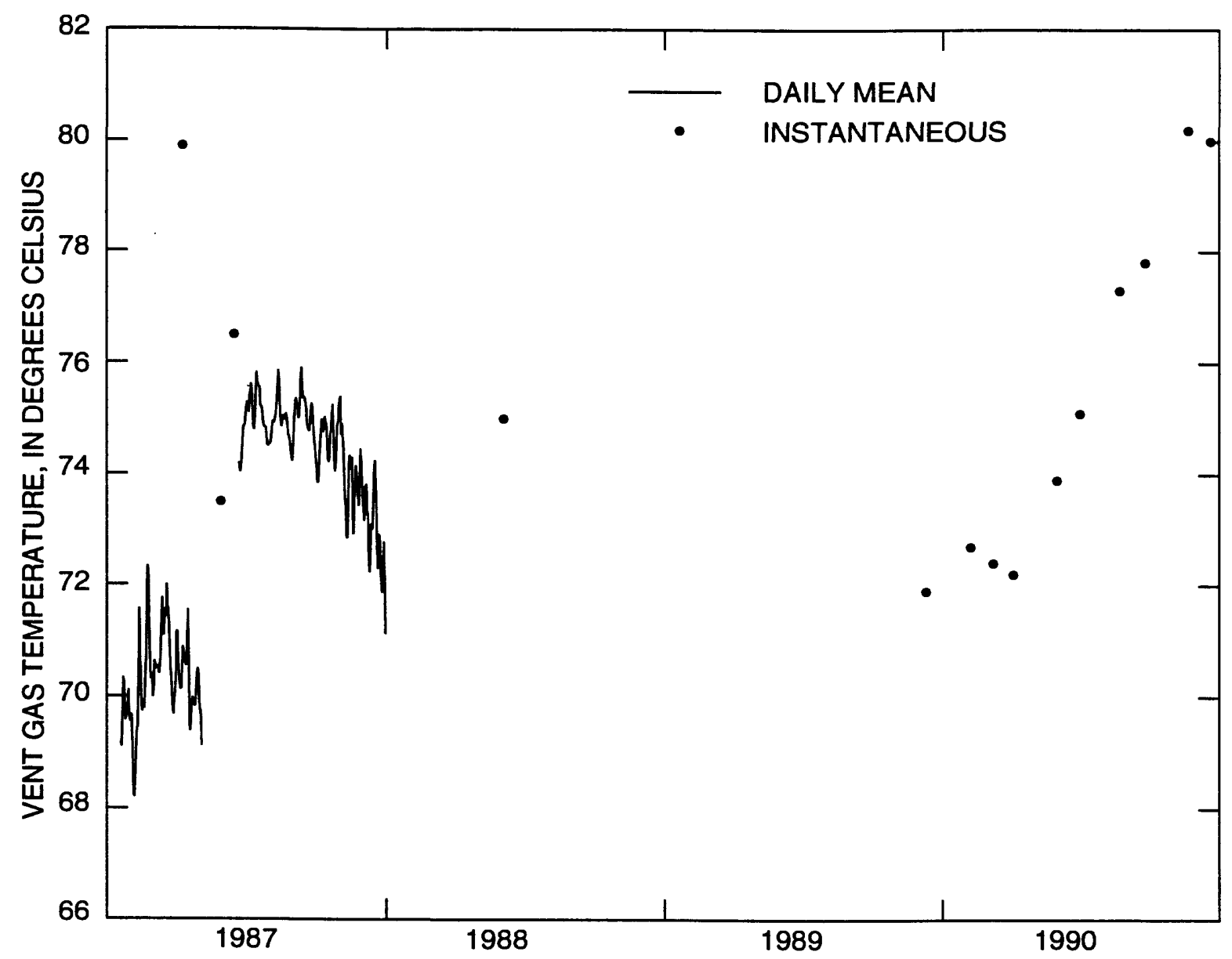

Figure 49. Daily mean and instantaneous measured vent gas temperatures, Basalt Fumarole (BSF), Long Valley Caldera, Mono County, California, 1987-90. 
Table 107. Daily mean vent gas temperature, Basalt Fumarole (BSF), Long Valley Caldera, Mono County, California, 1987

[Values are in degrees Celsius. Max, maximum; Min, minimum; -, no data]

\begin{tabular}{|c|c|c|c|c|c|c|c|c|c|c|c|c|}
\hline Day & Jan & Feb & Mar & Apr & May & June & July & Aug & Sept & Oct & Nov & Dec \\
\hline 1 & - & 69.6 & 70.4 & 70.1 & 70.5 & - & 74.9 & 74.5 & 74.2 & 74.4 & 75.3 & 73.6 \\
\hline 2 & - & 69.7 & 70.3 & 70.3 & 70.4 & - & 74.9 & 74.5 & 74.5 & 74.2 & 75.4 & 73.4 \\
\hline 3 & - & 69.7 & 70.0 & 71.2 & 69.9 & - & 75.2 & 74.5 & 74.8 & 73.9 & 74.7 & 73.2 \\
\hline 4 & - & 69.2 & 70.2 & 71.2 & 69.8 & - & 75.2 & 74.6 & 75.1 & 73.8 & 74.9 & 73.8 \\
\hline 5 & - & 68.5 & 70.6 & 70.8 & 69.7 & - & 75.3 & 74.7 & 75.3 & 74.0 & - & 73.8 \\
\hline 6 & - & 68.2 & 70.6 & 70.4 & 69.1 & - & 75.2 & 74.8 & 75.4 & 74.2 & 74.5 & 73.8 \\
\hline 7 & - & 68.2 & 70.5 & 70.2 & - & - & 75.1 & 75.0 & 75.3 & 74.6 & 73.9 & 73.3 \\
\hline 8 & - & 68.6 & 70.5 & 70.1 & - & - & 75.4 & 74.9 & 75.2 & 74.8 & 73.6 & 73.3 \\
\hline 9 & - & 69.1 & 70.5 & 70.1 & - & - & 75.5 & 74.9 & 75.0 & 75.0 & 73.4 & 72.6 \\
\hline 10 & - & 69.4 & 70.5 & 70.3 & - & - & 75.6 & 75.0 & 75.0 & 74.9 & 72.9 & 72.2 \\
\hline 11 & - & 69.5 & 70.4 & 70.9 & - & - & 75.5 & 75.1 & 75.3 & 74.8 & 72.8 & 72.4 \\
\hline 12 & - & 70.6 & 70.6 & 70.8 & - & - & 75.1 & 75.3 & 75.8 & 75.0 & 73.2 & 73.1 \\
\hline 13 & - & 71.6 & 70.8 & 70.7 & - & - & 74.8 & 75.6 & 75.9 & 75.0 & 74.1 & 73.2 \\
\hline 14 & - & 70.6 & 71.3 & 70.6 & - & - & 74.8 & 75.9 & 75.5 & 74.9 & 74.3 & 73.0 \\
\hline 15 & - & 70.5 & 71.8 & 70.6 & - & - & 75.0 & 75.7 & 75.4 & 74.9 & 74.1 & 73.5 \\
\hline 16 & - & 69.8 & 71.4 & 70.6 & - & - & 75.5 & 75.3 & 75.4 & 74.6 & 74.3 & 74.1 \\
\hline 17 & - & 69.7 & 71.1 & 71.1 & - & - & 75.8 & 75.0 & 75.4 & 74.3 & 74.3 & 74.3 \\
\hline 18 & - & 69.9 & 71.3 & 71.6 & - & - & 75.6 & 74.8 & 75.3 & 74.2 & 73.5 & 73.9 \\
\hline 19 & - & 69.8 & 71.6 & 70.8 & - & - & - & 74.9 & 75.2 & 74.3 & 72.9 & 73.7 \\
\hline 20 & 69.2 & 69.8 & 71.4 & 69.5 & - & - & 75.6 & 75.1 & 74.9 & 74.7 & 73.5 & 72.6 \\
\hline 21 & 69.1 & 70.4 & 72.0 & 69.2 & - & - & 75.6 & 75.1 & 74.8 & 74.8 & 73.8 & 72.3 \\
\hline 22 & 69.7 & 70.7 & 71.6 & 69.7 & - & - & 75.2 & 75.0 & 74.8 & 75.1 & 74.2 & 72.9 \\
\hline 23 & 70.3 & 72.0 & 71.6 & 69.9 & - & - & 75.2 & 75.0 & 74.8 & 75.3 & 74.0 & 72.9 \\
\hline 24 & 70.2 & 72.3 & 71.3 & 70.0 & - & 74.2 & 75.2 & 75.1 & 74.9 & 74.8 & 73.7 & 72.8 \\
\hline 25 & 69.9 & 72.2 & 70.8 & 70.0 & - & 74.2 & 75.0 & 75.0 & 75.2 & 74.2 & 73.6 & 72.2 \\
\hline 26 & 69.6 & 71.4 & 70.5 & 69.8 & - & 74.0 & 74.9 & 74.8 & 75.3 & 74.0 & 73.4 & 71.9 \\
\hline 27 & 69.7 & 70.7 & 70.3 & 69.8 & - & 74.1 & 74.9 & 74.7 & 75.1 & 74.2 & 73.8 & 71.9 \\
\hline 28 & 69.8 & 70.3 & 69.9 & 69.9 & - & 74.4 & 74.8 & 74.7 & 74.9 & 74.5 & 74.5 & 72.2 \\
\hline 29 & 69.9 & - & 69.8 & 70.2 & - & 74.6 & 74.8 & 74.6 & 74.7 & 74.9 & 74.3 & 72.8 \\
\hline 30 & 70.1 & - & 69.7 & 70.4 & - & 74.8 & 74.7 & 74.4 & 74.5 & 74.9 & 73.9 & 72.2 \\
\hline 31 & 69.6 & - & 69.9 & - & - & - & 74.5 & 74.3 & - & 75.3 & - & 71.1 \\
\hline Mean & 69.8 & 70.1 & 70.8 & 70.4 & 69.9 & 74.3 & 75.2 & 74.9 & 75.1 & 74.6 & 74.0 & 73.0 \\
\hline $\operatorname{Max}$ & 70.3 & 72.3 & 71.8 & 71.6 & 70.5 & 74.8 & 75.8 & 75.9 & 75.9 & 75.3 & 75.4 & 74.3 \\
\hline Min & 69.1 & 68.2 & 69.7 & 69.2 & 69.1 & 74.0 & 74.5 & 74.3 & 74.2 & 73.8 & 72.8 & 71.1 \\
\hline
\end{tabular}


Table 108. Miscellaneous instantaneous vent gas temperatures, Basalt Fumarole (BSF), Long Valley

Caldera, Mono County, California, 1987-90

[Vent gas temperature is in degrees Celsius]

\begin{tabular}{lc}
\hline Date & $\begin{array}{c}\text { Vent gas } \\
\text { temperature }\end{array}$ \\
\hline $04-11-87$ & 79.9 \\
$05-31-87$ & 73.5 \\
$06-18-87$ & 76.5 \\
$06-03-88$ & 75.0 \\
$12-11-89$ & 71.9 \\
$02-06-90$ & 72.7 \\
$03-07-90$ & 72.4 \\
$04-03-90$ & 72.2 \\
$05-30-90$ & 73.9 \\
$06-29-90$ & 75.1 \\
$08-21-90$ & 77.3 \\
$09-24-90$ & 77.8 \\
$11-18-90$ & 80.2 \\
$12-18-90$ & 80.0 \\
\hline
\end{tabular}




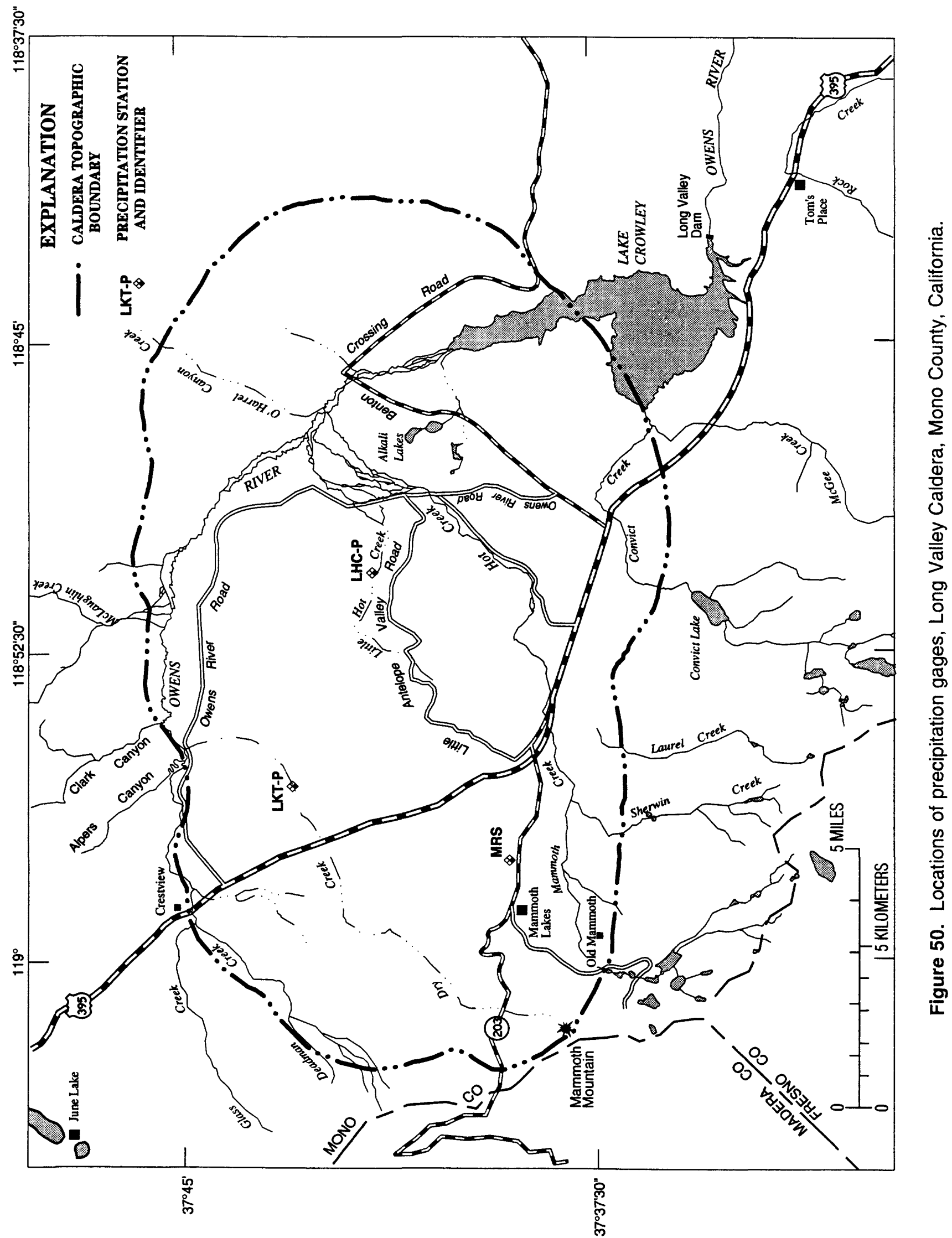




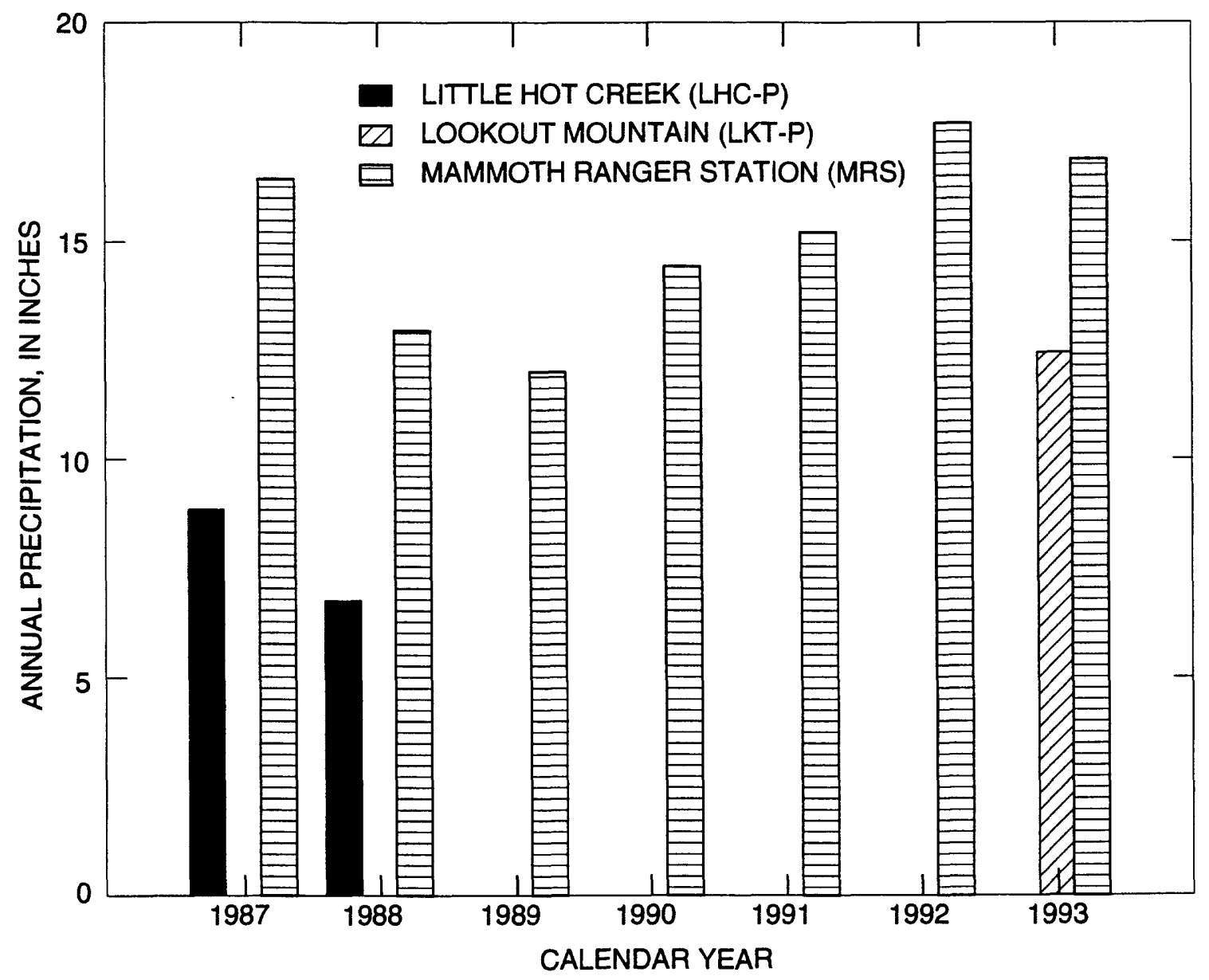

Figure 51. Annual precipitation at Little Hot Creek (LHC-P), Lookout Mountain (LKT-P), and Mammoth Ranger Station (MRS), 1987-93. 
Table 109. Daily precipitation, Little Hot Creek gage (LHC-P), Long Valley Caldera, Mono County, California, 1987

[Altitude of site: 6,960 feet. Instrument type: weighing-bucket gage. Precipitation in inches; calendar year January to December 1987; daily sum values. Max. maximum; *, no precipitation]

\begin{tabular}{|c|c|c|c|c|c|c|c|c|c|c|c|c|}
\hline Day & Jan & Feb & Mar & Apr & May & June & July & Aug & Sept & Oct & Nov & Dec \\
\hline 1 & $*$ & $*$ & $*$ & $*$ & $*$ & $*$ & $*$ & $*$ & * & $*$ & * & $*$ \\
\hline 2 & $*$ & * & $*$ & * & $*$ & $*$ & $*$ & $*$ & 0.07 & $*$ & 0.15 & $*$ \\
\hline 3 & 0.54 & $*$ & $*$ & 0.05 & $*$ & $*$ & $*$ & 0.10 & $*$ & $*$ & .15 & $*$ \\
\hline 4 & .59 & $*$ & $*$ & $*$ & $*$ & * & $*$ & $*$ & $*$ & $*$ & .35 & $*$ \\
\hline 5 & .02 & $*$ & 0.25 & $*$ & $*$ & 0.07 & $*$ & $*$ & $*$ & $*$ & .48 & $*$ \\
\hline 6 & .10 & $*$ & .03 & $*$ & $*$ & .21 & $*$ & $*$ & $*$ & $*$ & $*$ & 0.50 \\
\hline 7 & $*$ & $*$ & $*$ & $*$ & $*$ & $*$ & $*$ & $*$ & $*$ & $*$ & $*$ & .03 \\
\hline 8 & $*$ & $*$ & $*$ & $*$ & 0.10 & $*$ & $*$ & $*$ & $*$ & $*$ & $*$ & $*$ \\
\hline 9 & $*$ & $*$ & $*$ & $*$ & .03 & $*$ & $*$ & * & $*$ & $*$ & $*$ & $*$ \\
\hline 10 & $*$ & $*$ & $*$ & $*$ & .03 & $*$ & $*$ & $*$ & $*$ & $*$ & $*$ & $*$ \\
\hline 11 & $*$ & 0.02 & $*$ & $*$ & $*$ & * & $*$ & $*$ & * & $*$ & $*$ & $*$ \\
\hline 12 & $*$ & .66 & .09 & $*$ & .05 & $*$ & $*$ & $*$ & $*$ & 0.03 & $*$ & * \\
\hline 13 & $*$ & .49 & .06 & $*$ & $*$ & $*$ & $*$ & $*$ & $*$ & $*$ & .04 & $*$ \\
\hline 14 & $*$ & $*$ & .06 & $*$ & .10 & $*$ & $*$ & $*$ & $*$ & $*$ & $*$ & $*$ \\
\hline 15 & $*$ & $*$ & $*$ & $*$ & .15 & $*$ & 0.10 & $*$ & $*$ & $*$ & $*$ & .08 \\
\hline 16 & $*$ & $*$ & $*$ & $*$ & $*$ & $*$ & $*$ & $*$ & $*$ & $*$ & $*$ & .06 \\
\hline 17 & $*$ & $*$ & $*$ & $*$ & .13 & $*$ & $*$ & $*$ & $*$ & $*$ & .17 & $*$ \\
\hline 18 & $*$ & $*$ & $*$ & $*$ & $*$ & $*$ & $*$ & $*$ & $*$ & $*$ & $*$ & .08 \\
\hline 19 & $*$ & $*$ & $*$ & * & $*$ & $*$ & $*$ & $*$ & $*$ & $*$ & $*$ & .03 \\
\hline 20 & $*$ & $*$ & $*$ & $*$ & .52 & $*$ & $*$ & $*$ & $*$ & $*$ & .10 & $*$ \\
\hline 21 & $*$ & $*$ & .02 & $*$ & $*$ & $*$ & $*$ & * & $*$ & $*$ & .02 & $*$ \\
\hline 22 & $*$ & $*$ & $*$ & $*$ & * & $*$ & $*$ & $*$ & $*$ & .21 & $*$ & .06 \\
\hline 23 & $*$ & .15 & .06 & $*$ & $*$ & $*$ & $*$ & $*$ & $*$ & $*$ & $*$ & $*$ \\
\hline 24 & $*$ & $*$ & .06 & $*$ & $*$ & $*$ & $*$ & $*$ & $*$ & .05 & $*$ & $*$ \\
\hline 25 & $*$ & .02 & $*$ & $*$ & $*$ & $*$ & $*$ & $*$ & $*$ & $*$ & $*$ & $*$ \\
\hline 26 & $*$ & $*$ & $*$ & * & $*$ & .08 & $*$ & $*$ & * & $*$ & $*$ & $*$ \\
\hline 27 & .19 & $*$ & $*$ & $*$ & .20 & $*$ & $*$ & $*$ & $*$ & .06 & $*$ & $*$ \\
\hline 28 & $*$ & $*$ & $*$ & $*$ & $*$ & $*$ & $*$ & $*$ & $*$ & .09 & $*$ & .40 \\
\hline 29 & $*$ & $*$ & $*$ & .05 & * & $*$ & $*$ & $*$ & $*$ & $*$ & $*$ & .15 \\
\hline 30 & $*$ & $*$ & $*$ & $*$ & $*$ & $*$ & $*$ & $*$ & $*$ & $*$ & $*$ & $*$ \\
\hline 31 & * & $*$ & $*$ & $*$ & $*$ & $*$ & $*$ & $*$ & $*$ & .10 & $*$ & $*$ \\
\hline Total & 1.44 & 1.34 & .63 & .10 & 1.31 & .36 & .10 & .10 & .07 & .54 & 1.46 & 1.39 \\
\hline Max & .59 & .66 & .25 & .05 & .52 & .21 & .10 & .10 & .07 & .21 & .48 & .50 \\
\hline
\end{tabular}


Table 110. Daily precipitation, Little Hot Creek gage (LHC-P), Long Valley Caldera, Mono County, California, 1988

[Altitude of site: 6,960 feet. Instrument type: weighing-bucket gage. Precipitation in inches; calendar year January to December 1988; daily sum values. No record July 22 to August 3 and November 7 to December 6 due to recorder malfunction. Max, maximum; *, no precipitation; -, no data]

\begin{tabular}{|c|c|c|c|c|c|c|c|c|c|c|c|c|}
\hline Day & Jan & $\mathrm{Feb}$ & Mar & Apr & May & June & July & Aug & Sept & Oct & Nov & Dec \\
\hline 1 & $*$ & $*$ & 0.11 & $*$ & $*$ & $*$ & $*$ & - & 0.17 & $*$ & $*$ & - \\
\hline 2 & $*$ & $*$ & $*$ & $*$ & $*$ & * & $*$ & - & $*$ & $*$ & $*$ & - \\
\hline 3 & $*$ & $*$ & $*$ & $*$ & $*$ & $*$ & $*$ & - & $*$ & $*$ & * & - \\
\hline 4 & 0.40 & $*$ & $*$ & $*$ & $*$ & $*$ & $*$ & 0.09 & $*$ & $*$ & $*$ & - \\
\hline 5 & .43 & $*$ & $*$ & $*$ & $*$ & 0.02 & $*$ & $*$ & $*$ & 0.05 & $*$ & - \\
\hline 6 & $*$ & $*$ & $*$ & $*$ & $*$ & $*$ & $*$ & $*$ & $*$ & $*$ & $*$ & - \\
\hline 7 & $*$ & $*$ & $*$ & $*$ & $*$ & $*$ & $*$ & $*$ & $*$ & $*$ & - & $*$ \\
\hline 8 & $*$ & $*$ & $*$ & $*$ & $*$ & $*$ & $*$ & $*$ & $*$ & $*$ & - & * \\
\hline 9 & $*$ & $*$ & $*$ & $*$ & $*$ & $*$ & $*$ & $*$ & $*$ & $*$ & - & $*$ \\
\hline 10 & $*$ & $*$ & $*$ & $*$ & $*$ & $*$ & $*$ & $*$ & $*$ & $*$ & - & $*$ \\
\hline 11 & .05 & $*$ & $*$ & * & $*$ & * & * & $*$ & $*$ & $*$ & - & $*$ \\
\hline 12 & $*$ & $*$ & $*$ & $*$ & $*$ & $*$ & $*$ & $*$ & $*$ & $*$ & - & $*$ \\
\hline 13 & $*$ & $*$ & $*$ & $*$ & $*$ & $*$ & $*$ & $*$ & $*$ & $*$ & - & $*$ \\
\hline 14 & $*$ & $*$ & $*$ & 0.10 & $*$ & $*$ & $*$ & $*$ & $*$ & $*$ & - & $*$ \\
\hline 15 & .32 & $*$ & $*$ & .07 & $*$ & $*$ & $*$ & $*$ & $*$ & $*$ & - & $*$ \\
\hline 16 & .02 & $*$ & $*$ & $*$ & $*$ & $*$ & $*$ & $*$ & $*$ & $*$ & - & 0.30 \\
\hline 17 & .52 & $*$ & $*$ & $*$ & $*$ & $*$ & $*$ & $*$ & $*$ & $*$ & - & .20 \\
\hline 18 & $*$ & $*$ & $*$ & $*$ & $*$ & $*$ & * & $*$ & $*$ & $*$ & - & $*$ \\
\hline 19 & $*$ & $*$ & $*$ & .10 & $*$ & .37 & $*$ & $*$ & .02 & $*$ & - & * \\
\hline 20 & $*$ & $*$ & $*$ & .05 & $*$ & .10 & $*$ & $*$ & .09 & $*$ & - & .17 \\
\hline 21 & $*$ & $*$ & $*$ & $*$ & $*$ & $*$ & $*$ & $*$ & $*$ & $*$ & - & .02 \\
\hline 22 & $*$ & $*$ & $*$ & $*$ & $*$ & $*$ & - & $*$ & $*$ & $*$ & - & .16 \\
\hline 23 & $*$ & $*$ & $*$ & .04 & $*$ & $*$ & - & $*$ & $*$ & $*$ & - & .40 \\
\hline 24 & $*$ & $*$ & $*$ & * & $*$ & $*$ & - & $*$ & $*$ & $*$ & - & .75 \\
\hline 25 & $*$ & $*$ & $*$ & $*$ & $*$ & $*$ & - & $*$ & $*$ & $*$ & - & .02 \\
\hline 26 & $*$ & $*$ & $*$ & $*$ & $*$ & $*$ & - & .80 & $*$ & $*$ & - & .01 \\
\hline 27 & $*$ & 0.12 & $*$ & $*$ & $*$ & $*$ & - & $*$ & $*$ & $*$ & - & .01 \\
\hline 28 & $*$ & .04 & $*$ & $*$ & 0.35 & $*$ & - & $*$ & $*$ & $*$ & - & .01 \\
\hline 29 & $*$ & .07 & $*$ & $*$ & .02 & $*$ & - & $*$ & $*$ & * & - & $*$ \\
\hline 30 & $*$ & * & $*$ & * & $*$ & * & - & .04 & $*$ & $*$ & - & .09 \\
\hline 31 & $*$ & $*$ & $*$ & $*$ & $*$ & $*$ & - & $*$ & $*$ & .04 & - & .01 \\
\hline Total & 1.74 & .23 & .11 & .36 & .37 & .49 & 1 & 1.93 & .28 & .09 & 1 & ${ }^{1} 2.15$ \\
\hline $\operatorname{Max}$ & .52 & .12 & .11 & .10 & .35 & .37 & 1 & ${ }^{1} .80$ & .17 & .05 & 1 & ${ }^{1} .75$ \\
\hline
\end{tabular}

'Incomplete record. 
Table 111. Daily precipitation, Lookout Mountain gage (LKT-P), Long Valley Caldera, Mono County,

California, 1993

[Altitude of site: 7,350 feet. Instrument type: tipping-bucket gage. Values are in inches. Max, maximum; *, no precipitation; -, no data]

\begin{tabular}{|c|c|c|c|c|c|c|c|c|c|c|c|c|}
\hline Day & Jan & Feb & Mar & Apr & May & June & July & Aug & Sept & Oct & Nov & Dec \\
\hline 1 & - & - & $*$ & $*$ & $*$ & - & $*$ & - & - & - & - & - \\
\hline 2 & - & - & * & * & $*$ & - & $*$ & - & - & - & - & * \\
\hline 3 & - & - & $*$ & $*$ & 0.04 & - & $*$ & - & - & - & - & $*$ \\
\hline 4 & - & $*$ & $*$ & 0.08 & $*$ & - & $*$ & - & - & - & - & 0.04 \\
\hline 5 & - & 0.04 & $*$ & .04 & $*$ & - & $*$ & - & - & - & - & $*$ \\
\hline 6 & $*$ & .04 & * & $*$ & $*$ & - & * & - & - & - & - & $*$ \\
\hline 7 & $*$ & $*$ & $*$ & $*$ & $*$ & - & $*$ & - & - & - & - & * \\
\hline 8 & 0.04 & .12 & $*$ & $*$ & $*$ & - & * & - & - & - & - & $*$ \\
\hline 9 & $*$ & .04 & $*$ & $*$ & $*$ & - & $*$ & - & - & - & - & $*$ \\
\hline 10 & .39 & $*$ & $*$ & $*$ & $*$ & - & * & - & - & - & - & $*$ \\
\hline 11 & * & $*$ & $*$ & $*$ & $*$ & - & $*$ & - & - & - & - & .35 \\
\hline 12 & .39 & * & $*$ & $*$ & - & - & * & - & - & - & - & .23 \\
\hline 13 & .58 & $*$ & $*$ & $*$ & - & - & $*$ & - & - & - & - & $*$ \\
\hline 14 & 1.3 & * & * & $*$ & - & - & * & - & - & - & - & $*$ \\
\hline 15 & .74 & $*$ & $*$ & $*$ & - & - & $*$ & - & - & - & - & $*$ \\
\hline 16 & .35 & .04 & $*$ & $*$ & - & $*$ & $*$ & - & - & - & - & .04 \\
\hline 17 & .51 & $*$ & $*$ & .23 & - & $*$ & * & - & - & - & - & $*$ \\
\hline 18 & .16 & 1.1 & * & .08 & - & $*$ & * & - & - & - & - & $*$ \\
\hline 19 & .08 & .74 & $*$ & $*$ & - & $*$ & $*$ & - & - & - & - & $*$ \\
\hline 20 & * & .58 & $*$ & $*$ & - & $*$ & - & - & - & - & - & $*$ \\
\hline 21 & .04 & * & $*$ & $*$ & - & 0.04 & - & - & - & - & - & $*$ \\
\hline 22 & .35 & .16 & $*$ & $*$ & - & $*$ & - & - & - & - & - & $*$ \\
\hline 23 & * & 1.5 & $*$ & $*$ & - & $*$ & - & - & - & - & - & * \\
\hline 24 & * & 1.0 & $*$ & $*$ & - & $*$ & - & - & - & - & - & .04 \\
\hline 25 & * & $*$ & 0.23 & $*$ & - & $*$ & - & - & - & - & - & $*$ \\
\hline 26 & $*$ & .16 & .39 & $*$ & - & $*$ & - & - & - & - & - & $*$ \\
\hline 27 & - & .04 & $*$ & $*$ & - & * & - & - & - & - & - & .04 \\
\hline 28 & - & $*$ & .19 & $*$ & - & $*$ & - & - & - & - & - & $*$ \\
\hline 29 & - & $*$ & $*$ & * & - & * & - & - & - & - & - & $*$ \\
\hline 30 & - & $*$ & $*$ & $*$ & - & $*$ & - & - & - & - & - & $*$ \\
\hline 31 & - & $*$ & * & $*$ & - & $*$ & - & - & - & - & - & $*$ \\
\hline Total & $4.93^{1}$ & $5.56^{1}$ & .81 & .43 & $.04^{1}$ & $.04^{1}$ & 1 & - & - & - & - & $.74^{1}$ \\
\hline Max & $1.30^{1}$ & $1.50^{1}$ & .39 & .23 & $.04^{1}$ & $.04^{1}$ & 1 & - & - & - & - & $.35^{1}$ \\
\hline
\end{tabular}

'Incomplete record. 
Table 112. Monthly precipitation at Mammoth Ranger Station gage (MRS), Long Valley Caldera, Mono County, California, 1987-93

[Altitude of site: 7,800 feet. Instrument type: weighing-bucket recording gage. Data provided by: Thom Heller, U.S. Forest Service. Values are in inches. Max, maximum]

\begin{tabular}{|c|c|c|c|c|c|c|c|}
\hline & 1987 & 1988 & 1989 & 1990 & 1991 & 1992 & 1993 \\
\hline January & 3.13 & 3.26 & 0.57 & 4.89 & 0.93 & 1.38 & 4.99 \\
\hline February & 2.98 & .56 & 1.60 & 4.05 & .32 & 3.50 & 6.80 \\
\hline March & 2.00 & .15 & 3.59 & .86 & 9.12 & .80 & 1.03 \\
\hline April & .61 & .87 & .37 & 1.12 & .18 & .00 & .32 \\
\hline May & 1.09 & .93 & .81 & .80 & .21 & .02 & .00 \\
\hline June & .47 & .66 & .43 & .67 & .02 & 1.02 & 1.16 \\
\hline July & .00 & .31 & .07 & .50 & .25 & .86 & .00 \\
\hline August & .00 & .52 & 1.09 & .16 & .17 & .39 & .00 \\
\hline September & .05 & .31 & 1.59 & .54 & .54 & .30 & .00 \\
\hline October & .88 & .03 & .93 & .00 & .39 & .73 & .26 \\
\hline November & 1.90 & 2.33 & .71 & .48 & 1.01 & .00 & .76 \\
\hline December & 3.30 & 3.00 & .23 & .35 & 1.85 & 8.68 & 1.52 \\
\hline Total & 16.41 & 12.93 & 11.99 & 14.42 & 14.99 & 17.68 & 16.84 \\
\hline $\operatorname{Max}$ & 3.30 & 3.26 & 3.59 & 4.89 & 9.12 & 8.68 & 6.80 \\
\hline
\end{tabular}




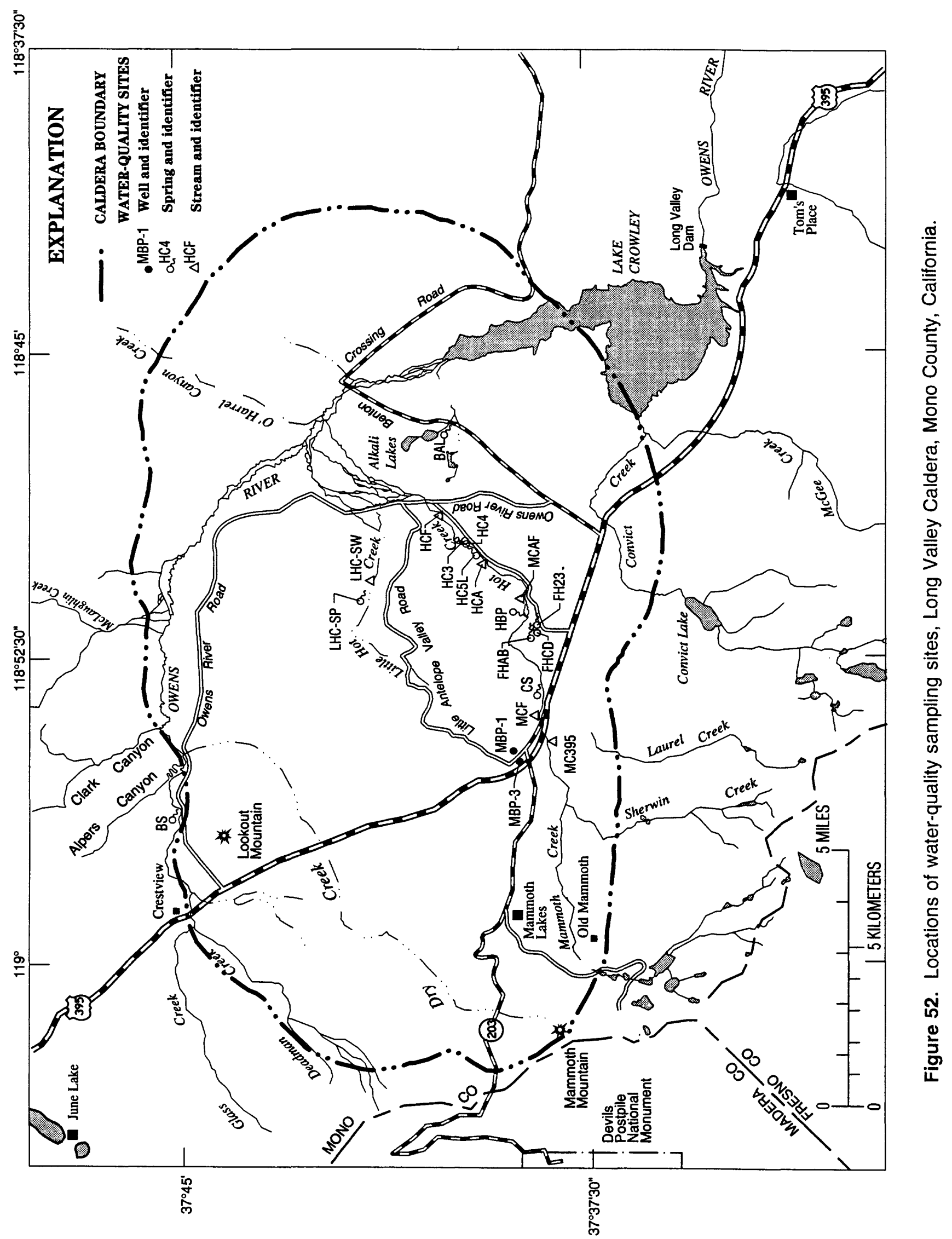


Table 113. Chemical and isotopic analyses of water samples collected at selected surface-water sites, springs, and wells, Long Valley Caldera, Mono County, California, 1987-93

$\left[{ }^{\circ} \mathrm{C}\right.$, degrees Celsius; $\mu \mathrm{S} / \mathrm{cm}$, microsiemens per centimeter at $25^{\circ} \mathrm{C}$; alkalinity is total, whole water, fixed end point titration to $\mathrm{pH} 4.5 ; \mathrm{mg} / \mathrm{L}$, milligrams per liter; $\mu \mathrm{g} / \mathrm{L}$, micrograms per liter; ratio per mil, parts per thousand difference from Standard Mean Ocean Water; - , no data; <, less than]

\begin{tabular}{|c|c|c|c|c|c|c|c|c|c|}
\hline $\begin{array}{l}\text { Station } \\
\text { identifier }\end{array}$ & Date & $\begin{array}{l}\text { Temper- } \\
\text { ature, } \\
\text { water } \\
\left({ }^{\circ} \mathrm{C}\right)\end{array}$ & $\begin{array}{c}\text { Specific } \\
\text { con- } \\
\text { duct- } \\
\text { ance } \\
(\mu \mathrm{S} / \mathrm{cm})\end{array}$ & $\begin{array}{c}\mathrm{pH}, \\
\text { water } \\
\text { whole } \\
\text { field } \\
\text { (standard } \\
\text { units) }\end{array}$ & $\begin{array}{c}\text { Alka- } \\
\text { linity, } \\
\text { field, } \\
\text { as } \mathrm{CaCO}_{3} \\
(\mathrm{mg} / \mathrm{L})\end{array}$ & $\begin{array}{c}\text { Alka- } \\
\text { linity } \\
\text { lab, } \\
\text { as } \mathrm{CaCO}_{3} \\
(\mathrm{mg} / \mathrm{L})\end{array}$ & $\begin{array}{l}\text { Chlo- } \\
\text { ride, } \\
\text { dis- } \\
\text { solved } \\
(\mathrm{mg} / \mathrm{L})\end{array}$ & $\begin{array}{l}\text { Fluo- } \\
\text { ride, } \\
\text { dis- } \\
\text { solved } \\
(\mathrm{mg} / \mathrm{L})\end{array}$ & $\begin{array}{c}\text { Nitro- } \\
\text { gen, } \\
\text { ammonia, } \\
\text { dis- } \\
\text { solved } \\
(\mathrm{mg} / \mathrm{L})\end{array}$ \\
\hline MC395 & $\begin{array}{l}07-13-88 \\
10-18-88 \\
01-11-89 \\
04-11-89 \\
07-18-89\end{array}$ & $\begin{array}{r}13.0 \\
5.5 \\
.0 \\
5.5 \\
13.5\end{array}$ & $\begin{array}{r}82 \\
140 \\
165 \\
143 \\
90\end{array}$ & $\begin{array}{l}7.9 \\
8.2 \\
7.6 \\
7.7 \\
7.2\end{array}$ & $\begin{array}{l}42 \\
73 \\
78 \\
69 \\
49\end{array}$ & $\begin{array}{l}30 \\
70 \\
79 \\
72 \\
43\end{array}$ & $\begin{array}{r}0.30 \\
.40 \\
.40 \\
.40 \\
.30\end{array}$ & $\begin{array}{r}0.20 \\
.10 \\
.30 \\
.20 \\
.10\end{array}$ & $\begin{array}{r}0.010 \\
<.010 \\
<.010 \\
.010 \\
.020\end{array}$ \\
\hline & $\begin{array}{c}10-18-89 \\
01-09-90 \\
04-25-90 \\
07-17-90 \\
10-16-90\end{array}$ & $\begin{array}{r}4.0 \\
.0 \\
3.0 \\
17.5 \\
8.0\end{array}$ & $\begin{array}{r}137 \\
143 \\
124 \\
84 \\
103\end{array}$ & $\begin{array}{l}8.2 \\
7.9 \\
8.0 \\
8.0 \\
8.1\end{array}$ & $\begin{array}{l}- \\
86 \\
64 \\
45 \\
64\end{array}$ & $\begin{array}{l}65 \\
79 \\
44 \\
68\end{array}$ & $\begin{array}{l}1.0 \\
.40 \\
.50 \\
<.10 \\
1.2\end{array}$ & $\begin{array}{l}.10 \\
.10 \\
.10 \\
.20 \\
.30\end{array}$ & $\begin{array}{r}<.010 \\
.020 \\
<.010 \\
.020 \\
<.050\end{array}$ \\
\hline & $\begin{array}{l}01-17-91 \\
04-16-91 \\
07-22-91 \\
10-22-91 \\
01-13-92\end{array}$ & $\begin{array}{r}.0 \\
1.0 \\
17.0 \\
9.5 \\
.5\end{array}$ & $\begin{array}{r}165 \\
161 \\
71 \\
119 \\
137\end{array}$ & $\begin{array}{l}8.0 \\
8.1 \\
7.3 \\
8.3 \\
8.2\end{array}$ & $\begin{array}{l}81 \\
82 \\
35 \\
63 \\
70\end{array}$ & $\begin{array}{l}85 \\
85 \\
35 \\
60 \\
72\end{array}$ & $\begin{array}{r}.20 \\
.20 \\
<.10 \\
.70 \\
.50\end{array}$ & $\begin{array}{r}<.10 \\
.10 \\
.10 \\
.20 \\
.20\end{array}$ & $\begin{array}{r}.040 \\
<.010 \\
.020 \\
.020 \\
.010\end{array}$ \\
\hline & $\begin{array}{l}04-14-92 \\
07-06-92 \\
10-19-92 \\
01-25-93 \\
04-12-93\end{array}$ & $\begin{array}{r}1.0 \\
15.0 \\
9.5 \\
.0 \\
4.5\end{array}$ & $\begin{array}{r}159 \\
88 \\
121 \\
121 \\
161\end{array}$ & $\begin{array}{l}8.4 \\
8.1 \\
8.1 \\
7.9 \\
8.2\end{array}$ & $\begin{array}{l}80 \\
42 \\
62 \\
63 \\
79\end{array}$ & $\begin{array}{l}81 \\
42 \\
60 \\
61 \\
79\end{array}$ & $\begin{array}{r}.10 \\
<.10 \\
.40 \\
.40 \\
.70\end{array}$ & $\begin{array}{r}<.10 \\
<.10 \\
.10 \\
.10 \\
.10\end{array}$ & $\begin{array}{r}.020 \\
.010 \\
.020 \\
<.010 \\
.020\end{array}$ \\
\hline & $\begin{array}{l}07-12-93 \\
10-13-93\end{array}$ & $\begin{array}{r}17.0 \\
9.0\end{array}$ & $\begin{array}{r}42 \\
101\end{array}$ & $\begin{array}{l}7.8 \\
8.1\end{array}$ & $\begin{array}{l}20 \\
49\end{array}$ & $\begin{array}{l}21 \\
52\end{array}$ & $\begin{array}{l}.20 \\
.30\end{array}$ & $\begin{array}{r}<.10 \\
.10\end{array}$ & $\begin{array}{l}.020 \\
.030\end{array}$ \\
\hline MCF & $\begin{array}{l}07-13-88 \\
10-19-88 \\
01-11-89 \\
04-11-89 \\
07-18-89\end{array}$ & $\begin{array}{r}17.0 \\
5.0 \\
.0 \\
7.0 \\
17.5\end{array}$ & $\begin{array}{r}84 \\
140 \\
165 \\
144 \\
88\end{array}$ & $\begin{array}{l}8.2 \\
7.6 \\
7.6 \\
7.9 \\
7.3\end{array}$ & $\begin{array}{l}38 \\
78 \\
76 \\
71 \\
47\end{array}$ & $\begin{array}{l}38 \\
70 \\
80 \\
72 \\
43\end{array}$ & $\begin{array}{l}.50 \\
.50 \\
.40 \\
.50 \\
.30\end{array}$ & $\begin{array}{l}.10 \\
.10 \\
.10 \\
.10 \\
.20\end{array}$ & $\begin{array}{r}<.010 \\
<.010 \\
<.010 \\
.010 \\
.010\end{array}$ \\
\hline & $\begin{array}{l}10-18-89 \\
01-09-90 \\
04-25-90\end{array}$ & $\begin{array}{r}5.5 \\
.0 \\
5.5\end{array}$ & $\begin{array}{l}131 \\
147 \\
131\end{array}$ & $\begin{array}{l}8.2 \\
8.0 \\
8.3\end{array}$ & $\begin{array}{l}\overline{83} \\
53\end{array}$ & $\begin{array}{l}64 \\
80 \\
63\end{array}$ & $\begin{array}{c}1.2 \\
.50 \\
.50\end{array}$ & $\begin{array}{l}.10 \\
.10 \\
.10\end{array}$ & $\begin{array}{r}<.010 \\
.020 \\
<.010\end{array}$ \\
\hline MCAF & $\begin{array}{l}07-13-88 \\
10-19-88 \\
04-11-89 \\
07-18-89 \\
10-18-89\end{array}$ & $\begin{array}{r}25.5 \\
1.0 \\
14.5 \\
23.5 \\
12.0\end{array}$ & $\begin{array}{l}146 \\
221 \\
165 \\
135 \\
202\end{array}$ & $\begin{array}{l}8.7 \\
8.3 \\
8.2 \\
8.6 \\
8.6\end{array}$ & $\begin{array}{l}57 \\
88 \\
73 \\
58 \\
\end{array}$ & $\begin{array}{l}54 \\
89 \\
75 \\
54 \\
82\end{array}$ & $\begin{array}{c}6.1 \\
11 \\
3.3 \\
5.6 \\
9.6\end{array}$ & $\begin{array}{l}.10 \\
.30 \\
.20 \\
.30 \\
.20\end{array}$ & $\begin{array}{l}<.010 \\
<.010 \\
<.010 \\
<.010 \\
<.010\end{array}$ \\
\hline
\end{tabular}


Table 113. Chemical and isotopic analyses of water samples collected at selected surface-water sites, springs, and wells, Long Valley Caldera, Mono County, California, 1987-93-Continued

\begin{tabular}{|c|c|c|c|c|c|c|c|c|c|c|c|}
\hline $\begin{array}{l}\text { Station } \\
\text { identifier }\end{array}$ & Date & $\begin{array}{l}\text { Nitro- } \\
\text { gen, } \\
\text { nitrite } \\
\text { dis- } \\
\text { solved } \\
(\mathrm{mg} / \mathrm{L})\end{array}$ & $\begin{array}{c}\text { Nitro- } \\
\text { gen. } \\
\mathrm{NO}_{2}+\mathrm{NO}_{3} \\
\text { dis- } \\
\text { solved } \\
(\mathrm{mg} / \mathrm{L})\end{array}$ & $\begin{array}{l}\text { Phos- } \\
\text { phorus } \\
\text { Ortho, } \\
\text { dis- } \\
\text { solved } \\
(\mathrm{mg} / \mathrm{L})\end{array}$ & $\begin{array}{l}\text { Sulfate, } \\
\text { dis- } \\
\text { solved } \\
(\mathrm{mg} / \mathrm{L})\end{array}$ & $\begin{array}{l}\text { Calcium, } \\
\text { dis- } \\
\text { solved } \\
(\mathrm{mg} / \mathrm{L})\end{array}$ & $\begin{array}{l}\text { Magne- } \\
\text { sium, } \\
\text { dis- } \\
\text { solved } \\
(\mathrm{mg} / \mathrm{L})\end{array}$ & $\begin{array}{l}\text { Stron- } \\
\text { tium, } \\
\text { dis- } \\
\text { solved } \\
(\mu \mathrm{g} / \mathrm{L})\end{array}$ & $\begin{array}{l}\text { Sodium, } \\
\text { dis- } \\
\text { solved } \\
(\mathrm{mg} / \mathrm{L})\end{array}$ & $\begin{array}{l}\text { Potas- } \\
\text { sium, } \\
\text { dis- } \\
\text { solved } \\
(\mathrm{mg} / \mathrm{L})\end{array}$ & $\begin{array}{c}\text { Silica, } \\
\text { dis- } \\
\text { solved } \\
(\mathrm{mg} / \mathrm{L})\end{array}$ \\
\hline \multirow[t]{22}{*}{ MC395 } & $07-13-88$ & 0.010 & 0.100 & 0.021 & 3.1 & 7.8 & 2.4 & 28 & 4.8 & 1.1 & 9.4 \\
\hline & $10-18-88$ & $<.010$ & $<.100$ & .030 & 5.8 & 11 & 5.6 & 46 & 9.3 & 2.3 & 17 \\
\hline & $01-11-89$ & $<.010$ & $<.100$ & .050 & 6.8 & 13 & 6.3 & 52 & 11 & 2.6 & 30 \\
\hline & $04-11-89$ & $<.010$ & $<.100$ & .060 & 6.3 & 11 & 6.0 & 46 & 11 & 2.4 & 27 \\
\hline & $07-18-89$ & $<.010$ & $<.100$ & .010 & 4.0 & 8.5 & 2.9 & 32 & 5.8 & 1.2 & 11 \\
\hline & $10-18-89$ & $<.010$ & $<.100$ & .030 & 6.0 & 11 & 4.8 & 46 & 9.3 & 2.1 & 17 \\
\hline & $01-09-90$ & $<.010$ & $<.100$ & .040 & 6.0 & 14 & 6.2 & - & 11 & 3.0 & 30 \\
\hline & $04-25-90$ & .020 & .500 & .040 & 5.1 & 10 & 4.7 & - & 8.6 & 2.0 & 21 \\
\hline & $07-17-90$ & $<.010$ & $<.100$ & .030 & 3.6 & 8.5 & 2.8 & - & 6.0 & 1.1 & 12 \\
\hline & $10-16-90$ & $<.010$ & $<.100$ & .040 & 4.3 & 11 & 5.0 & - & 9.6 & 2.2 & 18 \\
\hline & $01-17-91$ & $<.010$ & $<.100$ & .040 & 5.9 & 14 & 6.4 & - & 12 & 2.8 & 30 \\
\hline & $04-16-91$ & $<.010$ & .110 & .040 & 7.4 & 13 & 6.7 & - & 13 & 2.8 & 30 \\
\hline & $07-22-91$ & $<.010$ & $<.050$ & .010 & 3.1 & 7.8 & 2.1 & - & 4.9 & 1.0 & 12 \\
\hline & $10-22-91$ & $<.010$ & $<.050$ & .030 & 5.1 & 9.7 & 4.4 & - & 7.5 & 1.9 & 14 \\
\hline & $01-13-92$ & $<.010$ & .079 & .040 & 5.3 & 12 & 5.1 & - & 9.4 & 2.1 & 27 \\
\hline & $04-14-92$ & $<.010$ & $<.050$ & .060 & 6.6 & 12 & 6.8 & - & 13 & 2.6 & 28 \\
\hline & $07-06-92$ & $<.010$ & $<.050$ & .020 & 3.5 & 8.3 & 2.6 & - & 5.3 & 1.1 & 9.8 \\
\hline & $10-19-92$ & $<.010$ & $<.050$ & .020 & 4.3 & 11 & 4.3 & - & 7.9 & 2.0 & 14 \\
\hline & $01-25-93$ & $<.010$ & .069 & .080 & 5.4 & 11 & 4.4 & - & 8.3 & 1.9 & 22 \\
\hline & $04-12-93$ & $<.010$ & .200 & .050 & 7.2 & 12 & 6.3 & - & 12 & 2.6 & 30 \\
\hline & $07-12-93$ & $<.010$ & $<.050$ & .020 & 1.7 & 5.5 & .92 & - & 2.1 & .60 & 8.8 \\
\hline & $10-13-93$ & $<.010$ & $<.050$ & .020 & 4.1 & 9.1 & 3.7 & - & 6.1 & 1.6 & 16 \\
\hline \multirow[t]{8}{*}{$\mathrm{MCF}$} & $07-13-88$ & $<.010$ & $<.100$ & .020 & 4.1 & 8.0 & 2.5 & 31 & 5.4 & 1.1 & 9.3 \\
\hline & $10-19-88$ & $<.010$ & $<.100$ & .040 & 5.6 & 11 & 5.6 & 47 & 9.4 & 2.3 & 17 \\
\hline & $01-11-89$ & $<.010$ & $<.100$ & .050 & 6.9 & 13 & 6.3 & 52 & 11 & 2.6 & 30 \\
\hline & $04-11-89$ & $<.010$ & $<.100$ & .050 & 6.4 & 11 & 5.9 & 46 & 11 & 2.3 & 27 \\
\hline & $07-18-89$ & $<.010$ & $<.100$ & .020 & 4.0 & 8.6 & 3.0 & 33 & 6.0 & 1.1 & 11 \\
\hline & $10-18-89$ & $<.010$ & $<.100$ & .030 & 5.0 & 10 & 4.8 & 45 & 9.3 & 2.0 & 17 \\
\hline & $01-09-90$ & $<.010$ & $<.100$ & .040 & 6.0 & 14 & 6.2 & - & 11 & 2.8 & 30 \\
\hline & $04-25-90$ & .020 & .200 & .040 & 5.4 & 9.9 & 4.9 & - & 16 & 3.4 & 31 \\
\hline \multirow[t]{5}{*}{ MCAF } & $07-13-88$ & $<.010$ & $<.100$ & .050 & 8.4 & 9.0 & 3.5 & 39 & 13 & 2.8 & 25 \\
\hline & $10-19-88$ & $<.010$ & $<.100$ & .040 & 12 & 12 & 6.7 & 69 & 23 & 4.9 & 41 \\
\hline & $04-11-89$ & $<.010$ & $<.100$ & .060 & 7.7 & 11 & 5.9 & 51 & 15 & 3.0 & 31 \\
\hline & $07-18-89$ & $<.010$ & $<.100$ & .060 & 7.0 & 8.3 & 3.6 & 41 & 13 & 3.2 & 26 \\
\hline & $10-18-89$ & $<.010$ & $<.100$ & .040 & 10 & 12 & 5.8 & 64 & 20 & 5.0 & 40 \\
\hline
\end{tabular}


Table 113. Chemical and isotopic analyses of water samples collected at selected surface-water sites, springs, and wells, Long Valley Caldera, Mono County, California, 1987-93-Continued

\begin{tabular}{|c|c|c|c|c|c|c|c|c|c|c|}
\hline $\begin{array}{l}\text { Station } \\
\text { identifier }\end{array}$ & Date & $\begin{array}{c}\text { Boron, } \\
\text { dis- } \\
\text { solved } \\
(\mu \mathrm{g} / \mathrm{L})\end{array}$ & $\begin{array}{l}\text { Arsenic, } \\
\text { dis- } \\
\text { solved } \\
(\mu \mathrm{g} / \mathrm{L})\end{array}$ & $\begin{array}{l}\text { Lithium, } \\
\text { dis- } \\
\text { solved } \\
(\mu \mathrm{g} / \mathrm{L})\end{array}$ & $\begin{array}{c}\text { Mercury, } \\
\text { dis- } \\
\text { solved } \\
(\mu \mathrm{g} / \mathrm{L})\end{array}$ & $\begin{array}{l}\text { Iron, } \\
\text { dis- } \\
\text { solved } \\
(\mu \mathrm{g} / \mathrm{L})\end{array}$ & $\begin{array}{l}\text { Manga- } \\
\text { nese, } \\
\text { dis- } \\
\text { solved } \\
(\mu \mathrm{g} / \mathrm{L})\end{array}$ & $\begin{array}{l}\text { Solids, } \\
\text { residue } \\
\text { at } 180^{\circ} \mathrm{C} \\
\text { dis- } \\
\text { solved } \\
(\mathrm{mg} / \mathrm{L})\end{array}$ & $\begin{array}{c}\mathrm{H}-2 / \mathrm{H}-1 \\
\text { stable } \\
\text { isotope } \\
\text { ratio } \\
\text { per } \\
\text { mil }\end{array}$ & $\begin{array}{c}\text { O-18/O-16 } \\
\text { stable } \\
\text { isotope } \\
\text { ratio } \\
\text { per } \\
\text { mil }\end{array}$ \\
\hline \multirow[t]{22}{*}{ MC395 } & $07-13-88$ & $<10$ & 3 & 10 & $<0.1$ & 15 & 2 & 50 & - & - \\
\hline & $10-18-88$ & $<10$ & 4 & 30 & $<.1$ & 20 & 2 & 95 & - & - \\
\hline & $01-11-89$ & 20 & 4 & 40 & $<.1$ & 17 & $<1$ & 109 & - & - \\
\hline & $04-11-89$ & 10 & 21 & 40 & $<.1$ & 26 & 2 & 108 & - & - \\
\hline & $07-18-89$ & $<10$ & 4 & 20 & $<.1$ & 37 & 4 & 86 & - & - \\
\hline & $10-18-89$ & 10 & 3 & 30 & $<.1$ & 21 & 2 & 76 & - & - \\
\hline & $01-09-90$ & 10 & 29 & 30 & $<.1$ & 26 & 3 & 116 & - & - \\
\hline & $04-25-90$ & $<10$ & 4 & 20 & $<.1$ & 21 & 2 & 88 & - & - \\
\hline & $07-17-90$ & $<10$ & 3 & 20 & $<.1$ & 38 & 4 & 63 & - & - \\
\hline & $10-16-90$ & $<10$ & 3 & 20 & $<.1$ & 22 & 3 & 81 & - & - \\
\hline & $01-17-91$ & 10 & 4 & 30 & $<.1$ & 16 & 4 & 99 & - & - \\
\hline & $04-16-91$ & $<10$ & 4 & 30 & $<.1$ & 28 & 2 & 109 & - & - \\
\hline & $07-22-91$ & 20 & 4 & 20 & $<.1$ & 35 & 5 & 46 & - & - \\
\hline & $10-22-91$ & $<10$ & 4 & 20 & $<.1$ & 23 & 4 & 66 & -104.0 & -13.95 \\
\hline & $01-13-92$ & $<10$ & 2 & 30 & $<.1$ & 12 & $<1$ & 95 & - & - \\
\hline & $04-14-92$ & 20 & 5 & 40 & $<.1$ & 20 & 2 & 108 & - & - \\
\hline & $07-06-92$ & $<10$ & 2 & 10 & $<.1$ & 29 & 3 & 48 & - & - \\
\hline & $10-19-92$ & $<10$ & 3 & 20 & $<.1$ & 20 & 4 & 99 & - & - \\
\hline & $01-25-93$ & $<10$ & 3 & 20 & $<.1$ & 18 & $<1$ & 80 & - & - \\
\hline & $04-12-93$ & 10 & 5 & 30 & $<.1$ & 19 & 2 & 98 & -112.0 & -15.32 \\
\hline & $07-12-93$ & 10 & 2 & $<10$ & $<.1$ & 36 & 7 & 30 & -114.0 & -15.62 \\
\hline & $10-13-93$ & $<10$ & 3 & 10 & $<.1$ & 30 & 5 & 70 & - & - \\
\hline \multirow[t]{8}{*}{$\mathrm{MCF}$} & $07-13-88$ & 10 & 5 & 10 & $<.1$ & 15 & 16 & 56 & - & - \\
\hline & $10-19-88$ & $<10$ & 4 & 30 & $<.1$ & 18 & 3 & 94 & - & - \\
\hline & $01-11-89$ & $<10$ & 4 & 40 & $<.1$ & 17 & 4 & 113 & - & - \\
\hline & $04-11-89$ & $<10$ & 4 & 40 & - & 30 & 4 & 105 & - & - \\
\hline & $07-18-89$ & $<10$ & 4 & 20 & $<.1$ & 39 & 8 & 71 & - & - \\
\hline & $10-18-89$ & $<10$ & 3 & 30 & $<.1$ & 21 & 4 & 84 & - & - \\
\hline & $01-09-90$ & $<10$ & 1 & - & - & 21 & 3 & 120 & - & - \\
\hline & $04-25-90$ & $<10$ & 30 & 60 & $<.1$ & 59 & 52 & 87 & - & - \\
\hline \multirow[t]{5}{*}{ MCAF } & $07-13-88$ & 250 & 46 & 50 & $<.1$ & 55 & 15 & 100 & - & - \\
\hline & $10-19-88$ & 500 & 40 & 100 & $<.1$ & 55 & 44 & 163 & - & - \\
\hline & $04-11-89$ & 140 & 20 & 60 & $<.1$ & 43 & 70 & 123 & - & - \\
\hline & $07-18-89$ & 240 & 52 & 60 & $<.1$ & 56 & 10 & 113 & - & - \\
\hline & $10-18-89$ & 440 & 38 & 80 & $<.1$ & 33 & 20 & 118 & - & - \\
\hline
\end{tabular}


Table 113. Chemical and isotopic analyses of water samples collected at selected surface-water sites, springs, and wells, Long Valley Caldera, Mono County, California, 1987-93-Continued

\begin{tabular}{|c|c|c|c|c|c|c|c|c|c|}
\hline $\begin{array}{l}\text { Station } \\
\text { identifier }\end{array}$ & Date & $\begin{array}{l}\text { Temper- } \\
\text { ature, } \\
\text { water } \\
\left({ }^{\circ} \mathrm{C}\right)\end{array}$ & $\begin{array}{l}\text { Spe- } \\
\text { cific } \\
\text { con- } \\
\text { duct- } \\
\text { ance } \\
(\mu \mathrm{S} / \mathrm{cm})\end{array}$ & $\begin{array}{c}\mathrm{pH}, \\
\text { water } \\
\text { whole } \\
\text { field } \\
\text { (standard } \\
\text { units) }\end{array}$ & $\begin{array}{c}\text { Alka- } \\
\text { linity, } \\
\text { field, } \\
\text { as } \mathrm{CaCO}_{3} \\
(\mathrm{mg} / \mathrm{L})\end{array}$ & $\begin{array}{c}\text { Alka- } \\
\text { linity } \\
\text { lab, } \\
\text { as } \mathrm{CaCO}_{3} \\
(\mathrm{mg} / \mathrm{L})\end{array}$ & $\begin{array}{l}\text { Chlo- } \\
\text { ride, } \\
\text { dis- } \\
\text { solved } \\
(\mathrm{mg} / \mathrm{L})\end{array}$ & $\begin{array}{l}\text { Fluo- } \\
\text { ride, } \\
\text { dis- } \\
\text { solved } \\
(\mathrm{mg} / \mathrm{L})\end{array}$ & $\begin{array}{c}\text { Nitro- } \\
\text { gen, } \\
\text { ammonia, } \\
\text { dis- } \\
\text { solved } \\
(\mathrm{mg} / \mathrm{L})\end{array}$ \\
\hline \multirow[t]{16}{*}{$\mathrm{MCAF}$} & $01-09-90$ & 2.5 & 192 & 8.0 & 87 & 84 & 6.3 & 0.20 & 0.020 \\
\hline & $04-25-90$ & 17.0 & 168 & 8.7 & 70 & - & 6.0 & .30 & $<.010$ \\
\hline & $07-17-90$ & 24.0 & 142 & 8.9 & 61 & 60 & 7.2 & .20 & .020 \\
\hline & $10-16-90$ & 12.5 & 195 & 8.5 & 88 & 88 & 12 & $<.10$ & $<.020$ \\
\hline & $01-17-91$ & 5.5 & 251 & 8.0 & 101 & 100 & 17 & .40 & .080 \\
\hline & $04-16-91$ & 13.5 & 195 & 7.5 & 85 & 87 & 7.4 & .20 & $<.010$ \\
\hline & $07-22-91$ & 24.5 & 155 & 8.2 & 63 & 62 & 8.3 & .30 & .040 \\
\hline & $10-22-91$ & 13.5 & 154 & 8.7 & 67 & 65 & 5.8 & .30 & .010 \\
\hline & $01-13-92$ & 0.5 & 165 & 8.0 & 76 & 77 & 4.5 & .20 & $<.010$ \\
\hline & $04-14-92$ & 15.5 & 187 & 8.4 & 83 & 84 & 4.8 & .20 & .010 \\
\hline & $07-06-92$ & 22.0 & 105 & 8.9 & 43 & 43 & 2.2 & $<.10$ & .010 \\
\hline & $10-19-92$ & 14.5 & 156 & 8.4 & 69 & 68 & 4.6 & .20 & .020 \\
\hline & $01-25-93$ & 2.5 & 220 & 7.7 & 79 & 78 & 13 & .20 & .040 \\
\hline & $04-12-93$ & 14.0 & 187 & 8.3 & 79 & 81 & 5.2 & .20 & .020 \\
\hline & $07-13-93$ & 13.0 & 71 & 7.6 & 33 & 35 & 1.2 & $<.10$ & .030 \\
\hline & $10-13-93$ & 10.0 & 120 & 7.7 & 54 & 56 & 2.4 & .10 & .030 \\
\hline \multirow[t]{7}{*}{$\mathrm{HCA}$} & $06-22-87$ & - & - & - & - & - & 5.9 & .30 & - \\
\hline & $09-26-90$ & 10.5 & 231 & - & - & - & 7.8 & .20 & - \\
\hline & $10-17-90$ & 12.0 & 219 & - & - & - & 9.2 & .20 & - \\
\hline & $11-15-90$ & 11.0 & 240 & - & - & - & 9.7 & .50 & - \\
\hline & $12-19-90$ & 4.0 & 246 & - & - & - & 9.9 & .30 & - \\
\hline & $01-17-91$ & 14.0 & 236 & - & - & - & 9.5 & .40 & - \\
\hline & $02-15-91$ & 11.0 & 240 & - & - & - & 9.4 & .30 & - \\
\hline \multirow[t]{11}{*}{$\mathrm{HCF}$} & $07-27-87$ & 36.0 & 579 & - & - & 176 & 50 & .50 & .030 \\
\hline & $07-12-88$ & 34.0 & 470 & 7.8 & 140 & 147 & 40 & 1.6 & $<.010$ \\
\hline & $07-19-89$ & 37.0 & 586 & 7.7 & 171 & 173 & 52 & 2.4 & $<.010$ \\
\hline & $07-18-90$ & 33.0 & 591 & 7.6 & 192 & 183 & 57 & 2.5 & .020 \\
\hline & $09-26-90$ & 29.5 & 612 & - & - & - & 62 & 2.7 & - \\
\hline & $10-17-90$ & 33.0 & 600 & 7.9 & 194 & - & 59 & 2.5 & - \\
\hline & $11-15-90$ & 31.5 & 612 & - & - & - & 57 & 2.4 & - \\
\hline & $12-19-90$ & 25.5 & 656 & - & - & - & 63 & 2.7 & - \\
\hline & $01-17-91$ & 34.0 & 603 & 7.7 & 195 & - & 65 & 2.8 & - \\
\hline & $02-15-91$ & 33.5 & 652 & - & - & - & 65 & 3.1 & - \\
\hline & $07-08-92$ & 33.5 & 561 & 8.0 & 168 & 171 & 54 & 2.5 & .020 \\
\hline
\end{tabular}


Table 113. Chemical and isotopic analyses of water samples collected at selected surface-water sites, springs, and wells, Long Valley Caldera, Mono County, California, 1987-93-Continued

\begin{tabular}{|c|c|c|c|c|c|c|c|c|c|c|c|}
\hline $\begin{array}{l}\text { Station } \\
\text { identifier }\end{array}$ & Date & $\begin{array}{c}\text { Nitro- } \\
\text { gen, } \\
\text { nitrite } \\
\text { dis- } \\
\text { solved } \\
(\mathrm{mg} / \mathrm{L})\end{array}$ & $\begin{array}{c}\text { Nitro- } \\
\text { gen, } \\
\mathrm{NO}_{2}+\mathrm{NO}_{3} \\
\text { dis- } \\
\text { solved } \\
(\mathrm{mg} / \mathrm{L})\end{array}$ & $\begin{array}{c}\text { Phos- } \\
\text { phorus } \\
\text { ortho, } \\
\text { dis- } \\
\text { solved } \\
(\mathrm{mg} / \mathrm{L})\end{array}$ & $\begin{array}{c}\text { Sulfate, } \\
\text { dis- } \\
\text { solved } \\
(\mathrm{mg} / \mathrm{L})\end{array}$ & $\begin{array}{l}\text { Calcium, } \\
\text { dis- } \\
\text { solved } \\
(\mathrm{mg} / \mathrm{L})\end{array}$ & $\begin{array}{l}\text { Magne- } \\
\text { sium, } \\
\text { dis- } \\
\text { solved } \\
(\mathrm{mg} / \mathrm{L})\end{array}$ & $\begin{array}{l}\text { Stron- } \\
\text { tium. } \\
\text { dis- } \\
\text { solved } \\
(\mu \mathrm{g} / \mathrm{L})\end{array}$ & $\begin{array}{c}\text { Sodium, } \\
\text { dis- } \\
\text { solved } \\
(\mathrm{mg} / \mathrm{L})\end{array}$ & $\begin{array}{l}\text { Potas- } \\
\text { sium, } \\
\text { dis- } \\
\text { solved } \\
(\mathrm{mg} / \mathrm{L})\end{array}$ & $\begin{array}{c}\text { Silica, } \\
\text { dis- } \\
\text { solved } \\
(\mathrm{mg} / \mathrm{L})\end{array}$ \\
\hline \multirow{16}{*}{ MCAF } & $01-09-90$ & $<0.010$ & 0.130 & 0.040 & 9.0 & 13 & 6.5 & - & 18 & 4.5 & 40 \\
\hline & $04-25-90$ & .020 & 1.50 & .060 & 8.2 & 10 & 4.8 & - & 8.6 & 2.0 & 21 \\
\hline & $07-17-90$ & $<.010$ & $<.100$ & .100 & 6.1 & 9.1 & 3.7 & - & 15 & 3.5 & 24 \\
\hline & $10-16-90$ & $<.010$ & $<.100$ & .050 & 14 & 12 & 6.2 & - & 23 & 5.5 & 44 \\
\hline & $01-17-91$ & $<.010$ & $<.100$ & .070 & 15 & 13 & 7.0 & - & 29 & 6.7 & 56 \\
\hline & $04-16-91$ & $<.010$ & $<.050$ & .040 & 10 & 13 & 6.7 & - & 19 & 4.2 & 36 \\
\hline & $07-22-91$ & $<.010$ & $<.050$ & .050 & 7.6 & 9.2 & 3.8 & - & 16 & 3.7 & 32 \\
\hline & $10-22-91$ & $<.010$ & .051 & .030 & 7.1 & 9.4 & 4.4 & - & 14 & 3.6 & 27 \\
\hline & $01-13-92$ & $<.010$ & $<.050$ & .040 & 7.5 & 12 & 5.4 & - & 15 & 3.5 & 35 \\
\hline & $04-14-92$ & $<.010$ & $<.050$ & .070 & 8.2 & 12 & 6.5 & - & 17 & 3.3 & 33 \\
\hline & $07-06-92$ & $<.010$ & $<.050$ & .040 & 5.0 & 7.6 & 2.7 & - & 8.4 & 2.1 & 15 \\
\hline & $10-19-92$ & $<.010$ & $<.050$ & .040 & 6.7 & 11 & 4.8 & - & 14 & 3.5 & 26 \\
\hline & $01-25-93$ & .020 & .120 & .070 & 18 & 13 & 6.0 & - & 25 & 5.3 & 40 \\
\hline & $04-12-93$ & $<.010$ & .050 & .050 & 10 & 12 & 6.3 & - & 15 & 3.6 & 32 \\
\hline & $07-13-93$ & $<.010$ & $<.050$ & .030 & 2.3 & 6.8 & 2.0 & - & 4.4 & 1.2 & 14 \\
\hline & $10-13-93$ & $<.010$ & $<.050$ & .030 & 5.0 & 10 & 4.0 & - & 8.6 & 2.3 & 20 \\
\hline \multirow[t]{7}{*}{$\mathrm{HCA}$} & $06-22-87$ & - & - & - & - & - & - & - & - & - & 13 \\
\hline & $09-26-90$ & - & - & - & 19 & - & - & - & - & - & - \\
\hline & $10-17-90$ & - & - & - & 10 & - & - & - & - & - & - \\
\hline & $11-15-90$ & - & - & - & 12 & - & - & - & - & - & - \\
\hline & $12-19-90$ & - & - & - & 15 & - & - & - & - & - & - \\
\hline & $01-17-91$ & - & - & - & 14 & - & - & - & - & - & - \\
\hline & $02-15-91$ & - & - & - & 14 & - & - & - & - & - & - \\
\hline \multirow[t]{11}{*}{$\mathrm{HCF}$} & $07-27-87$ & - & .260 & - & 30 & 13 & 6.0 & - & 95 & 8.5 & 68 \\
\hline & $07-12-88$ & .020 & .430 & .160 & 24 & 12 & 5.5 & 110 & 78 & 7.0 & 58 \\
\hline & $07-19-89$ & $<.010$ & .120 & .170 & 28 & 13 & 5.7 & 120 & 98 & 8.2 & 67 \\
\hline & $07-18-90$ & .040 & $<.100$ & .240 & 32 & 13 & 6.0 & - & 100 & 9.0 & 69 \\
\hline & $09-26-90$ & - & - & - & 38 & - & - & - & - & - & - \\
\hline & $10-17-90$ & - & - & - & 30 & - & - & - & - & - & - \\
\hline & $11-15-90$ & - & - & - & 31 & - & - & - & - & - & - \\
\hline & $12-19-90$ & - & - & - & 36 & - & - & - & - & - & - \\
\hline & $01-17-91$ & - & - & - & 34 & - & - & - & - & - & - \\
\hline & $02-15-91$ & - & - & - & 35 & - & - & - & - & - & - \\
\hline & $07-08-92$ & .020 & .160 & .190 & 31 & 12 & 5.9 & - & 96 & 7.3 & - \\
\hline
\end{tabular}


Table 113. Chemical and isotopic analyses of water samples collected at selected surface-water sites, springs, and wells, Long Valley Caldera, Mono County, California, 1987-93-Continued

\begin{tabular}{|c|c|c|c|c|c|c|c|c|c|c|}
\hline $\begin{array}{l}\text { Station } \\
\text { identifier }\end{array}$ & Date & $\begin{array}{c}\text { Boron, } \\
\text { dis- } \\
\text { solved } \\
(\mu \mathrm{g} / \mathrm{L})\end{array}$ & $\begin{array}{l}\text { Arsenic, } \\
\text { dis- } \\
\text { solved } \\
(\mu \mathrm{g} / \mathrm{L})\end{array}$ & $\begin{array}{l}\text { Lithium, } \\
\text { dis- } \\
\text { solved } \\
(\mu \mathrm{g} / \mathrm{L})\end{array}$ & $\begin{array}{c}\text { Mercury, } \\
\text { dis- } \\
\text { solved } \\
(\mu \mathrm{g} / \mathrm{L})\end{array}$ & $\begin{array}{l}\text { Iron, } \\
\text { dis- } \\
\text { solved } \\
(\mu \mathrm{g} / \mathrm{L})\end{array}$ & $\begin{array}{l}\text { Manga- } \\
\text { nese, } \\
\text { dis- } \\
\text { solved } \\
(\mu \mathrm{g} / \mathrm{L})\end{array}$ & $\begin{array}{c}\text { Solids, } \\
\text { residue } \\
\text { at } 180^{\circ} \mathrm{C} \\
\text { dis- } \\
\text { solved } \\
(\mathrm{mg} / \mathrm{L})\end{array}$ & $\begin{array}{c}\mathrm{H}-2 / \mathrm{H}-1 \\
\text { stable } \\
\text { isotope } \\
\text { ratio } \\
\text { per } \\
\text { mil }\end{array}$ & $\begin{array}{c}\text { O-18/O-16 } \\
\text { stable } \\
\text { isotope } \\
\text { ratio } \\
\text { per } \\
\text { mil }\end{array}$ \\
\hline \multirow{16}{*}{ MCAF } & $01-09-90$ & 290 & 21 & 60 & $<0.1$ & 35 & 26 & 152 & - & - \\
\hline & $04-25-90$ & - & 3 & 20 & - & 22 & 5 & 110 & - & - \\
\hline & $07-17-90$ & 290 & 52 & 70 & $<.1$ & 65 & 20 & 107 & - & - \\
\hline & $10-16-90$ & 500 & 46 & 90 & .1 & 64 & 81 & 155 & - & - \\
\hline & $01-17-91$ & 640 & 54 & 120 & $<.1$ & 280 & 1000 & 197 & - & - \\
\hline & $04-16-91$ & 230 & 29 & 70 & $<.1$ & 130 & 220 & 146 & - & - \\
\hline & $07-22-91$ & 360 & 43 & 60 & $<.1$ & 44 & 9 & 98 & - & - \\
\hline & $10-22-91$ & 240 & 27 & 50 & $<.1$ & 41 & 18 & 99 & -107.0 & -13.95 \\
\hline & $01-13-92$ & 200 & 20 & 50 & $<.1$ & 68 & 96 & 118 & - & - \\
\hline & $04-14-92$ & 170 & 29 & 60 & $<.1$ & 68 & 92 & 136 & - & - \\
\hline & $07-06-92$ & 110 & 19 & 30 & $<.1$ & 66 & 35 & 68 & - & - \\
\hline & $10-19-92$ & 200 & 26 & 50 & $<.1$ & 34 & 24 & 134 & - & - \\
\hline & $01-25-93$ & 520 & 60 & 100 & $<.1$ & 230 & 240 & 162 & - & - \\
\hline & $04-12-93$ & 180 & 24 & 60 & $<.1$ & 110 & 120 & 118 & -112.0 & -15.18 \\
\hline & $07-13-93$ & 60 & 11 & 10 & $<.1$ & 75 & 71 & 54 & -113.0 & -15.39 \\
\hline & $10-13-93$ & 70 & 11 & 20 & $<.1$ & 61 & 64 & 74 & - & - \\
\hline \multirow[t]{7}{*}{$\mathrm{HCA}$} & $06-22-87$ & 250 & - & - & - & - & - & - & - & - \\
\hline & $09-26-90$ & 310 & - & - & - & - & - & - & - & - \\
\hline & $10-17-90$ & 330 & - & - & - & - & - & - & - & - \\
\hline & $11-15-90$ & 320 & - & - & - & - & - & - & - & - \\
\hline & $12-19-90$ & 340 & - & - & - & - & - & - & - & - \\
\hline & $01-17-91$ & 350 & - & - & - & - & - & - & - & - \\
\hline & $02-15-91$ & 350 & - & - & - & - & - & - & - & - \\
\hline \multirow[t]{11}{*}{$\mathrm{HCF}$} & $07-27-87$ & 2,300 & 110 & 590 & .3 & 6 & 31 & 369 & - & - \\
\hline & $07-12-88$ & 1,700 & 230 & 450 & .1 & 17 & 10 & 323 & - & - \\
\hline & $07-19-89$ & 2,300 & 270 & 600 & $<.1$ & 10 & 9 & 388 & - & - \\
\hline & $07-18-90$ & 2,600 & 280 & 600 & .1 & 16 & 20 & 408 & - & - \\
\hline & $09-26-90$ & 2,500 & - & - & - & - & - & - & - & - \\
\hline & $10-17-90$ & 2,600 & - & - & - & - & - & - & - & - \\
\hline & $11-15-90$ & 2,600 & - & - & - & - & - & - & - & - \\
\hline & $12-19-90$ & 2,900 & - & - & - & - & - & - & - & - \\
\hline & $01-17-91$ & 2,800 & - & - & - & - & - & - & - & - \\
\hline & $02-15-91$ & 2,900 & - & - & - & - & - & - & - & - \\
\hline & $07-08-92$ & 2,400 & 280 & 560 & $<.1$ & 10 & $<10$ & 346 & - & - \\
\hline
\end{tabular}


Table 113. Chemical and isotopic analyses of water samples collected at selected surface-water sites, springs, and wells, Long Valley Caldera, Mono County, California, 1987-93-Continued

\begin{tabular}{|c|c|c|c|c|c|c|c|c|c|}
\hline $\begin{array}{l}\text { Station } \\
\text { identifier }\end{array}$ & Date & $\begin{array}{l}\text { Temper- } \\
\text { ature, } \\
\text { water } \\
\left({ }^{\circ} \mathrm{C}\right)\end{array}$ & $\begin{array}{l}\text { Specific } \\
\text { con- } \\
\text { duct- } \\
\text { ance } \\
(\mu \mathrm{S} / \mathrm{cm})\end{array}$ & $\begin{array}{c}\mathrm{pH}, \\
\text { water } \\
\text { whole } \\
\text { field } \\
\text { (standard } \\
\text { units) }\end{array}$ & $\begin{array}{l}\text { Alka- } \\
\text { linity, } \\
\text { field, } \\
\text { as } \mathrm{CaCO}_{3} \\
(\mathrm{mg} / \mathrm{L})\end{array}$ & $\begin{array}{c}\text { Alka- } \\
\text { linity } \\
\text { lab, } \\
\text { as } \mathrm{CaCO}_{3} \\
(\mathrm{mg} / \mathrm{L})\end{array}$ & $\begin{array}{l}\text { Chlo- } \\
\text { ride, } \\
\text { dis- } \\
\text { solved } \\
(\mathrm{mg} / \mathrm{L})\end{array}$ & $\begin{array}{l}\text { Fluo- } \\
\text { ride, } \\
\text { dis- } \\
\text { solved } \\
(\mathrm{mg} / \mathrm{L})\end{array}$ & $\begin{array}{l}\text { Nitro- } \\
\text { gen, } \\
\text { ammonia } \\
\text { dis- } \\
\text { solved } \\
(\mathrm{mg} / \mathrm{L})\end{array}$ \\
\hline \multirow[t]{2}{*}{ LHC-SW } & $07-18-90$ & 52.5 & - & 8.1 & 626 & 609 & 210 & 8.7 & 0.020 \\
\hline & $07-08-92$ & 54.0 & 1,940 & 8.4 & 611 & 597 & 210 & 9.7 & .020 \\
\hline \multirow[t]{22}{*}{ FHCD } & $10-21-88$ & 15.0 & 185 & 7.3 & 108 & 103 & 4.5 & .30 & .021 \\
\hline & $01-10-89$ & 14.0 & 229 & 7.3 & 108 & 104 & 5.6 & .30 & .020 \\
\hline & $04-12-89$ & 15.0 & 231 & 7.3 & 108 & 106 & 5.6 & .30 & $<.010$ \\
\hline & $07-20-89$ & 15.5 & 236 & 7.2 & 108 & 105 & 5.7 & .40 & .020 \\
\hline & $10-19-89$ & 15.0 & 232 & 7.5 & - & 104 & 5.4 & .30 & .020 \\
\hline & $10-19-89$ & 15.0 & 232 & 7.5 & - & - & - & - & .020 \\
\hline & $01-08-90$ & 15.5 & 213 & 7.3 & 105 & 105 & 5.2 & .30 & .040 \\
\hline & $04-24-90$ & 15.5 & 243 & 7.4 & 99 & 106 & 7.2 & .30 & $<.010$ \\
\hline & $07-17-90$ & 15.5 & 230 & 7.2 & 104 & 105 & 7.0 & .10 & .010 \\
\hline & $10-16-90$ & 15.5 & 222 & 7.2 & 108 & 108 & 6.3 & .30 & .020 \\
\hline & $01-17-91$ & 16.0 & 246 & 7.4 & $\overline{0}$ & 110 & 8.2 & .40 & .010 \\
\hline & $04-16-91$ & 16.0 & 263 & 7.3 & 108 & 110 & 8.0 & .30 & $<.010$ \\
\hline & $07-23-91$ & 16.0 & 235 & 7.3 & 105 & 105 & 6.9 & .30 & .030 \\
\hline & $10-23-91$ & 15.5 & 239 & 7.5 & 111 & 108 & 6.1 & .30 & .030 \\
\hline & $01-14-92$ & 16.0 & 243 & 7.5 & 106 & 109 & 7.3 & .40 & $<.010$ \\
\hline & $04-15-92$ & 16.0 & 240 & 7.7 & 109 & 106 & 6.4 & .40 & .010 \\
\hline & $07-07-92$ & 16.5 & 246 & 7.5 & 106 & 108 & 6.5 & .40 & .030 \\
\hline & $10-20-92$ & 15.5 & 244 & 7.4 & 107 & 110 & 5.9 & .40 & .030 \\
\hline & $01-26-93$ & 16.0 & 250 & 7.6 & 113 & 113 & 5.9 & .30 & $<.010$ \\
\hline & $04-13-93$ & 15.5 & 255 & 7.5 & 107 & 111 & 6.7 & .30 & .020 \\
\hline & $07-13-93$ & 17.0 & 214 & 7.4 & 97 & 98 & 4.2 & .30 & .030 \\
\hline & $10-13-93$ & 15.0 & 237 & 7.2 & 104 & 108 & 4.3 & .30 & .040 \\
\hline \multirow[t]{8}{*}{ CS } & $08-29-87$ & 93.0 & - & 8.2 & - & 400 & 250 & 12 & $<.010$ \\
\hline & $07-14-88$ & 93.0 & - & 8.5 & 402 & 389 & 270 & 9.5 & .060 \\
\hline & $01-12-89$ & 92.5 & - & 8.4 & 390 & 383 & 270 & 11 & .050 \\
\hline & $07-18-89$ & 93.0 & - & 8.3 & 384 & 410 & 270 & 11 & .060 \\
\hline & $01-10-90$ & 93.0 & - & 8.6 & 408 & 400 & 260 & 12 & .080 \\
\hline & $07-18-90$ & 93.0 & - & 8.6 & 403 & 399 & 230 & 9.1 & $<.010$ \\
\hline & $01-18-91$ & 92.5 & - & 8.8 & 386 & 382 & 260 & 12 & .020 \\
\hline & $04-17-91$ & 92.5 & - & 8.7 & 357 & 354 & 260 & 11 & .100 \\
\hline \multirow[t]{5}{*}{ FHAB } & $07-11-88$ & 16.0 & 270 & 7.1 & - & 109 & 10 & .20 & .020 \\
\hline & $10-18-88$ & 16.5 & 267 & 7.2 & 114 & 112 & 11 & .30 & $<.010$ \\
\hline & $01-10-89$ & 16.0 & 272 & 7.1 & 116 & 112 & 11 & .30 & $<.010$ \\
\hline & $04-12-89$ & 16.0 & 278 & 7.2 & 115 & 112 & 11 & .20 & $<.010$ \\
\hline & $07-20-89$ & 16.5 & 276 & 7.1 & 105 & 109 & 15 & .30 & $<.010$ \\
\hline
\end{tabular}


Table 113. Chemical and isotopic analyses of water samples collected at selected surface-water sites, springs, and wells, Long Valley Caldera, Mono County, California, 1987-93-Continued

\begin{tabular}{|c|c|c|c|c|c|c|c|c|c|c|c|}
\hline $\begin{array}{l}\text { Station } \\
\text { identifier }\end{array}$ & Date & $\begin{array}{l}\text { Nitro- } \\
\text { gen, } \\
\text { nitrite } \\
\text { dis- } \\
\text { solved } \\
(\mathrm{mg} / \mathrm{L})\end{array}$ & $\begin{array}{c}\text { Nitro- } \\
\text { gen, } \\
\mathrm{NO}_{2}+\mathrm{NO}_{3} \\
\text { dis- } \\
\text { solved } \\
(\mathrm{mg} / \mathrm{L})\end{array}$ & $\begin{array}{l}\text { Phos- } \\
\text { phorus } \\
\text { ortho, } \\
\text { dis- } \\
\text { solved } \\
(\mathrm{mg} / \mathrm{L})\end{array}$ & $\begin{array}{l}\text { Sulfate } \\
\text { dis- } \\
\text { solved } \\
(\mathrm{mg} / \mathrm{L})\end{array}$ & $\begin{array}{l}\text { Calcium } \\
\text { dis- } \\
\text { solved } \\
(\mathrm{mg} / \mathrm{L})\end{array}$ & $\begin{array}{l}\text { Magne- } \\
\text { sium, } \\
\text { dis- } \\
\text { solved } \\
(\mathrm{mg} / \mathrm{L})\end{array}$ & $\begin{array}{l}\text { Stron- } \\
\text { tium, } \\
\text { dis- } \\
\text { solved } \\
(\mu \mathrm{g} / \mathrm{L})\end{array}$ & $\begin{array}{c}\text { Sodium, } \\
\text { dis- } \\
\text { solved } \\
(\mathrm{mg} / \mathrm{L})\end{array}$ & $\begin{array}{l}\text { Potas- } \\
\text { sium, } \\
\text { dis- } \\
\text { solved } \\
(\mathrm{mg} / \mathrm{L})\end{array}$ & $\begin{array}{c}\text { Silica, } \\
\text { dis- } \\
\text { solved } \\
(\mathrm{mg} / \mathrm{L})\end{array}$ \\
\hline \multirow[t]{2}{*}{ LHC-SW } & $07-18-90$ & $<0.010$ & $<0.100$ & 0.040 & 100 & 20 & 0.60 & - & 420 & 28 & 93 \\
\hline & $07-08-92$ & $<.010$ & $<.050$ & .030 & 110 & 16 & .50 & - & 440 & 28 & - \\
\hline \multirow[t]{22}{*}{ FHCD } & $10-21-88$ & $<.010$ & .300 & .160 & 11 & 12 & 9.4 & 69 & 24 & 4.4 & 57 \\
\hline & $01-10-89$ & $<.010$ & .320 & .180 & 11 & 12 & 9.0 & 70 & 22 & 5.1 & 57 \\
\hline & $04-12-89$ & $<.010$ & .290 & .180 & 11 & 12 & 9.0 & 70 & 23 & 4.7 & 58 \\
\hline & $07-20-89$ & $<.010$ & .240 & .170 & 10 & 12 & 9.0 & 72 & 23 & 5.1 & 59 \\
\hline & $10-19-89$ & $<.010$ & .270 & .170 & 11 & 12 & 8.6 & 72 & 23 & 4.6 & 57 \\
\hline & $10-19-89$ & .010 & .270 & .170 & - & - & - & - & - & - & - \\
\hline & $01-08-90$ & $<.010$ & .430 & .170 & 11 & 12 & 9.0 & - & 24 & 5.5 & 60 \\
\hline & $04-24-90$ & .020 & .300 & .190 & 10 & 12 & 9.1 & - & 25 & 5.2 & 60 \\
\hline & $07-17-90$ & $<.010$ & .300 & .190 & 11 & 12 & 8.8 & - & 24 & 5.1 & 59 \\
\hline & $10-16-90$ & $<.010$ & .300 & .170 & 11 & 12 & 9.1 & - & 24 & 4.9 & 59 \\
\hline & $01-17-91$ & $<.010$ & .300 & .180 & 14 & 13 & 9.5 & - & 25 & 5.1 & 58 \\
\hline & $04-16-91$ & $<.010$ & .300 & .160 & 10 & 13 & 9.6 & - & 25 & 5.1 & 59 \\
\hline & $07-23-91$ & $<.010$ & .330 & .170 & 11 & 12 & 8.9 & - & 25 & 5.0 & 59 \\
\hline & $10-23-91$ & $<.010$ & .410 & .110 & 13 & 12 & 9.4 & - & 26 & 5.2 & 59 \\
\hline & $01-14-92$ & $<.010$ & .320 & .180 & 15 & 12 & 9.2 & - & 24 & 5.1 & 62 \\
\hline & $04-15-92$ & $<.010$ & .330 & .190 & 13 & 12 & 9.3 & - & 24 & 5.1 & 55 \\
\hline & $07-07-92$ & $<.010$ & .330 & .180 & 12 & 12 & 9.1 & - & 25 & 5.2 & 58 \\
\hline & $10-20-92$ & $<.010$ & .280 & .180 & 11 & 12 & 9.3 & - & 25 & 5.3 & 58 \\
\hline & $01-26-93$ & .020 & .340 & .180 & 11 & 13 & 10 & - & 27 & 5.3 & 62 \\
\hline & $04-13-93$ & .010 & .400 & .180 & 11 & 14 & 9.5 & - & 23 & 5.2 & 58 \\
\hline & $07-13-93$ & .010 & .330 & .180 & 11 & 11 & 8.1 & - & 22 & 4.5 & 58 \\
\hline & $10-13-93$ & $<.010$ & .280 & .150 & 11 & 11 & 8.0 & - & 23 & 5.3 & 58 \\
\hline \multirow[t]{8}{*}{$\mathrm{CS}$} & $08-29-87$ & - & .120 & - & 140 & 1.6 & .01 & - & 380 & 30 & 190 \\
\hline & $07-14-88$ & .030 & $<.100$ & .110 & 130 & 1.5 & $<.10$ & 110 & 370 & 31 & 260 \\
\hline & $01-12-89$ & .040 & $<.100$ & .090 & 130 & 1.5 & .02 & 110 & 370 & 31 & 250 \\
\hline & $07-18-89$ & .030 & $<.100$ & .100 & 140 & 1.6 & .04 & 110 & 390 & 32 & 260 \\
\hline & $01-10-90$ & .050 & $<.100$ & .100 & 140 & 1.5 & $<.01$ & - & 390 & 34 & 260 \\
\hline & $07-18-90$ & .030 & $<.100$ & .110 & 120 & 1.4 & $<.02$ & - & 380 & 29 & 250 \\
\hline & $01-18-91$ & .050 & $<.100$ & .060 & 120 & 1.3 & $<.01$ & - & 380 & 28 & 250 \\
\hline & $04-17-91$ & .040 & $<.050$ & .280 & 140 & 1.2 & .02 & - & 370 & 29 & 260 \\
\hline \multirow[t]{5}{*}{ FHAB } & $07-11-88$ & $<.010$ & .340 & .150 & 14 & 14 & 10 & 91 & 26 & 5.0 & 60 \\
\hline & $10-18-88$ & $<.010$ & .340 & .140 & 13 & 14 & 11 & 96 & 29 & 5.2 & 61 \\
\hline & $01-10-89$ & $<.010$ & .350 & .160 & 14 & 14 & 10 & 97 & 26 & 6.0 & 60 \\
\hline & $04-12-89$ & $<.010$ & .320 & .150 & 14 & 14 & 10 & 99 & 27 & 5.6 & 61 \\
\hline & $07-20-89$ & $<.010$ & .270 & .140 & 13 & 14 & 9.9 & 98 & 28 & 6.0 & 62 \\
\hline
\end{tabular}


Table 113. Chemical and isotopic analyses of water samples collected at selected surface-water sites, springs, and wells, Long Valley Caldera, Mono County, California, 1987-93-Continued

\begin{tabular}{|c|c|c|c|c|c|c|c|c|c|c|}
\hline $\begin{array}{l}\text { Station } \\
\text { identifier }\end{array}$ & Date & $\begin{array}{c}\text { Boron, } \\
\text { dis- } \\
\text { solved } \\
(\mu \mathrm{g} / \mathrm{L})\end{array}$ & $\begin{array}{l}\text { Arsenic, } \\
\text { dis- } \\
\text { solved } \\
(\mu \mathrm{g} / \mathrm{L})\end{array}$ & $\begin{array}{l}\text { Lithium, } \\
\text { dis- } \\
\text { solved } \\
(\mu \mathrm{g} / \mathrm{L})\end{array}$ & $\begin{array}{c}\text { Mercury, } \\
\text { dis- } \\
\text { solved } \\
(\mu \mathrm{g} / \mathrm{L})\end{array}$ & $\begin{array}{l}\text { Iron, } \\
\text { dis- } \\
\text { solved } \\
(\mu \mathrm{g} / \mathrm{L})\end{array}$ & $\begin{array}{l}\text { Manga- } \\
\text { nese, } \\
\text { dis- } \\
\text { solved } \\
(\mu \mathrm{g} / \mathrm{L})\end{array}$ & $\begin{array}{c}\text { Solids, } \\
\text { residue } \\
\text { at } 180^{\circ} \mathrm{C} \\
\text { dis- } \\
\text { solved } \\
(\mathrm{mg} / \mathrm{L})\end{array}$ & $\begin{array}{c}\mathrm{H}-2 / \mathrm{H}-1 \\
\text { stable } \\
\text { isotope } \\
\text { ratio } \\
\text { per } \\
\text { mil }\end{array}$ & $\begin{array}{c}\mathrm{O}-18 / \mathrm{O}-16 \\
\text { stable } \\
\text { isotope } \\
\text { ratio } \\
\text { per } \\
\text { mil }\end{array}$ \\
\hline \multirow[t]{2}{*}{ LHC-SW } & $07-18-90$ & 9,100 & 710 & 3000 & $<0.1$ & 16 & 170 & 1,280 & -123.0 & -15.00 \\
\hline & & & & & & 20 & & 1,250 & - & - \\
\hline \multirow[t]{22}{*}{ FHCD } & $10-21-88$ & 230 & 57 & 70 & $<.1$ & 8 & $<1$ & 181 & - & - \\
\hline & $01-10-89$ & 240 & 62 & 80 & $<.1$ & 8 & 3 & 171 & - & - \\
\hline & $04-12-89$ & 240 & 54 & 90 & $<.1$ & 7 & $<1$ & 181 & - & - \\
\hline & $07-20-89$ & 260 & 49 & 80 & $<.1$ & 6 & 3 & 188 & - & - \\
\hline & $10-19-89$ & 270 & 54 & 70 & $<.1$ & 7 & $<1$ & 154 & - & - \\
\hline & $10-19-89$ & - & - & - & - & - & - & - & - & - \\
\hline & $01-08-90$ & 260 & 30 & 70 & $<.1$ & 16 & 1 & 180 & -116.5 & -15.85 \\
\hline & $04-24-90$ & 270 & 54 & 70 & $<.1$ & 7 & $<1$ & 182 & -117.0 & -15.80 \\
\hline & $07-17-90$ & 280 & 55 & 80 & $<.1$ & $<3$ & 1 & 187 & -117.0 & -15.75 \\
\hline & $10-16-90$ & 270 & 66 & 70 & $<.1$ & 9 & $<1$ & 181 & -118.0 & -15.80 \\
\hline & $01-17-91$ & 320 & 48 & 80 & $<.1$ & 6 & 2 & 163 & -115.0 & -15.80 \\
\hline & $04-16-91$ & 310 & 52 & 80 & $<.1$ & 4 & 1 & 176 & -117.0 & -15.80 \\
\hline & $07-23-91$ & 280 & 85 & 80 & $<.1$ & 5 & $<1$ & 184 & - & -15.65 \\
\hline & $10-23-91$ & 260 & 46 & 70 & $<.1$ & $<3$ & $<1$ & 173 & -116.0 & -15.70 \\
\hline & $01-14-92$ & 300 & 50 & 80 & $<.1$ & 7 & 2 & 170 & -115.0 & -15.60 \\
\hline & $04-15-92$ & 300 & 56 & 70 & $<.1$ & 3 & $<1$ & 194 & -117.0 & -15.75 \\
\hline & $07-07-92$ & 320 & 54 & 80 & $<.1$ & 3 & $<1$ & 182 & -116.0 & -15.65 \\
\hline & $10-20-92$ & 290 & 53 & 80 & $<.1$ & $<3$ & 2 & 204 & -116.0 & -15.70 \\
\hline & $01-26-93$ & 290 & 55 & 80 & $<.1$ & 5 & $<1$ & 190 & -115.0 & -15.76 \\
\hline & $04-13-93$ & 300 & 51 & 80 & $<.1$ & 12 & $<1$ & 180 & -117.0 & -15.82 \\
\hline & $07-13-93$ & 250 & 46 & 70 & $<.1$ & $<3$ & 1 & 168 & -116.0 & -15.82 \\
\hline & $10-13-93$ & 210 & 40 & 90 & $<.1$ & $<3$ & $<1$ & 174 & - & - \\
\hline \multirow[t]{8}{*}{$\mathrm{CS}$} & $08-29-87$ & 11,000 & 1,900 & 2,400 & .2 & 10 & 13 & 1,370 & -115.0 & -14.25 \\
\hline & $07-14-88$ & 11,000 & 2,200 & 2,800 & .3 & $<3$ & 6 & 1,360 & - & - \\
\hline & $01-12-89$ & 11,000 & 2,100 & 3,000 & .3 & $<3$ & 5 & 1,360 & - & - \\
\hline & $07-18-89$ & 11,000 & 1,800 & 3,100 & .2 & 4 & 6 & 1,250 & - & - \\
\hline & $01-10-90$ & 11,000 & 1,700 & 2,900 & .5 & 9 & 5 & 1,370 & -114.0 & -14.20 \\
\hline & $07-18-90$ & 12,000 & 1,200 & 3,000 & .3 & $<3$ & 5 & 1,240 & -113.0 & -14.25 \\
\hline & $01-18-91$ & 12,000 & 1,600 & 4,400 & .5 & 8 & 4 & 1,340 & - & - \\
\hline & $04-17-91$ & 12,000 & 1,800 & 2,900 & .1 & 11 & 4 & 1,410 & -115.0 & -13.90 \\
\hline \multirow[t]{5}{*}{ FHAB } & $07-11-88$ & 440 & 56 & 90 & $<.1$ & 3 & $<1$ & 201 & -114.0 & -15.20 \\
\hline & $10-18-88$ & 470 & 51 & 90 & $<.1$ & 3 & $<1$ & 203 & - & - \\
\hline & $01-10-89$ & 460 & 56 & 100 & $<.1$ & $<3$ & $<1$ & 192 & - & - \\
\hline & $04-12-89$ & 550 & 26 & 110 & $<.1$ & 6 & $<1$ & 209 & - & - \\
\hline & $07-20-89$ & 590 & 50 & 110 & $<.1$ & 4 & $<1$ & 214 & - & - \\
\hline
\end{tabular}


Table 113. Chemical and isotopic analyses of water samples collected at selected surface-water sites, springs, and wells, Long Valley Caldera, Mono County, California, 1987-93-Continued

\begin{tabular}{|c|c|c|c|c|c|c|c|c|c|}
\hline $\begin{array}{l}\text { Station } \\
\text { identifier }\end{array}$ & Date & $\begin{array}{c}\text { Temper- } \\
\text { ature, } \\
\text { water } \\
\left({ }^{\circ} \mathrm{C}\right)\end{array}$ & $\begin{array}{l}\text { Spe- } \\
\text { cific } \\
\text { con- } \\
\text { duct- } \\
\text { ance } \\
(\mu \mathrm{S} / \mathrm{cm})\end{array}$ & $\begin{array}{c}\text { pH, } \\
\text { water } \\
\text { whole } \\
\text { field } \\
\text { (standard } \\
\text { units) }\end{array}$ & $\begin{array}{c}\text { Alka- } \\
\text { linity, } \\
\text { field, } \\
\text { as } \mathrm{CaCO}_{3} \\
(\mathrm{mg} / \mathrm{L})\end{array}$ & $\begin{array}{c}\text { Alka- } \\
\text { linity } \\
\text { lab, } \\
\text { as } \mathrm{CaCO}{ }_{3} \\
(\mathrm{mg} / \mathrm{L})\end{array}$ & $\begin{array}{l}\text { Chlo- } \\
\text { ride, } \\
\text { dis- } \\
\text { solved } \\
(\mathrm{mg} / \mathrm{L})\end{array}$ & $\begin{array}{c}\text { Fluo- } \\
\text { ride, } \\
\text { dis- } \\
\text { solved } \\
(\mathrm{mg} / \mathrm{L})\end{array}$ & $\begin{array}{c}\text { Nitro- } \\
\text { gen, } \\
\text { ammonia, } \\
\text { dis- } \\
\text { solved } \\
(\mathrm{mg} / \mathrm{L})\end{array}$ \\
\hline FHAB & $\begin{array}{c}10-19-89 \\
01-8-90 \\
04-24-90 \\
07-17-90 \\
10-17-90\end{array}$ & $\begin{array}{l}17.0 \\
16.5 \\
17.0 \\
16.5 \\
17.0\end{array}$ & $\begin{array}{l}280 \\
254 \\
290 \\
273 \\
281\end{array}$ & $\begin{array}{l}7.2 \\
7.1 \\
7.3 \\
7.2 \\
7.1\end{array}$ & $\begin{array}{l}-\overline{111} \\
103 \\
111 \\
113\end{array}$ & $\begin{array}{l}110 \\
110 \\
113 \\
112 \\
114\end{array}$ & $\begin{array}{l}12 \\
13 \\
14 \\
14 \\
18\end{array}$ & $\begin{array}{r}0.30 \\
.30 \\
.30 \\
.20 \\
.30\end{array}$ & $\begin{array}{r}<0.010 \\
.020 \\
<.010 \\
.010 \\
.020\end{array}$ \\
\hline & $\begin{array}{l}01-16-91 \\
04-16-91 \\
07-23-91 \\
10-23-91 \\
01-14-92\end{array}$ & $\begin{array}{l}17.5 \\
16.5 \\
16.5 \\
17.5 \\
17.0\end{array}$ & $\begin{array}{l}295 \\
302 \\
264 \\
275 \\
274\end{array}$ & $\begin{array}{l}7.2 \\
7.1 \\
7.1 \\
7.2 \\
7.4\end{array}$ & $\begin{array}{l}109 \\
113 \\
102 \\
105 \\
106\end{array}$ & $\begin{array}{l}114 \\
116 \\
100 \\
109 \\
108\end{array}$ & $\begin{array}{l}18 \\
16 \\
13 \\
15 \\
12\end{array}$ & $\begin{array}{l}.30 \\
.20 \\
.30 \\
.30 \\
.30\end{array}$ & $\begin{array}{r}.010 \\
<.010 \\
.030 \\
.010 \\
<.010\end{array}$ \\
\hline & $\begin{array}{l}04-15-92 \\
07-07-92 \\
10-20-92 \\
01-26-93 \\
04-13-93\end{array}$ & $\begin{array}{l}17.0 \\
17.5 \\
17.5 \\
17.5 \\
16.5\end{array}$ & $\begin{array}{l}276 \\
268 \\
289 \\
287 \\
289\end{array}$ & $\begin{array}{l}7.4 \\
7.2 \\
7.4 \\
7.5 \\
7.3\end{array}$ & $\begin{array}{l}108 \\
101 \\
115 \\
116 \\
105\end{array}$ & $\begin{array}{l}107 \\
102 \\
116 \\
116 \\
107\end{array}$ & $\begin{array}{l}14 \\
16 \\
14 \\
13 \\
14\end{array}$ & $\begin{array}{l}.40 \\
.30 \\
.30 \\
.30 \\
.30\end{array}$ & $\begin{array}{r}<.010 \\
.020 \\
.020 \\
<.010 \\
.010\end{array}$ \\
\hline & $\begin{array}{l}07-13-93 \\
10-13-93\end{array}$ & $\begin{array}{l}16.5 \\
17.5\end{array}$ & $\begin{array}{l}226 \\
239\end{array}$ & $\begin{array}{l}7.3 \\
7.2\end{array}$ & $\begin{array}{l}92 \\
97\end{array}$ & $\begin{array}{r}93 \\
100\end{array}$ & $\begin{array}{l}9.1 \\
7.5\end{array}$ & $\begin{array}{l}.30 \\
.30\end{array}$ & $\begin{array}{l}.010 \\
.030\end{array}$ \\
\hline $\mathrm{FH} 23$ & $\begin{array}{l}07-11-88 \\
10-18-88 \\
01-10-89 \\
04-11-89 \\
07-20-89\end{array}$ & $\begin{array}{l}11.0 \\
11.0 \\
11.0 \\
11.0 \\
11.0\end{array}$ & $\begin{array}{l}193 \\
187 \\
182 \\
186 \\
190\end{array}$ & $\begin{array}{l}7.2 \\
7.1 \\
7.2 \\
7.2 \\
7.1\end{array}$ & $\begin{array}{l}- \\
81 \\
73 \\
77\end{array}$ & $\begin{array}{l}75 \\
75 \\
75 \\
76 \\
79\end{array}$ & $\begin{array}{l}6.2 \\
5.4 \\
5.4 \\
5.4 \\
5.4\end{array}$ & $\begin{array}{l}.30 \\
.20 \\
.20 \\
.20 \\
.30\end{array}$ & $\begin{array}{r}.010 \\
<.010 \\
<.010 \\
<.010 \\
.010\end{array}$ \\
\hline & $\begin{array}{l}10-18-89 \\
01-09-90 \\
04-24-90 \\
07-17-90 \\
10-16-90\end{array}$ & $\begin{array}{l}11.0 \\
11.0 \\
11.0 \\
11.5 \\
11.5\end{array}$ & $\begin{array}{l}190 \\
179 \\
199 \\
192 \\
166\end{array}$ & $\begin{array}{l}7.3 \\
7.2 \\
7.2 \\
7.1 \\
7.1\end{array}$ & $\begin{array}{l}- \\
73 \\
74 \\
77 \\
79\end{array}$ & $\begin{array}{l}77 \\
76 \\
76 \\
78 \\
78\end{array}$ & $\begin{array}{l}5.6 \\
5.2 \\
7.1 \\
8.0 \\
7.3\end{array}$ & $\begin{array}{l}.20 \\
.20 \\
.20 \\
.10 \\
.40\end{array}$ & $\begin{array}{r}<.010 \\
.020 \\
<.010 \\
.020 \\
<.020\end{array}$ \\
\hline & $\begin{array}{l}01-16-91 \\
04-16-91 \\
07-23-91 \\
10-23-91 \\
01-14-92\end{array}$ & $\begin{array}{l}11.5 \\
11.0 \\
11.5 \\
11.5 \\
11.5\end{array}$ & $\begin{array}{l}177 \\
190 \\
198 \\
201 \\
200\end{array}$ & $\begin{array}{l}7.2 \\
7.1 \\
7.0 \\
7.2 \\
7.3\end{array}$ & $\begin{array}{l}- \\
76 \\
79 \\
75 \\
79\end{array}$ & $\begin{array}{l}77 \\
76 \\
79 \\
81 \\
81\end{array}$ & $\begin{array}{l}5.7 \\
6.9 \\
7.9 \\
8.0 \\
7.0\end{array}$ & $\begin{array}{r}<.10 \\
.20 \\
.20 \\
.20 \\
.20\end{array}$ & $\begin{array}{r}<.010 \\
<.010 \\
.020 \\
.010 \\
<.010\end{array}$ \\
\hline & $\begin{array}{l}04-15-92 \\
07-07-92 \\
10-20-92 \\
01-26-93 \\
04-13-93\end{array}$ & $\begin{array}{l}11.0 \\
11.0 \\
11.5 \\
11.5 \\
11.5\end{array}$ & $\begin{array}{l}210 \\
213 \\
210 \\
213 \\
241\end{array}$ & $\begin{array}{l}7.4 \\
7.2 \\
7.2 \\
7.4 \\
7.1\end{array}$ & $\begin{array}{l}82 \\
84 \\
85 \\
88 \\
86\end{array}$ & $\begin{array}{l}80 \\
84 \\
84 \\
86 \\
90\end{array}$ & $\begin{array}{r}9.4 \\
8.1 \\
8.3 \\
8.2 \\
11\end{array}$ & $\begin{array}{l}.10 \\
.10 \\
.20 \\
.20 \\
.20\end{array}$ & $\begin{array}{r}<.010 \\
.020 \\
.030 \\
<.010 \\
<.010\end{array}$ \\
\hline
\end{tabular}


Table 113. Chemical and isotopic analyses of water samples collected at selected surface-water sites, springs, and wells, Long Valley Caldera, Mono County, California, 1987-93-Continued

\begin{tabular}{|c|c|c|c|c|c|c|c|c|c|c|c|}
\hline $\begin{array}{l}\text { Station } \\
\text { identifier }\end{array}$ & Date & $\begin{array}{l}\text { Nitro- } \\
\text { gen, } \\
\text { nitrite } \\
\text { dis- } \\
\text { solved } \\
(\mathrm{mg} / \mathrm{L})\end{array}$ & $\begin{array}{c}\text { Nitro- } \\
\text { gen, } \\
\mathrm{NO}_{2}+\mathrm{NO}_{3} \\
\text { dis- } \\
\text { solved } \\
(\mathrm{mg} / \mathrm{L})\end{array}$ & $\begin{array}{l}\text { Phos- } \\
\text { phorus } \\
\text { ortho, } \\
\text { dis- } \\
\text { solved } \\
(\mathrm{mg} / \mathrm{L})\end{array}$ & $\begin{array}{l}\text { Sulfate } \\
\text { dis- } \\
\text { solved } \\
(\mathrm{mg} / \mathrm{L})\end{array}$ & $\begin{array}{l}\text { Calcium } \\
\text { dis- } \\
\text { solved } \\
(\mathrm{mg} / \mathrm{L})\end{array}$ & $\begin{array}{l}\text { Magne- } \\
\text { sium, } \\
\text { dis- } \\
\text { solved } \\
(\mathrm{mg} / \mathrm{L})\end{array}$ & $\begin{array}{l}\text { Stron- } \\
\text { tium, } \\
\text { dis- } \\
\text { solved } \\
(\mu \mathrm{g} / \mathrm{L})\end{array}$ & $\begin{array}{l}\text { Sodium, } \\
\text { dis- } \\
\text { solved } \\
(\mathrm{mg} / \mathrm{L})\end{array}$ & $\begin{array}{l}\text { Potas- } \\
\text { sium, } \\
\text { dis- } \\
\text { solved } \\
(\mathrm{mg} / \mathrm{L})\end{array}$ & $\begin{array}{c}\text { Silica, } \\
\text { dis- } \\
\text { solved } \\
(\mathrm{mg} / \mathrm{L})\end{array}$ \\
\hline \multirow[t]{17}{*}{ FHAB } & $10-19-89$ & $<0.010$ & 0.300 & 0.150 & 13 & 14 & 9.7 & 100 & 28 & 6.0 & 60 \\
\hline & $01-08-90$ & $<.010$ & .320 & .150 & 14 & 14 & 10 & - & 29 & 6.4 & 63 \\
\hline & $04-24-90$ & .020 & .300 & .150 & 14 & 14 & 10 & - & 29 & 6.3 & 62 \\
\hline & $07-17-90$ & $<.010$ & .300 & .160 & 9.5 & 15 & 10 & - & 31 & 6.4 & 65 \\
\hline & $10-17-90$ & $<.010$ & .300 & .140 & 14 & 14 & 10 & - & 29 & 6.0 & 63 \\
\hline & $01-16-91$ & $<.010$ & .300 & .130 & 18 & 15 & 11 & - & 30 & 6.1 & 62 \\
\hline & $04-16-91$ & $<.010$ & .310 & .130 & 16 & 15 & 11 & - & 30 & 6.2 & 63 \\
\hline & $07-23-91$ & $<.010$ & .370 & .150 & 14 & 13 & 9.0 & - & 29 & 5.6 & 62 \\
\hline & $10-23-91$ & $<.010$ & .520 & .130 & 17 & 14 & 9.2 & 一 & 26 & 6.7 & 61 \\
\hline & $01-14-92$ & $<.010$ & .280 & .150 & 18 & 13 & 9.3 & - & 28 & 6.0 & 66 \\
\hline & $04-15-92$ & $<.010$ & .290 & .160 & 19 & 14 & 9.9 & 一 & 29 & 5.8 & 61 \\
\hline & $07-07-92$ & $<.010$ & .300 & .150 & 16 & 12 & 8.8 & - & 28 & 5.9 & 63 \\
\hline & $10-20-92$ & $<.010$ & .290 & .150 & 15 & 14 & 10 & - & 31 & 6.6 & 63 \\
\hline & $01-26-93$ & .010 & .320 & .150 & 14 & 14 & 11 & - & 31 & 6.4 & 67 \\
\hline & $04-13-93$ & $<.010$ & .780 & .160 & 16 & 14 & 10 & 一 & 28 & 6.0 & 63 \\
\hline & $07-13-93$ & $<.010$ & .440 & .160 & 12 & 9.4 & 7.1 & - & 21 & 4.6 & 56 \\
\hline & $10-13-93$ & $<.010$ & .330 & .140 & 12 & 12 & 8.9 & - & 22 & 5.1 & 53 \\
\hline \multirow[t]{20}{*}{ FH23 } & $07-11-88$ & $<.010$ & .310 & .080 & 12 & 16 & 5.6 & 57 & 13 & 3.0 & 39 \\
\hline & $10-18-88$ & $<.010$ & .290 & .090 & 12 & 16 & 5.9 & 58 & 15 & 3.2 & 39 \\
\hline & $01-10-89$ & $<.010$ & .290 & .090 & 12 & 16 & 5.4 & 55 & 13 & 3.4 & 38 \\
\hline & $04-11-89$ & $<.010$ & .330 & .100 & 12 & 16 & 5.4 & 56 & 13 & 3.3 & 38 \\
\hline & $07-20-89$ & $<.010$ & .260 & .090 & 11 & 16 & 5.5 & 59 & 14 & 3.5 & 39 \\
\hline & $10-18-89$ & .010 & .270 & .100 & 11 & 16 & 5.3 & 58 & 14 & 3.5 & 38 \\
\hline & $01-09-90$ & $<.010$ & .340 & .090 & 6.0 & 16 & 5.3 & - & 14 & 3.6 & 40 \\
\hline & $04-24-90$ & .020 & .700 & .100 & 13 & 16 & 5.6 & - & 15 & 3.5 & 40 \\
\hline & $07-17-90$ & $<.010$ & .500 & .100 & 11 & 17 & 5.5 & - & 15 & 3.4 & 40 \\
\hline & $10-16-90$ & $<.010$ & .300 & $<.090$ & 12 & 16 & 5.4 & - & 14 & 3.4 & 39 \\
\hline & $01-16-91$ & $<.010$ & .300 & .100 & 11 & 16 & 5.2 & - & 14 & 3.2 & 38 \\
\hline & $04-16-91$ & $<.010$ & .630 & .080 & 11 & 16 & 5.4 & - & 15 & 3.5 & 39 \\
\hline & $07-23-91$ & $<.010$ & .520 & .090 & 13 & 17 & 5.7 & - & 16 & 3.5 & 39 \\
\hline & $10-23-91$ & $<.010$ & .550 & .080 & 12 & 17 & 5.8 & - & 16 & 3.7 & 39 \\
\hline & $01-14-92$ & $<.010$ & .330 & .100 & 12 & 17 & 5.6 & - & 15 & 3.5 & 41 \\
\hline & $04-15-92$ & $<.010$ & .500 & .100 & 12 & 18 & 6.2 & - & 16 & 3.5 & 38 \\
\hline & $07-07-92$ & $<.010$ & .400 & .100 & 12 & 18 & 5.9 & - & 16 & 3.6 & 39 \\
\hline & $10-20-92$ & $<.010$ & .500 & .100 & 12 & 18 & 5.9 & - & 16 & 3.7 & 39 \\
\hline & $01-26-93$ & .010 & .350 & .100 & 12 & 18 & 6.3 & - & 18 & 3.7 & 41 \\
\hline & $04-13-93$ & $<.010$ & .530 & .100 & 13 & 20 & 6.8 & - & 17 & 3.8 & 40 \\
\hline
\end{tabular}


Table 113. Chemical and isotopic analyses of water samples collected at selected surface-water sites, springs, and wells, Long Valley Caldera, Mono County, California, 1987-93-Continued

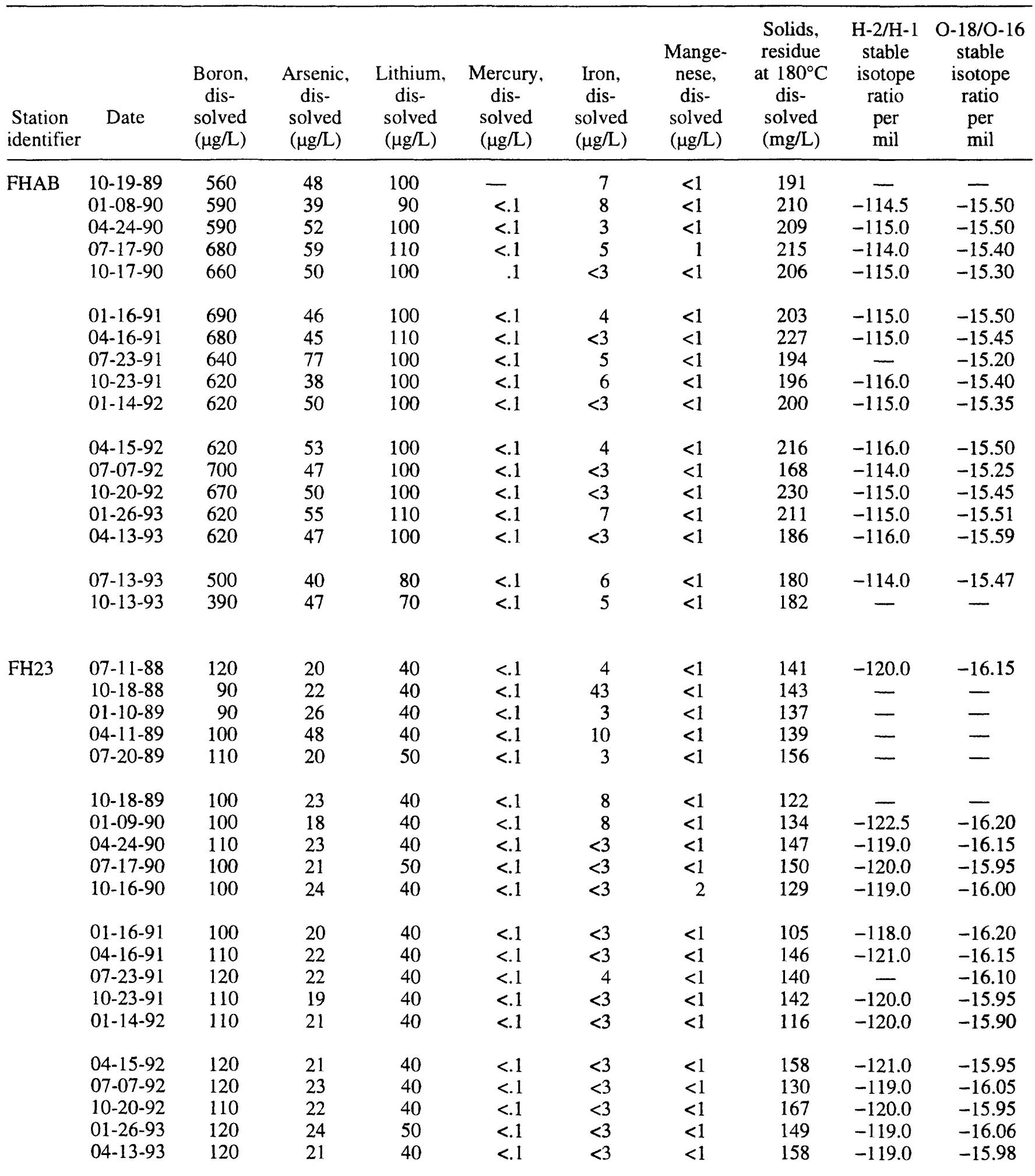


Table 113. Chemical and isotopic analyses of water samples collected at selected surface-water sites, springs, and wells, Long Valley Caldera, Mono County, California, 1987-93-Continued

\begin{tabular}{|c|c|c|c|c|c|c|c|c|c|}
\hline $\begin{array}{c}\text { Station } \\
\text { identifier }\end{array}$ & Date & $\begin{array}{l}\text { Temper- } \\
\text { ature, } \\
\text { water } \\
\left({ }^{\circ} \mathrm{C}\right)\end{array}$ & $\begin{array}{l}\text { Specific } \\
\text { con- } \\
\text { duct- } \\
\text { ance } \\
(\mu \mathrm{S} / \mathrm{cm})\end{array}$ & $\begin{array}{c}\mathrm{pH} \\
\text { water } \\
\text { whole } \\
\text { field } \\
\text { (standard } \\
\text { units) }\end{array}$ & $\begin{array}{c}\text { Alka- } \\
\text { linity, } \\
\text { field, } \\
\text { as } \mathrm{CaCO}_{3} \\
(\mathrm{mg} / \mathrm{L})\end{array}$ & $\begin{array}{c}\text { Alka- } \\
\text { linity } \\
\text { lab, } \\
\text { as } \mathrm{CaCO} \\
(\mathrm{mg} / \mathrm{L})\end{array}$ & $\begin{array}{l}\text { Chlo- } \\
\text { ride, } \\
\text { dis- } \\
\text { solved } \\
(\mathrm{mg} / \mathrm{L})\end{array}$ & $\begin{array}{l}\text { Fluo- } \\
\text { ride, } \\
\text { dis- } \\
\text { solved } \\
(\mathrm{mg} / \mathrm{L})\end{array}$ & $\begin{array}{c}\text { Nitro- } \\
\text { gen, } \\
\text { ammonia, } \\
\text { dis- } \\
\text { solved } \\
(\mathrm{mg} / \mathrm{L})\end{array}$ \\
\hline $\mathrm{FH} 23$ & $\begin{array}{l}07-13-93 \\
10-13-93\end{array}$ & $\begin{array}{l}11.5 \\
12.0\end{array}$ & $\begin{array}{l}230 \\
234\end{array}$ & $\begin{array}{l}7.1 \\
7.1\end{array}$ & $\begin{array}{l}94 \\
92\end{array}$ & $\begin{array}{l}95 \\
95\end{array}$ & $\begin{array}{l}8.6 \\
8.3\end{array}$ & $\begin{array}{r}0.20 \\
.20\end{array}$ & $\begin{array}{r}0.020 \\
.030\end{array}$ \\
\hline MBP-3 & $\begin{array}{l}07-30-87 \\
12-17-87 \\
04-19-88 \\
04-19-88 \\
07-13-88\end{array}$ & $\begin{array}{l}171.0 \\
170.0 \\
168.0 \\
168.0 \\
169.0\end{array}$ & $\begin{array}{l}- \\
z \\
-\end{array}$ & $\begin{array}{l}6.1 \\
6.5 \\
6.3 \\
6.3 \\
6.5\end{array}$ & $\begin{array}{r}356 \\
360 \\
358 \\
-360\end{array}$ & $\begin{array}{l}365 \\
370 \\
361 \\
360 \\
365\end{array}$ & $\begin{array}{l}240 \\
240 \\
220 \\
240 \\
240\end{array}$ & $\begin{array}{c}11 \\
9.0 \\
11 \\
11 \\
9.8\end{array}$ & $\begin{array}{r}.320 \\
.260 \\
.290 \\
-\quad \\
.330\end{array}$ \\
\hline & $\begin{array}{l}01-12-89 \\
07-21-89 \\
01-10-90 \\
07-10-90\end{array}$ & $\begin{array}{c}168.0 \\
163.5 \\
\overline{161.0}\end{array}$ & $\begin{array}{r}- \\
1,610 \\
1,710\end{array}$ & $\begin{array}{l}6.4 \\
6.1 \\
6.7 \\
6.3\end{array}$ & $\begin{array}{r}443 \\
354 \\
387 \\
-\end{array}$ & $\begin{array}{l}357 \\
367 \\
367 \\
377\end{array}$ & $\begin{array}{l}240 \\
260 \\
240 \\
240\end{array}$ & $\begin{array}{c}8.6 \\
10 \\
10 \\
8.0\end{array}$ & $\begin{array}{l}.250 \\
.220 \\
.250 \\
.210\end{array}$ \\
\hline MBP-1 & $\begin{array}{l}12-17-87 \\
12-17-87 \\
04-19-88 \\
04-19-88 \\
07-13-88\end{array}$ & $\begin{array}{l}- \\
\overline{164.0} \\
164.0 \\
163.0\end{array}$ & $\begin{array}{l}- \\
- \\
-\end{array}$ & $\begin{array}{l}- \\
6.5 \\
6.5 \\
6.5\end{array}$ & $\frac{-}{\overline{350}} \frac{-}{335}$ & $\begin{array}{l}353 \\
353 \\
346 \\
344 \\
341\end{array}$ & $\begin{array}{l}290 \\
260 \\
250 \\
250 \\
250\end{array}$ & $\begin{array}{l}11 \\
11 \\
11 \\
11 \\
11\end{array}$ & $\begin{array}{r}.360 \\
-\quad .400 \\
-.440\end{array}$ \\
\hline & $\begin{array}{l}01-12-89 \\
07-21-89 \\
01-10-90 \\
07-10-90\end{array}$ & $\begin{array}{c}163.0 \\
160.0 \\
- \\
158.0\end{array}$ & $\begin{array}{r}- \\
1,630 \\
1,690\end{array}$ & $\begin{array}{l}6.8 \\
6.8 \\
6.5 \\
6.8\end{array}$ & $\begin{array}{r}348 \\
344 \\
347 \\
-\end{array}$ & $\begin{array}{l}340 \\
334 \\
337 \\
351\end{array}$ & $\begin{array}{l}250 \\
250 \\
240 \\
260\end{array}$ & $\begin{array}{l}9.6 \\
10 \\
11 \\
11\end{array}$ & $\begin{array}{l}.370 \\
.320 \\
.330 \\
.280\end{array}$ \\
\hline HBP & $\begin{array}{l}07-10-90 \\
04-15-92\end{array}$ & $\begin{array}{l}64.5 \\
87.5\end{array}$ & $\begin{array}{l}1,730 \\
1,830\end{array}$ & $\begin{array}{l}7.7 \\
7.7\end{array}$ & $\overline{382}$ & $\begin{array}{l}399 \\
385\end{array}$ & $\begin{array}{l}240 \\
240\end{array}$ & $\begin{array}{l}11 \\
12\end{array}$ & $\begin{array}{l}.100 \\
.140\end{array}$ \\
\hline HC-5L & 07-08-92 & 83.0 & 1,720 & 8.6 & 466 & 459 & 210 & 10 & .030 \\
\hline HC-3 & $\begin{array}{c}07-28-87 \\
07-11-88 \\
07-19-89 \\
07-18-90 \\
10-17-90\end{array}$ & $\begin{array}{l}93.5 \\
93.0 \\
95.0 \\
95.5 \\
93.0\end{array}$ & $\begin{array}{l}- \\
z \\
z\end{array}$ & $\begin{array}{l}7.6 \\
8.1 \\
7.8 \\
8.1 \\
8.2\end{array}$ & $\begin{array}{l}\overline{473} \\
\overline{503} \\
486\end{array}$ & $\begin{array}{r}472 \\
476 \\
479 \\
475 \\
-\end{array}$ & $\begin{array}{l}220 \\
220 \\
220 \\
220 \\
220\end{array}$ & $\begin{array}{c}11 \\
9.9 \\
9.7 \\
9.9 \\
11\end{array}$ & $\begin{array}{r}.110 \\
.060 \\
.020 \\
.040 \\
-\end{array}$ \\
\hline & $\begin{array}{l}01-17-91 \\
04-16-92\end{array}$ & $\begin{array}{l}94.0 \\
81.0\end{array}$ & $\overline{1,830}$ & $\begin{array}{l}8.2 \\
7.6\end{array}$ & 472 & $\overline{475}$ & $\begin{array}{l}210 \\
230\end{array}$ & $\begin{array}{c}9.8 \\
12\end{array}$ & -.110 \\
\hline
\end{tabular}


Table 113. Chemical and isotopic analyses of water samples collected at selected surface-water sites, springs, and wells, Long Valley Caldera, Mono County, California, 1987-93-Continued

\begin{tabular}{|c|c|c|c|c|c|c|c|c|c|c|c|}
\hline $\begin{array}{l}\text { Station } \\
\text { identifier }\end{array}$ & Date & $\begin{array}{l}\text { Nitro- } \\
\text { gen. } \\
\text { nitrite } \\
\text { dis- } \\
\text { solved } \\
(\mathrm{mg} / \mathrm{L})\end{array}$ & $\begin{array}{c}\text { Nitro- } \\
\text { gen, } \\
\mathrm{NO}_{2}+\mathrm{NO}_{3} \\
\text { dis- } \\
\text { solved } \\
(\mathrm{mg} / \mathrm{L})\end{array}$ & $\begin{array}{l}\text { Phos- } \\
\text { phorus } \\
\text { ortho, } \\
\text { dis- } \\
\text { solved } \\
\text { (mg/L) }\end{array}$ & $\begin{array}{c}\text { Sulfate, } \\
\text { dis- } \\
\text { solved } \\
(\mathrm{mg} / \mathrm{L})\end{array}$ & $\begin{array}{l}\text { Calcium, } \\
\text { dis- } \\
\text { solved } \\
(\mathrm{mg} / \mathrm{L})\end{array}$ & $\begin{array}{l}\text { Magne- } \\
\text { sium, } \\
\text { dis- } \\
\text { solved } \\
(\mathrm{mg} / \mathrm{L})\end{array}$ & $\begin{array}{l}\text { Stron- } \\
\text { tium, } \\
\text { dis- } \\
\text { solved } \\
(\mu \mathrm{g} / L)\end{array}$ & $\begin{array}{l}\text { Sodium, } \\
\text { dis- } \\
\text { solved } \\
(\mathrm{mg} / \mathrm{L})\end{array}$ & $\begin{array}{l}\text { Potas- } \\
\text { sium, } \\
\text { dis- } \\
\text { solved } \\
(\mathrm{mg} / \mathrm{L})\end{array}$ & $\begin{array}{c}\text { Silica, } \\
\text { dis- } \\
\text { solved } \\
(\mathrm{mg} / \mathrm{L})\end{array}$ \\
\hline \multirow[t]{2}{*}{$\mathrm{FH} 23$} & $07-13-93$ & $<0.010$ & 0.460 & 0.100 & 13 & 21 & 6.9 & - & 17 & 3.8 & 41 \\
\hline & $10-13-93$ & $<.010$ & .440 & .100 & 12 & 19 & 6.5 & - & 17 & 3.9 & 38 \\
\hline \multirow[t]{9}{*}{ MBP-3 } & $07-30-87$ & - & $<.100$ & - & 120 & 5.3 & .22 & - & 330 & 36 & 240 \\
\hline & $12-17-87$ & - & $<.100$ & - & 110 & 6.7 & .22 & 220 & 330 & 36 & 230 \\
\hline & $04-19-88$ & $<.010$ & $<.100$ & .010 & 140 & 2.6 & .12 & 100 & 320 & 33 & 250 \\
\hline & $04-19-88$ & - & - & - & 120 & 2.9 & .09 & 110 & 350 & 30 & 240 \\
\hline & $07-13-88$ & $<.010$ & .130 & .030 & 110 & 5.8 & .19 & 200 & 340 & 22 & 240 \\
\hline & $01-12-89$ & $<.010$ & $<.100$ & $<.010$ & 110 & 2.1 & .20 & 86 & 330 & 30 & 230 \\
\hline & $07-21-89$ & $<.010$ & $<.100$ & $<.010$ & 110 & 5.9 & .25 & 190 & 330 & 30 & 230 \\
\hline & $01-10-90$ & $<.010$ & $<.100$ & .010 & 120 & 6.1 & .22 & - & 340 & 35 & 230 \\
\hline & $07-10-90$ & $<.010$ & $<.100$ & $<.010$ & 110 & 7.4 & $<.02$ & - & 330 & 31 & 210 \\
\hline \multirow[t]{9}{*}{ MBP-1 } & $12-17-87$ & - & $<.100$ & 一 & 120 & 5.0 & .18 & 170 & 320 & 37 & 230 \\
\hline & $12-17-87$ & - & - & - & 120 & 5.4 & .18 & 180 & 340 & 37 & 240 \\
\hline & $04-19-88$ & $<.010$ & $<.100$ & .020 & 120 & 4.7 & .12 & 160 & 340 & 24 & 220 \\
\hline & $04-19-88$ & - & - & - & 120 & 4.9 & .04 & 170 & 360 & 30 & 230 \\
\hline & $07-13-88$ & $<.010$ & $<.100$ & .010 & 120 & 5.3 & .10 & 170 & 350 & 23 & 240 \\
\hline & $01-12-89$ & .010 & $<.100$ & $<.010$ & 110 & 5.7 & .26 & 190 & 330 & 30 & 240 \\
\hline & $07-21-89$ & $<.010$ & $<.100$ & .020 & 120 & 5.1 & .15 & 170 & 340 & - & 230 \\
\hline & $01-10-90$ & $<.010$ & $<.100$ & .020 & 120 & 5.3 & .08 & - & 340 & 33 & 230 \\
\hline & $07-10-90$ & $<.010$ & $<.100$ & .010 & 120 & 7.9 & .11 & - & 360 & 30 & 220 \\
\hline \multirow[t]{2}{*}{ HBP } & $07-10-90$ & .040 & $<.100$ & .100 & 120 & 8.0 & $<.02$ & - & 360 & 22 & 200 \\
\hline & $04-15-92$ & $<.010$ & $<.050$ & .120 & 110 & 6.8 & .16 & - & 350 & 23 & 260 \\
\hline HC-5L & $07-08-92$ & $<.010$ & $<.050$ & .070 & 97 & 1.4 & .12 & - & 370 & 21 & 140 \\
\hline \multirow[t]{7}{*}{ HC-3 } & $07-28-87$ & - & $<.100$ & - & 97 & 3.8 & .15 & - & 380 & 23 & $<150$ \\
\hline & $07-11-88$ & .020 & $<.100$ & .040 & 96 & 3.8 & .17 & 260 & 390 & 17 & 150 \\
\hline & $07-19-89$ & $<.010$ & $<.100$ & .050 & 96 & 3.2 & .27 & 280 & 380 & 26 & 140 \\
\hline & $07-18-90$ & .010 & $<.100$ & .060 & 94 & 3.2 & .21 & - & 380 & 24 & 140 \\
\hline & $10-17-90$ & - & - & - & 91 & - & - & - & - & - & - \\
\hline & $01-17-91$ & - & - & - & 89 & - & - & - & - & - & - \\
\hline & $04-16-92$ & $<.010$ & $<.050$ & .070 & 110 & 3.2 & .07 & - & 370 & 12 & 160 \\
\hline
\end{tabular}


Table 113. Chemical and isotopic analyses of water samples collected at selected surface-water sites, springs, and wells, Long Valley Caldera, Mono County, California, 1987-93-Continued

\begin{tabular}{|c|c|c|c|c|c|c|c|c|c|c|}
\hline $\begin{array}{l}\text { Station } \\
\text { identifier }\end{array}$ & Date & $\begin{array}{c}\text { Boron, } \\
\text { dis- } \\
\text { solved } \\
(\mu \mathrm{g} / \mathrm{L})\end{array}$ & $\begin{array}{l}\text { Arsenic, } \\
\text { dis- } \\
\text { solved } \\
(\mu \mathrm{g} / \mathrm{L})\end{array}$ & $\begin{array}{l}\text { Lithium, } \\
\text { dis- } \\
\text { solved } \\
(\mu \mathrm{g} / \mathrm{L})\end{array}$ & $\begin{array}{l}\text { Mercury, } \\
\text { dis- } \\
\text { solved } \\
(\mu \mathrm{g} / \mathrm{L})\end{array}$ & $\begin{array}{l}\text { Iron, } \\
\text { dis- } \\
\text { solved } \\
(\mu \mathrm{g} / \mathrm{L})\end{array}$ & $\begin{array}{l}\text { Manga- } \\
\text { nese, } \\
\text { dis- } \\
\text { solved } \\
(\mu \mathrm{g} / \mathrm{L})\end{array}$ & $\begin{array}{c}\text { Solids, } \\
\text { residue } \\
\text { at } 180^{\circ} \mathrm{C} \\
\text { dis- } \\
\text { solved } \\
(\mathrm{mg} / \mathrm{L})\end{array}$ & $\begin{array}{c}\mathrm{H}-2 / \mathrm{H}-1 \\
\text { stable } \\
\text { isotope } \\
\text { ratio } \\
\text { per } \\
\text { mil }\end{array}$ & $\begin{array}{c}\text { O-18/O-16 } \\
\text { stable } \\
\text { isotope } \\
\text { ratio } \\
\text { per } \\
\text { mil }\end{array}$ \\
\hline \multirow[t]{2}{*}{$\mathrm{FH} 23$} & $07-13-93$ & 130 & 20 & 40 & - & $<3$ & $<1$ & 164 & -118.0 & -15.99 \\
\hline & $10-13-93$ & 110 & 22 & 40 & $<0.1$ & 4 & $<1$ & 166 & - & - \\
\hline \multirow[t]{9}{*}{ MBP-3 } & $07-30-87$ & 9,900 & 190 & 2,400 & 5.3 & 120 & 23 & 1,200 & -118.0 & -15.00 \\
\hline & $12-17-87$ & 9,600 & 1,800 & 2,500 & 2.8 & 38 & 22 & 1,210 & -118.5 & -14.90 \\
\hline & $04-19-88$ & 9,400 & 310 & 2,600 & 2.4 & 11 & 2 & 1,210 & - & - \\
\hline & 04-19-88 & 9,900 & 1,200 & 2,600 & - & 17 & 2 & 1,190 & - & - \\
\hline & $07-13-88$ & 9,600 & 1,700 & 2,400 & - & 290 & 6 & 1,220 & -118.5 & -14.80 \\
\hline & $01-12-89$ & 9,500 & 420 & 2,600 & 2.4 & 9 & 9 & 1,200 & - & - \\
\hline & $07-21-89$ & 9,600 & 1,000 & 2,700 & 1.0 & 22 & 20 & 1,240 & - & - \\
\hline & $01-10-90$ & 10,000 & 260 & 2,500 & 1.2 & 18 & 25 & 1,230 & -117.5 & -14.95 \\
\hline & $07-10-90$ & 10,000 & 340 & 2,600 & 5.2 & 28 & 27 & 1,230 & -116.0 & -14.70 \\
\hline \multirow[t]{9}{*}{ MBP-1 } & $12-17-87$ & 10,000 & 440 & 2,500 & 8.1 & 22 & 18 & 1,240 & -117.0 & -14.70 \\
\hline & $12-17-87$ & 11,000 & 1,900 & 2,500 & - & 13 & 17 & 1,220 & - & - \\
\hline & $04-19-88$ & 10,000 & 2,200 & 260 & 11 & 74 & 16 & 1,230 & - & - \\
\hline & $04-19-88$ & 11,000 & 1,600 & 2,600 & - & 15 & 19 & 1,220 & - & - \\
\hline & $07-13-88$ & 9,900 & 1,800 & 2,400 & - & 12 & 6 & 1,250 & -116.5 & -14.65 \\
\hline & $01-12-89$ & 9,900 & 1,200 & 2,700 & 4.5 & 13 & 18 & 1,200 & - & - \\
\hline & $07-21-89$ & 11,000 & 1,700 & 2,600 & 1.0 & 8 & 13 & 1,200 & -117.0 & -14.70 \\
\hline & $01-10-90$ & 10,000 & 1,800 & 2,400 & 6.8 & 31 & 18 & 1,210 & -119.0 & -14.80 \\
\hline & $07-10-90$ & 10,000 & 510 & 2,400 & 2.1 & 21 & 25 & 1,250 & -117.0 & -14.65 \\
\hline \multirow[t]{2}{*}{ HBP } & $07-10-90$ & 12,000 & 1,500 & 2,700 & .3 & 5 & 41 & 1,240 & -113.0 & -13.10 \\
\hline & $04-15-92$ & 11,000 & 1,500 & 4,300 & .2 & 5 & 22 & 1,230 & -114.0 & -13.85 \\
\hline HC-5L & $07-08-92$ & 10,000 & 1,100 & 2,300 & $<.1$ & 20 & 4 & 1,110 & -120.0 & -14.95 \\
\hline \multirow[t]{7}{*}{$\mathrm{HC}-3$} & $07-28-87$ & & 1,100 & 2,300 & .6 & $<3$ & $<1$ & 1,180 & -120.5 & -15.10 \\
\hline & $07-11-88$ & 9,700 & 1,300 & 2,400 & 1.0 & $<3$ & $<1$ & 1,200 & -119.5 & -15.00 \\
\hline & $07-19-89$ & 10,000 & 1,100 & 2,500 & $<.1$ & 4 & 2 & 1,190 & - & - \\
\hline & $07-18-90$ & 9,900 & 1,000 & 2,300 & .2 & $<3$ & 2 & 1,200 & -119.0 & -15.05 \\
\hline & $10-17-90$ & 10,000 & - & - & - & - & - & - & - & - \\
\hline & $01-17-91$ & 9,900 & - & - & - & - & - & - & -119.0 & -14.90 \\
\hline & $04-16-92$ & 10,000 & 1,100 & 4,000 & .2 & 7 & 4 & 1,200 & -120.0 & -14.65 \\
\hline
\end{tabular}


Table 113. Chemical and isotopic analyses of water samples collected at selected surface-water sites, springs, and wells, Long Valley Caldera, Mono County, California, 1987-93-Continued

\begin{tabular}{|c|c|c|c|c|c|c|c|c|c|}
\hline $\begin{array}{l}\text { Station } \\
\text { identifier }\end{array}$ & Date & $\begin{array}{c}\text { Temper- } \\
\text { ature, } \\
\text { water } \\
\left({ }^{\circ} \mathrm{C}\right)\end{array}$ & $\begin{array}{c}\text { Specific } \\
\text { con- } \\
\text { duct- } \\
\text { ance } \\
(\mu \mathrm{S} / \mathrm{cm})\end{array}$ & $\begin{array}{c}\text { pH, } \\
\text { water } \\
\text { whole } \\
\text { field } \\
\text { (standard } \\
\text { units) }\end{array}$ & $\begin{array}{c}\text { Alka- } \\
\text { linity, } \\
\text { field, } \\
\text { as } \mathrm{CaCO} \\
(\mathrm{mg} / \mathrm{L})\end{array}$ & $\begin{array}{c}\text { Alka- } \\
\text { linity } \\
\text { lab, } \\
\text { as } \mathrm{CaCO}_{3} \\
(\mathrm{mg} / \mathrm{L})\end{array}$ & $\begin{array}{l}\text { Chlo- } \\
\text { ride, } \\
\text { dis- } \\
\text { solved } \\
(\mathrm{mg} / \mathrm{L})\end{array}$ & $\begin{array}{l}\text { Fluo- } \\
\text { ride, } \\
\text { dis- } \\
\text { solved } \\
(\mathrm{mg} / \mathrm{L})\end{array}$ & $\begin{array}{c}\text { Nitro- } \\
\text { gen, } \\
\text { ammonia, } \\
\text { dis- } \\
\text { solved } \\
(\mathrm{mg} / \mathrm{L})\end{array}$ \\
\hline BAL & $\begin{array}{l}08-29-87 \\
07-14-88 \\
01-12-89 \\
07-17-89 \\
01-10-90\end{array}$ & $\begin{array}{l}58.5 \\
57.5 \\
57.5 \\
57.5 \\
57.0\end{array}$ & $\frac{-}{-}$ & $\begin{array}{l}6.8 \\
7.0 \\
7.0 \\
6.7 \\
7.0\end{array}$ & $\begin{array}{l}- \\
719 \\
693 \\
645\end{array}$ & $\begin{array}{l}594 \\
642 \\
645 \\
648 \\
638\end{array}$ & $\begin{array}{l}150 \\
150 \\
150 \\
160 \\
150\end{array}$ & $\begin{array}{l}3.2 \\
4.8 \\
3.7 \\
4.9 \\
4.4\end{array}$ & $\begin{array}{r}0.120 \\
.130 \\
.100 \\
.100 \\
.110\end{array}$ \\
\hline LHC-SP & $\begin{array}{l}08-29-87 \\
07-14-88 \\
01-13-89 \\
07-17-89 \\
01-09-90\end{array}$ & $\begin{array}{l}83.0 \\
83.0 \\
82.5 \\
82.5 \\
82.5\end{array}$ & $\begin{array}{r}\overline{-} \\
\bar{z} \\
2,100\end{array}$ & $\begin{array}{l}6.6 \\
7.0 \\
6.8 \\
6.7 \\
7.1\end{array}$ & $\begin{array}{l}5 \overline{590} \\
630 \\
631 \\
574\end{array}$ & $\begin{array}{l}548 \\
599 \\
592 \\
603 \\
594\end{array}$ & $\begin{array}{l}200 \\
200 \\
200 \\
200 \\
200\end{array}$ & $\begin{array}{l}7.2 \\
7.8 \\
7.2 \\
7.9 \\
8.5\end{array}$ & $\begin{array}{l}.140 \\
.150 \\
.140 \\
.110 \\
.150\end{array}$ \\
\hline & $\begin{array}{l}07-18-90 \\
01-18-91 \\
07-24-91 \\
01-15-92 \\
04-16-92\end{array}$ & $\begin{array}{l}83.0 \\
82.5 \\
83.0 \\
82.5 \\
82.5\end{array}$ & $\begin{array}{r}-\overline{1} \\
1,990 \\
2,030 \\
1,920 \\
1,980\end{array}$ & $\begin{array}{l}7.0 \\
6.9 \\
7.6 \\
8.0 \\
7.6\end{array}$ & $\begin{array}{l}595 \\
566 \\
602 \\
601 \\
598\end{array}$ & $\begin{array}{l}595 \\
553 \\
567 \\
606 \\
597\end{array}$ & $\begin{array}{l}200 \\
200 \\
200 \\
210 \\
200\end{array}$ & $\begin{array}{l}8.4 \\
8.5 \\
8.1 \\
8.1 \\
9.4\end{array}$ & $\begin{array}{l}.110 \\
.130 \\
.150 \\
.050 \\
.130\end{array}$ \\
\hline & $\begin{array}{l}07-08-92 \\
01-26-93 \\
07-15-93\end{array}$ & $\begin{array}{l}82.5 \\
83.0 \\
82.5\end{array}$ & $\begin{array}{l}1,920 \\
1,900 \\
1,910\end{array}$ & $\begin{array}{l}7.7 \\
7.1 \\
7.8\end{array}$ & $\begin{array}{r}600 \\
585 \\
-\end{array}$ & $\begin{array}{l}570 \\
599 \\
602\end{array}$ & $\begin{array}{l}200 \\
200 \\
190\end{array}$ & $\begin{array}{l}5.8 \\
8.7 \\
8.7\end{array}$ & $\begin{array}{l}.110 \\
.140 \\
.120\end{array}$ \\
\hline BS & $\begin{array}{l}08-29-87 \\
07-14-88 \\
07-19-90\end{array}$ & $\begin{array}{l}11.0 \\
10.5 \\
11.0\end{array}$ & $\begin{array}{l}189 \\
192 \\
191\end{array}$ & $\begin{array}{l}7.2 \\
7.3 \\
7.3\end{array}$ & $\begin{array}{l}\overline{82} \\
84\end{array}$ & $\begin{array}{l}83 \\
82 \\
85\end{array}$ & $\begin{array}{l}5.4 \\
6.5 \\
9.1\end{array}$ & $\begin{array}{l}.50 \\
.40 \\
.30\end{array}$ & $\begin{array}{r}.010 \\
<.010 \\
.030\end{array}$ \\
\hline
\end{tabular}


Table 113. Chemical and isotopic analyses of water samples collected at selected surface-water sites, springs, and wells, Long Valley Caldera, Mono County, California, 1987-93-Continued

\begin{tabular}{|c|c|c|c|c|c|c|c|c|c|c|c|}
\hline $\begin{array}{l}\text { Station } \\
\text { identifier }\end{array}$ & Date & $\begin{array}{l}\text { Nitro- } \\
\text { gen, } \\
\text { nitrite } \\
\text { dis- } \\
\text { solved } \\
(\mathrm{mg} / \mathrm{L})\end{array}$ & $\begin{array}{c}\text { Nitro- } \\
\text { gen, } \\
\mathrm{NO}_{2}+\mathrm{NO}_{3} \\
\text { dis- } \\
\text { solved } \\
(\mathrm{mg} / \mathrm{L})\end{array}$ & $\begin{array}{l}\text { Phos- } \\
\text { phorus } \\
\text { ortho, } \\
\text { dis- } \\
\text { solved } \\
(\mathrm{mg} / \mathrm{L})\end{array}$ & $\begin{array}{c}\text { Sulfate } \\
\text { dis- } \\
\text { solved } \\
(\mathrm{mg} / \mathrm{L})\end{array}$ & $\begin{array}{l}\text { Calcium } \\
\text { dis- } \\
\text { solved } \\
(\mathrm{mg} / \mathrm{L})\end{array}$ & $\begin{array}{l}\text { Magna- } \\
\text { sium, } \\
\text { dis- } \\
\text { solved } \\
(\mathrm{mg} / \mathrm{L})\end{array}$ & $\begin{array}{l}\text { Stron- } \\
\text { tium, } \\
\text { dis- } \\
\text { solved } \\
(\mu \mathrm{g} / \mathrm{L})\end{array}$ & $\begin{array}{l}\text { Sodium, } \\
\text { dis- } \\
\text { solved } \\
(\mathrm{mg} / \mathrm{L})\end{array}$ & $\begin{array}{l}\text { Potas- } \\
\text { sium, } \\
\text { dis- } \\
\text { solved } \\
(\mathrm{mg} / \mathrm{L})\end{array}$ & $\begin{array}{c}\text { Silica, } \\
\text { dis- } \\
\text { solved } \\
(\mathrm{mg} / \mathrm{L})\end{array}$ \\
\hline \multirow[t]{5}{*}{ BAL } & $08-29-87$ & $<0.010$ & $<0.100$ & 0.040 & 63 & 24 & 0.44 & - & 360 & 33 & 190 \\
\hline & $07-14-88$ & $<.010$ & $<.100$ & .050 & 61 & 25 & .62 & 180 & 360 & 31 & 200 \\
\hline & $01-12-89$ & $<.010$ & $<.100$ & .060 & 64 & 25 & .54 & 180 & 350 & 31 & 190 \\
\hline & $07-17-89$ & .010 & $<.100$ & .060 & 64 & 25 & .61 & 180 & 360 & 35 & 190 \\
\hline & $01-10-90$ & $<.010$ & $<.100$ & .050 & 64 & 24 & .66 & - & 370 & 38 & 190 \\
\hline \multirow[t]{13}{*}{ LHC-SP } & $08-29-87$ & $<.010$ & $<.100$ & .010 & 99 & 22 & .42 & - & 390 & 30 & 87 \\
\hline & $07-14-88$ & $<.010$ & $<.100$ & .040 & 95 & 23 & .58 & 580 & 370 & 24 & 90 \\
\hline & $01-13-89$ & $<.010$ & $<.100$ & .020 & 98 & 22 & .55 & 560 & 350 & 24 & 81 \\
\hline & $07-17-89$ & $<.010$ & $<.100$ & .030 & 100 & 23 & .62 & 540 & 390 & 22 & 88 \\
\hline & $01-09-90$ & $<.010$ & $<.100$ & .040 & 100 & 23 & .65 & - & 400 & 30 & 87 \\
\hline & $07-18-90$ & $<.010$ & $<.100$ & .040 & 98 & 23 & .69 & - & 390 & 28 & 87 \\
\hline & $01-18-91$ & $<.010$ & $<.100$ & .020 & 99 & 23 & .63 & - & 390 & 27 & 82 \\
\hline & $07-24-91$ & $<.010$ & $<.050$ & .150 & 100 & 21 & .63 & - & 370 & 25 & 82 \\
\hline & $01-15-92$ & $<.010$ & $<.050$ & .060 & 99 & 22 & .42 & - & 390 & 25 & 89 \\
\hline & $04-16-92$ & $<.010$ & $<.050$ & .060 & 100 & 22 & .61 & - & 380 & 28 & 82 \\
\hline & $07-08-92$ & $<.010$ & $<.050$ & .040 & 100 & 23 & .62 & - & 400 & 27 & 87 \\
\hline & $01-26-93$ & .020 & $<.050$ & .040 & 100 & 23 & .63 & - & 400 & 22 & 90 \\
\hline & $07-15-93$ & $<.010$ & $<.050$ & .030 & 100 & 23 & .62 & - & 390 & 24 & 86 \\
\hline \multirow[t]{3}{*}{ BS } & $08-29-87$ & - & $<.100$ & - & 7.3 & 5.1 & 6.4 & - & 24 & 3.8 & 54 \\
\hline & $07-14-88$ & $<.010$ & .130 & .340 & 7.0 & 5.4 & 6.6 & - & 24 & 4.0 & 55 \\
\hline & $07-19-90$ & $<.010$ & .100 & .340 & 6.3 & 6.0 & 6.7 & - & 26 & 4.4 & 54 \\
\hline
\end{tabular}


Table 113. Chemical and isotopic analyses of water samples collected at selected surface-water sites, springs, and wells, Long Valley Caldera, Mono County, California, 1987-93-Continued

\begin{tabular}{|c|c|c|c|c|c|c|c|c|c|c|}
\hline $\begin{array}{l}\text { Station } \\
\text { identifier }\end{array}$ & Date & $\begin{array}{c}\text { Boron, } \\
\text { dis- } \\
\text { solved } \\
(\mu \mathrm{g} / \mathrm{L})\end{array}$ & $\begin{array}{l}\text { Arsenic, } \\
\text { dis- } \\
\text { solved } \\
(\mu \mathrm{g} / \mathrm{L})\end{array}$ & $\begin{array}{l}\text { Lithium, } \\
\text { dis- } \\
\text { solved } \\
(\mu \mathrm{g} / \mathrm{L})\end{array}$ & $\begin{array}{c}\text { Mercury, } \\
\text { dis- } \\
\text { solved } \\
(\mu \mathrm{g} / \mathrm{L})\end{array}$ & $\begin{array}{l}\text { Iron, } \\
\text { dis- } \\
\text { solved } \\
(\mu \mathrm{g} / \mathrm{L})\end{array}$ & $\begin{array}{l}\text { Manga- } \\
\text { nese, } \\
\text { dis- } \\
\text { solved } \\
(\mu \mathrm{g} / L)\end{array}$ & $\begin{array}{c}\text { Solids, } \\
\text { residue } \\
\text { at } 180^{\circ} \mathrm{C} \\
\text { dis- } \\
\text { solved } \\
(\mathrm{mg} / \mathrm{L})\end{array}$ & $\begin{array}{c}\mathrm{H}-2 / \mathrm{H}-1 \\
\text { stable } \\
\text { isotope } \\
\text { ratio } \\
\text { per } \\
\text { mil }\end{array}$ & $\begin{array}{c}\text { O-18/O-16 } \\
\text { stable } \\
\text { isotope } \\
\text { ratio } \\
\text { per } \\
\text { mil }\end{array}$ \\
\hline BAL & $\begin{array}{l}08-29-87 \\
07-14-88 \\
01-12-89 \\
07-17-89 \\
01-10-90\end{array}$ & $\begin{array}{l}6,300 \\
6,100 \\
6,000 \\
6,600 \\
6,400\end{array}$ & $\begin{array}{r}86 \\
380 \\
470 \\
200 \\
150\end{array}$ & $\begin{array}{l}1,600 \\
1,500 \\
1,600 \\
1,700 \\
1,500\end{array}$ & $\begin{array}{r}0.3 \\
.3 \\
<.1 \\
<.1 \\
.1\end{array}$ & $\begin{array}{l}170 \\
180 \\
160 \\
180 \\
190\end{array}$ & $\begin{array}{l}150 \\
160 \\
150 \\
150 \\
160\end{array}$ & $\begin{array}{l}1,260 \\
1,260 \\
1,230 \\
1,270 \\
1,260\end{array}$ & $\begin{array}{c}-125.0 \\
- \\
-1 \overline{27} .5\end{array}$ & $\begin{array}{l}-16.35 \\
- \\
-16.25\end{array}$ \\
\hline LHC-SP & $\begin{array}{l}08-29-87 \\
07-14-88 \\
01-13-89 \\
07-17-89 \\
01-09-90\end{array}$ & $\begin{array}{l}8,900 \\
8,500 \\
8,500 \\
8,800 \\
9,000\end{array}$ & $\begin{array}{l}650 \\
590 \\
850 \\
300 \\
700\end{array}$ & $\begin{array}{l}2,800 \\
2,600 \\
2,700 \\
2,900 \\
2,700\end{array}$ & $\begin{array}{r}.2 \\
.4 \\
.5 \\
<.1 \\
.1\end{array}$ & $\begin{array}{l}44 \\
31 \\
36 \\
36 \\
37\end{array}$ & $\begin{array}{l}210 \\
210 \\
200 \\
200 \\
210\end{array}$ & $\begin{array}{l}1,240 \\
1,250 \\
1,230 \\
1,260 \\
1,240\end{array}$ & $\begin{array}{c}-124.0 \\
\overline{-} \\
\overline{-} \\
-123.5\end{array}$ & $\begin{array}{l}-15.75 \\
\overline{-} \\
-\overline{15.75}\end{array}$ \\
\hline & $\begin{array}{l}07-18-90 \\
01-18-91 \\
07-24-91 \\
01-15-92 \\
04-16-92\end{array}$ & $\begin{array}{l}8,800 \\
8,700 \\
9,200 \\
8,900 \\
9,100\end{array}$ & $\begin{array}{l}610 \\
700 \\
710 \\
710 \\
710\end{array}$ & $\begin{array}{l}2,800 \\
4,200 \\
3,800 \\
3,600 \\
4,500\end{array}$ & $\begin{array}{r}.1 \\
.7 \\
.2 \\
.1 \\
<.1\end{array}$ & $\begin{array}{l}15 \\
32 \\
33 \\
36 \\
29\end{array}$ & $\begin{array}{l}210 \\
200 \\
190 \\
200 \\
200\end{array}$ & $\begin{array}{l}1,250 \\
1,230 \\
1,220 \\
1,150 \\
1,220\end{array}$ & $\begin{array}{c}-122.0 \\
-122.0 \\
-120.0 \\
-123.0 \\
-\end{array}$ & $\begin{array}{l}-15.75 \\
-15.65 \\
-15.65 \\
-15.60 \\
-\end{array}$ \\
\hline & $\begin{array}{l}07-08-92 \\
01-26-93 \\
07-15-93\end{array}$ & $\begin{array}{l}9,100 \\
9,200 \\
9,700\end{array}$ & $\begin{array}{l}690 \\
780 \\
650\end{array}$ & $\begin{array}{l}2,800 \\
2,700 \\
2,900\end{array}$ & $\begin{array}{r}<.1 \\
.2 \\
<.1\end{array}$ & $\begin{array}{l}50 \\
36 \\
35\end{array}$ & $\begin{array}{l}200 \\
210 \\
200\end{array}$ & $\begin{array}{l}1,200 \\
1,220 \\
1,230\end{array}$ & $\begin{array}{c}-123.0 \\
-124.0 \\
-\end{array}$ & $\begin{array}{l}-15.65 \\
-15.71 \\
-\end{array}$ \\
\hline BS & $\begin{array}{l}08-29-87 \\
07-14-88 \\
07-19-90\end{array}$ & $\begin{array}{l}260 \\
300 \\
380\end{array}$ & $\begin{array}{l}18 \\
19 \\
18\end{array}$ & $\begin{array}{l}40 \\
40 \\
30\end{array}$ & $\begin{array}{l}<.1 \\
- \\
<.1\end{array}$ & $\begin{array}{l}<3 \\
<3 \\
<3\end{array}$ & $\begin{array}{l}<1 \\
<1 \\
2\end{array}$ & $\begin{array}{l}139 \\
153 \\
153\end{array}$ & $\begin{array}{c}-114.5 \\
-115.0\end{array}$ & $\begin{array}{l}-15.85 \\
-15.75\end{array}$ \\
\hline
\end{tabular}


Table 114. Partial chemical analyses of water samples collected at selected surface-water sites and springs, Long Valley Caldera, Mono County, California, 1987-93

$\left[{ }^{\circ} \mathrm{C}\right.$, degrees Celsius; $\mu \mathrm{S} / \mathrm{cm}$, microsiemens per centimeter at $25^{\circ} \mathrm{C} ; \mathrm{mg} / \mathrm{L}$, milligrams per liter; $\mu \mathrm{g} / \mathrm{L}$, micrograms per liter; - , no data]

\begin{tabular}{|c|c|c|c|c|c|c|}
\hline $\begin{array}{l}\text { Station } \\
\text { identifier }\end{array}$ & Date & $\begin{array}{c}\text { Temperature } \\
\text { water } \\
\left({ }^{\circ} \mathrm{C}\right)\end{array}$ & $\begin{array}{l}\text { Specific } \\
\text { conductance } \\
(\mu \mathrm{S} / \mathrm{cm})\end{array}$ & $\begin{array}{c}\text { Specific } \\
\text { conductance } \\
\text { lab } \\
(\mu \mathrm{S} / \mathrm{cm})\end{array}$ & $\begin{array}{l}\text { Chloride, } \\
\text { dissolved } \\
(\mathrm{mg} / \mathrm{L})\end{array}$ & $\begin{array}{c}\text { Boron, } \\
\text { dissolved } \\
(\mu \mathrm{g} / \mathrm{L})\end{array}$ \\
\hline \multirow{40}{*}{$\mathrm{HCA}$} & $01-16-87$ & - & - & 219 & 5.9 & 250 \\
\hline & $01-16-87$ & - & - & 222 & 5.7 & 250 \\
\hline & $02-17-87$ & - & - & 230 & 7.7 & 280 \\
\hline & $03-20-87$ & - & - & 218 & 5.5 & 250 \\
\hline & $04-20-87$ & - & 240 & - & - & - \\
\hline & $05-22-87$ & - & - & 199 & 6.2 & 240 \\
\hline & $06-22-87$ & - & - & 210 & 6.0 & 240 \\
\hline & $07-17-87$ & - & - & 218 & 6.3 & 260 \\
\hline & $08-20-87$ & - & - & - & 6.0 & 270 \\
\hline & $08-27-87$ & 16.0 & 222 & 223 & 7.0 & 250 \\
\hline & $09-21-87$ & - & - & 221 & 6.5 & 250 \\
\hline & $10-17-87$ & - & 223 & 226 & 5.9 & 260 \\
\hline & $10-20-87$ & - & - & 225 & 6.4 & 260 \\
\hline & $11-30-87$ & - & - & 232 & - & 280 \\
\hline & $12-15-87$ & 10.0 & 237 & 238 & - & 260 \\
\hline & $01-08-88$ & - & - & 225 & 7.4 & 260 \\
\hline & $02-26-88$ & - & - & 241 & 8.3 & 300 \\
\hline & $03-15-88$ & - & - & 235 & 7.0 & 270 \\
\hline & $04-05-88$ & - & - & 229 & 7.5 & 280 \\
\hline & $04-20-88$ & 14.5 & 226 & 229 & 8.0 & 270 \\
\hline & $05-10-88$ & - & - & 226 & 7.6 & 270 \\
\hline & $05-10-88$ & - & - & 227 & 7.2 & 270 \\
\hline & $06-10-88$ & - & - & 202 & 6.8 & 270 \\
\hline & $06-20-88$ & 20.0 & 201 & 211 & 7.6 & 270 \\
\hline & $07-07-88$ & - & - & 205 & 6.2 & 240 \\
\hline & $07-12-88$ & 14.0 & 201 & 198 & 6.1 & 230 \\
\hline & $08-03-88$ & - & - & 227 & 7.4 & 270 \\
\hline & $08-10-88$ & 15.0 & 218 & 225 & 7.1 & 270 \\
\hline & $09-06-88$ & - & - & 221 & 6.8 & 260 \\
\hline & $09-13-88$ & 12.0 & 214 & 224 & 7.0 & 280 \\
\hline & $09-13-88$ & 12.0 & 214 & 227 & 6.7 & 260 \\
\hline & $10-19-88$ & 16.5 & 222 & 228 & 7.2 & 290 \\
\hline & $11-21-88$ & 11.0 & 221 & 231 & 7.0 & 250 \\
\hline & $12-14-88$ & 8.0 & 224 & 234 & 7.0 & 280 \\
\hline & $01-09-89$ & 11.5 & 226 & 239 & 8.7 & 300 \\
\hline & $02-15-89$ & 13.0 & 217 & 230 & 7.6 & 280 \\
\hline & $03-21-89$ & 15.5 & 217 & 230 & 7.0 & 280 \\
\hline & $04-12-89$ & 15.0 & 211 & 219 & 6.5 & 270 \\
\hline & $05-16-89$ & 19.0 & 183 & 195 & 6.1 & 260 \\
\hline & $06-27-89$ & 12.5 & 209 & 220 & 7.4 & 310 \\
\hline
\end{tabular}


Table 114. Partial chemical analyses of water samples collected at selected surface-water sites and springs, Long Valley Caldera, Mono County, California, 1987-93-Continued

\begin{tabular}{|c|c|c|c|c|c|c|}
\hline $\begin{array}{c}\text { Station } \\
\text { identifier }\end{array}$ & Date & $\begin{array}{c}\text { Temperature } \\
\text { water } \\
\left({ }^{\circ} \mathrm{C}\right)\end{array}$ & $\begin{array}{c}\text { Specific } \\
\text { conductance } \\
(\mu \mathrm{S} / \mathrm{cm})\end{array}$ & $\begin{array}{c}\text { Specific } \\
\text { conductance } \\
\text { lab } \\
(\mu \mathrm{S} / \mathrm{cm})\end{array}$ & $\begin{array}{l}\text { Chloride, } \\
\text { dissolved } \\
\text { (mg/L) }\end{array}$ & $\begin{array}{l}\text { Boron, } \\
\text { dissolved } \\
(\mu \mathrm{g} / \mathrm{L})\end{array}$ \\
\hline \multirow[t]{45}{*}{$\mathrm{HCA}$} & $07-19-89$ & 15.5 & 216 & 225 & 7.4 & 300 \\
\hline & $08-15-89$ & 14.0 & 228 & 234 & 7.6 & 290 \\
\hline & $09-19-89$ & 10.5 & 218 & 223 & 6.6 & 260 \\
\hline & $10-17-89$ & 10.0 & 236 & 239 & 7.1 & 290 \\
\hline & $11-21-89$ & 7.5 & 226 & 236 & 6.9 & 280 \\
\hline & $12-20-89$ & 7.5 & 230 & 238 & 7.2 & 320 \\
\hline & $01-10-90$ & 8.0 & 227 & 242 & 6.7 & 300 \\
\hline & $02-21-90$ & 8.5 & 229 & 239 & 6.8 & 300 \\
\hline & $03-20-90$ & 10.5 & 229 & 241 & 7.7 & 330 \\
\hline & $04-24-90$ & 17.0 & 230 & 231 & 7.6 & 300 \\
\hline & $05-25-90$ & 10.0 & 223 & 213 & 9.7 & 300 \\
\hline & $06-11-90$ & 17.5 & 177 & 185 & 7.6 & 250 \\
\hline & $07-18-90$ & - & 219 & 234 & 9.5 & 330 \\
\hline & $08-28-90$ & 12.5 & 223 & 226 & 7.1 & 290 \\
\hline & $09-26-90$ & 10.5 & 231 & 228 & 7.8 & 310 \\
\hline & $10-17-90$ & 12.0 & 219 & 238 & 9.2 & 330 \\
\hline & $11-15-90$ & 11.0 & 240 & 238 & 9.7 & 320 \\
\hline & $12-19-90$ & 4.0 & 246 & 245 & 9.9 & 340 \\
\hline & $01-17-91$ & 14.0 & 236 & 248 & 9.5 & 350 \\
\hline & $02-15-91$ & 11.0 & 240 & 246 & 9.4 & 350 \\
\hline & $03-21-91$ & 16.5 & 241 & 254 & 8.0 & 380 \\
\hline & $04-17-91$ & 9.5 & 263 & 255 & 10 & 360 \\
\hline & $05-16-91$ & 20.0 & 232 & 232 & 9.0 & 330 \\
\hline & $06-14-91$ & 20.0 & 236 & 227 & - & - \\
\hline & $06-21-91$ & 11.5 & 166 & 168 & 5.9 & 240 \\
\hline & $07-23-91$ & 22.0 & 235 & 237 & 9.5 & 350 \\
\hline & $08-22-91$ & 17.5 & 216 & 222 & 8.4 & 310 \\
\hline & $09-18-91$ & 14.0 & 223 & 224 & 6.6 & 290 \\
\hline & $10-23-91$ & 13.5 & 226 & 229 & 8.0 & 310 \\
\hline & $11-21-91$ & 9.5 & 213 & 221 & 6.4 & 260 \\
\hline & $11-21-91$ & 9.5 & 213 & - & 7.0 & 250 \\
\hline & $12-12-91$ & 8.0 & 227 & 238 & 8.9 & 320 \\
\hline & $01-14-92$ & 12.5 & 228 & 236 & 8.8 & 290 \\
\hline & $02-20-92$ & 13.5 & 253 & 247 & 10 & 310 \\
\hline & $03-12-92$ & 15.0 & 263 & 258 & 11 & 360 \\
\hline & $04-16-92$ & 11.0 & 240 & 247 & 10 & 340 \\
\hline & $05-13-92$ & 19.0 & 172 & 170 & 6.7 & 190 \\
\hline & $06-09-92$ & 20.0 & 201 & 201 & 7.6 & 270 \\
\hline & $07-08-92$ & 14.5 & 219 & - & 6.9 & 290 \\
\hline & $08-04-92$ & 21.0 & 217 & 221 & 7.8 & 270 \\
\hline & $09-15-92$ & 18.0 & 228 & 231 & 8.2 & 280 \\
\hline & $10-21-92$ & 10.5 & 232 & 242 & 7.7 & 290 \\
\hline & $11-17-92$ & 12.0 & 229 & 233 & 7.3 & 290 \\
\hline & $12-23-92$ & - & 241 & 249 & 8.4 & 340 \\
\hline & $01-27-93$ & - & 253 & 257 & 9.2 & 360 \\
\hline
\end{tabular}


Table 114. Partial chemical analyses of water samples collected at selected surface-water sites and springs, Long Valley Caldera, Mono County, California, 1987-93-Continued

\begin{tabular}{|c|c|c|c|c|c|c|}
\hline $\begin{array}{l}\text { Station } \\
\text { identifier }\end{array}$ & Date & $\begin{array}{c}\text { Temperature } \\
\text { water } \\
\left({ }^{\circ} \mathrm{C}\right)\end{array}$ & $\begin{array}{c}\text { Specific } \\
\text { conductance } \\
(\mu \mathrm{S} / \mathrm{cm})\end{array}$ & $\begin{array}{c}\text { Specific } \\
\text { conductance } \\
\text { lab } \\
(\mu \mathrm{S} / \mathrm{cm})\end{array}$ & $\begin{array}{l}\text { Chloride, } \\
\text { dissolved } \\
\text { (mg/L) }\end{array}$ & $\begin{array}{c}\text { Boron, } \\
\text { dissolved } \\
(\mu \mathrm{g} / \mathrm{L})\end{array}$ \\
\hline \multirow[t]{11}{*}{$\mathrm{HCA}$} & $03-02-93$ & 12.5 & 257 & 258 & 9.4 & 350 \\
\hline & $03-24-93$ & 12.0 & 307 & 311 & 13 & - \\
\hline & $04-13-93$ & 15.5 & 251 & 244 & 8.9 & 280 \\
\hline & $05-20-93$ & 12.0 & 136 & 140 & 4.1 & 140 \\
\hline & $06-15-93$ & 20.5 & 122 & 122 & 3.1 & 120 \\
\hline & $07-14-93$ & 17.0 & 126 & 131 & 3.3 & 170 \\
\hline & $08-19-93$ & 19.5 & 208 & 210 & 8.7 & 210 \\
\hline & $09-08-93$ & 16.0 & 254 & 265 & 23 & 230 \\
\hline & $10-14-93$ & 13.0 & 205 & 215 & 5.9 & 220 \\
\hline & $11-18-93$ & 9.0 & 222 & 227 & 5.3 & 240 \\
\hline & $12-14-93$ & 9.0 & 221 & 229 & 5.9 & 240 \\
\hline \multirow[t]{30}{*}{$\mathrm{HCF}$} & $01-14-87$ & 25.0 & 510 & 543 & 47 & 2,100 \\
\hline & $01-14-87$ & 25.0 & 510 & 541 & 48 & 2,100 \\
\hline & $01-16-87$ & - & - & 533 & 45 & 2,000 \\
\hline & $01-16-87$ & - & - & 534 & 45 & 2,100 \\
\hline & $02-10-87$ & 26.0 & 637 & 526 & 46 & 2,300 \\
\hline & $02-10-87$ & 26.0 & 637 & 527 & 46 & 2,200 \\
\hline & $02-17-87$ & - & - & 545 & 49 & 2,300 \\
\hline & $03-20-87$ & - & - & 562 & 52 & 2,400 \\
\hline & $04-20-87$ & 35.0 & 565 & 570 & 51 & 2,300 \\
\hline & $04-20-87$ & 35.0 & 565 & 576 & 51 & 2,300 \\
\hline & $05-22-87$ & - & - & 422 & 32 & 1,500 \\
\hline & $05-27-87$ & 28.0 & 458 & 460 & 37 & 1,700 \\
\hline & $05-27-87$ & 28.0 & 458 & 463 & 39 & 1,700 \\
\hline & $06-18-87$ & 27.5 & 452 & 470 & 38 & 1,800 \\
\hline & $06-22-87$ & - & - & 498 & 42 & 1,900 \\
\hline & $07-17-87$ & - & - & 550 & 53 & 2,300 \\
\hline & $07-27-87$ & 36.0 & 579 & 560 & 51 & 2,300 \\
\hline & $08-20-87$ & - & - & - & 52 & 2,500 \\
\hline & $08-26-87$ & 36.0 & 595 & 580 & 55 & 2,400 \\
\hline & $08-26-87$ & 36.0 & 595 & 582 & 55 & 2,400 \\
\hline & $09-21-87$ & - & - & 578 & 58 & 2,400 \\
\hline & $10-17-87$ & - & 594 & 573 & 51 & 2,500 \\
\hline & $10-17-87$ & - & 594 & 541 & 51 & 2,400 \\
\hline & $10-20-87$ & - & - & 577 & 51 & 2,400 \\
\hline & $11-14-87$ & - & 578 & 570 & 50 & 2,300 \\
\hline & $11-14-87$ & 一 & 578 & 568 & 51 & 2,300 \\
\hline & $11-30-87$ & 一 & - & 592 & 51 & 2,400 \\
\hline & $12-15-87$ & 32.0 & 604 & 595 & 54 & 2,400 \\
\hline & $12-15-87$ & 32.0 & - & 594 & 54 & 2,400 \\
\hline & $01-08-88$ & - & - & 547 & 49 & 2,200 \\
\hline
\end{tabular}


Table 114. Partial chemical analyses of water samples collected at selected surface-water sites and springs, Long Valley Caldera, Mono County, California, 1987-93-Continued

\begin{tabular}{|c|c|c|c|c|c|c|}
\hline $\begin{array}{c}\text { Station } \\
\text { identifier }\end{array}$ & Date & $\begin{array}{c}\text { Temperature } \\
\text { water } \\
\left({ }^{\circ} \mathrm{C}\right)\end{array}$ & $\begin{array}{c}\text { Specific } \\
\text { conductance } \\
(\mu \mathrm{S} / \mathrm{cm})\end{array}$ & $\begin{array}{c}\text { Specific } \\
\text { conductance } \\
\text { lab } \\
(\mu \mathrm{S} / \mathrm{cm})\end{array}$ & $\begin{array}{c}\text { Chloride, } \\
\text { dissolved } \\
(\mathrm{mg} / \mathrm{L})\end{array}$ & $\begin{array}{c}\text { Boron, } \\
\text { dissolved } \\
(\mu \mathrm{g} / \mathrm{L})\end{array}$ \\
\hline \multirow[t]{45}{*}{$\mathrm{HCF}$} & $02-26-88$ & - & - & 557 & 48 & 2,200 \\
\hline & $03-03-88$ & 30.0 & 569 & 567 & 49 & 2,300 \\
\hline & $03-15-88$ & - & - & 579 & 49 & 2,300 \\
\hline & $04-05-88$ & - & - & 564 & 48 & 2,300 \\
\hline & $04-20-88$ & 29.0 & 561 & 568 & 50 & 2,200 \\
\hline & $04-20-88$ & 29.0 & 561 & 563 & 51 & 2,300 \\
\hline & $05-10-88$ & - & - & 566 & 48 & 2,300 \\
\hline & $05-10-88$ & - & - & 555 & 50 & 2,200 \\
\hline & $06-10-88$ & - & - & 459 & 38 & 1,800 \\
\hline & $06-21-88$ & 31.0 & 432 & 432 & 35 & 1,600 \\
\hline & $07-07-88$ & - & - & 477 & 39 & 1,700 \\
\hline & $08-03-88$ & - & - & 567 & 50 & 2,200 \\
\hline & $08-10-88$ & 35.5 & 576 & 569 & 52 & 2,200 \\
\hline & $09-06-88$ & - & - & 563 & 50 & 2,300 \\
\hline & $09-13-88$ & 35.5 & 602 & 571 & 53 & 2,500 \\
\hline & $09-13-88$ & 35.5 & 602 & 578 & 53 & 2,300 \\
\hline & $10-19-88$ & 36.0 & 620 & 597 & 56 & 2,600 \\
\hline & $11-21-88$ & 27.5 & 525 & 539 & 46 & 2,100 \\
\hline & $12-14-88$ & 29.5 & 587 & 609 & 56 & 2,500 \\
\hline & $01-09-89$ & 30.5 & 607 & 608 & 57 & 2,400 \\
\hline & $02-15-89$ & 32.0 & 603 & 596 & 55 & 2,400 \\
\hline & $03-21-89$ & 34.0 & 577 & 574 & 53 & 2,400 \\
\hline & $04-12-89$ & 32.5 & 584 & 557 & 48 & 2,300 \\
\hline & $04-12-89$ & 34.0 & 587 & 560 & 49 & 2,300 \\
\hline & $05-16-89$ & 32.0 & 434 & 461 & 38 & 1,900 \\
\hline & $06-27-89$ & 28.0 & 476 & 500 & 41 & 2,000 \\
\hline & $08-15-89$ & 36.5 & 596 & 598 & 55 & 2,400 \\
\hline & $09-19-89$ & 30.0 & 522 & 569 & 51 & 2,300 \\
\hline & $10-17-89$ & 34.0 & 619 & 619 & 55 & 2,700 \\
\hline & $11-21-89$ & 29.0 & 554 & 591 & 51 & 2,500 \\
\hline & $12-20-89$ & 29.0 & 613 & 627 & 59 & 2,700 \\
\hline & $01-10-90$ & 29.5 & 591 & 627 & 59 & 2,600 \\
\hline & $02-21-90$ & 30.5 & 575 & 608 & 56 & 2,500 \\
\hline & $03-20-90$ & 32.5 & 571 & 609 & 57 & 2,700 \\
\hline & $04-24-90$ & 34.5 & 618 & 609 & 62 & 2,700 \\
\hline & $05-25-90$ & 28.0 & 543 & 521 & 51 & 2,200 \\
\hline & $06-11-90$ & 30.5 & 417 & 431 & 39 & 1,700 \\
\hline & $08-28-90$ & 34.5 & 584 & 596 & 57 & 2,600 \\
\hline & $09-26-90$ & 29.5 & 612 & 587 & 62 & 2,500 \\
\hline & $10-17-90$ & 33.0 & 600 & 606 & 59 & 2,600 \\
\hline & $11-15-90$ & 31.5 & 612 & 602 & 57 & 2,600 \\
\hline & $12-19-90$ & 25.5 & 656 & 646 & 63 & 2,900 \\
\hline & $01-17-91$ & 34.0 & 603 & 655 & 65 & 2,800 \\
\hline & $02-15-91$ & 33.5 & 652 & 655 & 65 & 2,900 \\
\hline & $03-21-91$ & 33.5 & 599 & 634 & 59 & 2,800 \\
\hline
\end{tabular}


Table 114. Partial chemical analyses of water samples collected at selected surtace-water sites and springs, Long Valley Caldera, Mono County, California, 1987-93-Continued

\begin{tabular}{|c|c|c|c|c|c|c|}
\hline $\begin{array}{l}\text { Station } \\
\text { identifier }\end{array}$ & Date & $\begin{array}{c}\text { Temperature } \\
\text { water } \\
\left({ }^{\circ} \mathrm{C}\right)\end{array}$ & $\begin{array}{c}\text { Specific } \\
\text { conductance } \\
(\mu \mathrm{S} / \mathrm{cm})\end{array}$ & $\begin{array}{c}\text { Specific } \\
\text { conductance } \\
\text { lab } \\
(\mu \mathrm{S} / \mathrm{cm})\end{array}$ & $\begin{array}{l}\text { Chloride, } \\
\text { dissolved } \\
(\mathrm{mg} / \mathrm{L})\end{array}$ & $\begin{array}{c}\text { Boron, } \\
\text { dissolved } \\
(\mu \mathrm{g} / \mathrm{L})\end{array}$ \\
\hline \multirow[t]{33}{*}{$\mathrm{HCF}$} & $04-17-91$ & 32.0 & 654 & 641 & 60 & 2,800 \\
\hline & $05-16-91$ & 38.0 & 605 & 611 & 55 & 2,700 \\
\hline & $06-14-91$ & 27.0 & 299 & 293 & 26 & - \\
\hline & $06-21-91$ & 22.0 & 348 & 357 & 32 & 1,400 \\
\hline & $07-23-91$ & 37.0 & 569 & 564 & 55 & 2,400 \\
\hline & $08-22-91$ & 36.0 & 526 & 549 & 54 & 2,300 \\
\hline & $09-18-91$ & 34.0 & 569 & 574 & 53 & 2,500 \\
\hline & $10-23-91$ & 32.0 & 605 & 618 & 60 & 2,700 \\
\hline & $11-21-91$ & 26.0 & 559 & 570 & 51 & 2,400 \\
\hline & $12-12-91$ & 30.0 & 609 & 627 & 61 & 2,800 \\
\hline & 01-14-92 & 33.0 & 617 & 620 & 58 & 2,700 \\
\hline & $02-20-92$ & 33.0 & 633 & 616 & 59 & 2,600 \\
\hline & $03-12-92$ & 35.0 & 621 & 619 & 62 & 2,600 \\
\hline & $04-16-92$ & 34.0 & 657 & 643 & 71 & 2,900 \\
\hline & $05-13-92$ & 33.0 & 438 & 442 & 42 & 1,800 \\
\hline & $06-09-92$ & 35.0 & 520 & 527 & 52 & 2,300 \\
\hline & 08-04-92 & 39.0 & 609 & 609 & 64 & 2,600 \\
\hline & $09-15-92$ & 39.0 & 639 & 642 & 63 & 2,800 \\
\hline & $10-21-92$ & 33.0 & 646 & 640 & 64 & 2,800 \\
\hline & $11-17-92$ & 32.0 & 626 & 625 & 59 & 2,700 \\
\hline & $12-23-92$ & - & 666 & 673 & 62 & 3,100 \\
\hline & $01-27-93$ & 27.5 & 634 & 645 & 62 & 2,800 \\
\hline & $03-02-93$ & 30.0 & 637 & 637 & 60 & 2,700 \\
\hline & $03-24-93$ & 25.0 & 514 & 513 & 42 & 1,900 \\
\hline & $04-13-93$ & 24.5 & 547 & 555 & 51 & 2,200 \\
\hline & $05-20-93$ & 18.0 & 285 & 288 & 21 & 1,000 \\
\hline & $06-15-93$ & - & 238 & 237 & 15 & 820 \\
\hline & $07-14-93$ & 21.0 & 258 & 269 & 15 & 1,000 \\
\hline & $08-19-93$ & 30.5 & 445 & 443 & 38 & 1,600 \\
\hline & $09-08-93$ & 30.5 & 435 & 447 & 36 & 1,700 \\
\hline & $10-14-93$ & 27.0 & 504 & 508 & 41 & 2,000 \\
\hline & $11-18-93$ & 27.0 & 553 & 559 & 49 & 2,300 \\
\hline & $12-14-93$ & 26.5 & 549 & 558 & 46 & 2,300 \\
\hline \multirow[t]{10}{*}{ FHCD } & $10-19-89$ & 15.0 & 232 & - & - & - \\
\hline & $02-19-92$ & 16.0 & 253 & 249 & 7.8 & 270 \\
\hline & $03-12-92$ & 15.5 & 258 & 251 & 7.2 & 300 \\
\hline & $05-13-92$ & 16.5 & 252 & 254 & 7.4 & 320 \\
\hline & $05-27-92$ & 16.0 & 253 & 260 & 8.9 & - \\
\hline & $06-09-92$ & 16.0 & 253 & 257 & 7.8 & 350 \\
\hline & $08-04-92$ & 16.0 & 244 & 250 & 7.3 & 280 \\
\hline & $09-15-92$ & 16.0 & 244 & 250 & 6.9 & 280 \\
\hline & $11-17-92$ & 15.5 & 251 & 251 & 6.1 & 290 \\
\hline & $12-16-92$ & 15.5 & 250 & 260 & 6.3 & 310 \\
\hline
\end{tabular}


Table 114. Partial chemical analyses of water samples collected at selected surface-water sites and springs, Long Valley Caldera, Mono County, California, 1987-93-Continued

\begin{tabular}{|c|c|c|c|c|c|c|}
\hline $\begin{array}{l}\text { Station } \\
\text { identifier }\end{array}$ & Date & $\begin{array}{c}\text { Temperature } \\
\text { water } \\
\left({ }^{\circ} \mathrm{C}\right)\end{array}$ & $\begin{array}{c}\text { Specific } \\
\text { conductance } \\
(\mu \mathrm{S} / \mathrm{cm})\end{array}$ & $\begin{array}{c}\text { Specific } \\
\text { conductance } \\
\text { lab } \\
(\mu \mathrm{S} / \mathrm{cm})\end{array}$ & $\begin{array}{l}\text { Chloride, } \\
\text { dissolved } \\
(\mathrm{mg} / \mathrm{L})\end{array}$ & $\begin{array}{l}\text { Boron, } \\
\text { dissolved } \\
(\mu \mathrm{g} / \mathrm{L})\end{array}$ \\
\hline \multirow{8}{*}{ FHCD } & $02-23-93$ & 15.0 & 257 & 260 & 6.0 & 290 \\
\hline & $03-24-93$ & 15.5 & 265 & 268 & 8.8 & 300 \\
\hline & $05-19-93$ & 16.5 & 249 & 258 & 6.0 & 290 \\
\hline & $06-15-93$ & 16.0 & 242 & 246 & 5.3 & 270 \\
\hline & $08-18-93$ & 15.5 & 230 & 231 & 3.7 & 200 \\
\hline & $09-08-93$ & 15.0 & 223 & 233 & 4.1 & 200 \\
\hline & $11-17-93$ & 15.5 & 240 & 235 & 3.6 & 230 \\
\hline & $12-14-93$ & 15.0 & 239 & 246 & 4.0 & 240 \\
\hline \multirow[t]{22}{*}{ FHAB } & $04-21-87$ & - & - & 267 & 8.8 & 340 \\
\hline & $05-27-87$ & 15.0 & 274 & 274 & 10 & 410 \\
\hline & $07-31-87$ & 17.5 & 251 & 249 & 7.7 & 320 \\
\hline & $03-02-88$ & 16.5 & 266 & 272 & 7.4 & 390 \\
\hline & $12-13-90$ & 17.5 & - & - & - & - \\
\hline & $02-19-92$ & 17.5 & - & 281 & 15 & 580 \\
\hline & $03-10-92$ & 17.5 & 282 & 284 & 14 & 610 \\
\hline & $05-13-92$ & 17.5 & 282 & 286 & 15 & 610 \\
\hline & $05-27-92$ & 17.0 & 280 & 288 & 16 & - \\
\hline & $06-09-92$ & 17.0 & 270 & 276 & 18 & 680 \\
\hline & $08-04-92$ & 18.0 & 278 & 285 & 18 & 660 \\
\hline & $09-15-92$ & 18.0 & 283 & 291 & 15 & 650 \\
\hline & $11-17-92$ & 18.0 & 298 & 300 & 14 & 640 \\
\hline & $12-16-92$ & 17.5 & 289 & 293 & 14 & 630 \\
\hline & $02-23-93$ & 17.0 & 285 & 288 & 13 & 600 \\
\hline & $03-24-93$ & 17.5 & 285 & 287 & 13 & 590 \\
\hline & $05-19-93$ & 17.0 & 279 & 287 & 14 & 630 \\
\hline & $06-15-93$ & 18.0 & 261 & 260 & 11 & 580 \\
\hline & $08-18-93$ & 17.0 & 231 & 234 & 7.3 & 380 \\
\hline & $09-08-93$ & 17.0 & 225 & 233 & 7.4 & 400 \\
\hline & $11-17-93$ & 18.0 & 245 & 249 & 7.3 & 430 \\
\hline & $12-14-93$ & 17.5 & 249 & 255 & 8.6 & 440 \\
\hline \multirow[t]{5}{*}{ FH23 } & $04-21-87$ & - & - & 188 & 4.5 & 80 \\
\hline & $05-27-87$ & 11.0 & 172 & 189 & 4.3 & 90 \\
\hline & $06-18-87$ & 11.0 & 179 & 182 & 5.0 & 100 \\
\hline & $07-31-87$ & 11.0 & 181 & 182 & 4.4 & 80 \\
\hline & $03-02-88$ & 11.0 & 183 & 187 & 4.3 & 90 \\
\hline
\end{tabular}


Table 114. Partial chemical analyses of water samples collected at selected surface-water sites and springs, Long Valley Caldera, Mono County, California, 1987-93-Continued

\begin{tabular}{|c|c|c|c|c|c|c|}
\hline $\begin{array}{c}\text { Station } \\
\text { identifier }\end{array}$ & Date & $\begin{array}{c}\text { Temperature } \\
\text { water } \\
\left({ }^{\circ} \mathrm{C}\right)\end{array}$ & $\begin{array}{c}\text { Specific } \\
\text { conductance } \\
(\mu \mathrm{S} / \mathrm{cm})\end{array}$ & $\begin{array}{c}\text { Specific } \\
\text { conductance } \\
\text { lab } \\
(\mu \mathrm{S} / \mathrm{cm})\end{array}$ & $\begin{array}{l}\text { Chloride, } \\
\text { dissolved } \\
(\mathrm{mg} / \mathrm{L})\end{array}$ & $\begin{array}{c}\text { Boron, } \\
\text { dissolved } \\
(\mu \mathrm{g} / \mathrm{L})\end{array}$ \\
\hline \multirow[t]{9}{*}{ HC-5L } & $01-17-91$ & 92.0 & - & - & - & - \\
\hline & $04-17-91$ & 91.5 & - & 1,730 & 210 & 10,000 \\
\hline & $07-24-91$ & 92.5 & - & 1,730 & 220 & 10,000 \\
\hline & $10-23-91$ & 95.0 & 1,710 & 1,740 & 220 & 10,000 \\
\hline & $01-14-92$ & 94.0 & 1,710 & 1,730 & 210 & 9,700 \\
\hline & $04-16-92$ & 91.0 & 1,810 & 1,710 & 240 & 10,000 \\
\hline & $10-21-92$ & 93.5 & 1,740 & 1,730 & 220 & 9,800 \\
\hline & $07-14-93$ & 92.5 & 1,680 & 1,720 & 200 & 11,000 \\
\hline & $10-14-93$ & 91.0 & 1,770 & 1,720 & 200 & 9,700 \\
\hline \multirow[t]{5}{*}{$\mathrm{HC}-4$} & $10-21-92$ & 93.5 & 1,800 & 1,780 & 220 & 1,000 \\
\hline & $01-27-93$ & 92.5 & 1,730 & 1,760 & 210 & 10,000 \\
\hline & $04-13-93$ & - & 1,810 & 1,780 & 220 & 10,000 \\
\hline & $07-14-93$ & 92.0 & 1,660 & 1,710 & 200 & 10,000 \\
\hline & $10-14-93$ & 89.0 & 1,750 & 1,700 & 190 & 9,500 \\
\hline \multirow[t]{15}{*}{$\mathrm{HIC}-3$} & $02-10-87$ & 90.0 & 1,710 & 1,810 & 230 & 11,000 \\
\hline & $07-28-87$ & - & - & 1,760 & 230 & 9,900 \\
\hline & $03-03-88$ & 90.0 & - & 1,810 & 220 & 11,000 \\
\hline & $04-20-88$ & 91.5 & - & 1,780 & 220 & 9,800 \\
\hline & $10-19-88$ & 94.0 & - & 1,770 & 210 & 10,000 \\
\hline & $01-09-89$ & 94.0 & - & 1,760 & 210 & 9,600 \\
\hline & $04-12-89$ & 94.0 & - & 1,770 & 220 & 10,000 \\
\hline & $10-17-89$ & 95.0 & - & 1,820 & 210 & 10,000 \\
\hline & $01-10-90$ & 94.0 & - & 1,820 & 210 & 9,600 \\
\hline & $04-24-90$ & 91.5 & - & 1,770 & 230 & 10,000 \\
\hline & $07-18-90$ & - & - & - & - & - \\
\hline & $10-17-90$ & 93.0 & - & 1,750 & 220 & 10,000 \\
\hline & $01-17-91$ & 94.0 & - & 1,760 & 210 & 9,900 \\
\hline & $04-17-91$ & 89.0 & - & 1,790 & 210 & 10,000 \\
\hline & $07-24-91$ & 90.0 & - & 1,790 & 220 & 9,500 \\
\hline
\end{tabular}




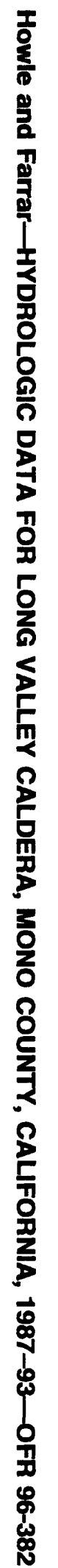

